\title{
From the Vlasov-Maxwell-Boltzmann system to incompressible viscous electro-magneto-hydrodynamics
}

\author{
Diogo Arsénio \\ Laure Saint-Raymond
}

\begin{abstract}
CNRS \& Université Paris Diderot, Institut de Mathématiques de Jussieu - Paris Rive Gauche, Bâtiment Sophie Germain, 8 place Aurélie Nemours, 75205 Paris Cedex 13, France

E-mail address: Diogo.Arsenio@imj-prg.fr

Université Pierre et Marie Curie \& Ecole Normale Supérieure, Département de Mathématiques et Applications, 45 rue D’Ulm, 75230 Paris Cedex 05, France

E-mail address: Laure.Saint-Raymond@ens.fr
\end{abstract}


Key words and phrases. Boltzmann equation, kinetic theory, hydrodynamic limits, Maxwell's equations, MHD, Vlasov-Maxwell-Boltzmann equations, Navier-Stokes equations, plasma physics, non-equilibrium statistical mechanics 


\section{Contents}

Preface vii

Part 1. Formal derivations and macroscopic weak stability 1

Chapter 1. The Vlasov-Maxwell-Boltzmann system 3

1.1. The Boltzmann collision operator

1.2. Formal macroscopic properties 7

1.3. The mathematical framework

Chapter 2. Scalings and formal limits 13

2.1. Incompressible viscous regimes 13

2.2. Scalings for the electromagnetic field

2.3. Formal analysis of the one species asymptotics 17

2.3.1. Thermodynamic equilibrium 18

2.3.2. Macroscopic constraints 20

2.3.3. Evolution equations 21

2.3.4. Summary 25

2.3.5. The Vlasov-Poisson-Boltzmann system 28

2.4. Formal analysis of the two species asymptotics 29

2.4.1. Thermodynamic equilibrium 30

2.4.2. The case of very weak interspecies collisions 35

2.4.3. Macroscopic hydrodynamic constraints 42

2.4.4. Hydrodynamic evolution equations 43

2.4.5. Macroscopic electrodynamic constraints and evolution 45

2.4.6. Summary 50]

2.4.7. The two species Vlasov-Poisson-Boltzmann system 53

Chapter 3. Weak stability of the limiting macroscopic systems 57

3.1. The incompressible quasi-static Navier-Stokes-Fourier-Maxwell-
Poisson system

3.2. The two-fluid incompressible Navier-Stokes-Fourier-Maxwell system with (solenoidal) Ohm's law

3.2.1. Large global solutions in two dimensions 63

3.2.2. Small global solutions in three dimensions 64

3.2.3. Weak-strong stability and dissipative solutions 65

3.2.3.1. The incompressible Navier-Stokes-Maxwell system 66

3.2.3.2. The two-fluid incompressible Navier-Stokes-Maxwell system with

Ohm's law

3.2.3.3. The two-fluid incompressible Navier-Stokes-Maxwell system with solenoidal Ohm's law

Part 2. Conditional convergence results

Chapter 4. Two typical regimes 
4.1. Renormalized solutions $\quad 96$

4.1.1. The Vlasov-Boltzmann equation 96

4.1.2. Coupling the Boltzmann equation with Maxwell's equations

4.1.3. The setting of our conditional study 103

4.1.4. Macroscopic conservation laws 105

4.2. The incompressible quasi-static Navier-Stokes-Fourier-MaxwellPoisson system

4.3. The two-fluid incompressible Navier-Stokes-Fourier-Maxwell system with (solenoidal) Ohm's law

4.3.1. Weak interactions

4.3.2. Strong interactions 117

4.4. Outline of proofs 120

Chapter 5. Weak compactness and relaxation estimates 123

5.1. Controls from the relative entropy bound 123

5.2. Controls from the entropy dissipation bound 126

5.3. Relaxation towards thermodynamic equilibrium 128

5.3.1. Infinitesimal Maxwellians 132

5.3.2. Bulk velocity and temperature 133

5.4. Improved integrability in velocity 137

Chapter 6. Lower order linear constraint equations and energy inequalities 143

6.1. Macroscopic constraint equations for one species 143

6.2. Macroscopic constraint equations for two species, weak interactions $\square$

6.3. Energy inequalities 148

6.4. The limiting Maxwell's equations 153

Chapter 7. Strong compactness and hypoellipticity 157

7.1. Compactness with respect to $v \quad 158$

7.1.1. Compactness of the gain term 158

7.1.2. Relative entropy, entropy dissipation and strong compactness 160

7.2. Compactness with respect to $x \quad 164$

7.2.1. Hypoellipticity and the transfer of compactness 164

7.2.2. Compactness of fluctuations for one species 167

7.2.3. Compactness of fluctuations for two species 173

Chapter 8. Higher order and nonlinear constraint equations 181

8.1. Macroscopic constraint equations for two species, weak interactions 181

8.1.1. Proof of Proposition 8.1 183

8.1.1.1. An admissible renormalization 183

8.1.1.2. Convergence of collision integrals 184

8.1.1.3. Decomposition of flux terms 188

8.1.1.4. Decomposition of acceleration terms 190

8.1.1.5. Convergence 192

8.2. Macroscopic constraint equations for two species, strong interactions 192

8.3. Energy inequalities 200

Chapter 9. Approximate macroscopic equations 205

9.1. Approximate conservation of mass, momentum and energy for one species

9.1.1. Conservation defects

9.1.2. Decomposition of flux terms 211

9.1.3. Decomposition of acceleration terms 215 
9.2. Approximate conservation of mass, momentum and energy for two species

9.2.1. Conservation defects

\begin{tabular}{l} 
9.2.2. Decomposition of flux terms 226 \\
\hline
\end{tabular}

9.2.3. Decomposition of acceleration terms 228

9.2.4. Proof of Proposition 9.6 230

9.2.5. Proofs of Lemmas 9.7, 9.8, 9.9 and 9.10 232

Chapter 10. Acoustic and electromagnetic waves 243

10.1. Formal filtering of oscillations 244

10.2. Rigorous filtering of oscillations 247

Chapter 11. Grad's moment method 251

11.1. Proof of Theorem 4.4 251

11.1.1. Weak convergence of fluctuations, collision integrands and electromagnetic fields $\quad 251$

11.1.2. Constraint equations, Maxwell's system and energy inequality $\quad 252$

11.1.3. Evolution equations 253

11.1.4. Temporal continuity, initial data and conclusion of proof 255

Chapter 12. The renormalized relative entropy method 257

12.1. The relative entropy method : old and new 257

12.2. Proof of Theorem 4.5 on weak interactions 258

12.2.1. Weak convergence of fluctuations, collision integrands and electromagnetic fields

12.2.2. Constraint equations, Maxwell's system and energy inequality

12.2.4. Convergence and conclusion of proof 276

12.3. Proof of Theorem 4.6 on strong interactions 276

12.3.1. Weak convergence of fluctuations, collision integrands and electromagnetic fields

12.3.2. Constraint equations, Maxwell's system and energy inequality

$\begin{array}{lll}\text { 12.3.3. } & \text { The renormalized modulated entropy inequality } & 279 \\ \text { 12.3.4. } & \text { Convergence and conclusion of proof } & 295\end{array}$

Part 3. Unconditional convergence results : long-range interactions 297

Part 4. Unconditional convergence results : short-range interactions

Appendix A. Cross-section for momentum and energy transfer

Appendix B. Young inequalitites

Appendix C. End of proof of Lemma 7.7 on hypoelliptic transfer of compactness 



\section{Preface}

The present book aims at presenting in a systematic, painstaking and rather exhaustive way the incompressible viscous fluid limits of the Vlasov-Maxwell-Boltzmann system for one or two species. In these regimes, the evolution of the fluid is governed by equations of Navier-Stokes-Fourier type, with some electromagnetic forcing. Depending on the precise scaling, this forcing term takes on various forms : it may be linear or nonlinear, electrostatic or governed by some hyperbolic wave equations, possibly constrained by some relation of Ohm's type.

From the mathematical point of view, these models have very different behaviors ; in particular, the existence and stability of solutions require sometimes very weak notions of solutions. The asymptotic analysis, which consists most often in retrieving the structure of the limiting system in the scaled Vlasov-MaxwellBoltzmann system, uses therefore various mathematical methods with important technical refinements. Thus, in order to make the reading easier, different tools will be presented in separated chapters.

The first part of this work is devoted to the systematic formal analysis of viscous hydrodynamic limits. Chapter 1 introduces the Vlasov-Maxwell-Boltzmann system as well as its formal properties. An important point to be noted is that the a priori bounds coming from these physical laws are not enough to prove the existence of global solutions, even in the renormalized sense of DiPerna and Lions [30, which is a major difficulty for the study of fast relaxation limits. This actually explains the dividing of the three other parts of this book, of increasing difficulty, giving rigorous convergence results in more and more general settings.

Chapter 2 introduces the different scaling parameters arising in the system, and details the formal steps leading to the constraint relations and the evolution equations in each regime. We therefore obtain a rather precise classification of physically relevant models for viscous incompressible plasmas, some of which actually do not seem to have been previously described in the literature.

Chapter 3 presents a mathematical analysis of these different models. The most singular of them have a behavior which is actually more similar to the incompressible Euler equations than to the Navier-Stokes equations : the lack of weak stability does not allow to prove the existence of global solutions, with the exception of very weak solutions in the spirit of dissipative solutions introduced by Lions for the Euler equations [57. This lack of stability for limiting systems is the second major difficulty for the study of hydrodynamic limits.

The goal of the second part is to make precise and rigorous the convergence results described formally in the first part. In order to isolate the difficulties which are specific to the asymptotic analysis, we choose here to prove first conditional results, i.e. to consider the convergence of renormalized solutions even though their existence is not known. This of course does not imply the convergence of weaker solutions which will be studied in the sequel (renormalized solutions with defect measure, and a fortiori solutions with Young measures), but most of the proof will 
remain unchanged. The important point is that the analysis is based essentially on the uniform estimates coming from the scaled entropy inequality, which holds in all situations.

Furthermore, we will focus exclusively on two typical regimes, namely leading :

- from the one species Vlasov-Maxwell-Boltzmann equations to the incompressible quasi-static Navier-Stokes-Fourier-Maxwell-Poisson system ;

- from the two species Vlasov-Maxwell-Boltzmann equations to the twofluid incompressible Navier-Stokes-Fourier-Maxwell system with Ohm's law in the case of strong interspecies collisions, or to the two-fluid incompressible Navier-Stokes-Fourier-Maxwell system with solenoidal Ohm's law in the case of weak interspecies collisions.

These asymptotic regimes are critical, in the sense that they are the most singular ones among the formal asymptotics mentioned in Chapter 2 and that all remaining regimes can be treated rigorously by similar or even simpler arguments.

We will not detail in this preface the content of all chapters of the second part, but rather insist on the main points requiring a treatment different from usual hydrodynamic limits 68. In the case with only one species, the main difference is due to the fact that the transport equation contains force terms involving a derivative with respect to $v$, which does not allow to transfer equi-integrability from the $v$-variable to the $x$-variable as in [37. This is a major complication. The new idea here consists in getting first some strong compactness in $v$ by using regularizing properties of the gain operator 51 and, then, in transferring this strong compactness onto the spatial variable by means of refined hypoelliptic arguments developed in $\mathbf{6}$. The second important difference comes from the fast temporal oscillations which couple acoustic and electromagnetic modes. Note that we introduce here a simple method to avoid dealing with non local projections.

Overall, we are eventually able to establish through weak compactness methods a very general result (Theorem 4.4) asserting the convergence of renormalized solutions of the one species Vlasov-Maxwell-Boltzmann system towards weak solutions of corresponding macroscopic systems.

In the case of two species, the situation not only requires to exploit the methods for one species, it is considerably more complex :

- First of all, there is an additional scaling parameter measuring the strength of interspecies interactions (and, incidentally, the typical size of the electric current, which can be much smaller than the bulk velocities of each of the two species of particles) : this implies that the (formal) expansions involve a larger number of terms (for instance, the constraint equations are derived at different orders).

- Secondly, the linearized collision operator has a more complicated vectorial structure. The inversion of fluxes and the computation of dissipation terms in the limiting energy inequalities are therefore substantially more technical.

- In the most singular regimes, we get nonlinear constraint equations. This means that renormalization methods, compensated compactness techniques and controls on the conservation defects are already required at this stage of the proof.

- We have no sufficient uniform a priori bound on the electric current to handle nonlinear terms, which prevents from taking limits in the approximate conservation of momentum law. To avoid this difficulty we need to introduce a modified conservation law involving the Poynting vector. 
- Even in this more suitable form, the evolution equations are not stable under weak convergence, and we have no equi-integrability in these singular regimes. We develop therefore some improved modulated entropy method, which allows to consider renormalized solutions without important restriction on the initial data. Note that this renormalized modulated entropy method should also lead to some improvements concerning the convergence of the Boltzmann equation (without any electromagnetic field) to the Navier-Stokes equations for ill-prepared initial data.

The third and fourth parts (which will be published in a second volume) are more technical. They show how to adapt the arguments presented in the conditional case of the second part to take into account the state of the art about the Cauchy theory for the Vlasov-Maxwell-Boltzmann system.

In the case of long-range microscopic interactions giving rise to a collision crosssection with a singularity for grazing collisions, treated in the third part, we start by proving the existence of renormalized solutions with a defect measure in the spirit of the construction by Alexandre and Villani 1. This result, which is important independently of the study of hydrodynamic limits, has been addressed in the note [7. The study of hydrodynamic limits follows then essentially the lines of [4] (combined with the results of the conditional part). We would like however to mention some important contributions :

- The first one concerns the estimate of the defect measure. A refined analysis of the convergence of approximate solutions to the VlasovMaxwell-Boltzmann system shows that the defect measure can be controlled by the entropy dissipation. This remark allows for a simplification of the proofs from [4, especially the passage to the limit in the kinetic equation leading to the characterization of the limiting form of the dissipation, and the control of conservation defects.

- The other simplification is related to the renormalization process. Here we choose a decomposition of the renormalized collision operator which allows both to control the singularity due to the collision cross-section, and to preserve the good scalings for the fluctuation. In particular, the same decomposition can be used for the control of the transport and of the conservation defects (with a loop estimate).

In the case of general microscopic interactions (including for instance the case of hard spheres), it is not known how to prove the convergence of approximation schemes of the Vlasov-Maxwell-Boltzmann system, due to a lack of compactness produced by the electromagnetic interaction. The existence of renormalized solutions is therefore still an open problem. Nevertheless, Lions [53 has defined a very weak notion of solution - the measure-valued renormalized solutions - defined as limit of approximate solutions : the equation to be satisfied involves indeed Young measures.

In the fourth part, we begin by refining the control of Young measures for such solutions by the entropy inequality. We then proceed by showing that the estimates obtained in the second part are very stable, so that they can be generalized with Young measures. By using convexity properties and Jensen inequalities, we can extend all the arguments, and operate both the moment method and the entropy method in more singular regimes. This extension to solutions of the VlasovMaxwell-Boltzmann system defined in a very weak sense shows that the methods 
based on the entropy inequality are extremely robust, and that the convergence is essentially determined by the limiting system.

These good asymptotic properties seem to further indicate that the measurevalued solutions defined by Lions (which have never been really studied from the qualitative point of view) are relevant in some sense.

Paris, France,

Diogo Arsénio \& Laure Saint-Raymond

January 2016 
Part 1

Formal derivations and macroscopic weak stability 



\section{CHAPTER 1}

\section{The Vlasov-Maxwell-Boltzmann system}

In the present monograph, we intend to investigate in a rather systematic way the scaling limits of the Vlasov-Maxwell-Boltzmann system

$$
\left\{\begin{array}{rlrl}
\partial_{t} f+v \cdot \nabla_{x} f+\frac{q}{m}(E+v \wedge B) \cdot \nabla_{v} f & =Q(f, f), & & \\
\mu_{0} \epsilon_{0} \partial_{t} E-\operatorname{rot} B & =-\mu_{0} q \int_{\mathbb{R}^{3}} f v d v, & \\
\partial_{t} B+\operatorname{rot} E & =0, & \\
\text { (Vlasov-Boltzmann) } & \text { (Ampère) } \\
\operatorname{div} E & =\frac{q}{\epsilon_{0}}\left(\int_{\mathbb{R}^{3}} f d v-1\right), & \\
\operatorname{div} B & =0, & & \text { (Faraday) } \\
\text { (Gauss) } & \text { (Gauss) }
\end{array}\right.
$$

leading to viscous incompressible magnetohydrodynamics, and to justify rigorously the corresponding asymptotics.

More precisely, the Vlasov-Maxwell-Boltzmann system describes the evolution of a gas of one species of charged particles (cations and anions (or electrons), i.e. positively and negatively charged ions, respectively) of mass $m>0$ and charge $q \in \mathbb{R}$, subject to auto-induced electromagnetic forces. Such a gas of charged particles, under a global neutrality condition, is called a plasma. The particle number density $f(t, x, v) \geq 0$, where $t \in[0, \infty), x \in \Omega \subset \mathbb{R}^{3}$ and $v \in \mathbb{R}^{3}$, represents the distribution of particles which, at time $t$, are at position $x$ and have velocity $v$.

The evolution of the density $f$ is governed by the Vlasov-Boltzmann equation, which is the first line of 11.1 . In essence, it tells that the variation of the density $f$ along the trajectories of the particles (represented by the transport term $\partial_{t} f+$ $\left.v \cdot \nabla_{x} f\right)$ is subject to the influence of a Lorentz force $q(E+v \wedge B)$ (represented by the Vlasov term $\left.\frac{q}{m}(E+v \wedge B) \cdot \nabla_{v} f\right)$ and inter-particle collisions in the gas (represented by the Boltzmann collision operator $Q(f, f)$ ).

The Lorentz force acting on the gas is auto-induced. That is, the electric field $E(t, x)$ and the magnetic field $B(t, x)$ are generated by the motion of the particles in the plasma itself. Their evolution is governed by Maxwell's equations, which are the remaining lines of (1.1), namely Ampère's equation, Faraday's equation and Gauss' laws. Here, the physical constants $\mu_{0}, \epsilon_{0}>0$ are, respectively, the vacuum permeability (or magnetic constant) and the vacuum permittivity (or electric constant). Recall that the speed of light is determined by the formula $c=\frac{1}{\sqrt{\mu_{0} \epsilon_{0}}}$. 

$(1.2)$

We will also consider the two species Vlasov-Maxwell-Boltzmann system

$$
\left\{\begin{aligned}
\partial_{t} f^{+}+v \cdot \nabla_{x} f^{+}+\frac{q^{+}}{m^{+}}(E+v \wedge B) \cdot \nabla_{v} f^{+} & =Q\left(f^{+}, f^{+}\right)+Q\left(f^{+}, f^{-}\right), \\
& \text {(Vlasov-Boltzmann for cations) } \\
\partial_{t} f^{-}+v \cdot \nabla_{x} f^{-}-\frac{q^{-}}{m^{-}}(E+v \wedge B) \cdot \nabla_{v} f^{-} & =Q\left(f^{-}, f^{-}\right)+Q\left(f^{-}, f^{+}\right), \\
& (\text {Vlasov-Boltzmann for anions) } \\
\mu_{0} \epsilon_{0} \partial_{t} E-\operatorname{rot} B= & -\mu_{0} \int_{\mathbb{R}^{3}}\left(q^{+} f^{+}-q^{-} f^{-}\right) v d v, \\
\partial_{t} B+\operatorname{rot} E=0, & \text { (Ampère) } \\
\operatorname{div} E=\frac{1}{\epsilon_{0}} \int_{\mathbb{R}^{3}}\left(q^{+} f^{+}-q^{-} f^{-}\right) d v, & \text { (Faraday) } \\
\operatorname{div} B=0, & \text { (Gauss) }
\end{aligned}\right.
$$

which is more physically accurate, since it describes the evolution of a gas of two species of oppositely charged particles (cations of charge $q^{+}>0$ and mass $m^{+}>0$, and anions of charge $-q^{-}<0$ and $m^{-}>0$ ), subject to auto-induced electromagnetic forces.

Thus, the particle number density $f^{+}(t, x, v) \geq 0$ represents the distribution of the positively charged ions (i.e. cations), while the particle number density $f^{-}(t, x, v) \geq 0$ represents the distribution of the negatively charged ions (i.e. anions). Note that the collision operators $Q\left(f^{+}, f^{-}\right)$and $Q\left(f^{-}, f^{+}\right)$have been added to the right-hand sides of the respective Vlasov-Boltzmann equations in $(1.2)$ in order to account for the variations in the densities $f^{+}$and $f^{-}$due to interspecies collisions.

We refer to 62 for a discussion of the validity of such systems from a physical viewpoint.

Henceforth, for the mere sake of mathematical convenience, we will make the simplification that both kinds of particles have the exact same mass $m^{ \pm}=m>0$ and charge $q^{ \pm}=q>0$. Even though this reduction may first appear rather unphysical, it remains nevertheless a reasonable approximation since the mass of cations and anions only differs by the mass of a few electrons, which is several orders of magnitude less than that of atomic nuclei. Anyway, we believe that the essential mathematical difficulties are contained in this case, and we expect that most of the analysis contained in this work carries over to the case of distinct masses, as long as they remain of a comparable order of magnitude. We refer to [46] for an independent formal study of some hydrodynamic limits of the two species Vlasov-Maxwell-Boltzmann system including the case of unequal masses, leading in particular to a formal justification of the Hall effect, which we will not address here.

The mathematical framework we shall consider is the one defined by physical a priori estimates, namely entropy and energy bounds, which corresponds to renormalized or even weaker solutions of the Vlasov-Maxwell-Boltzmann systems. For the sake of simplicity, we will consider, throughout this work, that the spatial domain is, in fact, the whole space $\Omega=\mathbb{R}^{3}$, thus avoiding the complicated discussion of boundary conditions. 
The general strategy that will be used to study magnetohydrodynamic limits is therefore based on uniform a priori bounds, weak compactness and either the moment method of Grad, or some generalized relative entropy method, which are the only way to deal with very weak solutions : strong convergence requires indeed - at least - that local conservation laws are satisfied.

\subsection{The Boltzmann collision operator}

The Boltzmann collision operator, present in the right-hand side of the VlasovBoltzmann equations in 1.1 and 1.2 , is the quadratic form, acting on the velocity variable, associated to the bilinear operator

$$
Q(f, h)=\int_{\mathbb{R}^{3}} \int_{\mathbb{S}^{2}}\left(f^{\prime} h_{*}^{\prime}-f h_{*}\right) b\left(v-v_{*}, \sigma\right) d \sigma d v_{*},
$$

where we have used the standard abbreviations

$$
f=f(v), \quad f^{\prime}=f\left(v^{\prime}\right), \quad h_{*}=h\left(v_{*}\right), \quad h_{*}^{\prime}=h\left(v_{*}^{\prime}\right),
$$

with $\left(v^{\prime}, v_{*}^{\prime}\right)$ given by

$$
v^{\prime}=\frac{v+v_{*}}{2}+\frac{\left|v-v_{*}\right|}{2} \sigma, \quad v_{*}^{\prime}=\frac{v+v_{*}}{2}-\frac{\left|v-v_{*}\right|}{2} \sigma .
$$

One can easily show that the quadruple $\left(v, v_{*}, v^{\prime}, v_{*}^{\prime}\right)$ parametrized by $\sigma \in \mathbb{S}^{2}$ provides the family of all solutions to the system of four equations

$$
\begin{aligned}
v+v_{*} & =v^{\prime}+v_{*}^{\prime}, \\
|v|^{2}+\left|v_{*}\right|^{2} & =\left|v^{\prime}\right|^{2}+\left|v_{*}^{\prime}\right|^{2} .
\end{aligned}
$$

At the kinetic level, these relations express the fact that interparticle collisions are assumed to be elastic and thus conserve momentum and energy, where $\left(v, v_{*}\right)$ denote the pre-collisional velocities and $\left(v^{\prime}, v_{*}^{\prime}\right)$ denote the post-collisional velocities of two interacting particles. Notice that the transformation $\left(v, v_{*}, \sigma\right) \mapsto\left(v^{\prime}, v_{*}^{\prime}, \frac{v-v_{*}}{\left|v-v_{*}\right|}\right)$ is involutional.

It is to be emphasized that the definition of the Boltzmann operator for interspecies collisions with distinct masses is more complex. Indeed, in this case, the microscopic conservations of momentum and energy are

$$
\begin{aligned}
m_{+} v_{+}+m_{-} v_{-} & =m_{+} v_{+}^{\prime}+m_{-} v_{-}^{\prime}, \\
m_{+}\left|v_{+}\right|^{2}+m_{-}\left|v_{-}\right|^{2} & =m_{+}\left|v_{+}^{\prime}\right|^{2}+m_{-}\left|v_{-}^{\prime}\right|^{2} .
\end{aligned}
$$

Therefore, the masses must appear in the convolution relations defining the mixed collision operators, which become highly singular whenever the mass ratio tends to infinity or to zero. Again, for mathematical convenience, we will not deal with this case and stick to equal masses.

The Boltzmann collision operator can therefore be split, at least formally, into a gain term and a loss term

$$
\begin{aligned}
Q(f, h) & =Q^{+}(f, h)-Q^{-}(f, h) \\
& =\int_{\mathbb{R}^{3} \times \mathbb{S}^{2}} f^{\prime} h_{*}^{\prime} b d v_{*} d \sigma-\int_{\mathbb{R}^{3} \times \mathbb{S}^{2}} f h_{*} b d v_{*} d \sigma .
\end{aligned}
$$

The loss term counts all collisions in which a given particle of velocity $v$ will encounter another particle, of velocity $v_{*}$, and thus will change its velocity leading to a loss of particles of velocity $v$, whereas the gain term measures the number of particles of velocity $v$ which are created due to some collision between particles of velocities $v^{\prime}$ and $v_{*}^{\prime}$. 
The cross-section $b=b(z, \sigma)$, or collision kernel, where $(z, \sigma) \in \mathbb{R}^{3} \times \mathbb{S}^{2}$, present in the integrand of 1.3 , is a measurable function positive almost everywhere, which somehow measures the statistical repartition of post-collisional velocities $\left(v^{\prime}, v_{*}^{\prime}\right)$ given the pre-collisional velocities $\left(v, v_{*}\right)$. Its precise form depends crucially on the nature of the microscopic interactions, thus, it is determined by the intermolecular forces that are being considered. However, due to the Galilean invariance of collisions, it only depends on the magnitude of the relative velocity $|z|$ and on the deviation angle $\theta$, or deflection (scattering) angle, defined by $\cos \theta=k \cdot \sigma$ where $k=\frac{z}{|z|}$. We will therefore sometimes abuse notation and write $b(z, \sigma)=b(|z|, \cos \theta)$ without any confusion since the arguments of $b$ are then either vectors or scalars.

It is a common mathematical simplification, called the cutoff assumption, to suppose that the cross-section is at least locally integrable, i.e. $b(z, \sigma) \in L_{\text {loc }}^{1}\left(\mathbb{R}^{3} \times \mathbb{S}^{2}\right)$. However, this hypothesis fails to hold when long-range interaction forces are present between the particles in the gas. Thus, in this non-cutoff case, the collision kernel is non-integrable. This is due to a strong singularity of the kernel in the angular variable created by the enormous amount of grazing collisions in the gas, i.e. collisions whose deflection angle is almost null.

For instance, if the particles are assumed to interact via a given repulsive potential $\Phi(r)$, where $r>0$ denotes the distance between two interacting particles, then the post-collisional velocities and especially the deviation angle $\theta$ can be computed in terms of the impact parameter $\beta$, i.e. the distance of closest approach if the particles were not to interact, and the relative velocity $z=v-v_{*}$ as the result of a classical scattering problem (see [19] for instance) :

$$
\theta(\beta, z)=\pi-2 \int_{0}^{\frac{\beta}{s_{0}}} \frac{d u}{\sqrt{1-u^{2}-\frac{4}{|z|^{2}} \Phi\left(\frac{\beta}{u}\right)}}
$$

where $s_{0}$ is the positive root of

$$
1-\frac{\beta^{2}}{s_{0}^{2}}-4 \frac{\Phi\left(s_{0}\right)}{|z|^{2}}=0 .
$$

Then the cross-section $b$ is implicitly defined by

$$
b(|z|, \cos \theta)=\frac{\beta}{\sin \theta} \frac{\partial \beta}{\partial \theta}|z| .
$$

It can be made fully explicit in the case of hard spheres

$$
b(|z|, \cos \theta)=a^{2}|z|,
$$

where $a>0$ is the (scaled) radius of the spheres.

As shown by Maxwell, it is possible to obtain a rather explicit expression for a wide class of physically relevant collision kernels (see [74] and references therein), namely the so-called inverse power kernels. This terminology stems from the fact that these kernels model a gas whose particles interact according to an inverse power potential $\Phi(r)=\frac{1}{r^{s-1}}$, where $r>0$ represents the distance between two particles and $s>2$. Maxwell's calculations show that in such a case one has

$$
b(|z|, \cos \theta)=|z|^{\gamma} b_{0}(\cos \theta), \quad \gamma=\frac{s-5}{s-1},
$$

where the angular cross-section $b_{0}(\cos \theta)$ is smooth on $\theta \in(0, \pi)$ and has a nonintegrable singularity at $\theta=0$ behaving as

$$
b(\cos \theta) \sin \theta \sim \frac{1}{\theta^{1+\nu}}, \quad \nu=\frac{2}{s-1},
$$


where the factor $\sin \theta$ accounts for the Jacobian determinant of spherical coordinates. Notice that, in this particular situation, $b(z, \sigma)$ is thus not locally integrable, which is not due to the specific form of inverse power potential. In fact, one can show (see [74]) that a non-integrable singularity arises if and only if forces of infinite range are present in the gas.

The case of Maxwellian molecules $s=5$ corresponds to $\gamma=0$, which is not physically relevant but enables one to perform many explicit calculations in agreement with physical observations. It is customary to loosely classify cross-sections into two categories : hard and soft, respectively corresponding to the super-Maxwellian $(s>5)$ and the sub-Maxwellian cases $(s<5)$. We will however not employ this dichotomy since our hypotheses will allow us to treat all hard and soft kernels in a single unified theory.

It turns out that the limiting case $s=2$, which corresponds to Coulombian interactions, is not well suited for Boltzmann's equation as the Boltzmann collision operator should be replaced by the Landau operator in order to handle that situation (see [74]). The other limiting case $s=\infty$ corresponds formally to the hard spheres case.

\subsection{Formal macroscopic properties}

Using the well-known facts (see [20]) that transforming $\left(v, v_{*}, \sigma\right) \mapsto\left(v_{*}, v,-\sigma\right)$ and $\left(v, v_{*}, \sigma\right) \mapsto\left(v^{\prime}, v_{*}^{\prime}, \frac{v-v_{*}}{\left|v-v_{*}\right|}\right)$ merely induces mappings with unit Jacobian determinants, known as the pre-post-collisional changes of variables or simply collisional symmetries, one can show that

$$
\begin{aligned}
& \int_{\mathbb{R}^{3}} Q(f, f)(v) \varphi(v) d v \\
& =\frac{1}{4} \int_{\mathbb{R}^{3} \times \mathbb{R}^{3} \times \mathbb{S}^{2}}\left(f^{\prime} f_{*}^{\prime}-f f_{*}\right) b\left(v-v_{*}, \sigma\right)\left(\varphi+\varphi_{*}-\varphi^{\prime}-\varphi_{*}^{\prime}\right) d v d v_{*} d \sigma
\end{aligned}
$$

for all $f(v)$ and $\varphi(v)$ regular enough. It then follows from 1.4 that the above integral vanishes if and only if $\varphi(v)$ is a collision invariant, i.e. any linear combination of $\left\{1, v_{1}, v_{2}, v_{3},|v|^{2}\right\}$.

Thus, successively multiplying the Vlasov-Boltzmann equation in (1.1) by the collision invariants and then integrating in velocity yields formally the local conservation laws

$$
\partial_{t} \int_{\mathbb{R}^{3}} f\left(\begin{array}{c}
1 \\
v \\
\frac{|v|^{2}}{2}
\end{array}\right) d v+\nabla_{x} \cdot \int_{\mathbb{R}^{3}} f\left(\begin{array}{c}
v \\
v \otimes v \\
\frac{|v|^{2}}{2} v
\end{array}\right) d v=\frac{q}{m} \int_{\mathbb{R}^{3}} f\left(\begin{array}{c}
0 \\
E+v \wedge B \\
E \cdot v
\end{array}\right) d v
$$

which provide the link to a macroscopic description of the gas.

In the case of two species 1.2 , we obtain (recall that we are assuming equal masses $m^{ \pm}=m$ and charges $q^{ \pm}=q$ )

$$
\partial_{t} \int_{\mathbb{R}^{3}} f^{ \pm} d v+\nabla_{x} \cdot \int_{\mathbb{R}^{3}} f^{ \pm} v d v=0
$$

and

$$
\begin{array}{r}
\partial_{t} \int_{\mathbb{R}^{3}}\left(f^{+}+f^{-}\right)\left(\begin{array}{c}
v \\
\frac{|v|^{2}}{2}
\end{array}\right) d v+\nabla_{x} \cdot \int_{\mathbb{R}^{3}}\left(f^{+}+f^{-}\right)\left(\begin{array}{c}
v \otimes v \\
\frac{|v|^{2}}{2} v
\end{array}\right) d v \\
=\frac{q}{m} \int_{\mathbb{R}^{3}}\left(f^{+}-f^{-}\right)\left(\begin{array}{c}
E+v \wedge B \\
E \cdot v
\end{array}\right) d v
\end{array}
$$

On the other hand, the standard energy estimates for Maxwell's system in 1.1 and (1.2) (we refer to [45] for more details on Maxwell's equations) are obtained, 
first, by taking the scalar product of the Ampère and Faraday equations with $E$ and $B$, respectively, and summing the resulting quantities, which yields the conservation laws for one species

$$
\partial_{t}\left(\frac{\mu_{0} \epsilon_{0}|E|^{2}+|B|^{2}}{2}\right)+\nabla_{x} \cdot(E \wedge B)=-\mu_{0} q \int_{\mathbb{R}^{3}} f E \cdot v d v
$$

and for two species

$$
\partial_{t}\left(\frac{\mu_{0} \epsilon_{0}|E|^{2}+|B|^{2}}{2}\right)+\nabla_{x} \cdot(E \wedge B)=-\mu_{0} q \int_{\mathbb{R}^{3}}\left(f^{+}-f^{-}\right) E \cdot v d v .
$$

Second, by taking the vector product of the Ampère and Faraday equations with $B$ and $E$, respectively, employing Gauss' laws when necessary and summing the resulting quantities, which yields the conservation laws for one species

$$
\begin{aligned}
\mu_{0} \epsilon_{0} \partial_{t}(E \wedge B)+\nabla_{x}\left(\frac{\mu_{0} \epsilon_{0}|E|^{2}+|B|^{2}}{2}\right) & -\nabla_{x} \cdot\left(\mu_{0} \epsilon_{0} E \otimes E+B \otimes B\right) \\
& =-\mu_{0} q \int_{\mathbb{R}^{3}} f(E+v \wedge B) d v+\mu_{0} q E,
\end{aligned}
$$

and for two species

$$
\begin{aligned}
\mu_{0} \epsilon_{0} \partial_{t}(E \wedge B)+\nabla_{x}\left(\frac{\mu_{0} \epsilon_{0}|E|^{2}+|B|^{2}}{2}\right) & -\nabla_{x} \cdot\left(\mu_{0} \epsilon_{0} E \otimes E+B \otimes B\right) \\
& =-\mu_{0} q \int_{\mathbb{R}^{3}}\left(f^{+}-f^{-}\right)(E+v \wedge B) d v,
\end{aligned}
$$

Notice the similitude of the source terms in $(1.6),(1.9), 1.11$, and in $1.8,1.10$, 1.12 .

The other very important feature of the Boltzmann equation comes also from the symmetries of the collision operator. Without caring about integrability issues, we plug $\varphi=\log f$ into the symmetrized integral 1.5 and use the properties of the logarithm to find

$$
\begin{aligned}
D(f) & \stackrel{\text { def }}{=}-\int_{\mathbb{R}^{3}} Q(f, f) \log f d v \\
& =\frac{1}{4} \int_{\mathbb{R}^{3} \times \mathbb{R}^{3} \times \mathbb{S}^{2}}\left(f^{\prime} f_{*}^{\prime}-f f_{*}\right) \log \left(\frac{f^{\prime} f_{*}^{\prime}}{f f_{*}}\right) b\left(v-v_{*}, \sigma\right) d v d v_{*} d \sigma \geq 0 .
\end{aligned}
$$

The so defined entropy dissipation $\int_{\mathbb{R}^{3}} D(f)(t, x) d x$ is non-negative and the functional $\int_{0}^{t} \int_{\mathbb{R}^{3}} D(f)(s, x) d x d s$ is therefore nondecreasing on $t>0$.

This leads to Boltzmann's $H$-theorem, also known as the second principle of thermodynamics, stating that the entropy

$$
\int_{\mathbb{R}^{3}} f \log f d v
$$

is (at least formally) a Lyapunov functional for the Boltzmann equation. Indeed, formally multiplying the Vlasov-Boltzmann equation in 1.1 by $\log f$ and then integrating in space and velocity clearly leads to

$$
\frac{d}{d t} \int_{\mathbb{R}^{3}} f \log f(t, x, v) d v+\nabla_{x} \cdot \int_{\mathbb{R}^{3}} f \log f(t, x, v) v d v+D(f)(t, x)=0 .
$$


A similar procedure on the two species Vlasov-Boltzmann equations in (1.2) yields

$$
\begin{aligned}
\frac{d}{d t} \int_{\mathbb{R}^{3}} & \left(f^{+} \log f^{+}+f^{-} \log f^{-}\right)(t, x, v) d v \\
& +\nabla_{x} \cdot \int_{\mathbb{R}^{3}}\left(f^{+} \log f^{+}+f^{-} \log f^{-}\right)(t, x, v) v d v \\
& +\left(D\left(f^{+}\right)+D\left(f^{-}\right)+D\left(f^{+}, f^{-}\right)\right)(t, x)=0
\end{aligned}
$$

where we have denoted the mixed entropy dissipation

$$
\begin{aligned}
D(f, h) & \stackrel{\text { def }}{=}-\int_{\mathbb{R}^{3}} Q(f, h) \log f+Q(h, f) \log h d v \\
& =\frac{1}{2} \int_{\mathbb{R}^{3} \times \mathbb{R}^{3} \times \mathbb{S}^{2}}\left(f^{\prime} h_{*}^{\prime}-f h_{*}\right) \log \left(\frac{f^{\prime} h_{*}^{\prime}}{f h_{*}}\right) b\left(v-v_{*}, \sigma\right) d v d v_{*} d \sigma \geq 0 .
\end{aligned}
$$

As for the equation $Q(f, f)=0$, it is possible to show, since necessarily $D(f)=$ 0 in this case, that it is only satisfied by the so-called Maxwellian distributions $M_{R, U, T}$ defined by

$$
M_{R, U, T}(v)=\frac{R}{(2 \pi T)^{\frac{3}{2}}} e^{-\frac{|v-U|^{2}}{2 T}},
$$

where $R \in \mathbb{R}_{+}, U \in \mathbb{R}^{3}$ and $T \in \mathbb{R}_{+}$are respectively the macroscopic density, bulk velocity and temperature, under some appropriate choice of units. The relation $Q(f, f)=0$ expresses the fact that collisions are no longer responsible for any variation in the density and so, that the gas has reached statistical equilibrium. In fact, it is possible to show that if the density $f$ is a Maxwellian distribution for some $R(t, x), U(t, x)$ and $T(t, x)$, then the macroscopic conservation laws 1.6$)$ turn out to constitute a compressible Euler system with electromagnetic forcing terms.

Similarly, for two species of particles, if the plasma reaches thermodynamic equilibrium so that the equations $Q\left(f^{+}, f^{+}\right)+Q\left(f^{+}, f^{-}\right)=0$ and $Q\left(f^{-}, f^{-}\right)+$ $Q\left(f^{-}, f^{+}\right)=0$ are solved simultaneously, then necessarily $D\left(f^{+}\right)+D\left(f^{-}\right)+$ $D\left(f^{+}, f^{-}\right)=0$, which implies that $f^{+}=M_{R^{+}, U^{+}, T^{+}}$and $f^{-}=M_{R^{-}, U^{-}, T^{-}}$with $U^{+}=U^{-}$and $T^{+}=T^{-}$, but not necessarily equal masses. In this case, it is possible to show that the macroscopic system of conservation laws 1.7$)-(1.8)$ constitute a compressible Euler system with electromagnetic forcing terms.

Finally, we define the (global) relative entropy, for any particle number density $f \geq 0$ and any Maxwellian distribution $M_{R, U, T}$, by

$$
H\left(f \mid M_{R, U, T}\right)(t)=\int_{\mathbb{R}^{3} \times \mathbb{R}^{3}}\left(f \log \frac{f}{M_{R, U, T}}-f+M_{R, U, T}\right)(t) d x d v \geq 0 .
$$

We will more simply denote the relative entropy by $H(f)$, whenever the relative Maxwellian distribution is clearly implied. The global control of the relative entropies follows then from the non-negativity of the entropies dissipations. Indeed, combining the $H$-theorem 1.14 with the global conservation of mass and energy from (1.6) and Maxwell's energy conservation (1.9), it is in general possible to establish for one species (see $\mathbf{3 2}$, for instance), further integrating in time and space, by virtue of the convexity properties of the entropies and the entropy dissipations, 
the following weaker relative entropy inequality, for any $t>0$,

$$
\begin{aligned}
\int_{\mathbb{R}^{3} \times \mathbb{R}^{3}} & \left(f \log \frac{f}{M}-f+M\right)(t) d x d v \\
& +\frac{1}{2} \int_{\mathbb{R}^{3}}\left(\frac{\epsilon_{0}}{m}|E|^{2}+\frac{1}{m \mu_{0}}|B|^{2}\right)(t) d x+\int_{0}^{t} \int_{\mathbb{R}^{3}} D(f)(s) d x d s \\
& \leq \int_{\mathbb{R}^{3} \times \mathbb{R}^{3}}\left(f^{\text {in }} \log \frac{f^{\text {in }}}{M}-f^{\text {in }}+M\right) d x d v \\
& +\frac{1}{2} \int_{\mathbb{R}^{3}}\left(\frac{\epsilon_{0}}{m}\left|E^{\text {in }}\right|^{2}+\frac{1}{m \mu_{0}}\left|B^{\text {in }}\right|^{2}\right) d x,
\end{aligned}
$$

where $\left(f^{\text {in }}, E^{\text {in }}, B^{\text {in }}\right)$ denotes the initial data and $M$ denotes a global normalized Maxwellian distribution

$$
M=M_{1,0,1}=\frac{1}{(2 \pi)^{\frac{3}{2}}} e^{-\frac{|v|^{2}}{2}} .
$$

Similarly, for two species, combining the $H$-theorem 1.15 with the global conservation of mass and energy from (1.7)- 1.8 and Maxwell's energy conservation 1.10, we get the entropy inequality, for all $t>0$,

$$
\begin{aligned}
\int_{\mathbb{R}^{3} \times \mathbb{R}^{3}} & \left(f^{+} \log \frac{f^{+}}{M}-f^{+}+M\right)(t)+\left(f^{-} \log \frac{f^{-}}{M}-f^{-}+M\right)(t) d x d v \\
& +\frac{1}{2} \int_{\mathbb{R}^{3}}\left(\frac{\epsilon_{0}}{m}|E|^{2}+\frac{1}{m \mu_{0}}|B|^{2}\right)(t) d x \\
& +\int_{0}^{t} \int_{\mathbb{R}^{3}}\left(D\left(f^{+}\right)+D\left(f^{-}\right)+D\left(f^{+}, f^{-}\right)\right)(s) d x d s \\
& \leq \int_{\mathbb{R}^{3} \times \mathbb{R}^{3}}\left(f^{+\mathrm{in}} \log \frac{f^{+\mathrm{in}}}{M}-f^{+\mathrm{in}}+M\right) d x d v \\
& +\int_{\mathbb{R}^{3} \times \mathbb{R}^{3}}\left(f^{-\mathrm{in}} \log \frac{f^{- \text {in }}}{M}-f^{-\mathrm{in}}+M\right) d x d v \\
& +\frac{1}{2} \int_{\mathbb{R}^{3}}\left(\frac{\epsilon_{0}}{m}\left|E^{\mathrm{in}}\right|^{2}+\frac{1}{m \mu_{0}}\left|B^{\mathrm{in}}\right|^{2}\right) d x,
\end{aligned}
$$

where $\left(f^{+ \text {in }}, f^{- \text {in }}, E^{\text {in }}, B^{\text {in }}\right)$ denotes the initial data.

Generally speaking, the $H$-theorem and the entropy inequalities 1.18 and (1.19) together with the conservation laws 1.6 and $(1.7)-(1.8)$ constitute key elements in the study of hydrodynamic limits.

\subsection{The mathematical framework}

The construction of suitable global solutions to the Vlasov-Maxwell-Boltzmann system 1.1

$$
\left\{\begin{aligned}
\partial_{t} f+v \cdot \nabla_{x} f+(E+v \wedge B) \cdot \nabla_{v} f & =Q(f, f), \\
\partial_{t} E-\operatorname{rot} B & =-\int_{\mathbb{R}^{3}} f v d v, \\
\partial_{t} B+\operatorname{rot} E & =0 \\
\operatorname{div} E & =\int_{\mathbb{R}^{3}} f d v-1, \\
\operatorname{div} B & =0
\end{aligned}\right.
$$


or to the two species Vlasov-Maxwell-Boltzmann system 1.2

$$
\left\{\begin{aligned}
\partial_{t} f^{ \pm}+v \cdot \nabla_{x} f^{ \pm} \pm(E+v \wedge B) \cdot \nabla_{v} f^{ \pm} & =Q\left(f^{ \pm}, f^{ \pm}\right)+Q\left(f^{ \pm}, f^{\mp}\right), \\
\partial_{t} E-\operatorname{rot} B & =-\int_{\mathbb{R}^{3}}\left(f^{+}-f^{-}\right) v d v \\
\partial_{t} B+\operatorname{rot} E & =0 \\
\operatorname{div} E & =\int_{\mathbb{R}^{3}}\left(f^{+}-f^{-}\right) d v \\
\operatorname{div} B & =0
\end{aligned}\right.
$$

for large initial data is considered of outstanding difficulty, due to the lack of dissipative phenomena in Maxwell's equations, which are hyperbolic. Here, for the sake of simplicity, we have discarded all free parameters, since these are irrelevant for the existence theory. Thus, so far, the only known answer to this problem is due to Lions in [53, where a rather weak notion of solutions was derived : the so-called measure-valued renormalized solutions. However, these solutions failed to reach mathematical consensus on their usefulness due to their very weak aspect.

It should be mentioned that an alternative approach yielding strong solutions, provided smallness and regularity assumptions on the initial data are satisfied, was obtained more recently by Guo in [4]. But such solutions fall out of the scope of our derivation of hydrodynamic limits since they are not based on the physical entropy and energy estimates. Anyway, were we to consider such strong solution, our approach and strategy would remain strictly the same, for, as we are about to see in Chapter 2 below, the only uniform bounds valid in the hydrodynamic limit are precisely the physical entropy and energy estimates.

This poor understanding of the mathematical theory of the Vlasov-MaxwellBoltzmann system is the reason why getting rigorous convergence results is so complex. For the sake of readability, we have therefore decided to separate the different kinds of difficulties.

- In a first part, we will prove conditional convergence results restricting our attention to the case of Maxwellian cross-sections, i.e. $b \equiv 1$, for mere technical simplicity, and assuming the existence of renormalized solutions to (1.1) and (1.2), which is actually not known. It is to be emphasized that, even if this notion of solution is relatively rough, the convergence proof in this weak case has no purely technical difficulty specific to this roughness. Indeed, were we to deal with stronger solutions, the strategy of proof would not be any different or easier because we are considering here only the uniform bounds which come from physical estimates.

In this framework, we can focus on the key arguments of the convergence proof, which are not so different from the ones used for hydrodynamic limits of neutral gases. A crucial point is to understand how to get strong compactness on macroscopic fields, which cannot be dealt with using $L^{1}$ mixing lemma such as in [37] because of the electromagnetic forcing terms. We will thus first prove strong compactness with respect to velocity, and then use refined hypoelliptic estimates established in [6] in order to transfer the strong compactness to the spatial variable (Chapter 7).

The other key point which requires a specific treatment is the study of fast time oscillations insofar as they possibly couple weak compressibility with strong electromagnetic effects (Chapter 10 . 
The second and third part will be then devoted to the understanding of additional technical difficulties related to the fact that we are not able to build renormalized solutions to the Vlasov-Maxwell-Boltzmann systems, but only even weaker solutions.

- In the case of singular collision kernels, using the regularizing properties of the collision operator with respect to $v$, we will actually show the existence of renormalized solutions with a defect measure in the sense of Alexandre and Villani. The major change is the fact that the renormalized kinetic equation is replaced by an inequality (the consistency coming from the conservation of mass). This leads to the introduction of a defect measure.

The important new step of the convergence proof is then to establish that this defect measure vanishes in the fast relaxation limit, which comes from refined entropy dissipation estimates.

There are also many additional technical steps due to the singularity of the collision kernel, which makes the control of the conservation defects and the hypoelliptic transfer of compactness more difficult.

- In the apparently simpler case of cutoff collision kernels, because of the lack of strong compactness estimates, we are not able to prove that approximate solutions $f_{N}$ to the Vlasov-Maxwell-Boltzmann systems (1.1) and 1.2 converge to actual renormalized solutions. Indeed, without strong compactness properties, it is not possible to establish that $\beta\left(f_{N}\right) \rightarrow \beta(f)$ for any renormalization $\beta$, which accounts for the introduction of Young measures and the definition of a very rough notion of solution, namely the measure-valued renormalized solutions. Of course the physical meaning of such weak solutions is unclear, which probably explains why they have not been studied so far.

Nevertheless, we will establish here that - in the fast relaxation limit - they exhibit the expected behavior, converging to the relevant magnetohydrodynamic model, which can be considered as an indication of their physical relevance.

The key point of the proof will be to obtain integrated versions of all estimates with respect to the Young measures, and to prove that asymptotically the Young measures are not seen by the limiting equation, even though they do not converge to Dirac masses due to lack of uniqueness of solutions in the limiting systems. 


\section{CHAPTER 2}

\section{Scalings and formal limits}

In view of what is known on hydrodynamic limits of the Boltzmann equation (see 69] and the references therein), which corresponds to the particular case where particles are not charged, i.e. $q=0$ in $(1.1)$, we will focus on incompressible diffusive regimes, since we do not expect to be able to obtain a complete mathematical derivation for other choices of scalings.

\subsection{Incompressible viscous regimes}

In the absence of electromagnetic field, the Boltzmann equation can be rewritten in non-dimensional variables

$$
\mathrm{St}_{t} f+v \cdot \nabla_{x} f=\frac{1}{\mathrm{Kn}} Q(f, f),
$$

where we have introduced the following parameters :

- the Knudsen number $\mathrm{Kn}=\frac{\lambda_{0}}{l_{0}}$, measuring the ratio of the mean free path $\lambda_{0}$ to the observation length scale $l_{0}$;

- the Strouhal number $\mathrm{St}=\frac{l_{0}}{c_{0} t_{0}}$, measuring the ratio of the observation length scale $l_{0}$ to the typical length $c_{0} t_{0}$ run by a particle during a unit of time $t_{0}$, where $c_{0}$ is the speed of sound (or thermal speed) ;

- choosing the length $l_{0}$, time $t_{0}$ and velocity scales $u_{0}$ in such a way that we observe a macroscopic motion, i.e. $u_{0}=\frac{l_{0}}{t_{0}}$, we have the identity $\mathrm{St}=\mathrm{Ma}$ where the Mach number $\mathrm{Ma}=\frac{u_{0}}{c_{0}}$ is defined as the ratio of the bulk velocity $u_{0}$ to the thermal speed.

Hydrodynamic approximations are obtained in the fast relaxation limit $\mathrm{Kn} \rightarrow$ 0 , which precisely corresponds to the asymptotic regime where the fluid under consideration satisfies the continuum hypothesis, for the mean free path becomes infinitesimally small. Because of the von Kármán relation for perfect gases, we then expect the flow to be dissipative when the Reynolds number

$$
\mathrm{Re} \approx \frac{\mathrm{Ma}}{\mathrm{Kn}}
$$

measuring the inverse kinematic viscosity of the gas, is of order 1, i.e. when the Mach number also tends to 0.

In order to ensure the consistency of these scaling assumptions, we will consider - as usual - data which are fluctuations $g$ of order Ma

$$
f=M(1+\operatorname{Ma} g),
$$

around a global normalized Maxwellian equilibrium

$$
M(v)=\frac{1}{(2 \pi)^{\frac{3}{2}}} e^{-\frac{|v|^{2}}{2}},
$$

of density 1 , bulk velocity 0 and temperature 1 .

Thus, as is well-known since the works of Bardos, Golse and Levermore 9, 10, the viscous incompressible hydrodynamic regimes of collisional kinetic systems 
are obtained in the fast relaxation limit when the above-mentioned dimensionless numbers Kn, St and Ma, are all of the same order $\epsilon>0$, say. In the sequel, we will therefore restrict our attention to the scaled Vlasov-Maxwell-Boltzmann system

$$
\left\{\begin{aligned}
\epsilon \partial_{t} f+v \cdot \nabla_{x} f+\frac{q l_{0}}{m c_{0}^{2}}\left(E+c_{0} v \wedge B\right) \cdot \nabla_{v} f & =\frac{1}{\epsilon} Q(f, f), \\
f & =M(1+\epsilon g), \\
\epsilon c_{0} \mu_{0} \epsilon_{0} \partial_{t} E-\operatorname{rot} B & =-\mu_{0} q c_{0} l_{0} \int_{\mathbb{R}^{3}} f v d v, \\
\epsilon c_{0} \partial_{t} B+\operatorname{rot} E & =0, \\
\operatorname{div} E & =\frac{q l_{0}}{\epsilon_{0}}\left(\int_{\mathbb{R}^{3}} f d v-1\right), \\
\operatorname{div} B & =0,
\end{aligned}\right.
$$

and to the scaled two species Vlasov-Maxwell-Boltzmann system

$$
\left\{\begin{aligned}
\epsilon \partial_{t} f^{ \pm}+v \cdot \nabla_{x} f^{ \pm} \pm \frac{q l_{0}}{m c_{0}^{2}}\left(E+c_{0} v \wedge B\right) & \cdot \nabla_{v} f^{ \pm} \\
& =\frac{1}{\epsilon} Q\left(f^{ \pm}, f^{ \pm}\right)+\frac{\delta^{2}}{\epsilon} Q\left(f^{ \pm}, f^{\mp}\right) \\
f^{ \pm} & =M\left(1+\epsilon g^{ \pm}\right), \\
\epsilon c_{0} \mu_{0} \epsilon_{0} \partial_{t} E-\operatorname{rot} B & =-\mu_{0} q c_{0} l_{0} \int_{\mathbb{R}^{3}}\left(f^{+}-f^{-}\right) v d v \\
\epsilon c_{0} \partial_{t} B+\operatorname{rot} E & =0, \\
\operatorname{div} E & =\frac{q l_{0}}{\epsilon_{0}} \int_{\mathbb{R}^{3}}\left(f^{+}-f^{-}\right) d v \\
\operatorname{div} B & =0
\end{aligned}\right.
$$

where we have introduced another bounded parameter $\delta>0$ in front of the interspecies collision operator to differentiate the strength of interactions. The size of the parameter $\delta$ will be compared to the Knudsen number $\mathrm{Kn}=\epsilon$ and we will distinguish three cases, due to their distinct asymptotic behavior :

- $\delta \sim 1$, strong interspecies interactions ;

- $\delta=o(1)$ and $\frac{\delta}{\epsilon}$ unbounded, weak interspecies interactions ;

- $\delta=O(\epsilon)$, very weak interspecies interactions.

Notice also that we have performed the same nondimensionalization on the whole Vlasov-Maxwell-Boltzmann systems, which explains the presence of the parameters $\epsilon, c_{0}$ and $l_{0}$ in Maxwell's equations.

\subsection{Scalings for the electromagnetic field}

First, from 1.18, we get the scaled entropy inequality for one species, for all $t>0$,

$$
\begin{aligned}
\frac{1}{\epsilon^{2}} \int_{\mathbb{R}^{3} \times \mathbb{R}^{3}} & \left(f \log \frac{f}{M}-f+M\right)(t) d x d v \\
& +\frac{1}{2 c_{0}^{2} \epsilon^{2}} \int_{\mathbb{R}^{3}}\left(\frac{\epsilon_{0}}{m}|E|^{2}+\frac{1}{m \mu_{0}}|B|^{2}\right)(t) d x+\frac{1}{\epsilon^{4}} \int_{0}^{t} \int_{\mathbb{R}^{3}} D(f)(s) d x d s \\
& \leq \frac{1}{\epsilon^{2}} \int_{\mathbb{R}^{3} \times \mathbb{R}^{3}}\left(f^{\mathrm{in}} \log \frac{f^{\mathrm{in}}}{M}-f^{\mathrm{in}}+M\right) d x d v \\
& +\frac{1}{2 c_{0}^{2} \epsilon^{2}} \int_{\mathbb{R}^{3}}\left(\frac{\epsilon_{0}}{m}\left|E^{\mathrm{in}}\right|^{2}+\frac{1}{m \mu_{0}}\left|B^{\mathrm{in}}\right|^{2}\right) d x
\end{aligned}
$$


where $\left(f^{\text {in }}, E^{\text {in }}, B^{\text {in }}\right)$ denotes the initial data.

As for the two species case, from 1.19, we get the scaled entropy inequality, for all $t>0$,

$$
\begin{aligned}
\frac{1}{\epsilon^{2}} \int_{\mathbb{R}^{3} \times \mathbb{R}^{3}} & \left(f^{+} \log \frac{f^{+}}{M}-f^{+}+M\right)(t)+\left(f^{-} \log \frac{f^{-}}{M}-f^{-}+M\right)(t) d x d v \\
& +\frac{1}{2 c_{0}^{2} \epsilon^{2}} \int_{\mathbb{R}^{3}}\left(\frac{\epsilon_{0}}{m}|E|^{2}+\frac{1}{m \mu_{0}}|B|^{2}\right)(t) d x \\
& +\frac{1}{\epsilon^{4}} \int_{0}^{t} \int_{\mathbb{R}^{3}}\left(D\left(f^{+}\right)+D\left(f^{-}\right)+\delta^{2} D\left(f^{+}, f^{-}\right)\right)(s) d x d s \\
& \leq \frac{1}{\epsilon^{2}} \int_{\mathbb{R}^{3} \times \mathbb{R}^{3}}\left(f^{+ \text {in }} \log \frac{f^{+ \text {in }}}{M}-f^{+ \text {in }}+M\right) d x d v \\
& +\frac{1}{\epsilon^{2}} \int_{\mathbb{R}^{3} \times \mathbb{R}^{3}}\left(f^{- \text {in }} \log \frac{f^{- \text {in }}}{M}-f^{- \text {in }}+M\right) d x d v \\
& +\frac{1}{2 c_{0}^{2} \epsilon^{2}} \int_{\mathbb{R}^{3}}\left(\frac{\epsilon_{0}}{m}\left|E^{\text {in }}\right|^{2}+\frac{1}{m \mu_{0}}\left|B^{\text {in }}\right|^{2}\right) d x,
\end{aligned}
$$

where $\left(f^{+ \text {in }}, f^{- \text {in }}, E^{\text {in }}, B^{\text {in }}\right)$ denotes the initial data.

Note that the entropy inequalities (2.1) and (2.2) are the only uniform controls we have on the particle number densities and on the electric and magnetic fields, meaning that whatever the repartition of the free energy at the initial time, all the contributions are expected to be of the same order.

Thus, up to a change of units in $E$ and $B$, namely setting

$$
\tilde{E}=\frac{1}{c_{0} \epsilon} \sqrt{\frac{\epsilon_{0}}{m}} E, \quad \tilde{B}=\frac{1}{c_{0} \epsilon \sqrt{m \mu_{0}}} B,
$$

so that $\tilde{E}$ and $\tilde{B}$ are uniformly controlled by the scaled entropy inequalities (2.1) or (2.2), we have (dropping the tildes for the sake of readability), for one species,

$$
\left\{\begin{aligned}
\epsilon \partial_{t} f+v \cdot \nabla_{x} f+(\alpha E+\beta v \wedge B) \cdot \nabla_{v} f & =\frac{1}{\epsilon} Q(f, f), \\
f & =M(1+\epsilon g), \\
\gamma \partial_{t} E-\operatorname{rot} B & =-\frac{\beta}{\epsilon^{2}} \int_{\mathbb{R}^{3}} f v d v, \\
\gamma \partial_{t} B+\operatorname{rot} E & =0, \\
\operatorname{div} E & =\frac{\alpha}{\epsilon^{2}}\left(\int_{\mathbb{R}^{3}} f d v-1\right), \\
\operatorname{div} B & =0,
\end{aligned}\right.
$$

and, for two species,

$$
\left\{\begin{aligned}
\epsilon \partial_{t} f^{ \pm}+v \cdot \nabla_{x} f^{ \pm} \pm(\alpha E+\beta v \wedge B) \cdot \nabla_{v} f^{ \pm} & =\frac{1}{\epsilon} Q\left(f^{ \pm}, f^{ \pm}\right)+\frac{\delta^{2}}{\epsilon} Q\left(f^{ \pm}, f^{\mp}\right), \\
f^{ \pm} & =M\left(1+\epsilon g^{ \pm}\right), \\
\gamma \partial_{t} E-\operatorname{rot} B & =-\frac{\beta}{\epsilon^{2}} \int_{\mathbb{R}^{3}}\left(f^{+}-f^{-}\right) v d v, \\
\gamma \partial_{t} B+\operatorname{rot} E & =0, \\
\operatorname{div} E & =\frac{\alpha}{\epsilon^{2}} \int_{\mathbb{R}^{3}}\left(f^{+}-f^{-}\right) d v, \\
\operatorname{div} B & =0,
\end{aligned}\right.
$$


where there are only three free parameters left (else that $\epsilon$ and $\delta$ ) to describe the qualitative behaviors of the systems, namely :

- $\alpha=\epsilon \frac{q l_{0}}{c_{0} \sqrt{m \epsilon_{0}}}$ measuring the electric repulsion according to Gauss' law ;

- $\beta=\epsilon q l_{0} \sqrt{\frac{\mu_{0}}{m}}$ measuring the magnetic induction according to Ampère's law ;

- $\gamma=\epsilon c_{0} \sqrt{\epsilon_{0} \mu_{0}}=u_{0} \sqrt{\epsilon_{0} \mu_{0}}$ which is nothing else than the ratio of the bulk velocity to the speed of light.

Notice that these parameters are naturally constrained to the relation

$$
\beta=\frac{\alpha \gamma}{\epsilon} \text {. }
$$

We will impose some natural restrictions on the size of $\alpha, \beta$ and $\gamma$. First of all, we will require that $\gamma=O(1)$. Note, however, that an unbounded $\frac{\gamma}{\epsilon}=$ $c_{0} \sqrt{\epsilon_{0} \mu_{0}}$ may seem physically unrealistic since it corresponds to a regime where the thermal speed (i.e. the speed of sound) exceeds the speed of light. As usual, such situations should only be interpreted as asymptotic regimes where appropriate physical approximations are valid.

Moreover, in the one species case, we will demand that $\alpha$ and $\beta$ are of order $O(\epsilon)$, so that electric and magnetic forces create bounded acceleration terms in the Vlasov-Boltzmann equation in 2.3.

Situations where one of these parameters is large compared to $\epsilon$ are much more complicated. Indeed, we expect the Lorentz force to strongly penalize the system, leading asymptotically to some nonlinear macroscopic constraint that we are not able to deal with in the one species case. Actually, as far as we know, there is no systematic mathematical method to investigate such problems of nonlinear singular perturbation. For instance, understanding the dynamo effect is a related question which remains challenging.

Thus, on the whole, for one species, we will consider bounded parameters $\alpha, \beta$ and $\gamma$ satisfying

$$
\alpha=O(\epsilon), \quad \beta=O(\epsilon), \quad \gamma=O(1) \quad \text { and } \quad \beta=\frac{\alpha \gamma}{\epsilon} .
$$

We will then distinguish two critical cases, namely

(1) $\alpha=\epsilon, \beta=\epsilon, \gamma=\epsilon$,

(2) $\alpha=\epsilon^{2}, \beta=\epsilon, \gamma=1$,

and will explain how all other cases can be easily deduced from the above, just eliminating lower order terms which are too small. The full range of parameters will be described later on by the Figure 1 on page 26. For the moment, we merely emphasize that the above-mentioned critical cases correspond exactly to the vertices of the domain represented in Figure 1

For two species, the restrictions on the size of the parameters $\alpha, \beta$ and $\gamma$ are not so explicitly deduced by inspection of the system (2.4), except in the case $\delta=O(\epsilon)$, which lowers the order of the interspecies collision term $\frac{\delta^{2}}{\epsilon} Q\left(f^{ \pm}, f^{\mp}\right)$ in (2.4) and is thus analog to the one species case. However, when $\frac{\delta}{\epsilon}$ is unbounded, the interspecies collision term $\frac{\delta^{2}}{\epsilon} Q\left(f^{ \pm}, f^{\mp}\right)$ becomes a singular perturbation and, as a matter of fact, the need of asymptotically bounded acceleration terms in the macroscopic laws associated with the Vlasov-Boltzmann equations in (2.4) leads us to require that $\alpha=O(\epsilon)$ and $\beta=O(\delta)$. Note that $\beta=O(\epsilon)$ is not required in this case, which is in sharp contrast with the one species case.

Thus, on the whole, for two species, we will consider bounded parameters $\alpha$, $\beta, \gamma$ and $\delta$ satisfying either

$$
\alpha=O(\epsilon), \quad \beta=O(\epsilon), \quad \gamma=O(1) \quad \text { and } \quad \beta=\frac{\alpha \gamma}{\epsilon},
$$


when $\delta=O(\epsilon)$, or

$$
\alpha=O(\epsilon), \quad \beta=O(\delta), \quad \gamma=O(1) \quad \text { and } \quad \beta=\frac{\alpha \gamma}{\epsilon},
$$

otherwise.

We will then distinguish two critical cases, namely

(1) $\alpha=\epsilon, \beta=\epsilon, \gamma=\epsilon$,

(2) $\alpha=\epsilon^{2}, \beta=\epsilon, \gamma=1$,

when $\delta=O(\epsilon)$, and

(1) $\alpha=\epsilon, \beta=\delta, \gamma=\delta$,

(2) $\alpha=\delta \epsilon, \beta=\delta, \gamma=1$,

when $\frac{\delta}{\epsilon}$ is unbounded (note that the latter two cases coincide when $\delta \sim 1$ ), and will explain how all other cases can be easily deduced from the above, just eliminating lower order terms which are too small. Thus, as for one species, when $\delta=O(\epsilon)$, the full range of parameters will be described by the Figure 1 on page 26. Furthermore, when $\delta \sim 1$, the range of parameters will be represented by the Figure 2 on page 50 . while the case $\delta=o(1)$ with $\frac{\delta}{\epsilon}$ unbounded will be described by the Figure 3 on page 52 Again, we merely emphasize, for the moment, that the above-mentioned critical cases correspond exactly to the vertices of the respective domains represented in Figures 1, 2 and 3 .

\subsection{Formal analysis of the one species asymptotics}

Thus, for a plasma of one species of particles, our starting point is the scaled system

$$
\left\{\begin{aligned}
\epsilon \partial_{t} f_{\epsilon}+v \cdot \nabla_{x} f_{\epsilon}+\left(\alpha E_{\epsilon}+\beta v \wedge B_{\epsilon}\right) \cdot \nabla_{v} f_{\epsilon} & =\frac{1}{\epsilon} Q\left(f_{\epsilon}, f_{\epsilon}\right), \\
f_{\epsilon} & =M\left(1+\epsilon g_{\epsilon}\right), \\
\gamma \partial_{t} E_{\epsilon}-\operatorname{rot} B_{\epsilon} & =-\frac{\beta}{\epsilon} \int_{\mathbb{R}^{3}} g_{\epsilon} v M d v, \\
\gamma \partial_{t} B_{\epsilon}+\operatorname{rot} E_{\epsilon} & =0, \\
\operatorname{div} E_{\epsilon} & =\frac{\alpha}{\epsilon} \int_{\mathbb{R}^{3}} g_{\epsilon} M d v, \\
\operatorname{div} B_{\epsilon} & =0,
\end{aligned}\right.
$$

supplemented with some initial data satisfying

$$
\frac{1}{\epsilon^{2}} H\left(f_{\epsilon}^{\mathrm{in}}\right)+\frac{1}{2} \int_{\mathbb{R}^{3}}\left|E_{\epsilon}^{\mathrm{in}}\right|^{2}+\left|B_{\epsilon}^{\mathrm{in}}\right|^{2} d x<\infty,
$$

where $H\left(f_{\epsilon}^{\text {in }}\right)=H\left(f_{\epsilon}^{\text {in }} \mid M\right)$. In particular, the corresponding scaled entropy inequality, where $t>0$,

$$
\begin{aligned}
\frac{1}{\epsilon^{2}} H\left(f_{\epsilon}\right)+\frac{1}{2} \int_{\mathbb{R}^{3}}\left|E_{\epsilon}\right|^{2}+\left|B_{\epsilon}\right|^{2} d x & +\frac{1}{\epsilon^{4}} \int_{0}^{t} \int_{\mathbb{R}^{3}} D\left(f_{\epsilon}\right)(s) d x d s \\
& \leq \frac{1}{\epsilon^{2}} H\left(f_{\epsilon}^{\text {in }}\right)+\frac{1}{2} \int_{\mathbb{R}^{3}}\left|E_{\epsilon}^{\text {in }}\right|^{2}+\left|B_{\epsilon}^{\text {in }}\right|^{2} d x,
\end{aligned}
$$

guarantees that the solution will remain - for all non-negative times - a fluctuation of order $\epsilon$ around the global equilibrium $M$ :

$$
f_{\epsilon}=M\left(1+\epsilon g_{\epsilon}\right) \text {. }
$$

Note that the kinetic equation in 2.5 can then be rewritten, in terms of the fluctuation $g_{\epsilon}$, as

(2.7) $\epsilon \partial_{t} g_{\epsilon}+v \cdot \nabla_{x} g_{\epsilon}+\left(\alpha E_{\epsilon}+\beta v \wedge B_{\epsilon}\right) \cdot \nabla_{v} g_{\epsilon}-\frac{\alpha}{\epsilon} E_{\epsilon} \cdot v\left(1+\epsilon g_{\epsilon}\right)=-\frac{1}{\epsilon} \mathcal{L} g_{\epsilon}+\mathcal{Q}\left(g_{\epsilon}, g_{\epsilon}\right)$, 
where we denote

$$
\mathcal{L} g=-\frac{1}{M}(Q(M g, M)+Q(M, M g)) \quad \text { and } \quad \mathcal{Q}(g, g)=\frac{1}{M} Q(M g, M g) .
$$

2.3.1. Thermodynamic equilibrium. The entropy inequality 2.6 provides uniform bounds on $E_{\epsilon}, B_{\epsilon}$ and $g_{\epsilon}$. Therefore, assuming some formal compactness, up to extraction of subsequences, one has

$$
E_{\epsilon} \rightarrow E, \quad B_{\epsilon} \rightarrow B, \quad g_{\epsilon} \rightarrow g,
$$

in a weak sense to be rigorously detailed in a subsequent chapter.

Then, multiplying (2.7) by $\epsilon$, and taking formal limits as $\epsilon \rightarrow 0$ shows that $\mathcal{L} g=0$. It can be shown (see Proposition 5.5 , below) that the kernel of the linearized Boltzmann operator $\mathcal{L}$ coincides exactly with the vector space spanned by the collision invariants $\left\{1, v_{1}, v_{2}, v_{3},|v|^{2}\right\}$. Thus, we conclude that $g$ is an infinitesimal Maxwellian, that is a linear combination of collision invariants

$$
g=\rho+u \cdot v+\theta\left(\frac{|v|^{2}}{2}-\frac{3}{2}\right),
$$

where $\rho \in \mathbb{R}, u \in \mathbb{R}^{3}$ and $\theta \in \mathbb{R}$ only depend on $t$ and $x$, and are respectively the fluctuations of density, bulk velocity and temperature.

The fact that the fluctuations assume the infinitesimal Maxwellian form describes that the gas reaches thermodynamic (or statistical) equilibrium, in the fast relaxation limit.

We define now the macroscopic fluctuations of density $\rho_{\epsilon}$, bulk velocity $u_{\epsilon}$ and temperature $\theta_{\epsilon}$ by

$$
\begin{aligned}
\rho_{\epsilon} & =\int_{\mathbb{R}^{3}} g_{\epsilon} M d v, \\
u_{\epsilon} & =\int_{\mathbb{R}^{3}} g_{\epsilon} v M d v, \\
\theta_{\epsilon} & =\int_{\mathbb{R}^{3}} g_{\epsilon}\left(\frac{|v|^{2}}{3}-1\right) M d v,
\end{aligned}
$$

and the hydrodynamic projection $\Pi g_{\epsilon}$ of $g_{\epsilon}$ by

$$
\Pi g_{\epsilon}=\rho_{\epsilon}+u_{\epsilon} \cdot v+\theta_{\epsilon}\left(\frac{|v|^{2}}{2}-\frac{3}{2}\right)
$$

which is nothing but the orthogonal projection of $g_{\epsilon}$ onto the kernel of $\mathcal{L}$ in $L^{2}(M d v)$.

Note that the previous step establishing the convergence of $g_{\epsilon}$ towards thermodynamic equilibrium yields, in fact, the uniform boundedness of $\frac{1}{\epsilon} \mathcal{L} g_{\epsilon}$, which implies, at least formally, that

$$
g_{\epsilon}-\Pi g_{\epsilon}=O(\epsilon)
$$

This convergence may also be derived directly from the uniform control of the entropy dissipation $\frac{1}{\epsilon^{4}} D\left(f_{\epsilon}\right)$ in the entropy inequality 2.6$)$, provided we can control the large values of the fluctuations. Indeed, according to 1.13 , we write

$$
D\left(f_{\epsilon}\right)=\frac{1}{4} \int_{\mathbb{R}^{3} \times \mathbb{R}^{3} \times \mathbb{S}^{2}}\left(\frac{f_{\epsilon}^{\prime} f_{\epsilon *}^{\prime}-f_{\epsilon} f_{\epsilon *}}{f_{\epsilon} f_{\epsilon *}}\right) \log \left(1+\frac{f_{\epsilon}^{\prime} f_{\epsilon *}^{\prime}-f_{\epsilon} f_{\epsilon *}}{f_{\epsilon} f_{\epsilon *}}\right) f_{\epsilon} f_{\epsilon *} b d v d v_{*} d \sigma .
$$

Therefore, since the non-negative function $z \log (1+z)$ behaves essentially as $z^{2}$, for small values of $|z|$, we deduce a formal control on

$$
\frac{1}{4 \epsilon^{4}} \int_{\mathbb{R}^{3} \times \mathbb{R}^{3} \times \mathbb{S}^{2}}\left(\frac{f_{\epsilon}^{\prime} f_{\epsilon *}^{\prime}-f_{\epsilon} f_{\epsilon *}}{f_{\epsilon} f_{\epsilon *}}\right)^{2} f_{\epsilon} f_{\epsilon *} b d v d v_{*} d \sigma .
$$


Then, since

$$
f_{\epsilon}^{\prime} f_{\epsilon *}^{\prime}-f_{\epsilon} f_{\epsilon *}=\epsilon\left(g_{\epsilon}^{\prime}+g_{\epsilon *}^{\prime}-g_{\epsilon}-g_{\epsilon *}\right)+\epsilon^{2}\left(g_{\epsilon}^{\prime} g_{\epsilon *}^{\prime}-g_{\epsilon} g_{\epsilon *}\right),
$$

we infer that $\frac{1}{\epsilon}\left(g_{\epsilon}^{\prime}+g_{\epsilon *}^{\prime}-g_{\epsilon}-g_{\epsilon *}\right)$ is uniformly bounded, which, in other words, amounts to a control on $\frac{1}{\epsilon} \mathcal{L} g_{\epsilon}$.

The asymptotic dynamics of $\left(\rho_{\epsilon}, u_{\epsilon}, \theta_{\epsilon}\right)$ is then governed by fluid equations, to be obtained from the moment equations associated with 2.7). Thus, successively multiplying 2.7 by the collision invariants $1, v$ and $\frac{|v|^{2}}{2}$, and integrating in $M d v$, yields

$$
\left\{\begin{aligned}
\partial_{t} \rho_{\epsilon}+\frac{1}{\epsilon} \operatorname{div} u_{\epsilon} & =0 \\
\partial_{t} u_{\epsilon}+\frac{1}{\epsilon} \nabla_{x}\left(\rho_{\epsilon}+\theta_{\epsilon}\right)-\frac{\alpha}{\epsilon^{2}} E_{\epsilon} & =\left(\frac{\alpha}{\epsilon} \rho_{\epsilon} E_{\epsilon}+\frac{\beta}{\epsilon} u_{\epsilon} \wedge B_{\epsilon}\right)-\frac{1}{\epsilon} \operatorname{div} \int_{\mathbb{R}^{3}} g_{\epsilon} \phi M d v \\
\frac{3}{2} \partial_{t}\left(\rho_{\epsilon}+\theta_{\epsilon}\right)+\frac{5}{2 \epsilon} \operatorname{div} u_{\epsilon} & =\frac{\alpha}{\epsilon} u_{\epsilon} \cdot E_{\epsilon}-\frac{1}{\epsilon} \operatorname{div} \int_{\mathbb{R}^{3}} g_{\epsilon} \psi M d v
\end{aligned}\right.
$$

where

$$
\phi(v)=v \otimes v-\frac{|v|^{2}}{3} \mathrm{Id}, \quad \psi(v)=\left(\frac{|v|^{2}}{2}-\frac{5}{2}\right) v .
$$

Recall that we are assuming $\alpha=O(\epsilon)$ and $\beta=O(\epsilon)$. Hence, the nonlinear terms in the right-hand side of 2.11 containing the electromagnetic fields are expected to be bounded. Furthermore, notice that the polynomials $\phi(v)$ and $\psi(v)$ are orthogonal to the collision invariants in the $L^{2}(M d v)$ inner-product. That is to say $\int_{\mathbb{R}^{3}} \varphi \phi M d v=0$ and $\int_{\mathbb{R}^{3}} \varphi \psi M d v=0$, for all collision invariants $\varphi(v)$. Since, according to 2.10, $g_{\epsilon}$ converges towards an infinitesimal Maxwellian with a rate $O(\epsilon)$, it is therefore natural to expect, at least formally, that the terms

$$
\begin{aligned}
& \frac{1}{\epsilon} \int_{\mathbb{R}^{3}} g_{\epsilon} \phi M d v=\frac{1}{\epsilon} \int_{\mathbb{R}^{3}}\left(g_{\epsilon}-\Pi g_{\epsilon}\right) \phi M d v, \\
& \frac{1}{\epsilon} \int_{\mathbb{R}^{3}} g_{\epsilon} \psi M d v=\frac{1}{\epsilon} \int_{\mathbb{R}^{3}}\left(g_{\epsilon}-\Pi g_{\epsilon}\right) \psi M d v,
\end{aligned}
$$

in 2.11 are bounded and have a limit.

More precisely, it can be shown that, in general, the linearized Boltzmann operator $\mathcal{L}$ is self-adjoint and Fredholm of index zero on $L^{2}(M d v)$ (or a variant of it depending on the cross-section). Therefore, its range is exactly the orthogonal complement of its kernel. It follows that $\phi \in L^{2}(M d v)$ and $\psi \in L^{2}(M d v)$ belong to the range of $\mathcal{L}$ and, thus, that there are inverses $\tilde{\phi} \in L^{2}(M d v)$ and $\tilde{\psi} \in L^{2}(M d v)$ such that

$$
\phi=\mathcal{L} \tilde{\phi} \quad \text { and } \quad \psi=\mathcal{L} \tilde{\psi}
$$

which can be uniquely determined by the fact that they are orthogonal to the kernel of $\mathcal{L}$ (i.e. to the collision invariants).

Consequently, the macroscopic system (2.11) can be recast as

$$
\left\{\begin{aligned}
\partial_{t} \rho_{\epsilon}+\frac{1}{\epsilon} \operatorname{div} u_{\epsilon} & =0 \\
\partial_{t} u_{\epsilon}+\frac{1}{\epsilon} \nabla_{x}\left(\rho_{\epsilon}+\theta_{\epsilon}\right)-\frac{\alpha}{\epsilon^{2}} E_{\epsilon} & =\left(\frac{\alpha}{\epsilon} \rho_{\epsilon} E_{\epsilon}+\frac{\beta}{\epsilon} u_{\epsilon} \wedge B_{\epsilon}\right)-\frac{1}{\epsilon} \operatorname{div} \int_{\mathbb{R}^{3}} \mathcal{L} g_{\epsilon} \tilde{\phi} M d v \\
\frac{3}{2} \partial_{t} \theta_{\epsilon}+\frac{1}{\epsilon} \operatorname{div} u_{\epsilon} & =\frac{\alpha}{\epsilon} u_{\epsilon} \cdot E_{\epsilon}-\frac{1}{\epsilon} \operatorname{div} \int_{\mathbb{R}^{3}} \mathcal{L} g_{\epsilon} \tilde{\psi} M d v
\end{aligned}\right.
$$


where the terms $\frac{1}{\epsilon} \mathcal{L} g_{\epsilon}$ will be expressed employing the Vlasov-Boltzmann equation 2.7). The above macroscopic system 2.14 is coupled with Maxwell's equations on $E_{\epsilon}$ and $B_{\epsilon}$ :

$$
\left\{\begin{aligned}
\gamma \partial_{t} E_{\epsilon}-\operatorname{rot} B_{\epsilon} & =-\frac{\beta}{\epsilon} u_{\epsilon} \\
\gamma \partial_{t} B_{\epsilon}+\operatorname{rot} E_{\epsilon} & =0 \\
\operatorname{div} E_{\epsilon} & =\frac{\alpha}{\epsilon} \rho_{\epsilon} \\
\operatorname{div} B_{\epsilon} & =0
\end{aligned}\right.
$$

A careful formal analysis of the whole coupled macroscopic system 2.14 -2.15 will yield the asymptotic dynamics of $(\rho, u, \theta, E, B)$.

2.3.2. Macroscopic constraints. At leading order, the system (2.14)- 2.15 describes the propagation of acoustic $\left(\rho_{\epsilon}, u_{\epsilon}, \sqrt{\frac{3}{2}} \theta_{\epsilon}\right)$ and electromagnetic $\left(E_{\epsilon}, B_{\epsilon}\right)$ waves :

$$
\partial_{t}\left(\begin{array}{c}
\rho_{\epsilon} \\
u_{\epsilon} \\
\sqrt{\frac{3}{2}} \theta_{\epsilon} \\
E_{\epsilon} \\
B_{\epsilon}
\end{array}\right)+W_{\epsilon}\left(\begin{array}{c}
\rho_{\epsilon} \\
u_{\epsilon} \\
\sqrt{\frac{3}{2}} \theta_{\epsilon} \\
E_{\epsilon} \\
B_{\epsilon}
\end{array}\right)=O(1),
$$

where the wave operator $W_{\epsilon}$, containing the singular terms from (2.14)-(2.15) and defined explicitly below, is antisymmetric (with respect to the $L^{2}(d x)$ innerproduct) and, therefore, can only have purely imaginary eigenvalues. The semigroup generated by this operator may thus produce fast time oscillations, which we are about to discuss briefly.

(1) When $\gamma \sim 1$ (so that $\alpha=O\left(\epsilon^{2}\right)$ ), we have

$$
W_{\epsilon}=\left(\begin{array}{ccccc}
0 & \frac{1}{\epsilon} \operatorname{div} & 0 & 0 & 0 \\
\frac{1}{\epsilon} \nabla_{x} & 0 & \frac{1}{\epsilon} \sqrt{\frac{2}{3}} \nabla_{x} & 0 & 0 \\
0 & \frac{1}{\epsilon} \sqrt{\frac{2}{3}} \operatorname{div} & 0 & 0 & 0 \\
0 & 0 & 0 & 0 & 0 \\
0 & 0 & 0 & 0 & 0
\end{array}\right) .
$$

Thus, the singular perturbation creates only high frequency acoustic waves. Consequently, averaging over fast time oscillations as $\epsilon \rightarrow 0$, we get the macroscopic constraints

$$
\operatorname{div} u=0, \quad \rho+\theta=0,
$$

respectively referred to as incompressibility and Boussinesq relations. These are supplemented by the asymptotic constraints coming from Gauss' laws in 2.15

$$
\operatorname{div} E=0, \quad \operatorname{div} B=0 .
$$

(2) When $\gamma=o(1)$ and $\alpha=O\left(\epsilon^{2}\right)$, we have

$$
W_{\epsilon}=\left(\begin{array}{ccccc}
0 & \frac{1}{\epsilon} \operatorname{div} & 0 & 0 & 0 \\
\frac{1}{\epsilon} \nabla_{x} & 0 & \frac{1}{\epsilon} \sqrt{\frac{2}{3}} \nabla_{x} & 0 & 0 \\
0 & \frac{1}{\epsilon} \sqrt{\frac{2}{3}} \operatorname{div} & 0 & 0 & 0 \\
0 & 0 & 0 & 0 & -\frac{1}{\gamma} \operatorname{rot} \\
0 & 0 & 0 & \frac{1}{\gamma} \operatorname{rot} & 0
\end{array}\right) .
$$


Thus, the singular perturbation creates both high frequency acoustic and electromagnetic waves. However, these waves remain decoupled and have a comparable frequency of oscillation if and only if $\gamma \sim \epsilon$. By averaging these fast time oscillations as $\epsilon \rightarrow 0$, we get the macroscopic constraints

$$
\begin{aligned}
\operatorname{div} u=0, & \rho+\theta=0, \\
\operatorname{rot} B=0, & \operatorname{rot} E=0 .
\end{aligned}
$$

These are supplemented by the asymptotic constraints coming from Gauss' laws in 2.15

$$
\operatorname{div} E=0, \quad \operatorname{div} B=0 .
$$

Hence,

$$
E=0, \quad B=0 .
$$

(3) When $\gamma=o(1)$ and $\frac{\alpha}{\epsilon^{2}}$ is unbounded, we have

$$
W_{\epsilon}=\left(\begin{array}{ccccc}
0 & \frac{1}{\epsilon} \operatorname{div} & 0 & 0 & 0 \\
\frac{1}{\epsilon} \nabla_{x} & 0 & \frac{1}{\epsilon} \sqrt{\frac{2}{3}} \nabla_{x} & -\frac{\alpha}{\epsilon^{2}} \text { Id } & 0 \\
0 & \frac{1}{\epsilon} \sqrt{\frac{2}{3}} \operatorname{div} & 0 & 0 & 0 \\
0 & \frac{\alpha}{\epsilon^{2}} \mathrm{Id} & 0 & 0 & -\frac{1}{\gamma} \operatorname{rot} \\
0 & 0 & 0 & \frac{1}{\gamma} \operatorname{rot} & 0
\end{array}\right) .
$$

Thus, the singular perturbation creates both high frequency acoustic and electromagnetic waves, which are coupled. These waves may or may not have comparable frequency of oscillation. By averaging these fast time oscillations as $\epsilon \rightarrow 0$, we get the macroscopic constraints

$$
\begin{aligned}
\operatorname{div} u & =0, & \nabla_{x}(\rho+\theta) & =\left[\frac{\alpha}{\epsilon}\right] E, \\
\operatorname{rot} B & =\left[\frac{\beta}{\epsilon}\right] u, & \operatorname{rot} E & =0,
\end{aligned}
$$

where we have denoted by $\left[\frac{\alpha}{\epsilon}\right]$ and $\left[\frac{\beta}{\epsilon}\right]$ the respective limits of $\frac{\alpha}{\epsilon}$ and $\frac{\beta}{\epsilon}$ as $\epsilon \rightarrow 0$. As usual, when $\alpha=o(\epsilon)$, the weak Boussinesq relation $\nabla_{x}(\rho+\theta)=0$ can be improved to the strong Boussinesq relation $\rho+\theta=0$, assuming $\rho$ and $\theta$ enjoy enough integrability. These are supplemented by the asymptotic constraints coming from Gauss' laws in 2.15

$$
\operatorname{div} E=\left[\frac{\alpha}{\epsilon}\right] \rho, \quad \operatorname{div} B=0 .
$$

The exact nature of time oscillations produced by the system 2.16, in the limit $\epsilon \rightarrow 0$, will be rigorously discussed, with greater detail, later on in Chapter 10

2.3.3. Evolution equations. The previous step shows that, since $W_{\epsilon}$ is singular, the asymptotic dynamics of $\left(\rho_{\epsilon}, u_{\epsilon}, \sqrt{\frac{3}{2}} \theta_{\epsilon}, E_{\epsilon}, B_{\epsilon}\right)$ becomes constrained to the kernel Ker $W_{\epsilon}$ as $\epsilon \rightarrow 0$. Moreover, since $W_{\epsilon}$ is antisymmetric, its range is necessarily orthogonal to its kernel. Therefore, in order to get the asymptotic evolution equations for $\left(\rho, u, \sqrt{\frac{3}{2}} \theta, E, B\right)$, it is natural to project the system 2.16 onto Ker $W_{\epsilon}$, which will rid us of all the singular terms in $(2.16)$ and allow us to pass to the limit. In other words, we will obtain the limiting dynamics of the system 2.16 by testing it against functions in $\operatorname{Ker} W_{\epsilon}$.

We will denote by $P: L^{2}(d x) \rightarrow L^{2}(d x)$ the Leray projector onto solenoidal vector fields and $P^{\perp}=\mathrm{Id}-P$ the projector onto the orthogonal complement, that is $P=-\Delta^{-1}$ rot rot and $P^{\perp}=\Delta^{-1} \nabla$ div. 
(1) When $\gamma \sim 1$, the kernel of $W_{\epsilon}$, defined in 2.17), is obviously determined by all $\left(\rho_{\epsilon}^{0}, u_{\epsilon}^{0}, \sqrt{\frac{3}{2}} \theta_{\epsilon}^{0}, E_{\epsilon}^{0}, B_{\epsilon}^{0}\right)$ which satisfy

$$
\operatorname{div} u_{\epsilon}^{0}=0 \quad \text { and } \quad \rho_{\epsilon}^{0}+\theta_{\epsilon}^{0}=0 .
$$

It is then readily seen that its orthogonal complement $\operatorname{Ker} W_{\epsilon}^{\perp}$ is determined by all $\left(\tilde{\rho}_{\epsilon}, \tilde{u}_{\epsilon}, \sqrt{\frac{3}{2}} \tilde{\theta}_{\epsilon}, \tilde{E}_{\epsilon}, \tilde{B}_{\epsilon}\right)$ such that

$$
P \tilde{u}_{\epsilon}=0 \quad \text { and } \quad \frac{3}{2} \tilde{\theta}_{\epsilon}-\tilde{\rho}_{\epsilon}=0 .
$$

Hence, projecting the system $2.14-2.15$ onto Ker $W_{\epsilon}^{\perp}$ yields

$$
\left\{\begin{aligned}
\partial_{t} P u_{\epsilon}+\frac{1}{\epsilon} P \operatorname{div} \int_{\mathbb{R}^{3}} \mathcal{L} g_{\epsilon} \tilde{\phi} M d v & =P\left(\frac{\alpha}{\epsilon^{2}} E_{\epsilon}+\frac{\alpha}{\epsilon} \rho_{\epsilon} E_{\epsilon}+\frac{\beta}{\epsilon} u_{\epsilon} \wedge B_{\epsilon}\right), \\
\partial_{t}\left(\frac{3}{2} \theta_{\epsilon}-\rho_{\epsilon}\right)+\frac{1}{\epsilon} \operatorname{div} \int_{\mathbb{R}^{3}} \mathcal{L} g_{\epsilon} \tilde{\psi} M d v & =\frac{\alpha}{\epsilon} u_{\epsilon} \cdot E_{\epsilon} .
\end{aligned}\right.
$$

(2) When $\gamma=o(1)$ and $\alpha=O\left(\epsilon^{2}\right)$, the kernel of $W_{\epsilon}$, defined in 2.19, is obviously determined by all $\left(\rho_{\epsilon}^{0}, u_{\epsilon}^{0}, \sqrt{\frac{3}{2}} \theta_{\epsilon}^{0}, E_{\epsilon}^{0}, B_{\epsilon}^{0}\right)$ which satisfy

$$
\begin{aligned}
& \operatorname{div} u_{\epsilon}^{0}=0, \quad \rho_{\epsilon}^{0}+\theta_{\epsilon}^{0}=0, \\
& \operatorname{rot} E_{\epsilon}^{0}=0, \quad \operatorname{rot} B_{\epsilon}^{0}=0 .
\end{aligned}
$$

It is then readily seen that its orthogonal complement $\operatorname{Ker} W_{\epsilon}^{\perp}$ is determined by all $\left(\tilde{\rho}_{\epsilon}, \tilde{u}_{\epsilon}, \sqrt{\frac{3}{2}} \tilde{\theta}_{\epsilon}, \tilde{E}_{\epsilon}, \tilde{B}_{\epsilon}\right)$ such that

$$
\begin{aligned}
P \tilde{u}_{\epsilon} & =0, & \frac{3}{2} \tilde{\theta}_{\epsilon}-\tilde{\rho}_{\epsilon} & =0, \\
P^{\perp} \tilde{E}_{\epsilon} & =0, & P^{\perp} \tilde{B}_{\epsilon} & =0 .
\end{aligned}
$$

Hence, projecting the system (2.14) onto $\operatorname{Ker} W_{\epsilon}^{\perp}$ also yields the system 2.23. Moreover, in view of Gauss' laws, the projection of Maxwell's equations 2.15 onto Ker $W_{\epsilon}^{\perp}$ yields no useful information.

(3) When $\gamma=o(1)$ and $\frac{\alpha}{\epsilon^{2}}$ is unbounded, the wave operator $W_{\epsilon}$ is defined by 2.21. Notice then that Gauss' laws from 2.15 are invariant under the action of the wave operator $W_{\epsilon}$. Consequently, it is enough to consider the restriction of $W_{\epsilon}$ to electromagnetic fields which verify Gauss' laws. It follows that the kernel of $W_{\epsilon}$ is obviously determined by all $\left(\rho_{\epsilon}^{0}, u_{\epsilon}^{0}, \sqrt{\frac{3}{2}} \theta_{\epsilon}^{0}, E_{\epsilon}^{0}, B_{\epsilon}^{0}\right)$ which satisfy

$$
\begin{aligned}
\operatorname{rot} B_{\epsilon}^{0} & =\frac{\beta}{\epsilon} u_{\epsilon}^{0}, & \nabla_{x}\left(\rho_{\epsilon}^{0}+\theta_{\epsilon}^{0}\right) & =\frac{\alpha}{\epsilon} E_{\epsilon}^{0}, \\
\operatorname{div} E_{\epsilon}^{0} & =\frac{\alpha}{\epsilon} \rho_{\epsilon}^{0}, & \operatorname{div} B_{\epsilon}^{0} & =0 .
\end{aligned}
$$

It is then readily seen that its orthogonal complement $\operatorname{Ker} W_{\epsilon}^{\perp}$ is determined by all $\left(\tilde{\rho}_{\epsilon}, \tilde{u}_{\epsilon}, \sqrt{\frac{3}{2}} \tilde{\theta}_{\epsilon}, \tilde{E}_{\epsilon}, \tilde{B}_{\epsilon}\right)$ such that

$$
\begin{array}{rlrl}
\frac{3}{2} \tilde{\theta}_{\epsilon}-\tilde{\rho}_{\epsilon} & =0, & \operatorname{rot} \tilde{u}_{\epsilon}+\frac{\beta}{\epsilon} \tilde{B}_{\epsilon} & =0, \\
\operatorname{div} \tilde{E}_{\epsilon} & =\frac{\alpha}{\epsilon} \tilde{\rho}_{\epsilon}, & \operatorname{div} \tilde{B}_{\epsilon}=0 .
\end{array}
$$

Considering the magnetic potential $\tilde{B}_{\epsilon}=\operatorname{rot} \tilde{A}_{\epsilon}$, uniquely determined if $\operatorname{div} \tilde{A}_{\epsilon}=0$ (i.e. fixing the Coulomb gauge), the above set of constraints 
can be rephrased as

$$
\begin{array}{rlrl}
\frac{3}{2} \tilde{\theta}_{\epsilon}-\tilde{\rho}_{\epsilon} & =0, & P \tilde{u}_{\epsilon}+\frac{\beta}{\epsilon} \tilde{A}_{\epsilon} & =0, \\
\operatorname{div} \tilde{E}_{\epsilon} & =\frac{\alpha}{\epsilon} \tilde{\rho}_{\epsilon}, & \operatorname{div} \tilde{A}_{\epsilon}=0 .
\end{array}
$$

Hence, projecting the system (2.14) onto Ker $W_{\epsilon}^{\perp}$ yields

$$
\left\{\begin{aligned}
\partial_{t}\left(P u_{\epsilon}+\frac{\beta}{\epsilon} A_{\epsilon}\right)+\frac{1}{\epsilon} P \operatorname{div} \int_{\mathbb{R}^{3}} \mathcal{L} g_{\epsilon} \tilde{\phi} M d v & =P\left(\frac{\alpha}{\epsilon} \rho_{\epsilon} E_{\epsilon}+\frac{\beta}{\epsilon} u_{\epsilon} \wedge B_{\epsilon}\right), \\
\partial_{t}\left(\frac{3}{2} \theta_{\epsilon}-\rho_{\epsilon}\right)+\frac{1}{\epsilon} \operatorname{div} \int_{\mathbb{R}^{3}} \mathcal{L} g_{\epsilon} \tilde{\psi} M d v & =\frac{\alpha}{\epsilon} u_{\epsilon} \cdot E_{\epsilon},
\end{aligned}\right.
$$

where $B_{\epsilon}=\operatorname{rot} A_{\epsilon}$ and $\operatorname{div} A_{\epsilon}=0$, and where we have used that Faraday's equation from 2.15 implies

$$
\frac{\beta}{\epsilon} \partial_{t} A_{\epsilon}+\frac{\alpha}{\epsilon^{2}} P E_{\epsilon}=0 \text {. }
$$

There only remains to evaluate the flux terms $\frac{1}{\epsilon} \int_{\mathbb{R}^{3}} \mathcal{L} g_{\epsilon} \tilde{\phi} M d v$ and $\frac{1}{\epsilon} \int_{\mathbb{R}^{3}} \mathcal{L} g_{\epsilon} \tilde{\psi} M d v$ in 2.23) and 2.24). Following [9, 10, this is done by employing 2.7) to evaluate that

$$
\frac{1}{\epsilon} \mathcal{L} g_{\epsilon}=\mathcal{Q}\left(g_{\epsilon}, g_{\epsilon}\right)-v \cdot \nabla_{x} g_{\epsilon}+\frac{\alpha}{\epsilon} E_{\epsilon} \cdot v+O(\epsilon)
$$

which yields formally in the limit, by virtue of the infinitesimal Maxwellian form 2.9 ,

$$
\begin{aligned}
\lim _{\epsilon \rightarrow 0} \frac{1}{\epsilon} \mathcal{L} g_{\epsilon} & =\mathcal{Q}(g, g)-v \cdot \nabla_{x} g+\left[\frac{\alpha}{\epsilon}\right] E \cdot v \\
& =\frac{1}{2} \mathcal{L}\left(g^{2}\right)-v \cdot \nabla_{x} g+\left[\frac{\alpha}{\epsilon}\right] E \cdot v \\
& =\frac{1}{2} u^{t} \mathcal{L}(\phi) u+\theta u \cdot \mathcal{L}(\psi)+\frac{1}{2} \theta^{2} \mathcal{L}\left(\frac{|v|^{4}}{4}\right) \\
& -\operatorname{div}\left((\rho+\theta) v+\frac{|v|^{2}}{3} u+\phi u+\theta \psi\right)+\left[\frac{\alpha}{\epsilon}\right] E \cdot v \\
& =\frac{1}{2} u^{t} \mathcal{L}(\phi) u+\theta u \cdot \mathcal{L}(\psi)+\frac{1}{2} \theta^{2} \mathcal{L}\left(\frac{|v|^{4}}{4}\right)-\operatorname{div}(\phi u+\theta \psi)
\end{aligned}
$$

where we have used, in the last line, that $\operatorname{div} u=0$ and $\nabla_{x}(\rho+\theta)=\left[\frac{\alpha}{\epsilon}\right] E$, whatever the asymptotic regime.

Next, we use that $\tilde{\phi}$ and $\tilde{\psi}$ have similar symmetry properties as $\phi$ and $\psi$, thanks to the rotational invariance of $\mathcal{L}$. More precisely, following [28, it can be shown (see also [13, Section 2.2.3]) that there exist two scalar valued functions $\alpha, \beta:[0, \infty) \rightarrow \mathbb{R}$ such that

$$
\tilde{\phi}(v)=\alpha(|v|) \phi(v) \quad \text { and } \quad \tilde{\psi}(v)=\beta(|v|) \psi(v),
$$

which implies (see [9, Lemma 4.4]) that

$$
\begin{aligned}
\int_{\mathbb{R}^{3}} \phi_{i j} \tilde{\phi}_{k l} M d v & =\mu\left(\delta_{i k} \delta_{j l}+\delta_{i l} \delta_{j k}-\frac{2}{3} \delta_{i j} \delta_{k l}\right), \\
\int_{\mathbb{R}^{3}} \psi_{i} \tilde{\psi}_{j} M d v & =\frac{5}{2} \kappa \delta_{i j},
\end{aligned}
$$

where

$$
\mu=\frac{1}{10} \int_{\mathbb{R}^{3}} \phi: \tilde{\phi} M d v \quad \text { and } \quad \kappa=\frac{2}{15} \int_{\mathbb{R}^{3}} \psi \cdot \tilde{\psi} M d v
$$


Hence, we conclude through tedious but straightforward calculations that

$$
\begin{aligned}
\lim _{\epsilon \rightarrow 0} \frac{1}{\epsilon} \int_{\mathbb{R}^{3}} \mathcal{L} g_{\epsilon} \tilde{\phi} M d v & =\int_{\mathbb{R}^{3}} \frac{1}{2}\left(u^{t} \phi u\right) \phi M d v-\int_{\mathbb{R}^{3}} \operatorname{div}(\phi u) \tilde{\phi} M d v \\
& =u \otimes u-\frac{|u|^{2}}{3} \operatorname{Id}-\mu\left(\nabla_{x} u+\nabla_{x}^{t} u\right) \\
\lim _{\epsilon \rightarrow 0} \frac{1}{\epsilon} \int_{\mathbb{R}^{3}} \mathcal{L} g_{\epsilon} \tilde{\psi} M d v & =\int_{\mathbb{R}^{3}} \theta u \cdot \psi \psi M d v-\int_{\mathbb{R}^{3}} \operatorname{div}(\theta \psi) \tilde{\psi} M d v \\
& =\frac{5}{2} \theta u-\frac{5}{2} \kappa \nabla_{x} \theta .
\end{aligned}
$$

We finally identify the advection and diffusion terms

$$
\begin{aligned}
\lim _{\epsilon \rightarrow 0} \frac{1}{\epsilon} P \operatorname{div} \int_{\mathbb{R}^{3}} \mathcal{L} g_{\epsilon} \tilde{\phi} M d v & =P\left(u \cdot \nabla_{x} u\right)-\mu \Delta_{x} u, \\
\lim _{\epsilon \rightarrow 0} \frac{1}{\epsilon} \operatorname{div} \int_{\mathbb{R}^{3}} \mathcal{L} g_{\epsilon} \tilde{\psi} M d v & =\frac{5}{2} u \cdot \nabla_{x} \theta-\frac{5}{2} \kappa \Delta_{x} \theta .
\end{aligned}
$$

On the whole, provided nonlinear terms remain stable in the limiting process, we obtain the following asymptotic systems :

(1) When $\gamma \sim 1$, letting $\epsilon$ tend to zero in the system 2.23 coupled with Maxwell's equations 2.15 yields

$$
\left\{\begin{aligned}
\partial_{t} u+u \cdot \nabla_{x} u-\mu \Delta_{x} u & =-\nabla_{x} p+\left[\frac{\alpha}{\epsilon^{2}}\right] E+\left[\frac{\beta}{\epsilon}\right] u \wedge B \\
\partial_{t} \theta+u \cdot \nabla_{x} \theta-\kappa \Delta_{x} \theta & =0 \\
{[\gamma] \partial_{t} E-\operatorname{rot} B } & =-\left[\frac{\beta}{\epsilon}\right] u \\
{[\gamma] \partial_{t} B+\operatorname{rot} E } & =0
\end{aligned}\right.
$$

with the constraints from 2.18

$$
\begin{aligned}
\operatorname{div} u=0, & \rho+\theta=0, \\
\operatorname{div} E=0, & \operatorname{div} B=0 .
\end{aligned}
$$

(2) When $\gamma=o(1)$ and $\alpha=O\left(\epsilon^{2}\right)$, letting $\epsilon$ tend to zero in the system 2.23 coupled with Maxwell's equations 2.15) yields

$$
\left\{\begin{array}{c}
\partial_{t} u+u \cdot \nabla_{x} u-\mu \Delta_{x} u=-\nabla_{x} p, \\
\partial_{t} \theta+u \cdot \nabla_{x} \theta-\kappa \Delta_{x} \theta=0,
\end{array}\right.
$$

with the constraints from 2.20

$$
\begin{aligned}
\operatorname{div} u & =0, & \rho+\theta & =0, \\
E & =0, & B & =0 .
\end{aligned}
$$

(3) When $\gamma=o(1)$ and $\frac{\alpha}{\epsilon^{2}}$ is unbounded, letting $\epsilon$ tend to zero in the system 2.24 coupled with Maxwell's equations 2.15 yields

$$
\left\{\begin{array}{l}
\partial_{t}\left(u+\left[\frac{\beta}{\epsilon}\right] A\right)+u \cdot \nabla_{x} u-\mu \Delta_{x} u=-\nabla_{x} p+\left[\frac{\alpha}{\epsilon}\right] \rho E+\left[\frac{\beta}{\epsilon}\right] u \wedge B, \\
\partial_{t}\left(\frac{3}{2} \theta-\rho\right)+\frac{5}{2} u \cdot \nabla_{x} \theta-\frac{5}{2} \kappa \Delta_{x} \theta=\left[\frac{\alpha}{\epsilon}\right] u \cdot E,
\end{array}\right.
$$


with the constraints from 2.22

$$
\begin{aligned}
\operatorname{div} u & =0, & \nabla_{x}(\rho+\theta) & =\left[\frac{\alpha}{\epsilon}\right] E, \\
\operatorname{rot} B & =\left[\frac{\beta}{\epsilon}\right] u, & \operatorname{rot} E & =0, \\
\operatorname{div} E & =\left[\frac{\alpha}{\epsilon}\right] \rho, & \operatorname{div} B & =0, \\
\operatorname{rot} A & =B, & \operatorname{div} A & =0 .
\end{aligned}
$$

The above system can be rewritten more explicitly by defining the adjusted electric field $\tilde{E}=-\partial_{t} A+E$. It then holds that

$$
\left\{\begin{aligned}
\partial_{t} u+u \cdot \nabla_{x} u-\mu \Delta_{x} u & =-\nabla_{x} p+\left[\frac{\beta}{\epsilon}\right] \tilde{E}+\rho \nabla_{x} \theta+\left[\frac{\beta}{\epsilon}\right] u \wedge B, \\
\partial_{t}\left(\frac{3}{2} \theta-\rho\right)+u \cdot \nabla_{x}\left(\frac{3}{2} \theta-\rho\right)-\frac{5}{2} \kappa \Delta_{x} \theta & =0 \\
\partial_{t} B+\operatorname{rot} \tilde{E} & =0
\end{aligned}\right.
$$

with the constraints

$$
\begin{array}{rlrl}
\operatorname{div} u & =0, & \Delta_{x}(\rho+\theta) & =\left[\frac{\alpha}{\epsilon}\right]^{2} \rho, \\
\operatorname{rot} B & =\left[\frac{\beta}{\epsilon}\right] u, & \operatorname{div} B & =0, \\
\operatorname{div} \tilde{E} & =\left[\frac{\alpha}{\epsilon}\right] \rho .
\end{array}
$$

Notice, finally, that if further $\alpha=o(\epsilon)$, then the above system is greatly simplified and becomes

$$
\left\{\begin{aligned}
\partial_{t} u+u \cdot \nabla_{x} u-\mu \Delta_{x} u & =-\nabla_{x} p+\left[\frac{\beta}{\epsilon}\right] \tilde{E}+\left[\frac{\beta}{\epsilon}\right] u \wedge B \\
\partial_{t} \theta+u \cdot \nabla_{x} \theta-\kappa \Delta_{x} \theta & =0 \\
\partial_{t} B+\operatorname{rot} \tilde{E} & =0
\end{aligned}\right.
$$

with the constraints

$$
\begin{aligned}
\operatorname{div} u & =0, & \rho+\theta & =0, \\
\operatorname{div} \tilde{E} & =0, & \operatorname{div} B & =0, \\
\operatorname{rot} B & =\left[\frac{\beta}{\epsilon}\right] u, & E & =0 .
\end{aligned}
$$

2.3.4. Summary. At last, we see that the asymptotics of the Vlasov-MaxwellBoltzmann system 2.5 can be depicted in terms of the limits of the following parameters :

- the strength of the electric induction $\alpha$,

- the strength of the magnetic induction $\beta=\frac{\alpha \gamma}{\epsilon}$,

- the ratio of the bulk velocity to the speed of light $\gamma$.

Figure 1 summarizes the different asymptotic regimes, on a logarithmic scale, of the Vlasov-Maxwell-Boltzmann system (2.5).

Thus, up to multiplicative constants, we reach the following asymptotic systems of equations :

(1) If $\alpha=o(\epsilon)$ and $\beta=o(\epsilon)$, we obtain the incompressible Navier-StokesFourier system :

$$
\left\{\begin{aligned}
\partial_{t} u+u \cdot \nabla_{x} u-\mu \Delta_{x} u & =-\nabla_{x} p, & & \operatorname{div} u=0 \\
\partial_{t} \theta+u \cdot \nabla_{x} \theta-\kappa \Delta_{x} \theta & =0, & & \rho+\theta=0 .
\end{aligned}\right.
$$




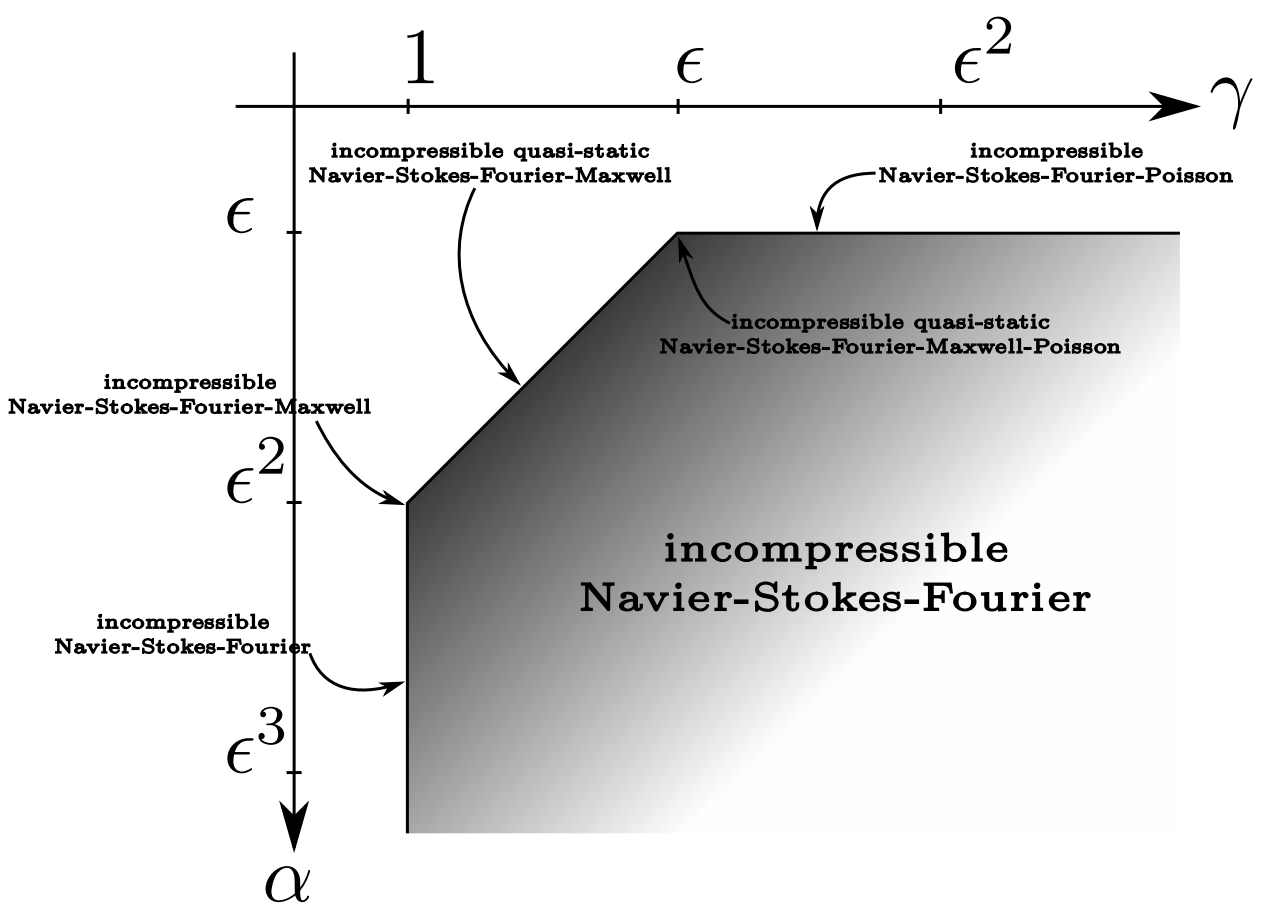

Figure 1. Asymptotic regimes of the one species Vlasov-Maxwell-

Boltzmann system (2.5).

This system satisfies the following formal energy conservation laws :

$$
\begin{aligned}
& \frac{1}{2} \frac{d}{d t}\|u\|_{L_{x}^{2}}^{2}+\mu\left\|\nabla_{x} u\right\|_{L_{x}^{2}}^{2}=0, \\
& \frac{1}{2} \frac{d}{d t}\|\theta\|_{L_{x}^{2}}^{2}+\kappa\left\|\nabla_{x} \theta\right\|_{L_{x}^{2}}^{2}=0 .
\end{aligned}
$$

(2) If $\alpha=\epsilon^{2}$ and $\gamma=1$, we obtain the incompressible Navier-Stokes-FourierMaxwell system :

$$
\left\{\begin{aligned}
\partial_{t} u+u \cdot \nabla_{x} u-\mu \Delta_{x} u & =-\nabla_{x} p+E+u \wedge B, & & \operatorname{div} u=0, \\
\partial_{t} \theta+u \cdot \nabla_{x} \theta-\kappa \Delta_{x} \theta & =0, & \rho+\theta & =0, \\
\partial_{t} E-\operatorname{rot} B & =-u, & \operatorname{div} E & =0, \\
\partial_{t} B+\operatorname{rot} E & =0, & \operatorname{div} B & =0 .
\end{aligned}\right.
$$

This system satisfies the following formal energy conservation laws :

$$
\begin{aligned}
\frac{1}{2} \frac{d}{d t}\left(\|u\|_{L_{x}^{2}}^{2}+\|E\|_{L_{x}^{2}}^{2}\right. & \left.+\|B\|_{L_{x}^{2}}^{2}\right)+\mu\left\|\nabla_{x} u\right\|_{L_{x}^{2}}^{2}=0 \\
\frac{1}{2} \frac{d}{d t}\|\theta\|_{L_{x}^{2}}^{2}+\kappa\left\|\nabla_{x} \theta\right\|_{L_{x}^{2}}^{2} & =0 .
\end{aligned}
$$

(3) If $\alpha=o(\epsilon), \beta=\epsilon$ and $\gamma=o(1)$, we obtain the incompressible quasi-static Navier-Stokes-Fourier-Maxwell system :

$$
\left\{\begin{aligned}
\partial_{t} u+u \cdot \nabla_{x} u-\mu \Delta_{x} u & =-\nabla_{x} p+E+u \wedge B, & & \operatorname{div} u=0, \\
\partial_{t} \theta+u \cdot \nabla_{x} \theta-\kappa \Delta_{x} \theta & =0, & \rho+\theta & =0, \\
\operatorname{rot} B & =u, & \operatorname{div} E & =0, \\
\partial_{t} B+\operatorname{rot} E & =0, & \operatorname{div} B & =0 .
\end{aligned}\right.
$$


This system satisfies the following formal energy conservation laws :

$$
\begin{aligned}
\frac{1}{2} \frac{d}{d t}\left(\|u\|_{L_{x}^{2}}^{2}+\|B\|_{L_{x}^{2}}^{2}\right)+\mu\left\|\nabla_{x} u\right\|_{L_{x}^{2}}^{2} & =0, \\
\frac{1}{2} \frac{d}{d t}\|\theta\|_{L_{x}^{2}}^{2}+\kappa\left\|\nabla_{x} \theta\right\|_{L_{x}^{2}}^{2} & =0 .
\end{aligned}
$$

Here, the electric field is defined indirectly as a mere distribution, through Faraday's equation, by

$$
E=-\partial_{t} A
$$

where $B=\operatorname{rot} A$ and $\operatorname{div} A=0$.

(4) If $\alpha=\epsilon$ and $\gamma=\epsilon$, we obtain the incompressible quasi-static NavierStokes-Fourier-Maxwell-Poisson system :

$$
\left\{\begin{array}{rlrl}
\partial_{t} u+u \cdot \nabla_{x} u-\mu \Delta_{x} u & =-\nabla_{x} p+E+\rho \nabla_{x} \theta+u \wedge B & \\
\partial_{t}\left(\frac{3}{2} \theta-\rho\right)+u \cdot \nabla_{x}\left(\frac{3}{2} \theta-\rho\right)-\frac{5}{2} \kappa \Delta_{x} \theta & =0, & \operatorname{div} u & =0, \\
\operatorname{rot} B & =u, & \Delta_{x}(\rho+\theta) & =\rho, \\
\partial_{t} B+\operatorname{rot} E & =0, & \operatorname{div} E & =\rho, \\
\operatorname{div} B & =0 .
\end{array}\right.
$$

This system satisfies the following formal energy conservation law (see Proposition 3.1 for an explicit computation of the energy) :

$$
\begin{aligned}
\frac{1}{2} \frac{d}{d t}\left(\|\rho\|_{L_{x}^{2}}^{2}+\|u\|_{L_{x}^{2}}^{2}+\frac{3}{2}\|\theta\|_{L_{x}^{2}}^{2}+\left\|P^{\perp} E\right\|_{L_{x}^{2}}^{2}+\|B\|_{L_{x}^{2}}^{2}\right) & \\
& +\mu\left\|\nabla_{x} u\right\|_{L_{x}^{2}}^{2}+\frac{5}{2} \kappa\left\|\nabla_{x} \theta\right\|_{L_{x}^{2}}^{2}=0 .
\end{aligned}
$$

Here, the solenoidal component of the electric field is defined indirectly as a mere distribution, through Faraday's equation, by

$$
P E=-\partial_{t} A,
$$

where $B=\operatorname{rot} A$ and $\operatorname{div} A=0$, while its irrotational component is determined, through Gauss' law, by

$$
P^{\perp} E=\nabla_{x}(\rho+\theta) .
$$

Notice that the equations in this system are all coupled.

(5) If $\alpha=\epsilon$ and $\gamma=o(\epsilon)$, we obtain the incompressible Navier-Stokes-FourierPoisson system :

$$
\left\{\begin{aligned}
\partial_{t} u+u \cdot \nabla_{x} u-\mu \Delta_{x} u & =-\nabla_{x} p+\rho \nabla_{x} \theta, & \operatorname{div} u & =0, \\
\partial_{t}\left(\frac{3}{2} \theta-\rho\right)+u \cdot \nabla_{x}\left(\frac{3}{2} \theta-\rho\right)-\frac{5}{2} \kappa \Delta_{x} \theta & =0, & \Delta_{x}(\rho+\theta) & =\rho .
\end{aligned}\right.
$$

This system satisfies the following formal energy conservation law :

$$
\begin{aligned}
\frac{1}{2} \frac{d}{d t}\left(\|\rho\|_{L_{x}^{2}}^{2}+\|u\|_{L_{x}^{2}}^{2}+\frac{3}{2}\|\theta\|_{L_{x}^{2}}^{2}+\left\|\nabla_{x}(\rho+\theta)\right\|_{L_{x}^{2}}^{2}\right) & \\
+\mu\left\|\nabla_{x} u\right\|_{L_{x}^{2}}^{2} & +\frac{5}{2} \kappa\left\|\nabla_{x} \theta\right\|_{L_{x}^{2}}^{2}=0 .
\end{aligned}
$$

Physically, in this system, the fluid is subject to a self-induced static electric field $E$ determined by

$$
\operatorname{rot} E=0, \quad \operatorname{div} E=\rho,
$$


hence

$$
E=\nabla_{x}(\rho+\theta)
$$

Notice that the equations in this system are all coupled.

2.3.5. The Vlasov-Poisson-Boltzmann system. The Vlasov-Poisson-Boltzmann system describes the evolution of a gas of one species of charged particles (ions or electrons) subject to an auto-induced electrostatic force. This system is obtained formally from the Vlasov-Maxwell-Boltzmann system by letting the speed of light tend to infinity while all other parameters remain fixed. Accordingly, setting $\gamma=0$ in 2.5 yields the scaled Vlasov-Poisson-Boltzmann system :

$$
\left\{\begin{aligned}
\epsilon \partial_{t} f_{\epsilon}+v \cdot \nabla_{x} f_{\epsilon}+\alpha \nabla_{x} \phi_{\epsilon} \cdot \nabla_{v} f_{\epsilon} & =\frac{1}{\epsilon} Q\left(f_{\epsilon}, f_{\epsilon}\right), \\
f_{\epsilon} & =M\left(1+\epsilon g_{\epsilon}\right), \\
\Delta_{x} \phi_{\epsilon} & =\frac{\alpha}{\epsilon} \int_{\mathbb{R}^{3}} g_{\epsilon} M d v .
\end{aligned}\right.
$$

Here, the plasma is subject to a self-induced electrostatic field $E_{\epsilon}$ determined by

$$
\operatorname{rot} E_{\epsilon}=0, \quad \operatorname{div} E_{\epsilon}=\frac{\alpha}{\epsilon} \int_{\mathbb{R}^{3}} g_{\epsilon} M d v,
$$

hence

$$
E_{\epsilon}=\nabla_{x} \phi_{\epsilon}
$$

The above system is supplemented with some initial data satisfying

$$
\frac{1}{\epsilon^{2}} H\left(f_{\epsilon}^{\mathrm{in}}\right)+\frac{1}{2} \int_{\mathbb{R}^{3}}\left|E_{\epsilon}^{\mathrm{in}}\right|^{2} d x<\infty .
$$

In particular, solutions of 2.31 satisfy the corresponding scaled entropy inequality, where $t>0$,

$$
\begin{aligned}
\frac{1}{\epsilon^{2}} H\left(f_{\epsilon}\right)+\frac{1}{2} \int_{\mathbb{R}^{3}}\left|E_{\epsilon}\right|^{2} d x & +\frac{1}{\epsilon^{4}} \int_{0}^{t} \int_{\mathbb{R}^{3}} D\left(f_{\epsilon}\right)(s) d x d s \\
& \leq \frac{1}{\epsilon^{2}} H\left(f_{\epsilon}^{\mathrm{in}}\right)+\frac{1}{2} \int_{\mathbb{R}^{3}}\left|E_{\epsilon}^{\mathrm{in}}\right|^{2} d x .
\end{aligned}
$$

Thus, the formal asymptotic analysis of 2.31) is contained in our analysis of the Vlasov-Maxwell-Boltzmann system 2.5. Specifically, setting $\gamma=\beta=0$ in the limiting systems obtained in Section 2.3.3, we see that the Vlasov-PoissonBoltzmann system 2.31 converges, when $\alpha=o(\epsilon)$, towards the incompressible Navier-Stokes-Fourier system in a Boussinesq regime, with $E=0$ :

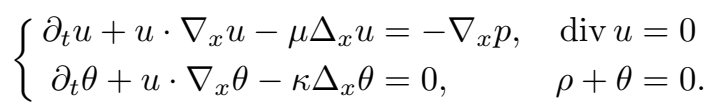

While, when $\left[\frac{\alpha}{\epsilon}\right] \neq 0$, we find the convergence towards the incompressible NavierStokes-Fourier-Poisson system :

$$
\left\{\begin{aligned}
\partial_{t} u+u \cdot \nabla_{x} u-\mu \Delta_{x} u & =-\nabla_{x} p \\
& +\rho \nabla_{x} \theta, \quad \operatorname{div} u=0, \\
\partial_{t}\left(\frac{3}{2} \theta-\rho\right)+u \cdot \nabla_{x}\left(\frac{3}{2} \theta-\rho\right)-\frac{5}{2} \kappa \Delta_{x} \theta & =0, \quad \Delta_{x}(\rho+\theta)=\left[\frac{\alpha}{\epsilon}\right]^{2} \rho,
\end{aligned}\right.
$$

where the electrostatic field is determined by $\left[\frac{\alpha}{\epsilon}\right] E=\nabla_{x}(\rho+\theta)$. 
In fact, the Vlasov-Poisson-Boltzmann system is inherently simpler than the Vlasov-Maxwell-Boltzmann system, because it couples the Vlasov-Boltzmann equation with a simple elliptic equation, namely Poisson's equation, while the VlasovMaxwell-Boltzmann system couples the Vlasov-Boltzmann equation with an hyperbolic system, namely Maxwell's system of equations. Thus, the rigorous mathematical analysis on the Vlasov-Maxwell-Boltzmann system, presented in the remainder of this work, will also apply to the Vlasov-Poisson-Boltzmann system and, therefore, analog results will hold.

\subsection{Formal analysis of the two species asymptotics}

We turn now to the formal asymptotic study of the incompressible viscous regimes of the two species Vlasov-Maxwell-Boltzmann system (1.2). Recall that we are only considering the case of equal masses and opposite charges. The analysis follows exactly the same steps as in the one species case (1.1). However, the situation obviously becomes now more complex and general.

For a plasma of two species of particles, our starting point is the scaled system $(2.32)$

$$
\left\{\begin{aligned}
\epsilon \partial_{t} f_{\epsilon}^{ \pm}+v \cdot \nabla_{x} f_{\epsilon}^{ \pm} \pm\left(\alpha E_{\epsilon}+\beta v \wedge B_{\epsilon}\right) \cdot \nabla_{v} f_{\epsilon}^{ \pm} & =\frac{1}{\epsilon} Q\left(f_{\epsilon}^{ \pm}, f_{\epsilon}^{ \pm}\right)+\frac{\delta^{2}}{\epsilon} Q\left(f_{\epsilon}^{ \pm}, f_{\epsilon}^{\mp}\right), \\
f_{\epsilon}^{ \pm} & =M\left(1+\epsilon g_{\epsilon}^{ \pm}\right), \\
\gamma \partial_{t} E_{\epsilon}-\operatorname{rot} B_{\epsilon} & =-\frac{\beta}{\epsilon} \int_{\mathbb{R}^{3}}\left(g_{\epsilon}^{+}-g_{\epsilon}^{-}\right) v M d v, \\
\gamma \partial_{t} B_{\epsilon}+\operatorname{rot} E_{\epsilon} & =0, \\
\operatorname{div} E_{\epsilon} & =\frac{\alpha}{\epsilon} \int_{\mathbb{R}^{3}}\left(g_{\epsilon}^{+}-g_{\epsilon}^{-}\right) M d v, \\
\operatorname{div} B_{\epsilon} & =0,
\end{aligned}\right.
$$

supplemented with some initial data satisfying

$$
\frac{1}{\epsilon^{2}} H\left(f_{\epsilon}^{+\mathrm{in}}\right)+\frac{1}{\epsilon^{2}} H\left(f_{\epsilon}^{-\mathrm{in}}\right)+\frac{1}{2} \int_{\mathbb{R}^{3}}\left|E_{\epsilon}^{\mathrm{in}}\right|^{2}+\left|B_{\epsilon}^{\mathrm{in}}\right|^{2} d x<\infty,
$$

where $H\left(f_{\epsilon}^{ \pm \mathrm{in}}\right)=H\left(f_{\epsilon}^{ \pm \mathrm{in}} \mid M\right)$. In particular, the corresponding scaled entropy inequality, where $t>0$,

$$
\begin{aligned}
\frac{1}{\epsilon^{2}} H\left(f_{\epsilon}^{+}\right)+\frac{1}{\epsilon^{2}} H\left(f_{\epsilon}^{-}\right) & +\frac{1}{2} \int_{\mathbb{R}^{3}}\left|E_{\epsilon}\right|^{2}+\left|B_{\epsilon}\right|^{2} d x \\
& +\frac{1}{\epsilon^{4}} \int_{0}^{t} \int_{\mathbb{R}^{3}}\left(D\left(f_{\epsilon}^{+}\right)+D\left(f_{\epsilon}^{-}\right)+\delta^{2} D\left(f_{\epsilon}^{+}, f_{\epsilon}^{-}\right)\right)(s) d x d s \\
& \leq \frac{1}{\epsilon^{2}} H\left(f_{\epsilon}^{+\mathrm{in}}\right)+\frac{1}{\epsilon^{2}} H\left(f_{\epsilon}^{-\mathrm{in}}\right)+\frac{1}{2} \int_{\mathbb{R}^{3}}\left|E_{\epsilon}^{\mathrm{in}}\right|^{2}+\left|B_{\epsilon}^{\mathrm{in}}\right|^{2} d x,
\end{aligned}
$$

guarantees that the solution will remain - for all non-negative times - a fluctuation of order $\epsilon$ around the global equilibrium $M$ :

$$
f_{\epsilon}^{ \pm}=M\left(1+\epsilon g_{\epsilon}^{ \pm}\right) .
$$

Note that the kinetic equations in 2.32 can then be rewritten, in terms of the fluctuation $g_{\epsilon}$, as

$$
\begin{aligned}
& \epsilon \partial_{t}\left(\begin{array}{c}
g_{\epsilon}^{+} \\
g_{\epsilon}^{-}
\end{array}\right)+v \cdot \nabla_{x}\left(\begin{array}{c}
g_{\epsilon}^{+} \\
g_{\epsilon}^{-}
\end{array}\right)+\left(\alpha E_{\epsilon}+\beta v \wedge B_{\epsilon}\right) \cdot \nabla_{v}\left(\begin{array}{c}
g_{\epsilon}^{+} \\
-g_{\epsilon}^{-}
\end{array}\right)-\frac{\alpha}{\epsilon} E_{\epsilon} \cdot v\left(\begin{array}{c}
1+\epsilon g_{\epsilon}^{+} \\
-1-\epsilon g_{\epsilon}^{-}
\end{array}\right) \\
&=-\frac{1}{\epsilon}\left(\begin{array}{c}
\mathcal{L} g_{\epsilon}^{+}+\delta^{2} \mathcal{L}\left(g_{\epsilon}^{+}, g_{\epsilon}^{-}\right) \\
\mathcal{L} g_{\epsilon}^{-}+\delta^{2} \mathcal{L}\left(g_{\epsilon}^{-}, g_{\epsilon}^{+}\right)
\end{array}\right)+\left(\begin{array}{c}
\mathcal{Q}\left(g_{\epsilon}^{+}, g_{\epsilon}^{+}\right)+\delta^{2} \mathcal{Q}\left(g_{\epsilon}^{+}, g_{\epsilon}^{-}\right) \\
\mathcal{Q}\left(g_{\epsilon}^{-}, g_{\epsilon}^{-}\right)+\delta^{2} \mathcal{Q}\left(g_{\epsilon}^{-}, g_{\epsilon}^{+}\right)
\end{array}\right),
\end{aligned}
$$


where we denote

$$
\mathcal{L}(g, h)=-\frac{1}{M}(Q(M g, M)+Q(M, M h)) \quad \text { and } \quad \mathcal{Q}(g, h)=\frac{1}{M} Q(M g, M h) .
$$

It turns out that, in the limit $\epsilon \rightarrow 0$, we will have now three types of constraints :

- conditions on the velocity profiles coming from the fast relaxation towards thermodynamic equilibrium (i.e. small Knudsen regime, see Section 2.4.1) ;

- linear macroscopic hydrodynamic constraints due to the weak compressibility (i.e. small Mach regime, see Section 2.4.3) ;

- nonlinear macroscopic electrodynamic constraints coming from momentum and energy exchange between species due to interspecies collisions (see Section 2.4.5).

As in the case of one species of charged particles, we expect the first two types of constraints to be weakly stable, and thus to be derived from simple uniform a priori estimates. The procedure leading to the last couple of electrodynamic constraint equations (including Ohm's law) is a little bit more complex and will depend on the strength of interspecies collisional interactions, that is to say, on the size of $\delta>0$ compared to $\epsilon>0$. In fact, the nature of the whole asymptotic systems obtained in the limit $\epsilon \rightarrow 0$ will be conditioned by the size of $\delta$, and we will therefore distinguish three different asymptotic regimes :

- Very weak interspecies collisional interactions, $\delta=O(\epsilon)$; in this regime, the interspecies collision operators $\frac{\delta^{2}}{\epsilon} Q\left(f_{\epsilon}^{ \pm}, f_{\epsilon}^{\mp}\right)$ in 2.32 are a regular perturbation. Therefore, the corresponding limiting systems will be composed of two hydrodynamic systems - one for each species - coupled mainly through the mean field interactions of the electromagnetic forces. The derivation of these regimes will be easily deduced from the asymptotic analysis for one species from Section 2.3 and will therefore be treated first in Section 2.4.2

- Weak interspecies collisional interactions, $\delta=o(1)$ and $\frac{\delta}{\epsilon}$ unbounded ; in this regime, the interspecies collision operators $\frac{\delta^{2}}{\epsilon} Q\left(f_{\epsilon}^{ \pm}, f_{\epsilon}^{\mp}\right)$ in 2.32 are a singular perturbation, whose order may vary from the other singular perturbations present in the system 2.32. In particular, it is not the most singular perturbation of 2.32.

- Strong interspecies collisional interactions, $\delta \sim 1$; in this regime, the interspecies collision operators $\frac{\delta^{2}}{\epsilon} Q\left(f_{\epsilon}^{ \pm}, f_{\epsilon}^{\mp}\right)$ in 2.32 are a singular perturbation of the most singular order present in the system 2.32 .

2.4.1. Thermodynamic equilibrium. The entropy inequality 2.33 provides uniform bounds on $E_{\epsilon}, B_{\epsilon}, g_{\epsilon}^{+}$and $g_{\epsilon}^{-}$. Therefore, assuming some formal compactness, up to extraction of subsequences, one has

$$
E_{\epsilon} \rightarrow E, \quad B_{\epsilon} \rightarrow B, \quad g_{\epsilon}^{ \pm} \rightarrow g^{ \pm},
$$

in a weak sense to be rigorously detailed in a subsequent chapter.

Then, multiplying (2.34) by $\epsilon$, and taking formal limits as $\epsilon \rightarrow 0$ shows that

$$
\lim _{\epsilon \rightarrow 0} \mathbf{L}_{\delta}\left(\begin{array}{c}
g_{\epsilon}^{+} \\
g_{\epsilon}^{-}
\end{array}\right)=\mathbf{L}_{[\delta]}\left(\begin{array}{c}
g^{+} \\
g^{-}
\end{array}\right)=\left(\begin{array}{l}
0 \\
0
\end{array}\right)
$$

where

$$
\mathbf{L}_{\delta}\left(\begin{array}{l}
g \\
h
\end{array}\right)=\left(\begin{array}{c}
\mathcal{L} g+\delta^{2} \mathcal{L}(g, h) \\
\mathcal{L} h+\delta^{2} \mathcal{L}(h, g)
\end{array}\right)=\left(\begin{array}{c}
\mathcal{L} g+\delta^{2} \int_{\mathbb{R}^{3} \times \mathbb{S}^{2}}\left(g+h_{*}-g^{\prime}-h_{*}^{\prime}\right) b M_{*} d v_{*} d \sigma \\
\mathcal{L} h+\delta^{2} \int_{\mathbb{R}^{3} \times \mathbb{S}^{2}}\left(h+g_{*}-h^{\prime}-g_{*}^{\prime}\right) b M_{*} d v_{*} d \sigma
\end{array}\right)
$$


and

$\mathbf{L}_{[\delta]}\left(\begin{array}{l}g \\ h\end{array}\right)=\left(\begin{array}{l}\mathcal{L} g+[\delta]^{2} \mathcal{L}(g, h) \\ \mathcal{L} h+[\delta]^{2} \mathcal{L}(h, g)\end{array}\right)=\left(\begin{array}{l}\mathcal{L} g+[\delta]^{2} \int_{\mathbb{R}^{3} \times \mathbb{S}^{2}}\left(g+h_{*}-g^{\prime}-h_{*}^{\prime}\right) b M_{*} d v_{*} d \sigma \\ \mathcal{L} h+[\delta]^{2} \int_{\mathbb{R}^{3} \times \mathbb{S}^{2}}\left(h+g_{*}-h^{\prime}-g_{*}^{\prime}\right) b M_{*} d v_{*} d \sigma\end{array}\right)$.

It can be shown (see Proposition 5.7) that, when $[\delta] \neq 0$, the kernel of the vectorial linearized Boltzmann operators $\mathbf{L}_{\delta}$ and $\mathbf{L}_{[\delta]}$ coincide exactly with the vector space spanned by the set

$$
\left\{\left(\begin{array}{l}
1 \\
0
\end{array}\right),\left(\begin{array}{l}
0 \\
1
\end{array}\right),\left(\begin{array}{l}
v_{1} \\
v_{1}
\end{array}\right),\left(\begin{array}{l}
v_{2} \\
v_{2}
\end{array}\right),\left(\begin{array}{l}
v_{3} \\
v_{3}
\end{array}\right),\left(\begin{array}{l}
|v|^{2} \\
|v|^{2}
\end{array}\right)\right\} .
$$

However, when $[\delta]=0$, the kernel of $\mathbf{L}_{[\delta]}$ is larger and is composed of all vectors $\left(\begin{array}{l}\varphi_{1}(v) \\ \varphi_{2}(v)\end{array}\right)$ such that $\varphi_{1}(v)$ and $\varphi_{2}(v)$ are collision invariants whose coefficients are independent.

Thus, we conclude, if $[\delta] \neq 0$, that $g^{ \pm}$is an infinitesimal Maxwellian of the form

$$
\left(\begin{array}{l}
g^{+} \\
g^{-}
\end{array}\right)=\left(\begin{array}{l}
\rho^{+}+u \cdot v+\theta\left(\frac{|v|^{2}}{2}-\frac{3}{2}\right) \\
\rho^{-}+u \cdot v+\theta\left(\frac{|v|^{2}}{2}-\frac{3}{2}\right)
\end{array}\right)
$$

while, if $[\delta]=0$,

$$
\left(\begin{array}{l}
g^{+} \\
g^{-}
\end{array}\right)=\left(\begin{array}{l}
\rho^{+}+u^{+} \cdot v+\theta^{+}\left(\frac{|v|^{2}}{2}-\frac{3}{2}\right) \\
\rho^{-}+u^{-} \cdot v+\theta^{-}\left(\frac{|v|^{2}}{2}-\frac{3}{2}\right)
\end{array}\right)
$$

where $\rho^{+}, \rho^{-} \in \mathbb{R}, u, u^{+}, u^{-} \in \mathbb{R}^{3}$ and $\theta, \theta^{+}, \theta^{-} \in \mathbb{R}$ only depend on $t$ and $x$, and are respectively the fluctuations of density, bulk velocity and temperature.

In fact, whenever $\frac{\delta}{\epsilon}$ is unbounded, we show below that necessarily $u^{+}=u^{-}$and $\theta^{+}=\theta^{-}$, as well, because of higher order singular limiting constraints. Therefore, the infinitesimal Maxwellian form (2.38) will be assumed by the limiting fluctuations in the case $\delta=O(\epsilon)$ only, that is in the case of very weak interspecies collisions.

The fact that the fluctuations assume the infinitesimal Maxwellian form describes that the gas reaches thermodynamic (or statistical) equilibrium, in the fast relaxation limit.

We define now the macroscopic fluctuations of density $\rho_{\epsilon}^{ \pm}$, bulk velocity $u_{\epsilon}^{ \pm}$ and temperature $\theta_{\epsilon}^{ \pm}$by

$$
\begin{aligned}
& \rho_{\epsilon}^{ \pm}=\int_{\mathbb{R}^{3}} g_{\epsilon}^{ \pm} M d v, \\
& u_{\epsilon}^{ \pm}=\int_{\mathbb{R}^{3}} g_{\epsilon}^{ \pm} v M d v, \\
& \theta_{\epsilon}^{ \pm}=\int_{\mathbb{R}^{3}} g_{\epsilon}^{ \pm}\left(\frac{|v|^{2}}{3}-1\right) M d v,
\end{aligned}
$$

and the hydrodynamic projection $\Pi g_{\epsilon}^{ \pm}$of $g_{\epsilon}^{ \pm}$by

$$
\Pi g_{\epsilon}^{ \pm}=\rho_{\epsilon}^{ \pm}+u_{\epsilon}^{ \pm} \cdot v+\theta_{\epsilon}^{ \pm}\left(\frac{|v|^{2}}{2}-\frac{3}{2}\right)
$$

which is nothing but the orthogonal projection of $g_{\epsilon}^{ \pm}$onto the kernel of $\mathcal{L}$ in $L^{2}(M d v)$.

Note that the previous step establishing the convergence of $g_{\epsilon}^{ \pm}$towards thermodynamic equilibrium yields, in fact, the uniform boundedness of $\frac{1}{\epsilon} \mathbf{L}_{\delta}\left(\begin{array}{l}g_{\epsilon}^{+} \\ g_{\epsilon}^{-}\end{array}\right)$. 
Therefore, if $[\delta] \neq 0$, we deduce, at least formally, that

$$
\left(\begin{array}{c}
g_{\epsilon}^{+} \\
g_{\epsilon}^{-}
\end{array}\right)-\left(\begin{array}{c}
\frac{\rho_{\epsilon}^{+}-\rho_{\epsilon}^{-}}{2}+\Pi \frac{g_{\epsilon}^{+}+g_{\epsilon}^{-}}{2} \\
\frac{\rho_{\epsilon}^{-}-\rho_{\epsilon}^{+}}{2}+\Pi \frac{g_{\epsilon}^{+}+g_{\epsilon}^{-}}{2}
\end{array}\right)=O(\epsilon)
$$

where $\left(\begin{array}{l}\frac{\rho_{\epsilon}^{+}-\rho_{\epsilon}^{-}}{2}+\Pi \frac{g_{\epsilon}^{+}+g_{\epsilon}^{-}}{2} \\ \frac{\rho_{\epsilon}^{-}-\rho_{\epsilon}^{+}}{2}+\Pi \frac{g_{\epsilon}^{+}+g_{\epsilon}^{-}}{2}\end{array}\right)$ clearly defines the orthogonal projection of $\left(\begin{array}{l}g_{\epsilon}^{+} \\ g_{\epsilon}^{-}\end{array}\right)$onto the kernel of $\mathbf{L}_{\delta}$, which is spanned by 2.36. This bound implies, in particular, that

$$
\left(g_{\epsilon}^{+}-\rho_{\epsilon}^{+}\right)-\left(g_{\epsilon}^{-}-\rho_{\epsilon}^{-}\right)=O(\epsilon) \quad \text { and } \quad g_{\epsilon}^{ \pm}-\Pi g_{\epsilon}^{ \pm}=O(\epsilon) .
$$

However, if $[\delta]=0$, we can only formally deduce, for the moment, that

$$
g_{\epsilon}^{ \pm}-\Pi g_{\epsilon}^{ \pm}=O(\epsilon) \text {. }
$$

Just as in the one species case (see Section 2.3.1), the convergence of $g_{\epsilon}^{+}$and $g_{\epsilon}^{-}$with a rate $O(\epsilon)$ towards their hydrodynamic projections $\Pi g_{\epsilon}^{+}$and $\Pi g_{\epsilon}^{-}$can also be inferred, at least formally, from the uniform control of the entropies dissipations $\frac{1}{\epsilon^{4}} D\left(f_{\epsilon}^{+}\right)$and $\frac{1}{\epsilon^{4}} D\left(f_{\epsilon}^{-}\right)$in 2.33 . We are now going to show how the exact same formal reasoning applied to the control of the mixed entropy dissipation $\frac{\delta^{2}}{\epsilon^{4}} D\left(f_{\epsilon}^{+}, f_{\epsilon}^{-}\right)$ in 2.33 yields formally that

$$
\left(g_{\epsilon}^{+}-\rho_{\epsilon}^{+}\right)-\left(g_{\epsilon}^{-}-\rho_{\epsilon}^{-}\right)=O\left(\frac{\epsilon}{\delta}\right),
$$

which is not so readily deduced by direct inspection of (2.34). Note that this control is relevant in the cases of weak or strong interspecies interactions only, that is when $\frac{\delta}{\epsilon}$ is unbounded.

Thus, as in Section 2.3.1. formally approximating $z \log (1+z)$ by $z^{2}$, which is valid in a neighborhood of $z=0$, in the definition 1.16 of the mixed entropy dissipation, we deduce a control of

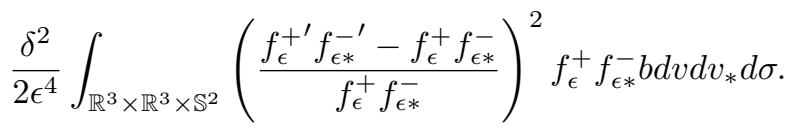

Then, since

$$
\begin{aligned}
& f_{\epsilon}^{+^{\prime}} f_{\epsilon *}^{{ }^{\prime}}-f_{\epsilon}^{+} f_{\epsilon *}^{-} \\
& \quad=\epsilon\left(\left(\Pi g_{\epsilon}^{+}\right)^{\prime}+\left(\Pi g_{\epsilon}^{-}\right)_{*}^{\prime}-\left(\Pi g_{\epsilon}^{+}\right)-\left(\Pi g_{\epsilon}^{-}\right)_{*}\right) \\
& \quad+\epsilon^{2}\left(\left(\frac{g_{\epsilon}^{+}-\Pi g_{\epsilon}^{+}}{\epsilon}\right)^{\prime}+\left(\frac{g_{\epsilon}^{-}-\Pi g_{\epsilon}^{-}}{\epsilon}\right)_{*}^{\prime}-\left(\frac{g_{\epsilon}^{+}-\Pi g_{\epsilon}^{+}}{\epsilon}\right)-\left(\frac{g_{\epsilon}^{-}-\Pi g_{\epsilon}^{-}}{\epsilon}\right)_{*}\right) \\
& \quad+\epsilon^{2}\left(g_{\epsilon}^{+^{\prime}} g_{\epsilon *}^{-{ }^{\prime}}-g_{\epsilon}^{+} g_{\epsilon *}^{-}\right),
\end{aligned}
$$

we infer that $\frac{\delta}{\epsilon}\left(\left(\Pi g_{\epsilon}^{+}\right)^{\prime}+\left(\Pi g_{\epsilon}^{-}\right)_{*}^{\prime}-\left(\Pi g_{\epsilon}^{+}\right)-\left(\Pi g_{\epsilon}^{-}\right)_{*}\right)$ is uniformly bounded. Finally, a direct computation of the integral

$$
\frac{\delta^{2}}{\epsilon^{2}} \int_{\mathbb{R}^{3} \times \mathbb{R}^{3} \times \mathbb{S}^{2}}\left(\left(\Pi g_{\epsilon}^{+}\right)^{\prime}+\left(\Pi g_{\epsilon}^{-}\right)_{*}^{\prime}-\left(\Pi g_{\epsilon}^{+}\right)-\left(\Pi g_{\epsilon}^{-}\right)_{*}\right)^{2} b d v d v_{*} d \sigma
$$

shows that

$$
u^{+}-u^{-}=O\left(\frac{\epsilon}{\delta}\right) \quad \text { and } \quad \theta^{+}-\theta^{-}=O\left(\frac{\epsilon}{\delta}\right)
$$

which incidentally establishes 2.39 . Of course, the rigorous demonstration of such bounds, later on in Section 5.3.2 will necessitate the control of the large values of the fluctuations in order to justify the formal approximation of $z \log (1+z)$ by $z^{2}$. 
On the whole, we have shown that, for all cases of strong, weak and very weak interspecies interactions, it holds

$$
g_{\epsilon}^{ \pm}-\Pi g_{\epsilon}^{ \pm}=O(\epsilon) \quad \text { and } \quad\left(g_{\epsilon}^{+}-\rho_{\epsilon}^{+}\right)-\left(g_{\epsilon}^{-}-\rho_{\epsilon}^{-}\right)=O\left(\frac{\epsilon}{\delta}\right) .
$$

Note that this implies that

$$
\begin{aligned}
\left(\begin{array}{c}
g_{\epsilon}^{+} \\
g_{\epsilon}^{-}
\end{array}\right) & -\left(\begin{array}{l}
\frac{\rho_{\epsilon}^{+}-\rho_{\epsilon}^{-}}{2}+\Pi \frac{g_{\epsilon}^{+}+g_{\epsilon}^{-}}{2} \\
\frac{\rho_{\epsilon}^{-}-\rho_{\epsilon}^{+}}{2}+\Pi \frac{g_{\epsilon}^{+}+g_{\epsilon}^{-}}{2}
\end{array}\right) \\
& =\left(\begin{array}{c}
g_{\epsilon}^{+} \\
g_{\epsilon}^{-}
\end{array}\right)-\left(\begin{array}{l}
\Pi g_{\epsilon}^{+} \\
\Pi g_{\epsilon}^{-}
\end{array}\right)+\left(\begin{array}{c}
\Pi \frac{\left(g_{\epsilon}^{+}-\rho_{\epsilon}^{+}\right)-\left(g_{\epsilon}^{-}-\rho_{\epsilon}^{-}\right)}{2} \\
-\Pi \frac{\left(g_{\epsilon}^{+}-\rho_{\epsilon}^{+}\right)-\left(g_{\epsilon}^{-}-\rho_{\epsilon}^{-}\right)}{2}
\end{array}\right)=O\left(\frac{\epsilon}{\delta}\right) .
\end{aligned}
$$

We will therefore henceforth denote, when considering weak or strong interspecies collisions,

$$
\left(\begin{array}{c}
h_{\epsilon}^{+} \\
h_{\epsilon}^{-}
\end{array}\right)=\frac{\delta}{\epsilon}\left[\left(\begin{array}{c}
g_{\epsilon}^{+} \\
g_{\epsilon}^{-}
\end{array}\right)-\left(\begin{array}{c}
\frac{\rho_{\epsilon}^{+}-\rho_{\epsilon}^{-}}{2}+\Pi \frac{g_{\epsilon}^{+}+g_{\epsilon}^{-}}{2} \\
\frac{\rho_{\epsilon}^{-}-\rho_{\epsilon}^{+}}{2}+\Pi \frac{g_{\epsilon}^{+}+g_{\epsilon}^{-}}{2}
\end{array}\right)\right] .
$$

In particular, further note that, for weak interspecies collisions, that is whenever $\delta=o(1)$ and $\frac{\delta}{\epsilon}$ is unbounded,

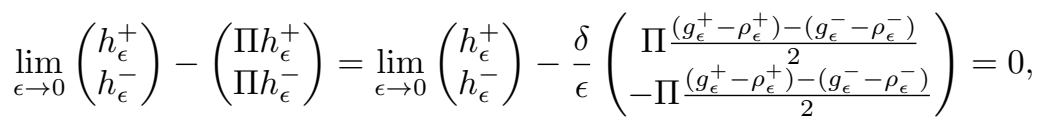

so that the weak limits $h_{\epsilon}^{ \pm} \rightarrow h^{ \pm}$are necessarily infinitesimal Maxwellians. But this does not seem to hold for strong interspecies interactions, that is $\delta \sim 1$.

In light of the above formal controls, we define new macroscopic hydrodynamic variables

$$
\rho_{\epsilon}=\frac{\rho_{\epsilon}^{+}+\rho_{\epsilon}^{-}}{2}, \quad u_{\epsilon}=\frac{u_{\epsilon}^{+}+u_{\epsilon}^{-}}{2}, \quad \theta_{\epsilon}=\frac{\theta_{\epsilon}^{+}+\theta_{\epsilon}^{-}}{2},
$$

and electrodynamic variables (irrelevant for very weak interspecies collisions because $\frac{\delta}{\epsilon}$ is bounded in this case)

$$
n_{\epsilon}=\rho_{\epsilon}^{+}-\rho_{\epsilon}^{-}, \quad j_{\epsilon}=\frac{\delta}{\epsilon}\left(u_{\epsilon}^{+}-u_{\epsilon}^{-}\right), \quad w_{\epsilon}=\frac{\delta}{\epsilon}\left(\theta_{\epsilon}^{+}-\theta_{\epsilon}^{-}\right),
$$

namely the electric charge $n_{\epsilon}$, the electric current $j_{\epsilon}$ and the internal electric energy $w_{\epsilon}$. We will also consider their formal weak limits

$$
\rho_{\epsilon} \rightarrow \rho, \quad u_{\epsilon} \rightarrow u, \quad \theta_{\epsilon} \rightarrow \theta, \quad n_{\epsilon} \rightarrow n, \quad j_{\epsilon} \rightarrow j, \quad w_{\epsilon} \rightarrow w .
$$

Notice that

$$
\Pi h_{\epsilon}^{ \pm}= \pm \frac{1}{2}\left(j_{\epsilon} \cdot v+w_{\epsilon}\left(\frac{|v|^{2}}{2}-\frac{3}{2}\right)\right),
$$

hence, for weak interspecies collisions,

$$
\lim _{\epsilon \rightarrow 0} h_{\epsilon}^{ \pm}=h^{ \pm}= \pm \frac{1}{2}\left(j \cdot v+w\left(\frac{|v|^{2}}{2}-\frac{3}{2}\right)\right),
$$

whereas, for strong interspecies collisions, we only have that

$$
\lim _{\epsilon \rightarrow 0} \Pi h_{\epsilon}^{ \pm}=\Pi h^{ \pm}= \pm \frac{1}{2}\left(j \cdot v+w\left(\frac{|v|^{2}}{2}-\frac{3}{2}\right)\right) .
$$

The asymptotic dynamics of $\left(\rho_{\epsilon}^{+}, u_{\epsilon}^{+}, \theta_{\epsilon}^{+}, \rho_{\epsilon}^{-}, u_{\epsilon}^{-}, \theta_{\epsilon}^{-}\right)$, or equivalently $\left(\rho_{\epsilon}, u_{\epsilon}, \theta_{\epsilon}, q_{\epsilon}, j_{\epsilon}, w_{\epsilon}\right)$, is then governed by fluid equations, to be obtained from the moments equations 
associated with 2.34). Thus, successively multiplying (2.34 by the collision invariants $1, v$ and $\frac{|v|^{2}}{2}$, and integrating in $M d v$, yields

$(2.43)$

$$
\left\{\begin{array}{c}
\partial_{t} \rho_{\epsilon}^{ \pm}+\frac{1}{\epsilon} \operatorname{div} u_{\epsilon}^{ \pm}=0 \\
\partial_{t} u_{\epsilon}^{ \pm}+\frac{1}{\epsilon} \nabla_{x}\left(\rho_{\epsilon}^{ \pm}+\theta_{\epsilon}^{ \pm}\right) \mp \frac{\alpha}{\epsilon^{2}} E_{\epsilon}+\frac{\delta^{2}}{\epsilon^{2}} \int_{\mathbb{R}^{3}} \mathcal{L}\left(g_{\epsilon}^{ \pm}, g_{\epsilon}^{\mp}\right) v M d v= \\
\pm\left(\frac{\alpha}{\epsilon} \rho_{\epsilon}^{ \pm} E_{\epsilon}+\frac{\beta}{\epsilon} u_{\epsilon}^{ \pm} \wedge B_{\epsilon}\right) \\
-\frac{1}{\epsilon} \operatorname{div} \int_{\mathbb{R}^{3}} g_{\epsilon}^{ \pm} \phi M d v+\frac{\delta^{2}}{\epsilon} \int_{\mathbb{R}^{3}} \mathcal{Q}\left(g_{\epsilon}^{ \pm}, g_{\epsilon}^{\mp}\right) v M d v \\
\frac{3}{2} \partial_{t}\left(\rho_{\epsilon}^{ \pm}+\theta_{\epsilon}^{ \pm}\right)+\frac{5}{2 \epsilon} \operatorname{div} u_{\epsilon}^{ \pm}+\frac{\delta^{2}}{\epsilon^{2}} \int_{\mathbb{R}^{3}} \mathcal{L}\left(g_{\epsilon}^{ \pm}, g_{\epsilon}^{\mp}\right) \frac{|v|^{2}}{2} M d v= \pm \frac{\alpha}{\epsilon} u_{\epsilon}^{ \pm} \cdot E_{\epsilon} \\
-\frac{1}{\epsilon} \operatorname{div} \int_{\mathbb{R}^{3}} g_{\epsilon}^{ \pm} \psi M d v+\frac{\delta^{2}}{\epsilon} \int_{\mathbb{R}^{3}} \mathcal{Q}\left(g_{\epsilon}^{ \pm}, g_{\epsilon}^{\mp}\right) \frac{|v|^{2}}{2} M d v
\end{array}\right.
$$

where $\phi(v)$ and $\psi(v)$ have already been defined in 2.12 . The above system will be used in the case of very weak interspecies interactions only. For weak and strong interspecies interactions, the evolution equations can then be recast, in terms of the new hydrodynamic and electrodynamic variables, as

$$
\left\{\begin{aligned}
\partial_{t} \rho_{\epsilon}+\frac{1}{\epsilon} \operatorname{div} u_{\epsilon} & =0 \\
\partial_{t} u_{\epsilon}+\frac{1}{\epsilon} \nabla_{x}\left(\rho_{\epsilon}+\theta_{\epsilon}\right) & =\left(\frac{\alpha}{2 \epsilon} n_{\epsilon} E_{\epsilon}+\frac{\beta}{2 \delta} j_{\epsilon} \wedge B_{\epsilon}\right) \\
& -\frac{1}{\epsilon} \operatorname{div} \int_{\mathbb{R}^{3}} \frac{g_{\epsilon}^{+}+g_{\epsilon}^{-}}{2} \phi M d v \\
\frac{3}{2} \partial_{t} \theta_{\epsilon}+\frac{1}{\epsilon} \operatorname{div} u_{\epsilon} & =\frac{\alpha}{2 \delta} j_{\epsilon} \cdot E_{\epsilon}-\frac{1}{\epsilon} \operatorname{div} \int_{\mathbb{R}^{3}} \frac{g_{\epsilon}^{+}+g_{\epsilon}^{-}}{2} \psi M d v .
\end{aligned}\right.
$$

Recall that we are assuming $\alpha=O(\epsilon)$ and $\beta=O(\epsilon)$ for very weak interspecies collisions, i.e. when $\delta=O(\epsilon)$, and $\alpha=O(\epsilon)$ and $\beta=O(\delta)$ for weak and strong interspecies collisions, i.e. when $\frac{\delta}{\epsilon}$ is unbounded. Hence, the nonlinear terms in the right-hand side of (2.43), for very weak interspecies collisions, and (2.44), for weak and strong interspecies collisions, containing the electromagnetic fields are expected to be bounded. Furthermore, just as in the case of one species, the polynomials $\phi(v)$ and $\psi(v)$ are orthogonal to the collision invariants in the $L^{2}(M d v)$ inner-product. That is to say $\int_{\mathbb{R}^{3}} \varphi \phi M d v=0$ and $\int_{\mathbb{R}^{3}} \varphi \psi M d v=0$, for all collision invariants $\varphi(v)$. Since, according to 2.40 , the fluctuations $g_{\epsilon}^{+}$and $g_{\epsilon}^{-}$converge towards infinitesimal Maxwellians with a rate $O(\epsilon)$, it is therefore natural to expect, at least formally, that the terms

$$
\begin{aligned}
& \frac{1}{\epsilon} \int_{\mathbb{R}^{3}} g_{\epsilon}^{ \pm} \phi M d v=\frac{1}{\epsilon} \int_{\mathbb{R}^{3}}\left(g_{\epsilon}^{ \pm}-\Pi g_{\epsilon}^{ \pm}\right) \phi M d v, \\
& \frac{1}{\epsilon} \int_{\mathbb{R}^{3}} g_{\epsilon}^{ \pm} \psi M d v=\frac{1}{\epsilon} \int_{\mathbb{R}^{3}}\left(g_{\epsilon}^{ \pm}-\Pi g_{\epsilon}^{ \pm}\right) \psi M d v
\end{aligned}
$$

in 2.43 and 2.44 are bounded and have a limit. 
Thus, following the strategy for one species in Section 2.3.1, we rewrite 2.43 as

$$
\left\{\begin{array}{c}
\partial_{t} \rho_{\epsilon}^{ \pm}+\frac{1}{\epsilon} \operatorname{div} u_{\epsilon}^{ \pm}=0 \\
\partial_{t} u_{\epsilon}^{ \pm}+\frac{1}{\epsilon} \nabla_{x}\left(\rho_{\epsilon}^{ \pm}+\theta_{\epsilon}^{ \pm}\right) \mp \frac{\alpha}{\epsilon^{2}} E_{\epsilon}+\frac{\delta^{2}}{\epsilon^{2}} \int_{\mathbb{R}^{3}} \mathcal{L}\left(g_{\epsilon}^{ \pm}, g_{\epsilon}^{\mp}\right) v M d v= \\
\pm\left(\frac{\alpha}{\epsilon} \rho_{\epsilon}^{ \pm} E_{\epsilon}+\frac{\beta}{\epsilon} u_{\epsilon}^{ \pm} \wedge B_{\epsilon}\right) \\
-\frac{1}{\epsilon} \operatorname{div} \int_{\mathbb{R}^{3}} \mathcal{L} g_{\epsilon}^{ \pm} \tilde{\phi} M d v+\frac{\delta^{2}}{\epsilon} \int_{\mathbb{R}^{3}} \mathcal{Q}\left(g_{\epsilon}^{ \pm}, g_{\epsilon}^{\mp}\right) v M d v \\
\frac{3}{2} \partial_{t} \theta_{\epsilon}^{ \pm}+\frac{1}{\epsilon} \operatorname{div} u_{\epsilon}^{ \pm}+\frac{\delta^{2}}{\epsilon^{2}} \int_{\mathbb{R}^{3}} \mathcal{L}\left(g_{\epsilon}^{ \pm}, g_{\epsilon}^{\mp}\right) \frac{|v|^{2}}{2} M d v= \pm \frac{\alpha}{\epsilon} u_{\epsilon}^{ \pm} \cdot E_{\epsilon} \\
-\frac{1}{\epsilon} \operatorname{div} \int_{\mathbb{R}^{3}} \mathcal{L} g_{\epsilon}^{ \pm} \tilde{\psi} M d v+\frac{\delta^{2}}{\epsilon} \int_{\mathbb{R}^{3}} \mathcal{Q}\left(g_{\epsilon}^{ \pm}, g_{\epsilon}^{\mp}\right) \frac{|v|^{2}}{2} M d v
\end{array}\right.
$$

and (2.44) as

$$
\left\{\begin{aligned}
\partial_{t} \rho_{\epsilon}+\frac{1}{\epsilon} \operatorname{div} u_{\epsilon} & =0 \\
\partial_{t} u_{\epsilon}+\frac{1}{\epsilon} \nabla_{x}\left(\rho_{\epsilon}+\theta_{\epsilon}\right) & =\left(\frac{\alpha}{2 \epsilon} n_{\epsilon} E_{\epsilon}+\frac{\beta}{2 \delta} j_{\epsilon} \wedge B_{\epsilon}\right) \\
& -\frac{1}{2 \epsilon} \operatorname{div} \int_{\mathbb{R}^{3}} \mathcal{L}\left(g_{\epsilon}^{+}+g_{\epsilon}^{-}\right) \tilde{\phi} M d v \\
\frac{3}{2} \partial_{t} \theta_{\epsilon}+\frac{1}{\epsilon} \operatorname{div} u_{\epsilon} & =\frac{\alpha}{2 \delta} j_{\epsilon} \cdot E_{\epsilon}-\frac{1}{2 \epsilon} \operatorname{div} \int_{\mathbb{R}^{3}} \mathcal{L}\left(g_{\epsilon}^{+}+g_{\epsilon}^{-}\right) \tilde{\psi} M d v
\end{aligned}\right.
$$

where $\tilde{\phi}$ and $\tilde{\psi}$ are the pseudo-inverses of $\phi$ and $\psi$, respectively, defined in (2.13), and where the terms $\frac{1}{\epsilon} \mathcal{L} g_{\epsilon}^{ \pm}$will be expressed employing the Vlasov-Boltzmann equations (2.34). Each of the above macroscopic systems (2.45) and (2.46) is coupled with Maxwell's equations on $E_{\epsilon}$ and $B_{\epsilon}$ :

$$
\left\{\begin{aligned}
\gamma \partial_{t} E_{\epsilon}-\operatorname{rot} B_{\epsilon} & =-\frac{\beta}{\epsilon}\left(u_{\epsilon}^{+}-u_{\epsilon}^{-}\right)=-\frac{\beta}{\delta} j_{\epsilon}, \\
\gamma \partial_{t} B_{\epsilon}+\operatorname{rot} E_{\epsilon} & =0 \\
\operatorname{div} E_{\epsilon} & =\frac{\alpha}{\epsilon}\left(\rho_{\epsilon}^{+}-\rho_{\epsilon}^{-}\right)=\frac{\alpha}{\epsilon} n_{\epsilon} \\
\operatorname{div} B_{\epsilon} & =0
\end{aligned}\right.
$$

A careful formal analysis of the whole coupled macroscopic systems 2.45)(2.47), for very weak interspecies collisions, and 2.46-2.47), for weak and strong interspecies collisions, will yield the asymptotic dynamics of $\left(\rho^{ \pm}, u^{ \pm}, \theta^{ \pm}, E, B\right)$ and $(\rho, u, \theta, E, B)$, respectively. However, note that, in the case of weak or strong interspecies collisions only, the above coupled system 2.46)-2.47) remains underdetermined, as the evolution for $n_{\epsilon}, j_{\epsilon}$ and $w_{\epsilon}$ is missing. It turns out that the electrodynamic variables will be determined by nonlinear constraint equations. In particular, $j_{\epsilon}$ will be asymptotically determined by the so-called Ohm's law, which we derive below in Section 2.4.5.

2.4.2. The case of very weak interspecies collisions. The reader should, at this point, take some time to compare the two species system $2.45-(2.47)$ with the one species system (2.14)-2.15). When $\delta=O(\epsilon)$, the coupling between cations and anions in the two species system $2.45-(2.47)$ is caused only by the mean field interaction of the electromagnetic field $(E, B)$ and by the low order interspecies 
collision terms

$$
\frac{\delta^{2}}{\epsilon^{2}} \int_{\mathbb{R}^{3}} \mathcal{L}\left(g_{\epsilon}^{ \pm}, g_{\epsilon}^{\mp}\right)\left(\begin{array}{c}
v \\
\frac{|v|^{2}}{2}
\end{array}\right) M d v \quad \text { and } \quad \frac{\delta^{2}}{\epsilon} \int_{\mathbb{R}^{3}} \mathcal{Q}\left(g_{\epsilon}^{ \pm}, g_{\epsilon}^{\mp}\right)\left(\begin{array}{c}
v \\
\frac{|v|^{2}}{2}
\end{array}\right) M d v
$$

As we are about to discuss, the system 2.45-2.47 essentially behaves, in the limit $\epsilon \rightarrow 0$, as two coupled one species systems of the kind (2.14)-(2.15).

Indeed, when compared with (2.14), the only additional terms that one finds in 2.45 are :

- The linear interspecies collision terms $\frac{\delta^{2}}{\epsilon^{2}} \int_{\mathbb{R}^{3}} \mathcal{L}\left(g_{\epsilon}^{ \pm}, g_{\epsilon}^{\mp}\right)\left(\begin{array}{c}v \\ \frac{|v|^{2}}{2}\end{array}\right) M d v$, which converge, as $\epsilon \rightarrow 0$, towards

$$
\left[\frac{\delta}{\epsilon}\right]^{2} \int_{\mathbb{R}^{3}} \mathcal{L}\left(g^{ \pm}, g^{\mp}\right)\left(\begin{array}{c}
v \\
\frac{|v|^{2}}{2}
\end{array}\right) M d v= \pm\left[\begin{array}{c}
\delta \\
\epsilon
\end{array}\right]^{2}\left(\begin{array}{c}
\sigma^{-1}\left(u^{+}-u^{-}\right) \\
\frac{5}{2} \lambda^{-1}\left(\theta^{+}-\theta^{-}\right)
\end{array}\right)
$$

where the electrical conductivity $\sigma>0$ and the energy conductivity $\lambda>0$ are constants defined by

$$
\begin{aligned}
\frac{1}{\sigma} & =\frac{1}{2} \int_{\mathbb{R}^{3}} v \cdot \mathcal{L}(v,-v) M d v \\
& =\frac{1}{2} \int_{\mathbb{R}^{3} \times \mathbb{R}^{3} \times \mathbb{S}^{2}}\left|v-v^{\prime}\right|^{2} b\left(v-v_{*}, \sigma\right) M M_{*} d v d v_{*} d \sigma \\
& =\frac{1}{2} \int_{\mathbb{R}^{3} \times \mathbb{R}^{3}}\left|v-v_{*}\right|^{2} m\left(v-v_{*}\right) M M_{*} d v d v_{*},
\end{aligned}
$$

and

$$
\begin{aligned}
\frac{1}{\lambda} & =\frac{1}{20} \int_{\mathbb{R}^{3}}|v|^{2} \mathcal{L}\left(|v|^{2},-|v|^{2}\right) M d v \\
& =\frac{1}{20} \int_{\mathbb{R}^{3} \times \mathbb{R}^{3} \times \mathbb{S}^{2}}\left(|v|^{2}-\left|v^{\prime}\right|^{2}\right)^{2} b\left(v-v_{*}, \sigma\right) M M_{*} d v d v_{*} d \sigma \\
& =\frac{1}{20} \int_{\mathbb{R}^{3} \times \mathbb{R}^{3}}\left(|v|^{2}-\left|v_{*}\right|^{2}\right)^{2} m\left(v-v_{*}\right) M M_{*} d v d v_{*},
\end{aligned}
$$

where the cross-section for momentum and energy transfer $m\left(v-v_{*}\right)$ is defined in Proposition A.1.

- The nonlinear interspecies collisions terms $\frac{\delta^{2}}{\epsilon} \int_{\mathbb{R}^{3}} \mathcal{Q}\left(g_{\epsilon}^{ \pm}, g_{\epsilon}^{\mp}\right)\left(\begin{array}{c}v \\ \frac{|v|^{2}}{2}\end{array}\right) M d v$, which are at least of formal order $O(\epsilon)$ and thus, vanish in the limit $\epsilon \rightarrow 0$.

Thus, the remainder of the formal asymptotic analysis of the two species system 2.45- -2.47 follows exactly the same steps as the analysis of the one species system (2.14)-2.15 performed in Sections 2.3.2 and 2.3.3, which we somewhat detail now.

Note first that the system (2.45)-(2.47) can be rewritten as a singular perturbation

$$
\partial_{t}\left(\begin{array}{c}
\rho_{\epsilon}^{+} \\
u_{\epsilon}^{+} \\
\sqrt{\frac{3}{2}} \theta_{\epsilon}^{+} \\
\rho_{\epsilon}^{-} \\
u_{\epsilon}^{-} \\
\sqrt{\frac{3}{2}} \theta_{\epsilon}^{-} \\
E_{\epsilon} \\
B_{\epsilon}
\end{array}\right)+W_{\epsilon}\left(\begin{array}{c}
\rho_{\epsilon}^{+} \\
u_{\epsilon}^{+} \\
\sqrt{\frac{3}{2}} \theta_{\epsilon}^{+} \\
\rho_{\epsilon}^{-} \\
u_{\epsilon}^{-} \\
\sqrt{\frac{3}{2}} \theta_{\epsilon}^{-} \\
E_{\epsilon} \\
B_{\epsilon}
\end{array}\right)=O(1)
$$


which describes the propagation of waves in the system, where the wave operator is given by

$$
W_{\epsilon}=\left(\begin{array}{cccccccc}
0 & \frac{1}{\epsilon} \operatorname{div} & 0 & 0 & 0 & 0 & 0 & 0 \\
\frac{1}{\epsilon} \nabla_{x} & 0 & \frac{1}{\epsilon} \sqrt{\frac{2}{3}} \nabla_{x} & 0 & 0 & 0 & -\frac{\alpha}{\epsilon^{2}} \operatorname{Id} & 0 \\
0 & \frac{1}{\epsilon} \sqrt{\frac{2}{3}} \operatorname{div} & 0 & 0 & 0 & 0 & 0 & 0 \\
0 & 0 & 0 & 0 & \frac{1}{\epsilon} \operatorname{div} & 0 & 0 & 0 \\
0 & 0 & 0 & \frac{1}{\epsilon} \nabla_{x} & 0 & \frac{1}{\epsilon} \sqrt{\frac{2}{3}} \nabla_{x} & \frac{\alpha}{\epsilon^{2}} \mathrm{Id} & 0 \\
0 & 0 & 0 & 0 & \frac{1}{\epsilon} \sqrt{\frac{2}{3}} \operatorname{div} & 0 & 0 & 0 \\
0 & \frac{\alpha}{\epsilon^{2}} \mathrm{Id} & 0 & 0 & -\frac{\alpha}{\epsilon^{2}} \mathrm{Id} & 0 & 0 & -\frac{1}{\gamma} \operatorname{rot} \\
0 & 0 & 0 & 0 & 0 & 0 & \frac{1}{\gamma} \operatorname{rot} & 0
\end{array}\right) .
$$

We derive then the macroscopic constraint equations on $\left(\rho^{ \pm}, u^{ \pm}, \theta^{ \pm}, E, B\right)$ reproducing the reasoning from Section 2.3.2.

(1) When $\gamma \sim 1$ (so that $\alpha=O\left(\epsilon^{2}\right)$ ), averaging over fast time oscillations as $\epsilon \rightarrow 0$, we get the macroscopic constraints

$$
\operatorname{div} u^{ \pm}=0, \quad \rho^{ \pm}+\theta^{ \pm}=0,
$$

respectively referred to as incompressibility and Boussinesq relations. These are supplemented by the asymptotic constraints coming from Gauss' laws in 2.47

$$
\operatorname{div} E=0, \quad \operatorname{div} B=0 .
$$

(2) When $\gamma=o(1)$ and $\alpha=O\left(\epsilon^{2}\right)$, averaging over fast time oscillations as $\epsilon \rightarrow 0$, we get the macroscopic constraints

$$
\begin{aligned}
\operatorname{div} u^{ \pm} & =0, & \rho^{ \pm}+\theta^{ \pm} & =0, \\
\operatorname{rot} B & =0, & \operatorname{rot} E & =0 .
\end{aligned}
$$

These are supplemented by the asymptotic constraints coming from Gauss' laws in 2.47)

$$
\operatorname{div} E=0, \quad \operatorname{div} B=0 .
$$

Hence,

$$
E=0, \quad B=0 .
$$

(3) When $\gamma=o(1)$ and $\frac{\alpha}{\epsilon^{2}}$ is unbounded, averaging over fast time oscillations as $\epsilon \rightarrow 0$, we get the macroscopic constraints

$$
\begin{aligned}
\operatorname{div} u^{ \pm} & =0, & \nabla_{x}\left(\rho^{ \pm}+\theta^{ \pm}\right) & = \pm\left[\frac{\alpha}{\epsilon}\right] E, \\
\operatorname{rot} B & =\left[\frac{\beta}{\epsilon}\right]\left(u^{+}-u^{-}\right), & \operatorname{rot} E & =0 .
\end{aligned}
$$

As usual, when $\alpha=o(\epsilon)$, the weak Boussinesq relation $\nabla_{x}\left(\rho^{ \pm}+\theta^{ \pm}\right)=0$ can be improved to the strong Boussinesq relation $\rho^{ \pm}+\theta^{ \pm}=0$, assuming $\rho^{ \pm}$and $\theta^{ \pm}$enjoy enough integrability. These are supplemented by the asymptotic constraints coming from Gauss' laws in 2.47

$$
\operatorname{div} E=\left[\frac{\alpha}{\epsilon}\right]\left(\rho^{+}-\rho^{-}\right), \quad \operatorname{div} B=0 .
$$

Next, following the reasoning from Section 2.3.3, we derive the asymptotic evolution equations associated with the two species system (2.45)-2.47). To this end, notice that 2.34 implies, in particular, that

$$
\frac{1}{\epsilon} \mathcal{L} g_{\epsilon}^{ \pm}=\mathcal{Q}\left(g_{\epsilon}^{ \pm}, g_{\epsilon}^{ \pm}\right)-v \cdot \nabla_{x} g_{\epsilon}^{ \pm} \pm \frac{\alpha}{\epsilon} E_{\epsilon} \cdot v+O(\epsilon),
$$


which is analog to 2.25 in the one species case. Hence, we obtain the advection and diffusion terms, as in Section 2.3.3

$$
\begin{aligned}
\lim _{\epsilon \rightarrow 0} \frac{1}{\epsilon} P \operatorname{div} \int_{\mathbb{R}^{3}} \mathcal{L} g_{\epsilon}^{ \pm} \tilde{\phi} M d v & =P\left(u^{ \pm} \cdot \nabla_{x} u^{ \pm}\right)-\mu \Delta_{x} u^{ \pm}, \\
\lim _{\epsilon \rightarrow 0} \frac{1}{\epsilon} \operatorname{div} \int_{\mathbb{R}^{3}} \mathcal{L} g_{\epsilon}^{ \pm} \tilde{\psi} M d v & =\frac{5}{2} u^{ \pm} \cdot \nabla_{x} \theta^{ \pm}-\frac{5}{2} \kappa \Delta_{x} \theta^{ \pm},
\end{aligned}
$$

where

$$
\mu=\frac{1}{10} \int_{\mathbb{R}^{3}} \phi: \tilde{\phi} M d v \quad \text { and } \quad \kappa=\frac{2}{15} \int_{\mathbb{R}^{3}} \psi \cdot \tilde{\psi} M d v .
$$

We are now in a position to obtain the limiting evolution of the system 2.45)-

(1) When $\gamma \sim 1$, letting $\epsilon$ tend to zero in the system 2.45)-2.47) yields

$$
\left\{\begin{aligned}
& \partial_{t} u^{ \pm}+u^{ \pm} \cdot \nabla_{x} u^{ \pm}-\mu \Delta_{x} u^{ \pm} \pm\left[\frac{\delta}{\epsilon}\right]^{2} \frac{1}{\sigma}\left(u^{+}-u^{-}\right)=-\nabla_{x} p^{ \pm} \pm\left[\frac{\alpha}{\epsilon^{2}}\right] E \pm\left[\frac{\beta}{\epsilon}\right] u^{ \pm} \wedge B \\
& \partial_{t} \theta^{ \pm}+u^{ \pm} \cdot \nabla_{x} \theta^{ \pm}-\kappa \Delta_{x} \theta^{ \pm} \\
& \pm\left[\frac{\delta}{\epsilon}\right]^{2} \frac{1}{\lambda}\left(\theta^{+}-\theta^{-}\right)=0, \\
& {[\gamma] \partial_{t} E-\operatorname{rot} B=-\left[\frac{\beta}{\epsilon}\right]\left(u^{+}-u^{-}\right), } \\
& {[\gamma] \partial_{t} B+\operatorname{rot} E=0, }
\end{aligned}\right.
$$

with the constraints

$$
\begin{aligned}
\operatorname{div} u^{ \pm} & =0, \quad \rho^{ \pm}+\theta^{ \pm}=0, \\
\operatorname{div} E & =0, \quad \operatorname{div} B=0 .
\end{aligned}
$$

(2) When $\gamma=o(1)$ and $\alpha=O\left(\epsilon^{2}\right)$, letting $\epsilon$ tend to zero in the system (2.45)-(2.47) yields

$$
\left\{\begin{array}{l}
\partial_{t} u^{ \pm}+u^{ \pm} \cdot \nabla_{x} u^{ \pm}-\mu \Delta_{x} u^{ \pm} \pm\left[\frac{\delta}{\epsilon}\right]^{2} \frac{1}{\sigma}\left(u^{+}-u^{-}\right)=-\nabla_{x} p^{ \pm}, \\
\partial_{t} \theta^{ \pm}+u^{ \pm} \cdot \nabla_{x} \theta^{ \pm}-\kappa \Delta_{x} \theta^{ \pm} \pm\left[\frac{\delta}{\epsilon}\right]^{2} \frac{1}{\lambda}\left(\theta^{+}-\theta^{-}\right)=0,
\end{array}\right.
$$

with the constraints

$$
\begin{aligned}
\operatorname{div} u^{ \pm} & =0, & \rho^{ \pm}+\theta^{ \pm} & =0, \\
E & =0, & B & =0 .
\end{aligned}
$$

(3) When $\gamma=o(1)$ and $\frac{\alpha}{\epsilon^{2}}$ is unbounded, letting $\epsilon$ tend to zero in the system 2.45-2.47) yields

$$
\left\{\begin{aligned}
\partial_{t}\left(u^{ \pm} \pm\left[\frac{\beta}{\epsilon}\right] A\right) & +u^{ \pm} \cdot \nabla_{x} u^{ \pm}-\mu \Delta_{x} u^{ \pm} \\
& \pm\left[\frac{\delta}{\epsilon}\right]^{2} \frac{1}{\sigma}\left(u^{+}-u^{-}\right)=-\nabla_{x} p^{ \pm} \pm\left[\frac{\alpha}{\epsilon}\right] \rho^{ \pm} E \pm\left[\frac{\beta}{\epsilon}\right] u^{ \pm} \wedge B \\
\partial_{t}\left(\frac{3}{2} \theta^{ \pm}-\rho^{ \pm}\right)+ & \frac{5}{2} u^{ \pm} \cdot \nabla_{x} \theta^{ \pm}-\frac{5}{2} \kappa \Delta_{x} \theta^{ \pm} \\
\pm & \frac{5}{2}\left[\frac{\delta}{\epsilon}\right]^{2} \frac{1}{\lambda}\left(\theta^{+}-\theta^{-}\right)= \pm\left[\frac{\alpha}{\epsilon}\right] u^{ \pm} \cdot E
\end{aligned}\right.
$$


with the constraints

$$
\begin{aligned}
\operatorname{div} u^{ \pm} & =0, & \nabla_{x}\left(\rho^{ \pm}+\theta^{ \pm}\right) & = \pm\left[\frac{\alpha}{\epsilon}\right] E, \\
\operatorname{rot} B & =\left[\frac{\beta}{\epsilon}\right]\left(u^{+}-u^{-}\right), & \operatorname{rot} E & =0, \\
\operatorname{div} E & =\left[\frac{\alpha}{\epsilon}\right]\left(\rho^{+}-\rho^{-}\right), & \operatorname{div} B & =0, \\
\operatorname{rot} A & =B, & \operatorname{div} A & =0 .
\end{aligned}
$$

The above system can be rewritten more explicitly by defining the adjusted electric field $\tilde{E}=-\partial_{t} A+E$. It then holds that

$$
\left\{\begin{aligned}
\partial_{t} u^{ \pm}+u^{ \pm} \cdot \nabla_{x} u^{ \pm}-\mu \Delta_{x} u^{ \pm} \pm\left[\frac{\delta}{\epsilon}\right]^{2} \frac{1}{\sigma}\left(u^{+}-u^{-}\right) & =-\nabla_{x} p^{ \pm} \pm\left[\frac{\beta}{\epsilon}\right] \tilde{E} \\
& +\rho^{ \pm} \nabla_{x} \theta^{ \pm} \pm\left[\frac{\beta}{\epsilon}\right] u^{ \pm} \wedge B, \\
\partial_{t}\left(\frac{3}{2} \theta^{ \pm}-\rho^{ \pm}\right)+u^{ \pm} \cdot \nabla_{x}\left(\frac{3}{2} \theta^{ \pm}-\rho^{ \pm}\right)-\frac{5}{2} \kappa \Delta_{x} \theta^{ \pm} & \\
\pm \frac{5}{2}\left[\frac{\delta}{\epsilon}\right]^{2} \frac{1}{\lambda}\left(\theta^{+}-\theta^{-}\right) & =0, \\
\partial_{t} B+\operatorname{rot} \tilde{E} & =0,
\end{aligned}\right.
$$

with the constraints

$$
\begin{array}{rlrl}
\operatorname{div} u^{ \pm} & =0, & \Delta_{x}\left(\rho^{ \pm}+\theta^{ \pm}\right) & = \pm\left[\frac{\alpha}{\epsilon}\right]^{2}\left(\rho^{+}-\rho^{-}\right), \\
\operatorname{rot} B & =\left[\frac{\beta}{\epsilon}\right]\left(u^{+}-u^{-}\right), & \operatorname{div} B & =0, \\
\operatorname{div} \tilde{E} & =\left[\frac{\alpha}{\epsilon}\right]\left(\rho^{+}-\rho^{-}\right) .
\end{array}
$$

Notice, finally, that if further $\alpha=o(\epsilon)$, then the above system is greatly simplified and becomes

$$
\left\{\begin{aligned}
\partial_{t} u^{ \pm}+u^{ \pm} \cdot \nabla_{x} u^{ \pm}- & \mu \Delta_{x} u^{ \pm} \\
& \pm\left[\frac{\delta}{\epsilon}\right]^{2} \frac{1}{\sigma}\left(u^{+}-u^{-}\right)=-\nabla_{x} p^{ \pm} \pm\left[\frac{\beta}{\epsilon}\right] \tilde{E} \pm\left[\frac{\beta}{\epsilon}\right] u^{ \pm} \wedge B \\
\partial_{t} \theta^{ \pm}+u^{ \pm} \cdot \nabla_{x} \theta^{ \pm}-\kappa \Delta_{x} \theta^{ \pm} & \pm\left[\frac{\delta}{\epsilon}\right]^{2} \frac{1}{\lambda}\left(\theta^{+}-\theta^{-}\right)=0 \\
\partial_{t} B+\operatorname{rot} \tilde{E} & =0
\end{aligned}\right.
$$

with the constraints

$$
\begin{aligned}
\operatorname{div} u^{ \pm} & =0, & \rho^{ \pm}+\theta^{ \pm} & =0, \\
\operatorname{div} \tilde{E} & =0, & \operatorname{div} B & =0, \\
\operatorname{rot} B & =\left[\frac{\beta}{\epsilon}\right]\left(u^{+}-u^{-}\right), & E & =0 .
\end{aligned}
$$

On the whole, we conclude that, in the case of very weak interspecies collisions $\delta=O(\epsilon)$, the parameters $\alpha, \beta$ and $\gamma$ determine the asymptotics of the two species Vlasov-Maxwell-Boltzmann system 2.32 exactly as they do determine the asymptotics of the one species Vlasov-Maxwell-Boltzmann system treated in Section 2.3. More precisely, the limiting two fluid macroscopic systems we obtain here can always be interpreted as two systems for one species - one for cations and one 
for anions - coupled through their mean field interaction with the electromagnetic field $(E, B)$ and, whenever $\delta \sim \epsilon$, by an interspecies exchange of momentum and energy expressed by the linear terms $\frac{1}{\sigma}\left(u^{+}-u^{-}\right)$and $\frac{1}{\lambda}\left(\theta^{+}-\theta^{-}\right)$. Therefore, the different asymptotic regimes for two species are also described by Figure 1 on page 26.

Thus, when $\delta \sim \epsilon$, up to multiplicative constants, we reach the following asymptotic systems of equations :

(1) If $\alpha=o(\epsilon)$ and $\beta=o(\epsilon)$, we obtain the two fluid incompressible NavierStokes-Fourier system :

$$
\begin{cases}\partial_{t} u^{ \pm}+u^{ \pm} \cdot \nabla_{x} u^{ \pm}-\mu \Delta_{x} u^{ \pm} \pm \frac{1}{\sigma}\left(u^{+}-u^{-}\right)=-\nabla_{x} p^{ \pm}, & \operatorname{div} u^{ \pm}=0 \\ \partial_{t} \theta^{ \pm}+u^{ \pm} \cdot \nabla_{x} \theta^{ \pm}-\kappa \Delta_{x} \theta^{ \pm} \pm \frac{1}{\lambda}\left(\theta^{+}-\theta^{-}\right)=0, & \rho^{ \pm}+\theta^{ \pm}=0 .\end{cases}
$$

This system satisfies the following formal energy conservation laws :

$$
\begin{aligned}
& \frac{1}{2} \frac{d}{d t}\left(\left\|u^{+}\right\|_{L_{x}^{2}}^{2}+\left\|u^{-}\right\|_{L_{x}^{2}}^{2}\right)+\mu\left(\left\|\nabla_{x} u^{+}\right\|_{L_{x}^{2}}^{2}+\left\|\nabla_{x} u^{-}\right\|_{L_{x}^{2}}^{2}\right)+\frac{1}{\sigma}\left\|u^{+}-u^{-}\right\|_{L_{x}^{2}}^{2}=0 \\
& \frac{1}{2} \frac{d}{d t}\left(\left\|\theta^{+}\right\|_{L_{x}^{2}}^{2}+\left\|\theta^{-}\right\|_{L_{x}^{2}}^{2}\right)+\kappa\left(\left\|\nabla_{x} \theta^{+}\right\|_{L_{x}^{2}}^{2}+\left\|\nabla_{x} \theta^{-}\right\|_{L_{x}^{2}}^{2}\right)+\frac{1}{\lambda}\left\|\theta^{+}-\theta^{-}\right\|_{L_{x}^{2}}^{2}=0 .
\end{aligned}
$$

(2) If $\alpha=\epsilon^{2}$ and $\gamma=1$, we obtain the two fluid incompressible Navier-StokesFourier-Maxwell system :

$$
\left\{\begin{array}{rlrl}
\partial_{t} u^{ \pm}+u^{ \pm} \cdot \nabla_{x} u^{ \pm}-\mu \Delta_{x} u^{ \pm} & \\
\pm \frac{1}{\sigma}\left(u^{+}-u^{-}\right) & =-\nabla_{x} p^{ \pm} \pm E \pm u^{ \pm} \wedge B, & & \operatorname{div} u^{ \pm}=0, \\
\partial_{t} \theta^{ \pm}+u^{ \pm} \cdot \nabla_{x} \theta^{ \pm}-\kappa \Delta_{x} \theta^{ \pm} & \\
\pm \frac{1}{\lambda}\left(\theta^{+}-\theta^{-}\right) & =0, & \rho^{ \pm}+\theta^{ \pm}=0, \\
\partial_{t} E-\operatorname{rot} B & =-\left(u^{+}-u^{-}\right), & \operatorname{div} E & =0, \\
\partial_{t} B+\operatorname{rot} E & =0, & \operatorname{div} B & =0 .
\end{array}\right.
$$

This system satisfies the following formal energy conservation laws :

$$
\begin{aligned}
\frac{1}{2} \frac{d}{d t}\left(\left\|u^{+}\right\|_{L_{x}^{2}}^{2}+\left\|u^{-}\right\|_{L_{x}^{2}}^{2}+\right. & \left.\|E\|_{L_{x}^{2}}^{2}+\|B\|_{L_{x}^{2}}^{2}\right) \\
& +\mu\left(\left\|\nabla_{x} u^{+}\right\|_{L_{x}^{2}}^{2}+\left\|\nabla_{x} u^{-}\right\|_{L_{x}^{2}}^{2}\right)+\frac{1}{\sigma}\left\|u^{+}-u^{-}\right\|_{L_{x}^{2}}^{2}=0 \\
\frac{1}{2} \frac{d}{d t}\left(\left\|\theta^{+}\right\|_{L_{x}^{2}}^{2}+\left\|\theta^{-}\right\|_{L_{x}^{2}}^{2}\right) & +\kappa\left(\left\|\nabla_{x} \theta^{+}\right\|_{L_{x}^{2}}^{2}+\left\|\nabla_{x} \theta^{-}\right\|_{L_{x}^{2}}^{2}\right)+\frac{1}{\lambda}\left\|\theta^{+}-\theta^{-}\right\|_{L_{x}^{2}}^{2}=0 .
\end{aligned}
$$

(3) If $\alpha=o(\epsilon), \beta=\epsilon$ and $\gamma=o(1)$, we obtain the two-fluid incompressible quasi-static Navier-Stokes-Fourier-Maxwell system :

$$
\left\{\begin{array}{rlrl}
\partial_{t} u^{ \pm}+u^{ \pm} \cdot \nabla_{x} u^{ \pm}-\mu \Delta_{x} u^{ \pm} & \\
\pm \frac{1}{\sigma}\left(u^{+}-u^{-}\right) & =-\nabla_{x} p^{ \pm} \pm E \pm u^{ \pm} \wedge B, & & \operatorname{div} u^{ \pm}=0, \\
\partial_{t} \theta^{ \pm}+u^{ \pm} \cdot \nabla_{x} \theta^{ \pm}-\kappa \Delta_{x} \theta^{ \pm} & \\
\pm \frac{1}{\lambda}\left(\theta^{+}-\theta^{-}\right) & =0, & & \rho^{ \pm}+\theta^{ \pm}=0, \\
\operatorname{rot} B & =u^{+}-u^{-}, & \operatorname{div} E=0, \\
\partial_{t} B+\operatorname{rot} E & =0, & \operatorname{div} B=0 .
\end{array}\right.
$$


This system satisfies the following formal energy conservation laws :

$$
\begin{aligned}
& \frac{1}{2} \frac{d}{d t}\left(\left\|u^{+}\right\|_{L_{x}^{2}}^{2}+\left\|u^{-}\right\|_{L_{x}^{2}}^{2}+\|B\|_{L_{x}^{2}}^{2}\right) \\
& +\mu\left(\left\|\nabla_{x} u^{+}\right\|_{L_{x}^{2}}^{2}+\left\|\nabla_{x} u^{-}\right\|_{L_{x}^{2}}^{2}\right)+\frac{1}{\sigma}\left\|u^{+}-u^{-}\right\|_{L_{x}^{2}}^{2}=0, \\
& \frac{1}{2} \frac{d}{d t}\left(\left\|\theta^{+}\right\|_{L_{x}^{2}}^{2}+\left\|\theta^{-}\right\|_{L_{x}^{2}}^{2}\right)+\kappa\left(\left\|\nabla_{x} \theta^{+}\right\|_{L_{x}^{2}}^{2}+\left\|\nabla_{x} \theta^{-}\right\|_{L_{x}^{2}}^{2}\right)+\frac{1}{\lambda}\left\|\theta^{+}-\theta^{-}\right\|_{L_{x}^{2}}^{2}=0 .
\end{aligned}
$$

Here, the electric field is defined indirectly as a mere distribution, through Faraday's equation, by

$$
E=-\partial_{t} A
$$

where $B=\operatorname{rot} A$ and $\operatorname{div} A=0$.

(4) If $\alpha=\epsilon$ and $\gamma=\epsilon$, we obtain the two fluid incompressible quasi-static Navier-Stokes-Fourier-Maxwell-Poisson system :

$$
\begin{aligned}
\partial_{t} u^{ \pm}+u^{ \pm} \cdot \nabla_{x} u^{ \pm}-\mu \Delta_{x} u^{ \pm} & \\
\pm \frac{1}{\sigma}\left(u^{+}-u^{-}\right) & =-\nabla_{x} p^{ \pm} \pm E+\rho^{ \pm} \nabla_{x} \theta^{ \pm} \pm u^{ \pm} \wedge B, \\
\operatorname{div} u^{ \pm} & =0, \\
\partial_{t}\left(\frac{3}{2} \theta^{ \pm}-\rho^{ \pm}\right)+u^{ \pm} \cdot \nabla_{x}\left(\frac{3}{2} \theta^{ \pm}-\rho^{ \pm}\right) & -\frac{5}{2} \kappa \Delta_{x} \theta^{ \pm} \\
\pm \frac{5}{2 \lambda}\left(\theta^{+}-\theta^{-}\right) & =0, \\
\Delta_{x}\left(\rho^{ \pm}+\theta^{ \pm}\right) & = \pm\left(\rho^{+}-\rho^{-}\right), \\
\operatorname{rot} B & =u^{+}-u^{-}, \\
\partial_{t} B+\operatorname{rot} E & =0, \\
\operatorname{div} E & =\rho^{+}-\rho^{-}, \\
\operatorname{div} B & =0 .
\end{aligned}
$$

This system satisfies the following formal energy conservation law :

$$
\begin{aligned}
\frac{1}{2} \frac{d}{d t}\left(\left\|\rho^{+}\right\|_{L_{x}^{2}}^{2}\right. & +\left\|\rho^{-}\right\|_{L_{x}^{2}}^{2}+\left\|u^{+}\right\|_{L_{x}^{2}}^{2}+\left\|u^{-}\right\|_{L_{x}^{2}}^{2} \\
& \left.+\frac{3}{2}\left\|\theta^{+}\right\|_{L_{x}^{2}}^{2}+\frac{3}{2}\left\|\theta^{-}\right\|_{L_{x}^{2}}^{2}+\left\|P^{\perp} E\right\|_{L_{x}^{2}}^{2}+\|B\|_{L_{x}^{2}}^{2}\right) \\
& +\mu\left(\left\|\nabla_{x} u^{+}\right\|_{L_{x}^{2}}^{2}+\left\|\nabla_{x} u^{-}\right\|_{L_{x}^{2}}^{2}\right)+\frac{5}{2} \kappa\left(\left\|\nabla_{x} \theta^{+}\right\|_{L_{x}^{2}}^{2}+\left\|\nabla_{x} \theta^{-}\right\|_{L_{x}^{2}}^{2}\right) \\
& +\frac{1}{\sigma}\left\|u^{+}-u^{-}\right\|_{L_{x}^{2}}^{2}+\frac{5}{2 \lambda}\left\|\theta^{+}-\theta^{-}\right\|_{L_{x}^{2}}^{2}=0 .
\end{aligned}
$$

Here, the solenoidal component of the electric field is defined indirectly as a mere distribution, through Faraday's equation, by

$$
P E=-\partial_{t} A,
$$

where $B=\operatorname{rot} A$ and $\operatorname{div} A=0$, while its irrotational component is determined, through Gauss' law, by

$$
P^{\perp} E= \pm \nabla_{x}\left(\rho^{ \pm}+\theta^{ \pm}\right) .
$$

Notice that the equations in this system are all coupled. 
(5) If $\alpha=\epsilon$ and $\gamma=o(\epsilon)$, we obtain the two fluid incompressible NavierStokes-Fourier-Poisson system :

$$
\left\{\begin{aligned}
\partial_{t} u^{ \pm}+u^{ \pm} \cdot \nabla_{x} u^{ \pm}-\mu \Delta_{x} u^{ \pm} \pm \frac{1}{\sigma}\left(u^{+}-u^{-}\right) & =-\nabla_{x} p^{ \pm}+\rho^{ \pm} \nabla_{x} \theta^{ \pm}, \\
\operatorname{div} u^{ \pm} & =0, \\
\partial_{t}\left(\frac{3}{2} \theta^{ \pm}-\rho^{ \pm}\right)+u^{ \pm} \cdot \nabla_{x}\left(\frac{3}{2} \theta^{ \pm}-\rho^{ \pm}\right)-\frac{5}{2} \kappa \Delta_{x} \theta^{ \pm} & \\
\pm \frac{5}{2 \lambda}\left(\theta^{+}-\theta^{-}\right) & =0, \\
\Delta_{x}\left(\rho^{ \pm}+\theta^{ \pm}\right) & = \pm\left(\rho^{+}-\rho^{-}\right) .
\end{aligned}\right.
$$

This system satisfies the following formal energy conservation law :

$$
\begin{aligned}
\frac{1}{2} \frac{d}{d t}\left(\left\|\rho^{+}\right\|_{L_{x}^{2}}^{2}\right. & +\left\|\rho^{-}\right\|_{L_{x}^{2}}^{2}+\left\|u^{+}\right\|_{L_{x}^{2}}^{2}+\left\|u^{-}\right\|_{L_{x}^{2}}^{2} \\
& \left.+\frac{3}{2}\left\|\theta^{+}\right\|_{L_{x}^{2}}^{2}+\frac{3}{2}\left\|\theta^{-}\right\|_{L_{x}^{2}}^{2}+\left\|\nabla_{x}\left(\rho^{ \pm}+\theta^{ \pm}\right)\right\|_{L_{x}^{2}}^{2}\right) \\
& +\mu\left(\left\|\nabla_{x} u^{+}\right\|_{L_{x}^{2}}^{2}+\left\|\nabla_{x} u^{-}\right\|_{L_{x}^{2}}^{2}\right)+\frac{5}{2} \kappa\left(\left\|\nabla_{x} \theta^{+}\right\|_{L_{x}^{2}}^{2}+\left\|\nabla_{x} \theta^{-}\right\|_{L_{x}^{2}}^{2}\right) \\
& +\frac{1}{\sigma}\left\|u^{+}-u^{-}\right\|_{L_{x}^{2}}^{2}+\frac{5}{2 \lambda}\left\|\theta^{+}-\theta^{-}\right\|_{L_{x}^{2}}^{2}=0 .
\end{aligned}
$$

Physically, in this system, the fluid is subject to a self-induced static electric field $E$ determined by

$$
\operatorname{rot} E=0, \quad \operatorname{div} E=\rho^{+}-\rho^{-},
$$

hence

$$
E= \pm \nabla_{x}\left(\rho^{ \pm}+\theta^{ \pm}\right) .
$$

Notice that the equations in this system are all coupled.

When $\delta=o(\epsilon)$, one obtains the corresponding asymptotic systems by simply discarding the linear terms $\pm \frac{1}{\sigma}\left(u^{+}-u^{-}\right)$and $\pm \frac{1}{\lambda}\left(\theta^{+}-\theta^{-}\right)$in the preceding systems.

The above interpretation of two fluid systems as a coupling of one fluid systems will no longer hold for the more singular case of weak and strong interactions, i.e. when $\frac{\delta}{\epsilon}$ is unbounded, which we treat next.

2.4.3. Macroscopic hydrodynamic constraints. Let us focus now on the analysis of the weak and strong interspecies collisional interactions. Contrary to the one species case, in the two species case, when $\frac{\delta}{\epsilon}$ is unbounded, the acoustic waves are always decoupled from the electromagnetic waves, which we treat below in Section 2.4.5. We deal now with the acoustic waves. waves :

At leading order, the system 2.46 describes the propagation of acoustic $\left(\rho_{\epsilon}, u_{\epsilon}, \sqrt{\frac{3}{2}} \theta_{\epsilon}\right)$

$$
\partial_{t}\left(\begin{array}{c}
\rho_{\epsilon} \\
u_{\epsilon} \\
\sqrt{\frac{3}{2}} \theta_{\epsilon}
\end{array}\right)+W_{\epsilon}\left(\begin{array}{c}
\rho_{\epsilon} \\
u_{\epsilon} \\
\sqrt{\frac{3}{2}} \theta_{\epsilon}
\end{array}\right)=O(1),
$$

where the wave operator $W_{\epsilon}$, containing the singular terms from 2.46$]$, is defined by

$$
W_{\epsilon}=\left(\begin{array}{ccc}
0 & \frac{1}{\epsilon} \operatorname{div} & 0 \\
\frac{1}{\epsilon} \nabla_{x} & 0 & \frac{1}{\epsilon} \sqrt{\frac{2}{3}} \nabla_{x} \\
0 & \frac{1}{\epsilon} \sqrt{\frac{2}{3}} \operatorname{div} & 0
\end{array}\right)
$$


The wave operator $W_{\epsilon}$ is antisymmetric (with respect to the $L^{2}(d x)$ innerproduct) and, therefore, can only have purely imaginary eigenvalues. The semigroup generated by this operator may thus produce fast time oscillations. Consequently, averaging over fast time oscillations as $\epsilon \rightarrow 0$, we get the macroscopic constraints

$$
\operatorname{div} u=0, \quad \rho+\theta=0,
$$

respectively referred to as incompressibility and Boussinesq relations. The exact nature of time oscillations produced by the system 2.52 , in the limit $\epsilon \rightarrow 0$, will be rigorously discussed, with greater detail, later on in Chapter 10 .

2.4.4. Hydrodynamic evolution equations. The previous step shows that, since $W_{\epsilon}$ is singular, the asymptotic dynamics of $\left(\rho_{\epsilon}, u_{\epsilon}, \sqrt{\frac{3}{2}} \theta_{\epsilon}\right)$ becomes constrained to the kernel Ker $W_{\epsilon}$ as $\epsilon \rightarrow 0$. Moreover, since $W_{\epsilon}$ is antisymmetric, its range is necessarily orthogonal to its kernel. Therefore, in order to get the asymptotic evolution equations for $\left(\rho, u, \sqrt{\frac{3}{2}} \theta\right)$, it is natural to project the system 2.52 onto Ker $W_{\epsilon}$, which will rid us of all the singular terms in 2.52 and allow us to pass to the limit. In other words, we will obtain the limiting dynamics of the system 2.52 by testing it against functions in $\operatorname{Ker} W_{\epsilon}$.

The kernel of $W_{\epsilon}$, defined in 2.53 , is obviously determined by all $\left(\rho_{\epsilon}^{0}, u_{\epsilon}^{0}, \sqrt{\frac{3}{2}} \theta_{\epsilon}^{0}\right)$ which satisfy

$$
\operatorname{div} u_{\epsilon}^{0}=0 \quad \text { and } \quad \rho_{\epsilon}^{0}+\theta_{\epsilon}^{0}=0 .
$$

It is then readily seen that its orthogonal complement $\operatorname{Ker} W_{\epsilon}^{\perp}$ is determined by all $\left(\tilde{\rho}_{\epsilon}, \tilde{u}_{\epsilon}, \sqrt{\frac{3}{2}} \tilde{\theta}_{\epsilon}\right)$ such that

$$
P \tilde{u}_{\epsilon}=0 \quad \text { and } \quad \frac{3}{2} \tilde{\theta}_{\epsilon}-\tilde{\rho}_{\epsilon}=0
$$

Hence, projecting the system 2.46 onto Ker $W_{\epsilon}^{\perp}$ yields

$$
\left\{\begin{aligned}
\partial_{t} P u_{\epsilon}+\frac{1}{\epsilon} P \operatorname{div} \int_{\mathbb{R}^{3}} \mathcal{L}\left(\frac{g_{\epsilon}^{+}+g_{\epsilon}^{-}}{2}\right) \tilde{\phi} M d v & =P\left(\frac{\alpha}{2 \epsilon} n_{\epsilon} E_{\epsilon}+\frac{\beta}{2 \delta} j_{\epsilon} \wedge B_{\epsilon}\right), \\
\partial_{t}\left(\frac{3}{2} \theta_{\epsilon}-\rho_{\epsilon}\right)+\frac{1}{\epsilon} \operatorname{div} \int_{\mathbb{R}^{3}} \mathcal{L}\left(\frac{g_{\epsilon}^{+}+g_{\epsilon}^{-}}{2}\right) \tilde{\psi} M d v & =\frac{\alpha}{2 \delta} j_{\epsilon} \cdot E_{\epsilon} .
\end{aligned}\right.
$$

There only remains to evaluate the flux terms $\frac{1}{\epsilon} \int_{\mathbb{R}^{3}} \mathcal{L}\left(\frac{g_{\epsilon}^{+}+g_{\epsilon}^{-}}{2}\right) \tilde{\phi} M d v$ and $\frac{1}{\epsilon} \int_{\mathbb{R}^{3}} \mathcal{L}\left(\frac{g_{\epsilon}^{+}+g_{\epsilon}^{-}}{2}\right) \tilde{\psi} M d v$ in 2.55). Just as for one species in Section 2.3.3 following 9, 10, this is done by employing 2.34 to evaluate that

$$
\begin{aligned}
\frac{1+\delta^{2}}{\epsilon} \mathcal{L}\left(g_{\epsilon}^{+}+g_{\epsilon}^{-}\right) & =\left[\mathcal{Q}\left(g_{\epsilon}^{+}, g_{\epsilon}^{+}\right)+\mathcal{Q}\left(g_{\epsilon}^{-}, g_{\epsilon}^{-}\right)\right]+\delta^{2}\left[\mathcal{Q}\left(g_{\epsilon}^{+}, g_{\epsilon}^{-}\right)+\mathcal{Q}\left(g_{\epsilon}^{-}, g_{\epsilon}^{+}\right)\right] \\
& -v \cdot \nabla_{x}\left(g_{\epsilon}^{+}+g_{\epsilon}^{-}\right)+O(\epsilon)
\end{aligned}
$$


which yields formally in the limit, by virtue of the infinitesimal Maxwellian form (2.37),

$$
\begin{aligned}
\lim _{\epsilon \rightarrow 0} \frac{1}{\epsilon} \mathcal{L}\left(\frac{g_{\epsilon}^{+}+g_{\epsilon}^{-}}{2}\right) & =\frac{1}{2\left(1+[\delta]^{2}\right)}\left[\mathcal{Q}\left(g^{+}, g^{+}\right)+\mathcal{Q}\left(g^{-}, g^{-}\right)\right] \\
& +\frac{[\delta]^{2}}{2\left(1+[\delta]^{2}\right)}\left[\mathcal{Q}\left(g^{+}, g^{-}\right)+\mathcal{Q}\left(g^{-}, g^{+}\right)\right] \\
& -\frac{1}{1+[\delta]^{2}} v \cdot \nabla_{x}\left(\frac{g^{+}+g^{-}}{2}\right) \\
& =\frac{1}{4}\left[\mathcal{L}\left(g^{+2}\right)+\mathcal{L}\left(g^{-2}\right)\right]-\frac{1}{1+[\delta]^{2}} v \cdot \nabla_{x}\left(\frac{g^{+}+g^{-}}{2}\right) \\
& =\frac{1}{2} u^{t} \mathcal{L}(\phi) u+\theta u \cdot \mathcal{L}(\psi)+\frac{1}{2} \theta^{2} \mathcal{L}\left(\frac{|v|^{4}}{4}\right) \\
& -\frac{1}{1+[\delta]^{2}} \operatorname{div}\left((\rho+\theta) v+\frac{|v|^{2}}{3} u+\phi u+\theta \psi\right) \\
& =\frac{1}{2} u^{t} \mathcal{L}(\phi) u+\theta u \cdot \mathcal{L}(\psi)+\frac{1}{2} \theta^{2} \mathcal{L}\left(\frac{|v|^{4}}{4}\right) \\
& -\frac{1}{1+[\delta]^{2}} \operatorname{div}(\phi u+\theta \psi),
\end{aligned}
$$

where we have used, in the last line, that $\operatorname{div} u=0$ and $\nabla_{x}(\rho+\theta)=0$

Next, we use that $\tilde{\phi}$ and $\tilde{\psi}$ have similar symmetry properties as $\phi$ and $\psi$, thanks to the rotational invariance of $\mathcal{L}$. More precisely, following $2 \mathbf{2 8}$, it can be shown (see also [13, Section 2.2.3]) that there exist two scalar valued functions $\alpha, \beta:[0, \infty) \rightarrow \mathbb{R}$ such that

$$
\tilde{\phi}(v)=\alpha(|v|) \phi(v) \quad \text { and } \quad \tilde{\psi}(v)=\beta(|v|) \psi(v),
$$

which implies (see [9, Lemma 4.4]) that

$$
\begin{aligned}
\int_{\mathbb{R}^{3}} \phi_{i j} \tilde{\phi}_{k l} M d v & =\left(1+[\delta]^{2}\right) \mu\left(\delta_{i k} \delta_{j l}+\delta_{i l} \delta_{j k}-\frac{2}{3} \delta_{i j} \delta_{k l}\right), \\
\int_{\mathbb{R}^{3}} \psi_{i} \tilde{\psi}_{j} M d v & =\left(1+[\delta]^{2}\right) \frac{5}{2} \kappa \delta_{i j},
\end{aligned}
$$

where

(2.56) $\quad \mu=\frac{1}{10\left(1+[\delta]^{2}\right)} \int_{\mathbb{R}^{3}} \phi: \tilde{\phi} M d v \quad$ and $\quad \kappa=\frac{2}{15\left(1+[\delta]^{2}\right)} \int_{\mathbb{R}^{3}} \psi \cdot \tilde{\psi} M d v$.

Hence, we conclude through tedious but straightforward calculations that

$$
\begin{aligned}
\lim _{\epsilon \rightarrow 0} \frac{1}{\epsilon} \int_{\mathbb{R}^{3}} \mathcal{L}\left(\frac{g_{\epsilon}^{+}+g_{\epsilon}^{-}}{2}\right) \tilde{\phi} M d v & =\int_{\mathbb{R}^{3}} \frac{1}{2}\left(u^{t} \phi u\right) \phi M d v \\
& -\frac{1}{1+[\delta]^{2}} \int_{\mathbb{R}^{3}} \operatorname{div}(\phi u) \tilde{\phi} M d v \\
& =u \otimes u-\frac{|u|^{2}}{3} \mathrm{Id}-\mu\left(\nabla_{x} u+\nabla_{x}^{t} u\right) \\
\lim _{\epsilon \rightarrow 0} \frac{1}{\epsilon} \int_{\mathbb{R}^{3}} \mathcal{L}\left(\frac{g_{\epsilon}^{+}+g_{\epsilon}^{-}}{2}\right) \tilde{\psi} M d v & =\int_{\mathbb{R}^{3}} \theta u \cdot \psi \psi M d v \\
& -\frac{1}{1+[\delta]^{2}} \int_{\mathbb{R}^{3}} \operatorname{div}(\theta \psi) \tilde{\psi} M d v \\
& =\frac{5}{2} \theta u-\frac{5}{2} \kappa \nabla_{x} \theta
\end{aligned}
$$


We finally identify the advection and diffusion terms

$$
\begin{aligned}
\lim _{\epsilon \rightarrow 0} \frac{1}{\epsilon} P \operatorname{div} \int_{\mathbb{R}^{3}} \mathcal{L}\left(\frac{g_{\epsilon}^{+}+g_{\epsilon}^{-}}{2}\right) \tilde{\phi} M d v & =P\left(u \cdot \nabla_{x} u\right)-\mu \Delta_{x} u, \\
\lim _{\epsilon \rightarrow 0} \frac{1}{\epsilon} \operatorname{div} \int_{\mathbb{R}^{3}} \mathcal{L}\left(\frac{g_{\epsilon}^{+}+g_{\epsilon}^{-}}{2}\right) \tilde{\psi} M d v & =\frac{5}{2} u \cdot \nabla_{x} \theta-\frac{5}{2} \kappa \Delta_{x} \theta .
\end{aligned}
$$

On the whole, provided nonlinear terms remain stable in the limiting process, we obtain the following asymptotic system :

$$
\left\{\begin{array}{l}
\partial_{t} u+u \cdot \nabla_{x} u-\mu \Delta_{x} u=-\nabla_{x} p+\frac{1}{2}\left[\frac{\alpha}{\epsilon}\right] n E+\frac{1}{2}\left[\frac{\beta}{\delta}\right] j \wedge B, \\
\partial_{t} \theta+u \cdot \nabla_{x} \theta-\kappa \Delta_{x} \theta=0,
\end{array}\right.
$$

with the constraints from 2.54

$$
\operatorname{div} u=0, \quad \rho+\theta=0 .
$$

Unfortunately, as will be discussed later on in Section 3.2, the rigorous weak stability of the nonlinear terms $n_{\epsilon} E_{\epsilon} \rightarrow n E$ and $j_{\epsilon} \wedge B_{\epsilon} \rightarrow j \wedge B$ remains unclear, in general. This will be, in fact, one of the main reasons for the breakdown of the weak compactness method in the most singular cases of hydrodynamic limits of the two species Vlasov-Maxwell-Boltzmann system 2.32, which will lead us to develop new relative entropy methods and consider dissipative solutions (see Section 3.2.3 and Chapter 12 .

There only remains now to formally establish the asymptotic system for the electrodynamic variables $(n, j, w)$ and the electromagnetic field $(E, B)$, which we do next.

2.4.5. Macroscopic electrodynamic constraints and evolution. The constraint equations for the electrodynamic variables will be obtained from the analysis of the difference of both components of 2.34) :

$(2.59)$

$$
\begin{aligned}
\frac{\epsilon}{\delta} \partial_{t}\left(g_{\epsilon}^{+}-g_{\epsilon}^{-}\right) & +\frac{1}{\delta} v \cdot \nabla_{x}\left(g_{\epsilon}^{+}-g_{\epsilon}^{-}\right) \\
& +\left(\frac{\alpha}{\delta} E_{\epsilon}+\frac{\beta}{\delta} v \wedge B_{\epsilon}\right) \cdot \nabla_{v}\left(g_{\epsilon}^{+}+g_{\epsilon}^{-}\right)-\frac{\alpha}{\delta \epsilon} E_{\epsilon} \cdot v\left(2+\epsilon\left(g_{\epsilon}^{+}+g_{\epsilon}^{-}\right)\right) \\
& =-\frac{1}{\delta^{2}} \mathcal{L}\left(h_{\epsilon}^{+}-h_{\epsilon}^{-}\right)-\mathcal{L}\left(h_{\epsilon}^{+}-h_{\epsilon}^{-}, h_{\epsilon}^{-}-h_{\epsilon}^{+}\right) \\
& +\frac{1-\delta^{2}}{2 \delta} \mathcal{Q}\left(g_{\epsilon}^{+}+g_{\epsilon}^{-}, n_{\epsilon}\right)+\frac{1+\delta^{2}}{2 \delta} \mathcal{Q}\left(n_{\epsilon}, g_{\epsilon}^{+}+g_{\epsilon}^{-}\right) \\
& +\epsilon \frac{1-\delta^{2}}{2 \delta^{2}} \mathcal{Q}\left(g_{\epsilon}^{+}+g_{\epsilon}^{-}, h_{\epsilon}^{+}-h_{\epsilon}^{-}\right)+\epsilon \frac{1+\delta^{2}}{2 \delta^{2}} \mathcal{Q}\left(h_{\epsilon}^{+}-h_{\epsilon}^{-}, g_{\epsilon}^{+}+g_{\epsilon}^{-}\right)
\end{aligned}
$$

However, the analysis in the case $\delta \sim 1$ will slightly differ from the case $\delta=o(1)$, with $\frac{\delta}{\epsilon}$ unbounded.

We begin with the case $\delta \sim 1$ of strong interspecies interactions. First, integrating 2.59 in $M d v$ and letting $\epsilon \rightarrow 0$ easily yields the continuity equation

$$
\partial_{t} n+\frac{1}{[\delta]} \operatorname{div} j=0
$$


Moreover, the above equation 2.59) contains no singular term in this situation. Therefore, letting $\epsilon \rightarrow 0$ yields, employing (2.37),

$$
\begin{aligned}
\frac{1}{[\delta]} v \cdot \nabla_{x} n & -2\left[\frac{\beta}{\delta}\right](u \wedge B) \cdot v-2\left[\frac{\alpha}{\delta \epsilon}\right] E \cdot v \\
& =-\frac{1}{[\delta]^{2}} \mathcal{L}\left(h^{+}-h^{-}\right)-\mathcal{L}\left(h^{+}-h^{-}, h^{-}-h^{+}\right) \\
& +[\delta] n \mathcal{L}\left(u \cdot v+\theta \frac{|v|^{2}}{2},-u \cdot v-\theta \frac{|v|^{2}}{2}\right) .
\end{aligned}
$$

Further defining the linear operator

$$
\mathfrak{L} g=\mathcal{L}(g,-g),
$$

we have

$$
\begin{aligned}
\left(\frac{1}{[\delta]} \nabla_{x} n\right. & \left.-2\left[\frac{\beta}{\delta}\right](u \wedge B)-2\left[\frac{\alpha}{\delta \epsilon}\right] E\right) \cdot v \\
= & {[\delta] n \mathfrak{L}\left(u \cdot v+\theta \frac{|v|^{2}}{2}\right)-\frac{1}{[\delta]^{2}} \mathcal{L}\left(h^{+}-h^{-}\right)-\mathfrak{L}\left(h^{+}-h^{-}\right) . }
\end{aligned}
$$

Now, it can be shown that, in general, the linear operator $\frac{1}{[\delta]^{2}} \mathcal{L}+\mathfrak{L}$ is selfadjoint and Fredholm of index zero on $L^{2}(M d v)$ (or a variant of it depending on the cross-section, see Propositions 5.4 and 5.8. Therefore, its range is exactly the orthogonal complement of its kernel, which is composed of all constant functions (see Proposition 5.9. It follows that $\Phi(v)=v \in L^{2}(M d v)$ and $\Psi(v)=\frac{|v|^{2}}{2}-$ $\frac{3}{2} \in L^{2}(M d v)$ belong to the range of $\frac{1}{[\delta]^{2}} \mathcal{L}+\mathfrak{L}$ and, thus, that there are inverses $\tilde{\Phi} \in L^{2}(M d v)$ and $\tilde{\Psi} \in L^{2}(M d v)$ such that

$$
\Phi=\frac{1}{[\delta]^{2}} \mathcal{L} \tilde{\Phi}+\mathfrak{L} \tilde{\Phi} \quad \text { and } \quad \Psi=\frac{1}{[\delta]^{2}} \mathcal{L} \tilde{\Psi}+\mathfrak{L} \tilde{\Psi}
$$

which can be uniquely determined by the fact that they are orthogonal to the kernel of $\frac{1}{[\delta]^{2}} \mathcal{L}+\mathfrak{L}$ (i.e. to constant functions). Furthermore, it can be shown that $\tilde{\Phi}$ and $\tilde{\Psi}$ have similar symmetry properties as $\Phi$ and $\Psi$, thanks to the rotational invariance of $\mathcal{L}$ and $\mathfrak{L}$. More precisely, employing methods from [28] (see also [13, Section $2.2 .3]$ ), one verifies that there exist two scalar valued functions $\alpha, \beta:[0, \infty) \rightarrow \mathbb{R}$ such that

$$
\tilde{\Phi}(v)=\alpha(|v|) \Phi(v) \quad \text { and } \quad \tilde{\Psi}(v)=\beta(|v|) \Psi(v),
$$

which implies that

$$
\int_{\mathbb{R}^{3}} \Phi_{i} \tilde{\Phi}_{j} M d v=\frac{1}{2} \sigma \delta_{i j}
$$

where

$$
\sigma=\frac{2}{3} \int_{\mathbb{R}^{3}} \Phi \cdot \tilde{\Phi} M d v
$$

defines the electrical conductivity $\sigma>0$. For completeness, we also define the energy conductivity $\lambda>0$ by

$$
\lambda=\int_{\mathbb{R}^{3}} \Psi \tilde{\Psi} M d v
$$

Then, multiplying 2.62 by $\tilde{\Phi}$, integrating in $M d v$, exploiting the self-adjointness of $\mathcal{L}$ and $\mathfrak{L}$ and the limiting representation 2.42 of $\Pi h^{ \pm}$, yields Ohm's law

$$
j-[\delta] n u=\sigma\left(-\frac{1}{2[\delta]} \nabla_{x} n+\left[\frac{\alpha}{\delta \epsilon}\right] E+\left[\frac{\beta}{\delta}\right] u \wedge B\right) .
$$


Similarly, multiplying 2.62 by $\tilde{\Psi}$, we obtain the energy equivalence relation

$$
w=[\delta] n \theta
$$

Finally, in the case $\delta \sim 1$, the whole asymptotic system 2.57-2.58-2.602.67)-2.68 will be fully determined when considering the coupling with the limiting Maxwell's equations from 2.47) :

$$
\left\{\begin{aligned}
{[\gamma] \partial_{t} E-\operatorname{rot} B } & =-\left[\frac{\beta}{\delta}\right] j \\
{[\gamma] \partial_{t} B+\operatorname{rot} E } & =0 \\
\operatorname{div} E & =\left[\frac{\alpha}{\epsilon}\right] n \\
\operatorname{div} B & =0
\end{aligned}\right.
$$

Let us focus now on the case $\delta=o(1)$, which turns out to be more complicated than the case $\delta \sim 1$, for it contains yet another singular limit, as we are about to see. Indeed, the most singular term in 2.59$)$ being $-\frac{1}{\delta^{2}} \mathcal{L}\left(h_{\epsilon}^{+}-h_{\epsilon}^{-}\right)$, we begin by projecting 2.59 onto the collision invariants in order to eliminate this singular term. This yields (this system may also be deduced directly from (2.43) by considering the difference of the equations for cations and anions)

$$
\left\{\begin{aligned}
\partial_{t} n_{\epsilon}+\frac{1}{\delta} \operatorname{div} j_{\epsilon} & =0 \\
\frac{\epsilon^{2}}{\delta^{2}} \partial_{t} j_{\epsilon}+\frac{1}{\delta} \nabla_{x}\left(n_{\epsilon}+\frac{\epsilon}{\delta} w_{\epsilon}\right) & -\frac{2 \alpha}{\delta \epsilon} E_{\epsilon}+\int_{\mathbb{R}^{3}} \mathcal{L}\left(h_{\epsilon}^{+}-h_{\epsilon}^{-}, h_{\epsilon}^{-}-h_{\epsilon}^{+}\right) v M d v \\
& =\left(\frac{2 \alpha}{\delta} \rho_{\epsilon} E_{\epsilon}+\frac{2 \beta}{\delta} u_{\epsilon} \wedge B_{\epsilon}\right) \\
& -\frac{\epsilon}{\delta} \operatorname{div} \int_{\mathbb{R}^{3}} \frac{1}{\epsilon}\left(g_{\epsilon}^{+}-\Pi g_{\epsilon}^{+}-g_{\epsilon}^{-}+\Pi g_{\epsilon}^{-}\right) \phi M d v \\
& +\delta \int_{\mathbb{R}^{3}}\left[\mathcal{Q}\left(g_{\epsilon}^{+}, g_{\epsilon}^{-}\right)-\mathcal{Q}\left(g_{\epsilon}^{-}, g_{\epsilon}^{+}\right)\right] v M d v \\
\frac{3 \epsilon^{2}}{2 \delta^{2}} \partial_{t} w_{\epsilon}+\frac{\epsilon}{\delta^{2}} \operatorname{div} j_{\epsilon} & +\int_{\mathbb{R}^{3}} \mathcal{L}\left(h_{\epsilon}^{+}-h_{\epsilon}^{-}, h_{\epsilon}^{-}-h_{\epsilon}^{+}\right) \frac{|v|^{2}}{2} M d v \\
& =\frac{2 \alpha}{\delta} u_{\epsilon} \cdot E_{\epsilon} \\
& -\frac{\epsilon}{\delta} \operatorname{div} \int_{\mathbb{R}^{3}} \frac{1}{\epsilon}\left(g_{\epsilon}^{+}-\Pi g_{\epsilon}^{+}-g_{\epsilon}^{-}+\Pi g_{\epsilon}^{-}\right) \psi M d v \\
& +\delta \int_{\mathbb{R}^{3}}\left[\mathcal{Q}\left(g_{\epsilon}^{+}, g_{\epsilon}^{-}\right)-\mathcal{Q}\left(g_{\epsilon}^{-}, g_{\epsilon}^{+}\right)\right] \frac{|v|^{2}}{2} M d v
\end{aligned}\right.
$$

Then, since $\Pi h_{\epsilon}^{ \pm}= \pm \frac{1}{2}\left(j_{\epsilon} \cdot v+w_{\epsilon}\left(\frac{|v|^{2}}{2}-\frac{3}{2}\right)\right)$, straightforward computations based on symmetry of integrands show that

$$
\begin{aligned}
\int_{\mathbb{R}^{3}} \mathcal{L}\left(\Pi\left(h_{\epsilon}^{+}-h_{\epsilon}^{-}\right), \Pi\left(h_{\epsilon}^{-}-h_{\epsilon}^{+}\right)\right) v M d v & =\frac{2}{\sigma} j_{\epsilon} \\
\int_{\mathbb{R}^{3}} \mathcal{L}\left(\Pi\left(h_{\epsilon}^{+}-h_{\epsilon}^{-}\right), \Pi\left(h_{\epsilon}^{-}-h_{\epsilon}^{+}\right)\right) \frac{|v|^{2}}{2} M d v & =\frac{1}{\lambda} w_{\epsilon}
\end{aligned}
$$


where the electrical conductivity $\sigma>0$ and the energy conductivity $\lambda>0$ are constants defined by

$$
\begin{aligned}
\frac{1}{\sigma} & =\frac{1}{6} \int_{\mathbb{R}^{3}} v \cdot \mathcal{L}(v,-v) M d v \\
& =\frac{1}{6} \int_{\mathbb{R}^{3} \times \mathbb{R}^{3} \times \mathbb{S}^{2}}\left|v-v^{\prime}\right|^{2} b\left(v-v_{*}, \sigma\right) M M_{*} d v d v_{*} d \sigma \\
& =\frac{1}{6} \int_{\mathbb{R}^{3} \times \mathbb{R}^{3}}\left|v-v_{*}\right|^{2} m\left(v-v_{*}\right) M M_{*} d v d v_{*},
\end{aligned}
$$

and

$$
\begin{aligned}
\frac{1}{\lambda} & =\frac{1}{4} \int_{\mathbb{R}^{3}}|v|^{2} \mathcal{L}\left(|v|^{2},-|v|^{2}\right) M d v \\
& =\frac{1}{4} \int_{\mathbb{R}^{3} \times \mathbb{R}^{3} \times \mathbb{S}^{2}}\left(|v|^{2}-\left|v^{\prime}\right|^{2}\right)^{2} b\left(v-v_{*}, \sigma\right) M M_{*} d v d v_{*} d \sigma \\
& =\frac{1}{4} \int_{\mathbb{R}^{3} \times \mathbb{R}^{3}}\left(|v|^{2}-\left|v_{*}\right|^{2}\right)^{2} m\left(v-v_{*}\right) M M_{*} d v d v_{*},
\end{aligned}
$$

where the cross-section for momentum and energy transfer $m\left(v-v_{*}\right)$ is defined in Proposition A.1. It follows that the system 2.69 may be rewritten as

$(2.72)$

$$
\left\{\begin{aligned}
\partial_{t} n_{\epsilon} & +\frac{1}{\delta} \operatorname{div} j_{\epsilon}=0 \\
\frac{2}{\sigma} j_{\epsilon} & +\frac{1}{\delta} \nabla_{x}\left(n_{\epsilon}+\frac{\epsilon}{\delta} w_{\epsilon}\right)-\frac{2 \alpha}{\delta \epsilon} E_{\epsilon} \\
& =\left(\frac{2 \alpha}{\delta} \rho_{\epsilon} E_{\epsilon}+\frac{2 \beta}{\delta} u_{\epsilon} \wedge B_{\epsilon}\right)-\frac{\epsilon^{2}}{\delta^{2}} \partial_{t} j_{\epsilon} \\
& -\frac{\epsilon}{\delta} \operatorname{div} \int_{\mathbb{R}^{3}} \frac{1}{\epsilon}\left(g_{\epsilon}^{+}-\Pi g_{\epsilon}^{+}-g_{\epsilon}^{-}+\Pi g_{\epsilon}^{-}\right) \phi M d v \\
& -\int_{\mathbb{R}^{3}} \mathcal{L}\left(h_{\epsilon}^{+}-\Pi h_{\epsilon}^{+}-h_{\epsilon}^{-}+\Pi h_{\epsilon}^{-}, h_{\epsilon}^{-}-\Pi h_{\epsilon}^{-}-h_{\epsilon}^{+}+\Pi h_{\epsilon}^{+}\right) v M d v \\
& +\delta \int_{\mathbb{R}^{3}}\left[\mathcal{Q}\left(g_{\epsilon}^{+}, g_{\epsilon}^{-}\right)-\mathcal{Q}\left(g_{\epsilon}^{-}, g_{\epsilon}^{+}\right)\right] v M d v, \\
\frac{1}{\lambda} w_{\epsilon} & +\frac{\epsilon}{\delta^{2}} \operatorname{div} j_{\epsilon} \\
& =\frac{2 \alpha}{\delta} u_{\epsilon} \cdot E_{\epsilon}-\frac{3 \epsilon^{2}}{2 \delta^{2}} \partial_{t} w_{\epsilon} \\
& -\frac{\epsilon}{\delta} \operatorname{div} \int_{\mathbb{R}^{3}} \frac{1}{\epsilon}\left(g_{\epsilon}^{+}-\Pi g_{\epsilon}^{+}-g_{\epsilon}^{-}+\Pi g_{\epsilon}^{-}\right) \psi M d v \\
& -\int_{\mathbb{R}^{3}} \mathcal{L}\left(h_{\epsilon}^{+}-\Pi h_{\epsilon}^{+}-h_{\epsilon}^{-}+\Pi h_{\epsilon}^{-}, h_{\epsilon}^{-}-\Pi h_{\epsilon}^{-}-h_{\epsilon}^{+}+\Pi h_{\epsilon}^{+}\right) \frac{|v|^{2}}{2} M d v \\
& +\delta \int_{\mathbb{R}^{3}}\left[\mathcal{Q}\left(g_{\epsilon}^{+}, g_{\epsilon}^{-}\right)-\mathcal{Q}\left(g_{\epsilon}^{-}, g_{\epsilon}^{+}\right)\right] \frac{|v|^{2}}{2} M d v .
\end{aligned}\right.
$$

In fact, the system 2.72 coupled with Maxwell's equations 2.47) still contains a singular perturbation, which will be treated much like the singular perturbation of the one species case in Sections 2.3.2 and 2.3.3. Thus, by virtue of (2.41), it is 
readily seen that the system 2.72 may be further simplified to

$$
\left\{\begin{aligned}
\operatorname{div} j_{\epsilon} & =O(\delta) \\
\nabla_{x}\left(n_{\epsilon}+\frac{\epsilon}{\delta} w_{\epsilon}\right) & =\frac{2 \alpha}{\epsilon} E_{\epsilon}+O(\delta) \\
P j_{\epsilon} & =\sigma P\left(\frac{\alpha}{\delta \epsilon} E_{\epsilon}+\frac{\beta}{\delta} u_{\epsilon} \wedge B_{\epsilon}\right)+o(1) \\
w_{\epsilon} & =o(1)
\end{aligned}\right.
$$

We discuss now the limit $\epsilon \rightarrow 0$ of the coupled system $(2.47)-(2.73)$.

(1) When $\gamma \sim 1$ (so that $\alpha=O(\delta \epsilon)$ ), letting $\epsilon \rightarrow 0$ in 2.47)-(2.73), we obtain

$$
\left\{\begin{aligned}
{[\gamma] \partial_{t} E-\operatorname{rot} B } & =-\left[\frac{\beta}{\delta}\right] j, & \operatorname{div} E & =0 \\
{[\gamma] \partial_{t} B+\operatorname{rot} E } & =0, & \operatorname{div} B & =0 \\
j & =\sigma\left(-\nabla_{x} \bar{p}+\left[\frac{\alpha}{\delta \epsilon}\right] E+\left[\frac{\beta}{\delta}\right] u \wedge B\right), & \operatorname{div} j & =0 \\
n & =0, & w & =0,
\end{aligned}\right.
$$

where $\bar{p}$ is an electrodynamic pressure.

(2) When $\gamma=o(1)$ and $\alpha=O(\delta \epsilon)$, letting $\epsilon \rightarrow 0$ in 2.47)-2.73), we obtain

$$
\left\{\begin{array}{rlrl}
E & =0 \\
\operatorname{rot} B & =\left[\frac{\beta}{\delta}\right] j, & \operatorname{div} B & =0 \\
j & =\sigma\left(-\nabla_{x} \bar{p}+\left[\frac{\beta}{\delta}\right] u \wedge B\right), \\
n & =0, & \operatorname{div} j & =0 \\
w & =0
\end{array}\right.
$$

where $\bar{p}$ is an electrodynamic pressure.

(3) When $\gamma=o(1)$ and $\frac{\alpha}{\delta \epsilon}$ is unbounded, we need to further use Faraday's equation from 2.47, as in Section 2.3.3, to write that

$$
\frac{\beta}{\delta} \partial_{t} A_{\epsilon}+\frac{\alpha}{\delta \epsilon} P E_{\epsilon}=0
$$

where $B_{\epsilon}=\operatorname{rot} A_{\epsilon}$ and $\operatorname{div} A_{\epsilon}=0$. Thus, letting $\epsilon \rightarrow 0$ in 2.47)-2.73, we obtain

$$
\left\{\begin{aligned}
\operatorname{div} E & =\left[\frac{\alpha}{\epsilon}\right] n, & \operatorname{rot} E & =0, \\
\operatorname{rot} B & =\left[\frac{\beta}{\delta}\right] j, & \operatorname{div} B & =0, \\
\operatorname{rot} A & =B, & \operatorname{div} A & =0, \\
j & =\sigma\left(-\nabla_{x} \bar{p}-\left[\frac{\beta}{\delta}\right] \partial_{t} A+\left[\frac{\beta}{\delta}\right] u \wedge B\right), & \operatorname{div} j & =0, \\
\nabla_{x} n & =2\left[\frac{\alpha}{\epsilon}\right] E, & w & =0,
\end{aligned}\right.
$$

where $A_{\epsilon} \rightarrow A$ and $\bar{p}$ is an electrodynamic pressure. Note that $\Delta_{x} n=$ $2\left[\frac{\alpha}{\epsilon}\right]^{2} n$, so that necessarily $n=0$ and $E=0$. The above system can be rewritten more explicitly by defining the adjusted electric field $\tilde{E}=-\partial_{t} A$. 
It then holds that

$$
\left\{\begin{aligned}
\operatorname{rot} B & =\left[\frac{\beta}{\delta}\right] j, & \operatorname{div} B & =0, \\
\partial_{t} B+\operatorname{rot} \tilde{E} & =0, & \operatorname{div} \tilde{E} & =0, \\
j & =\sigma\left(-\nabla_{x} \bar{p}+\left[\frac{\beta}{\delta}\right] \tilde{E}+\left[\frac{\beta}{\delta}\right] u \wedge B\right), & \operatorname{div} j & =0, \\
n & =0, & w & =0 .
\end{aligned}\right.
$$

2.4.6. Summary. At last, we see that the asymptotics of the two species Vlasov-Maxwell-Boltzmann system 2.32 can be depicted in terms of the limits of the following parameters :

- the strength of the electric induction $\alpha$,

- the strength of the magnetic induction $\beta=\frac{\alpha \gamma}{\epsilon}$,

- the ratio of the bulk velocity to the speed of light $\gamma$,

- the strength of the interspecies collisional interactions $\delta$.

The case of very weak interspecies collisions has already been discussed in Section 2.4 .2 and is analogous to the one species case. Regarding the weak and strong interspecies collisions, Figures 2 and 3 summarize the different asymptotic regimes, on a logarithmic scale, of the two species Vlasov-Maxwell-Boltzmann system 2.32 .

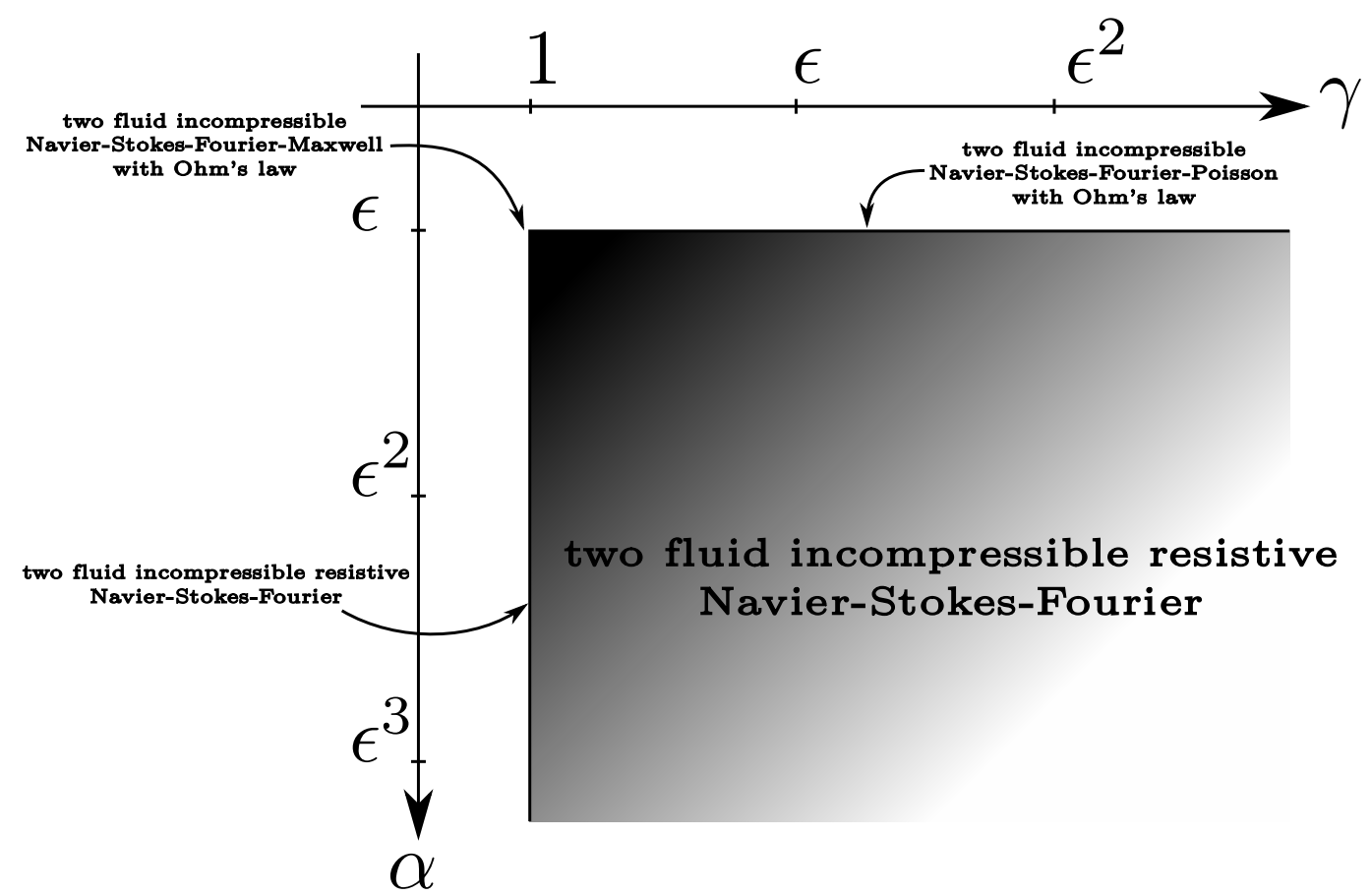

Figure 2. Asymptotic regimes of the two species Vlasov-MaxwellBoltzmann system 2.32 for strong interspecies interactions.

Thus, up to multiplicative constants, in the case of strong interactions $\delta=1$, we reach the following asymptotic systems of equations : 
(1) If $\alpha=o(\epsilon)$, we obtain the two fluid incompressible resistive Navier-StokesFourier system :

$$
\left\{\begin{aligned}
\partial_{t} u+u \cdot \nabla_{x} u-\mu \Delta_{x} u & =-\nabla_{x} p, & & \operatorname{div} u=0, \\
\partial_{t} \theta+u \cdot \nabla_{x} \theta-\kappa \Delta_{x} \theta & =0, & \rho+\theta & =0, \\
\partial_{t} n+u \cdot \nabla_{x} n-\frac{\sigma}{2} \Delta_{x} n & =0, & j-n u & =-\frac{\sigma}{2} \nabla_{x} n, \quad w=n \theta .
\end{aligned}\right.
$$

This system satisfies the following formal energy conservation laws :

$$
\begin{aligned}
& \frac{1}{2} \frac{d}{d t}\|u\|_{L_{x}^{2}}^{2}+\mu\left\|\nabla_{x} u\right\|_{L_{x}^{2}}^{2}=0, \\
& \frac{1}{2} \frac{d}{d t}\|\theta\|_{L_{x}^{2}}^{2}+\kappa\left\|\nabla_{x} \theta\right\|_{L_{x}^{2}}^{2}=0, \\
& \frac{1}{2} \frac{d}{d t}\|n\|_{L_{x}^{2}}^{2}+\frac{\sigma}{2}\left\|\nabla_{x} n\right\|_{L_{x}^{2}}^{2}=0 .
\end{aligned}
$$

(2) If $\alpha=\epsilon$ and $\gamma=1$, we obtain the two fluid incompressible Navier-StokesFourier-Maxwell system with Ohm's law :

$$
\left\{\begin{aligned}
\partial_{t} u+u \cdot \nabla_{x} u-\mu \Delta_{x} u & =-\nabla_{x} p+\frac{1}{2}(n E+j \wedge B), & & \operatorname{div} u=0, \\
\partial_{t} \theta+u \cdot \nabla_{x} \theta-\kappa \Delta_{x} \theta & =0, & \rho+\theta & =0, \\
\partial_{t} E-\operatorname{rot} B & =-j, & \operatorname{div} E & =n, \\
\partial_{t} B+\operatorname{rot} E & =0, & \operatorname{div} B & =0, \\
j-n u & =\sigma\left(-\frac{1}{2} \nabla_{x} n+E+u \wedge B\right), & & =n \theta .
\end{aligned}\right.
$$

This system satisfies the following formal energy conservation laws (see Proposition 3.3 for an explicit computation of the energy) :

$$
\begin{array}{r}
\frac{1}{4} \frac{d}{d t}\left(2\|u\|_{L_{x}^{2}}^{2}+\frac{1}{2}\|n\|_{L_{x}^{2}}^{2}+\|E\|_{L_{x}^{2}}^{2}+\|B\|_{L_{x}^{2}}^{2}\right)+\mu\left\|\nabla_{x} u\right\|_{L_{x}^{2}}^{2}+\frac{1}{2 \sigma}\|j-n u\|_{L_{x}^{2}}^{2}=0 \\
\frac{1}{2} \frac{d}{d t}\|\theta\|_{L_{x}^{2}}^{2}+\kappa\left\|\nabla_{x} \theta\right\|_{L_{x}^{2}}^{2}=0 .
\end{array}
$$

(3) If $\alpha=\epsilon$ and $\gamma=o(1)$, we obtain the two fluid incompressible NavierStokes-Fourier-Poisson system with Ohm's law :

$$
\left\{\begin{aligned}
\partial_{t} u+u \cdot \nabla_{x} u-\mu \Delta_{x} u & =-\nabla_{x} p+\frac{1}{2} n \nabla_{x} \phi, & \operatorname{div} u & =0, \\
\partial_{t} \theta+u \cdot \nabla_{x} \theta-\kappa \Delta_{x} \theta & =0, & \rho+\theta & =0, \\
\partial_{t} n+u \cdot \nabla_{x} n-\frac{\sigma}{2} \Delta_{x} n+\sigma n & =0, & \Delta_{x} \phi & =n, \\
j-n u & =\sigma \nabla_{x}\left(\phi-\frac{1}{2} n\right), & w & =n \theta .
\end{aligned}\right.
$$

This system satisfies the following formal energy conservation laws :

$$
\begin{array}{r}
\frac{1}{4} \frac{d}{d t}\left(2\|u\|_{L_{x}^{2}}^{2}+\frac{1}{2}\|n\|_{L_{x}^{2}}^{2}+\left\|\nabla_{x} \phi\right\|_{L_{x}^{2}}^{2}\right)+\mu\left\|\nabla_{x} u\right\|_{L_{x}^{2}}^{2}+\frac{1}{2 \sigma}\|j-n u\|_{L_{x}^{2}}^{2}=0 \\
\frac{1}{2} \frac{d}{d t}\|n\|_{L_{x}^{2}}^{2}+\frac{\sigma}{2}\left\|\nabla_{x} n\right\|_{L_{x}^{2}}^{2}+\sigma\|n\|_{L_{x}^{2}}^{2}=0 \\
\frac{1}{2} \frac{d}{d t}\|\theta\|_{L_{x}^{2}}^{2}+\kappa\left\|\nabla_{x} \theta\right\|_{L_{x}^{2}}^{2}=0 .
\end{array}
$$

Finally, up to multiplicative constants, in the case of weak interactions $\delta=o(1)$, we reach the following asymptotic systems of equations : 


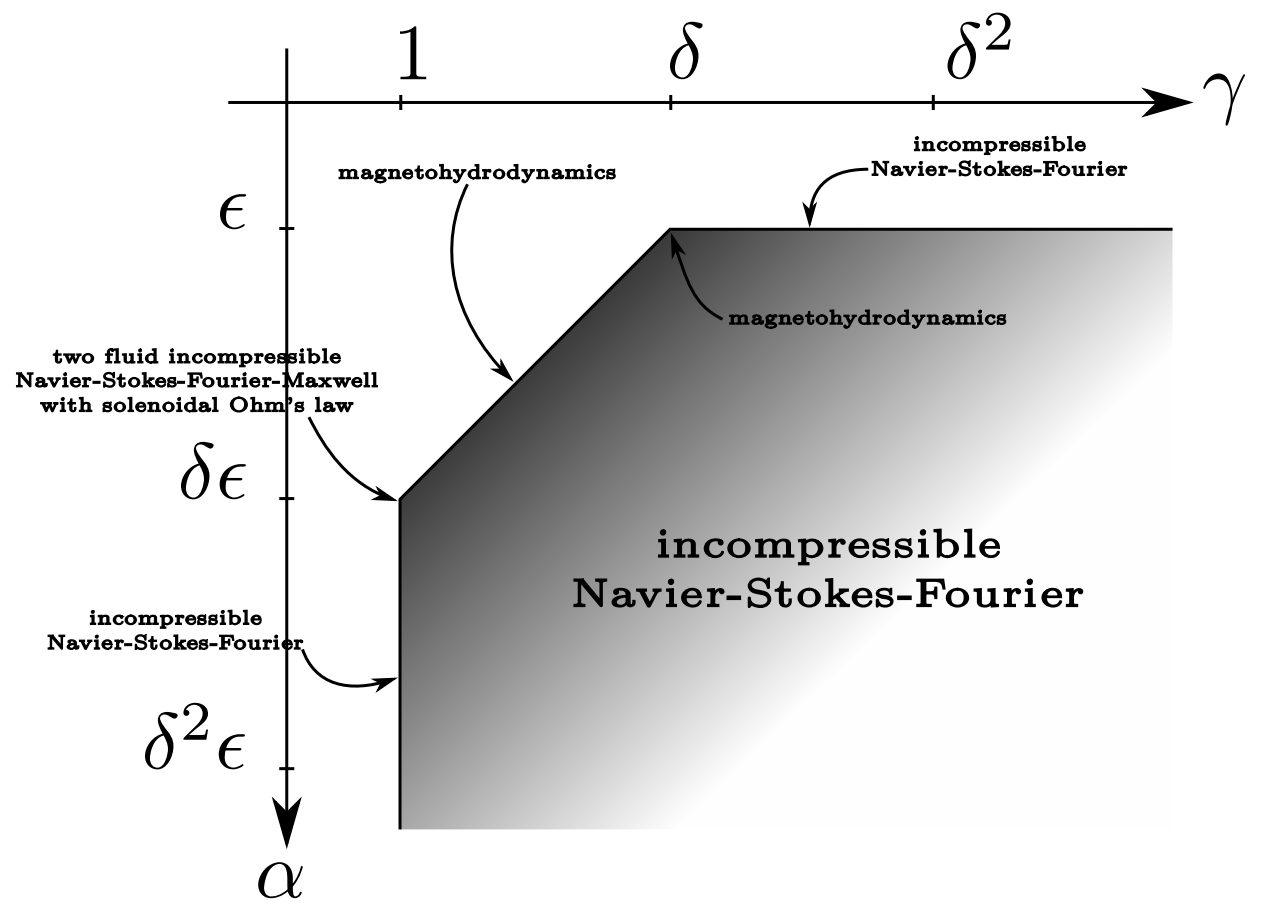

Figure 3. Asymptotic regimes of the two species Vlasov-MaxwellBoltzmann system 2.32 for weak interspecies interactions.

(1) If $\beta=o(\delta)$, we obtain the incompressible Navier-Stokes-Fourier system :

$$
\left\{\begin{aligned}
\partial_{t} u+u \cdot \nabla_{x} u-\mu \Delta_{x} u & =-\nabla_{x} p, & \operatorname{div} u & =0, \\
\partial_{t} \theta+u \cdot \nabla_{x} \theta-\kappa \Delta_{x} \theta & =0, & \rho+\theta & =0, \\
n & =0, & j & =0, \quad w=0 .
\end{aligned}\right.
$$

This system satisfies the following formal energy conservation laws :

$$
\begin{aligned}
& \frac{1}{2} \frac{d}{d t}\|u\|_{L_{x}^{2}}^{2}+\mu\left\|\nabla_{x} u\right\|_{L_{x}^{2}}^{2}=0, \\
& \frac{1}{2} \frac{d}{d t}\|\theta\|_{L_{x}^{2}}^{2}+\kappa\left\|\nabla_{x} \theta\right\|_{L_{x}^{2}}^{2}=0 .
\end{aligned}
$$

(2) If $\alpha=\delta \epsilon$ and $\gamma=1$, we obtain the two fluid incompressible Navier-StokesFourier-Maxwell system with solenoidal Ohm's law :

$$
\left\{\begin{aligned}
\partial_{t} u+u \cdot \nabla_{x} u-\mu \Delta_{x} u & =-\nabla_{x} p+\frac{1}{2} j \wedge B, & \operatorname{div} u & =0, \\
\partial_{t} \theta+u \cdot \nabla_{x} \theta-\kappa \Delta_{x} \theta & =0, & \rho+\theta & =0, \\
\partial_{t} E-\operatorname{rot} B & =-j, & \operatorname{div} E & =0, \\
\partial_{t} B+\operatorname{rot} E & =0, & \operatorname{div} B & =0, \\
j & =\sigma\left(-\nabla_{x} \bar{p}+E+u \wedge B\right), & \operatorname{div} j & =0, \\
n & =0, & w & =0 .
\end{aligned}\right.
$$

This system satisfies the following formal energy conservation laws (see Proposition 3.3 for an explicit computation of the energy) :

$$
\begin{aligned}
\frac{1}{4} \frac{d}{d t}\left(2\|u\|_{L_{x}^{2}}^{2}+\|E\|_{L_{x}^{2}}^{2}+\|B\|_{L_{x}^{2}}^{2}\right)+\mu\left\|\nabla_{x} u\right\|_{L_{x}^{2}}^{2}+\frac{1}{2 \sigma}\|j\|_{L_{x}^{2}}^{2} & =0 \\
\frac{1}{2} \frac{d}{d t}\|\theta\|_{L_{x}^{2}}^{2}+\kappa\left\|\nabla_{x} \theta\right\|_{L_{x}^{2}}^{2} & =0 .
\end{aligned}
$$


(3) If $\beta=\delta$ and $\gamma=o(1)$, we obtain the two fluid incompressible quasi-static Navier-Stokes-Fourier-Maxwell system with solenoidal Ohm's law :

$$
\left\{\begin{aligned}
\partial_{t} u+u \cdot \nabla_{x} u-\mu \Delta_{x} u & =-\nabla_{x} p+\frac{1}{2} j \wedge B, & & \operatorname{div} u=0, \\
\partial_{t} \theta+u \cdot \nabla_{x} \theta-\kappa \Delta_{x} \theta & =0, & \rho+\theta & =0, \\
\operatorname{rot} B & =j, & \operatorname{div} E & =0, \\
\partial_{t} B+\operatorname{rot} E & =0, & \operatorname{div} B & =0, \\
j & =\sigma\left(-\nabla_{x} \bar{p}+E+u \wedge B\right), & \operatorname{div} j & =0, \\
n & =0, & w & =0 .
\end{aligned}\right.
$$

This system satisfies the following formal energy conservation laws :

$$
\begin{aligned}
& \frac{1}{4} \frac{d}{d t}\left(2\|u\|_{L_{x}^{2}}^{2}+\|B\|_{L_{x}^{2}}^{2}\right)+ \mu\left\|\nabla_{x} u\right\|_{L_{x}^{2}}^{2}+\frac{1}{2 \sigma}\|j\|_{L_{x}^{2}}^{2}=0 \\
& \frac{1}{2} \frac{d}{d t}\|\theta\|_{L_{x}^{2}}^{2}+\kappa\left\|\nabla_{x} \theta\right\|_{L_{x}^{2}}^{2}=0 .
\end{aligned}
$$

Here, the electric field is defined indirectly as a mere distribution, through Faraday's equation, by

$$
E=-\partial_{t} A
$$

where $B=\operatorname{rot} A$ and $\operatorname{div} A=0$. Note that the above system can be rewritten as

$\left\{\begin{aligned} \partial_{t} u+u \cdot \nabla_{x} u-\mu \Delta_{x} u & =-\nabla_{x} p+\frac{1}{2} \operatorname{rot} B \wedge B, & & \operatorname{div} u=0, \\ \partial_{t} \theta+u \cdot \nabla_{x} \theta-\kappa \Delta_{x} \theta & =0, & & \rho+\theta=0, \\ \partial_{t} B+u \cdot \nabla_{x} B-\frac{1}{\sigma} \Delta_{x} B & =B \cdot \nabla_{x} u, & & \operatorname{div} B=0,\end{aligned}\right.$

which is nothing but the well-known magnetohydrodynamic system. The rigorous derivation of this system starting from other macroscopic systems such as 2.48 and 2.75 as been investigated in [5].

2.4.7. The two species Vlasov-Poisson-Boltzmann system. The two species Vlasov-Poisson-Boltzmann system describes the evolution of a gas of two species of charged particles (cations and anions) subject to an auto-induced electrostatic force. This system is obtained formally from the two species VlasovMaxwell-Boltzmann system by letting the speed of light tend to infinity while all other parameters remain fixed. Accordingly, setting $\gamma=0$ in 2.32 yields the scaled Vlasov-Poisson-Boltzmann system :

$$
\left\{\begin{aligned}
\epsilon \partial_{t} f_{\epsilon}^{ \pm}+v \cdot \nabla_{x} f_{\epsilon}^{ \pm} \pm \alpha \nabla_{x} \phi_{\epsilon} \cdot \nabla_{v} f_{\epsilon}^{ \pm} & =\frac{1}{\epsilon} Q\left(f_{\epsilon}^{ \pm}, f_{\epsilon}^{ \pm}\right)+\frac{\delta^{2}}{\epsilon} Q\left(f_{\epsilon}^{ \pm}, f_{\epsilon}^{\mp}\right), \\
f_{\epsilon}^{ \pm} & =M\left(1+\epsilon g_{\epsilon}^{ \pm}\right), \\
\Delta_{x} \phi_{\epsilon} & =\frac{\alpha}{\epsilon} \int_{\mathbb{R}^{3}}\left(g_{\epsilon}^{+}-g_{\epsilon}^{-}\right) M d v,
\end{aligned}\right.
$$

Here, the plasma is subject to a self-induced electrostatic field $E_{\epsilon}$ determined by

$$
\operatorname{rot} E_{\epsilon}=0, \quad \operatorname{div} E_{\epsilon}=\frac{\alpha}{\epsilon} \int_{\mathbb{R}^{3}}\left(g_{\epsilon}^{+}-g_{\epsilon}^{-}\right) M d v,
$$

hence

$$
E_{\epsilon}=\nabla_{x} \phi_{\epsilon} .
$$

The above system is supplemented with some initial data satisfying

$$
\frac{1}{\epsilon^{2}} H\left(f_{\epsilon}^{+ \text {in }}\right)+\frac{1}{\epsilon^{2}} H\left(f_{\epsilon}^{- \text {in }}\right)+\frac{1}{2} \int_{\mathbb{R}^{3}}\left|E_{\epsilon}^{\text {in }}\right|^{2} d x<\infty .
$$


In particular, solutions of 2.76 satisfy the corresponding scaled entropy inequality, where $t>0$,

$$
\begin{aligned}
\frac{1}{\epsilon^{2}} H\left(f_{\epsilon}^{+}\right)+\frac{1}{\epsilon^{2}} H\left(f_{\epsilon}^{-}\right) & +\frac{1}{2} \int_{\mathbb{R}^{3}}\left|E_{\epsilon}\right|^{2} d x \\
& +\frac{1}{\epsilon^{4}} \int_{0}^{t} \int_{\mathbb{R}^{3}}\left(D\left(f_{\epsilon}^{+}\right)+D\left(f_{\epsilon}^{-}\right)+\delta^{2} D\left(f_{\epsilon}^{+}, f_{\epsilon}^{-}\right)\right)(s) d x d s \\
& \leq \frac{1}{\epsilon^{2}} H\left(f_{\epsilon}^{+\mathrm{in}}\right)+\frac{1}{\epsilon^{2}} H\left(f_{\epsilon}^{-\mathrm{in}}\right)+\frac{1}{2} \int_{\mathbb{R}^{3}}\left|E_{\epsilon}^{\mathrm{in}}\right|^{2} d x,
\end{aligned}
$$

Thus, the formal asymptotic analysis of 2.76 is contained in our analysis of the two species Vlasov-Maxwell-Boltzmann system (2.32). Specifically, setting $\gamma=\beta=$ 0 in the limiting systems first obtained in Section 2.4 .2 , for very weak interspecies collisions, we see that the two species Vlasov-Poisson-Boltzmann system 2.76 . converges, when $\alpha=o(\epsilon)$ and $\delta=O(\epsilon)$, towards the two fluid incompressible Navier-Stokes-Fourier system in a Boussinesq regime, with $E=0$ :

$$
\begin{cases}\partial_{t} u^{ \pm}+u^{ \pm} \cdot \nabla_{x} u^{ \pm}-\mu \Delta_{x} u^{ \pm} \pm\left[\frac{\delta}{\epsilon}\right]^{2} \frac{1}{\sigma}\left(u^{+}-u^{-}\right)=-\nabla_{x} p^{ \pm}, & \operatorname{div} u^{ \pm}=0 \\ \partial_{t} \theta^{ \pm}+u^{ \pm} \cdot \nabla_{x} \theta^{ \pm}-\kappa \Delta_{x} \theta^{ \pm} \pm\left[\frac{\delta}{\epsilon}\right]^{2} \frac{1}{\kappa}\left(\theta^{+}-\theta^{-}\right)=0, & \rho^{ \pm}+\theta^{ \pm}=0 .\end{cases}
$$

While, when $\left[\frac{\alpha}{\epsilon}\right] \neq 0$ and $\delta=O(\epsilon)$, we find the convergence towards the two fluid incompressible Navier-Stokes-Fourier-Poisson system :

$$
\left\{\begin{aligned}
\partial_{t} u^{ \pm}+u^{ \pm} \cdot \nabla_{x} u^{ \pm}-\mu \Delta_{x} u^{ \pm} \pm\left[\frac{\delta}{\epsilon}\right]^{2} \frac{1}{\sigma}\left(u^{+}-u^{-}\right) & =-\nabla_{x} p^{ \pm}+\rho^{ \pm} \nabla_{x} \theta^{ \pm}, \\
\operatorname{div} u^{ \pm} & =0, \\
\partial_{t}\left(\frac{3}{2} \theta^{ \pm}-\rho^{ \pm}\right)+u^{ \pm} \cdot \nabla_{x}\left(\frac{3}{2} \theta^{ \pm}-\rho^{ \pm}\right)-\frac{5}{2} \kappa \Delta_{x} \theta^{ \pm} & \\
\pm \frac{5}{2}\left[\frac{\delta}{\epsilon}\right]^{2} \frac{1}{\lambda}\left(\theta^{+}-\theta^{-}\right) & =0, \\
\Delta_{x}\left(\rho^{ \pm}+\theta^{ \pm}\right) & = \pm\left[\frac{\alpha}{\epsilon}\right]^{2}\left(\rho^{+}-\rho^{-}\right),
\end{aligned}\right.
$$

where the electrostatic field is determined by $\left[\frac{\alpha}{\epsilon}\right] E= \pm \nabla_{x}\left(\rho^{ \pm}+\theta^{ \pm}\right)$.

Regarding weak interspecies interactions, setting $\gamma=\beta=0$ in the corresponding limiting systems obtained in Sections 2.4.4 and 2.4.5. we see that the two species Vlasov-Poisson-Boltzmann system 2.76 always converges, when $\delta=o(1)$ and $\frac{\delta}{\epsilon}$ is unbounded, towards the incompressible Navier-Stokes-Fourier system in a Boussinesq regime, with $E=0$ :

$$
\left\{\begin{aligned}
\partial_{t} u+u \cdot \nabla_{x} u-\mu \Delta_{x} u & =-\nabla_{x} p, & \operatorname{div} u & =0, \\
\partial_{t} \theta+u \cdot \nabla_{x} \theta-\kappa \Delta_{x} \theta & =0, & \rho+\theta & =0, \\
n & =0, & j & =0, \quad w=0 .
\end{aligned}\right.
$$

Finally, in the case of strong interspecies interactions, setting $\gamma=\beta=0$ in corresponding the limiting systems obtained in Sections 2.4.4 and 2.4.5, we see that the two species Vlasov-Poisson-Boltzmann system 2.76 converges, when $\alpha=o(\epsilon)$ and $\delta=1$, towards the two fluid incompressible resistive Navier-Stokes-Fourier system in a Boussinesq regime, with $E=0$ :

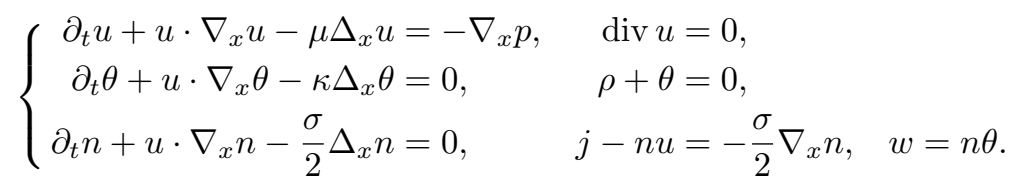


While, when $\left[\frac{\alpha}{\epsilon}\right] \neq 0$ and $\delta=1$, we find the convergence towards the two fluid incompressible Navier-Stokes-Fourier-Poisson system with Ohm's law :

$$
\left\{\begin{aligned}
\partial_{t} u+u \cdot \nabla_{x} u-\mu \Delta_{x} u & =-\nabla_{x} p+\frac{1}{2}\left[\frac{\alpha}{\epsilon}\right] n \nabla_{x} \phi, & \operatorname{div} u & =0, \\
\partial_{t} \theta+u \cdot \nabla_{x} \theta-\kappa \Delta_{x} \theta & =0, & \rho+\theta & =0, \\
\partial_{t} n+u \cdot \nabla_{x} n-\frac{\sigma}{2} \Delta_{x} n+\sigma\left[\frac{\alpha}{\epsilon}\right]^{2} n & =0, & \Delta_{x} \phi & =\left[\frac{\alpha}{\epsilon}\right] n, \\
j-n u & =\sigma \nabla_{x}\left(\left[\frac{\alpha}{\epsilon}\right] \phi-\frac{1}{2} n\right), & w & =n \theta,
\end{aligned}\right.
$$

where the electrostatic field is determined by $E=\nabla_{x} \phi$.

In fact, the two species Vlasov-Poisson-Boltzmann system is inherently simpler than the two species Vlasov-Maxwell-Boltzmann system, because it couples the Vlasov-Boltzmann equations with a simple elliptic equation, namely Poisson's equation, while the two species Vlasov-Maxwell-Boltzmann system couples the Vlasov-Boltzmann equations with an hyperbolic system, namely Maxwell's system of equations. Thus, the rigorous mathematical analysis on the two species VlasovMaxwell-Boltzmann system, presented in the remainder of this work, will also apply to the two species Vlasov-Poisson-Boltzmann system and, therefore, analog results will hold. 



\section{CHAPTER 3}

\section{Weak stability of the limiting macroscopic systems}

In the previous chapter, we have formally derived numerous viscous incompressible systems for plasmas starting from Vlasov-Maxwell-Boltzmann systems and we intend to provide, in the remainder of our work, justifications of these derivations. Nevertheless, prior to any rigorous proof of hydrodynamic limit, it is crucial to understand the well-posedness of the asymptotic macroscopic models and to study their stability properties.

Describing the Cauchy problem of each single macroscopic system from Chapter 2 would be unreasonable. Rather, we are now going to focus on the following three systems found therein :

- the incompressible quasi-static Navier-Stokes-Fourier-Maxwell-Poisson system 2.29,

- the two-fluid incompressible Navier-Stokes-Fourier-Maxwell system with Ohm's law 2.74,

- the two-fluid incompressible Navier-Stokes-Fourier-Maxwell system with solenoidal Ohm's law 2.75,

and establish the existence of weak or dissipative solutions to their respective initial value problems. In fact, these three systems are among the most singular ones found in Chapter 2. Thus, we hope the reader will find it clear that the existence of appropriate weak or dissipative solutions to the remaining macroscopic systems from Chapter 2 will then follow from straightforward adjustments of the existence theories presented here.

In the remaining Parts 2, 3 and 4 of our work, we will also focus on the three aforementioned systems and give complete justifications of their derivation from hydrodynamic limits of Vlasov-Maxwell-Boltzmann systems.

\subsection{The incompressible quasi-static Navier-Stokes-Fourier-Maxwell-Poisson system}

We are first concerned here with the incompressible quasi-static Navier-StokesFourier-Maxwell-Poisson system 2.29 , which we rewrite, for mere convenience :

$$
\left\{\begin{array}{rlrl}
\partial_{t} u+u \cdot \nabla_{x} u-\mu \Delta_{x} u & =-\nabla_{x} p+E+\rho \nabla_{x} \theta+u \wedge B & \\
\partial_{t}\left(\frac{3}{2} \theta-\rho\right)+u \cdot \nabla_{x}\left(\frac{3}{2} \theta-\rho\right)-\frac{5}{2} \kappa \Delta_{x} \theta & =0, & \operatorname{div} u & =0, \\
\operatorname{rot} B & =u, & \Delta_{x}(\rho+\theta) & =\rho, \\
\partial_{t} B+\operatorname{rot} E & =0, & \operatorname{div} E & =\rho, \\
\operatorname{div} B & =0 .
\end{array}\right.
$$

Although it looks more complicated because it involves more terms, the system (3.1) has the same structure as the incompressible Navier-Stokes equations : it is indeed a system of parabolic equations, in which the nonlinear advection terms are well-defined by the energy estimate.

The following formal proposition shows how to compute the energy. 
Proposition 3.1. Let $(\rho, u, \theta, B)$ be a smooth solution to the incompressible quasi-static Navier-Stokes-Fourier-Maxwell-Poisson system (3.1).

Then, the following global energy inequality holds :

$$
\begin{aligned}
& \frac{1}{2}\left(\|\rho(t)\|_{L_{x}^{2}}^{2}+\|u(t)\|_{L_{x}^{2}}^{2}+\frac{3}{2}\|\theta(t)\|_{L_{x}^{2}}^{2}+\left\|\nabla_{x}(\rho+\theta)(t)\right\|_{L_{x}^{2}}^{2}+\|B(t)\|_{L_{x}^{2}}^{2}\right) \\
& \quad+\int_{0}^{t} \mu\left\|\nabla_{x} u(s)\right\|_{L_{x}^{2}}^{2}+\frac{5}{2} \kappa\left\|\nabla_{x} \theta(s)\right\|_{L_{x}^{2}}^{2} d s \\
& \quad \leq \frac{1}{2}\left(\left\|\rho^{\mathrm{in}}\right\|_{L_{x}^{2}}^{2}+\left\|u^{\mathrm{in}}\right\|_{L_{x}^{2}}^{2}+\frac{3}{2}\left\|\theta^{\mathrm{in}}\right\|_{L_{x}^{2}}^{2}+\left\|\nabla_{x}\left(\rho^{\mathrm{in}}+\theta^{\mathrm{in}}\right)\right\|_{L_{x}^{2}}^{2}+\left\|B^{\mathrm{in}}\right\|_{L_{x}^{2}}^{2}\right)
\end{aligned}
$$

where $\nabla_{x}(\rho+\theta)=P^{\perp} E \neq E$.

Proof. Multiplying the equation expressing the conservation of momentum in (3.1) by $u$ and integrating with respect to space variables, we get

$$
\begin{aligned}
\frac{1}{2} \frac{d}{d t}\|u\|_{L_{x}^{2}}^{2}+\mu\left\|\nabla_{x} u\right\|_{L_{x}^{2}}^{2} & =\int_{\mathbb{R}^{3}} u \cdot E+\rho u \cdot \nabla_{x} \theta d x \\
& =\int_{\mathbb{R}^{3}} \operatorname{rot} B \cdot E+\rho u \cdot \nabla_{x} \theta d x \\
& =-\frac{1}{2} \frac{d}{d t}\|B\|_{L_{x}^{2}}^{2}+\int_{\mathbb{R}^{3}} \rho u \cdot \nabla_{x} \theta d x
\end{aligned}
$$

Then, multiplying the equation expressing the conservation of energy by $\theta$, we get similarly

$$
\begin{aligned}
\frac{3}{4} \frac{d}{d t}\|\theta\|_{L_{x}^{2}}^{2}+\frac{5}{2} \kappa\left\|\nabla_{x} \theta\right\|_{L_{x}^{2}}^{2} & =\int_{\mathbb{R}^{3}} \theta \partial_{t} \rho+\theta u \cdot \nabla_{x} \rho d x \\
& =-\frac{1}{2} \frac{d}{d t}\|\rho\|_{L_{x}^{2}}^{2}+\int_{\mathbb{R}^{3}}(\rho+\theta) \partial_{t} \rho-\rho u \cdot \nabla_{x} \theta d x \\
& =-\frac{1}{2} \frac{d}{d t}\|\rho\|_{L_{x}^{2}}^{2}-\frac{1}{2} \frac{d}{d t}\left\|\nabla_{x}(\rho+\theta)\right\|_{L_{x}^{2}}^{2}-\int_{\mathbb{R}^{3}} \rho u \cdot \nabla_{x} \theta d x .
\end{aligned}
$$

Summing the above identities, we obtain the expected global conservation of energy.

Using the a priori estimates provided by the energy inequality $(3.2)$ and reproducing the arguments of Leray [48, we can easily establish the global existence of weak solutions. Indeed, combining first the bound on $\nabla_{x}(\rho+\theta)$ with the additional spatial regularity on $u$ and $\theta$, coming from the dissipation terms in the energy inequality (3.2), we infer that all three terms $\rho, u$ and $\theta$ enjoy some spatial regularity. More precisely, they are all uniformly bounded in $L_{\text {loc }}^{2}\left(d t ; H^{1}(d x)\right)$. Furthermore, recalling that $P E=-\partial_{t} A$, where $B=\operatorname{rot} A$ with $\operatorname{div} A=0$, some temporal regularity on $u+A$ and $\frac{3}{2} \theta-\rho$ is clearly inherited from the evolution equations, which allows us to establish, invoking a classical compactness result by Aubin and Lions 8, 50. (see also [71] for a sharp compactness criterion), that $u+A$ and $\frac{3}{2} \theta-\rho$ are strongly relatively compact in all variables in $L_{\text {loc }}^{2}(d t d x)$. Finally, noticing that one may express

$$
\begin{aligned}
\rho & =\frac{2 \Delta_{x}}{3-5 \Delta_{x}}\left(\frac{3}{2} \theta-\rho\right), & u & =\frac{-\Delta_{x}}{1-\Delta_{x}}(u+A), \\
\theta & =\frac{2-2 \Delta_{x}}{3-5 \Delta_{x}}\left(\frac{3}{2} \theta-\rho\right), & B & =\frac{\operatorname{rot}}{1-\Delta_{x}}(u+A),
\end{aligned}
$$

using Poisson's equations

$$
-\Delta_{x} A=u, \quad \Delta_{x}(\rho+\theta)=\rho,
$$


we easily find that all four observables $\rho, u, \theta$ and $B$ belong to a compact subset of $L_{\text {loc }}^{2}(d t d x)$.

The above compactness properties, which also hold for the similar systems (2.28), 2.30), (2.49), (2.50) and (2.51), allow us to prove the weak stability of the nonlinear terms in (3.1) and, therefore, to take weak limits in any suitable approximation scheme to establish the existence of weak solutions. Analogous existence results hold for systems (2.28), 2.30, 2.49, 2.50 and (2.51), as well.

Henceforth, we will utilize the prefixes $w$ - or $w^{*}$ - to express that a given space is endowed with its weak or weak-* topology, respectively.

THEOREM 3.2. Let $\left(\rho^{\text {in }}, u^{\text {in }}, \theta^{\text {in }}, B^{\text {in }}\right) \in L^{2}\left(\mathbb{R}^{3}, d x\right)$ be such that

$$
\operatorname{div} u^{\text {in }}=0, \quad \operatorname{div} B^{\text {in }}=0, \quad \operatorname{rot} B^{\text {in }}=u^{\text {in }}, \quad \Delta_{x}\left(\rho^{\text {in }}+\theta^{\text {in }}\right)=\rho^{\text {in }} .
$$
[48])

Then, there exists (at least) one global weak solution (in the sense of Leray

$$
\begin{aligned}
(\rho, u, \theta, B) & \in C\left([0, \infty) ; w-L^{2}\left(\mathbb{R}^{3}, d x\right)\right) \cap L^{\infty}\left([0, \infty), d t ; L^{2}\left(\mathbb{R}^{3}, d x\right)\right), \\
(u, \theta) & \in L^{2}\left([0, \infty), d t ; \dot{H}^{1}\left(\mathbb{R}^{3}, d x\right)\right),
\end{aligned}
$$

to the incompressible quasi-static Navier-Stokes-Fourier-Maxwell-Poisson system (3.1). Furthermore, it satisfies the energy inequality (3.2).

As usual for such weak solutions, uniqueness is not known to hold. To prove that the system (3.1) is well-posed in the sense of Hadamard, we would have to deal with a stronger notion of solution. Note however that, by modulating the energy inequality, we can establish some weak-strong uniqueness principle, meaning that if a somewhat regular solution to (3.1) is known to exist, then any weak solution with matching initial data coincides with the smooth one as long as it exists. We refer to the next Section 3.2 for details on how to modulate the energy and, thus, establish such weak-strong uniqueness principles.

\subsection{The two-fluid incompressible Navier-Stokes-Fourier-Maxwell system with (solenoidal) Ohm's law}

We focus now on the two-fluid incompressible Navier-Stokes-Fourier-Maxwell system with Ohm's law 2.74 :

$$
\left\{\begin{aligned}
\partial_{t} u+u \cdot \nabla_{x} u-\mu \Delta_{x} u & =-\nabla_{x} p+\frac{1}{2}(n E+j \wedge B), & & \operatorname{div} u=0, \\
\partial_{t} \theta+u \cdot \nabla_{x} \theta-\kappa \Delta_{x} \theta & =0, & & \rho+\theta=0, \\
\partial_{t} E-\operatorname{rot} B & =-j, & \operatorname{div} E & =n, \\
\partial_{t} B+\operatorname{rot} E & =0, & \operatorname{div} B & =0, \\
j-n u & =\sigma\left(-\frac{1}{2} \nabla_{x} n+E+u \wedge B\right), & & =n \theta,
\end{aligned}\right.
$$

and on the two-fluid incompressible Navier-Stokes-Fourier-Maxwell system with solenoidal Ohm's law 2.75 :

$$
\left\{\begin{aligned}
\partial_{t} u+u \cdot \nabla_{x} u-\mu \Delta_{x} u & =-\nabla_{x} p+\frac{1}{2} j \wedge B, & \operatorname{div} u & =0, \\
\partial_{t} \theta+u \cdot \nabla_{x} \theta-\kappa \Delta_{x} \theta & =0, & \rho+\theta & =0, \\
\partial_{t} E-\operatorname{rot} B & =-j, & \operatorname{div} E & =0, \\
\partial_{t} B+\operatorname{dot} E & =0, & \operatorname{div} B & =0, \\
j & =\sigma\left(-\nabla_{x} \bar{p}+E+u \wedge B\right), & \operatorname{div} j & =0, \\
n & =0, & w & =0 .
\end{aligned}\right.
$$


The above models (3.3) and (3.4) are not stable under weak convergence in the energy space and, thus, share more similarities with the three-dimensional incompressible Euler equations, as we are about to discuss.

To this end, note first that the advection-diffusion equation on $\theta$ is not really coupled with the other equations on $(u, n, j, E, B)$ in $(3.3)$ and $(3.4)$, and that it is linear provided the velocity field $u$ is given. It is therefore sufficient to focus on the reduced systems of equations

$$
\left\{\begin{aligned}
\partial_{t} u+u \cdot \nabla_{x} u-\mu \Delta_{x} u & =-\nabla_{x} p+\frac{1}{2}(n E+j \wedge B), & & \operatorname{div} u=0, \\
\partial_{t} E-\operatorname{rot} B & =-j, & & \operatorname{div} E=n, \\
\partial_{t} B+\operatorname{rot} E & =0, & & \operatorname{div} B=0 \\
j-n u & =\sigma\left(-\frac{1}{2} \nabla_{x} n+E+u \wedge B\right), & &
\end{aligned}\right.
$$

and

$$
\left\{\begin{aligned}
\partial_{t} u+u \cdot \nabla_{x} u-\mu \Delta_{x} u & =-\nabla_{x} p+\frac{1}{2} j \wedge B, & \operatorname{div} u & =0, \\
\partial_{t} E-\operatorname{rot} B & =-j, & \operatorname{div} E & =0, \\
\partial_{t} B+\operatorname{rot} E & =0, & \operatorname{div} B & =0, \\
j & =\sigma\left(-\nabla_{x} \bar{p}+E+u \wedge B\right), & \operatorname{div} j & =0 .
\end{aligned}\right.
$$

REMARK. The system (3.6) can be viewed as an asymptotic regime of system 3.5. Indeed, at least formally, it is obtained, as $\delta \rightarrow 0$, from the system

$$
\left\{\begin{array}{rlrl}
\partial_{t} u+u \cdot \nabla_{x} u-\mu \Delta_{x} u & =-\nabla_{x} p+\frac{1}{2}(\delta n E+j \wedge B), & & \operatorname{div} u=0, \\
\partial_{t} E-\operatorname{rot} B & =-j, & & \operatorname{div} E=\delta n, \\
\partial_{t} B+\operatorname{rot} E & =0, & & \operatorname{div} B=0, \\
j-\delta n u & =\sigma\left(-\frac{1}{2 \delta} \nabla_{x} n+E+u \wedge B\right), &
\end{array}\right.
$$

which is consistent with the formal derivations from Section 2.4 .

A natural framework to study these equations (coming from physics) should be the energy space, i.e. the functional space defined by the (formal) energy conservation. We indeed expect solutions in this space to be global.

The following formal proposition shows how to compute the energy of the twofluid incompressible Navier-Stokes-Maxwell system with Ohm's law (3.5), or with solenoidal Ohm's law (3.6.

Proposition 3.3. Let $(u, E, B)$ be a smooth solution to the two-fluid incompressible Navier-Stokes-Maxwell system with Ohm's law 3.5), or with solenoidal Ohm's law (3.6.).

Then the following global conservation of energy holds :

$$
\mathcal{E}(t)+\int_{0}^{t} \mathcal{D}(s) d s=\mathcal{E}(0), \quad \text { for all } t>0
$$

where the energy $\mathcal{E}$ and the energy dissipation $\mathcal{D}$ are given by, for the system $(3.5)$,

$$
\begin{aligned}
& \mathcal{E}(t)=\frac{1}{2}\|u(t)\|_{L_{x}^{2}}^{2}+\frac{1}{8}\|n(t)\|_{L_{x}^{2}}^{2}+\frac{1}{4}\|E(t)\|_{L_{x}^{2}}^{2}+\frac{1}{4}\|B(t)\|_{L_{x}^{2}}^{2}, \\
& \mathcal{D}(t)=\mu\left\|\nabla_{x} u(t)\right\|_{L_{x}^{2}}^{2}+\frac{1}{2 \sigma}\|(j-n u)(t)\|_{L_{x}^{2}}^{2},
\end{aligned}
$$


or, for the system (3.6),

$$
\begin{aligned}
& \mathcal{E}(t)=\frac{1}{2}\|u(t)\|_{L_{x}^{2}}^{2}+\frac{1}{4}\|E(t)\|_{L_{x}^{2}}^{2}+\frac{1}{4}\|B(t)\|_{L_{x}^{2}}^{2}, \\
& \mathcal{D}(t)=\mu\left\|\nabla_{x} u(t)\right\|_{L_{x}^{2}}^{2}+\frac{1}{2 \sigma}\|j(t)\|_{L_{x}^{2}}^{2} .
\end{aligned}
$$

Proof. We consider the system (3.5) first. Multiplying the equation expressing the conservation of momentum in (3.5) by $u$ and integrating with respect to space variables, we get

$$
\frac{1}{2} \frac{d}{d t}\|u\|_{L_{x}^{2}}^{2}+\mu\left\|\nabla_{x} u\right\|_{L_{x}^{2}}^{2}=\frac{1}{2} \int_{\mathbb{R}^{3}}(n E+j \wedge B) \cdot u d x,
$$

while multiplying Ohm's law in 3.5 by $j-n u$ and integrating in space yields the identity

$$
\frac{1}{\sigma}\|j-n u\|_{L_{x}^{2}}^{2}=\int_{\mathbb{R}^{3}}\left(\frac{1}{2} n \operatorname{div} j+E \cdot j-(n E+j \wedge B) \cdot u\right) d x
$$

where we have employed the incompressibility of the velocity field. Hence, we obtain, further exploiting the continuity equation $\partial_{t} n+\operatorname{div} j=0$ (deduced by taking the divergence of Ampère's equation and from Gauss' law), that

$$
\begin{aligned}
\frac{1}{2} \frac{d}{d t}\|u\|_{L_{x}^{2}}^{2}+\mu\left\|\nabla_{x} u\right\|_{L_{x}^{2}}^{2}+\frac{1}{2 \sigma}\|j-n u\|_{L_{x}^{2}}^{2} & =\int_{\mathbb{R}^{3}} \frac{1}{4} n \operatorname{div} j+\frac{1}{2} E \cdot j d x \\
& =-\frac{1}{8} \frac{d}{d t}\|n\|_{L_{x}^{2}}^{2}+\int_{\mathbb{R}^{3}} \frac{1}{2} E \cdot j d x .
\end{aligned}
$$

As for the system (3.6), similar and, actually, simpler computations yield that

$$
\frac{1}{2} \frac{d}{d t}\|u\|_{L_{x}^{2}}^{2}+\mu\left\|\nabla_{x} u\right\|_{L_{x}^{2}}^{2}+\frac{1}{2 \sigma}\|j\|_{L_{x}^{2}}^{2}=\int_{\mathbb{R}^{3}} \frac{1}{2} E \cdot j d x .
$$

Next, for both systems (3.5) and (3.6), the conservation of the electromagnetic energy is given by Maxwell's equations

$$
\frac{1}{2} \frac{d}{d t}\left(\|E\|_{L_{x}^{2}}^{2}+\|B\|_{L_{x}^{2}}^{2}\right)=-\int_{\mathbb{R}^{3}} E \cdot j d x .
$$

Summing the above formal identities leads to the expected global conservation of energy.

The uniform bounds resulting from the energy conservations in Proposition 3.3 imply that all the terms in systems (3.5) and (3.6) make sense, especially the nonlinear terms in the motion equations and in Ohm's laws.

Notice, however, that it is at first not clear that the Lorentz force $n E+j \wedge$ $B$ in $\sqrt{3.5}$ is a well-defined distribution, based on the natural a priori estimates provided by the energy and energy dissipation, because $j$ does not necessarily lie in $L_{t}^{1} L_{x}^{2}$. Nevertheless, it is possible to give it a rigorous sense by exploiting simple identities. A first approach consists in identifying the force term $n E+j \wedge B$ with the conservation law for the electromagnetic energy flux $E \wedge B$ (also called the Poynting vector, see [45, Section 6.7])

$$
\partial_{t}(E \wedge B)+\frac{1}{2} \nabla_{x}\left(E^{2}+B^{2}\right)-\nabla_{x} \cdot(E \otimes E+B \otimes B)=-n E-j \wedge B
$$

derived directly from Maxwell's equations in (3.5) (see the derivation of (1.11) and $(1.12$ ), so that the force makes sense in some Sobolev space with negative 
regularity index. In fact, it will be much more appropriate to estimate the Lorentz force directly using Ohm's law from (3.5) as follows

$$
\begin{aligned}
n E+j \wedge B & =(j-n u) \wedge B+n(E+u \wedge B) \\
& =(j-n u) \wedge B+\frac{1}{\sigma} n(j-n u)+\frac{1}{4} \nabla_{x}\left(n^{2}\right),
\end{aligned}
$$

so that the force is now understood as the sum of a locally integrable function and a pressure gradient. All other terms from (3.5) and (3.6) are obviously well-defined.

Unfortunately, the uniform energy bounds do not guarantee the weak stability of the nonlinear terms $n E$ and $j \wedge B$ composing the Lorentz forces. This is a major obstacle to establishing the global existence of weak solutions in the spirit of Leray 48, which are therefore not known to exist in general.

There are two evident strategies, which unfortunately turn out to be unsuccessful, that one would want to apply here in order to circumvent the lack of weak stability of the Lorentz forces in systems $(3.5)$ and $(3.6)$.

The first one consists in propagating strong compactness or regularity in Maxwell's equations, which are indeed the archetype of hyperbolic equations, meaning that singularities are propagated. In general, these singularities, or oscillations, may be created either by boundary data, by initial data or by the source terms, and they remain localized on the corresponding light cones. Here, we are not considering boundaries and the initial data can always be well-prepared. However, it remains unclear how to prevent the emergence of oscillations from the source term $-j$ in Maxwell's equations, which is determined by the nonlinear Ohm's laws in (3.5) and (3.6). Therefore, we do not expect to gain regularity (or even compactness) on the electromagnetic field $(E, B)$. So, this strategy fails in general.

It is to be noted, though, that this approach has been successfully applied by Masmoudi [58 to a slightly different system coupling the incompressible NavierStokes equations with Maxwell's equations in the two-dimensional case. Since the equations studied therein are very similar to (3.5) and (3.6), we present Masmoudi's result below in Section 3.2.1 in order to emphasize the mathematical difficulties inherent to the coupling with Maxwell's equations through Ohm's law and its similarities with the two-dimensional Euler equations. Also, we believe that similar results on systems (3.5) and (3.6) can be achieved.

The second strategy consists in utilizing the linear structure of Maxwell's equations with the specific quadratic structure of the Lorentz force to apply the theory of compensated compactness of Murat and Tartar [63, 64, 73. (see also 72 for an introduction to the subject) and, thus, filter any undesired nonlinear resonances. This approach plainly fails and it seems that it can only potentially succeed by exploiting the full nonlinear structure of the whole systems $(3.5)$ and $(3.6)$. But we are not aware of such successful nonlinear treatment of resonances. We refer to [5] for some more details about the failure of the method of compensated compactness in the electromagnetic setting.

Following the concise Section 3.2.1 below, where we present the main result from [58. on the well-posedness of an incompressible Navier-Stokes-Maxwell system in two dimensions, we will discuss very briefly in Section 3.2.2 the well-posedness of the same system in three dimensions and for small initial data. Finally, in Section 3.2.3. we will introduce the dissipative solutions of the systems (3.5) and (3.6) and justify their global existence in any dimension, which will be particularly relevant to our work. 
3.2.1. Large global solutions in two dimensions. In [58, Masmoudi studied the following incompressible Navier-Stokes-Maxwell system :

$$
\left\{\begin{aligned}
\partial_{t} u+u \cdot \nabla_{x} u-\mu \Delta_{x} u & =-\nabla_{x} p+j \wedge B, & \operatorname{div} u & =0, \\
\partial_{t} E-\operatorname{rot} B & =-j, & j & =\sigma(E+u \wedge B), \\
\partial_{t} B+\operatorname{rot} E & =0, & \operatorname{div} B & =0,
\end{aligned}\right.
$$

which is somewhat related to the systems 3.5$)$ and $(3.6)$, and satisfies the formal energy conservation

$$
\frac{1}{2} \frac{d}{d t}\left(\|u\|_{L_{x}^{2}}^{2}+\|E\|_{L_{x}^{2}}^{2}+\|B\|_{L_{x}^{2}}^{2}\right)+\mu\left\|\nabla_{x} u\right\|_{L_{x}^{2}}^{2}+\frac{1}{\sigma}\|j\|_{L_{x}^{2}}^{2}=0
$$

Notice that, in this system, there is no constraint on $\operatorname{div} E$ or $\operatorname{div} j$. He restricted his analysis to the two-dimensional case, which is obtained by assuming that

$$
u=\left(\begin{array}{c}
u_{1}\left(x_{1}, x_{2}\right) \\
u_{2}\left(x_{1}, x_{2}\right) \\
0
\end{array}\right), \quad E=\left(\begin{array}{c}
E_{1}\left(x_{1}, x_{2}\right) \\
E_{2}\left(x_{1}, x_{2}\right) \\
0
\end{array}\right) \quad \text { and } \quad B=\left(\begin{array}{c}
0 \\
0 \\
B_{3}\left(x_{1}, x_{2}\right)
\end{array}\right)
$$

In order to understand the propagation of singularities in Maxwell's system (in two or three dimensions), it is often convenient to express it using vector and scalar potentials in an equivalent form (see [45, Sections 6.2 and 6.3]). To this end, since the magnetic field $B$ is solenoidal, we may always write $B=\operatorname{rot} A$, for some vector potential $A$. Moreover, taking into account Faraday's equation, we see that necessarily $E=-\nabla_{x} \varphi-\partial_{t} A$ for some scalar potential $\varphi$. As a matter of fact, the potentials $A$ and $\varphi$ are not uniquely determined. Indeed, the electromagnetic field is invariant under the so-called gauge transformation $(A, \varphi) \mapsto\left(A+\nabla_{x} \psi, \varphi-\partial_{t} \psi\right)$.

This gauge invariance allows us to impose a further condition on the potentials. Typically, one may impose the so-called Coulomb gauge $\operatorname{div} A=0$, which is simple and natural for stationary settings. Another classical example of gauge fixing includes the Lorenz (not to be confused with Lorentz) gauge $\operatorname{div} A=-\partial_{t} \varphi$, which usually yields an evolution for the potentials governed by decoupled wave equations.

Here, for the Maxwell system in (3.8), we choose the slight variant of the Lorenz gauge

$$
\operatorname{div} A=-\partial_{t} \varphi-\sigma \varphi
$$

which yields the decoupled damped wave equation

$$
\partial_{t}^{2} A+\sigma \partial_{t} A-\Delta_{x} A=\sigma u \wedge(\operatorname{rot} A) .
$$

Note that it is always possible to find $A$ and $\varphi$ satisfying (3.10). Indeed, if (3.10) is not satisfied, one may always apply a gauge transformation with $\psi$ solving the damped wave equation $\partial_{t}^{2} \psi+\sigma \partial_{t} \psi-\Delta_{x} \psi=\operatorname{div} A+\partial_{t} \varphi+\sigma \varphi$ and produce new potentials for which 3.10 holds.

Now, if the velocity field $u$ is bounded in $L^{1}\left([0, T], d t ; L^{\infty}(d x)\right)$, for some $T>0$, it is possible to show, through standard energy estimates, that the damped wave equation (3.11), which is linear in $A$, propagates the strong compactness of $\partial_{t} A$ and $\nabla_{x} A$ in $L^{\infty}\left([0, T], d t ; L^{2}(d x)\right)$. This would obviously imply the propagation of strong compactness for the magnetic field $B$.

Unfortunately, in two dimensions of space, the $H^{1}$ estimate on the velocity field $u$ provided by the conservation of energy barely fails to yield, by Sobolev embedding, an $L^{\infty}$ bound on $u$. Masmoudi's idea was then to compensate this lack of critical embedding by placing the initial electromagnetic field in a better $H^{s}$ space, with $0<s<1$, and to propagate this initial regularity with Maxwell's equations at the same time that the parabolic regularity of the Stokes flow is employed to estimate 
the velocity field in a higher regularity space. This approach eventually allows to bound $u$ in $L_{t}^{1} L_{x}^{\infty}$ in terms of its $L_{t}^{2} \dot{H}_{x}^{1}$ norm with some logarithmic loss.

As a byproduct of these estimates, it is also possible to establish the exponential growth of the $H^{s}$ norms. In Masmoudi's own words : "One can compare this growth estimate with the double exponential growth estimate of the $H^{s}$ norms in the twodimensional incompressible Euler system."

Finally, it is interesting to note that Masmoudi's proof uses neither the divergence free condition of the magnetic field nor the decay property of the linear part coming from Maxwell's equations.

The following theorem contains the main well-posedness result from $\mathbf{5 8}$. Note that it gives the existence and uniqueness for initial data in a very large dense subspace of $L^{2}$, namely in $\cup_{0<s<1} H^{s}$, but it fails to guarantee the existence of a weak solution when the initial data lies merely in $L^{2}$.

Theorem $3.4([\mathbf{5 8}]$ ). Take $0<s<1$,

$$
u^{\text {in }} \in L^{2}\left(\mathbb{R}^{2}\right) \quad \text { and } \quad E^{\text {in }}, B^{\text {in }} \in H^{s}\left(\mathbb{R}^{2}\right) .
$$

Then, there exists a unique global solution $(u, E, B)$ of (3.8) such that for all $T>0$

$$
u \in C\left([0, T] ; L^{2}\right) \cap L^{2}\left([0, T] ; \dot{H}^{1}\right) \quad \text { and } \quad E, B \in C\left([0, T] ; H^{s}\right) .
$$

Moreover,

$$
j \in L^{2}\left([0, T] ; L^{2}\right) \cap L^{1}\left([0, T] ; H^{s}\right) \quad \text { and } \quad u \in L^{1}\left([0, T] ; H^{s^{\prime}}\right),
$$

for each $1<s^{\prime}<\min (2 s+1,2)$. In addition, the energy identity 3.9 holds and we have the following exponential growth estimate for all $t>0$ :

$$
\|u\|_{L^{1}\left([0, t] ; H^{s^{\prime}}\right)}+\|E(t)\|_{H^{s}}+\|B(t)\|_{H^{s}} \leq\left(1+\left\|E^{\mathrm{in}}\right\|_{H^{s}}+\left\|B^{\mathrm{in}}\right\|_{H^{s}}\right) e^{C^{\mathrm{in}}(1+t)},
$$

where $C^{\text {in }}=C\left(1+\left\|u^{\text {in }}\right\|_{L^{2}}^{2}+\left\|E^{\text {in }}\right\|_{L^{2}}^{2}+\left\|B^{\text {in }}\right\|_{L^{2}}^{2}\right)$ for some constant $C$.

3.2.2. Small global solutions in three dimensions. As we have seen, there are serious obstacles to the construction of global solutions of the system (3.8) for large initial data in the energy space. Nevertheless, it is in general possible to achieve the well-posedness of a system, globally in time, by showing its strong stability for small initial data in some space satisfying the same scaling invariance as the given system of equations.

This is precisely what Ibrahim and Keraani managed to obtain in [43] for the three-dimensional incompressible Navier-Stokes-Maxwell system (3.8) using the strategy of Fujita and Kato [35, which is based on refined a priori estimates obtained by paradifferential calculus and some fixed point argument. Note that the results from 43 do not imply the local existence of strong solutions for large data, which has been established in a separate work by Ibrahim and Yoneda in 44 .

These results have then been unified and extended to a more natural setting by Germain, Ibrahim and Masmoudi in [36.

We believe that the methods employed in [36, 43, 44] can potentially lead to similar results for the analogous incompressible Navier-Stokes-Maxwell systems (3.5) and (3.6). The main result in this three-dimensional setting is contained in the following theorem (we refer directly to [36] for definitions of the functional spaces).

TheOREM 3.5 ([36, 43, 44]). To any initial data

$$
u^{\text {in }}, E^{\text {in }}, B^{\text {in }} \in \dot{H}^{\frac{1}{2}}\left(\mathbb{R}^{3}\right),
$$


there corresponds an existence time $T>0$ and a unique local solution of 3.8

$$
u \in \tilde{L}^{\infty}\left((0, T) ; \dot{H}^{\frac{1}{2}}\right) \cap L^{2}\left((0, T) ; \dot{H}^{\frac{3}{2}} \cap L^{\infty}\right) \quad \text { and } \quad E, B \in \tilde{L}^{\infty}\left((0, T) ; \dot{H}^{\frac{1}{2}}\right) .
$$

Furthermore, the solution is global (i.e. $T=\infty$ ) if the initial data is sufficiently small.

3.2.3. Weak-strong stability and dissipative solutions. On the one hand, As already explained, there is no known global well-posedness theory for the systems (3.5), (3.6) and (3.8) in the energy space, due to their lack of weak stability. On the other hand, in Sections 3.2.1 and 3.2.2 we have briefly presented theorems on the existence and uniqueness of strong solutions to the system (3.8).

Around such smooth solutions and in order to circumvent the lack of weak stability, we introduce now the dissipative solutions of these incompressible NavierStokes-Maxwell systems. Generally speaking, the concept of dissipative solutions is based on the weak-strong stability, when available, of a given system, i.e. the uniqueness of all weak solutions provided at least one strong solution exists. It seems that such weak-strong stability principles were first introduced by Dafermos 24 in the context of conservation laws.

Dissipative solutions are not new in fluid and gas dynamics. They are precisely employed to treat the instability of nonlinear terms in the energy space. Lions first defined them for the Boltzmann equation in [52. He then established their existence for the incompressible Euler system in [57, Section 4.4], as an alternative to the very weak notion of measure-valued solutions introduced by DiPerna and Majda 34, which have later been shown in [15] to be actually stronger (at least not weaker, as each measure-valued solution is shown to be a dissipative solution, as well). It can more easily be shown that any weak solution of the incompressible Euler system is a dissipative solution (see [25. Appendix B] for a proof). This, however, is not known to hold in general for renormalized solutions of the Boltzmann equation, i.e. renormalized solutions are not known, in general, to be dissipative solutions as defined by Lions in [52.

It is sometimes argued that dissipative solutions are too weak and that they do not express any physical reality, because they are not shown to be unique in general. Even so, they do enjoy certain definite qualities :

- they exist globally in time for large initial data in the energy space ;

- they coincide with the unique strong solution when the latter exists ;

- they allow energy dissipation phenomena to occur.

The last property above is especially significant in light of recent results on the energy dissipation in the incompressible Euler flow establishing, in particular, the existence of weak solutions with kinetic energy strictly decaying (or increasing, which is equivalent since the Euler flow is reversible) over time (see [25, 170]). This energy dissipation cannot hold beyond a certain regularity threshold (see [21, $\mathbf{2 3}$. on Onsager's conjecture) and, therefore, it is crucial to consider rather low regularity weak solutions of the incompressible Euler system in order to understand energy dissipation and turbulent flow. In this context, we wish to mention the striking recent developments $[\mathbf{1 6}, \mathbf{1 7}, \mathbf{1 8}, \mathbf{2 2}, \mathbf{2 6}, \mathbf{2 7}]$ demonstrating the existence of energydissipating flows enjoying some Hölder regularity.

Dissipative solutions have found an important application in a wide range of asymptotic problems, for they are especially well adapted, through relative entropy methods (or modulated energy methods), to situations presenting a lack of compactness. In particular, they were employed by the second author in 67, 69. to establish the hydrodynamic convergence of renormalized solutions of the Boltzmann equation towards dissipative solutions of the incompressible Euler system (see also 
68. ). Another application by Brenier 14 concerns the convergence of the VlasovPoisson system towards the incompressible Euler equations in the quasi-neutral regime.

3.2.3.1. The incompressible Navier-Stokes-Maxwell system. We explain now how the energy 3.9 can be modulated and establish a weak-strong stability principle, which will eventually lead to a suitable notion of dissipative solution for the incompressible Navier-Stokes-Maxwell system (3.8) in any dimension. We will then move on to apply the same strategy to the more complex systems $\sqrt{3.5}$ and $(3.6)$ by modulating the energies from Proposition 3.3 and thus produce similar dissipative solutions.

Proposition 3.6. Let $(u, E, B)$ be a smooth solution to the incompressible Navier-Stokes-Maxwell system (3.8). Further consider test functions $(\bar{u}, \bar{j}, \bar{E}, \bar{B}) \in$ $C_{c}^{\infty}\left([0, \infty) \times \mathbb{R}^{3}\right)$ such that

$$
\left\{\begin{array}{l}
\partial_{t} \bar{E}-\operatorname{rot} \bar{B}=-\bar{j}, \quad \operatorname{div} \bar{u}=0 \\
\partial_{t} \bar{B}+\operatorname{rot} \bar{E}=0, \quad \operatorname{div} \bar{B}=0
\end{array}\right.
$$

We define the acceleration operator by

$$
\mathbf{A}(\bar{u}, \bar{j}, \bar{E}, \bar{B})=\left(\begin{array}{c}
-\partial_{t} \bar{u}-P\left(\bar{u} \cdot \nabla_{x} \bar{u}\right)+\mu \Delta_{x} \bar{u}+P(\bar{j} \wedge \bar{B}) \\
-\frac{1}{\sigma} \bar{j}+\bar{E}+\bar{u} \wedge \bar{B}
\end{array}\right)
$$

and the growth rate by

$$
\lambda(t)=\left(\frac{2}{\mu}+\sigma\right)\|\bar{u}(t)\|_{L_{x}^{\infty}}^{2}+\frac{2 C_{0}^{2}}{\mu}\|\bar{j}(t)\|_{L_{x}^{3}}^{2}
$$

where $C_{0}>0$ denotes the operator norm of the Sobolev embedding $\dot{H}^{1}\left(\mathbb{R}^{3}\right) \hookrightarrow$ $L^{6}\left(\mathbb{R}^{3}\right)$.

Then, one has the stability inequality

$$
\begin{aligned}
\delta \mathcal{E}(t)+ & \frac{1}{2} \int_{0}^{t} \delta \mathcal{D}(s) e^{\int_{s}^{t} \lambda(\sigma) d \sigma} d s \\
& \leq \delta \mathcal{E}(0) e^{\int_{0}^{t} \lambda(s) d s}+\int_{0}^{t}\left[\int_{\mathbb{R}^{3}} \mathbf{A} \cdot\left(\begin{array}{c}
u-\bar{u} \\
j-\bar{j}
\end{array}\right) d x\right](s) e^{\int_{s}^{t} \lambda(\sigma) d \sigma} d s
\end{aligned}
$$

where the modulated energy $\delta \mathcal{E}$ and energy dissipation $\delta \mathcal{D}$ are given by

$$
\begin{aligned}
\delta \mathcal{E}(t) & =\frac{1}{2}\|(u-\bar{u})(t)\|_{L_{x}^{2}}^{2}+\frac{1}{2}\|(E-\bar{E})(t)\|_{L_{x}^{2}}^{2}+\frac{1}{2}\|(B-\bar{B})(t)\|_{L_{x}^{2}}^{2}, \\
\delta \mathcal{D}(t) & =\mu\left\|\nabla_{x}(u-\bar{u})(t)\right\|_{L_{x}^{2}}^{2}+\frac{1}{\sigma}\|(j-\bar{j})(t)\|_{L_{x}^{2}}^{2} \cdot
\end{aligned}
$$

Proof. We have already formally established in 3.9 the conservation of the energy for the system (3.8). The very same computations applied to the test functions $(\bar{u}, \bar{j}, \bar{E}, \bar{B})$ yield the identity

$$
\frac{1}{2} \frac{d}{d t}\left(\|\bar{u}\|_{L_{x}^{2}}^{2}+\|\bar{E}\|_{L_{x}^{2}}^{2}+\|\bar{B}\|_{L_{x}^{2}}^{2}\right)+\mu\left\|\nabla_{x} \bar{u}\right\|_{L_{x}^{2}}^{2}+\frac{1}{\sigma}\|\bar{j}\|_{L_{x}^{2}}^{2}=-\int_{\mathbb{R}^{3}} \mathbf{A} \cdot\left(\begin{array}{l}
\bar{u} \\
\bar{j}
\end{array}\right) d x .
$$


Furthermore, another similar duality computation gives

$$
\begin{aligned}
\frac{d}{d t} \int_{\mathbb{R}^{3}} u & \cdot \bar{u}+E \cdot \bar{E}+B \cdot \bar{B} d x+\int_{\mathbb{R}^{3}} 2 \mu \nabla_{x} u: \nabla_{x} \bar{u}+\frac{2}{\sigma} j \cdot \bar{j} d x \\
& =-\int_{\mathbb{R}^{3}} \bar{u} \otimes(u-\bar{u}): \nabla_{x}(u-\bar{u}) d x \\
& +\int_{\mathbb{R}^{3}}((j-\bar{j}) \wedge(B-\bar{B})) \cdot \bar{u}+((u-\bar{u}) \wedge(B-\bar{B})) \cdot \bar{j} d x \\
& -\int_{\mathbb{R}^{3}} \mathbf{A} \cdot\left(\begin{array}{c}
u \\
j
\end{array}\right) d x .
\end{aligned}
$$

On the whole, combining the above identities with the formal energy conservation 3.9 , we find

$$
\begin{aligned}
\frac{1}{2} \frac{d}{d t} & \left(\|u-\bar{u}\|_{L_{x}^{2}}^{2}+\|E-\bar{E}\|_{L_{x}^{2}}^{2}+\|B-\bar{B}\|_{L_{x}^{2}}^{2}\right)+\mu\left\|\nabla_{x}(u-\bar{u})\right\|_{L_{x}^{2}}^{2}+\frac{1}{\sigma}\|j-\bar{j}\|_{L_{x}^{2}}^{2} \\
\quad & \int_{\mathbb{R}^{3}} \bar{u} \otimes(u-\bar{u}): \nabla_{x}(u-\bar{u}) d x \\
& +\int_{\mathbb{R}^{3}}(\bar{j} \wedge(B-\bar{B})) \cdot(u-\bar{u})-((j-\bar{j}) \wedge(B-\bar{B})) \cdot \bar{u} d x \\
& +\int_{\mathbb{R}^{3}} \mathbf{A} \cdot\left(\begin{array}{c}
u-\bar{u} \\
j-\bar{j}
\end{array}\right) d x .
\end{aligned}
$$

The next step consists in estimating the terms in the right-hand side above that are nonlinear in $(u, j, E, B)$ and to absorb the resulting expressions with the modulated energy $\delta \mathcal{E}(t)$ and the modulated energy dissipation $\delta \mathcal{D}(t)$ by suitable uses of Young's inequality and Grönwall's lemma. Thus, we obtain

$$
\begin{aligned}
\frac{d}{d t} \delta \mathcal{E}(t) & +\delta \mathcal{D}(t) \\
& \leq\|\bar{u}\|_{L_{x}^{\infty}}\|u-\bar{u}\|_{L_{x}^{2}}\left\|\nabla_{x}(u-\bar{u})\right\|_{L_{x}^{2}} \\
& +\|\bar{j}\|_{L_{x}^{3}}\|B-\bar{B}\|_{L_{x}^{2}}\|u-\bar{u}\|_{L_{x}^{6}}+\|\bar{u}\|_{L_{x}^{\infty}}\|B-\bar{B}\|_{L_{x}^{2}}\|j-\bar{j}\|_{L_{x}^{2}} \\
& +\int_{\mathbb{R}^{3}} \mathbf{A} \cdot\left(\begin{array}{c}
u-\bar{u} \\
j-\bar{j}
\end{array}\right) d x \\
& \leq \frac{1}{\mu}\|\bar{u}\|_{L_{x}^{\infty}}^{2}\|u-\bar{u}\|_{L_{x}^{2}}^{2}+\left(\frac{\sigma}{2}\|\bar{u}\|_{L_{x}^{\infty}}^{2}+\frac{C_{0}^{2}}{\mu}\|\bar{j}\|_{L_{x}^{3}}^{2}\right)\|B-\bar{B}\|_{L_{x}^{2}}^{2} \\
& +\frac{\mu}{2}\left\|\nabla_{x}(u-\bar{u})\right\|_{L_{x}^{2}}^{2}+\frac{1}{2 \sigma}\|j-\bar{j}\|_{L_{x}^{2}}^{2}+\int_{\mathbb{R}^{3}} \mathbf{A} \cdot\left(\begin{array}{c}
u-\bar{u} \\
j-\bar{j}
\end{array}\right) d x .
\end{aligned}
$$

Hence,

$$
\frac{d}{d t} \delta \mathcal{E}(t)+\frac{1}{2} \delta \mathcal{D}(t) \leq \lambda(t) \delta \mathcal{E}(t)+\int_{\mathbb{R}^{3}} \mathbf{A} \cdot\left(\begin{array}{c}
u-\bar{u} \\
j-\bar{j}
\end{array}\right) d x
$$

which concludes the proof of the proposition with a direct application of Grönwall's lemma.

Note that the test functions satisfying the linear constraints 3.12 are easily constructed by considering scalar potentials $\bar{\varphi} \in C_{c}^{\infty}\left([0, \infty) \times \mathbb{R}^{3}\right)$ and vector potentials $\bar{A} \in C_{c}^{\infty}\left([0, \infty) \times \mathbb{R}^{3}\right)$ and then setting

$$
\bar{E}=-\nabla_{x} \bar{\varphi}-\partial_{t} \bar{A} \quad \text { and } \quad \bar{B}=\operatorname{rot} \bar{A} .
$$

One may prefer, for various reasons, to deal, in a completely equivalent manner, with test functions $(\bar{u}, \bar{j}, \bar{E}, \bar{B}) \in C_{c}^{\infty}\left([0, \infty) \times \mathbb{R}^{3}\right)$ satisfying the stationary constraints

$$
\operatorname{div} \bar{u}=0, \quad \operatorname{div} \bar{B}=0, \quad \bar{j}=\sigma(\bar{E}+\bar{u} \wedge \bar{B}),
$$


rather than the constraints 3.12. In this case, instead of 3.13 , we obtain the stability inequality

$$
\begin{aligned}
\delta \mathcal{E}(t)+ & \frac{1}{2} \int_{0}^{t} \delta \mathcal{D}(s) e^{\int_{s}^{t} \lambda(\sigma) d \sigma} d s \\
& \leq \delta \mathcal{E}(0) e^{\int_{0}^{t} \lambda(s) d s}+\int_{0}^{t}\left[\int_{\mathbb{R}^{3}} \mathbf{A} \cdot\left(\begin{array}{c}
u-\bar{u} \\
E-\bar{E} \\
B-\bar{B}
\end{array}\right) d x\right](s) e^{\int_{s}^{t} \lambda(\sigma) d \sigma} d s,
\end{aligned}
$$

where the acceleration operator is now defined by

$$
\mathbf{A}(\bar{u}, \bar{j}, \bar{E}, \bar{B})=\left(\begin{array}{c}
-\partial_{t} \bar{u}-P\left(\bar{u} \cdot \nabla_{x} \bar{u}\right)+\mu \Delta_{x} \bar{u}+P(\bar{j} \wedge \bar{B}) \\
-\partial_{t} \bar{E}+\operatorname{rot} \bar{B}-\bar{j} \\
-\partial_{t} \bar{B}-\operatorname{rot} \bar{E}
\end{array}\right) .
$$

The preceding proposition provides an important weak-strong stability property for the incompressible Navier-Stokes-Maxwell system (3.8). Indeed, the stability inequality $(3.13)$ essentially implies that a solution $(\bar{u}, j, \bar{E}, \bar{B})$ of $(3.8)$ such that $\bar{u} \in L_{t}^{2} L_{x}^{\infty}$ and $j \in L_{t}^{2} L_{x}^{3}$, if it exists, is unique in the whole class of weak solutions in the energy space, for any given initial data.

REMARK. In order to impose minimal local integrability assumptions on the test function $\bar{u}$, it is tempting to employ the method of Lions and Masmoudi 56 for estimating the nonlinear term $[\bar{u} \otimes(u-\bar{u})]: \nabla_{x}(u-\bar{u})$ by splitting $\bar{u}=$ $\bar{u} \mathbb{1}_{\{|\bar{u}| \leq K\}}+\bar{u} \mathbb{1}_{\{|\bar{u}|>K\}}$, for some large $K>0$, which yields

$$
\begin{aligned}
\|[\bar{u} \otimes & (u-\bar{u})]: \nabla_{x}(u-\bar{u}) \|_{L_{x}^{1}} \\
& \leq K\|u-\bar{u}\|_{L_{x}^{2}}\left\|\nabla_{x}(u-\bar{u})\right\|_{L_{x}^{2}}+\left\|\bar{u} \mathbb{1}_{\{|\bar{u}|>K\}}\right\|_{L_{x}^{3}}\|u-\bar{u}\|_{L_{x}^{6}}\left\|\nabla_{x}(u-\bar{u})\right\|_{L_{x}^{2}} \\
& \leq \frac{1}{\nu} \frac{K^{2}}{4}\|u-\bar{u}\|_{L_{x}^{2}}^{2}+\nu\left\|\nabla_{x}(u-\bar{u})\right\|_{L_{x}^{2}}^{2}+C_{0}\left\|\bar{u} \mathbb{1}_{\{|\bar{u}|>K\}}\right\|_{L_{x}^{3}}\left\|\nabla_{x}(u-\bar{u})\right\|_{L_{x}^{2}}^{2},
\end{aligned}
$$

for any $\nu>0$, where $C_{0}>0$ denotes the operator norm of the Sobolev embedding $\dot{H}_{x}^{1} \hookrightarrow L_{x}^{6}$. Then, choosing $K>0$ large enough so that $\left\|\bar{u} \mathbb{1}_{\{|\bar{u}|>K\}}\right\|_{L_{x}^{3}}$ is arbitrarily small and setting $\nu>0$ small enough, it is readily seen that the last two terms above can be absorbed by the modulated entropy dissipation. Of course, the choice of the parameter $K$ is not uniform for all $\bar{u} \in L_{x}^{3}$. This approach definitely allows us to merely consider velocity fields $\bar{u} \in L_{t}^{2} L_{x}^{\infty}+L_{t}^{\infty} L_{x}^{3}$ when establishing weak-strong stability principles for the incompressible Navier-Stokes system (see [56 ). Here, however, considering the coupling of the fluid equations with Maxwell's system introduces other nonlinear terms in the estimates, which unfortunately require that $\bar{u} \in L_{t}^{2} L_{x}^{\infty}$ in order to be duly controlled.

By analogy with Lions' dissipative solutions to the incompressible Euler system 57. Section 4.4], we provide now a suitable notion of dissipative solution for the incompressible Navier-Stokes-Maxwell system (3.8), based on Proposition 3.6, and establish their existence next.

Definition. We say that

$$
(u, E, B) \in L^{\infty}\left([0, \infty) ; L^{2}\left(\mathbb{R}^{3}\right)\right) \cap C\left([0, \infty) ; w-L^{2}\left(\mathbb{R}^{3}\right)\right)
$$

such that

$$
\operatorname{div} u=0, \quad \operatorname{div} B=0,
$$

is a dissipative solution of the incompressible Navier-Stokes-Maxwell system (3.8), if it solves Maxwell's equations

$$
\left\{\begin{array}{l}
\partial_{t} E-\operatorname{rot} B=-j \\
\partial_{t} B+\operatorname{rot} E=0
\end{array}\right.
$$


with Ohm's law

$$
j=\sigma(E+u \wedge B)
$$

in the sense of distributions, and if, for any test functions $(\bar{u}, \bar{j}, \bar{E}, \bar{B}) \in C_{c}^{\infty}\left([0, \infty) \times \mathbb{R}^{3}\right)$ satisfying the linear constraints $(3.12)$, the stability inequality $(3.13)$ is verified.

As previously mentioned, dissipative solutions define actual solutions in the sense that they coincide with the unique strong solution when the latter exists. The following theorem asserts their existence.

TheOREM 3.7. For any initial data $\left(u^{\text {in }}, E^{\text {in }}, B^{\text {in }}\right) \in L^{2}\left(\mathbb{R}^{3}\right)$ such that

$$
\operatorname{div} u^{\text {in }}=0, \quad \operatorname{div} B^{\text {in }}=0
$$

there exists a dissipative solution to the incompressible Navier-Stokes-Maxwell system 3.8.

Proof. Following Lions [57, we easily build the dissipative solutions by introducing viscous approximations of the system (3.8). Thus, for each $\nu>0$, we consider weak solutions of the following system :

$$
\left\{\begin{aligned}
\partial_{t} u_{\nu}+u_{\nu} \cdot \nabla_{x} u_{\nu}-\mu \Delta_{x} u_{\nu} & =-\nabla_{x} p_{\nu}+j_{\nu} \wedge B_{\nu}, & & \operatorname{div} u_{\nu}=0 \\
\partial_{t} E_{\nu}-\operatorname{rot} B_{\nu} & =-j_{\nu}, & j_{\nu} & =\sigma\left(E_{\nu}+u_{\nu} \wedge B_{\nu}\right) \\
\partial_{t} B_{\nu}+\operatorname{rot} E_{\nu}-\nu \Delta_{x} B_{\nu} & =0, & \operatorname{div} B_{\nu} & =0
\end{aligned}\right.
$$

associated with the initial data $\left(u^{\text {in }}, E^{\text {in }}, B^{\text {in }}\right)$ and satisfying the energy inequality, for all $t>0$,

$$
\begin{aligned}
\frac{1}{2}\left(\left\|u_{\nu}\right\|_{L_{x}^{2}}^{2}\right. & \left.+\left\|E_{\nu}\right\|_{L_{x}^{2}}^{2}+\left\|B_{\nu}\right\|_{L_{x}^{2}}^{2}\right)(t) \\
& +\int_{0}^{t} \mu\left\|\nabla_{x} u_{\nu}(s)\right\|_{L_{x}^{2}}^{2}+\frac{1}{\sigma}\left\|j_{\nu}(s)\right\|_{L_{x}^{2}}^{2}+\nu\left\|\nabla_{x} B_{\nu}(s)\right\|_{L_{x}^{2}}^{2} d s \\
& \leq \frac{1}{2}\left(\left\|u^{\mathrm{in}}\right\|_{L_{x}^{2}}^{2}+\left\|E^{\mathrm{in}}\right\|_{L_{x}^{2}}^{2}+\left\|B^{\mathrm{in}}\right\|_{L_{x}^{2}}^{2}\right) .
\end{aligned}
$$

Such weak solutions are easily established following the method of Leray [48, for the nonlinear term $j_{\nu} \wedge B_{\nu}$ is now stable with respect to weak convergence in the energy space defined by the above energy inequality, thanks to the dissipation on $B_{\nu}$.

Then, repeating the computations of Proposition 3.6 it is readily seen that

$$
\begin{aligned}
\frac{d}{d t} \int_{\mathbb{R}^{3}} & u_{\nu} \cdot \bar{u}+E_{\nu} \cdot \bar{E}+B_{\nu} \cdot \bar{B} d x+\int_{\mathbb{R}^{3}} 2 \mu \nabla_{x} u_{\nu}: \nabla_{x} \bar{u}+\frac{2}{\sigma} j_{\nu} \cdot \bar{j} d x \\
& =-\int_{\mathbb{R}^{3}} \bar{u} \otimes\left(u_{\nu}-\bar{u}\right): \nabla_{x}\left(u_{\nu}-\bar{u}\right) d x \\
& +\int_{\mathbb{R}^{3}}\left(\left(j_{\nu}-\bar{j}\right) \wedge\left(B_{\nu}-\bar{B}\right)\right) \cdot \bar{u}+\left(\left(u_{\nu}-\bar{u}\right) \wedge\left(B_{\nu}-\bar{B}\right)\right) \cdot \bar{j} d x \\
& -\int_{\mathbb{R}^{3}} \mathbf{A} \cdot\left(\begin{array}{c}
u_{\nu} \\
j_{\nu}
\end{array}\right) d x-\int_{\mathbb{R}^{3}} \nu \nabla_{x} B_{\nu}: \nabla_{x} \bar{B} d x .
\end{aligned}
$$


Hence, defining the modulated energy $\delta \mathcal{E}_{\nu}(t)$ and modulated energy dissipation $\delta \mathcal{D}_{\nu}(t)$ by simply replacing $(u, j, E, B)$ by $\left(u_{\nu}, j_{\nu}, E_{\nu}, B_{\nu}\right)$ in 3.14, we infer that

$$
\begin{aligned}
\delta \mathcal{E}_{\nu}(t) & +\int_{0}^{t} \delta \mathcal{D}_{\nu}(s)+\nu\left\|\nabla_{x} B_{\nu}(s)\right\|_{L_{x}^{2}}^{2} d s \\
& \leq \delta \mathcal{E}_{\nu}(0)+\int_{0}^{t} \int_{\mathbb{R}^{3}} \bar{u} \otimes\left(u_{\nu}-\bar{u}\right): \nabla_{x}\left(u_{\nu}-\bar{u}\right) d x d s \\
& +\int_{0}^{t} \int_{\mathbb{R}^{3}}\left(\bar{j} \wedge\left(B_{\nu}-\bar{B}\right)\right) \cdot\left(u_{\nu}-\bar{u}\right)-\left(\left(j_{\nu}-\bar{j}\right) \wedge\left(B_{\nu}-\bar{B}\right)\right) \cdot \bar{u} d x d s \\
& +\int_{0}^{t} \int_{\mathbb{R}^{3}} \mathbf{A} \cdot\left(\begin{array}{c}
u_{\nu}-\bar{u} \\
j_{\nu}-\bar{j}
\end{array}\right)+\nu \nabla_{x} B_{\nu}: \nabla_{x} \bar{B} d x d s .
\end{aligned}
$$

Then, following the proof of Proposition 3.6, we arrive at

$$
\begin{aligned}
\delta \mathcal{E}_{\nu}(t) & +\int_{0}^{t} \frac{1}{2} \delta \mathcal{D}_{\nu}(s)+\nu\left\|\nabla_{x} B_{\nu}(s)\right\|_{L_{x}^{2}}^{2} d s \\
& \leq \delta \mathcal{E}_{\nu}(0)+\int_{0}^{t} \lambda(s) \delta \mathcal{E}_{\nu}(s)+\left[\int_{\mathbb{R}^{3}} \mathbf{A} \cdot\left(\begin{array}{c}
u_{\nu}-\bar{u} \\
j_{\nu}-\bar{j}
\end{array}\right)+\nu \nabla_{x} B_{\nu}: \nabla_{x} \bar{B} d x\right] d s \\
& \leq \delta \mathcal{E}_{\nu}(0)+\int_{0}^{t} \lambda(s) \delta \mathcal{E}_{\nu}(s)+\left[\int_{\mathbb{R}^{3}} \mathbf{A} \cdot\left(\begin{array}{c}
u_{\nu}-\bar{u} \\
j_{\nu}-\bar{j}
\end{array}\right) d x\right] d s \\
& +\int_{0}^{t} \frac{\nu}{2}\left\|\nabla_{x} B_{\nu}\right\|_{L_{x}^{2}}^{2}+\frac{\nu}{2}\left\|\nabla_{x} \bar{B}\right\|_{L_{x}^{2}}^{2} d s
\end{aligned}
$$

and an application of Grönwall's lemma yields

$$
\begin{aligned}
\delta \mathcal{E}_{\nu}(t)+ & \frac{1}{2} \int_{0}^{t} \delta \mathcal{D}_{\nu}(s) e^{\int_{s}^{t} \lambda(\sigma) d \sigma} d s \leq \delta \mathcal{E}_{\nu}(0) e^{\int_{0}^{t} \lambda(s) d s} \\
& +\int_{0}^{t}\left[\int_{\mathbb{R}^{3}} \mathbf{A} \cdot\left(\begin{array}{c}
u_{\nu}-\bar{u} \\
j_{\nu}-\bar{j}
\end{array}\right) d x+\frac{\nu}{2}\left\|\nabla_{x} \bar{B}\right\|_{L_{x}^{2}}^{2}\right](s) e^{\int_{s}^{t} \lambda(\sigma) d \sigma} d s .
\end{aligned}
$$

We may now pass to the limit in the above stability inequality. Thus, up to extraction of subsequences, we may assume that, as $\nu \rightarrow 0$,

$$
\begin{array}{ll}
u_{\nu} \stackrel{*}{\rightarrow}_{u} & \text { in } L_{t}^{\infty} L_{x}^{2} \cap L_{t}^{2} \dot{H}_{x}^{1}, \\
j_{\nu} \rightarrow j & \text { in } L_{t}^{2} L_{x}^{2}, \\
E_{\nu} \stackrel{*}{\rightarrow} E & \text { in } L_{t}^{\infty} L_{x}^{2}, \\
B_{\nu} \stackrel{*}{\rightarrow} B & \text { in } L_{t}^{\infty} L_{x}^{2} .
\end{array}
$$

Furthermore, noticing that $\partial_{t} u_{\nu}, \partial_{t} E_{\nu}$ and $\partial_{t} B_{\nu}$ are uniformly bounded, in $L_{\text {loc }}^{1}$ in time and in some negative index Sobolev space in $x$, it is possible to show (see [57. Appendix C]) that $\left(u_{\nu}, E_{\nu}, B_{\nu}\right)$ converges to $(u, E, B) \in C\left([0, \infty) ; w-L^{2}\left(\mathbb{R}^{3}\right)\right)$ weakly in $L_{x}^{2}$ uniformly locally in time. Then, by the weak lower semi-continuity of the norms, we obtain that, for every $t>0$,

$$
\delta \mathcal{E}(t)+\frac{1}{2} \int_{0}^{t} \delta \mathcal{D}(s) e^{\int_{s}^{t} \lambda(\sigma) d \sigma} d s \leq \liminf _{\nu \rightarrow 0} \delta \mathcal{E}_{\nu}(t)+\frac{1}{2} \int_{0}^{t} \delta \mathcal{D}_{\nu}(s) e^{\int_{s}^{t} \lambda(\sigma) d \sigma} d s
$$

Hence, the stability inequality 3.13 holds.

Finally, invoking a classical compactness result by Aubin and Lions [8, [50] (see also 71 for a sharp compactness criterion), we infer that the $u_{\nu}$ 's converge towards $u$ strongly in $L_{\text {loc }}^{2}(d t d x)$. Therefore, it is readily seen that Ohm's law is satisfied asymptotically, which concludes the proof of the theorem. 
3.2.3.2. The two-fluid incompressible Navier-Stokes-Maxwell system with Ohm's law. Following the strategy of Proposition 3.6, the next result establishes a crucial weak-strong stability principle for the two-fluid incompressible Navier-StokesMaxwell system with Ohm's law 3.5.

Proposition 3.8. Let $(u, n, E, B)$ be a smooth solution to the two-fluid incompressible Navier-Stokes-Maxwell system with Ohm's law (3.5). Further consider test functions $(\bar{u}, \bar{n}, \bar{j}, \bar{E}, \bar{B}) \in C_{c}^{\infty}\left([0, \infty) \times \mathbb{R}^{3}\right)$ such that

$$
\left\{\begin{aligned}
& \operatorname{div} \bar{u}=0 \\
\partial_{t} \bar{E}-\operatorname{rot} \bar{B}=-\bar{j}, & \operatorname{div} \bar{E}=\bar{n} \\
\partial_{t} \bar{B}+\operatorname{rot} \bar{E}=0, & \operatorname{div} \bar{B}=0
\end{aligned}\right.
$$

We define the acceleration operator by

$$
\mathbf{A}(\bar{u}, \bar{n}, \bar{j}, \bar{E}, \bar{B})=\left(\begin{array}{c}
-\partial_{t} \bar{u}-P\left(\bar{u} \cdot \nabla_{x} \bar{u}\right)+\mu \Delta_{x} \bar{u}+\frac{1}{2} P(\bar{n} \bar{E}+\bar{j} \wedge \bar{B}) \\
-\frac{1}{2 \sigma}(\bar{j}-\bar{n} \bar{u})+\frac{1}{2}\left(-\frac{1}{2} \nabla_{x} \bar{n}+\bar{E}+\bar{u} \wedge \bar{B}\right)
\end{array}\right)
$$

and the growth rate by

$$
\lambda(t)=\left(\frac{3}{\mu}+\frac{4}{\sigma}+2 \sigma\right)\|\bar{u}(t)\|_{L_{x}^{\infty}}^{2}+\frac{3 C_{0}^{2}}{\mu}\left(\left\|\left(\frac{1}{2} \nabla_{x} \bar{n}-\bar{E}\right)(t)\right\|_{L_{x}^{3}}^{2}+\frac{1}{2}\|\bar{j}(t)\|_{L_{x}^{3}}^{2}\right)
$$

where $C_{0}>0$ denotes the operator norm of the Sobolev embedding $\dot{H}^{1}\left(\mathbb{R}^{3}\right) \hookrightarrow$ $L^{6}\left(\mathbb{R}^{3}\right)$.

$$
\begin{aligned}
\delta \mathcal{E}(t)+ & \frac{1}{2} \int_{0}^{t} \delta \mathcal{D}(s) e^{\int_{s}^{t} \lambda(\sigma) d \sigma} d s \\
& \leq \delta \mathcal{E}(0) e^{\int_{0}^{t} \lambda(s) d s}+\int_{0}^{t}\left[\int_{\mathbb{R}^{3}} \mathbf{A} \cdot\left(\begin{array}{c}
u-\bar{u} \\
j-\bar{j}-n(u-\bar{u})
\end{array}\right) d x\right](s) e^{\int_{s}^{t} \lambda(\sigma) d \sigma} d s,
\end{aligned}
$$

where the modulated energy $\delta \mathcal{E}$ and energy dissipation $\delta \mathcal{D}$ are given by

$$
\begin{aligned}
\delta \mathcal{E}(t) & =\frac{1}{2}\|(u-\bar{u})(t)\|_{L_{x}^{2}}^{2}+\frac{1}{8}\|(n-\bar{n})(t)\|_{L_{x}^{2}}^{2} \\
& +\frac{1}{4}\|(E-\bar{E})(t)\|_{L_{x}^{2}}^{2}+\frac{1}{4}\|(B-\bar{B})(t)\|_{L_{x}^{2}}^{2}, \\
\delta \mathcal{D}(t) & =\mu\left\|\nabla_{x}(u-\bar{u})(t)\right\|_{L_{x}^{2}}^{2}+\frac{1}{2 \sigma}\|(j-n u-(\bar{j}-\bar{n} \bar{u}))(t)\|_{L_{x}^{2}}^{2} .
\end{aligned}
$$

Proof. We have already formally established in Proposition 3.3 the conservation of the energy for systems (3.5). The very same computations applied to the test functions $(\bar{u}, \bar{n}, \bar{j}, \bar{E}, \bar{B})$ yield the identity

$$
\frac{d}{d t} \overline{\mathcal{E}}(t)+\overline{\mathcal{D}}(t)=-\int_{\mathbb{R}^{3}} \mathbf{A} \cdot\left(\begin{array}{c}
\bar{u} \\
\bar{j}-\bar{n} \bar{u}
\end{array}\right) d x
$$

where the energy $\overline{\mathcal{E}}$ and energy dissipation $\overline{\mathcal{D}}$ are obtained simply by replacing the unknowns by the test functions in the respective definitions of Proposition 3.3 . 
Furthermore, other similar duality computations yield that

$$
\begin{aligned}
& \frac{d}{d t} \int_{\mathbb{R}^{3}}\left(u \cdot \bar{u}+\frac{1}{4} n \bar{n}+\frac{1}{2} E \cdot \bar{E}+\frac{1}{2} B \cdot \bar{B}\right) d x+\int_{\mathbb{R}^{3}} 2 \mu \nabla_{x} u: \nabla_{x} \bar{u} d x \\
& \quad=-\int_{\mathbb{R}^{3}} \bar{u} \otimes(u-\bar{u}): \nabla_{x}(u-\bar{u}) d x \\
& \quad+\frac{1}{2} \int_{\mathbb{R}^{3}}(n E+j \wedge B) \cdot \bar{u}+(\bar{n} \bar{E}+\bar{j} \wedge \bar{B}) \cdot u d x \\
& \quad-\frac{1}{2} \int_{\mathbb{R}^{3}} j \cdot\left(\bar{E}-\frac{1}{2} \nabla_{x} \bar{n}\right)+\bar{j} \cdot\left(E-\frac{1}{2} \nabla_{x} n\right) d x-\int_{\mathbb{R}^{3}} \mathbf{A} \cdot\left(\begin{array}{c}
u \\
0
\end{array}\right) d x
\end{aligned}
$$

and

$$
\begin{aligned}
& \frac{1}{\sigma}(j-n u) \cdot(\bar{j}-\bar{n} \bar{u}) \\
& \quad=\frac{1}{2 \sigma}(j-n u+(n-\bar{n}) \bar{u}) \cdot(\bar{j}-\bar{n} \bar{u})+\frac{1}{2 \sigma}(n-\bar{n}) \bar{u} \cdot(j-n u-(\bar{j}-\bar{n} \bar{u})) \\
& \quad+\frac{1}{2 \sigma}(j-n u) \cdot(\bar{j}-n \bar{u}) \\
& \quad=\frac{1}{2}(j-n u+(n-\bar{n}) \bar{u}) \cdot\left(-\frac{1}{2} \nabla_{x} \bar{n}+\bar{E}+\bar{u} \wedge \bar{B}\right)-\mathbf{A} \cdot\left(\begin{array}{c}
0 \\
j-n u+(n-\bar{n}) \bar{u}
\end{array}\right) \\
& \quad+\frac{1}{2 \sigma}(n-\bar{n}) \bar{u} \cdot(j-n u-(\bar{j}-\bar{n} \bar{u}))+\frac{1}{2}\left(-\frac{1}{2} \nabla_{x} n+E+u \wedge B\right) \cdot(\bar{j}-n \bar{u}),
\end{aligned}
$$

whence, considering the sum of the preceding relations,

$$
\begin{aligned}
\frac{d}{d t} & \int_{\mathbb{R}^{3}}\left(u \cdot \bar{u}+\frac{1}{4} n \bar{n}+\frac{1}{2} E \cdot \bar{E}+\frac{1}{2} B \cdot \bar{B}\right) d x \\
& +\int_{\mathbb{R}^{3}} 2 \mu \nabla_{x} u: \nabla_{x} \bar{u}+\frac{1}{\sigma}(j-n u) \cdot(\bar{j}-\bar{n} \bar{u}) d x \\
& =-\int_{\mathbb{R}^{3}} \bar{u} \otimes(u-\bar{u}): \nabla_{x}(u-\bar{u}) d x+\frac{1}{2} \int_{\mathbb{R}^{3}}(n-\bar{n})(u-\bar{u}) \cdot\left(\frac{1}{2} \nabla_{x} \bar{n}-\bar{E}\right) d x \\
& +\frac{1}{2} \int_{\mathbb{R}^{3}}((j-n u-(\bar{j}-\bar{n} \bar{u})) \wedge(B-\bar{B})) \cdot \bar{u}+((u-\bar{u}) \wedge(B-\bar{B})) \cdot \bar{j} d x \\
& +\frac{1}{2 \sigma} \int_{\mathbb{R}^{3}}(n-\bar{n})(j-n u-(\bar{j}-\bar{n} \bar{u})) \cdot \bar{u} d x-\int_{\mathbb{R}^{3}} \mathbf{A} \cdot\left(\begin{array}{c}
u \\
j-n u+(n-\bar{n}) \bar{u}
\end{array}\right) d x
\end{aligned}
$$

On the whole, combining the above identities with the energy decay imposed by the formal energy conservations from Proposition 3.3 , we find the following modulated energy inequality :

$$
\begin{aligned}
& \frac{d}{d t} \delta \mathcal{E}(t)+\delta \mathcal{D}(t) \\
& \quad \leq \int_{\mathbb{R}^{3}} \bar{u} \otimes(u-\bar{u}): \nabla_{x}(u-\bar{u}) d x-\frac{1}{2} \int_{\mathbb{R}^{3}}(n-\bar{n})(u-\bar{u}) \cdot\left(\frac{1}{2} \nabla_{x} \bar{n}-\bar{E}\right) d x \\
& \quad-\frac{1}{2} \int_{\mathbb{R}^{3}}((j-n u-(\bar{j}-\bar{n} \bar{u})) \wedge(B-\bar{B})) \cdot \bar{u}+((u-\bar{u}) \wedge(B-\bar{B})) \cdot \bar{j} d x \\
& \quad-\frac{1}{2 \sigma} \int_{\mathbb{R}^{3}}(n-\bar{n})(j-n u-(\bar{j}-\bar{n} \bar{u})) \cdot \bar{u} d x+\int_{\mathbb{R}^{3}} \mathbf{A} \cdot\left(\begin{array}{c}
u-\bar{u} \\
j-\bar{j}-n(u-\bar{u})
\end{array}\right) d x
\end{aligned}
$$

The next step consists in estimating the terms in the right-hand side above that are nonlinear in $(u, n, j, E, B)$ and to absorb the resulting expressions with the modulated energy $\delta \mathcal{E}(t)$ and the modulated energy dissipation $\delta \mathcal{D}(t)$ by suitable 
uses of Young's inequality and Grönwall's lemma. Thus, we obtain

$$
\begin{aligned}
\frac{d}{d t} & \delta \mathcal{E}(t)+\delta \mathcal{D}(t) \\
& \leq\|\bar{u}\|_{L_{x}^{\infty}}\|u-\bar{u}\|_{L_{x}^{2}}\left\|\nabla_{x}(u-\bar{u})\right\|_{L_{x}^{2}}+\frac{1}{2}\left\|\frac{1}{2} \nabla_{x} \bar{n}-\bar{E}\right\|_{L_{x}^{3}}\|n-\bar{n}\|_{L_{x}^{2}}\|u-\bar{u}\|_{L_{x}^{6}} \\
& +\frac{1}{2 \sigma}\|\bar{u}\|_{L_{x}^{\infty}}\left(\|n-\bar{n}\|_{L_{x}^{2}}+\sigma\|B-\bar{B}\|_{L_{x}^{2}}\right)\|j-n u-(\bar{j}-\bar{n} \bar{u})\|_{L_{x}^{2}} \\
& +\frac{1}{2}\|\bar{j}\|_{L_{x}^{3}}\|B-\bar{B}\|_{L_{x}^{2}}\|u-\bar{u}\|_{L_{x}^{6}}+\int_{\mathbb{R}^{3}} \mathbf{A} \cdot\left(\begin{array}{c}
j-\bar{j}-n(u-\bar{u}) \\
j
\end{array}\right) d x \\
& \leq \frac{3}{2 \mu}\|\bar{u}\|_{L_{x}^{\infty}}^{2}\|u-\bar{u}\|_{L_{x}^{2}}^{2}+\left(\frac{3 C_{0}^{2}}{8 \mu}\left\|\frac{1}{2} \nabla_{x} \bar{n}-\bar{E}\right\|_{L_{x}^{3}}^{2}+\frac{1}{2 \sigma}\|\bar{u}\|_{L_{x}^{\infty}}^{2}\right)\|n-\bar{n}\|_{L_{x}^{2}}^{2} \\
& +\left(\frac{\sigma}{2}\|\bar{u}\|_{L_{x}^{\infty}}^{2}+\frac{3 C_{0}^{2}}{8 \mu}\|\bar{j}\|_{L_{x}^{3}}^{2}\right)\|B-\bar{B}\|_{L_{x}^{2}}^{2} \\
& +\frac{\mu}{2}\left\|\nabla_{x}(u-\bar{u})\right\|_{L_{x}^{2}}^{2}+\frac{1}{4 \sigma}\|j-n u-(\bar{j}-\bar{n} \bar{u})\|_{L_{x}^{2}}^{2} \\
& +\int_{\mathbb{R}^{3}} \mathbf{A} \cdot\left(\begin{array}{c}
u-\bar{j}-n(u-\bar{u}) \\
j-\bar{j}
\end{array}\right) d x .
\end{aligned}
$$

Hence,

$$
\frac{d}{d t} \delta \mathcal{E}(t)+\frac{1}{2} \delta \mathcal{D}(t) \leq \lambda(t) \delta \mathcal{E}(t)+\int_{\mathbb{R}^{3}} \mathbf{A} \cdot\left(\begin{array}{c}
u-\bar{u} \\
j-\bar{j}-n(u-\bar{u})
\end{array}\right) d x,
$$

which concludes the proof of the proposition with a direct application of Grönwall's lemma.

Again, note that the test functions satisfying the linear constraints $(3.16)$ are easily constructed employing the relations (3.15). Now, one may prefer to deal, in a completely equivalent manner, with test functions $(\bar{u}, \bar{n}, \bar{j}, \bar{E}, \bar{B}) \in C_{c}^{\infty}\left([0, \infty) \times \mathbb{R}^{3}\right)$ satisfying the stationary constraints

$\operatorname{div} \bar{u}=0, \quad \operatorname{div} \bar{E}=\bar{n}, \quad \operatorname{div} \bar{B}=0, \quad \bar{j}-\bar{n} \bar{u}=\sigma\left(-\frac{1}{2} \nabla_{x} \bar{n}+\bar{E}+\bar{u} \wedge \bar{B}\right)$

rather than the constraints 3.16). In this case, instead of (3.17), we obtain the stability inequality

$$
\begin{aligned}
& \delta \mathcal{E}(t)+\frac{1}{2} \int_{0}^{t} \delta \mathcal{D}(s) e^{\int_{s}^{t} \lambda(\sigma) d \sigma} d s \\
& \leq \delta \mathcal{E}(0) e^{\int_{0}^{t} \lambda(s) d s}+\int_{0}^{t}\left[\int_{\mathbb{R}^{3}} \mathbf{A} \cdot\left(\begin{array}{c}
E-\frac{1}{2} \nabla_{x} n-\left(\bar{E}-\frac{1}{2} \nabla_{x} \bar{n}\right) \\
B-\bar{B}
\end{array}\right) d x\right](s) e^{\int_{s}^{t} \lambda(\sigma) d \sigma} d s,
\end{aligned}
$$

where the acceleration operator is now defined by

$$
\mathbf{A}(\bar{u}, \bar{n}, \bar{j}, \bar{E}, \bar{B})=\left(\begin{array}{c}
-\partial_{t} \bar{u}-P\left(\bar{u} \cdot \nabla_{x} \bar{u}\right)+\mu \Delta_{x} \bar{u}+\frac{1}{2} P(\bar{n} \bar{E}+\bar{j} \wedge \bar{B}) \\
\frac{1}{2}\left(-\partial_{t} \bar{E}+\operatorname{rot} \bar{B}-\bar{j}\right) \\
\frac{1}{2}\left(-\partial_{t} \bar{B}-\operatorname{rot} \bar{E}\right)
\end{array}\right) .
$$

The preceding proposition provides an important weak-strong stability property for the two-fluid incompressible Navier-Stokes-Maxwell system with Ohm's law (3.5). Indeed, the stability inequality 3.17) essentially implies that a solution $(\bar{u}, \bar{n}, j, E, \bar{B})$ of 3.5 such that $\bar{u} \in L_{t}^{2} L_{x}^{\infty}, j \in L_{t}^{2} L_{x}^{3}$ and $\frac{1}{2} \nabla_{x} \bar{n}-\bar{E} \in L_{t}^{2} L_{x}^{3}$, if it exists, is unique in the whole class of weak solutions in the energy space, for any given initial data. 
By analogy with Lions' dissipative solutions to the incompressible Euler system 57. Section 4.4], we provide now a suitable notion of dissipative solution for the two-fluid incompressible Navier-Stokes-Maxwell system with Ohm's law (3.5), based on Proposition 3.8, and establish their existence next.

Definition. We say that

$$
(u, n, E, B) \in L^{\infty}\left([0, \infty) ; L^{2}\left(\mathbb{R}^{3}\right)\right) \cap C\left([0, \infty) ; w-L^{2}\left(\mathbb{R}^{3}\right)\right)
$$

such that

$$
\operatorname{div} u=0, \quad \operatorname{div} E=n, \quad \operatorname{div} B=0,
$$

is a dissipative solution of the two-fluid incompressible Navier-StokesMaxwell system with Ohm's law (3.5), if it solves Maxwell's equations

$$
\left\{\begin{array}{l}
\partial_{t} E-\operatorname{rot} B=-j, \\
\partial_{t} B+\operatorname{rot} E=0,
\end{array}\right.
$$

with Ohm's law

$$
j-n u=\sigma\left(-\frac{1}{2} \nabla_{x} n+E+u \wedge B\right),
$$

in the sense of distributions, and if, for any test functions $(\bar{u}, \bar{n}, \bar{j}, \bar{E}, \bar{B}) \in C_{c}^{\infty}\left([0, \infty) \times \mathbb{R}^{3}\right)$ satisfying the linear constraints $(3.16)$, the stability inequality $(3.17)$ is verified.

As previously mentioned, dissipative solutions define actual solutions in the sense that they coincide with the unique strong solution when the latter exists. The following theorem asserts their existence.

TheOREM 3.9. For any initial data $\left(u^{\text {in }}, n^{\text {in }}, E^{\text {in }}, B^{\text {in }}\right) \in L^{2}\left(\mathbb{R}^{3}\right)$ such that

$$
\operatorname{div} u^{\text {in }}=0, \quad \operatorname{div} E^{\text {in }}=n^{\text {in }}, \quad \operatorname{div} B^{\text {in }}=0,
$$

there exists a dissipative solution to the two-fluid incompressible Navier-StokesMaxwell system with Ohm's law 3.5.

Proof. As in the proof of Theorem 3.7 it is possible, here, to justify the existence of dissipative solutions by introducing viscous approximations of the system (3.5). Thus, for each $\nu>0$, we consider weak solutions of the following system :

$$
\left\{\begin{array}{rlrl}
\partial_{t} u_{\nu}+u_{\nu} \cdot \nabla_{x} u_{\nu}-\mu \Delta_{x} u_{\nu} & =-\nabla_{x} p_{\nu}+\frac{1}{2}\left(n_{\nu} E_{\nu}+j_{\nu} \wedge B_{\nu}\right), & & \operatorname{div} u_{\nu}=0 \\
\partial_{t} E_{\nu}-\operatorname{rot} B_{\nu}-\nu \Delta_{x} E_{\nu} & =-j_{\nu}, & & \operatorname{div} E_{\nu}=n_{\nu} \\
\partial_{t} B_{\nu}+\operatorname{rot} E_{\nu}-\nu \Delta_{x} B_{\nu} & =0, & & \operatorname{div} B_{\nu}=0 \\
j_{\nu}-n_{\nu} u_{\nu} & =\sigma\left(-\frac{1}{2} \nabla_{x} n_{\nu}+E_{\nu}+u_{\nu} \wedge B_{\nu}\right) &
\end{array}\right.
$$

associated with the initial data $\left(u^{\text {in }}, n^{\text {in }}, E^{\text {in }}, B^{\text {in }}\right)$ and satisfying the energy inequality, for all $t>0$,

$$
\begin{aligned}
\left(\frac{1}{2}\left\|u_{\nu}\right\|_{L_{x}^{2}}^{2}+\frac{1}{8}\left\|n_{\nu}\right\|_{L_{x}^{2}}^{2}+\frac{1}{4}\left\|E_{\nu}\right\|_{L_{x}^{2}}^{2}+\frac{1}{4}\left\|B_{\nu}\right\|_{L_{x}^{2}}^{2}\right)(t) \\
+\int_{0}^{t}\left(\mu\left\|\nabla_{x} u_{\nu}\right\|_{L_{x}^{2}}^{2}+\frac{1}{2 \sigma}\left\|j_{\nu}-n_{\nu} u_{\nu}\right\|_{L_{x}^{2}}^{2}\right. \\
\left.+\frac{\nu}{4}\left\|\nabla_{x} n_{\nu}\right\|_{L_{x}^{2}}^{2}+\frac{\nu}{2}\left\|\nabla_{x} E_{\nu}\right\|_{L_{x}^{2}}^{2}+\frac{\nu}{2}\left\|\nabla_{x} B_{\nu}\right\|_{L_{x}^{2}}^{2}\right)(s) d s \\
\leq \frac{1}{2}\left\|u^{\mathrm{in}}\right\|_{L_{x}^{2}}^{2}+\frac{1}{8}\left\|n^{\mathrm{in}}\right\|_{L_{x}^{2}}^{2}+\frac{1}{4}\left\|E^{\mathrm{in}}\right\|_{L_{x}^{2}}^{2}+\frac{1}{4}\left\|B^{\mathrm{in}}\right\|_{L_{x}^{2}}^{2} .
\end{aligned}
$$

Such weak solutions are easily established following the method of Leray 48 , for the nonlinear terms $n_{\nu} E_{\nu}$ and $j_{\nu} \wedge B_{\nu}$ are now stable with respect to weak convergence 
in the energy space defined by the above energy inequality, thanks to the dissipation on $n_{\nu}, E_{\nu}$ and $B_{\nu}$.

Then, repeating the computations of Proposition 3.8 it is readily seen that

$$
\begin{aligned}
& \frac{d}{d t} \int_{\mathbb{R}^{3}}\left(u_{\nu} \cdot \bar{u}+\frac{1}{4} n_{\nu} \bar{n}+\frac{1}{2} E_{\nu} \cdot \bar{E}+\frac{1}{2} B_{\nu} \cdot \bar{B}\right) d x \\
& +\int_{\mathbb{R}^{3}} 2 \mu \nabla_{x} u_{\nu}: \nabla_{x} \bar{u}+\frac{1}{\sigma}\left(j_{\nu}-n_{\nu} u_{\nu}\right) \cdot(\bar{j}-\bar{n} \bar{u}) d x \\
& =-\int_{\mathbb{R}^{3}} \bar{u} \otimes\left(u_{\nu}-\bar{u}\right): \nabla_{x}\left(u_{\nu}-\bar{u}\right) d x+\frac{1}{2} \int_{\mathbb{R}^{3}}\left(n_{\nu}-\bar{n}\right)\left(u_{\nu}-\bar{u}\right) \cdot\left(\frac{1}{2} \nabla_{x} \bar{n}-\bar{E}\right) d x \\
& +\frac{1}{2} \int_{\mathbb{R}^{3}}\left(\left(j_{\nu}-n_{\nu} u_{\nu}-(\bar{j}-\bar{n} \bar{u})\right) \wedge\left(B_{\nu}-\bar{B}\right)\right) \cdot \bar{u}+\left(\left(u_{\nu}-\bar{u}\right) \wedge\left(B_{\nu}-\bar{B}\right)\right) \cdot \bar{j} d x \\
& +\frac{1}{2 \sigma} \int_{\mathbb{R}^{3}}\left(n_{\nu}-\bar{n}\right)\left(j_{\nu}-n_{\nu} u_{\nu}-(\bar{j}-\bar{n} \bar{u})\right) \cdot \bar{u} d x \\
& -\int_{\mathbb{R}^{3}} \mathbf{A} \cdot\left(u_{\nu}-n_{\nu} u_{\nu}+\left(n_{\nu}-\bar{n}\right) \bar{u}\right) d x \\
& -\int_{\mathbb{R}^{3}} \frac{\nu}{4} \nabla_{x} n_{\nu}: \nabla_{x} \bar{n}+\frac{\nu}{2} \nabla_{x} E_{\nu}: \nabla_{x} \bar{E}+\frac{\nu}{2} \nabla_{x} B_{\nu}: \nabla_{x} \bar{B} d x .
\end{aligned}
$$

Hence, defining the modulated energy $\delta \mathcal{E}_{\nu}(t)$ and modulated energy dissipation $\delta \mathcal{D}_{\nu}(t)$ by simply replacing $(u, n, j, E, B)$ by $\left(u_{\nu}, n_{\nu}, j_{\nu}, E_{\nu}, B_{\nu}\right)$ in (3.18), we infer that

$$
\begin{aligned}
\delta \mathcal{E}_{\nu}(t) & +\int_{0}^{t} \delta \mathcal{D}_{\nu}(s)+\frac{\nu}{2}\left(\frac{1}{2}\left\|\nabla_{x} n_{\nu}\right\|_{L_{x}^{2}}^{2}+\left\|\nabla_{x} E_{\nu}\right\|_{L_{x}^{2}}^{2}+\left\|\nabla_{x} B_{\nu}\right\|_{L_{x}^{2}}^{2}\right)(s) d s \\
& \leq \delta \mathcal{E}_{\nu}(0)+\int_{0}^{t} \int_{\mathbb{R}^{3}} \bar{u} \otimes\left(u_{\nu}-\bar{u}\right): \nabla_{x}\left(u_{\nu}-\bar{u}\right) d x d s \\
& -\frac{1}{2} \int_{0}^{t} \int_{\mathbb{R}^{3}}\left(n_{\nu}-\bar{n}\right)\left(u_{\nu}-\bar{u}\right) \cdot\left(\frac{1}{2} \nabla_{x} \bar{n}-\bar{E}\right) d x d s \\
& -\frac{1}{2} \int_{0}^{t} \int_{\mathbb{R}^{3}}\left(\left(j_{\nu}-n_{\nu} u_{\nu}-(\bar{j}-\bar{n} \bar{u})\right) \wedge\left(B_{\nu}-\bar{B}\right)\right) \cdot \bar{u} d x d s \\
& -\frac{1}{2} \int_{0}^{t} \int_{\mathbb{R}^{3}}\left(\left(u_{\nu}-\bar{u}\right) \wedge\left(B_{\nu}-\bar{B}\right)\right) \cdot \bar{j} d x d s \\
& -\frac{1}{2 \sigma} \int_{0}^{t} \int_{\mathbb{R}^{3}}\left(n_{\nu}-\bar{n}\right)\left(j_{\nu}-n_{\nu} u_{\nu}-(\bar{j}-\bar{n} \bar{u})\right) \cdot \bar{u} d x d s \\
& +\int_{0}^{t} \int_{\mathbb{R}^{3}} \mathbf{A} \cdot\left(\begin{array}{c}
u_{\nu}-\bar{u} \\
j_{\nu}-\bar{j}-n_{\nu}\left(u_{\nu}-\bar{u}\right)
\end{array}\right) d x d s \\
& +\int_{0}^{t} \int_{\mathbb{R}^{3}} \frac{\nu}{4} \nabla_{x} n_{\nu}: \nabla_{x} \bar{n}+\frac{\nu}{2} \nabla_{x} E_{\nu}: \nabla_{x} \bar{E}+\frac{\nu}{2} \nabla_{x} B_{\nu}: \nabla_{x} \bar{B} d x d s .
\end{aligned}
$$


Then, following the proof of Proposition 3.8 , we arrive at

$$
\begin{aligned}
\delta \mathcal{E}_{\nu}(t) & +\int_{0}^{t} \frac{1}{2} \delta \mathcal{D}_{\nu}(s)+\frac{\nu}{2}\left(\frac{1}{2}\left\|\nabla_{x} n_{\nu}\right\|_{L_{x}^{2}}^{2}+\left\|\nabla_{x} E_{\nu}\right\|_{L_{x}^{2}}^{2}+\left\|\nabla_{x} B_{\nu}\right\|_{L_{x}^{2}}^{2}\right)(s) d s \\
& \leq \delta \mathcal{E}_{\nu}(0)+\int_{0}^{t} \lambda(s) \delta \mathcal{E}_{\nu}(s)+\left[\int_{\mathbb{R}^{3}} \mathbf{A} \cdot\left(\begin{array}{c}
u_{\nu}-\bar{u} \\
j_{\nu}-\bar{j}-n_{\nu}\left(u_{\nu}-\bar{u}\right)
\end{array}\right) d x\right] d s \\
& +\frac{\nu}{2} \int_{0}^{t} \int_{\mathbb{R}^{3}} \frac{1}{2} \nabla_{x} n_{\nu}: \nabla_{x} \bar{n}+\nabla_{x} E_{\nu}: \nabla_{x} \bar{E}+\nabla_{x} B_{\nu}: \nabla_{x} \bar{B} d x d s \\
& \leq \delta \mathcal{E}_{\nu}(0)+\int_{0}^{t} \lambda(s) \delta \mathcal{E}_{\nu}(s)+\left[\int_{\mathbb{R}^{3}} \mathbf{A} \cdot\left(\begin{array}{c}
u_{\nu}-\bar{u} \\
j_{\nu}-\bar{j}-n_{\nu}\left(u_{\nu}-\bar{u}\right)
\end{array}\right) d x\right] d s \\
& +\frac{\nu}{4} \int_{0}^{t} \frac{1}{2}\left\|\nabla_{x} n_{\nu}\right\|_{L_{x}^{2}}^{2}+\left\|\nabla_{x} E_{\nu}\right\|_{L_{x}^{2}}^{2}+\left\|\nabla_{x} B_{\nu}\right\|_{L_{x}^{2}}^{2} d s \\
& +\frac{\nu}{4} \int_{0}^{t} \frac{1}{2}\left\|\nabla_{x} \bar{n}\right\|_{L_{x}^{2}}^{2}+\left\|\nabla_{x} \bar{E}\right\|_{L_{x}^{2}}^{2}+\left\|\nabla_{x} \bar{B}\right\|_{L_{x}^{2}}^{2} d s
\end{aligned}
$$

and an application of Grönwall's lemma yields

$$
\begin{aligned}
& \delta \mathcal{E}_{\nu}(t)+\frac{1}{2} \int_{0}^{t} \delta \mathcal{D}_{\nu}(s) e^{\int_{s}^{t} \lambda(\sigma) d \sigma} d s \leq \delta \mathcal{E}_{\nu}(0) e^{\int_{0}^{t} \lambda(s) d s} \\
& +\int_{0}^{t}\left[\int_{\mathbb{R}^{3}} \mathbf{A} \cdot\left(\begin{array}{c}
u_{\nu}-\bar{u} \\
j_{\nu}-\bar{j}-n_{\nu}\left(u_{\nu}-\bar{u}\right)
\end{array}\right) d x\right](s) e^{\int_{s}^{t} \lambda(\sigma) d \sigma} d s \\
& +\frac{\nu}{4} \int_{0}^{t}\left[\frac{1}{2}\left\|\nabla_{x} \bar{n}\right\|_{L_{x}^{2}}^{2}+\left\|\nabla_{x} \bar{E}\right\|_{L_{x}^{2}}^{2}+\left\|\nabla_{x} \bar{B}\right\|_{L_{x}^{2}}^{2}\right](s) e^{\int_{s}^{t} \lambda(\sigma) d \sigma} d s .
\end{aligned}
$$

We may now pass to the limit in the above stability inequality. Thus, up to extraction of subsequences, we may assume that, as $\nu \rightarrow 0$,

$$
\begin{array}{ll}
u_{\nu} \stackrel{*}{\rightarrow} u & \text { in } L_{t}^{\infty} L_{x}^{2} \cap L_{t}^{2} \dot{H}_{x}^{1}, \\
n_{\nu} \stackrel{*}{\rightarrow} n & \text { in } L_{t}^{\infty} L_{x}^{2}, \\
E_{\nu} \stackrel{*}{\rightarrow} E & \text { in } L_{t}^{\infty} L_{x}^{2}, \\
B_{\nu} \stackrel{*}{\rightarrow} B & \text { in } L_{t}^{\infty} L_{x}^{2} .
\end{array}
$$

Furthermore, noticing that $\partial_{t} u_{\nu}, \partial_{t} n_{\nu}, \partial_{t} E_{\nu}$ and $\partial_{t} B_{\nu}$ are uniformly bounded, in $L_{\text {loc }}^{1}$ in time and in some negative index Sobolev space in $x$, it is possible to show (see [57, Appendix C]) that $\left(u_{\nu}, n_{\nu}, E_{\nu}, B_{\nu}\right)$ converges to $(u, n, E, B) \in$ $C\left([0, \infty) ; w-L^{2}\left(\mathbb{R}^{3}\right)\right)$ weakly in $L_{x}^{2}$ uniformly locally in time. Moreover, invoking a classical compactness result by Aubin and Lions 8, 50 (see also 71 for a sharp compactness criterion), we infer that the $u_{\nu}$ 's converge towards $u$ strongly in $L_{\mathrm{loc}}^{2}(d t d x)$. In particular, it follows that, up to extraction,

$$
\begin{array}{cl}
n_{\nu} u_{\nu} \stackrel{*}{\rightarrow} n u & \text { in } L_{t}^{\infty} L_{x}^{1} \cap L_{t}^{2} L_{x}^{6}, \\
j_{\nu}-n_{\nu} u_{\nu} \rightarrow j-n u & \text { in } L_{t}^{2} L_{x}^{2} .
\end{array}
$$

Then, by the weak lower semi-continuity of the norms, we obtain that, for every $t>0$,

$$
\delta \mathcal{E}(t)+\frac{1}{2} \int_{0}^{t} \delta \mathcal{D}(s) e^{\int_{s}^{t} \lambda(\sigma) d \sigma} d s \leq \liminf _{\nu \rightarrow 0} \delta \mathcal{E}_{\nu}(t)+\frac{1}{2} \int_{0}^{t} \delta \mathcal{D}_{\nu}(s) e^{\int_{s}^{t} \lambda(\sigma) d \sigma} d s .
$$

Hence, the stability inequality (3.17) holds. Finally, it is readily seen that Ohm's law is satisfied asymptotically, which concludes the proof of the theorem. 
We present now an alternative kind of stability inequality for the two-fluid incompressible Navier-Stokes-Maxwell system with Ohm's law (3.5), whose understanding will be crucial for the relative entropy method - developed later on in Chapter 12 - in the hydrodynamic limit of the two species Vlasov-MaxwellBoltzmann system (2.32). It is based on the identity (3.7) linking the Lorentz force with the Poynting vector $E \wedge B$, which will allow us to stabilize the modulated nonlinear terms solely with the modulated energy $\delta \mathcal{E}$ (i.e. without absorbing nonlinear terms with the modulated dissipation $\delta \mathcal{D}$; note the different coefficient in front of $\delta \mathcal{D}$ in the stability inequalities (3.17) and (3.21), below).

Proposition 3.10. Let $(u, n, E, B)$ be a smooth solution to the two-fluid incompressible Navier-Stokes-Maxwell system with Ohm's law (3.5). Further consider test functions $(\bar{u}, \bar{n}, \bar{j}, \bar{E}, \bar{B}) \in C_{c}^{\infty}\left([0, \infty) \times \mathbb{R}^{3}\right)$ such that $\|\bar{u}\|_{L_{t, x}^{\infty}}<1$ and

$$
\left\{\begin{aligned}
& \operatorname{div} \bar{u}=0 \\
\partial_{t} \bar{E}-\operatorname{rot} \bar{B}=-\bar{j}, & \operatorname{div} \bar{E}=\bar{n} \\
\partial_{t} \bar{B}+\operatorname{rot} \bar{E}=0, & \operatorname{div} \bar{B}=0
\end{aligned}\right.
$$

We define the acceleration operator by

$$
\mathbf{A}(\bar{u}, \bar{n}, \bar{j}, \bar{E}, \bar{B})=\left(\begin{array}{c}
-\partial_{t} \bar{u}-P\left(\bar{u} \cdot \nabla_{x} \bar{u}\right)+\mu \Delta_{x} \bar{u}+\frac{1}{2} P(\bar{n} \bar{E}+\bar{j} \wedge \bar{B}) \\
-\frac{1}{2 \sigma}(\bar{j}-\bar{n} \bar{u})+\frac{1}{2}\left(-\frac{1}{2} \nabla_{x} \bar{n}+\bar{E}+\bar{u} \wedge \bar{B}\right)
\end{array}\right)
$$

and the growth rate by

$$
\lambda(t)=\frac{2\left\|\nabla_{t, x} \bar{u}(t)\right\|_{L_{x}^{\infty}}}{1-\|\bar{u}(t)\|_{L_{x}^{\infty}}}+\frac{\sqrt{2}\|(\bar{j}-\bar{n} \bar{u})(t)\|_{L_{x}^{\infty}}}{2\left(1-\|\bar{u}(t)\|_{L_{x}^{\infty}}\right)}+\left\|\left(\frac{1}{2} \nabla_{x} \bar{n}-\bar{E}-\bar{u} \wedge \bar{B}\right)(t)\right\|_{L_{x}^{\infty}} .
$$

$$
\begin{aligned}
\delta \mathcal{E}(t)+ & \int_{0}^{t} \delta \mathcal{D}(s) e^{\int_{s}^{t} \lambda(\sigma) d \sigma} d s \\
& \leq \delta \mathcal{E}(0) e^{\int_{0}^{t} \lambda(s) d s}+\int_{0}^{t}\left[\int_{\mathbb{R}^{3}} \mathbf{A} \cdot\left(\begin{array}{c}
u-\bar{u} \\
j-n u-(\bar{j}-\bar{n} \bar{u})
\end{array}\right) d x\right](s) e^{\int_{s}^{t} \lambda(\sigma) d \sigma} d s,
\end{aligned}
$$

where the modulated energy $\delta \mathcal{E}$ and energy dissipation $\delta \mathcal{D}$ are given by

$$
\begin{aligned}
\delta \mathcal{E}(t) & =\frac{1}{2}\|(u-\bar{u})(t)\|_{L_{x}^{2}}^{2}+\frac{1}{8}\|(n-\bar{n})(t)\|_{L_{x}^{2}}^{2} \\
& +\frac{1}{4}\|(E-\bar{E})(t)\|_{L_{x}^{2}}^{2}+\frac{1}{4}\|(B-\bar{B})(t)\|_{L_{x}^{2}}^{2} \\
& -\frac{1}{2} \int_{\mathbb{R}^{3}}((E-\bar{E})(t) \wedge(B-\bar{B})(t)) \cdot \bar{u}(t) d x, \\
\delta \mathcal{D}(t) & =\mu\left\|\nabla_{x}(u-\bar{u})(t)\right\|_{L_{x}^{2}}^{2}+\frac{1}{2 \sigma}\|(j-n u-(\bar{j}-\bar{n} \bar{u}))(t)\|_{L_{x}^{2}}^{2} .
\end{aligned}
$$


Proof. Following the proof of Proposition 3.8, using that $\operatorname{div} u=\operatorname{div} \bar{u}=0$, we consider first the identity

$$
\begin{aligned}
\frac{d}{d t} & \int_{\mathbb{R}^{3}}\left(u \cdot \bar{u}+\frac{1}{4} n \bar{n}+\frac{1}{2} E \cdot \bar{E}+\frac{1}{2} B \cdot \bar{B}\right) d x+\int_{\mathbb{R}^{3}} 2 \mu \nabla_{x} u: \nabla_{x} \bar{u} d x \\
& =\int_{\mathbb{R}^{3}}(u-\bar{u}) \otimes(u-\bar{u}): \nabla_{x} \bar{u} d x \\
& +\frac{1}{2} \int_{\mathbb{R}^{3}}(n E+j \wedge B) \cdot \bar{u}+(\bar{n} \bar{E}+\bar{j} \wedge \bar{B}) \cdot u d x \\
& -\frac{1}{2} \int_{\mathbb{R}^{3}} j \cdot\left(\bar{E}-\frac{1}{2} \nabla_{x} \bar{n}\right)+\bar{j} \cdot\left(E-\frac{1}{2} \nabla_{x} n\right) d x-\int_{\mathbb{R}^{3}} \mathbf{A} \cdot\left(\begin{array}{l}
u \\
0
\end{array}\right) d x \\
& =\int_{\mathbb{R}^{3}}(u-\bar{u}) \otimes(u-\bar{u}): \nabla_{x} \bar{u} d x \\
& +\frac{1}{2} \int_{\mathbb{R}^{3}}((n-\bar{n})(E-\bar{E})+(j-\bar{j}) \wedge(B-\bar{B})) \cdot \bar{u} d x \\
& +\frac{1}{2} \int_{\mathbb{R}^{3}}(\bar{j}-\bar{n} \bar{u}) \cdot((u-\bar{u}) \wedge(B-\bar{B})) d x \\
& +\frac{1}{2} \int_{\mathbb{R}^{3}}(n-\bar{n})(u-\bar{u}) \cdot\left(\frac{1}{2} \nabla_{x} \bar{n}-\bar{E}-\bar{u} \wedge \bar{B}\right) d x \\
& +\frac{1}{2} \int_{\mathbb{R}^{3}}(j-n u) \cdot\left(\frac{1}{2} \nabla_{x} \bar{n}-\bar{E}-\bar{u} \wedge \bar{B}\right)+(\bar{j}-\bar{n} \bar{u}) \cdot\left(\frac{1}{2} \nabla_{x} n-E-u \wedge B\right) d x \\
& -\int_{\mathbb{R}^{3}} \mathbf{A} \cdot\left(\begin{array}{l}
u \\
0
\end{array}\right) d x .
\end{aligned}
$$

Further using Ohm's laws, we find

$$
\begin{aligned}
\frac{d}{d t} \int_{\mathbb{R}^{3}} & \left(u \cdot \bar{u}+\frac{1}{4} n \bar{n}+\frac{1}{2} E \cdot \bar{E}+\frac{1}{2} B \cdot \bar{B}\right) d x \\
& +\int_{\mathbb{R}^{3}} 2 \mu \nabla_{x} u: \nabla_{x} \bar{u}+\frac{1}{\sigma}(j-n u) \cdot(\bar{j}-\bar{n} \bar{u}) d x \\
& =\int_{\mathbb{R}^{3}}(u-\bar{u}) \otimes(u-\bar{u}): \nabla_{x} \bar{u} d x \\
& +\frac{1}{2} \int_{\mathbb{R}^{3}}((n-\bar{n})(E-\bar{E})+(j-\bar{j}) \wedge(B-\bar{B})) \cdot \bar{u} d x \\
& +\frac{1}{2} \int_{\mathbb{R}^{3}}(\bar{j}-\bar{n} \bar{u}) \cdot((u-\bar{u}) \wedge(B-\bar{B})) d x \\
& +\frac{1}{2} \int_{\mathbb{R}^{3}}(n-\bar{n})(u-\bar{u}) \cdot\left(\frac{1}{2} \nabla_{x} \bar{n}-\bar{E}-\bar{u} \wedge \bar{B}\right) d x \\
& -\int_{\mathbb{R}^{3}} \mathbf{A} \cdot\left(\begin{array}{c}
u \\
j-n u
\end{array}\right) d x .
\end{aligned}
$$

Then, expressing the modulated Lorentz force with a modulated Poynting vector as

$$
\begin{aligned}
\partial_{t}((E-\bar{E}) \wedge(B-\bar{B})) & +\frac{1}{2} \nabla_{x}\left(|E-\bar{E}|^{2}+|B-\bar{B}|^{2}\right) \\
& -\nabla_{x} \cdot((E-\bar{E}) \otimes(E-\bar{E})+(B-\bar{B}) \otimes(B-\bar{B})) \\
& =-(n-\bar{n})(E-\bar{E})-(j-\bar{j}) \wedge(B-\bar{B})
\end{aligned}
$$


we arrive at the relation

$$
\begin{aligned}
\frac{d}{d t} \int_{\mathbb{R}^{3}} & \left(u \cdot \bar{u}+\frac{1}{4} n \bar{n}+\frac{1}{2} E \cdot \bar{E}+\frac{1}{2} B \cdot \bar{B}+\frac{1}{2}((E-\bar{E}) \wedge(B-\bar{B})) \cdot \bar{u}\right) d x \\
& -\int_{\mathbb{R}^{3}} \frac{1}{2}((E-\bar{E}) \wedge(B-\bar{B})) \cdot \partial_{t} \bar{u} d x \\
& +\int_{\mathbb{R}^{3}} 2 \mu \nabla_{x} u: \nabla_{x} \bar{u}+\frac{1}{\sigma}(j-n u) \cdot(\bar{j}-\bar{n} \bar{u}) d x \\
& =\int_{\mathbb{R}^{3}}(u-\bar{u}) \otimes(u-\bar{u}): \nabla_{x} \bar{u} d x \\
& -\frac{1}{2} \int_{\mathbb{R}^{3}}((E-\bar{E}) \otimes(E-\bar{E})+(B-\bar{B}) \otimes(B-\bar{B})): \nabla_{x} \bar{u} d x \\
& +\frac{1}{2} \int_{\mathbb{R}^{3}}(\bar{j}-\bar{n} \bar{u}) \cdot((u-\bar{u}) \wedge(B-\bar{B})) d x \\
& +\frac{1}{2} \int_{\mathbb{R}^{3}}(n-\bar{n})(u-\bar{u}) \cdot\left(\frac{1}{2} \nabla_{x} \bar{n}-\bar{E}-\bar{u} \wedge \bar{B}\right) d x \\
& -\int_{\mathbb{R}^{3}} \mathbf{A} \cdot\left(\begin{array}{c}
u \\
j-n u
\end{array}\right) d x .
\end{aligned}
$$

On the whole, combining the preceding identity with the energy conservation law for test functions (3.19) and the energy decay imposed by the formal energy conservations from Proposition 3.3 , we find the following modulated energy inequality :

$$
\begin{aligned}
\frac{d}{d t} \delta & \mathcal{E}(t)+\delta \mathcal{D}(t) \\
& \leq-\int_{\mathbb{R}^{3}}(u-\bar{u}) \otimes(u-\bar{u}): \nabla_{x} \bar{u} d x-\int_{\mathbb{R}^{3}} \frac{1}{2}((E-\bar{E}) \wedge(B-\bar{B})) \cdot \partial_{t} \bar{u} d x \\
& +\frac{1}{2} \int_{\mathbb{R}^{3}}((E-\bar{E}) \otimes(E-\bar{E})+(B-\bar{B}) \otimes(B-\bar{B})): \nabla_{x} \bar{u} d x \\
& -\frac{1}{2} \int_{\mathbb{R}^{3}}(\bar{j}-\bar{n} \bar{u}) \cdot((u-\bar{u}) \wedge(B-\bar{B})) d x \\
& -\frac{1}{2} \int_{\mathbb{R}^{3}}(n-\bar{n})(u-\bar{u}) \cdot\left(\frac{1}{2} \nabla_{x} \bar{n}-\bar{E}-\bar{u} \wedge \bar{B}\right) d x \\
& +\int_{\mathbb{R}^{3}} \mathbf{A} \cdot\left(\begin{array}{c}
u-\bar{u} \\
j-n u-(\bar{j}-\bar{n} \bar{u})
\end{array}\right) d x .
\end{aligned}
$$

The next step consists in estimating the terms in the right-hand side above that are nonlinear in $(u, n, j, E, B)$ and to absorb the resulting expressions with the modulated energy $\delta \mathcal{E}(t)$ by suitable uses of Young's inequality and Grönwall's lemma. 
Thus, we obtain

$$
\begin{aligned}
& \frac{d}{d t} \delta \mathcal{E}(t)+\delta \mathcal{D}(t) \\
& \leq\left\|\nabla_{t, x} \bar{u}\right\|_{L_{x}^{\infty}}\left(\|u-\bar{u}\|_{L_{x}^{2}}^{2}+\frac{1}{2}\|E-\bar{E}\|_{L_{x}^{2}}^{2}+\frac{1}{2}\|B-\bar{B}\|_{L_{x}^{2}}^{2}\right) \\
& +\frac{1}{2}\left(\|\bar{j}-\bar{n} \bar{u}\|_{L_{x}^{\infty}}\|B-\bar{B}\|_{L_{x}^{2}}+\left\|\frac{1}{2} \nabla_{x} \bar{n}-\bar{E}-\bar{u} \wedge \bar{B}\right\|_{L_{x}^{\infty}}\|n-\bar{n}\|_{L_{x}^{2}}\right)\|u-\bar{u}\|_{L_{x}^{2}} \\
& +\int_{\mathbb{R}^{3}} \mathbf{A} \cdot\left(\begin{array}{c}
u-\bar{u} \\
j-n u-(\bar{j}-\bar{n} \bar{u})
\end{array}\right) d x \\
& \leq\left\|\nabla_{t, x} \bar{u}\right\|_{L_{x}^{\infty}}\left(\|u-\bar{u}\|_{L_{x}^{2}}^{2}+\frac{1}{2}\|E-\bar{E}\|_{L_{x}^{2}}^{2}+\frac{1}{2}\|B-\bar{B}\|_{L_{x}^{2}}^{2}\right) \\
& +\frac{\sqrt{2}}{4}\|\bar{j}-\bar{n} \bar{u}\|_{L_{x}^{\infty}}\left(\|u-\bar{u}\|_{L_{x}^{2}}^{2}+\frac{1}{2}\|B-\bar{B}\|_{L_{x}^{2}}^{2}\right) \\
& +\frac{1}{2}\left\|\frac{1}{2} \nabla_{x} \bar{n}-\bar{E}-\bar{u} \wedge \bar{B}\right\|_{L_{x}^{\infty}}\left(\|u-\bar{u}\|_{L_{x}^{2}}^{2}+\frac{1}{4}\|n-\bar{n}\|_{L_{x}^{2}}^{2}\right) \\
& +\int_{\mathbb{R}^{3}} \mathbf{A} \cdot\left(\begin{array}{c}
u-\bar{u} \\
j-n u-(\bar{j}-\bar{n} \bar{u})
\end{array}\right) d x .
\end{aligned}
$$

Hence, further noticing that

$$
\begin{aligned}
\left(1-\|\bar{u}\|_{L_{x}^{\infty}}\right) & \left(\frac{1}{2}\|u-\bar{u}\|_{L_{x}^{2}}^{2}+\frac{1}{8}\|n-\bar{n}\|_{L_{x}^{2}}^{2}+\frac{1}{4}\|E-\bar{E}\|_{L_{x}^{2}}^{2}+\frac{1}{4}\|B-\bar{B}\|_{L_{x}^{2}}^{2}\right) \\
& \leq \frac{1}{2}\|u-\bar{u}\|_{L_{x}^{2}}^{2}+\frac{1}{8}\|n-\bar{n}\|_{L_{x}^{2}}^{2}+\frac{1}{4}\|E-\bar{E}\|_{L_{x}^{2}}^{2}+\frac{1}{4}\|B-\bar{B}\|_{L_{x}^{2}}^{2} \\
& -\frac{1}{2}\|\bar{u}\|_{L_{x}^{\infty}} \int_{\mathbb{R}^{3}}|E-\bar{E}||B-\bar{B}| d x \\
& \leq \frac{1}{2}\|u-\bar{u}\|_{L_{x}^{2}}^{2}+\frac{1}{8}\|n-\bar{n}\|_{L_{x}^{2}}^{2}+\frac{1}{4}\|E-\bar{E}\|_{L_{x}^{2}}^{2}+\frac{1}{4}\|B-\bar{B}\|_{L_{x}^{2}}^{2} \\
& -\frac{1}{2} \int_{\mathbb{R}^{3}}((E-\bar{E}) \wedge(B-\bar{B})) \cdot \bar{u} d x \\
& =\delta \mathcal{E}(t),
\end{aligned}
$$

we find, since $\|\bar{u}\|_{L_{t, x}^{\infty}}<1$, that

$$
\frac{d}{d t} \delta \mathcal{E}(t)+\delta \mathcal{D}(t) \leq \lambda(t) \delta \mathcal{E}(t)+\int_{\mathbb{R}^{3}} \mathbf{A} \cdot\left(\begin{array}{c}
u-\bar{u} \\
j-n u-(\bar{j}-\bar{n} \bar{u})
\end{array}\right) d x,
$$

which concludes the proof of the proposition with a direct application of Grönwall's lemma.

REMARK. Notice that the preceding method of modulation of the Poynting vector is not applicable to the incompressible Navier-Stokes-Maxwell system (3.8), for the divergence of the electric field $E$ is not determined therein, i.e. Gauss' law $\operatorname{div} E=n$ cannot be used to provide a bound on $\operatorname{div} E$.

The preceding proposition provides another weak-strong stability property for the two-fluid incompressible Navier-Stokes-Maxwell system with Ohm's law (3.5). Indeed, the stability inequality (3.21) essentially implies that a solution $(\bar{u}, \bar{n}, \bar{j}, \bar{E}, \bar{B})$ of 3.5 such that $\bar{u} \in L_{t, x}^{\infty}, \nabla_{t, x} \bar{u} \in L_{t}^{1} L_{x}^{\infty}, \bar{j}-\bar{n} \bar{u} \in L_{t}^{1} L_{x}^{\infty}$ and $\|\bar{u}\|_{L_{t, x}^{\infty}}<1$, if it exists, is unique in the whole class of weak solutions in the energy space, for any given initial data. 
We do not know whether the condition $\|\bar{u}\|_{L_{t, x}^{\infty}}<1$ in Proposition 3.10 is actually necessary or merely a technical limitation. Nevertheless, this result shows that such a condition has a stabilizing effect on the two-fluid incompressible NavierStokes-Maxwell system with Ohm's law (3.5). Furthermore, this restriction is physically relevant since it imposes that the modulus of the bulk velocity $\bar{u}$ remains everywhere and at all times below the speed of light. More precisely, keeping track of the relevant physical constants in the formal derivations of Chapter 2, we see that the system 3.5 can be recast as

$$
\left\{\begin{array}{rlrl}
\partial_{t} u+u \cdot \nabla_{x} u-\mu \Delta_{x} u & =-\nabla_{x} p+\frac{1}{2}(c n E+j \wedge B), & & \operatorname{div} u=0 \\
\frac{1}{c} \partial_{t} E-\operatorname{rot} B & =-j, & & \operatorname{div} E=c n \\
\frac{1}{c} \partial_{t} B+\operatorname{rot} E & =0, & \operatorname{div} B=0 \\
j-n u & =\sigma\left(-\frac{1}{2} \nabla_{x} n+c E+u \wedge B\right), &
\end{array}\right.
$$

where the constant $c>0$ denotes the speed of light. Notice that the formal energy conservation law satisfied by this system is independent of $c>0$ and is thus given by Proposition 3.3. Moreover, expressing the Lorentz force with the Poynting vector as in (3.7) yields now

$$
\frac{1}{c} \partial_{t}(E \wedge B)+\frac{1}{2} \nabla_{x}\left(E^{2}+B^{2}\right)-\nabla_{x} \cdot(E \otimes E+B \otimes B)=-c n E-j \wedge B .
$$

Therefore, applying the proof of Proposition 3.10 to the preceding system, we arrive at a stability inequality valid under the restriction that the bulk velocity remains bounded by the speed of light $\|\bar{u}\|_{L_{t, x}^{\infty}}<c$, which is natural.

Following the previous developments, it is also possible to use now the stability inequality 3.21 from Proposition 3.10 to define another notion of dissipative solutions for the two-fluid incompressible Navier-Stokes-Maxwell system with Ohm's law 3.5, whose existence is then established by reproducing the arguments from Theorem 3.9. Indeed, applying the computations from the proof of Proposition 3.10 to the viscous approximation $(3.20$ only produces new dissipative terms which are easily controlled in the limit $\nu \rightarrow 0$ (note that the condition $\|\bar{u}\|_{L_{t, x}^{\infty}}<1$ has to be used in order to absorb the dissipative terms produced by expressing the Lorentz force with the Poynting vector through the viscous Maxwell system from (3.20)). Thus, the only remaining argument from the proof of Theorem 3.9 that needs special care in order to conclude the existence of dissipative solutions resides in the weak lower semi-continuity of the modulated energy $\delta \mathcal{E}(t)$ defined by $(3.22)$, which we establish now.

To this end, let us consider

$$
\begin{array}{ll}
E_{\nu} \rightarrow E & \text { in } L_{x}^{2}, \\
B_{\nu} \rightarrow B & \text { in } L_{x}^{2},
\end{array}
$$

as $\nu \rightarrow 0$. It is enough to show that

$$
\begin{aligned}
\frac{1}{2}\|E\|_{L_{x}^{2}}^{2}+\frac{1}{2}\|B\|_{L_{x}^{2}}^{2} & -\int_{\mathbb{R}^{3}}(E \wedge B) \cdot \bar{u} d x \\
& \leq \liminf _{\nu \rightarrow 0}\left(\frac{1}{2}\left\|E_{\nu}\right\|_{L_{x}^{2}}^{2}+\frac{1}{2}\left\|B_{\nu}\right\|_{L_{x}^{2}}^{2}-\int_{\mathbb{R}^{3}}\left(E_{\nu} \wedge B_{\nu}\right) \cdot \bar{u} d x\right),
\end{aligned}
$$


provided $\|\bar{u}\|_{L_{x}^{\infty}}<1$, which will follow from a convexity argument. Indeed, defining the bilinear form $\mathcal{B}: \mathbb{R}^{6} \times \mathbb{R}^{6} \rightarrow \mathbb{R}$ by

$$
\mathcal{B}\left(\left(\begin{array}{l}
E \\
B
\end{array}\right),\left(\begin{array}{l}
E_{\nu} \\
B_{\nu}
\end{array}\right)\right)=E \cdot E_{\nu}+B \cdot B_{\nu}-\left(E \wedge B_{\nu}\right) \cdot \bar{u}-\left(E_{\nu} \wedge B\right) \cdot \bar{u}
$$

it is readily seen that $\mathcal{B}$ is symmetric and positive definite :

$$
\mathcal{B}\left(\left(\begin{array}{l}
E \\
B
\end{array}\right),\left(\begin{array}{l}
E \\
B
\end{array}\right)\right)=|E|^{2}+|B|^{2}-2(E \wedge B) \cdot \bar{u} \geq(1-|\bar{u}|)\left(|E|^{2}+|B|^{2}\right) \geq 0 .
$$

In particular, it follows that

$$
\mathcal{B}\left(\left(\begin{array}{l}
E \\
B
\end{array}\right),\left(\begin{array}{l}
E_{\nu} \\
B_{\nu}
\end{array}\right)\right) \leq \frac{1}{2} \mathcal{B}\left(\left(\begin{array}{c}
E \\
B
\end{array}\right),\left(\begin{array}{l}
E \\
B
\end{array}\right)\right)+\frac{1}{2} \mathcal{B}\left(\left(\begin{array}{l}
E_{\nu} \\
B_{\nu}
\end{array}\right),\left(\begin{array}{l}
E_{\nu} \\
B_{\nu}
\end{array}\right)\right)
$$

Hence, we deduce

$$
\begin{aligned}
\int_{\mathbb{R}^{3}} \mathcal{B}\left(\left(\begin{array}{l}
E \\
B
\end{array}\right),\left(\begin{array}{l}
E \\
B
\end{array}\right)\right) d x & =\lim _{\nu \rightarrow 0} \int_{\mathbb{R}^{3}} \mathcal{B}\left(\left(\begin{array}{l}
E \\
B
\end{array}\right),\left(\begin{array}{l}
E_{\nu} \\
B_{\nu}
\end{array}\right)\right) d x \\
& \leq \frac{1}{2} \int_{\mathbb{R}^{3}} \mathcal{B}\left(\left(\begin{array}{c}
E \\
B
\end{array}\right),\left(\begin{array}{c}
E \\
B
\end{array}\right)\right) d x \\
& +\lim _{\nu \rightarrow 0} \frac{1}{2} \int_{\mathbb{R}^{3}} \mathcal{B}\left(\left(\begin{array}{c}
E_{\nu} \\
B_{\nu}
\end{array}\right),\left(\begin{array}{c}
E_{\nu} \\
B_{\nu}
\end{array}\right)\right) d x
\end{aligned}
$$

which establishes (3.24).

3.2.3.3. The two-fluid incompressible Navier-Stokes-Maxwell system with solenoidal Ohm's law. Following the strategy of Propositions 3.6 and 3.8, the next result establishes a crucial weak-strong stability principle for the two-fluid incompressible Navier-Stokes-Maxwell system with solenoidal Ohm's law (3.6).

Proposition 3.11. Let $(u, E, B)$ be a smooth solution to the two-fluid incompressible Navier-Stokes-Maxwell system with solenoidal Ohm's law (3.6). Further consider test functions $(\bar{u}, \bar{j}, \bar{E}, \bar{B}) \in C_{c}^{\infty}\left([0, \infty) \times \mathbb{R}^{3}\right)$ such that

$$
\left\{\begin{aligned}
\operatorname{div} \bar{j} & =0, & & \operatorname{div} \bar{u}=0, \\
\partial_{t} \bar{E}-\operatorname{rot} \bar{B} & =-\bar{j}, & & \operatorname{div} \bar{E}=0, \\
\partial_{t} \bar{B}+\operatorname{rot} \bar{E} & =0, & & \operatorname{div} \bar{B}=0 .
\end{aligned}\right.
$$

We define the acceleration operator by

$$
\mathbf{A}(\bar{u}, \bar{j}, \bar{E}, \bar{B})=\left(\begin{array}{c}
-\partial_{t} \bar{u}-P\left(\bar{u} \cdot \nabla_{x} \bar{u}\right)+\mu \Delta_{x} \bar{u}+\frac{1}{2} P(\bar{j} \wedge \bar{B}) \\
-\frac{1}{2 \sigma} \bar{j}+\frac{1}{2} P(\bar{E}+\bar{u} \wedge \bar{B})
\end{array}\right),
$$

and the growth rate by

$$
\lambda(t)=\left(\frac{2}{\mu}+4 \sigma\right)\|\bar{u}(t)\|_{L_{x}^{\infty}}^{2}+\frac{2 C_{0}^{2}}{\mu}\|\bar{j}(t)\|_{L_{x}^{3}}^{2},
$$

where $C_{0}>0$ denotes the operator norm of the Sobolev embedding $\dot{H}^{1}\left(\mathbb{R}^{3}\right) \hookrightarrow$ $L^{6}\left(\mathbb{R}^{3}\right)$.

Then, one has the stability inequality

$$
\begin{aligned}
\delta \mathcal{E}(t)+ & \frac{1}{2} \int_{0}^{t} \delta \mathcal{D}(s) e^{\int_{s}^{t} \lambda(\sigma) d \sigma} d s \\
& \leq \delta \mathcal{E}(0) e^{\int_{0}^{t} \lambda(s) d s}+\int_{0}^{t}\left[\int_{\mathbb{R}^{3}} \mathbf{A} \cdot\left(\begin{array}{c}
u-\bar{u} \\
j-\bar{j}
\end{array}\right) d x\right](s) e^{\int_{s}^{t} \lambda(\sigma) d \sigma} d s
\end{aligned}
$$


where the modulated energy $\delta \mathcal{E}$ and energy dissipation $\delta \mathcal{D}$ are given by

$$
\begin{aligned}
\delta \mathcal{E}(t) & =\frac{1}{2}\|(u-\bar{u})(t)\|_{L_{x}^{2}}^{2}+\frac{1}{4}\|(E-\bar{E})(t)\|_{L_{x}^{2}}^{2}+\frac{1}{4}\|(B-\bar{B})(t)\|_{L_{x}^{2}}^{2}, \\
\delta \mathcal{D}(t) & =\mu\left\|\nabla_{x}(u-\bar{u})(t)\right\|_{L_{x}^{2}}^{2}+\frac{1}{2 \sigma}\|(j-\bar{j})(t)\|_{L_{x}^{2}}^{2} .
\end{aligned}
$$

Proof. We have already formally established in Proposition 3.3 the conservation of the energy for system (3.6). The very same computations applied to the test functions $(\bar{u}, \bar{j}, \bar{E}, \bar{B})$ yield the identity

$$
\frac{d}{d t} \overline{\mathcal{E}}(t)+\overline{\mathcal{D}}(t)=-\int_{\mathbb{R}^{3}} \mathbf{A} \cdot\left(\frac{\bar{u}}{\bar{j}}\right) d x
$$

where the energy $\overline{\mathcal{E}}$ and energy dissipation $\overline{\mathcal{D}}$ are obtained simply by replacing the unknowns by the test functions in the respective definitions of Proposition 3.3 .

Furthermore, another similar duality computation yields that

$$
\begin{aligned}
\frac{d}{d t} & \int_{\mathbb{R}^{3}}\left(u \cdot \bar{u}+\frac{1}{2} E \cdot \bar{E}+\frac{1}{2} B \cdot \bar{B}\right) d x+\int_{\mathbb{R}^{3}} 2 \mu \nabla_{x} u: \nabla_{x} \bar{u}+\frac{1}{\sigma} j \cdot \bar{j} d x \\
& =-\int_{\mathbb{R}^{3}} \bar{u} \otimes(u-\bar{u}): \nabla_{x}(u-\bar{u}) d x \\
& +\frac{1}{2} \int_{\mathbb{R}^{3}}((j-\bar{j}) \wedge(B-\bar{B})) \cdot \bar{u}+((u-\bar{u}) \wedge(B-\bar{B})) \cdot \bar{j} d x \\
& -\int_{\mathbb{R}^{3}} \mathbf{A} \cdot\left(\begin{array}{c}
u \\
j
\end{array}\right) d x .
\end{aligned}
$$

On the whole, combining the above identities with the energy decay imposed by the formal energy conservations from Proposition 3.3, we find the following modulated energy inequality :

$$
\begin{aligned}
\frac{d}{d t} \delta \mathcal{E}(t)+\delta \mathcal{D}(t) \\
\quad \leq \int_{\mathbb{R}^{3}} \bar{u} \otimes(u-\bar{u}): \nabla_{x}(u-\bar{u}) d x \\
\quad-\frac{1}{2} \int_{\mathbb{R}^{3}}((j-\bar{j}) \wedge(B-\bar{B})) \cdot \bar{u}+((u-\bar{u}) \wedge(B-\bar{B})) \cdot \bar{j} d x \\
\quad+\int_{\mathbb{R}^{3}} \mathbf{A} \cdot\left(\begin{array}{c}
u-\bar{u} \\
j-\bar{j}
\end{array}\right) d x .
\end{aligned}
$$

The next step consists in estimating the terms in the right-hand side above that are nonlinear in $(u, j, E, B)$ and to absorb the resulting expressions with the modulated energy $\delta \mathcal{E}(t)$ and the modulated energy dissipation $\delta \mathcal{D}(t)$ by suitable uses of Young's inequality and Grönwall's lemma. Thus, we obtain

$$
\begin{aligned}
\frac{d}{d t} & \delta \mathcal{E}(t)+\delta \mathcal{D}(t) \\
& \leq\|\bar{u}\|_{L_{x}^{\infty}}\|u-\bar{u}\|_{L_{x}^{2}}\left\|\nabla_{x}(u-\bar{u})\right\|_{L_{x}^{2}} \\
& +\frac{1}{2}\|\bar{j}\|_{L_{x}^{3}}\|B-\bar{B}\|_{L_{x}^{2}}\|u-\bar{u}\|_{L_{x}^{6}}+\frac{1}{2}\|\bar{u}\|_{L_{x}^{\infty}}\|B-\bar{B}\|_{L_{x}^{2}}\|j-\bar{j}\|_{L_{x}^{2}} \\
& +\int_{\mathbb{R}^{3}} \mathbf{A} \cdot\left(\begin{array}{c}
u-\bar{u} \\
j-\bar{j}
\end{array}\right) d x \\
& \leq \frac{1}{\mu}\|\bar{u}\|_{L_{x}^{\infty}}^{2}\|u-\bar{u}\|_{L_{x}^{2}}^{2}+\left(\sigma\|\bar{u}\|_{L_{x}^{\infty}}^{2}+\frac{C_{0}^{2}}{2 \mu}\|\bar{j}\|_{L_{x}^{3}}^{2}\right)\|B-\bar{B}\|_{L_{x}^{2}}^{2} \\
& +\frac{\mu}{2}\left\|\nabla_{x}(u-\bar{u})\right\|_{L_{x}^{2}}^{2}+\frac{1}{4 \sigma}\|j-\bar{j}\|_{L_{x}^{2}}^{2}+\int_{\mathbb{R}^{3}} \mathbf{A} \cdot\left(\begin{array}{c}
u-\bar{u} \\
j-\bar{j}
\end{array}\right) d x .
\end{aligned}
$$


Hence,

$$
\frac{d}{d t} \delta \mathcal{E}(t)+\frac{1}{2} \delta \mathcal{D}(t) \leq \lambda(t) \delta \mathcal{E}(t)+\int_{\mathbb{R}^{3}} \mathbf{A} \cdot\left(\begin{array}{c}
u-\bar{u} \\
j-\bar{j}
\end{array}\right) d x,
$$

which concludes the proof of the proposition with a direct application of Grönwall's lemma.

Note that the test functions satisfying the linear constraints 3.25 are easily constructed by considering vector potentials $\bar{A} \in C_{c}^{\infty}\left([0, \infty) \times \mathbb{R}^{3}\right)$ and then setting

$$
\bar{E}=-\partial_{t} \operatorname{rot} \bar{A} \quad \text { and } \quad \bar{B}=\operatorname{rot} \operatorname{rot} \bar{A} .
$$

Now, one may prefer to deal, in a completely equivalent manner, with test functions $(\bar{u}, \bar{E}, \bar{B}) \in C_{c}^{\infty}\left([0, \infty) \times \mathbb{R}^{3}\right)$ and $\bar{j} \in C^{\infty}\left([0, \infty) \times \mathbb{R}^{3}\right)$ (here, we cannot impose that $\bar{j}$ be compactly supported) satisfying the stationary constraints

$$
\operatorname{div} \bar{u}=0, \quad \operatorname{div} \bar{E}=0, \quad \operatorname{div} \bar{B}=0, \quad \bar{j}=\sigma P(\bar{E}+\bar{u} \wedge \bar{B}),
$$

rather than the constraints 3.25 . In this case, instead of 3.26 , we obtain the stability inequality

$$
\begin{aligned}
\delta \mathcal{E}(t)+ & \frac{1}{2} \int_{0}^{t} \delta \mathcal{D}(s) e^{\int_{s}^{t} \lambda(\sigma) d \sigma} d s \\
& \leq \delta \mathcal{E}(0) e^{\int_{0}^{t} \lambda(s) d s}+\int_{0}^{t}\left[\int_{\mathbb{R}^{3}} \mathbf{A} \cdot\left(\begin{array}{c}
u-\bar{u} \\
E-\bar{E} \\
B-\bar{B}
\end{array}\right) d x\right](s) e^{\int_{s}^{t} \lambda(\sigma) d \sigma} d s,
\end{aligned}
$$

where the acceleration operator is now defined by

$$
\mathbf{A}(\bar{u}, \bar{j}, \bar{E}, \bar{B})=\left(\begin{array}{c}
-\partial_{t} \bar{u}-P\left(\bar{u} \cdot \nabla_{x} \bar{u}\right)+\mu \Delta_{x} \bar{u}+\frac{1}{2} P(\bar{j} \wedge \bar{B}) \\
\frac{1}{2}\left(-\partial_{t} \bar{E}+\operatorname{rot} \bar{B}-\bar{j}\right) \\
\frac{1}{2}\left(-\partial_{t} \bar{B}-\operatorname{rot} \bar{E}\right)
\end{array}\right) .
$$

The preceding proposition provides an important weak-strong stability property for the two-fluid incompressible Navier-Stokes-Maxwell system with solenoidal Ohm's law (3.6). Indeed, the stability inequality 3.26 essentially implies that a solution $(\bar{u}, j, E, \bar{B})$ of (3.6) such that $\bar{u} \in L_{t}^{2} L_{x}^{\infty}$ and $\bar{j} \in L_{t}^{2} L_{x}^{3}$, if it exists, is unique in the whole class of weak solutions in the energy space, for any given initial data.

By analogy with Lions' dissipative solutions to the incompressible Euler system [57. Section 4.4], we provide now a suitable notion of dissipative solution for the two-fluid incompressible Navier-Stokes-Maxwell system with solenoidal Ohm's law (3.6), based on Proposition 3.11, and establish their existence next.

Definition. We say that

$$
(u, E, B) \in L^{\infty}\left([0, \infty) ; L^{2}\left(\mathbb{R}^{3}\right)\right) \cap C\left([0, \infty) ; w-L^{2}\left(\mathbb{R}^{3}\right)\right)
$$

such that

$$
\operatorname{div} u=0, \quad \operatorname{div} E=0, \quad \operatorname{div} B=0,
$$

is a dissipative solution of the two-fluid incompressible Navier-StokesMaxwell system with solenoidal Ohm's law (3.6), if it solves Maxwell's equations

with solenoidal Ohm's law

$$
\left\{\begin{array}{l}
\partial_{t} E-\operatorname{rot} B=-j, \\
\partial_{t} B+\operatorname{rot} E=0
\end{array}\right.
$$

$$
j=\sigma\left(-\nabla_{x} \bar{p}+E+u \wedge B\right),
$$

in the sense of distributions, and if, for any test functions $(\bar{u}, \bar{j}, \bar{E}, \bar{B}) \in C_{c}^{\infty}\left([0, \infty) \times \mathbb{R}^{3}\right)$ satisfying the linear constraints $(3.25)$, the stability inequality $(3.26)$ is verified. 
As previously mentioned, dissipative solutions define actual solutions in the sense that they coincide with the unique strong solution when the latter exists. The following theorem asserts their existence.

Theorem 3.12. For any initial data $\left(u^{\text {in }}, E^{\text {in }}, B^{\text {in }}\right) \in L^{2}\left(\mathbb{R}^{3}\right)$ such that

$$
\operatorname{div} u^{\text {in }}=0, \quad \operatorname{div} E^{\text {in }}=0, \quad \operatorname{div} B^{\text {in }}=0,
$$

there exists a dissipative solution to the two-fluid incompressible Navier-StokesMaxwell system with solenoidal Ohm's law (3.6).

Proof. As in the proof of Theorems 3.7 and 3.9, it is possible, here, to justify the existence of dissipative solutions by introducing viscous approximations of the system (3.6). However, it will be much more judicious to recover the system (3.6) as an asymptotic regime of the two fluid incompressible Navier-Stokes-Maxwell system (2.48) for very weak interspecies interactions, which we recast here, for all $\nu>0$, as

$$
\left\{\begin{array}{rlrl}
\partial_{t} u_{\nu}^{+}+u_{\nu}^{+} \cdot \nabla_{x} u_{\nu}^{+}-\mu \Delta_{x} u_{\nu}^{+} & \\
+\frac{1}{\sigma \nu^{2}}\left(u_{\nu}^{+}-u_{\nu}^{-}\right) & =-\nabla_{x} p_{\nu}^{+}+\frac{1}{\nu}\left(E_{\nu}+u_{\nu}^{+} \wedge B_{\nu}\right), & \operatorname{div} u_{\nu}^{+}=0, \\
\partial_{t} u_{\nu}^{-}+u_{\nu}^{-} \cdot \nabla_{x} u_{\nu}^{-}-\mu \Delta_{x} u_{\nu}^{-} & \\
-\frac{1}{\sigma \nu^{2}}\left(u_{\nu}^{+}-u_{\nu}^{-}\right)=-\nabla_{x} p_{\nu}^{-}-\frac{1}{\nu}\left(E_{\nu}+u_{\nu}^{-} \wedge B_{\nu}\right), & \operatorname{div} u_{\nu}^{-}=0, \\
\partial_{t} E_{\nu}-\operatorname{rot} B_{\nu}=-\frac{1}{\nu}\left(u_{\nu}^{+}-u_{\nu}^{-}\right), & \operatorname{div} E_{\nu}=0, \\
\partial_{t} B_{\nu}+\operatorname{rot} E_{\nu}=0, & \operatorname{div} B_{\nu}=0,
\end{array}\right.
$$

associated with an initial data $\left(u_{\nu}^{ \pm \text {in }}, E^{\text {in }}, B^{\text {in }}\right)$ satisfying

$$
u^{\mathrm{in}}=\frac{u_{\nu}^{+\mathrm{in}}+u_{\nu}^{-\mathrm{in}}}{2}
$$

The above two fluid system satisfies the energy inequality, for all $t>0$,

$$
\begin{aligned}
& \frac{1}{2}\left(\left\|u_{\nu}^{+}\right\|_{L_{x}^{2}}^{2}+\left\|u_{\nu}^{-}\right\|_{L_{x}^{2}}^{2}+\left\|E_{\nu}\right\|_{L_{x}^{2}}^{2}+\left\|B_{\nu}\right\|_{L_{x}^{2}}^{2}\right)(t) \\
& \quad+\int_{0}^{t} \mu\left(\left\|\nabla_{x} u_{\nu}^{+}(s)\right\|_{L_{x}^{2}}^{2}+\left\|\nabla_{x} u_{\nu}^{-}(s)\right\|_{L_{x}^{2}}^{2}\right)+\frac{1}{\sigma}\left\|\frac{u_{\nu}^{+}(s)-u_{\nu}^{-}(s)}{\nu}\right\|_{L_{x}^{2}}^{2} d s \\
& \quad \leq \frac{1}{2}\left(\left\|u_{\nu}^{+\mathrm{in}}\right\|_{L_{x}^{2}}^{2}+\left\|u_{\nu}^{-\mathrm{in}}\right\|_{L_{x}^{2}}^{2}+\left\|E^{\mathrm{in}}\right\|_{L_{x}^{2}}^{2}+\left\|B^{\mathrm{in}}\right\|_{L_{x}^{2}}^{2}\right) .
\end{aligned}
$$

Further defining the variables

$$
u_{\nu}=\frac{u_{\nu}^{+}+u_{\nu}^{-}}{2} \quad \text { and } \quad j_{\nu}=\frac{u_{\nu}^{+}-u_{\nu}^{-}}{\nu},
$$

the system 3.29 can be rewritten as

$$
\left\{\begin{array}{rlrl}
\partial_{t} u_{\nu}+u_{\nu} \cdot \nabla_{x} u_{\nu}+\frac{\nu^{2}}{4} j_{\nu} \cdot \nabla_{x} j_{\nu} & -\mu \Delta_{x} u_{\nu} & \\
& =-\nabla_{x} p_{\nu}+\frac{1}{2} j_{\nu} \wedge B_{\nu}, & & \operatorname{div} u_{\nu}=0 \\
\frac{\nu^{2}}{2}\left(\partial_{t} j_{\nu}+u_{\nu} \cdot \nabla_{x} j_{\nu}+j_{\nu} \cdot \nabla_{x} u_{\nu}\right. & \left.-\mu \Delta_{x} j_{\nu}\right) & \\
+\frac{1}{\sigma} j_{\nu} & =-\nabla_{x} \bar{p}_{\nu}+E_{\nu}+u_{\nu} \wedge B_{\nu}, & & \operatorname{div} j_{\nu}=0 \\
\partial_{t} E_{\nu}-\operatorname{rot} B_{\nu} & =-j_{\nu}, & \operatorname{div} E_{\nu}=0 \\
\partial_{t} B_{\nu}+\operatorname{rot} E_{\nu} & =0, & \operatorname{div} B_{\nu}=0,
\end{array}\right.
$$


and the corresponding energy inequality becomes, for all $t>0$,

$$
\begin{aligned}
& \left(\frac{1}{2}\left\|u_{\nu}\right\|_{L_{x}^{2}}^{2}+\frac{\nu^{2}}{8}\left\|j_{\nu}\right\|_{L_{x}^{2}}^{2}+\frac{1}{4}\left\|E_{\nu}\right\|_{L_{x}^{2}}^{2}+\frac{1}{4}\left\|B_{\nu}\right\|_{L_{x}^{2}}^{2}\right)(t) \\
& \quad+\int_{0}^{t} \mu\left(\left\|\nabla_{x} u_{\nu}(s)\right\|_{L_{x}^{2}}^{2}+\frac{\nu^{2}}{4}\left\|\nabla_{x} j_{\nu}(s)\right\|_{L_{x}^{2}}^{2}\right)+\frac{1}{2 \sigma}\left\|j_{\nu}(s)\right\|_{L_{x}^{2}}^{2} d s \\
& \quad \leq \frac{1}{2}\left\|u^{\mathrm{in}}\right\|_{L_{x}^{2}}^{2}+\frac{\nu^{2}}{8}\left\|j_{\nu}^{\mathrm{in}}\right\|_{L_{x}^{2}}^{2}+\frac{1}{4}\left\|E^{\mathrm{in}}\right\|_{L_{x}^{2}}^{2}+\frac{1}{4}\left\|B^{\mathrm{in}}\right\|_{L_{x}^{2}}^{2},
\end{aligned}
$$

where $j_{\nu}^{\text {in }}=\frac{u_{\nu}^{+ \text {in }}-u_{\nu}^{- \text {in }}}{\nu}$.

Weak solutions of the above systems $(3.29$ and $\sqrt{3.30}$ are easily established following the method of Leray [48, for the nonlinear terms $u_{\nu}^{ \pm} \wedge B_{\nu}$ (or, equivalently, $u_{\nu} \wedge B_{\nu}$ and $j_{\nu} \wedge B_{\nu}$ ) are stable with respect to weak convergence in the energy space defined by the above energy inequalities.

Now, for any test functions $(\bar{u}, \bar{j}, \bar{E}, \bar{B}) \in C_{c}^{\infty}\left([0, \infty) \times \mathbb{R}^{3}\right)$ satisfying the linear constraints 3.25 , we define the approximate acceleration operator by

$$
\mathbf{A}_{\nu}(\bar{u}, \bar{j}, \bar{E}, \bar{B})=\mathbf{A}(\bar{u}, \bar{j}, \bar{E}, \bar{B})-\frac{\nu^{2}}{4}\left(\begin{array}{c}
P\left(\bar{j} \cdot \nabla_{x} \bar{j}\right) \\
\partial_{t} \bar{j}+P\left(\bar{u} \cdot \nabla_{x} \bar{j}+\bar{j} \cdot \nabla_{x} \bar{u}\right)-\mu \Delta_{x} \bar{j}
\end{array}\right) .
$$

Then, a straightforward energy estimate yields that

$$
\begin{aligned}
& \frac{d}{d t}\left(\frac{1}{2}\|\bar{u}\|_{L_{x}^{2}}^{2}+\frac{\nu^{2}}{8}\|\bar{j}\|_{L_{x}^{2}}^{2}+\frac{1}{4}\|\bar{E}\|_{L_{x}^{2}}^{2}+\frac{1}{4}\|\bar{B}\|_{L_{x}^{2}}^{2}\right) \\
& \quad+\mu\left(\left\|\nabla_{x} \bar{u}\right\|_{L_{x}^{2}}^{2}+\frac{\nu^{2}}{4}\left\|\nabla_{x} \bar{j}\right\|_{L_{x}^{2}}^{2}\right)+\frac{1}{2 \sigma}\|\bar{j}\|_{L_{x}^{2}}^{2}=-\int_{\mathbb{R}^{3}} \mathbf{A}_{\nu} \cdot\left(\frac{\bar{u}}{\bar{j}}\right) d x
\end{aligned}
$$

Moreover, another similar duality computation gives that

$$
\begin{aligned}
\frac{d}{d t} \int_{\mathbb{R}^{3}} & \left(u_{\nu} \cdot \bar{u}+\frac{\nu^{2}}{4} j_{\nu} \cdot \bar{j}+\frac{1}{2} E_{\nu} \cdot \bar{E}+\frac{1}{2} B_{\nu} \cdot \bar{B}\right) d x \\
& +\int_{\mathbb{R}^{3}} 2 \mu \nabla_{x} u_{\nu}: \nabla_{x} \bar{u}+\frac{\mu \nu^{2}}{2} \nabla_{x} j_{\nu}: \nabla_{x} \bar{j}+\frac{1}{\sigma} j_{\nu} \cdot \bar{j} d x \\
& =-\int_{\mathbb{R}^{3}} \bar{u} \otimes\left(u_{\nu}-\bar{u}\right): \nabla_{x}\left(u_{\nu}-\bar{u}\right)+\frac{\nu^{2}}{4} \bar{u} \otimes\left(j_{\nu}-\bar{j}\right): \nabla_{x}\left(j_{\nu}-\bar{j}\right) d x \\
& -\frac{\nu^{2}}{4} \int_{\mathbb{R}^{3}} \bar{j} \otimes\left(j_{\nu}-\bar{j}\right): \nabla_{x}\left(u_{\nu}-\bar{u}\right)+\bar{j} \otimes\left(u_{\nu}-\bar{u}\right): \nabla_{x}\left(j_{\nu}-\bar{j}\right) d x \\
& +\frac{1}{2} \int_{\mathbb{R}^{3}}\left(\left(j_{\nu}-\bar{j}\right) \wedge\left(B_{\nu}-\bar{B}\right)\right) \cdot \bar{u}+\left(\left(u_{\nu}-\bar{u}\right) \wedge\left(B_{\nu}-\bar{B}\right)\right) \cdot \bar{j} d x \\
& -\int_{\mathbb{R}^{3}} \mathbf{A}_{\nu} \cdot\left(\begin{array}{c}
u_{\nu} \\
j_{\nu}
\end{array}\right) d x .
\end{aligned}
$$

Hence, defining the modulated energy $\delta \mathcal{E}_{\nu}(t)$ and modulated energy dissipation $\delta \mathcal{D}_{\nu}(t)$ by

$$
\begin{aligned}
\delta \mathcal{E}_{\nu}(t) & =\frac{1}{2}\left\|\left(u_{\nu}-\bar{u}\right)(t)\right\|_{L_{x}^{2}}^{2}+\frac{\nu^{2}}{8}\left\|\left(j_{\nu}-\bar{j}\right)(t)\right\|_{L_{x}^{2}}^{2} \\
& +\frac{1}{4}\left\|\left(E_{\nu}-\bar{E}\right)(t)\right\|_{L_{x}^{2}}^{2}+\frac{1}{4}\left\|\left(B_{\nu}-\bar{B}\right)(t)\right\|_{L_{x}^{2}}^{2}, \\
\delta \mathcal{D}_{\nu}(t) & =\mu\left\|\nabla_{x}\left(u_{\nu}-\bar{u}\right)(t)\right\|_{L_{x}^{2}}^{2}+\frac{\mu \nu^{2}}{4}\left\|\nabla_{x}\left(j_{\nu}-\bar{j}\right)(t)\right\|_{L_{x}^{2}}^{2}+\frac{1}{2 \sigma}\left\|\left(j_{\nu}-\bar{j}\right)(t)\right\|_{L_{x}^{2}}^{2},
\end{aligned}
$$


we find that

$$
\begin{aligned}
& \delta \mathcal{E}_{\nu}(t)+\int_{0}^{t} \delta \mathcal{D}_{\nu}(s) d s \\
& \leq \delta \mathcal{E}_{\nu}(0)+\int_{0}^{t} \int_{\mathbb{R}^{3}} \bar{u} \otimes\left(u_{\nu}-\bar{u}\right): \nabla_{x}\left(u_{\nu}-\bar{u}\right)+\frac{\nu^{2}}{4} \bar{u} \otimes\left(j_{\nu}-\bar{j}\right): \nabla_{x}\left(j_{\nu}-\bar{j}\right) d x d s \\
& +\frac{\nu^{2}}{4} \int_{0}^{t} \int_{\mathbb{R}^{3}} \bar{j} \otimes\left(j_{\nu}-\bar{j}\right): \nabla_{x}\left(u_{\nu}-\bar{u}\right)+\bar{j} \otimes\left(u_{\nu}-\bar{u}\right): \nabla_{x}\left(j_{\nu}-\bar{j}\right) d x d s \\
& -\frac{1}{2} \int_{0}^{t} \int_{\mathbb{R}^{3}}\left(\left(j_{\nu}-\bar{j}\right) \wedge\left(B_{\nu}-\bar{B}\right)\right) \cdot \bar{u}+\left(\left(u_{\nu}-\bar{u}\right) \wedge\left(B_{\nu}-\bar{B}\right)\right) \cdot \bar{j} d x d s \\
& +\int_{0}^{t} \int_{\mathbb{R}^{3}} \mathbf{A}_{\nu} \cdot\left(\begin{array}{c}
u_{\nu}-\bar{u} \\
j_{\nu}-\bar{j}
\end{array}\right) d x d s .
\end{aligned}
$$

The next step consists in estimating the terms in the right-hand side above that are nonlinear in $\left(u_{\nu}, j_{\nu}, E_{\nu}, B_{\nu}\right)$ and to absorb the resulting expressions with the modulated energy $\delta \mathcal{E}_{\nu}(t)$ and the modulated energy dissipation $\delta \mathcal{D}_{\nu}(t)$ by suitable uses of Young's inequality and Grönwall's lemma. Thus, we obtain

$$
\begin{aligned}
& \delta \mathcal{E}_{\nu}(t)+\int_{0}^{t} \delta \mathcal{D}_{\nu}(s) d s \\
& \leq \delta \mathcal{E}_{\nu}(0)+\int_{0}^{t}\|\bar{u}\|_{L_{x}^{\infty}}\left\|u_{\nu}-\bar{u}\right\|_{L_{x}^{2}}\left\|\nabla_{x}\left(u_{\nu}-\bar{u}\right)\right\|_{L_{x}^{2}} d s \\
& +\frac{\nu^{2}}{4} \int_{0}^{t}\|\bar{u}\|_{L_{x}^{\infty}}\left\|j_{\nu}-\bar{j}\right\|_{L_{x}^{2}}\left\|\nabla_{x}\left(j_{\nu}-\bar{j}\right)\right\|_{L_{x}^{2}} d s \\
& +\frac{\nu^{2}}{4} \int_{0}^{t}\|\bar{j}\|_{L_{x}^{\infty}}\left\|j_{\nu}-\bar{j}\right\|_{L_{x}^{2}}\left\|\nabla_{x}\left(u_{\nu}-\bar{u}\right)\right\|_{L_{x}^{2}}+\|\bar{j}\|_{L_{x}^{3}}\left\|u_{\nu}-\bar{u}\right\|_{L_{x}^{6}}\left\|\nabla_{x}\left(j_{\nu}-\bar{j}\right)\right\|_{L_{x}^{2}} d s \\
& +\frac{1}{2} \int_{0}^{t}\|\bar{j}\|_{L_{x}^{3}}\left\|B_{\nu}-\bar{B}\right\|_{L_{x}^{2}}\left\|u_{\nu}-\bar{u}\right\|_{L_{x}^{6}}+\|\bar{u}\|_{L_{x}^{\infty}}\left\|B_{\nu}-\bar{B}\right\|_{L_{x}^{2}}\left\|j_{\nu}-\bar{j}\right\|_{L_{x}^{2}} d s \\
& +\int_{0}^{t} \int_{\mathbb{R}^{3}} \mathbf{A}_{\nu} \cdot\left(\begin{array}{c}
u_{\nu}-\bar{u} \\
j_{\nu}-\bar{j}
\end{array}\right) d x d s \\
& \leq \delta \mathcal{E}_{\nu}(0)+\int_{0}^{t} \frac{1}{\mu}\|\bar{u}\|_{L_{x}^{\infty}}^{2}\left\|u_{\nu}-\bar{u}\right\|_{L_{x}^{2}}^{2}+\left(\sigma\|\bar{u}\|_{L_{x}^{\infty}}^{2}+\frac{C_{0}^{2}}{2 \mu}\|\bar{j}\|_{L_{x}^{3}}^{2}\right)\left\|B_{\nu}-\bar{B}\right\|_{L_{x}^{2}}^{2} d s \\
& +\int_{0}^{t}\left(\frac{\mu}{2}+\frac{\mu \nu^{2}}{16}+\frac{\nu^{2} C_{0}^{2}}{4 \mu}\|\bar{j}\|_{L_{x}^{3}}^{2}\right)\left\|\nabla_{x}\left(u_{\nu}-\bar{u}\right)\right\|_{L_{x}^{2}}^{2}+\frac{\mu \nu^{2}}{8}\left\|\nabla_{x}\left(j_{\nu}-\bar{j}\right)\right\|_{L_{x}^{2}}^{2} d s \\
& +\int_{0}^{t}\left(\frac{1}{4 \sigma}+\frac{\nu^{2}}{4 \mu}\|\bar{u}\|_{L_{x}^{\infty}}^{2}+\frac{\nu^{2}}{4 \mu}\|\bar{j}\|_{L_{x}^{\infty}}^{2}\right)\left\|j_{\nu}-\bar{j}\right\|_{L_{x}^{2}}^{2}+\left[\int_{\mathbb{R}^{3}} \mathbf{A}_{\nu} \cdot\left(\begin{array}{c}
u_{\nu}-\bar{u} \\
j_{\nu}-\bar{j}
\end{array}\right) d x\right] d s \\
& \leq \delta \mathcal{E}_{\nu}(0)+\int_{0}^{t} \lambda(s) \delta \mathcal{E}_{\nu}(s)+\left[\int_{\mathbb{R}^{3}} \mathbf{A}_{\nu} \cdot\left(\begin{array}{c}
u_{\nu}-\bar{u} \\
j_{\nu}-\bar{j}
\end{array}\right) d x\right] d s \\
& +\int_{0}^{t}\left(\frac{1}{2}+\nu^{2} \beta(s)\right) \delta \mathcal{D}_{\nu}(s) d s
\end{aligned}
$$

where

Hence,

$$
\beta(t)=\frac{1}{16}+\frac{C_{0}^{2}}{4 \mu^{2}}\|\bar{j}\|_{L_{x}^{3}}^{2}+\frac{\sigma}{2 \mu}\left(\|\bar{u}\|_{L_{x}^{\infty}}^{2}+\|\bar{j}\|_{L_{x}^{\infty}}^{2}\right) .
$$

$$
\begin{aligned}
\delta \mathcal{E}_{\nu}(t) & +\int_{0}^{t} \frac{1}{2} \delta \mathcal{D}_{\nu}(s) d s \\
& \leq \delta \mathcal{E}_{\nu}(0)+\int_{0}^{t} \lambda(s) \delta \mathcal{E}_{\nu}(s)+\left[\int_{\mathbb{R}^{3}} \mathbf{A}_{\nu} \cdot\left(\begin{array}{c}
u_{\nu}-\bar{u} \\
j_{\nu}-\bar{j}
\end{array}\right) d x\right]+\nu^{2} \beta(s) \delta \mathcal{D}_{\nu}(s) d s
\end{aligned}
$$


and an application of Grönwall's lemma yields

$$
\begin{aligned}
\delta \mathcal{E}_{\nu}(t) & +\frac{1}{2} \int_{0}^{t} \delta \mathcal{D}_{\nu}(s) e^{\int_{s}^{t} \lambda(\sigma) d \sigma} d s \leq \delta \mathcal{E}_{\nu}(0) e^{\int_{0}^{t} \lambda(s) d s} \\
& +\int_{0}^{t}\left[\left[\int_{\mathbb{R}^{3}} \mathbf{A}_{\nu} \cdot\left(\begin{array}{c}
u_{\nu}-\bar{u} \\
j_{\nu}-\bar{j}
\end{array}\right) d x\right](s)+\nu^{2} \beta(s) \delta \mathcal{D}_{\nu}(s)\right] e^{\int_{s}^{t} \lambda(\sigma) d \sigma} d s .
\end{aligned}
$$

We may now pass to the limit in the above stability inequality. Thus, up to extraction of subsequences, we may assume that, as $\nu \rightarrow 0$,

$$
\begin{array}{ll}
u_{\nu} \stackrel{*}{\rightarrow} u & \text { in } L_{t}^{\infty} L_{x}^{2} \cap L_{t}^{2} \dot{H}_{x}^{1}, \\
j_{\nu} \rightarrow j & \text { in } L_{t}^{2} L_{x}^{2}, \\
E_{\nu} \stackrel{*}{\rightarrow} E & \text { in } L_{t}^{\infty} L_{x}^{2}, \\
B_{\nu} \stackrel{*}{\rightarrow} B & \text { in } L_{t}^{\infty} L_{x}^{2} .
\end{array}
$$

Furthermore, noticing that $\partial_{t} u_{\nu}, \partial_{t} E_{\nu}$ and $\partial_{t} B_{\nu}$ are uniformly bounded, in $L_{\text {loc }}^{1}$ in time and in some negative index Sobolev space in $x$, it is possible to show (see 57. Appendix $\mathrm{C}])$ that $\left(u_{\nu}, E_{\nu}, B_{\nu}\right)$ converges to $(u, E, B) \in C\left([0, \infty) ; w-L^{2}\left(\mathbb{R}^{3}\right)\right)$ weakly in $L_{x}^{2}$ uniformly locally in time. Then, by the weak lower semi-continuity of the norms, we obtain that, for every $t>0$,

$$
\delta \mathcal{E}(t)+\frac{1}{2} \int_{0}^{t} \delta \mathcal{D}(s) e^{\int_{s}^{t} \lambda(\sigma) d \sigma} d s \leq \liminf _{\nu \rightarrow 0} \delta \mathcal{E}_{\nu}(t)+\frac{1}{2} \int_{0}^{t} \delta \mathcal{D}_{\nu}(s) e^{\int_{s}^{t} \lambda(\sigma) d \sigma} d s
$$

Hence, further assuming that $\delta \mathcal{E}_{\nu}(0) \rightarrow \delta \mathcal{E}(0)$, as $\nu \rightarrow 0$, the stability inequality (3.26) holds. Notice that the convergence of the initial data is satisfied whenever $\left\|u_{\nu}^{+ \text {in }}-u_{\nu}^{-\mathrm{in}}\right\|_{L_{x}^{2}} \rightarrow 0$, as $\nu \rightarrow 0$.

Finally, invoking a classical compactness result by Aubin and Lions [8, 50] (see also 71 for a sharp compactness criterion), we infer that the $u_{\nu}$ 's converge towards $u$ strongly in $L_{\text {loc }}^{2}(d t d x)$. Therefore, passing to the limit in the evolution equation for $j_{\nu}$ in (3.30), it is readily seen that Ohm's law is satisfied asymptotically, which concludes the proof of the theorem.

As before, we present now an alternative kind of stability inequality for the two-fluid incompressible Navier-Stokes-Maxwell system with solenoidal Ohm's law (3.6). It is a mere adaptation of Proposition 3.10 to the present case, which relies on the interpretation of the Lorentz force with the Poynting vector. We recall that this method allows us to stabilize the modulated nonlinear terms solely with the modulated energy $\delta \mathcal{E}$.

Proposition 3.13. Let $(u, E, B)$ be a smooth solution to the two-fluid incompressible Navier-Stokes-Maxwell system with solenoidal Ohm's law (3.6). Further consider test functions $(\bar{u}, \bar{j}, \bar{E}, \bar{B}) \in C_{c}^{\infty}\left([0, \infty) \times \mathbb{R}^{3}\right)$ such that $\|\bar{u}\|_{L_{t, x}^{\infty}}<1$ and

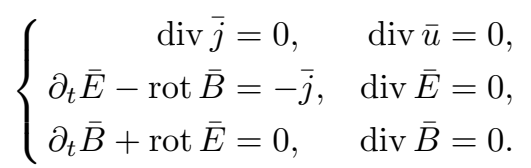

We define the acceleration operator by

$$
\mathbf{A}(\bar{u}, \bar{j}, \bar{E}, \bar{B})=\left(\begin{array}{c}
-\partial_{t} \bar{u}-P\left(\bar{u} \cdot \nabla_{x} \bar{u}\right)+\mu \Delta_{x} \bar{u}+\frac{1}{2} P(\bar{j} \wedge \bar{B}) \\
-\frac{1}{2 \sigma} \bar{j}+\frac{1}{2} P(\bar{E}+\bar{u} \wedge \bar{B})
\end{array}\right),
$$

and the growth rate by

$$
\lambda(t)=\frac{2\left\|\nabla_{t, x} \bar{u}(t)\right\|_{L_{x}^{\infty}}}{1-\|\bar{u}(t)\|_{L_{x}^{\infty}}}+\frac{\sqrt{2}\|\bar{j}(t)\|_{L_{x}^{\infty}}}{2\left(1-\|\bar{u}(t)\|_{L_{x}^{\infty}}\right)} .
$$


Then, one has the stability inequality

$$
\begin{aligned}
\delta \mathcal{E}(t)+ & \int_{0}^{t} \delta \mathcal{D}(s) e^{\int_{s}^{t} \lambda(\sigma) d \sigma} d s \\
& \leq \delta \mathcal{E}(0) e^{\int_{0}^{t} \lambda(s) d s}+\int_{0}^{t}\left[\int_{\mathbb{R}^{3}} \mathbf{A} \cdot\left(\begin{array}{c}
u-\bar{u} \\
j-\bar{j}
\end{array}\right) d x\right](s) e^{\int_{s}^{t} \lambda(\sigma) d \sigma} d s
\end{aligned}
$$

where the modulated energy $\delta \mathcal{E}$ and energy dissipation $\delta \mathcal{D}$ are given by

$$
\begin{aligned}
\delta \mathcal{E}(t) & =\frac{1}{2}\|(u-\bar{u})(t)\|_{L_{x}^{2}}^{2}+\frac{1}{4}\|(E-\bar{E})(t)\|_{L_{x}^{2}}^{2}+\frac{1}{4}\|(B-\bar{B})(t)\|_{L_{x}^{2}}^{2} \\
& -\frac{1}{2} \int_{\mathbb{R}^{3}}((E-\bar{E})(t) \wedge(B-\bar{B})(t)) \cdot \bar{u}(t) d x, \\
\delta \mathcal{D}(t) & =\mu\left\|\nabla_{x}(u-\bar{u})(t)\right\|_{L_{x}^{2}}^{2}+\frac{1}{2 \sigma}\|(j-\bar{j})(t)\|_{L_{x}^{2}}^{2} .
\end{aligned}
$$

Proof. Following the proof of Proposition 3.11, we consider first the identity

$$
\begin{aligned}
\frac{d}{d t} & \int_{\mathbb{R}^{3}}\left(u \cdot \bar{u}+\frac{1}{2} E \cdot \bar{E}+\frac{1}{2} B \cdot \bar{B}\right) d x+\int_{\mathbb{R}^{3}} 2 \mu \nabla_{x} u: \nabla_{x} \bar{u}+\frac{1}{\sigma} j \cdot \bar{j} d x \\
& =\int_{\mathbb{R}^{3}}(u-\bar{u}) \otimes(u-\bar{u}): \nabla_{x} \bar{u} d x \\
& +\frac{1}{2} \int_{\mathbb{R}^{3}}((j-\bar{j}) \wedge(B-\bar{B})) \cdot \bar{u}+((u-\bar{u}) \wedge(B-\bar{B})) \cdot \bar{j} d x \\
& -\int_{\mathbb{R}^{3}} \mathbf{A} \cdot\left(\begin{array}{c}
u \\
j
\end{array}\right) d x .
\end{aligned}
$$

Note that this relation can be recovered by formally discarding all terms involving the charge density $n$ in 3.23 .

Then, expressing the modulated Lorentz force with a modulated Poynting vector as

$$
\begin{aligned}
\partial_{t}((E-\bar{E}) \wedge(B-\bar{B})) & +\frac{1}{2} \nabla_{x}\left(|E-\bar{E}|^{2}+|B-\bar{B}|^{2}\right) \\
& -\nabla_{x} \cdot((E-\bar{E}) \otimes(E-\bar{E})+(B-\bar{B}) \otimes(B-\bar{B})) \\
& =-(j-\bar{j}) \wedge(B-\bar{B})
\end{aligned}
$$

we arrive at the relation

$$
\begin{aligned}
\frac{d}{d t} \int_{\mathbb{R}^{3}} & \left(u \cdot \bar{u}+\frac{1}{2} E \cdot \bar{E}+\frac{1}{2} B \cdot \bar{B}+\frac{1}{2}((E-\bar{E}) \wedge(B-\bar{B})) \cdot \bar{u}\right) d x \\
& -\int_{\mathbb{R}^{3}} \frac{1}{2}((E-\bar{E}) \wedge(B-\bar{B})) \cdot \partial_{t} \bar{u} d x+\int_{\mathbb{R}^{3}} 2 \mu \nabla_{x} u: \nabla_{x} \bar{u}+\frac{1}{\sigma} j \cdot \bar{j} d x \\
& =\int_{\mathbb{R}^{3}}(u-\bar{u}) \otimes(u-\bar{u}): \nabla_{x} \bar{u} d x \\
& -\frac{1}{2} \int_{\mathbb{R}^{3}}((E-\bar{E}) \otimes(E-\bar{E})+(B-\bar{B}) \otimes(B-\bar{B})): \nabla_{x} \bar{u} d x \\
& +\frac{1}{2} \int_{\mathbb{R}^{3}} \bar{j} \cdot((u-\bar{u}) \wedge(B-\bar{B})) d x-\int_{\mathbb{R}^{3}} \mathbf{A} \cdot\left(\begin{array}{c}
u \\
j
\end{array}\right) d x .
\end{aligned}
$$

On the whole, combining the preceding identity with the energy conservation law for test functions (3.27) and the energy decay imposed by the formal energy 
conservations from Proposition 3.3 , we find the following modulated energy inequality :

$$
\begin{aligned}
& \frac{d}{d t} \delta \mathcal{E}(t)+\delta \mathcal{D}(t) \\
& \quad \leq-\int_{\mathbb{R}^{3}}(u-\bar{u}) \otimes(u-\bar{u}): \nabla_{x} \bar{u} d x-\int_{\mathbb{R}^{3}} \frac{1}{2}((E-\bar{E}) \wedge(B-\bar{B})) \cdot \partial_{t} \bar{u} d x \\
& \quad+\frac{1}{2} \int_{\mathbb{R}^{3}}((E-\bar{E}) \otimes(E-\bar{E})+(B-\bar{B}) \otimes(B-\bar{B})): \nabla_{x} \bar{u} d x \\
& \quad-\frac{1}{2} \int_{\mathbb{R}^{3}} \bar{j} \cdot((u-\bar{u}) \wedge(B-\bar{B})) d x+\int_{\mathbb{R}^{3}} \mathbf{A} \cdot\left(\begin{array}{c}
u-\bar{u} \\
j-\bar{j}
\end{array}\right) d x .
\end{aligned}
$$

The next step consists in estimating the terms in the right-hand side above that are nonlinear in $(u, j, E, B)$ and to absorb the resulting expressions with the modulated energy $\delta \mathcal{E}(t)$ by suitable uses of Young's inequality and Grönwall's lemma. Thus, we obtain

$$
\begin{aligned}
& \frac{d}{d t} \delta \mathcal{E}(t)+\delta \mathcal{D}(t) \\
& \leq\left\|\nabla_{t, x} \bar{u}\right\|_{L_{x}^{\infty}}\left(\|u-\bar{u}\|_{L_{x}^{2}}^{2}+\frac{1}{2}\|E-\bar{E}\|_{L_{x}^{2}}^{2}+\frac{1}{2}\|B-\bar{B}\|_{L_{x}^{2}}^{2}\right) \\
& +\frac{1}{2}\|\bar{j}\|_{L_{x}^{\infty}}\|B-\bar{B}\|_{L_{x}^{2}}\|u-\bar{u}\|_{L_{x}^{2}}+\int_{\mathbb{R}^{3}} \mathbf{A} \cdot\left(\begin{array}{c}
u-\bar{u} \\
j-\bar{j}
\end{array}\right) d x \\
& \leq\left\|\nabla_{t, x} \bar{u}\right\|_{L_{x}^{\infty}}\left(\|u-\bar{u}\|_{L_{x}^{2}}^{2}+\frac{1}{2}\|E-\bar{E}\|_{L_{x}^{2}}^{2}+\frac{1}{2}\|B-\bar{B}\|_{L_{x}^{2}}^{2}\right) \\
& +\frac{\sqrt{2}}{4}\|\bar{j}\|_{L_{x}^{\infty}}\left(\|u-\bar{u}\|_{L_{x}^{2}}^{2}+\frac{1}{2}\|B-\bar{B}\|_{L_{x}^{2}}^{2}\right)+\int_{\mathbb{R}^{3}} \mathbf{A} \cdot\left(\begin{array}{c}
u-\bar{u} \\
j-\bar{j}
\end{array}\right) d x .
\end{aligned}
$$

Hence, further noticing that

$$
\begin{aligned}
\left(1-\|\bar{u}\|_{L_{x}^{\infty}}\right) & \left(\frac{1}{2}\|u-\bar{u}\|_{L_{x}^{2}}^{2}+\frac{1}{4}\|E-\bar{E}\|_{L_{x}^{2}}^{2}+\frac{1}{4}\|B-\bar{B}\|_{L_{x}^{2}}^{2}\right) \\
& \leq \frac{1}{2}\|u-\bar{u}\|_{L_{x}^{2}}^{2}+\frac{1}{4}\|E-\bar{E}\|_{L_{x}^{2}}^{2}+\frac{1}{4}\|B-\bar{B}\|_{L_{x}^{2}}^{2} \\
& -\frac{1}{2}\|\bar{u}\|_{L_{x}^{\infty}} \int_{\mathbb{R}^{3}}|E-\bar{E}||B-\bar{B}| d x \\
& \leq \frac{1}{2}\|u-\bar{u}\|_{L_{x}^{2}}^{2}+\frac{1}{4}\|E-\bar{E}\|_{L_{x}^{2}}^{2}+\frac{1}{4}\|B-\bar{B}\|_{L_{x}^{2}}^{2} \\
& -\frac{1}{2} \int_{\mathbb{R}^{3}}((E-\bar{E}) \wedge(B-\bar{B})) \cdot \bar{u} d x \\
& =\delta \mathcal{E}(t),
\end{aligned}
$$

we find, since $\|\bar{u}\|_{L_{t, x}^{\infty}}<1$, that

$$
\frac{d}{d t} \delta \mathcal{E}(t)+\delta \mathcal{D}(t) \leq \lambda(t) \delta \mathcal{E}(t)+\int_{\mathbb{R}^{3}} \mathbf{A} \cdot\left(\begin{array}{c}
u-\bar{u} \\
j-\bar{j}
\end{array}\right) d x
$$

which concludes the proof of the proposition with a direct application of Grönwall's lemma.

The preceding proposition provides another weak-strong stability property for the two-fluid incompressible Navier-Stokes-Maxwell system with solenoidal Ohm's law (3.6). Indeed, the stability inequality 3.31 essentially implies that a solution $(\bar{u}, \bar{j}, E, \bar{B})$ of 3.6 such that $\bar{u} \in L_{t, x}^{\infty}, \nabla_{t, x} \bar{u} \in L_{t}^{1} L_{x}^{\infty}, \bar{j} \in L_{t}^{1} L_{x}^{\infty}$ and $\|\bar{u}\|_{L_{t, x}^{\infty}}<1$, if it exists, is unique in the whole class of weak solutions in the energy space, for any given initial data. 
As in Proposition 3.10 , the condition $\|\bar{u}\|_{L_{t, x}^{\infty}}<1$ in Proposition 3.13 is physically relevant, for it imposes that the modulus of the bulk velocity $\bar{u}$ remains everywhere and at all times below the speed of light. More precisely, keeping track of the relevant physical constants in the formal derivations of Chapter 2, we see that the system $\sqrt{3.6}$ can be recast as

$$
\left\{\begin{array}{rlrl}
\partial_{t} u+u \cdot \nabla_{x} u-\mu \Delta_{x} u & =-\nabla_{x} p+\frac{1}{2} j \wedge B, & \operatorname{div} u=0, \\
\frac{1}{c} \partial_{t} E-\operatorname{rot} B & =-j, & \operatorname{div} E & =0, \\
\frac{1}{c} \partial_{t} B+\operatorname{rot} E & =0, & \operatorname{div} B & =0, \\
j & =\sigma\left(-\nabla_{x} \bar{p}+c E+u \wedge B\right), & \operatorname{div} j=0,
\end{array}\right.
$$

where the constant $c>0$ denotes the speed of light. Then, applying the proof of Proposition 3.13 to the preceding system, we arrive at a stability inequality valid under the restriction that the bulk velocity remains bounded by the speed of light $\|\bar{u}\|_{L_{t, x}^{\infty}}<c$, which is natural.

Following the previous developments, it is also possible to use now the stability inequality 3.31 from Proposition 3.13 to define another notion of dissipative solutions for the two-fluid incompressible Navier-Stokes-Maxwell system with solenoidal Ohm's law (3.6), whose existence is then established by reproducing the arguments from Theorem 3.12. The only argument from the proof of Theorem 3.12 that needs special care in order to conclude the existence of dissipative solutions resides in the weak lower semi-continuity of the modulated energy $\delta \mathcal{E}(t)$ defined by $(3.32)$, which we have already established in (3.24). 

Part 2

Conditional convergence results 



\section{CHAPTER 4}

\section{Two typical regimes}

We will now focus on two specific regimes which are critical, in the sense that all the formal asymptotics mentioned in Chapter 2 can be rigorously obtained by similar or even simpler arguments.

The first scaling we will investigate here is the one leading from the one species Vlasov-Maxwell-Boltzmann equations 2.5 to the incompressible quasistatic Navier-Stokes-Fourier-Maxwell-Poisson system 2.29). More precisely, we will set $\alpha=\epsilon, \beta=\epsilon$ and $\gamma=\epsilon$ in (2.5). As discussed in Section 3.1, the resulting limiting model is then very similar to the incompressible Navier-Stokes equations and, thus, the usual methods of hydrodynamic limits will apply. We shall focus specifically on the influence of the electromagnetic field, which induces numerous technical complications.

The second regime we will study is more singular since the magnetic forcing is much stronger. Specifically, we will consider the scaling leading from the two species Vlasov-Maxwell-Boltzmann equations 2.32 to the two-fluid incompressible NavierStokes-Fourier-Maxwell system with Ohm's law (2.74) in the case of strong interspecies collisions, or to the two-fluid incompressible Navier-Stokes-Fourier-Maxwell system with solenoidal Ohm's law 2.75 in the case of weak interspecies collisions. More precisely, we will set $\alpha=\delta \epsilon, \beta=\delta$ and $\gamma=1$ in 2.32 , with $\frac{\delta}{\epsilon}$ unbounded. Actually, as discussed in Section 3.2 the corresponding limiting models (2.74) and 2.75 are not stable under weak convergence in the energy space and, thus, share more similarities with the three-dimensional incompressible Euler equations. So will our proofs of hydrodynamic convergence in this setting.

All along this second part on rigorous hydrodynamic convergence proofs, we will consider renormalized solutions, whose definition we recall below in Section 4.1, of the Vlasov-Maxwell-Boltzmann systems for any number species. In fact, their existence is not established, which is precisely the reason why the convergence results presented here are deemed conditional, and remains a challenging open problem of outstanding difficulty.

Loosely speaking, the specific complexity of the Vlasov-Maxwell-Boltzmann system originates in the nonlinear coupling of the Vlasov-Boltzmann equation with a hyperbolic system, namely Maxwell's equations. This essential difficulty remains ubiquitous in our analysis of its hydrodynamic limits and is passed on to the most singular asymptotic models present in our work, such as the systems (2.74) and (2.75), whose well-posedness is not fully understood (see Section 3.2) and contains very challenging open questions, as well. 


\subsection{Renormalized solutions}

We are now going to recall the notion of renormalized solutions for the VlasovMaxwell-Boltzmann systems 2.5

$$
\left\{\begin{aligned}
\partial_{t} f+v \cdot \nabla_{x} f+(E+v \wedge B) \cdot \nabla_{v} f & =Q(f, f) \\
\partial_{t} E-\operatorname{rot} B & =-\int_{\mathbb{R}^{3}} f v d v \\
\partial_{t} B+\operatorname{rot} E & =0 \\
\operatorname{div} E & =\int_{\mathbb{R}^{3}} f d v-1, \\
\operatorname{div} B & =0
\end{aligned}\right.
$$

and 2.32

$$
\left\{\begin{aligned}
\partial_{t} f^{ \pm}+v \cdot \nabla_{x} f^{ \pm} \pm(E+v \wedge B) \cdot \nabla_{v} f^{ \pm} & =Q\left(f^{ \pm}, f^{ \pm}\right)+Q\left(f^{ \pm}, f^{\mp}\right), \\
\partial_{t} E-\operatorname{rot} B & =-\int_{\mathbb{R}^{3}}\left(f^{+}-f^{-}\right) v d v \\
\partial_{t} B+\operatorname{rot} E & =0 \\
\operatorname{div} E & =\int_{\mathbb{R}^{3}}\left(f^{+}-f^{-}\right) d v \\
\operatorname{div} B & =0
\end{aligned}\right.
$$

where we have discarded the free parameters.

4.1.1. The Vlasov-Boltzmann equation. Let us focus first on the simpler Vlasov-Boltzmann equation :

$$
\partial_{t} f+v \cdot \nabla_{x} f+F \cdot \nabla_{v} f=Q(f, f),
$$

with a given force field $F(t, x, v)$ satisfying, at least,

$$
F, \nabla_{v} \cdot F \in L_{\text {loc }}^{1}\left(d t d x ; L^{1}\left(M^{\alpha} d v\right)\right) \quad \text { for all } \alpha>0 .
$$

The above conditions on the force field are minimal requirements so that it is possible to define renormalized solutions of 4.3 (see definition below). We will, however, further restrict the range of applicability of force fields :

- we assume that $\nabla_{v} \cdot F=0$, so that the local conservation of mass is verified ;

- we assume that $F \cdot v=0$, so that the global Maxwellian $M(v)$ is an equilibrium state of 4.3 .

Renormalized solutions of 4.3 are known to exist since the late eighties, thanks to DiPerna and Lions 30 (at least for the Boltzmann equation, i.e. for the case $F=0$ ). We are going to briefly describe their derivation, their limitations and emphasize the main mathematical difficulties preventing their construction for the above Vlasov-Maxwell-Boltzmann systems.

Throughout this work, we are interested in the fluctuations of a density $f(t, x, v)$ around a global normalized Maxwellian $M(v)$, we will therefore conveniently employ the density $G(t, x, v)$ defined by $f=M G$. In this notation, the Vlasov-Boltzmann equation 4.3 reads

$$
\partial_{t} G+v \cdot \nabla_{x} G+F \cdot \nabla_{v} G=\mathcal{Q}(G, G),
$$

where we denote

$$
\mathcal{Q}(G, H)=\frac{1}{M} Q(M G, M H) .
$$

Thus, DiPerna and Lions formulated in $\mathbf{3 0}$ the first theory yielding global solutions to the Boltzmann equation (4.4), with $F=0$, for large initial data 
$G(0, x, v)=G^{\text {in }}(x, v) \geq 0$. Their construction heavily relied on a new notion of solutions, namely the renormalized solutions.

Recall that we utilize the prefixes $w$ - or $w^{*}$ - to express that a given space is endowed with its weak or weak-* topology, respectively.

Definition. We say that a nonlinearity $\beta \in C^{1}([0, \infty) ; \mathbb{R})$ is an admissible renormalization if it satisfies, for some $C>0$,

$$
\left|\beta^{\prime}(z)\right| \leq \frac{C}{(1+z)^{\frac{1}{2}}} \quad \text { for all } z \geq 0 .
$$

A density function $f(t, x, v)=M G(t, x, v) \geq 0$, where $(t, x, v) \in[0, \infty) \times \mathbb{R}^{3} \times$ $\mathbb{R}^{3}$, such that

$$
G \in C\left([0, \infty) ; w-L_{\text {loc }}^{1}(d x d v)\right) \cap L^{\infty}\left([0, \infty), d t ; L_{\text {loc }}^{1}\left(d x ; L^{1}\left(\left(1+|v|^{2}\right) M d v\right)\right)\right),
$$

is a renormalized solution of the Vlasov-Boltzmann equation (4.4) if it solves

$$
\partial_{t} \beta(G)+v \cdot \nabla_{x} \beta(G)+F \cdot \nabla_{v} \beta(G)=\beta^{\prime}(G) \mathcal{Q}(G, G)
$$

in the sense of distributions for any admissible renormalization, and satisfies the entropy inequality, for all $t>0$,

$$
H(f(t))+\int_{0}^{t} \int_{\mathbb{R}^{3}} D(f(s)) d x d s \leq H\left(f^{\text {in }}\right)<\infty,
$$

where $f^{\text {in }}=M G^{\text {in }}$ is the initial value of $f=M G$ and the relative entropy $H(f)=$ $H(f \mid M)$ is defined in (1.17), while the entropy dissipation $D(f)$ is defined in (1.13).

Note that the renormalized collision operator $\beta^{\prime}(G) \mathcal{Q}(G, G)$ is well-defined in $L_{\text {loc }}^{1}\left(d t d x ; L^{1}\left(M^{\alpha} d v\right)\right)$, with $\alpha>0$, for any admissible renormalization, any function in 4.5 and any integrable cross-section $b(z, \sigma) \in L_{\text {loc }}^{1}\left(\mathbb{R}^{3} \times \mathbb{S}^{2}\right)$ satisfying the so-called DiPerna-Lions assumption

$$
\lim _{|v| \rightarrow \infty} \frac{1}{|v|^{2}} \int_{K \times \mathbb{S}^{2}} b\left(v-v_{*}, \sigma\right) d v_{*} d \sigma=0,
$$

for any compact subset $K \subset \mathbb{R}^{3}$.

Indeed, it is possible to show directly from 4.7 that (see 4, for instance, for more details), for any $\alpha>0$,

$$
\lim _{|v| \rightarrow \infty} \frac{1}{|v|^{2}} \int_{\mathbb{R}^{3} \times \mathbb{S}^{2}} b\left(v-v_{*}, \sigma\right) M_{*}^{\alpha} d v_{*} d \sigma=0 .
$$

Therefore, considering first non-negative renormalizations satisfying $0 \leq \beta^{\prime}(z) \leq$ $\frac{C}{1+z}$, the renormalized loss part $\beta^{\prime}(G) \mathcal{Q}^{-}(G, G)$ is easily estimated as

$$
\begin{aligned}
& \int_{\mathbb{R}^{3}} \beta^{\prime}(G) \mathcal{Q}^{-}(G, G) M^{\alpha} d v \\
& \quad=\int_{\mathbb{R}^{3}} G_{*}\left(1+\left|v_{*}\right|^{2}\right) M_{*}\left[\frac{1}{1+\left|v_{*}\right|^{2}} \int_{\mathbb{R}^{3} \times \mathbb{S}^{2}} \beta^{\prime}(G) G b\left(v-v_{*}, \sigma\right) M^{\alpha} d v d \sigma\right] d v_{*} \\
& \quad \leq C\|G\|_{L^{1}\left(\left(1+|v|^{2}\right) M d v\right)}
\end{aligned}
$$

while the renormalized gain term $\beta^{\prime}(G) \mathcal{Q}^{+}(G, G)$ is well-defined in $L_{\text {loc }}^{1}\left(d t d x ; L^{1}\left(M^{\alpha} d v\right)\right)$ by the renormalized Vlasov-Boltzmann equation (4.6) because it is the only unestimated expression remaining and it is non-negative. These controls are easily extended to signed renormalizations satisfying $\left|\beta^{\prime}(z)\right| \leq \frac{C}{1+z}$, for the VlasovBoltzmann equation 4.6 is linear with respect to renormalizations so that we may decompose $\beta^{\prime}(z)$ with respect to its positive and negative parts. 
Alternatively and as was originally performed in [30, we could also use the elementary inequality (B.9), setting $z=\frac{G^{\prime} G_{*}^{\prime}}{G G_{*}}-1$ and $y=\log K$, with $K>1$, which implies that

$$
\begin{aligned}
\int_{\mathbb{R}^{3}} \beta^{\prime}(G) \mathcal{Q}^{+}(G, G) M d v \leq K \int_{\mathbb{R}^{3}} \beta^{\prime}(G) \mathcal{Q}^{-}(G, G) M d v \\
\quad+\frac{1}{\log K} \int_{\mathbb{R}^{3} \times \mathbb{R}^{3} \times \mathbb{S}^{2}} \beta^{\prime}(G)\left(G^{\prime} G_{*}^{\prime}-G G_{*}\right) \log \left(\frac{G^{\prime} G_{*}^{\prime}}{G G_{*}}\right) M M_{*} b d v d v_{*} d \sigma,
\end{aligned}
$$

to claim that the gain part belongs to $L_{\text {loc }}^{1}\left(d t d x ; L^{1}(M d v)\right)$, since it is natural to control the entropy dissipation term above.

Finally, it is possible to extend the definition of the renormalized collision operator $\beta^{\prime}(G) \mathcal{Q}(G, G)$ to all admissible renormalizations by decomposing the renormalized collision integrand as

$$
\begin{aligned}
\beta^{\prime}(G)\left(G^{\prime} G_{*}^{\prime}-G G_{*}\right) & =\beta^{\prime}(G)\left(\sqrt{G^{\prime} G_{*}^{\prime}}-\sqrt{G G_{*}}\right)^{2} \\
& +2 \beta^{\prime}(G) \sqrt{G G_{*}}\left(\sqrt{G^{\prime} G_{*}^{\prime}}-\sqrt{G G_{*}}\right),
\end{aligned}
$$

and noticing that

$$
\begin{aligned}
\int_{\mathbb{R}^{3} \times \mathbb{R}^{3} \times \mathbb{S}^{2}} & \left(\sqrt{f^{\prime} f_{*}^{\prime}}-\sqrt{f f_{*}}\right)^{2} b\left(v-v_{*}, \sigma\right) d v d v_{*} d \sigma \\
\leq & \leq \frac{1}{4} \int_{\mathbb{R}^{3} \times \mathbb{R}^{3} \times \mathbb{S}^{2}}\left(f^{\prime} f_{*}^{\prime}-f f_{*}\right) \log \left(\frac{f^{\prime} f_{*}^{\prime}}{f f_{*}}\right) b\left(v-v_{*}, \sigma\right) d v d v_{*} d \sigma=D(f),
\end{aligned}
$$

which follows from the elementary inequality (B.8).

Thus, by a solution $G$ of the renormalized equation (4.6), we naturally mean that $G$ should satisfy, for every $\alpha>0$ and any non-negative test functions $\rho(t, x) \in$ $C_{c}^{\infty}\left([0, \infty) \times \mathbb{R}^{3}\right)$ and $\varphi(v) \in W^{1, \infty}(d v)$, that

$$
\begin{aligned}
-\int_{\mathbb{R}^{3} \times \mathbb{R}^{3}} \beta & \left(G^{\mathrm{in}}\right) \rho(0, x) \varphi(v) M^{\alpha} d x d v \\
& -\int_{[0, \infty) \times \mathbb{R}^{3} \times \mathbb{R}^{3}} \beta(G)\left(\partial_{t}+v \cdot \nabla_{x}+F \cdot \nabla_{v}\right)\left[\rho(t, x) \varphi(v) M^{\alpha}\right] d t d x d v \\
& =\int_{[0, \infty) \times \mathbb{R}^{3} \times \mathbb{R}^{3}} \beta^{\prime}(G) \mathcal{Q}(G, G) \rho(t, x) \varphi(v) M^{\alpha} d t d x d v .
\end{aligned}
$$

The following theorem is a modern formulation of the existence result found in [30. The existence of renormalized solutions for Vlasov-Boltzmann systems where the force field derives from a self-induced potential, such as the Vlasov-PoissonBoltzmann system, has been established in [53], while the study of renormalized solutions close to Maxwellian equilibrium has been performed in [54].

THEOREM 4.1 ([30, [32]). Let $b(z, \sigma)$ be a locally integrable collision kernel satisfying the DiPerna-Lions assumption 4.7) and $F(t, x, v) \in L_{\mathrm{loc}}^{1}(d t d x d v)$ a given force field such that

$$
\nabla_{v} \cdot F=0, \quad F \cdot v=0 \quad \text { and } \quad F \in L_{\mathrm{loc}}^{1}\left(d t ; W_{\mathrm{loc}}^{1,1}(d x d v)\right) .
$$

Then, for any initial condition $f^{\text {in }}=M G^{\text {in }} \in L_{\text {loc }}^{1}\left(d x ; L^{1}\left(\left(1+|v|^{2}\right) d v\right)\right)$ such that $f^{\text {in }}=M G^{\text {in }} \geq 0$ and

$$
H\left(f^{\text {in }}\right)=H\left(f^{\text {in }} \mid M\right)=\int_{\mathbb{R}^{3} \times \mathbb{R}^{3}}\left(G^{\text {in }} \log G^{\text {in }}-G^{\text {in }}+1\right) M d x d v<\infty,
$$


there exists a renormalized solution $f(t, x, v)=M G(t, x, v)$ to the Vlasov-Boltzmann equation (4.4. Moreover, it satisfies the local conservation of mass

$$
\partial_{t} \int_{\mathbb{R}^{3}} f d v+\nabla_{x} \cdot \int_{\mathbb{R}^{3}} f v d v=0
$$

and the global entropy inequality, for any $t \geq 0$,

$$
H(f(t))+\int_{0}^{t} \int_{\mathbb{R}^{3}} D(f(s)) d x d s \leq H\left(f^{\mathrm{in}}\right) .
$$

The proof of the above theorem follows the usual steps found in the analysis of weak solutions of partial differential equations, that is to say, solving an approximate truncated equation, establishing uniform a priori estimates and the weak compactness of the approximate solutions, and finally passing to the limit (by showing the weak stability of nonlinear terms) and, thus, recovering the original equation. It is often the case that these steps reduce to the study of the crucial weak stability of solutions. Thus, for the Vlasov-Boltzmann equation (4.4), the above theorem naturally follows from the weak stability of renormalized solutions, or, in other words, from the weak stability of weak solutions of the renormalized equation 4.6 satisfying the uniform bounds provided by the entropy inequality (4.9).

DiPerna and Lions showed the weak stability of the Boltzmann equation, i.e. when $F=0$, in 30 and refined their result in 32 by establishing the entropy inequality (4.9). Note that, since we are assuming $\nabla_{v} \cdot F=0$ and $F \cdot v=0$, the entropy inequality (4.9) easily follows from formal estimates on the VlasovBoltzmann equation (4.4), even when $F \neq 0$. Later, Lions improved the method of proof in $[\mathbf{5 1}, \mathbf{5 2}, \mathbf{5 3}$. We briefly explain now Lions' strategy, which relies on velocity averaging lemmas, heavy renormalization techniques and, most importantly, on the compactifying (even regularizing, in some cases) effect of the gain term $Q^{+}(f, f)$ of the collision operator.

To this end, let us consider a sequence $\left\{f_{k}\right\}_{k \in \mathbb{N}}$ of actual renormalized solutions to 4.3 , with initial data $\left\{f_{k}^{\text {in }}\right\}_{k \in \mathbb{N}}$, which converges weakly (at least in $L_{\text {loc }}^{1}$, say) as $k \rightarrow \infty$ to $f^{\text {in }}$. We further assume that the initial data satisfies the following strong entropic convergence

$$
\lim _{k \rightarrow \infty} H\left(f_{k}^{\text {in }}\right)=H\left(f^{\text {in }}\right),
$$

so that the entropy inequality is uniformly satisfied

$$
H\left(f_{k}(t)\right)+\int_{0}^{t} \int_{\mathbb{R}^{3}} D\left(f_{k}(s)\right) d x d s \leq H\left(f_{k}^{\text {in }}\right) .
$$

Notice that a uniform bound on the entropies $H\left(f_{k}(t)\right)$ yields, with a direct application of the elementary Young inequality (B.3), a uniform bound on $f_{k}(t, x, v)$ in $L_{\text {loc }}^{\infty}\left(d t ; L_{\text {loc }}^{1}\left(d x ; L^{1}\left(\left(1+v^{2}\right) d v\right)\right)\right)$. Moreover, it is possible to show, with a slightly more refined application of the Young inequality (B.3) with the DunfordPettis compactness criterion (see [66] and Section 5.1 for details), that the $f_{k}$ 's are in fact weakly relatively compact in $L_{\text {loc }}^{1}\left(d t d x ; L^{1}(d v)\right)$. Therefore, up to extraction, we may assume that the sequence $\left\{f_{k}\right\}_{k \in \mathbb{N}}$ converges weakly, as $k \rightarrow \infty$, to some $f$ in $L_{\mathrm{loc}}^{1}\left(d t d x ; L^{1}(d v)\right)$.

Similarly, uniform bounds on the nonlinear terms

$$
\frac{Q^{ \pm}\left(f_{k}, f_{k}\right)}{1+\delta f_{k} * v\left[\int_{\mathbb{S}^{2}} b(\cdot, \sigma) d \sigma\right]} \quad \text { and } \quad \beta^{\prime}\left(G_{k}\right) Q^{ \pm}\left(f_{k}, f_{k}\right) \text {, }
$$


where $f_{k}=M G_{k}$, for any $\delta>0$ and any admissible nonlinearity $\beta(z) \in C^{1}([0, \infty) ; \mathbb{R})$, are easily obtained from 4.10 through the standard use of the elementary inequality (B.9), setting $z=\frac{f_{k}^{\prime} f_{k *}^{\prime}}{f_{k} f_{k *}}-1$ and $y=\log K$, with $K>1$, which yields

$$
f_{k}^{\prime} f_{k *}^{\prime} \leq K f_{k} f_{k *}+\frac{1}{\log K}\left(f_{k}^{\prime} f_{k *}^{\prime}-f_{k} f_{k *}\right) \log \left(\frac{f_{k}^{\prime} f_{k *}^{\prime}}{f_{k} f_{k *}}\right) .
$$

The above functional inequality further implies the weak compactness of the above nonlinear terms, thanks to the Dunford-Pettis compactness criterion (see 66 ).

At this point, using the convexity methods from [32, one can already establish the limiting entropy inequality (4.9), passing to the limit in 4.10).

Next, since each $f_{k}=M G_{k}$ is a weak solution of the renormalized equation (4.6), it is also possible to show, with a standard use of velocity averaging lemmas (one can use the results from [33, for instance, treating $F \cdot \nabla_{v} \beta\left(G_{k}\right)=\nabla_{v} \cdot\left(F \beta\left(G_{k}\right)\right.$ ) as a source term), that, for any admissible nonlinearity $\beta(z) \in C^{1}([0, \infty) ; \mathbb{R})$ and any cutoff $\varphi(v) \in C_{c}^{\infty}\left(\mathbb{R}^{3}\right)$,

$$
\int_{\mathbb{R}^{3}} \beta\left(G_{k}\right)(t, x, v) \varphi(v) d v \text { is relatively compact in } L_{\mathrm{loc}}^{1}(d t d x),
$$

and, up to further extraction of subsequences,

$$
f_{k} *_{v}\left[\int_{\mathbb{S}^{2}} b(\cdot, \sigma) d \sigma\right] \rightarrow f *_{v}\left[\int_{\mathbb{S}^{2}} b(\cdot, \sigma) d \sigma\right]
$$

as $k \rightarrow \infty$ in $L_{\text {loc }}^{1}(d t d x d v)$ and almost everywhere,

where $b(z, \sigma)$ may in fact be replaced by any collision kernel satisfying the DiPernaLions assumption 4.7). In particular, it follows that

$$
\begin{gathered}
\frac{Q^{ \pm}\left(f_{k}, f_{k}\right)}{1+\delta f_{k} *_{v}\left[\int_{\mathbb{S}^{2}} b(\cdot, \sigma) d \sigma\right]} \varphi(t, x) \rightarrow \frac{Q^{ \pm}(f, f)}{1+\delta f * v\left[\int_{\mathbb{S}^{2}} b(\cdot, \sigma) d \sigma\right]} \varphi(t, x) \\
\text { as } k \rightarrow \infty \text { in } w-L^{1}(d t d x d v),
\end{gathered}
$$

for any $\varphi(t, x) \in C_{c}^{\infty}\left([0, \infty) \times \mathbb{R}^{3}\right)$.

Lions showed in [51, using Fourier integral operators, that the weak convergence of $f_{k}$ towards $f$ in $L_{\text {loc }}^{1}(d t d x d v)$, the strong relative compactness of the velocity averages (4.11)- 4.12 and the uniform bounds from the entropy inequality (4.10 are sufficient to imply that, up to extraction of a subsequence, for every $\delta>0$,

$$
\begin{gathered}
\frac{Q^{+}\left(f_{k}, f_{k}\right)}{1+\delta f_{k} *_{v}\left[\int_{\mathbb{S}^{2}} b(\cdot, \sigma) d \sigma\right]} \varphi(t, x) \rightarrow \frac{Q^{+}(f, f)}{1+\delta f *_{v}\left[\int_{\mathbb{S}^{2}} b(\cdot, \sigma) d \sigma\right]} \varphi(t, x) \\
\text { as } k \rightarrow \infty \text { in } L^{1}(d t d x d v) \text { and almost everywhere, }
\end{gathered}
$$

for any $\varphi(t, x) \in C_{c}^{\infty}\left([0, \infty) \times \mathbb{R}^{3}\right)$. Therefore, it holds in particular that

$$
Q^{+}\left(f_{k}, f_{k}\right) \rightarrow Q^{+}(f, f) \text { almost everywhere. }
$$

Following [53, we fix now the specific renormalization $\beta_{\delta}(z)=\frac{z}{1+\delta z}$, for any $0<\delta<1$, and we assume, without loss of generality, up to extraction of subsequences, that, as $k \rightarrow \infty$,

$$
\begin{gathered}
\beta_{\delta}\left(G_{k}\right)=\frac{G_{k}}{1+\delta G_{k}} \rightarrow \beta_{\delta} \leq \beta_{\delta}(G), \\
\beta_{\delta}^{\prime}\left(G_{k}\right)=\frac{1}{\left(1+\delta G_{k}\right)^{2}} \rightarrow h_{\delta} \geq \beta_{\delta}^{\prime}(G), \\
\beta_{\delta}^{\prime}\left(G_{k}\right) G_{k}=\frac{G_{k}}{\left(1+\delta G_{k}\right)^{2}}=\beta_{\delta}\left(G_{k}\right)\left(1-\delta \beta_{\delta}\left(G_{k}\right)\right) \rightarrow g_{\delta} \leq \beta_{\delta}\left(1-\delta \beta_{\delta}\right),
\end{gathered}
$$


in $w^{*}-L_{\mathrm{loc}}^{\infty}(d t d x d v)$. Therefore, passing to the limit in 4.6 , we obtain, in view of the strong convergences 4.12 and 4.13 ,

$$
\partial_{t} \beta_{\delta}+v \cdot \nabla_{x} \beta_{\delta}+F \cdot \nabla_{v} \beta_{\delta}=h_{\delta} \mathcal{Q}^{+}(G, G)-g_{\delta} f *_{v}\left[\int_{\mathbb{S}^{2}} b(\cdot, \sigma) d \sigma\right],
$$

where the last term $h_{\delta} \mathcal{Q}^{+}(G, G)$ is well-defined in $L_{\text {loc }}^{1}(d t d x d v)$ by its mere nonnegativeness.

Note that, for any $\lambda>0$, choosing $K>0$ large enough so that, by equiintegrability of the $G_{k}$ 's,

$$
\sup _{k \in \mathbb{N}}\left\|G_{k} \mathbb{1}_{\left\{G_{k} \geq K\right\}}\right\|_{L_{\text {loc }}^{1}(d t d x d v)} \leq \lambda,
$$

we find

$$
\begin{aligned}
\left\|G-\beta_{\delta}\right\|_{L_{\mathrm{loc}}^{1}(d t d x d v)} & \leq \liminf _{k \rightarrow \infty}\left\|G_{k}-\beta_{\delta}\left(G_{k}\right)\right\|_{L_{\mathrm{loc}}^{1}(d t d x d v)} \\
& \leq \frac{\delta K}{1+\delta K} \sup _{k \in \mathbb{N}}\left\|G_{k}\right\|_{L_{\mathrm{loc}}^{1}(d t d x d v)}+\lambda
\end{aligned}
$$

and

$$
\begin{aligned}
\left\|G-g_{\delta}\right\|_{L_{\mathrm{loc}}^{1}(d t d x d v)} & \leq \liminf _{k \rightarrow \infty}\left\|G_{k}-\beta_{\delta}^{\prime}\left(G_{k}\right) G_{k}\right\|_{L_{\mathrm{loc}}^{1}(d t d x d v)} \\
& \leq \frac{\delta^{2} K^{2}+2 \delta K}{(1+\delta K)^{2}} \sup _{k \in \mathbb{N}}\left\|G_{k}\right\|_{L_{\mathrm{loc}}^{1}(d t d x d v)}+\lambda .
\end{aligned}
$$

Hence, by the arbitrariness of $\lambda>0$,

$$
\begin{aligned}
& \lim _{\delta \rightarrow 0}\left\|G-\beta_{\delta}\right\|_{L_{\mathrm{loc}}^{1}(d t d x d v)}=0, \\
& \lim _{\delta \rightarrow 0}\left\|G-g_{\delta}\right\|_{L_{\mathrm{loc}}^{1}(d t d x d v)}=0 .
\end{aligned}
$$

Similarly, it is readily seen that, for any $1 \leq p<\infty$,

$$
\lim _{\delta \rightarrow 0}\left\|1-h_{\delta}\right\|_{L_{\mathrm{loc}}^{p}(d t d x d v)}=0 .
$$

Finally, notice that $\beta_{\delta}, g_{\delta}$ and $h_{\delta}$ are all increasing as $\delta$ vanishes. Hence, as $\delta \rightarrow 0$, both $\beta_{\delta}$ and $g_{\delta}$ converge towards $G$ almost everywhere, while $h_{\delta}$ converges toward a constant almost everywhere.

Now comes a fundamental idea of Lions from [52, 53, which will be of particular interest to us and which has numerous qualitative consequences on renormalized solutions. This key idea consists in renormalizing equation (4.14) over again according to the following simple yet crucial lemma from [31].

Lemma 4.2 ([31, Theorem II.1, p. 516]). Let $f(t, x) \in L^{\infty}\left([0, T] ; L_{\mathrm{loc}}^{p}\left(\mathbb{R}^{n}\right)\right)$, with $1<p \leq \infty, T>0$ and $n \in \mathbb{N}$, be a solution of the linear transport equation

$$
\partial_{t} f+b \cdot \nabla_{x} f+c f=h,
$$

where

$$
\begin{aligned}
& b \in L^{\gamma}\left([0, T] ; W_{\mathrm{loc}}^{1, \alpha}\left(\mathbb{R}^{n}\right)\right), \\
& c \in L^{\gamma}\left([0, T] ; L_{\mathrm{loc}}^{\alpha}\left(\mathbb{R}^{n}\right)\right), \\
& h \in L^{\gamma}\left([0, T] ; L_{\mathrm{loc}}^{\beta}\left(\mathbb{R}^{n}\right)\right),
\end{aligned}
$$

for some $p^{\prime} \leq \alpha<\infty, \frac{1}{p}+\frac{1}{p^{\prime}}=1,1 \leq \gamma<\infty$ and $1 \leq \beta<\infty$ such that $\frac{1}{\beta}=\frac{1}{\alpha}+\frac{1}{p}$.

Then, for any $\chi_{\delta}(x)=\frac{1}{\delta^{n}} \chi\left(\frac{x}{\delta}\right)$, with $\chi \in C_{c}^{\infty}\left(\mathbb{R}^{n}\right), \chi \geq 0, \int_{\mathbb{R}^{n}} \chi(x) d x=1$ and $\delta>0$, the mollification $f_{\delta}=f * \chi_{\delta}$ satisfies

$$
\partial_{t} f_{\delta}+b \cdot \nabla_{x} f_{\delta}+c f_{\delta}=h+r_{\delta},
$$

where the remainder $r_{\delta}$ vanishes in $L^{\gamma}\left([0, T] ; L_{\mathrm{loc}}^{\beta}\left(\mathbb{R}^{n}\right)\right)$, as $\delta \rightarrow 0$. 
In particular, it follows that, for any renormalization $\beta \in C^{1}(\mathbb{R})$ such that $\beta^{\prime}$ is bounded on $\mathbb{R}$,

$$
\partial_{t} \beta(f)+b \cdot \nabla_{x} \beta(f)+c f \beta^{\prime}(f)=h \beta^{\prime}(f) .
$$

The above lemma has fundamental consequences in transport theory and in the theory of ordinary differential equations. Indeed, as established by DiPerna and Lions in 31, it can be shown that, loosely speaking, as soon as Lemma 4.2 applies, weak solutions of 4.15 are, in fact, renormalized solutions, unique and time continuous in the strong topology, and that the transport equation 4.15 propagates strong compactness. In turn, the properties of the transport equation have important consequences on ordinary differential equations, and the existence and uniqueness of a Lagrangian flow was also established in 31 under very weak assumptions on the corresponding Eulerian flow, which should typically be in $L^{1}\left([0, T] ; W_{\mathrm{loc}}^{1,1}\left(\mathbb{R}^{n}\right)\right)$.

Thus, in view of the regularity hypothesis 4.8) on the force field

$$
F \in L_{\mathrm{loc}}^{1}\left(d t ; W_{\mathrm{loc}}^{1,1}(d x d v)\right),
$$

applying Lemma 4.2 to the transport equation 4.14) (transport by the vector field $\left.(v, F(t, x, v)) \in \mathbb{R}^{6}\right)$ yields that $\beta_{\delta}$ is a renormalized solutions of 4.14 , that is to say, for any admissible renormalization $\beta$,

$$
\begin{aligned}
\partial_{t} \beta\left(\beta_{\delta}\right)+v \cdot \nabla_{x} \beta\left(\beta_{\delta}\right) & +F \cdot \nabla_{v} \beta\left(\beta_{\delta}\right) \\
& =\beta^{\prime}\left(\beta_{\delta}\right) h_{\delta} \mathcal{Q}^{+}(G, G)-\beta^{\prime}\left(\beta_{\delta}\right) g_{\delta} f *_{v}\left[\int_{\mathbb{S}^{2}} b(\cdot, \sigma) d \sigma\right] .
\end{aligned}
$$

Finally, we let $\delta \rightarrow 0$ in the above renormalized equation. To this end, notice that $\beta^{\prime}\left(\beta_{\delta}\right) g_{\delta}$ is bounded uniformly by a constant pointwise and converges almost everywhere to $\beta^{\prime}(G) G$. Therefore, the last term above converges towards the expected renormalized loss term while it remains uniformly locally integrable. Moreover, we see that, integrating (4.16) locally in all variables, the gain term $\beta^{\prime}\left(\beta_{\delta}\right) h_{\delta} \mathcal{Q}^{+}(G, G)$ remains uniformly locally integrable, so that it converges towards the expected renormalized gain term. On the whole, since the left-hand side of 4.16 is easily handled by the strong convergence of $\beta_{\delta}$ towards $G$, we conclude, letting $\delta \rightarrow 0$ in (4.16), that $G$ solves 4.6 in the sense of distributions, which completes the justification of Theorem 4.1 according to $\mathbf{3 0}, \mathbf{3 2}, \mathbf{5 1}, \mathbf{5 2}, \mathbf{5 3}$.

It is to be emphasized that Theorem 4.1 can be easily generalized to a system of Vlasov-Boltzmann equations for two species of particles.

4.1.2. Coupling the Boltzmann equation with Maxwell's equations. Thus, we see that the validity of Theorem 4.1 rests crucially upon Lemma 4.2 and, so, that the regularity hypothesis on the force field $F \in L_{\mathrm{loc}}^{1}\left(d t ; W_{\mathrm{loc}}^{1,1}(d x d v)\right)$ cannot be weakened, at least not with this method of proof. This is precisely the unique obstacle which prevents the construction of renormalized solutions for the Vlasov-Maxwell-Boltzmann systems (4.1) and (4.2), whose force fields are not regular.

As far as the existence theory of global solutions is concerned, notice that the nonlinear coupling of a kinetic equation with Maxwell's equations through the influence of a Lorentz force is not always a problem. In particular, it is possible to show the weak stability of the Vlasov-Maxwell system (without collisions) for densities in $L_{t}^{\infty} L_{x, v}^{2}$ and, therefore, to establish the existence of (non-renormalized) 
weak solutions for this system (see [29]). Indeed, neglecting the collision operators in (4.1) and 4.2), the only remaining nonlinear terms are

$$
(E+v \wedge B) \cdot \nabla_{v} f \quad \text { and } \quad(E+v \wedge B) \cdot \nabla_{v} f^{ \pm} .
$$

Since the densities $f$ in 4.1 and $f^{ \pm}$in 4.2 do enjoy some strong compactness (even some kind of regularity) in time and space by virtue of velocity averaging lemmas (see [33, for instance), while the Lorentz force $E+v \wedge B$ is smooth in velocity (obviously, $E$ and $B$ do not depend on $v$ ), it is clear that the above nonlinear electromagnetic forcing terms are weakly stable as long as no renormalization is required. In conclusion, problematic difficulties arise when entering the realm of collisional kinetic theory, where renormalizing becomes a necessity. Nevertheless, it is to be noted that the existence of renormalized solutions for such collisionless Vlasov-Maxwell systems remains unknown, as well.

In contrast with the Vlasov-Maxwell-Boltzmann systems, the Vlasov-PoissonBoltzmann systems (2.31) and 2.76) do enjoy the existence of renormalized solutions (see [53]). Indeed, thanks to Poisson's equation, the force fields therein have enough regularity to apply Lemma 4.2 and the strategy of proof of Theorem 4.1 applies.

Of course, since then, there have been generalizations of Lemma 4.2 and incidentally of the results from [31, most notably by Ambrosio [2], where the local Sobolev regularity of the vector field was relaxed to a local $B V$ regularity, and by Le Bris and Lions 47, where a specific structure of the vector field, which unfortunately doesn't match the structure of (4.14), was used in order to impose a mere partial $W_{\text {loc }}^{1,1}$ regularity on it. In any case, it is apparent, much like in the Cauchy-Lipschitz theorem on ordinary differential equations, that a minimum of a control on one full derivative of the vector field is necessary to crank the proof of Theorem 4.1, which is far from reach in the case of Vlasov-Maxwell-Boltzmann systems where $E, B \in L^{\infty}\left(d t ; L^{2}(d x)\right)$, at best. This viewpoint is also corroborated by the counterexamples presented at the end of $\mathbf{3 1}$.

Thus, it seems that any result confirming the existence of renormalized solutions for Vlasov-Maxwell-Boltzmann systems will have to exploit the very specific structure of the electromagnetic interaction within the plasma.

Surprisingly the situation is much better when the microscopic interactions described by the collision operator have infinite range so that the collisional crosssection has a singularity at grazing collisions : the entropy dissipation indeed controls some derivative with respect to $v$ in this case. Using the hypoellipticity of the kinetic transport operator, we can then transfer part of this regularity onto the $x$ variable. Following the strategy by Alexandre and Villani [1, and renormalizing the Vlasov-Boltzmann equation by concave functions, we thus get some global renormalized solutions involving a defect measure (which is formally 0 because of the conservation of mass). This construction has been sketched in [7. It will be detailed and used to obtain fully rigorous convergence results in Part 3 .

An alternative approach based on Young measures, as introduced by Lions in 53 . will be the focus of our work in Part 4. We will see that, even though the notion of solution is very poor, the asymptotic analysis is robust and leads to similar convergence results.

Note that Parts 3 and 4 will be more technical as we will have to deal with very weak solutions. However the strategy of proof as well as the main arguments will be similar to the ones presented here, this is why we start with conditional results.

4.1.3. The setting of our conditional study. We provide now a precise definition of renormalized solutions for the Vlasov-Maxwell-Boltzmann systems (4.1) and 4.2), even though their existence remains uncertain. 
Definition. We say that a density function $f(t, x, v)=M G(t, x, v) \geq 0$ and electromagnetic vector fields $E(t, x)$ and $B(t, x)$, where $(t, x, v) \in[0, \infty) \times \mathbb{R}^{3} \times \mathbb{R}^{3}$, such that

$$
\begin{aligned}
G & \in C\left([0, \infty) ; w-L_{\mathrm{loc}}^{1}(d x d v)\right) \cap L^{\infty}\left([0, \infty), d t ; L_{\mathrm{loc}}^{1}\left(d x ; L^{1}\left(\left(1+|v|^{2}\right) M d v\right)\right)\right), \\
E, B & \in C\left([0, \infty) ; w-L^{2}(d x)\right) \cap L^{\infty}\left([0, \infty), d t ; L^{2}(d x)\right),
\end{aligned}
$$

are a renormalized solution of the one species Vlasov-Maxwell-Boltzmann system 4.1 if they solve

$$
\left\{\begin{aligned}
\partial_{t} \beta(G)+v \cdot \nabla_{x} \beta(G)+(E+v \wedge B) \cdot \nabla_{v} \beta(G) & -E \cdot v \beta^{\prime}(G) G \\
& =\beta^{\prime}(G) \mathcal{Q}(G, G), \\
\partial_{t} E-\operatorname{rot} B & =-\int_{\mathbb{R}^{3}} M G v d v, \\
\partial_{t} B+\operatorname{rot} E & =0, \\
\operatorname{div} E & =\int_{\mathbb{R}^{3}} M G d v-1, \\
\operatorname{div} B & =0,
\end{aligned}\right.
$$

in the sense of distributions for any admissible renormalization, and satisfies the entropy inequality, for all $t>0$,

$$
\begin{aligned}
H(f)+\frac{1}{2} \int_{\mathbb{R}^{3}}\left(|E|^{2}+|B|^{2}\right) d x & +\int_{0}^{t} \int_{\mathbb{R}^{3}} D(f)(s) d x d s \\
& \leq H\left(f^{\text {in }}\right)+\frac{1}{2} \int_{\mathbb{R}^{3}}\left(\left|E^{\text {in }}\right|^{2}+\left|B^{\text {in }}\right|^{2}\right) d x<\infty
\end{aligned}
$$

where $f^{\text {in }}=M G^{\text {in }}$ is the initial value of $f=M G$ and the relative entropy $H(f)=$ $H(f \mid M)$ is defined in (1.17), while the entropy dissipation $D(f)$ is defined in (1.13).

Definition. We say that density functions $G^{+}(t, x, v) \geq 0$ and $G^{-}(t, x, v) \geq 0$, and electromagnetic vector fields $E(t, x)$ and $B(t, x)$, where $(t, x, v) \in[0, \infty) \times \mathbb{R}^{3} \times$ $\mathbb{R}^{3}$, such that

$$
\begin{aligned}
G^{ \pm} & \in C\left([0, \infty) ; w-L_{\mathrm{loc}}^{1}(d x d v)\right) \cap L^{\infty}\left([0, \infty), d t ; L_{\mathrm{loc}}^{1}\left(d x ; L^{1}\left(\left(1+|v|^{2}\right) M d v\right)\right)\right), \\
E, B & \in C\left([0, \infty) ; w-L^{2}(d x)\right) \cap L^{\infty}\left([0, \infty), d t ; L^{2}(d x)\right),
\end{aligned}
$$

are a renormalized solution of the two species Vlasov-Maxwell-Boltzmann system 4.2 if they solve

$$
\left\{\begin{aligned}
\partial_{t} \beta\left(G^{ \pm}\right)+v \cdot \nabla_{x} \beta\left(G^{ \pm}\right) & \pm(E+v \wedge B) \cdot \nabla_{v} \beta\left(G^{ \pm}\right) \mp E \cdot v \beta^{\prime}\left(G^{ \pm}\right) G^{ \pm} \\
& =\beta^{\prime}\left(G^{ \pm}\right) \mathcal{Q}\left(G^{ \pm}, G^{ \pm}\right)+\beta^{\prime}\left(G^{ \pm}\right) \mathcal{Q}\left(G^{ \pm}, G^{\mp}\right), \\
\partial_{t} E-\operatorname{rot} B & =-\int_{\mathbb{R}^{3}} M\left(G^{+}-G^{-}\right) v d v \\
\partial_{t} B+\operatorname{rot} E & =0, \\
\operatorname{div} E & =\int_{\mathbb{R}^{3}} M\left(G^{+}-G^{-}\right) d v \\
\operatorname{div} B & =0
\end{aligned}\right.
$$


in the sense of distributions for any admissible renormalization, and satisfy the entropy inequality, for all $t>0$,

$$
\begin{aligned}
H\left(f^{+}\right)+H\left(f^{-}\right) & +\frac{1}{2} \int_{\mathbb{R}^{3}}\left(|E|^{2}+|B|^{2}\right) d x \\
& +\int_{0}^{t} \int_{\mathbb{R}^{3}}\left(D\left(f^{+}\right)+D\left(f^{-}\right)+D\left(f^{+}, f^{-}\right)\right)(s) d x d s \\
& \leq H\left(f^{+\mathrm{in}}\right)+H\left(f^{-\mathrm{in}}\right)+\frac{1}{2} \int_{\mathbb{R}^{3}}\left(\left|E^{\mathrm{in}}\right|^{2}+\left|B^{\mathrm{in}}\right|^{2}\right) d x<\infty
\end{aligned}
$$

where $f^{ \pm \text {in }}=M G^{ \pm \text {in }}$ is the initial value of $f^{ \pm}=M G^{ \pm}$and the relative entropies $H\left(f^{ \pm}\right)=H\left(f^{ \pm} \mid M\right)$ are defined in (1.17), while the entropy dissipations $D\left(f^{ \pm}\right)$ and $D\left(f^{+}, f^{-}\right)$are defined in (1.13) and (1.16).

4.1.4. Macroscopic conservation laws. As already explained in Section 1.2 , the one species Vlasov-Maxwell-Boltzmann system (4.1) formally satisfies the macroscopic conservation laws

$$
\partial_{t} \int_{\mathbb{R}^{3}} f\left(\begin{array}{c}
1 \\
v \\
\frac{|v|^{2}}{2}
\end{array}\right) d v+\nabla_{x} \cdot \int_{\mathbb{R}^{3}} f\left(\begin{array}{c}
v \\
v \otimes v \\
\frac{|v|^{2}}{2} v
\end{array}\right) d v=\int_{\mathbb{R}^{3}} f\left(\begin{array}{c}
0 \\
E+v \wedge B \\
E \cdot v
\end{array}\right) d v,
$$

while the two species Vlasov-Maxwell-Boltzmann system 4.2 formally satisfies the macroscopic conservation laws

$$
\partial_{t} \int_{\mathbb{R}^{3}} f^{ \pm} d v+\nabla_{x} \cdot \int_{\mathbb{R}^{3}} f^{ \pm} v d v=0
$$

and

$$
\begin{aligned}
\partial_{t} \int_{\mathbb{R}^{3}}\left(f^{+}+f^{-}\right)\left(\begin{array}{c}
v \\
\frac{|v|^{2}}{2}
\end{array}\right) d v+\nabla_{x} \cdot \int_{\mathbb{R}^{3}}\left(f^{+}+f^{-}\right)\left(\begin{array}{c}
v \otimes v \\
\frac{|v|^{2}}{2} v
\end{array}\right) d v \\
=\int_{\mathbb{R}^{3}}\left(f^{+}-f^{-}\right)\left(\begin{array}{c}
E+v \wedge B \\
E \cdot v
\end{array}\right) d v
\end{aligned}
$$

However, it is at first unclear whether such formal laws are actually rigorously satisfied by the renormalized solutions defined in the previous section. It is therefore necessary to justify their validity.

To this end, we suppose now that such renormalized solutions $(f, E, B)$ and $\left(f^{ \pm}, E, B\right)$ have been previously obtained through an approximation procedure as detailed in Section 4.1.1. More precisely, we assume that there are sequences $\left\{\left(f_{k}, E_{k}, B_{k}\right)\right\}_{k \in \mathbb{N}}$ and $\left\{\left(f_{k}^{ \pm}, E_{k}, B_{k}\right)\right\}_{k \in \mathbb{N}}$ of smooth solutions to 4.1 and 4.2 (or appropriate approximations of these systems), for some uniformly bounded initial data, satisfying all macroscopic conservation laws and respectively converging in some suitable weak sense towards $(f, E, B)$ and $\left(f^{ \pm}, E, B\right)$. Therefore, in virtue of the uniform bounds provided by the entropy inequalities (4.17) and (4.18), it is readily seen that the terms $f_{k}, f_{k} v, f_{k}^{ \pm}$and $f_{k}^{ \pm} v$ are all respectively converging to $f, f v, f^{ \pm}$and $f^{ \pm} v$ weakly in $L_{\text {loc }}^{1}\left(d t d x ; L^{1}(d v)\right)$.

It follows that the conservations of mass

$$
\begin{array}{r}
\partial_{t} \int_{\mathbb{R}^{3}} f d v+\nabla_{x} \cdot \int_{\mathbb{R}^{3}} f v d v=0, \\
\partial_{t} \int_{\mathbb{R}^{3}} f^{ \pm} d v+\nabla_{x} \cdot \int_{\mathbb{R}^{3}} f^{ \pm} v d v=0,
\end{array}
$$

are easily established for renormalized solutions. This is in general the case when dealing with collisional kinetic equations. 
However, the same is unfortunately not true for the conservations of momentum and energy. Indeed, these laws involve higher moments of $f$ and $f^{ \pm}$, which may be singular due to high velocities build-up, as well as products of electromagnetic fields with particle densities, which may not even make sense if not renormalized.

In order to account for large velocities, we introduce now, following $\mathbf{5 5}$, since the terms $f_{k}|v|^{2}, f|v|^{2}, f_{k}^{ \pm}|v|^{2}$ and $f^{ \pm}|v|^{2}$ are uniformly bounded in $L_{\text {loc }}^{1}\left(d t d x ; L^{1}(d v)\right)$, the Radon measures $m_{i j} \in \mathcal{M}_{\text {loc }}\left([0, \infty) \times \mathbb{R}^{3}\right), i, j=1,2,3$, defined as the following defects in the limit $k \rightarrow \infty$ (up to extraction of subsequences) :

$$
\int_{\mathbb{R}^{3}} f_{k} v_{i} v_{j} d v \stackrel{*}{\rightarrow} \int_{\mathbb{R}^{3}} f v_{i} v_{j} d v+m_{i j} \quad \text { in } \mathcal{M}_{\mathrm{loc}}\left([0, \infty) \times \mathbb{R}^{3}\right),
$$

in the one species case, and

$$
\int_{\mathbb{R}^{3}}\left(f_{k}^{+}+f_{k}^{-}\right) v_{i} v_{j} d v \stackrel{*}{\rightarrow} \int_{\mathbb{R}^{3}}\left(f^{+}+f^{-}\right) v_{i} v_{j} d v+m_{i j} \quad \text { in } \mathcal{M}_{\mathrm{loc}}\left([0, \infty) \times \mathbb{R}^{3}\right),
$$

in the two species case. Note that the measures $m_{i j}$ are also defined by the limits, valid for any $R>0$,

$$
\begin{gathered}
\int_{\mathbb{R}^{3}} f_{k} v_{i} v_{j} \mathbb{1}_{\{|v| \geq R\}} d v \stackrel{*}{\rightarrow} \int_{\mathbb{R}^{3}} f v_{i} v_{j} \mathbb{1}_{\{|v| \geq R\}} d v+m_{i j}, \\
\int_{\mathbb{R}^{3}}\left(f_{k}^{+}+f_{k}^{-}\right) v_{i} v_{j} \mathbb{1}_{\{|v| \geq R\}} d v \stackrel{*}{\rightarrow} \int_{\mathbb{R}^{3}}\left(f^{+}+f^{-}\right) v_{i} v_{j} \mathbb{1}_{\{|v| \geq R\}} d v+m_{i j},
\end{gathered}
$$

in $\mathcal{M}_{\text {loc }}\left([0, \infty) \times \mathbb{R}^{3}\right)$. In particular, it follows that the matrix measure $m=$ $\left(m_{i j}\right)_{1 \leq i, j \leq 3}$ is symmetric and positive definite in the sense that, for any $\varphi \in$ $C_{c}\left([0, \infty) \times \mathbb{R}^{3} ; \mathbb{R}^{3}\right)$,

$$
\int_{[0, \infty) \times \mathbb{R}^{3}} \varphi^{t}(d m) \varphi=\sum_{i, j=1}^{3} \int_{[0, \infty) \times \mathbb{R}^{3}} \varphi_{i} \varphi_{j} d m_{i j} \geq 0,
$$

whence, for any $\varphi, \psi \in C_{c}\left([0, \infty) \times \mathbb{R}^{3} ; \mathbb{R}^{3}\right)$,

$$
\left|\int_{[0, \infty) \times \mathbb{R}^{3}} \varphi^{t}(d m) \psi\right| \leq \frac{1}{2} \int_{[0, \infty) \times \mathbb{R}^{3}} \varphi^{t}(d m) \varphi+\frac{1}{2} \int_{[0, \infty) \times \mathbb{R}^{3}} \psi^{t}(d m) \psi .
$$

Further substituting $\varphi$ and $\psi$ in the preceding inequality by $\lambda^{\frac{1}{2}} \varphi$ and $\lambda^{-\frac{1}{2}} \psi$, with $\lambda>0$, respectively, and then optimizing the resulting inequality in $\lambda>0$ yields that

$$
\left|\int_{[0, \infty) \times \mathbb{R}^{3}} \varphi^{t}(d m) \psi\right| \leq\left(\int_{[0, \infty) \times \mathbb{R}^{3}} \varphi^{t}(d m) \varphi\right)^{\frac{1}{2}}\left(\int_{[0, \infty) \times \mathbb{R}^{3}} \psi^{t}(d m) \psi\right)^{\frac{1}{2}},
$$

for any $\varphi, \psi \in C_{c}\left([0, \infty) \times \mathbb{R}^{3} ; \mathbb{R}^{3}\right)$.

The matrix measure $m$ will be used to characterize the flux terms in the conservation of momentum and the density terms in the conservation of energy. However, the flux terms in the conservation of energy contain higher order moments which cannot be handled and we will therefore simply leave them out of the analysis by only considering the global conservation of energy.

As for the forcing terms involving the electromagnetic fields, they do not even make sense with the sole use of the a priori estimates provided by the entropy inequalities (4.17) and 4.18). It is therefore necessary to use now the conservation laws of energy (1.9)- 1.10) and for the Poynting vector 1.11)- 1.12 in Maxwell's equations to recast these forcing terms with quadratic expressions involving the electromagnetic fields only. 
Thus, using the identities $1.9,1.10,1.11$ and 1.12 , the local conservations of momentum may be rewritten as

$$
\begin{aligned}
\partial_{t}\left(\int_{\mathbb{R}^{3}} f_{k} v d v+E_{k} \wedge B_{k}\right)+\nabla_{x} \cdot\left(\int_{\mathbb{R}^{3}} f_{k} v \otimes v d v-E_{k} \otimes E_{k}-B_{k} \otimes B_{k}\right) & \\
+\nabla_{x}\left(\frac{\left|E_{k}\right|^{2}+\left|B_{k}\right|^{2}}{2}\right) & =E_{k}
\end{aligned}
$$

in the one species case, and as

$$
\begin{aligned}
& \partial_{t}\left(\int_{\mathbb{R}^{3}}\left(f_{k}^{+}+f_{k}^{-}\right) v d v+E_{k} \wedge B_{k}\right) \\
& \quad+\nabla_{x} \cdot\left(\int_{\mathbb{R}^{3}}\left(f_{k}^{+}+f_{k}^{-}\right) v \otimes v d v-E_{k} \otimes E_{k}-B_{k} \otimes B_{k}\right) \\
& \quad+\nabla_{x}\left(\frac{\left|E_{k}\right|^{2}+\left|B_{k}\right|^{2}}{2}\right)=0,
\end{aligned}
$$

in the two species case, whereas the global conservations of energy may be expessed as

$$
\frac{d}{d t} \int_{\mathbb{R}^{3}}\left(\int_{\mathbb{R}^{3}} f_{k} \frac{|v|^{2}}{2} d v+\frac{\left|E_{k}\right|^{2}+\left|B_{k}\right|^{2}}{2}\right) d x=0
$$

in the one species case, and as

$$
\frac{d}{d t} \int_{\mathbb{R}^{3}}\left(\int_{\mathbb{R}^{3}}\left(f_{k}^{+}+f_{k}^{-}\right) \frac{|v|^{2}}{2} d v+\frac{\left|E_{k}\right|^{2}+\left|B_{k}\right|^{2}}{2}\right) d x=0
$$

in the two species case.

Passing to the limit $k \rightarrow \infty$ therefore requires the introduction of yet another set of Radon measures $a_{i j} \in \mathcal{M}_{\text {loc }}\left([0, \infty) \times \mathbb{R}^{3}\right), i, j=1,2,3,4,5,6$, where the matrix measure $a=\left(a_{i j}\right)_{1 \leq i, j \leq 6}$ is defined as the following defect :

$$
\left(\begin{array}{l}
E_{k} \\
B_{k}
\end{array}\right) \otimes\left(\begin{array}{l}
E_{k} \\
B_{k}
\end{array}\right) \stackrel{*}{\rightarrow}\left(\begin{array}{l}
E \\
B
\end{array}\right) \otimes\left(\begin{array}{l}
E \\
B
\end{array}\right)+a \text { in } \mathcal{M}_{\text {loc }}\left([0, \infty) \times \mathbb{R}^{3}\right) .
$$

Note that the matrix measure $n$ is also defined by the limit

$$
\left(\begin{array}{l}
E_{k}-E \\
B_{k}-B
\end{array}\right) \otimes\left(\begin{array}{l}
E_{k}-E \\
B_{k}-B
\end{array}\right) \stackrel{*}{\rightarrow} a \quad \text { in } \mathcal{M}_{\mathrm{loc}}\left([0, \infty) \times \mathbb{R}^{3}\right) .
$$

It then follows that, as before, the matrix measure $a$ is symmetric and positive definite in the sense that, for any $\varphi \in C_{c}\left([0, \infty) \times \mathbb{R}^{3} ; \mathbb{R}^{6}\right)$,

$$
\int_{[0, \infty) \times \mathbb{R}^{3}} \varphi^{t}(d a) \varphi=\sum_{i, j=1}^{6} \int_{[0, \infty) \times \mathbb{R}^{3}} \varphi_{i} \varphi_{j} d a_{i j} \geq 0
$$

whence (see the analogous inequality 4.19 )

$$
\left|\int_{[0, \infty) \times \mathbb{R}^{3}} \varphi^{t}(d a) \psi\right| \leq\left(\int_{[0, \infty) \times \mathbb{R}^{3}} \varphi^{t}(d a) \varphi\right)^{\frac{1}{2}}\left(\int_{[0, \infty) \times \mathbb{R}^{3}} \psi^{t}(d a) \psi\right)^{\frac{1}{2}}
$$

for any $\varphi, \psi \in C_{c}\left([0, \infty) \times \mathbb{R}^{3} ; \mathbb{R}^{6}\right)$.

For mere convenience of notation, we further introduce the matrix measures $e=\left(a_{i j}\right)_{1 \leq i, j \leq 3}$ and $b=\left(a_{(i+3)(j+3)}\right)_{1 \leq i, j \leq 3}$. 
Now, letting $k \rightarrow \infty$ in the conservation laws 4.20, 4.21, 4.22 and 4.23 respectively yields the local conservations of momentum

$$
\begin{aligned}
& \partial_{t}\left(\int_{\mathbb{R}^{3}} f v d v+E \wedge B+\left(\begin{array}{l}
a_{26}-a_{35} \\
a_{34}-a_{16} \\
a_{15}-a_{24}
\end{array}\right)\right) \\
& \quad+\nabla_{x} \cdot\left(\int_{\mathbb{R}^{3}} f v \otimes v d v+m-E \otimes E-e-B \otimes B-b\right) \\
& \quad+\nabla_{x}\left(\frac{|E|^{2}+|B|^{2}+\operatorname{Tr} a}{2}\right)=E
\end{aligned}
$$

in the one species case, and

$$
\begin{aligned}
& \partial_{t}\left(\int_{\mathbb{R}^{3}}\left(f^{+}+f^{-}\right) v d v+E \wedge B+\left(\begin{array}{l}
a_{26}-a_{35} \\
a_{34}-a_{16} \\
a_{15}-a_{24}
\end{array}\right)\right) \\
& \quad+\nabla_{x} \cdot\left(\int_{\mathbb{R}^{3}}\left(f^{+}+f^{-}\right) v \otimes v d v+m-E \otimes E-e-B \otimes B-b\right) \\
& \quad+\nabla_{x}\left(\frac{|E|^{2}+|B|^{2}+\operatorname{Tr} a}{2}\right)=0,
\end{aligned}
$$

in the two species case, as well as the global energy decay

$$
\begin{gathered}
\int_{\mathbb{R}^{3}}\left(\int_{\mathbb{R}^{3}} f \frac{|v|^{2}}{2} d v+\frac{\operatorname{Tr} m}{2}+\frac{|E|^{2}+|B|^{2}+\operatorname{Tr} a}{2}\right) d x \\
\leq \int_{\mathbb{R}^{3}}\left(\int_{\mathbb{R}^{3}} f^{\text {in }} \frac{|v|^{2}}{2} d v+\frac{\left|E^{\text {in }}\right|^{2}+\left|B^{\text {in }}\right|^{2}}{2}\right) d x,
\end{gathered}
$$

in the one species case, and

$$
\begin{gathered}
\int_{\mathbb{R}^{3}}\left(\int_{\mathbb{R}^{3}}\left(f^{+}+f^{-}\right) \frac{|v|^{2}}{2} d v+\frac{\operatorname{Tr} m}{2}+\frac{|E|^{2}+|B|^{2}+\operatorname{Tr} a}{2}\right) d x \\
\leq \int_{\mathbb{R}^{3}}\left(\int_{\mathbb{R}^{3}}\left(f^{+\mathrm{in}}+f^{-\mathrm{in}}\right) \frac{|v|^{2}}{2} d v+\frac{\left|E^{\mathrm{in}}\right|^{2}+\left|B^{\mathrm{in}}\right|^{2}}{2}\right) d x
\end{gathered}
$$

in the two species case.

Note that the above global energy decay containing the defect measures may be incorporated into the entropy inequalities (4.17) and (4.18), so that renormalized solutions of the Vlasov-Maxwell-Boltzmann systems (4.1) and (4.2) may be assumed to respectively satisfy the entropy inequalities

$$
\begin{aligned}
H(f)+\frac{1}{2} \int_{\mathbb{R}^{3}}\left(|E|^{2}+|B|^{2}\right) d x & +\frac{1}{2} \int_{\mathbb{R}^{3}} \operatorname{Tr}(m+e+b) d x+\int_{0}^{t} \int_{\mathbb{R}^{3}} D(f)(s) d x d s \\
& \leq H\left(f^{\mathrm{in}}\right)+\frac{1}{2} \int_{\mathbb{R}^{3}}\left(\left|E^{\mathrm{in}}\right|^{2}+\left|B^{\mathrm{in}}\right|^{2}\right) d x<\infty,
\end{aligned}
$$

in the one species case, and

$$
\begin{aligned}
H\left(f^{+}\right)+H\left(f^{-}\right) & +\frac{1}{2} \int_{\mathbb{R}^{3}}\left(|E|^{2}+|B|^{2}\right) d x+\frac{1}{2} \int_{\mathbb{R}^{3}} \operatorname{Tr}(m+e+b) d x \\
& +\int_{0}^{t} \int_{\mathbb{R}^{3}}\left(D\left(f^{+}\right)+D\left(f^{-}\right)+D\left(f^{+}, f^{-}\right)\right)(s) d x d s \\
& \leq H\left(f^{+\mathrm{in}}\right)+H\left(f^{-\mathrm{in}}\right)+\frac{1}{2} \int_{\mathbb{R}^{3}}\left(\left|E^{\mathrm{in}}\right|^{2}+\left|B^{\mathrm{in}}\right|^{2}\right) d x<\infty
\end{aligned}
$$

in the two species case. 
The preceding characterization of defects in macroscopic conservation laws will not be of further use in our study of the hydrodynamic limits of the one species Vlasov-Maxwell-Boltzmann system 4.1). It will, however, be of crucial utility in the renormalized relative entropy method developed later on in Chapter 12 in relation with hydrodynamic limits of the two species Vlasov-Maxwell-Boltzmann system 4.2.

Notice, finally, that the symmetry and the positive definiteness of the matrix measures $m$ and $a$ imply that the bounds on the non-negative measures $\operatorname{Tr}(m+e+b)$, provided a priori by the entropy inequalities 4.25 and $(4.26)$, are sufficient to control all components of $m$ and $a$. Indeed, the inequalities (4.19) and (4.24) provide all necessary estimates of $m$ and $a$ in terms of $\operatorname{Tr}(m+e+b)$.

In particular, it is readily seen that, for any $u \in C_{c}\left(\mathbb{R}^{3} ; \mathbb{R}^{3}\right)$,

$$
\left|\int_{\mathbb{R}^{3}} u \cdot\left(\begin{array}{l}
a_{26}-a_{35} \\
a_{34}-a_{16} \\
a_{15}-a_{24}
\end{array}\right) d x\right| \leq 6\|u\|_{L^{\infty}}\left(\int_{\mathbb{R}^{3}}(\operatorname{Tr} e) d x\right)^{\frac{1}{2}}\left(\int_{\mathbb{R}^{3}}(\operatorname{Tr} b) d x\right)^{\frac{1}{2}}
$$

Still, since the above defect stems from a vector product, it is possible to improve the constant in the preceding inequality. We record such improvement in the following result, for later use.

Lemma 4.3. For any $u \in C_{c}\left(\mathbb{R}^{3} ; \mathbb{R}^{3}\right)$, it holds that

$$
\left|\int_{\mathbb{R}^{3}} u \cdot\left(\begin{array}{l}
a_{26}-a_{35} \\
a_{34}-a_{16} \\
a_{15}-a_{24}
\end{array}\right) d x\right| \leq\|u\|_{L^{\infty}}\left(\int_{\mathbb{R}^{3}}(\operatorname{Tr} e) d x\right)^{\frac{1}{2}}\left(\int_{\mathbb{R}^{3}}(\operatorname{Tr} b) d x\right)^{\frac{1}{2}}
$$

Proof. Using 4.24, we first obtain

$$
\begin{aligned}
\left|\int_{\mathbb{R}^{3}} u \cdot\left(\begin{array}{l}
a_{26}-a_{35} \\
a_{34}-a_{16} \\
a_{15}-a_{24}
\end{array}\right) d x\right| & \leq\left|\int_{\mathbb{R}^{3}}\left(a_{15} u_{3}-a_{16} u_{2}\right) d x\right|+\left|\int_{\mathbb{R}^{3}}\left(a_{26} u_{1}-a_{24} u_{3}\right) d x\right| \\
& +\left|\int_{\mathbb{R}^{3}}\left(a_{34} u_{2}-a_{35} u_{1}\right) d x\right| \\
& \leq\left(\int_{\mathbb{R}^{3}} a_{11} d x\right)^{\frac{1}{2}}\left(\int_{\mathbb{R}^{3}}\left(a_{55} u_{3}^{2}-2 a_{56} u_{3} u_{2}+a_{66} u_{2}^{2}\right) d x\right)^{\frac{1}{2}} \\
& +\left(\int_{\mathbb{R}^{3}} a_{22} d x\right)^{\frac{1}{2}}\left(\int_{\mathbb{R}^{3}}\left(a_{66} u_{1}^{2}-2 a_{64} u_{1} u_{3}+a_{44} u_{3}^{2}\right) d x\right)^{\frac{1}{2}} \\
& +\left(\int_{\mathbb{R}^{3}} a_{33} d x\right)^{\frac{1}{2}}\left(\int_{\mathbb{R}^{3}}\left(a_{44} u_{2}^{2}-2 a_{45} u_{2} u_{1}+a_{55} u_{1}^{2}\right) d x\right)^{\frac{1}{2}} .
\end{aligned}
$$


It follows that, for any $\alpha>0$,

$$
\begin{aligned}
\left|\int_{\mathbb{R}^{3}} u \cdot\left(\begin{array}{l}
a_{26}-a_{35} \\
a_{34}-a_{16} \\
a_{15}-a_{24}
\end{array}\right) d x\right| & \leq \frac{\alpha}{2} \int_{\mathbb{R}^{3}}(\operatorname{Tr} e) d x \\
& +\frac{1}{2 \alpha} \int_{\mathbb{R}^{3}}\left(a_{55} u_{3}^{2}-2 a_{56} u_{3} u_{2}+a_{66} u_{2}^{2}\right) d x \\
& +\frac{1}{2 \alpha} \int_{\mathbb{R}^{3}}\left(a_{66} u_{1}^{2}-2 a_{64} u_{1} u_{3}+a_{44} u_{3}^{2}\right) d x \\
& +\frac{1}{2 \alpha} \int_{\mathbb{R}^{3}}\left(a_{44} u_{2}^{2}-2 a_{45} u_{2} u_{1}+a_{55} u_{1}^{2}\right) d x \\
& =\frac{\alpha}{2} \int_{\mathbb{R}^{3}}(\operatorname{Tr} e) d x+\frac{1}{2 \alpha} \int_{\mathbb{R}^{3}}(\operatorname{Tr} b)|u|^{2} d x \\
& -\frac{1}{2 \alpha} \int_{\mathbb{R}^{3}}\left(u^{t} b u\right) d x \\
& \leq \frac{\alpha}{2} \int_{\mathbb{R}^{3}}(\operatorname{Tr} e) d x+\frac{\|u\|_{L^{\infty}}^{2}}{2 \alpha} \int_{\mathbb{R}^{3}}(\operatorname{Tr} b) d x
\end{aligned}
$$

which, upon optimizing in $\alpha>0$, concludes the justification of the lemma.

\subsection{The incompressible quasi-static}

\section{Navier-Stokes-Fourier-Maxwell-Poisson system}

Henceforth, in this second part of our work on conditional results, unless otherwise stated, we will focus, for the mere sake of technical simplicity, on some Maxwellian cross-section, say $b \equiv 1$. All other mathematically and physically pertinent cross-sections (deriving from hard, soft, short-range and long-range interaction potentials) will be discussed and treated in full generality in the remaining parts of our work on unconditional results.

Following Section 2.3, we first consider a plasma constituted of a gas of cations (positively charged ions), with a uniform background of heavy anions (negatively charged ions) assumed to be at statistical equilibrium. Elementary interactions are taken into account by both a mean field term (corresponding to long-range interactions) and a local collision term (associated to short-range interactions) involving possibly different mean free paths. Thus, the charged particles evolve under the coupled effect of the Lorentz force due to the self-induced electromagnetic field, and of the collisions with other particles, according to the following scaled VlasovMaxwell-Boltzmann system :

$$
\left\{\begin{aligned}
\epsilon \partial_{t} f_{\epsilon}+v \cdot \nabla_{x} f_{\epsilon}+\epsilon\left(E_{\epsilon}+v \wedge B_{\epsilon}\right) \cdot \nabla_{v} f_{\epsilon} & =\frac{1}{\epsilon} Q\left(f_{\epsilon}, f_{\epsilon}\right), \\
f_{\epsilon} & =M\left(1+\epsilon g_{\epsilon}\right), \\
\epsilon \partial_{t} E_{\epsilon}-\operatorname{rot} B_{\epsilon} & =-\int_{\mathbb{R}^{3}} g_{\epsilon} v M d v, \\
\epsilon \partial_{t} B_{\epsilon}+\operatorname{rot} E_{\epsilon} & =0, \\
\operatorname{div} E_{\epsilon} & =\int_{\mathbb{R}^{3}} g_{\epsilon} M d v, \\
\operatorname{div} B_{\epsilon} & =0 .
\end{aligned}\right.
$$


In this scaling, the entropy inequality states that

$$
\begin{aligned}
\frac{1}{\epsilon^{2}} H\left(f_{\epsilon}\right)+\frac{1}{2} \int_{\mathbb{R}^{3}}\left(\left|E_{\epsilon}\right|^{2}+\left|B_{\epsilon}\right|^{2}\right) d x & +\frac{1}{\epsilon^{4}} \int_{0}^{t} \int_{\mathbb{R}^{3}} D\left(f_{\epsilon}\right)(s) d x d s \\
& \leq \frac{1}{\epsilon^{2}} H\left(f_{\epsilon}^{\text {in }}\right)+\frac{1}{2} \int_{\mathbb{R}^{3}}\left(\left|E_{\epsilon}^{\text {in }}\right|^{2}+\left|B_{\epsilon}^{\text {in }}\right|^{2}\right) d x
\end{aligned}
$$

where $H\left(f_{\epsilon}\right)=H\left(f_{\epsilon} \mid M\right)$. In particular, it yields uniform bounds on $E_{\epsilon}, B_{\epsilon}$ and $g_{\epsilon}$.

Since we are interested in the limiting fluctuation $g_{\epsilon} \rightarrow g$, it is then natural to rewrite the kinetic equation in terms of the fluctuations $g_{\epsilon}$,

$$
\epsilon \partial_{t} g_{\epsilon}+v \cdot \nabla_{x} g_{\epsilon}+\epsilon\left(E_{\epsilon}+v \wedge B_{\epsilon}\right) \cdot \nabla_{v} g_{\epsilon}-E_{\epsilon} \cdot v\left(1+\epsilon g_{\epsilon}\right)=-\frac{1}{\epsilon} \mathcal{L} g_{\epsilon}+\mathcal{Q}\left(g_{\epsilon}, g_{\epsilon}\right)
$$

According to the formal analysis from Section 2.3, we then expect the limiting macroscopic observables to solve the incompressible quasi-static Navier-StokesFourier-Maxwell-Poisson system :

$$
\left\{\begin{array}{rlrl}
\partial_{t} u+u \cdot \nabla_{x} u-\mu \Delta_{x} u & =-\nabla_{x} p+E+\rho \nabla_{x} \theta+u \wedge B & \\
\partial_{t}\left(\frac{3}{2} \theta-\rho\right)+u \cdot \nabla_{x}\left(\frac{3}{2} \theta-\rho\right)-\frac{5}{2} \kappa \Delta_{x} \theta & =0, & \operatorname{div} u & =0, \\
\operatorname{rot} B & =u, & \Delta_{x}(\rho+\theta) & =\rho, \\
\partial_{t} B+\operatorname{rot} E & =0, & \operatorname{div} E & =\rho, \\
\operatorname{div} B & =0 .
\end{array}\right.
$$

As discussed in Section 3.1, this system is similar to the usual Navier-Stokes equations, since it is weakly stable in the class of functions of finite energy.

Because of this crucial weak stability property, the study of hydrodynamic limits follows closely what has been previously done for the incompressible NavierStokes-Fourier limit of the Boltzmann equation (see [4, 68 and the references therein for a survey of related results). In particular, we will be able to prove a convergence result which :

- holds globally in time ;

- does not require any assumption on the initial velocity profile ;

- does not assume any constraint on the initial thermodynamic fields.

We would also be able to take into account boundary conditions, and describe their limiting form, but this point will not be dealt with here. We refer to $\mathbf{5 9}, \mathbf{6 8}$ for a complete treatment of boundary conditions in the viscous hydrodynamic limits of the Boltzmann equation, based on the renormalized solutions on bounded domains constructed by Mischler in 60, 61.

As we will see, if we assume that the Vlasov-Maxwell-Boltzmann system 4.27) has renormalized solutions (which, again, is not known), the main challenge here lies in understanding the influence of the electromagnetic force both on hypoelliptic processes of the kinetic transport equation and on fast time oscillations.

Our goal, here, is to establish the convergence of scaled families of renormalized solutions to the one species Vlasov-Maxwell-Boltzmann system (4.27) towards solutions of the incompressible quasi-static Navier-Stokes-Fourier-Maxwell-Poisson system 4.30, without any restriction on their size, regularity or well-preparedness of the initial data.

Following the program proposed by Bardos, Golse and Levermore in [9] (which relies essentially on weak compactness arguments), we can prove the following theorem. Recall that, in this second part, we are only considering the Maxwellian cross-section $b \equiv 1$. 
TheOrem 4.4. Let $\left(f_{\epsilon}^{\mathrm{in}}, E_{\epsilon}^{\mathrm{in}}, B_{\epsilon}^{\mathrm{in}}\right)$ be a family of initial data such that

$$
\frac{1}{\epsilon^{2}} H\left(f_{\epsilon}^{\text {in }}\right)+\frac{1}{2} \int_{\mathbb{R}^{3}}\left(\left|E_{\epsilon}^{\text {in }}\right|^{2}+\left|B_{\epsilon}^{\text {in }}\right|^{2}\right) d x \leq C^{\text {in }},
$$

for some $C^{\text {in }}>0$, and

$$
\operatorname{div} E_{\epsilon}^{\text {in }}=\int_{\mathbb{R}^{3}} g_{\epsilon}^{\text {in }} M d v, \quad \operatorname{div} B_{\epsilon}^{\text {in }}=0,
$$

where $f_{\epsilon}^{\text {in }}=M\left(1+\epsilon g_{\epsilon}^{\text {in }}\right)$. For any $\epsilon>0$, we assume the existence of a renormalized solution $\left(f_{\epsilon}, E_{\epsilon}, B_{\epsilon}\right)$ to the scaled one species Vlasov-Maxwell-Boltzmann system 4.27) (for the Maxwellian cross-section $b \equiv 1$ ) with initial data $\left(f_{\epsilon}^{\text {in }}, E_{\epsilon}^{\text {in }}, B_{\epsilon}^{\text {in }}\right)$. We define the macroscopic fluctuations of density $\rho_{\epsilon}$, bulk velocity $u_{\epsilon}$ and temperature $\theta_{\epsilon}$ by

$$
\begin{aligned}
\rho_{\epsilon} & =\int_{\mathbb{R}^{3}} g_{\epsilon} M d v, \\
u_{\epsilon} & =\int_{\mathbb{R}^{3}} g_{\epsilon} v M d v, \\
\theta_{\epsilon} & =\int_{\mathbb{R}^{3}} g_{\epsilon}\left(\frac{|v|^{2}}{3}-1\right) M d v,
\end{aligned}
$$

and denote their respective initial value by $\rho_{\epsilon}^{\text {in }}, u_{\epsilon}^{\text {in }}$ and $\theta_{\epsilon}^{\text {in }}$.

Then, the family $\left(\rho_{\epsilon}, u_{\epsilon}, \theta_{\epsilon}, B_{\epsilon}\right)$ is weakly relatively compact in $L_{\mathrm{loc}}^{1}(d t d x)$ (while the family of initial data $\left(\rho_{\epsilon}^{\text {in }}, u_{\epsilon}^{\text {in }}, \theta_{\epsilon}^{\text {in }}, B_{\epsilon}^{\text {in }}\right)$ is weakly relatively compact in $\left.L_{\text {loc }}^{1}(d x)\right)$ and any of its limit points $(\rho, u, \theta, B)$ is a weak solution of the incompressible quasistatic Navier-Stokes-Fourier-Maxwell-Poisson system 4.30 with initial data

$$
\begin{array}{ll}
\rho^{\text {in }}=\frac{\Delta_{x}}{3-5 \Delta_{x}}\left(3 \theta_{0}^{\text {in }}-2 \rho_{0}^{\text {in }}\right), & u^{\text {in }}=\frac{\operatorname{rot}}{1-\Delta_{x}}\left(\operatorname{rot} u_{0}^{\text {in }}+B_{0}^{\text {in }}\right), \\
\theta^{\text {in }}=\frac{1-\Delta_{x}}{3-5 \Delta_{x}}\left(3 \theta_{0}^{\text {in }}-2 \rho_{0}^{\text {in }}\right), & B^{\text {in }}=\frac{1}{1-\Delta_{x}}\left(\operatorname{rot} u_{0}^{\text {in }}+B_{0}^{\text {in }}\right),
\end{array}
$$

where $\left(\rho_{0}^{\mathrm{in}}, u_{0}^{\mathrm{in}}, \theta_{0}^{\mathrm{in}}, B_{0}^{\mathrm{in}}\right) \in L^{2}(d x)$ is the weak limit of $\left(\rho_{\epsilon}^{\mathrm{in}}, u_{\epsilon}^{\mathrm{in}}, \theta_{\epsilon}^{\mathrm{in}}, B_{\epsilon}^{\mathrm{in}}\right)$.

The proof of Theorem 4.4 is built over the course of the coming chapters and is per se the subject of Chapter 11 .

Note that, strictly speaking, the weak solution we obtain in the limit (and which depends in general on the subsequence under consideration) is not necessarily a Leray solution of the system 4.30 , since it is does not satisfy the energy inequality (3.2), but only a bound. However, it is possible to obtain asymptotically a Leray solution by strengthening the initial well-preparedness of the data. More precisely, one would have to impose that the initial data converges entropically (as introduced in [9]) in the sense that

$$
\begin{aligned}
\frac{1}{\epsilon^{2}} H\left(f_{\epsilon}^{\text {in }}\right) & +\frac{1}{2} \int_{\mathbb{R}^{3}}\left(\left|E_{\epsilon}^{\text {in }}\right|^{2}+\left|B_{\epsilon}^{\text {in }}\right|^{2}\right) d x \\
& \rightarrow \frac{1}{2} \int_{\mathbb{R}^{3}}\left(\rho^{\text {in }}{ }^{2}+\left|u^{\text {in }}\right|^{2}+\frac{3}{2} \theta^{\text {in }}{ }^{2}+\left|\nabla_{x}\left(\rho^{\text {in }}+\theta^{\text {in }}\right)\right|^{2}+\left|B^{\text {in }}\right|^{2}\right) d x
\end{aligned}
$$

In fact, the above entropic convergence has rather strong implications on the initial data. Indeed, further denoting by $g_{0}^{\text {in }}$ and $E_{0}^{\text {in }}$ the weak limits of $g_{\epsilon}^{\text {in }}$ and $E_{\epsilon}^{\text {in }}$, respectively, standard convexity arguments on weak convergence (see Lemma 
5.1 below, or [9, Proposition 3.1]) yield that

$$
\begin{aligned}
\frac{1}{2} \int_{\mathbb{R}^{3}}\left(\rho_{0}^{\text {in }}{ }^{2}+\left|u_{0}^{\text {in }}\right|^{2}+\frac{3}{2} \theta_{0}^{\text {in }}{ }^{2}\right) d x & =\frac{1}{2} \int_{\mathbb{R}^{3} \times \mathbb{R}^{3}}\left(\Pi g_{0}^{\text {in }}\right)^{2} M d v d x \\
& \leq \frac{1}{2} \int_{\mathbb{R}^{3} \times \mathbb{R}^{3}}\left(g_{0}^{\text {in }}\right)^{2} M d v d x \leq \liminf _{\epsilon \rightarrow 0} \frac{1}{\epsilon^{2}} H\left(f_{\epsilon}^{\text {in }}\right),
\end{aligned}
$$

and

$$
\frac{1}{2} \int_{\mathbb{R}^{3}}\left(\left|E_{0}^{\text {in }}\right|^{2}+\left|B_{0}^{\text {in }}\right|^{2}\right) d x \leq \liminf _{\epsilon \rightarrow 0} \frac{1}{2} \int_{\mathbb{R}^{3}}\left(\left|E_{\epsilon}^{\text {in }}\right|^{2}+\left|B_{\epsilon}^{\text {in }}\right|^{2}\right) d x .
$$

Moreover, one easily verifies that

$$
\left(\begin{array}{c}
\rho_{0}^{\text {in }} \\
\sqrt{\frac{3}{2}} \theta_{0}^{\text {in }} \\
E_{0}^{\text {in }}
\end{array}\right) \mapsto\left(\begin{array}{c}
\frac{3}{3-5 \Delta_{x}}\left(\rho_{0}^{\text {in }}-\operatorname{div} E_{0}^{\text {in }}\right)+\frac{\Delta_{x}}{3-5 \Delta_{x}}\left(3 \theta_{0}^{\text {in }}-2 \rho_{0}^{\text {in }}\right) \\
\frac{2}{3-5 \Delta_{x}} \sqrt{\frac{3}{2}}\left(\rho_{0}^{\text {in }}-\operatorname{div} E_{0}^{\text {in }}\right)+\frac{1-\Delta_{x}}{3-5 \Delta_{x}} \sqrt{\frac{3}{2}}\left(3 \theta_{0}^{\text {in }}-2 \rho_{0}^{\text {in }}\right) \\
\frac{5}{3-5 \Delta_{x}} \nabla_{x}\left(\rho_{0}^{\text {in }}-\operatorname{div} E_{0}^{\text {in }}\right)+\frac{1}{3-5 \Delta_{x}} \nabla_{x}\left(3 \theta_{0}^{\text {in }}-2 \rho_{0}^{\text {in }}\right)
\end{array}\right)
$$

defines the orthogonal projection onto the subspace of $L^{2}(d x)$ defined by the constraint

while

$$
\nabla_{x}\left(\rho_{0}^{\text {in }}+\theta_{0}^{\text {in }}\right)=E_{0}^{\text {in }}
$$

$$
\left(\begin{array}{c}
u_{0}^{\text {in }} \\
B_{0}^{\text {in }}
\end{array}\right) \mapsto\left(\begin{array}{c}
\frac{\operatorname{rot}}{1-\Delta_{x}}\left(\operatorname{rot} u_{0}^{\text {in }}+P B_{0}^{\text {in }}\right) \\
\frac{1}{1-\Delta_{x}}\left(\operatorname{rot} u_{0}^{\text {in }}+P B_{0}^{\text {in }}\right)
\end{array}\right)
$$

corresponds to the orthogonal projection onto the subspace of $L^{2}(d x)$ defined by the constraints

Therefore, it follows that

$$
\operatorname{rot} B_{0}^{\text {in }}=u_{0}^{\text {in }} \quad \text { and } \quad \operatorname{div} B_{0}^{\text {in }}=0 .
$$

$$
\begin{aligned}
\frac{1}{2} \int_{\mathbb{R}^{3}}\left(\rho^{\text {in }}+\left|u^{\text {in }}\right|^{2}\right. & \left.+\frac{3}{2} \theta^{\text {in }}{ }^{2}+\left|\nabla_{x}\left(\rho^{\text {in }}+\theta^{\text {in }}\right)\right|^{2}+\left|B^{\text {in }}\right|^{2}\right) d x \\
& \leq \frac{1}{2} \int_{\mathbb{R}^{3}}\left(\rho_{0}^{\text {in }}{ }^{2}+\left|u_{0}^{\text {in }}\right|^{2}+\frac{3}{2} \theta_{0}^{\text {in }}{ }^{2}+\left|E_{0}^{\text {in }}\right|^{2}+\left|B_{0}^{\text {in }}\right|^{2}\right) d x
\end{aligned}
$$

with equality if and only if $\rho^{\text {in }}=\rho_{0}^{\text {in }}, u^{\text {in }}=u_{0}^{\text {in }}, \theta^{\text {in }}=\theta_{0}^{\text {in }}, \nabla_{x}\left(\rho^{\text {in }}+\theta^{\text {in }}\right)=E_{0}^{\text {in }}$ and $B^{\text {in }}=B_{0}^{\text {in }}$, which, when combined with the above entropic convergence of the initial data and according to Proposition 4.11 from $\left[\mathbf{9}\right.$, implies that $\left(\rho_{\epsilon}^{\text {in }}, u_{\epsilon}^{\text {in }}, \theta_{\epsilon}^{\text {in }}, E_{\epsilon}^{\text {in }}, B_{\epsilon}^{\text {in }}\right)$ converges strongly to $\left(\rho^{\text {in }}, u^{\text {in }}, \theta^{\text {in }}, \nabla_{x}\left(\rho^{\text {in }}+\theta^{\text {in }}\right), B^{\text {in }}\right)$, where

$$
\text { (4.33) } \quad \operatorname{div} u^{\text {in }}=0, \quad \operatorname{div} B^{\text {in }}=0, \quad \operatorname{rot} B^{\text {in }}=u^{\text {in }}, \quad \Delta_{x}\left(\rho^{\text {in }}+\theta^{\text {in }}\right)=\rho^{\text {in }},
$$

and that $g_{\epsilon}^{\text {in }}$ converges strongly to

$$
\rho^{\text {in }}+u^{\text {in }} \cdot v+\theta^{\text {in }}\left(\frac{|v|^{2}}{2}-\frac{3}{2}\right), \quad \text { in } L_{\text {loc }}^{1}\left(d x ; L^{1}\left(\left(1+|v|^{2}\right) M d v\right)\right) .
$$

The strong convergence of $g_{\epsilon}^{\text {in }}$ towards an infinitesimal Maxwellian implies the vanishing of the initial relaxation layer, while the strong convergence of the initial macroscopic observables towards initial data satisfying the constraints 4.33 implies that there are no acoustic-electromagnetic waves. In fact, the weak convergence result in Theorem 4.4 could be strengthened into a strong convergence result, for well-prepared initial data and provided that the limiting system has a unique solution satisfying the energy equality (see [9, Theorem 7.4] on the strong Navier-Stokes limit).

It is to be emphasized that the generalized relative entropy method, which is developed later on in Chapter 12 and is used to prove Theorems 4.5 and 4.6 below, is also applicable to the asymptotic regime studied in Theorem 4.4. This method would provide some strong convergence result even for ill-prepared initial 
data provided we can build an approximate solution which is smooth and accounts for the corrections due to the initial layer and the acoustic-electromagnetic waves.

\subsection{The two-fluid incompressible Navier-Stokes-Fourier-Maxwell system with (solenoidal) Ohm's law}

According to Section 2.4, we consider now a plasma constituted of two species of oppositely charged particles with approximately equal mass, namely cations (positively charged ions) and anions (negatively charged ions). Elementary interactions are taken into account by both mean field terms (corresponding to long-range interactions) and some local collision terms (associated to short-range interactions) involving possibly different mean free paths. Thus, the charged particles evolve under the coupled effect of the Lorentz force due to the self-induced electromagnetic field, and of the collisions with other particles, according to the following scaled two species Vlasov-Maxwell-Boltzmann system :

$$
\left\{\begin{aligned}
\epsilon \partial_{t} f_{\epsilon}^{ \pm}+v \cdot \nabla_{x} f_{\epsilon}^{ \pm} \pm \delta\left(\epsilon E_{\epsilon}+v \wedge B_{\epsilon}\right) \cdot \nabla_{v} f_{\epsilon}^{ \pm} & =\frac{1}{\epsilon} Q\left(f_{\epsilon}^{ \pm}, f_{\epsilon}^{ \pm}\right)+\frac{\delta^{2}}{\epsilon} Q\left(f_{\epsilon}^{ \pm}, f_{\epsilon}^{\mp}\right), \\
f_{\epsilon}^{ \pm} & =M\left(1+\epsilon g_{\epsilon}^{ \pm}\right), \\
\partial_{t} E_{\epsilon}-\operatorname{rot} B_{\epsilon} & =-\frac{\delta}{\epsilon} \int_{\mathbb{R}^{3}}\left(g_{\epsilon}^{+}-g_{\epsilon}^{-}\right) v M d v, \\
\partial_{t} B_{\epsilon}+\operatorname{rot} E_{\epsilon} & =0, \\
\operatorname{div} E_{\epsilon} & =\delta \int_{\mathbb{R}^{3}}\left(g_{\epsilon}^{+}-g_{\epsilon}^{-}\right) M d v, \\
\operatorname{div} B_{\epsilon} & =0,
\end{aligned}\right.
$$

where $\frac{\delta}{\epsilon}$ is asymptotically unbounded. In this scaling, the entropy inequality states that

$$
\begin{aligned}
\frac{1}{\epsilon^{2}} H\left(f_{\epsilon}^{+}\right)+\frac{1}{\epsilon^{2}} H\left(f_{\epsilon}^{-}\right) & +\frac{1}{2 \epsilon^{2}} \int_{\mathbb{R}^{3}} \operatorname{Tr} m_{\epsilon} d x+\frac{1}{2} \int_{\mathbb{R}^{3}}\left(\left|E_{\epsilon}\right|^{2}+\left|B_{\epsilon}\right|^{2}+\operatorname{Tr} a_{\epsilon}\right) d x \\
& +\frac{1}{\epsilon^{4}} \int_{0}^{t} \int_{\mathbb{R}^{3}}\left(D\left(f_{\epsilon}^{+}\right)+D\left(f_{\epsilon}^{-}\right)+\delta^{2} D\left(f_{\epsilon}^{+}, f_{\epsilon}^{-}\right)\right)(s) d x d s \\
& \leq \frac{1}{\epsilon^{2}} H\left(f_{\epsilon}^{+\mathrm{in}}\right)+\frac{1}{\epsilon^{2}} H\left(f_{\epsilon}^{-\mathrm{in}}\right)+\frac{1}{2} \int_{\mathbb{R}^{3}}\left(\left|E_{\epsilon}^{\mathrm{in}}\right|^{2}+\left|B_{\epsilon}^{\mathrm{in}}\right|^{2}\right) d x
\end{aligned}
$$

where $H\left(f_{\epsilon}^{ \pm}\right)=H\left(f_{\epsilon}^{ \pm} \mid M\right)$ and the symmetric positive definite matrix measures $m_{\epsilon}$ and $a_{\epsilon}$ are the defects introduced in Section 4.1.4 stemming from the terms $\int_{\mathbb{R}^{3}}\left(f_{\epsilon}^{+}+f_{\epsilon}^{-}\right) v \otimes v d v$ and $\left(\begin{array}{c}E_{\epsilon} \\ B_{\epsilon}\end{array}\right) \otimes\left(\begin{array}{l}E_{\epsilon} \\ B_{\epsilon}\end{array}\right)$, respectively. In particular, it yields uniform bounds on $E_{\epsilon}, B_{\epsilon}$ and $g_{\epsilon}^{ \pm}$.

Since we are interested in the limiting fluctuation $g_{\epsilon}^{ \pm} \rightarrow g^{ \pm}$, it is then natural to rewrite the kinetic equations in terms of the fluctuations $g_{\epsilon}^{ \pm}$,

$$
\begin{aligned}
& \epsilon \partial_{t}\left(\begin{array}{c}
g_{\epsilon}^{+} \\
g_{\epsilon}^{-}
\end{array}\right)+v \cdot \nabla_{x}\left(\begin{array}{c}
g_{\epsilon}^{+} \\
g_{\epsilon}^{-}
\end{array}\right)+\delta\left(\epsilon E_{\epsilon}+v \wedge B_{\epsilon}\right) \cdot \nabla_{v}\left(\begin{array}{c}
g_{\epsilon}^{+} \\
-g_{\epsilon}^{-}
\end{array}\right)-\delta E_{\epsilon} \cdot v\left(\begin{array}{c}
1+\epsilon g_{\epsilon}^{+} \\
-1-\epsilon g_{\epsilon}^{-}
\end{array}\right) \\
&=-\frac{1}{\epsilon}\left(\begin{array}{c}
\mathcal{L} g_{\epsilon}^{+}+\delta^{2} \mathcal{L}\left(g_{\epsilon}^{+}, g_{\epsilon}^{-}\right) \\
\mathcal{L} g_{\epsilon}^{-}+\delta^{2} \mathcal{L}\left(g_{\epsilon}^{-}, g_{\epsilon}^{+}\right)
\end{array}\right)+\left(\begin{array}{c}
\mathcal{Q}\left(g_{\epsilon}^{+}, g_{\epsilon}^{+}\right)+\delta^{2} \mathcal{Q}\left(g_{\epsilon}^{+}, g_{\epsilon}^{-}\right) \\
\mathcal{Q}\left(g_{\epsilon}^{-}, g_{\epsilon}^{-}\right)+\delta^{2} \mathcal{Q}\left(g_{\epsilon}^{-}, g_{\epsilon}^{+}\right)
\end{array}\right) .
\end{aligned}
$$

According to the formal analysis from Section 2.4 we then expect the limiting macroscopic observables to solve, in the case of strong interspecies collisions $\delta=$ 1, the two-fluid incompressible Navier-Stokes-Fourier-Maxwell system with Ohm's 
law :

$$
\left\{\begin{aligned}
\partial_{t} u+u \cdot \nabla_{x} u-\mu \Delta_{x} u & =-\nabla_{x} p+\frac{1}{2}(n E+j \wedge B), & & \operatorname{div} u=0, \\
\partial_{t} \theta+u \cdot \nabla_{x} \theta-\kappa \Delta_{x} \theta & =0, & \rho+\theta & =0, \\
\partial_{t} E-\operatorname{rot} B & =-j, & \operatorname{div} E & =n, \\
\partial_{t} B+\operatorname{rot} E & =0, & \operatorname{div} B & =0, \\
j-n u & =\sigma\left(-\frac{1}{2} \nabla_{x} n+E+u \wedge B\right), & & =n \theta,
\end{aligned}\right.
$$

and, in the case of weak interspecies collisions $\delta=o(1)$, with $\frac{\delta}{\epsilon}$ unbounded, the twofluid incompressible Navier-Stokes-Fourier-Maxwell system with solenoidal Ohm's law :

$$
\left\{\begin{aligned}
\partial_{t} u+u \cdot \nabla_{x} u-\mu \Delta_{x} u & =-\nabla_{x} p+\frac{1}{2} j \wedge B, & \operatorname{div} u & =0, \\
\partial_{t} \theta+u \cdot \nabla_{x} \theta-\kappa \Delta_{x} \theta & =0, & \rho+\theta & =0, \\
\partial_{t} E-\operatorname{rot} B & =-j, & \operatorname{div} E & =0, \\
\partial_{t} B+\operatorname{rot} E & =0, & \operatorname{div} B & =0, \\
j & =\sigma\left(-\nabla_{x} \bar{p}+E+u \wedge B\right), & \operatorname{div} j & =0, \\
n & =0, & w & =0 .
\end{aligned}\right.
$$

As previously emphasized in Section 3.2, the above limiting models (4.37) and (4.38 are not stable under weak convergence in the energy space and, thus, share more similarities with the three-dimensional incompressible Euler equations.

Our goal, here, is to establish the convergence of scaled families of renormalized solutions to the two species Vlasov-Maxwell-Boltzmann system 4.34 towards dissipative solutions of the two-fluid incompressible Navier-Stokes-Fourier-Maxwell system with Ohm's law 4.37), in the case of strong interspecies interactions $\delta=1$, or with solenoidal Ohm's law 4.38, in the case of weak interspecies interactions $\delta=o(1)$, with $\frac{\delta}{\epsilon}$ unbounded, without any restriction on their size or regularity. We will, however, impose some well-preparedness of the initial data.

Improving on the program by the second author completed in 67, 69 (which relies essentially on modulated entropy arguments), we can prove the following theorems. Recall that, in this second part, we are only considering the Maxwellian cross-section $b \equiv 1$.

4.3.1. Weak interactions. In the case of weak interspecies interactions $\delta=$ $o(1)$, with $\frac{\delta}{\epsilon}$ unbounded, we have the following result.

THEOREM 4.5. Let $\left(f_{\epsilon}^{ \pm \mathrm{in}}, E_{\epsilon}^{\mathrm{in}}, B_{\epsilon}^{\mathrm{in}}\right)$ be a family of initial data such that

$$
\frac{1}{\epsilon^{2}} H\left(f_{\epsilon}^{+ \text {in }}\right)+\frac{1}{\epsilon^{2}} H\left(f_{\epsilon}^{- \text {in }}\right)+\frac{1}{2} \int_{\mathbb{R}^{3}}\left(\left|E_{\epsilon}^{\text {in }}\right|^{2}+\left|B_{\epsilon}^{\text {in }}\right|^{2}\right) d x \leq C^{\text {in }},
$$

for some $C^{\text {in }}>0$, and

$$
\operatorname{div} E_{\epsilon}^{\mathrm{in}}=\delta \int_{\mathbb{R}^{3}}\left(g_{\epsilon}^{+\mathrm{in}}-g_{\epsilon}^{-\mathrm{in}}\right) M d v, \quad \operatorname{div} B_{\epsilon}^{\mathrm{in}}=0,
$$

where $f_{\epsilon}^{ \pm \mathrm{in}}=M\left(1+\epsilon g_{\epsilon}^{ \pm \mathrm{in}}\right)$. We further assume that the initial data is wellprepared in the sense that, as $\epsilon \rightarrow 0$,

$$
g_{\epsilon}^{ \pm \mathrm{in}} \rightarrow g^{\mathrm{in}}=\rho^{\mathrm{in}}+u^{\mathrm{in}} \cdot v+\theta^{\mathrm{in}}\left(\frac{|v|^{2}}{2}-\frac{3}{2}\right) \quad \text { in } w-L_{\mathrm{loc}}^{1}(M d x d v),
$$

where $\rho^{\text {in }}, u^{\text {in }}, \theta^{\text {in }} \in L^{2}(d x)$ satisfy the incompressibility and Boussinesq constraints

$$
\operatorname{div} u^{\text {in }}=0, \quad \rho^{\text {in }}+\theta^{\text {in }}=0,
$$


and that the following strong convergences hold, as $\epsilon \rightarrow 0$,

$$
\begin{aligned}
\frac{1}{\epsilon^{2}} H\left(f_{\epsilon}^{+ \text {in }}\right)+\frac{1}{\epsilon^{2}} H\left(f_{\epsilon}^{- \text {in }}\right) & \rightarrow \int_{\mathbb{R}^{3} \times \mathbb{R}^{3}}\left(g^{\text {in }}\right)^{2} M d v d x=\int_{\mathbb{R}^{3}}\left|u^{\text {in }}\right|^{2}+\frac{5}{2}\left(\theta^{\text {in }}\right)^{2} d x, \\
E_{\epsilon}^{\text {in }} & \rightarrow E^{\text {in }}, \quad \text { in } L^{2}(d x), \\
B_{\epsilon}^{\text {in }} & \rightarrow B^{\text {in }}, \quad \text { in } L^{2}(d x),
\end{aligned}
$$

for some $E^{\text {in }}, B^{\text {in }} \in L^{2}(d x)$. In particular, in view of 4.40, it necessarily holds that

$$
\operatorname{div} E^{\text {in }}=0, \quad \operatorname{div} B^{\text {in }}=0 .
$$

For any $\epsilon>0$, we assume the existence of a renormalized solution $\left(f_{\epsilon}^{ \pm}, E_{\epsilon}, B_{\epsilon}\right)$ to the scaled two species Vlasov-Maxwell-Boltzmann system 4.34) (for the Maxwellian cross-section $b \equiv 1)$, where $\delta=o(1)$ and $\frac{\delta}{\epsilon}$ is asymptotically unbounded, with initial data $\left(f_{\epsilon}^{ \pm \mathrm{in}}, E_{\epsilon}^{\mathrm{in}}, B_{\epsilon}^{\mathrm{in}}\right)$. We define the macroscopic fluctuations of density $\rho_{\epsilon}^{ \pm}$, bulk velocity $u_{\epsilon}^{ \pm}$and temperature $\theta_{\epsilon}^{ \pm}$by

$$
\begin{aligned}
& \rho_{\epsilon}^{ \pm}=\int_{\mathbb{R}^{3}} g_{\epsilon}^{ \pm} M d v, \\
& u_{\epsilon}^{ \pm}=\int_{\mathbb{R}^{3}} g_{\epsilon}^{ \pm} v M d v, \\
& \theta_{\epsilon}^{ \pm}=\int_{\mathbb{R}^{3}} g_{\epsilon}^{ \pm}\left(\frac{|v|^{2}}{3}-1\right) M d v .
\end{aligned}
$$

We finally define the hydrodynamic variables

$$
\rho_{\epsilon}=\frac{\rho_{\epsilon}^{+}+\rho_{\epsilon}^{-}}{2}, \quad u_{\epsilon}=\frac{u_{\epsilon}^{+}+u_{\epsilon}^{-}}{2}, \quad \theta_{\epsilon}=\frac{\theta_{\epsilon}^{+}+\theta_{\epsilon}^{-}}{2},
$$

and electrodynamic variables

$$
n_{\epsilon}=\rho_{\epsilon}^{+}-\rho_{\epsilon}^{-}, \quad j_{\epsilon}=\frac{\delta}{\epsilon}\left(u_{\epsilon}^{+}-u_{\epsilon}^{-}\right), \quad w_{\epsilon}=\frac{\delta}{\epsilon}\left(\theta_{\epsilon}^{+}-\theta_{\epsilon}^{-}\right) .
$$

Then, the family $\left(\rho_{\epsilon}, u_{\epsilon}, \theta_{\epsilon}, n_{\epsilon}, j_{\epsilon}, w_{\epsilon}, E_{\epsilon}, B_{\epsilon}\right)$ is weakly relatively compact in $L_{\text {loc }}^{1}(d t d x)$ and any of its limit points $(\rho, u, \theta, n, j, w, E, B)$ is a dissipative solution of the two-fluid incompressible Navier-Stokes-Fourier-Maxwell system with solenoidal Ohm's law 4.38) with initial data $\left(u^{\text {in }}, \theta^{\text {in }}, E^{\text {in }}, B^{\text {in }}\right)$ - that is, it verifies the energy inequality corresponding to 4.38, it enjoys the weak temporal continuity $(u, \theta, E, B) \in C\left([0, \infty) ; w-L^{2}\left(\mathbb{R}^{3}\right)\right)$, it solves the system

$$
\left\{\begin{aligned}
\operatorname{div} u & =0, & \rho+\theta & =0, \\
\partial_{t} E-\operatorname{rot} B & =-j, & \operatorname{div} E & =0, \\
\partial_{t} B+\operatorname{rot} E & =0, & \operatorname{div} B & =0, \\
j & =\sigma\left(-\nabla_{x} \bar{p}+E+u \wedge B\right), & \operatorname{div} j & =0, \\
n & =0, & w & =0,
\end{aligned}\right.
$$

in the sense of distributions, and it satisfies the stability inequality

$$
\begin{aligned}
& \delta \mathcal{E}(t)+\frac{1}{2} \int_{0}^{t} \delta \mathcal{D}(s) e^{\int_{s}^{t} \lambda(\sigma) d \sigma} d s \\
& \leq \delta \mathcal{E}(0) e^{\int_{0}^{t} \lambda(s) d s}+\int_{0}^{t} \int_{\mathbb{R}^{3}} \mathbf{A} \cdot\left(\begin{array}{c}
u-\bar{u} \\
\frac{5}{2}(\theta-\bar{\theta}) \\
j-\bar{j} \\
E-\bar{E}+\bar{u} \wedge(B-\bar{B}) \\
B-\bar{B}+(E-\bar{E}) \wedge \bar{u}
\end{array}\right)(s) d x e^{\int_{s}^{t} \lambda(\sigma) d \sigma} d s,
\end{aligned}
$$


4.3. THE TWO-FLUID INCOMPRESSIBLE NAVIER-STOKES-FOURIER-MAXWELL. . 117

for any test functions $(\bar{u}, \bar{\theta}, \bar{j}, \bar{E}, \bar{B}) \in C_{c}^{\infty}\left([0, \infty) \times \mathbb{R}^{3}\right)$ with

$$
\operatorname{div} \bar{u}=\operatorname{div} \bar{j}=\operatorname{div} \bar{E}=\operatorname{div} \bar{B}=0 \quad \text { and } \quad\|\bar{u}\|_{L^{\infty}(d t d x)}<1,
$$

where the modulated energy and modulated energy dissipation are respectively given by

$$
\begin{aligned}
\delta \mathcal{E}(t) & =\|u-\bar{u}\|_{L^{2}(d x)}^{2}+\frac{5}{2}\|\theta-\bar{\theta}\|_{L^{2}(d x)}^{2}+\frac{1}{2}\|E-\bar{E}\|_{L^{2}(d x)}^{2}+\frac{1}{2}\|B-\bar{B}\|_{L^{2}(d x)}^{2} \\
& -\int_{\mathbb{R}^{3}}\left(\left(E_{\epsilon}-\bar{E}\right) \wedge\left(B_{\epsilon}-\bar{B}\right)\right) \cdot \bar{u} d x, \\
\delta \mathcal{D}(t) & =2 \mu\left\|\nabla_{x}(u-\bar{u})\right\|_{L_{x}^{2}}^{2}+5 \kappa\left\|\nabla_{x}(\theta-\bar{\theta})\right\|_{L_{x}^{2}}^{2}+\frac{1}{\sigma}\|j-\bar{j}\|_{L_{x}^{2}}^{2},
\end{aligned}
$$

the acceleration operator is defined by

$$
\mathbf{A}(\bar{u}, \bar{\theta}, \bar{j}, \bar{E}, \bar{B})=\left(\begin{array}{c}
-2\left(\partial_{t} \bar{u}+P\left(\bar{u} \cdot \nabla_{x} \bar{u}\right)-\mu \Delta_{x} \bar{u}\right)+P(\bar{j} \wedge \bar{B}) \\
-2\left(\partial_{t} \bar{\theta}+\bar{u} \cdot \nabla_{x} \bar{\theta}-\kappa \Delta_{x} \bar{\theta}\right) \\
-\frac{1}{\sigma} \bar{j}+P(\bar{E}+\bar{u} \wedge \bar{B}) \\
-\left(\partial_{t} \bar{E}-\operatorname{rot} \bar{B}+\bar{j}\right) \\
-\left(\partial_{t} \bar{B}+\operatorname{rot} \bar{E}\right)
\end{array}\right)
$$

and the growth rate is given by

$$
\left.\begin{array}{rl}
\lambda(t)=C\left(\frac{\|\bar{u}(t)\|_{W^{1, \infty}(d x)}+\left\|\partial_{t} \bar{u}(t)\right\|_{L^{\infty}(d x)}+\|\bar{j}(t)\|_{L^{\infty}(d x)}}{1-\|\bar{u}(t)\|_{L^{\infty}(d x)}}\right. & \\
+\|\bar{\theta}(t)\|_{W^{1, \infty}(d x)}+\|\bar{\theta}(t)\|_{W^{1, \infty}(d x)}^{2}
\end{array}\right),
$$

with a constant $C>0$ independent of test functions.

In particular, this dissipative solution coincides with the unique smooth solution with velocity field bounded pointwise by the speed of light (i.e. $\left.\|u\|_{L^{\infty}(d t d x)}<1\right)$ as long as the latter exists.

The proof of Theorem 4.5 is built over the course of the coming chapters and is per se the subject of Section 12.2 .

4.3.2. Strong interactions. In the case of strong interspecies interactions $\delta=1$, we have the following result.

THEOREM 4.6. Let $\left(f_{\epsilon}^{ \pm \mathrm{in}}, E_{\epsilon}^{\mathrm{in}}, B_{\epsilon}^{\mathrm{in}}\right)$ be a family of initial data such that

$$
\frac{1}{\epsilon^{2}} H\left(f_{\epsilon}^{+\mathrm{in}}\right)+\frac{1}{\epsilon^{2}} H\left(f_{\epsilon}^{-\mathrm{in}}\right)+\frac{1}{2} \int_{\mathbb{R}^{3}}\left(\left|E_{\epsilon}^{\mathrm{in}}\right|^{2}+\left|B_{\epsilon}^{\mathrm{in}}\right|^{2}\right) d x \leq C^{\mathrm{in}},
$$

for some $C^{\text {in }}>0$, and

$$
\operatorname{div} E_{\epsilon}^{\mathrm{in}}=\int_{\mathbb{R}^{3}}\left(g_{\epsilon}^{+\mathrm{in}}-g_{\epsilon}^{-\mathrm{in}}\right) M d v, \quad \operatorname{div} B_{\epsilon}^{\mathrm{in}}=0,
$$

where $f_{\epsilon}^{ \pm \mathrm{in}}=M\left(1+\epsilon g_{\epsilon}^{ \pm \mathrm{in}}\right)$. We further assume that the initial data is wellprepared in the sense that, as $\epsilon \rightarrow 0$,

$$
g_{\epsilon}^{ \pm \text {in }} \rightarrow g^{ \pm \text {in }}=\rho^{ \pm \text {in }}+u^{\text {in }} \cdot v+\theta^{\text {in }}\left(\frac{|v|^{2}}{2}-\frac{3}{2}\right) \quad \text { in } w-L_{\text {loc }}^{1}(M d x d v),
$$

where $\rho^{ \pm \text {in }}, u^{\text {in }}, \theta^{\text {in }} \in L^{2}(d x)$ satisfy the incompressibility and Boussinesq constraints, denoting $\rho^{\text {in }}=\frac{\rho^{+ \text {in }}+\rho^{- \text {in }}}{2}$,

$$
\operatorname{div} u^{\text {in }}=0, \quad \rho^{\text {in }}+\theta^{\text {in }}=0,
$$


and that the following strong convergences hold, as $\epsilon \rightarrow 0$,

$$
\begin{aligned}
\frac{1}{\epsilon^{2}} H\left(f_{\epsilon}^{+ \text {in }}\right)+\frac{1}{\epsilon^{2}} H\left(f_{\epsilon}^{- \text {in }}\right) & \rightarrow \int_{\mathbb{R}^{3} \times \mathbb{R}^{3}} \frac{1}{2}\left(g^{+ \text {in }}\right)^{2}+\frac{1}{2}\left(g^{- \text {in }}\right)^{2} M d v d x \\
& =\int_{\mathbb{R}^{3}} \frac{1}{4}\left(n^{\text {in }}\right)^{2}+\left|u^{\text {in }}\right|^{2}+\frac{5}{2}\left(\theta^{\text {in }}\right)^{2} d x, \\
E_{\epsilon}^{\text {in }} & \rightarrow E^{\text {in }}, \quad \text { in } L^{2}(d x), \\
B_{\epsilon}^{\text {in }} & \rightarrow B^{\text {in }}, \quad \text { in } L^{2}(d x),
\end{aligned}
$$

for some $E^{\mathrm{in}}, B^{\mathrm{in}} \in L^{2}(d x)$. In particular, in view of (4.43), it necessarily holds that, denoting $n^{\text {in }}=\rho^{+ \text {in }}-\rho^{- \text {in }}$,

$$
\operatorname{div} E^{\text {in }}=n^{\text {in }}, \quad \operatorname{div} B^{\text {in }}=0 .
$$

For any $\epsilon>0$, we assume the existence of a renormalized solution $\left(f_{\epsilon}^{ \pm}, E_{\epsilon}, B_{\epsilon}\right)$ to the scaled two species Vlasov-Maxwell-Boltzmann system (4.34) (for the Maxwellian cross-section $b \equiv 1)$, where $\delta=1$, with initial data $\left(f_{\epsilon}^{ \pm \mathrm{in}}, E_{\epsilon}^{\mathrm{in}}, B_{\epsilon}^{\mathrm{in}}\right)$. We define the macroscopic fluctuations of density $\rho_{\epsilon}^{ \pm}$, bulk velocity $u_{\epsilon}^{ \pm}$and temperature $\theta_{\epsilon}^{ \pm}$by

$$
\begin{aligned}
& \rho_{\epsilon}^{ \pm}=\int_{\mathbb{R}^{3}} g_{\epsilon}^{ \pm} M d v, \\
& u_{\epsilon}^{ \pm}=\int_{\mathbb{R}^{3}} g_{\epsilon}^{ \pm} v M d v, \\
& \theta_{\epsilon}^{ \pm}=\int_{\mathbb{R}^{3}} g_{\epsilon}^{ \pm}\left(\frac{|v|^{2}}{3}-1\right) M d v .
\end{aligned}
$$

We finally define the hydrodynamic variables

$$
\rho_{\epsilon}=\frac{\rho_{\epsilon}^{+}+\rho_{\epsilon}^{-}}{2}, \quad u_{\epsilon}=\frac{u_{\epsilon}^{+}+u_{\epsilon}^{-}}{2}, \quad \theta_{\epsilon}=\frac{\theta_{\epsilon}^{+}+\theta_{\epsilon}^{-}}{2},
$$

and electrodynamic variables

$$
n_{\epsilon}=\rho_{\epsilon}^{+}-\rho_{\epsilon}^{-}, \quad j_{\epsilon}=\frac{1}{\epsilon}\left(u_{\epsilon}^{+}-u_{\epsilon}^{-}\right), \quad w_{\epsilon}=\frac{1}{\epsilon}\left(\theta_{\epsilon}^{+}-\theta_{\epsilon}^{-}\right) .
$$

Then, the family $\left(\rho_{\epsilon}, u_{\epsilon}, \theta_{\epsilon}, n_{\epsilon}, j_{\epsilon}, E_{\epsilon}, B_{\epsilon}\right)$ (note that we have excluded the variable $w_{\epsilon}$ ) is relatively compact in the sense that for every sequence in this family there exists a subsequence such that

$$
\left(\rho_{\epsilon}, u_{\epsilon}, \theta_{\epsilon}, n_{\epsilon}, r_{\epsilon} j_{\epsilon}, E_{\epsilon}, B_{\epsilon}\right) \rightarrow(\rho, u, \theta, n, j, E, B) \quad \text { in } w-L_{\mathrm{loc}}^{1}(d t d x),
$$

where $r_{\epsilon}(t, x)$ is a sequence of mesurable scalar functions converging almost everywhere towards the constant function 1.

Moreover, up to further extraction of subsequences, one also has the convergence

$$
r_{\epsilon} \frac{1}{\epsilon}\left(g_{\epsilon}^{+}-g_{\epsilon}^{-}-n_{\epsilon}\right) \rightarrow h \quad \text { in } w-L_{\mathrm{loc}}^{1}\left(d t d x ; L^{1}((1+|v|) M d v)\right) .
$$

We set

$$
w=\int_{\mathbb{R}^{3}} h\left(\frac{|v|^{2}}{3}-1\right) M d v .
$$

Note that $w$ is not necessarily a limit point of $w_{\epsilon}$.

Any such limit point $(\rho, u, \theta, n, j, w, E, B)$ is a dissipative solution of the twofluid incompressible Navier-Stokes-Fourier-Maxwell system with Ohm's law (4.37) with initial data $\left(u^{\text {in }}, \theta^{\text {in }}, n^{\text {in }}, E^{\text {in }}, B^{\text {in }}\right)$ - that is, it verifies the energy inequality 
corresponding to 4.37), it enjoys the weak temporal continuity $(u, \theta, n, E, B) \in$ $C\left([0, \infty) ; w-L^{2}\left(\mathbb{R}^{3}\right)\right)$, it solves the system

$$
\left\{\begin{array}{rlrl}
\operatorname{div} u & =0, & \rho+\theta & =0, \\
\operatorname{div} E & =n, & \operatorname{div} B & =0, \\
\partial_{t} B+\operatorname{rot} E & =0, & j-n u & =\sigma\left(-\frac{1}{2} \nabla_{x} n+E+u \wedge B\right), \\
w & =n \theta,
\end{array}\right.
$$

in the sense of distributions (note that we have voluntarily left Ampère's equation out of the above system), and it satisfies the stability inequality

$$
\begin{aligned}
\delta \mathcal{E}(t) & +\frac{1}{2} \int_{0}^{t} \delta \mathcal{D}(s) e^{\int_{s}^{t} \lambda(\sigma) d \sigma} d s \\
& \leq \delta \mathcal{E}(0) e^{\int_{0}^{t} \lambda(s) d s} \\
& +\int_{0}^{t} \int_{\mathbb{R}^{3}} \mathbf{A} \cdot\left(\begin{array}{c}
u-\bar{u} \\
\frac{5}{2}(\theta-\bar{\theta}) \\
j-n u-(\bar{j}-\bar{n} \bar{u}) \\
E-\bar{E}+\bar{u} \wedge(B-\bar{B})-\frac{1}{2} \nabla_{x}(n-\bar{n}) \\
B-\bar{B}+(E-\bar{E}) \wedge \bar{u}
\end{array}\right)(s) d x e^{\int_{s}^{t} \lambda(\sigma) d \sigma} d s,
\end{aligned}
$$

for any test functions $(\bar{u}, \bar{\theta}, \bar{n}, \bar{j}, \bar{E}, \bar{B}) \in C_{c}^{\infty}\left([0, \infty) \times \mathbb{R}^{3}\right)$ with

$$
\operatorname{div} \bar{u}=\operatorname{div} \bar{B}=0, \quad \operatorname{div} \bar{E}=\bar{n} \quad \text { and } \quad\|\bar{u}\|_{L^{\infty}(d t d x)}<1,
$$

where the modulated energy and modulated energy dissipation are respectively given by

$$
\begin{aligned}
\delta \mathcal{E}(t) & =\frac{1}{4}\|n-\bar{n}\|_{L^{2}(d x)}^{2}+\|u-\bar{u}\|_{L^{2}(d x)}^{2}+\frac{5}{2}\|\theta-\bar{\theta}\|_{L^{2}(d x)}^{2} \\
& +\frac{1}{2}\|E-\bar{E}\|_{L^{2}(d x)}^{2}+\frac{1}{2}\|B-\bar{B}\|_{L^{2}(d x)}^{2}-\int_{\mathbb{R}^{3}}\left(\left(E_{\epsilon}-\bar{E}\right) \wedge\left(B_{\epsilon}-\bar{B}\right)\right) \cdot \bar{u} d x, \\
\delta \mathcal{D}(t) & =2 \mu\left\|\nabla_{x}(u-\bar{u})\right\|_{L_{x}^{2}}^{2}+5 \kappa\left\|\nabla_{x}(\theta-\bar{\theta})\right\|_{L_{x}^{2}}^{2}+\frac{1}{\sigma}\|(j-n u)-(\bar{j}-\bar{n} \bar{u})\|_{L_{x}^{2}}^{2},
\end{aligned}
$$

the acceleration operator is defined by

$$
\mathbf{A}(\bar{u}, \bar{\theta}, \bar{j}, \bar{E}, \bar{B})=\left(\begin{array}{c}
-2\left(\partial_{t} \bar{u}+P\left(\bar{u} \cdot \nabla_{x} \bar{u}\right)-\mu \Delta_{x} \bar{u}\right)+P(\bar{n} \bar{E}+\bar{j} \wedge \bar{B}) \\
-2\left(\partial_{t} \bar{\theta}+\bar{u} \cdot \nabla_{x} \bar{\theta}-\kappa \Delta_{x} \bar{\theta}\right) \\
-\frac{1}{\sigma}\left(\bar{j}-\bar{n} \bar{u}-\frac{1}{2} \nabla_{x} \bar{n}+\bar{E}+\bar{u} \wedge \bar{B}\right. \\
-\left(\partial_{t} \bar{E}-\operatorname{rot} \bar{B}+\bar{j}\right) \\
-\left(\partial_{t} \bar{B}+\operatorname{rot} \bar{E}\right)
\end{array}\right)
$$

and the growth rate is given by

$$
\begin{aligned}
& \lambda(t)= \\
& C\left(\frac{\|\bar{u}(t)\|_{W^{1, \infty}(d x)}+\left\|\partial_{t} \bar{u}(t)\right\|_{L^{\infty}(d x)}+\|\bar{\theta}(t)\|_{W^{1, \infty}(d x)}+\|(\bar{j}-\bar{n} \bar{u})(t)\|_{L^{\infty}(d x)}}{1-\|\bar{u}(t)\|_{L^{\infty}(d x)}}\right. \\
& \left.+\|\bar{\theta}(t)\|_{W^{1, \infty}(d x)}^{2}+\left\|\left(\frac{1}{2} \nabla_{x} \bar{n}-\bar{E}-\bar{u} \wedge \bar{B}\right)(t)\right\|_{L^{\infty}(d x)}\right),
\end{aligned}
$$

with a constant $C>0$ independent of test functions.

In particular, this dissipative solution coincides with the unique smooth solution with velocity field bounded pointwise by the speed of light (i.e. $\left.\|u\|_{L^{\infty}(d t d x)}<1\right)$ as long as the latter exists. 
The proof of Theorem 4.6 is built over the course of the coming chapters and is per se the subject of Section 12.3 .

In both Theorems 4.5 and 4.6, we focus on the case of well-prepared initial data. That is to say, we assume that the initial distribution has a velocity profile close to local thermodynamic equilibrium

$$
g^{ \pm \text {in }}=\rho^{ \pm \text {in }}+u^{\text {in }} \cdot v+\theta^{\text {in }}\left(\frac{|v|^{2}}{2}-\frac{3}{2}\right)
$$

(with $g^{+ \text {in }}=g^{- \text {in }}$ in the case of weak interactions) in order that there is no relaxation layer, and that the asymptotic initial thermodynamic fields satisfy the incompressibility and Boussinesq constraints

$$
\operatorname{div} u^{\text {in }}=0, \quad \rho^{\text {in }}+\theta^{\text {in }}=0,
$$

which ensures that there are no acoustic waves.

The case of ill-prepared initial data could be handled by constructing an accurate approximate solution as in 69. The corresponding result should be even better in the present viscous incompressible regime because we can control conservation defects and fluxes without any additional integrability assumptions on renormalized solutions to (4.34). Note, however, that such a result would still be conditional as the existence of renormalized solutions to 4.34 has to be assumed.

Relaxing the regularity assumption on the asymptotic solution would require new ideas : the stability in the energy and entropy methods is indeed controlled by higher integrability or regularity norms of the limiting fields. As discussed in Section 3.2 the two-fluid incompressible Navier-Stokes-Fourier-Maxwell systems with (solenoidal) Ohm's law (4.37) and 4.38) are not known to have weak solutions, so that we do not expect to extend our convergence results for distributional solutions with low regularity.

\subsection{Outline of proofs}

We expect the Vlasov-Maxwell-Boltzmann systems (4.27) and 4.34) to exhibit very different qualitative behaviors in the three asymptotic scalings we consider : one species, two species with weak interactions, and two species with strong interactions. However, estimates coming directly from the entropy inequalities 4.28 and 4.35 and leading to weak compactness results are similar in all regimes, so we will gather them in Chapter 5 . We will also obtain the thermodynamic equilibria coming from relaxation estimates in Chapter 5

Then, Chapter 6 will be devoted to the derivation of constraints which are stable under weak convergence and can be handled with the weak bounds from Chapter 5. These constraints include, for instance, some lower order macroscopic constraints (such as the Boussinesq and incompressibility relations) for one species or two species with weak interactions. We will also establish the limiting energy inequalities for one species and two species with weak interactions and discuss the limiting form of Maxwell's system. This chapter does not handle the constraints pertaining to two species with strong interactions, which will require the more advanced techniques of the following chapters.

A major difference between regimes appears in Chapter 7 regarding spatial regularity. The basic idea is to use the hypoellipticity of the free transport operator - as studied in [6] - to transfer regularity from the $v$ variable to the $x$ variable. But, because of the singularity in the Lorentz force, source terms in the kinetic equations are of different sizes so that different renormalizations of the kinetic equations will have to be considered for the three different regimes. Roughly speaking, we will be able to establish some strong compactness and equi-integrability on the fluctuations 
in the less singular regime with only one species, and only some weaker analog on some truncated fluctuations for two species (with a truncation depending on the asymptotic parameter $\delta$ ).

Another important difference comes from the nonlinear constraints (which occur only in the cases of two species). We will see in Chapter 8 that, for weak interactions, Ohm's law is obtained as a higher order singular perturbation. Its derivation will use the renormalized form of the kinetic equations as well as the partial equi-integrability established in Chapter 7. In the case of strong interactions, even though Ohm's law appears at leading order, proving its stability is much more intricate as it will require an additional macroscopic renormalization. Chapter 8 will also contain the derivation of the energy inequality for strong interactions, which will require the use of the strong compactness bounds from Chapter 7, as well.

The last pieces of information we will need to get the consistency of the hydrodynamic limits are the approximate conservation laws, for which we have to go even further in the asymptotic expansions (see Chapter 9). In the case of one species, this will require to use some suitable renormalization as well as the equi-integrability established in Chapter 7 . In the case of two species, such strong equi-integrability properties are no longer available and we will have to rely on weaker bounds. Thus, in this case, we will only obtain a conditional result, in the sense that the conservation defects and remainders will be controlled by some modulated entropy. We will therefore need, later on (in Chapter 12, , some loop argument based on Grönwall's lemma to prove both the consistency and the convergence in these regimes.

In view of these differences, the convergence proofs will follow different strategies.

In the case of one species, we will use a weak compactness method which relies on some precise study of acoustic and electromagnetic waves (described in Chapter 10 and compensated compactness.

The core of the proof of convergence for one species will then be the content of Chapter 11 .

For two species with both weak and strong interactions, we will finally introduce in Chapter 12 a novel renormalized relative entropy method, which will allow to get some stability without any a priori spatial regularity. 



\section{CHAPTER 5}

\section{Weak compactness and relaxation estimates}

In this chapter, we establish and recall, from previous works on the hydrodynamic limit of the Boltzmann equation, essential weak compactness estimates on the fluctuations based on the uniform bounds provided by the scaled relative entropy inequalities (4.28), in the case of Theorem 4.4 and 4.35), in the case of Theorems 4.5 and 4.6 .

The results presented here are somewhat preliminary to the core of the proofs of Theorems 4.4, 4.5 and 4.6. Thus, they include the first rigorous steps in the proofs of our main theorems and are sometimes straightforward adaptations of lemmas from previous works on the hydrodynamic limit of the Boltzmann equation, while some are new or non-trivial adaptations. In particular, the estimates for two species of particles presented below are all novel.

First, recall that we are considering, in Theorem 4.4 a sequence of renormalized solutions $\left(f_{\epsilon}, E_{\epsilon}, B_{\epsilon}\right)$ of the scaled one species Vlasov-Maxwell-Boltzmann system (4.27) with initial data $\left(f_{\epsilon}^{\text {in }}, E_{\epsilon}^{\text {in }}, B_{\epsilon}^{\text {in }}\right)$ satisfying the uniform bound (4.31), while in Theorems 4.5 and 4.6 , we consider a sequence of renormalized solutions $\left(f_{\epsilon}^{ \pm}, E_{\epsilon}, B_{\epsilon}\right)$ of the scaled two species Vlasov-Maxwell-Boltzmann system (4.34) with initial data $\left(f_{\epsilon}^{ \pm \text {in }}, E_{\epsilon}^{\text {in }}, B_{\epsilon}^{\text {in }}\right)$ satisfying the uniform bound 4.39 .

We will conveniently employ the notations for fluctuations

$$
\begin{aligned}
f_{\epsilon} & =M G_{\epsilon}=M\left(1+\epsilon g_{\epsilon}\right), \quad f_{\epsilon}^{\text {in }}=M G_{\epsilon}^{\text {in }}=M\left(1+\epsilon g_{\epsilon}^{\text {in }}\right), \\
f_{\epsilon}^{ \pm} & =M G_{\epsilon}^{ \pm}=M\left(1+\epsilon g_{\epsilon}^{ \pm}\right), \quad f_{\epsilon}^{ \pm \text {in }}=M G_{\epsilon}^{ \pm \text {in }}=M\left(1+\epsilon g_{\epsilon}^{ \pm \text {in }}\right),
\end{aligned}
$$

and for scaled collision integrands

$$
\begin{aligned}
q_{\epsilon} & =\frac{1}{\epsilon^{2}}\left(G_{\epsilon}^{\prime} G_{\epsilon *}^{\prime}-G_{\epsilon} G_{\epsilon *}\right), \\
q_{\epsilon}^{+} & =\frac{1}{\epsilon^{2}}\left(G_{\epsilon}^{+\prime} G_{\epsilon *}^{+\prime}-G_{\epsilon}^{+} G_{\epsilon *}^{+}\right), \\
q_{\epsilon}^{-} & =\frac{1}{\epsilon^{2}}\left(G_{\epsilon}^{-{ }^{\prime}} G_{\epsilon *}^{-\prime}-G_{\epsilon}^{-} G_{\epsilon *}^{-}\right), \\
q_{\epsilon}^{+,-} & =\frac{\delta}{\epsilon^{2}}\left(G_{\epsilon}^{+{ }^{\prime}} G_{\epsilon *}^{-\prime}-G_{\epsilon}^{+} G_{\epsilon *}^{-}\right), \\
q_{\epsilon}^{-,+} & =\frac{\delta}{\epsilon^{2}}\left(G_{\epsilon}^{-{ }^{\prime}} G_{\epsilon *}^{+\prime}-G_{\epsilon}^{-} G_{\epsilon *}^{+}\right) .
\end{aligned}
$$

\subsection{Controls from the relative entropy bound}

Following [9], we introduce the non-negative convex function

$$
h(z)=(1+z) \log (1+z)-z,
$$

defined over $(-1, \infty)$. We may then recast the entropy inequalities (4.28) and (4.35) utilizing this notation to get the relative entropy bounds, for all $t \geq 0$,

$$
\frac{1}{\epsilon^{2}} H\left(f_{\epsilon}\right)=\frac{1}{\epsilon^{2}} H\left(f_{\epsilon} \mid M\right)=\int_{\mathbb{R}^{3} \times \mathbb{R}^{3}} \frac{1}{\epsilon^{2}} h\left(\epsilon g_{\epsilon}\right) M d x d v \leq C^{\text {in }},
$$


and

$$
\frac{1}{\epsilon^{2}} H\left(f_{\epsilon}^{ \pm}\right)=\frac{1}{\epsilon^{2}} H\left(f_{\epsilon}^{ \pm} \mid M\right)=\int_{\mathbb{R}^{3} \times \mathbb{R}^{3}} \frac{1}{\epsilon^{2}} h\left(\epsilon g_{\epsilon}^{ \pm}\right) M d x d v \leq C^{\mathrm{in}} .
$$

The relative entropy bounds are expected to control the size of the fluctuations $g_{\epsilon}$ and $g_{\epsilon}^{ \pm}$since

$$
h(z) \sim \frac{1}{2} z^{2}, \quad \text { near } z=0 .
$$

However, this behavior only holds asymptotically, as $z \rightarrow 0$, and thus, in order to exploit the relative entropy bounds, we will have to rely crucially on Young's inequality $\mathrm{B} .3$ for $h(z)$, presented in Appendix B.

The following lemma is a mere reformulation of Proposition 3.1 from $\mathbf{9}$. It is a consequence solely of the fact that the fluctuations satisfy the entropy bounds (5.1) and 5.2.

LEMMA 5.1. Let $f_{\epsilon}(t, x, v)$ be a family of measurable, almost everywhere nonnegative distribution functions such that, for all $t \geq 0$,

$$
\frac{1}{\epsilon^{2}} H\left(f_{\epsilon}\right)(t)=\int_{\mathbb{R}^{3} \times \mathbb{R}^{3}} \frac{1}{\epsilon^{2}} h\left(\epsilon g_{\epsilon}\right)(t) M d x d v \leq C^{\text {in }} .
$$

Then, as $\epsilon \rightarrow 0$ :

(1) any subsequence of fluctuations $g_{\epsilon}$ is uniformly bounded in

$$
L^{\infty}\left(d t ; L_{\mathrm{loc}}^{1}\left(d x ; L^{1}\left(\left(1+|v|^{2}\right) M d v\right)\right)\right),
$$

and weakly relatively compact in

$$
L_{\mathrm{loc}}^{1}\left(d t d x ; L^{1}\left(\left(1+|v|^{2}\right) M d v\right)\right) .
$$

(2) if $g$ is a weak limit point in $L_{\mathrm{loc}}^{1}\left(d t d x ; L^{1}\left(\left(1+|v|^{2}\right) M d v\right)\right)$ of the family of fluctuations $g_{\epsilon}$, then $g$ belongs to $L^{\infty}\left(d t ; L^{2}(M d x d v)\right)$ and satisfies, for almost every $t \geq 0$,

$$
\frac{1}{2} \int_{\mathbb{R}^{3} \times \mathbb{R}^{3}} g(t)^{2} M d x d v \leq C^{\text {in }} .
$$

Proof. For the sake of completeness, we recall the main ideas from the proof of Proposition 3.1 in $[\mathbf{9}$, which is based on an application of inequality (B.3). Thus, setting $y=\frac{1}{4}\left(1+|v|^{2}\right), z=g_{\epsilon}, \beta=4$ and $\alpha \geq 4 \epsilon$ in (B.3) yields, almost everywhere in $(t, x, v)$,

$$
\left(1+|v|^{2}\right)\left|g_{\epsilon}\right| \leq \frac{\alpha}{\epsilon^{2}} h\left(\epsilon g_{\epsilon}\right)+\frac{16 e^{\frac{1}{4}}}{\alpha} e^{\frac{|v|^{2}}{4}} .
$$

This is then integrated in all variables on suitable sets to demonstrate, with the entropy bound, the equi-integrability and tightness of the sequences, and thus their weak compactness.

We set $\alpha=1$ first. Then, for each measurable set $E \subset \mathbb{R}^{3}$ of finite measure, it holds that, for every $0<\epsilon \leq \frac{1}{4}$, i.e. for all but a finite number of $\epsilon$ 's,

$$
\int_{E \times \mathbb{R}^{3}}\left(1+|v|^{2}\right)\left|g_{\epsilon}(t)\right| M d x d v \leq C^{\text {in }}+16 e^{\frac{1}{4}}|E| \int_{\mathbb{R}^{3}} e^{\frac{|v|^{2}}{4}} M d v .
$$

Hence, the family $\left(1+|v|^{2}\right) g_{\epsilon}$ is uniformly bounded in $L^{\infty}\left(d t ; L_{\text {loc }}^{1}\left(d x ; L^{1}(M d v)\right)\right)$.

Similarly, for arbitrary $\alpha \geq 4 \epsilon$ and for any measurable set $E \subset[0, T] \times \mathbb{R}^{3} \times \mathbb{R}^{3}$, where $T>0$, one has that

$$
\int_{E}\left(1+|v|^{2}\right)\left|g_{\epsilon}\right| M d t d x d v \leq \alpha T C^{\text {in }}+\frac{16 e^{\frac{1}{4}}}{\alpha}|E| .
$$


This shows, by the arbitrariness of $\alpha \geq 4 \epsilon$, that the family $\left(1+|v|^{2}\right) g_{\epsilon} M$ is uniformly integrable on $[0, T] \times \mathbb{R}^{3} \times \mathbb{R}^{3}$.

Finally, for arbitrary $\alpha \geq 4 \epsilon$, any time $T>0$, any compact set $K \subset \mathbb{R}^{3}$ and any large radius $R>0$, we find that

$$
\int_{[0, T] \times K \times\{|v| \geq R\}}\left(1+|v|^{2}\right)\left|g_{\epsilon}\right| M d t d x d v \leq \alpha T C^{\text {in }}+\frac{16 e^{\frac{1}{4}}}{\alpha} T|K| \int_{\{|v| \geq R\}} e^{\frac{|v|^{2}}{4}} M d v,
$$

which, by the arbitrariness of $\alpha \geq 4 \epsilon$, clearly implies the tightness in velocity of the family $\left(1+|v|^{2}\right) g_{\epsilon} M$.

On the whole, by virtue of the Dunford-Pettis criterion [66, we infer the weak relative compactness of the family $\left(1+|v|^{2}\right) g_{\epsilon} M$ in $L_{\text {loc }}^{1}\left(d t d x ; L^{1}(d v)\right)$, which concludes the demonstration of the first assertion of the lemma.

The second assertion will follow from a convexity analysis of the relative entropy functional $H\left(f_{\epsilon}\right)$. Indeed, by convexity of $h(z)$, it holds that

$$
\frac{1}{\epsilon^{2}} h(\epsilon g)+\frac{1}{\epsilon} h^{\prime}(\epsilon g)\left(g_{\epsilon}-g\right) \leq \frac{1}{\epsilon^{2}} h\left(\epsilon g_{\epsilon}\right) .
$$

Hence, for any large $\lambda>0$, any times $0 \leq t_{1}<t_{2}$ and any compact set $K \subset \mathbb{R}^{3}$, by the non-negativity of $h(z)$, we have that, for $\epsilon<\frac{1}{\lambda}$ (so that $\epsilon g>-1$ ),

$$
\begin{aligned}
\int_{t_{1}}^{t_{2}} \int_{K \times \mathbb{R}^{3}}\left(\frac{1}{\epsilon^{2}} h(\epsilon g)+\frac{1}{\epsilon} h^{\prime}(\epsilon g)\left(g_{\epsilon}-g\right)\right) & \mathbb{1}_{\{|g| \leq \lambda\}} M d x d v d t \\
& \leq \int_{t_{1}}^{t_{2}} \int_{\mathbb{R}^{3} \times \mathbb{R}^{3}} \frac{1}{\epsilon^{2}} h\left(\epsilon g_{\epsilon}\right) M d x d v d t
\end{aligned}
$$

Furthermore, notice that $h(z)=\frac{1}{2} z^{2}-\int_{0}^{z} \frac{1}{(1+y)^{2}} \frac{(y-z)^{2}}{2} d y$ and $h^{\prime}(z)=z-\int_{0}^{z} \frac{1}{(1+y)^{2}}(y-$ $z) d y$, from which we easily deduce the strong convergences

$$
\frac{1}{\epsilon^{2}} h(\epsilon g) \mathbb{1}_{\{|g| \leq \lambda\}} \rightarrow \frac{1}{2} g^{2} \mathbb{1}_{\{|g| \leq \lambda\}} \text { and } \frac{1}{\epsilon} h^{\prime}(\epsilon g) \mathbb{1}_{\{|g| \leq \lambda\}} \rightarrow g \mathbb{1}_{\{|g| \leq \lambda\}} \text { in } L^{\infty}(d t d x d v) \text {. }
$$

Therefore, taking weak limits in the above convexity inequality yields

$$
\begin{aligned}
\int_{t_{1}}^{t_{2}} \int_{K \times \mathbb{R}^{3}} \frac{1}{2} g^{2} \mathbb{1}_{\{|g| \leq \lambda\}} M d x d v d t & \leq \liminf _{\epsilon \rightarrow 0} \int_{t_{1}}^{t_{2}} \int_{\mathbb{R}^{3} \times \mathbb{R}^{3}} \frac{1}{\epsilon^{2}} h\left(\epsilon g_{\epsilon}\right) M d x d v d t \\
& \leq C^{\mathrm{in}}\left(t_{2}-t_{1}\right),
\end{aligned}
$$

which, by monotonicity of the integrands, gives

$$
\int_{t_{1}}^{t_{2}} \int_{\mathbb{R}^{3} \times \mathbb{R}^{3}} \frac{1}{2} g^{2} M d x d v d t \leq C^{\mathrm{in}}\left(t_{2}-t_{1}\right)
$$

Finally, the proof of the lemma is concluded by the arbitrariness of $t_{1}$ and $t_{2}$.

The second assertion of the preceding lemma shows that, in the vanishing $\epsilon$ limit, the limiting fluctuation belongs to $L^{2}(M d x d v)$ uniformly in $t$. Hence, the weighted $L^{1}$-bound implied by the first assertion of Lemma 5.1 is certainly not optimal.

Thus, in order to refine our understanding of the limit $\epsilon \rightarrow 0$, we consider the following renormalized fluctuations

$$
\begin{array}{ll}
\sqrt{G_{\epsilon}}=1+\frac{\epsilon}{2} \hat{g}_{\epsilon}, & \sqrt{G_{\epsilon}^{\text {in }}}=1+\frac{\epsilon}{2} \hat{g}_{\epsilon}^{\text {in }}, \\
\sqrt{G_{\epsilon}^{ \pm}}=1+\frac{\epsilon}{2} \hat{g}_{\epsilon}^{ \pm}, & \sqrt{G_{\epsilon}^{ \pm \mathrm{in}}}=1+\frac{\epsilon}{2} \hat{g}_{\epsilon}^{ \pm \mathrm{in}},
\end{array}
$$


or, equivalently,

$$
\begin{aligned}
\hat{g}_{\epsilon} & =\frac{2}{\epsilon}\left(\sqrt{G_{\epsilon}}-1\right), & \hat{g}_{\epsilon}^{\text {in }} & =\frac{2}{\epsilon}\left(\sqrt{G_{\epsilon}^{\text {in }}}-1\right), \\
\hat{g}_{\epsilon}^{ \pm} & =\frac{2}{\epsilon}\left(\sqrt{G_{\epsilon}^{ \pm}}-1\right), & \hat{g}_{\epsilon}^{\text {in } \pm} & =\frac{2}{\epsilon}\left(\sqrt{G_{\epsilon}^{\text {in }}}-1\right) .
\end{aligned}
$$

Such square root renormalizations have already been used in previous works on hydrodynamic limits. The advantages of these renormalized fluctuations over the original ones become apparent in the coming lemma, which is, essentially, a modern reformulation of Corollary 3.2 from $[\mathbf{9}$.

LEMMA 5.2. Let $f_{\epsilon}(t, x, v)$ be a family of measurable, almost everywhere nonnegative distribution functions such that, for all $t \geq 0$,

$$
\frac{1}{\epsilon^{2}} H\left(f_{\epsilon}\right)(t)=\int_{\mathbb{R}^{3} \times \mathbb{R}^{3}} \frac{1}{\epsilon^{2}} h\left(\epsilon g_{\epsilon}\right)(t) M d x d v \leq C^{\text {in }} .
$$

Then, as $\epsilon \rightarrow 0$, any subsequence of renormalized fluctuations $\hat{g}_{\epsilon}$ is uniformly bounded in $L^{\infty}\left(d t ; L^{2}(M d x d v)\right)$.

Proof. The elementary inequality B.5 implies that, for all $t \geq 0$,

$$
\begin{aligned}
\int_{\mathbb{R}^{3} \times \mathbb{R}^{3}} \hat{g}_{\epsilon}^{2}(t) M d x d v & =\int_{\mathbb{R}^{3} \times \mathbb{R}^{3}} \frac{4}{\epsilon^{2}}\left(\sqrt{1+\epsilon g_{\epsilon}}-1\right)^{2}(t) M d x d v \\
& \leq \frac{4}{\epsilon^{2}} H\left(f_{\epsilon}\right)(t) \leq 4 C^{\mathrm{in}},
\end{aligned}
$$

which is the announced result.

The simple Lemma 5.2 provides important information on any subsequence of fluctuations $g_{\epsilon}$. Indeed, a very natural application of this refined a priori estimate follows from decomposing the fluctuations as

$$
g_{\epsilon}=\hat{g}_{\epsilon}+\frac{\epsilon}{4} \hat{g}_{\epsilon}^{2} \in L^{\infty}\left(d t ; L^{2}(M d x d v)\right)+\epsilon L^{\infty}\left(d t ; L^{1}(M d x d v)\right) .
$$

Therefore, we see from Lemma 5.2 , that the fluctuations $g_{\epsilon}$ are uniformly bounded in $L^{\infty}\left(d t ; L^{2}(M d x d v)\right)$, up to a remainder of order $\epsilon$ in $L^{\infty}\left(d t ; L^{1}(M d x d v)\right)$. In particular, according to Lemma 5.1 , if $g$ is a weak limit point in $L_{\text {loc }}^{1}\left(d t d x ; L^{1}\left(\left(1+|v|^{2}\right) M d v\right)\right)$ of a converging subsequence of fluctuations $g_{\epsilon}$, then $\hat{g}_{\epsilon}$ also converges towards $g$ in the weak-* topology of $L^{\infty}\left(d t ; L^{2}(M d x d v)\right)$.

As we will see later on, it will be crucial to establish sharper properties of tightness and equi-integrability on the sequence of integrable functions $\hat{g}_{\epsilon}^{2}$. These refinements will follow from the joint control of the fluctuations by the entropy and the entropy dissipation bounds.

\subsection{Controls from the entropy dissipation bound}

Following [9], again, we introduce the non-negative convex function

$$
r(z)=z \log (1+z),
$$

defined over $(-1, \infty)$. We may then recast the entropy inequalities 4.28 and 4.35 utilizing this notation to get the entropy dissipation bounds (here, exceptionally, we consider any cross-section $b \geq 0$, for more generality), for all $t \geq 0$,

$$
\begin{aligned}
\frac{1}{\epsilon^{4}} \int_{0}^{t} \int_{\mathbb{R}^{3}} D & \left(f_{\epsilon}\right)(s) d x d s \\
& =\int_{0}^{t} \int_{\mathbb{R}^{3}} \int_{\mathbb{R}^{3} \times \mathbb{R}^{3} \times \mathbb{S}^{2}} \frac{1}{4 \epsilon^{4}} r\left(\frac{\epsilon^{2} q_{\epsilon}}{G_{\epsilon} G_{\epsilon *}}\right) f_{\epsilon} f_{\epsilon *} b d v d v_{*} d \sigma d x d s \leq C^{\mathrm{in}},
\end{aligned}
$$


and

$$
\begin{aligned}
\frac{1}{\epsilon^{4}} \int_{0}^{t} \int_{\mathbb{R}^{3}} & \left(D\left(f_{\epsilon}^{+}\right)+D\left(f_{\epsilon}^{-}\right)+\delta^{2} D\left(f_{\epsilon}^{+}, f_{\epsilon}^{-}\right)\right)(s) d x d s \\
= & \int_{0}^{t} \int_{\mathbb{R}^{3}} \int_{\mathbb{R}^{3} \times \mathbb{R}^{3} \times \mathbb{S}^{2}} \frac{1}{4 \epsilon^{4}} r\left(\frac{\epsilon^{2} q_{\epsilon}^{+}}{G_{\epsilon}^{+} G_{\epsilon *}^{+}}\right) f_{\epsilon}^{+} f_{\epsilon *}^{+} b d v d v_{*} d \sigma d x d s \\
& +\int_{0}^{t} \int_{\mathbb{R}^{3}} \int_{\mathbb{R}^{3} \times \mathbb{R}^{3} \times \mathbb{S}^{2}} \frac{1}{4 \epsilon^{4}} r\left(\frac{\epsilon^{2} q_{\epsilon}^{-}}{G_{\epsilon}^{-} G_{\epsilon *}^{-}}\right) f_{\epsilon}^{-} f_{\epsilon *}^{-} b d v d v_{*} d \sigma d x d s \\
& +\int_{0}^{t} \int_{\mathbb{R}^{3}} \int_{\mathbb{R}^{3} \times \mathbb{R}^{3} \times \mathbb{S}^{2}} \frac{\delta^{2}}{2 \epsilon^{4}} r\left(\frac{\epsilon^{2} q_{\epsilon}^{+,-}}{\delta G_{\epsilon}^{+} G_{\epsilon *}^{-}}\right) f_{\epsilon}^{+} f_{\epsilon *}^{-} b d v d v_{*} d \sigma d x d s \leq C^{\mathrm{in}}
\end{aligned}
$$

The entropy dissipation bounds are expected to control the size of the collision integrands $q_{\epsilon}, q_{\epsilon}^{+}, q_{\epsilon}^{-}, q_{\epsilon}^{ \pm}$and $q_{\epsilon}^{\mp}$ since

$$
r(z) \sim z^{2}, \quad \text { near } z=0
$$

However, this behavior only holds asymptotically, as $z \rightarrow 0$, and thus, in order to exploit the entropy dissipation bounds, we will have to rely crucially on Young's inequality (B.4) for $r(z)$ and on inequality (B.8), presented in Appendix B. Furthermore, when coupled with a coercivity estimate for some suitable non-singular linearized collision operator, the entropy dissipation bounds will actually provide some control on the relaxation to equilibrium of the fluctuations $g_{\epsilon}$ and $g_{\epsilon}^{ \pm}$(see Section 5.3 below).

In order to refine our understanding of the limit $\epsilon \rightarrow 0$, we consider the following renormalized collision integrands

$$
\begin{aligned}
\hat{q}_{\epsilon} & =\frac{2}{\epsilon^{2}}\left(\sqrt{G_{\epsilon}^{\prime} G_{\epsilon *}^{\prime}}-\sqrt{G_{\epsilon} G_{\epsilon *}}\right), \\
\hat{q}_{\epsilon}^{+} & =\frac{2}{\epsilon^{2}}\left(\sqrt{G_{\epsilon}^{+\prime} G_{\epsilon *}^{+\prime}}-\sqrt{G_{\epsilon}^{+} G_{\epsilon *}^{+}}\right), \\
\hat{q}_{\epsilon}^{-} & =\frac{2}{\epsilon^{2}}\left(\sqrt{G_{\epsilon}^{-\prime} G_{\epsilon *}^{-\prime}}-\sqrt{G_{\epsilon}^{-} G_{\epsilon *}^{-}}\right), \\
\hat{q}_{\epsilon}^{+,-} & =\frac{2 \delta}{\epsilon^{2}}\left(\sqrt{G_{\epsilon}^{+\prime} G_{\epsilon *}^{-\prime}}-\sqrt{G_{\epsilon}^{+} G_{\epsilon *}^{-}}\right), \\
\hat{q}_{\epsilon}^{-,+} & =\frac{2 \delta}{\epsilon^{2}}\left(\sqrt{G_{\epsilon}^{-\prime} G_{\epsilon *}^{+\prime}}-\sqrt{G_{\epsilon}^{-} G_{\epsilon *}^{+}}\right) .
\end{aligned}
$$

The advantages of these renormalized collision integrands over the original ones become apparent in the coming lemma.

LEMMA 5.3. Let $f_{\epsilon}^{+}(t, x, v)$ and $f_{\epsilon}^{-}(t, x, v)$ be two families of measurable, almost everywhere non-negative distribution functions such that, for all $t \geq 0$,

$$
\begin{aligned}
\frac{\delta^{2}}{\epsilon^{4}} \int_{0}^{t} \int_{\mathbb{R}^{3}} D & \left(f_{\epsilon}^{+}, f_{\epsilon}^{-}\right)(s) d x d s \\
& =\int_{0}^{t} \int_{\mathbb{R}^{3}} \int_{\mathbb{R}^{3} \times \mathbb{R}^{3} \times \mathbb{S}^{2}} \frac{\delta^{2}}{2 \epsilon^{4}} r\left(\frac{\epsilon^{2} q_{\epsilon}^{+,-}}{\delta G_{\epsilon}^{+} G_{\epsilon *}^{-}}\right) f_{\epsilon}^{+} f_{\epsilon *}^{-} b d v d v_{*} d \sigma d x d s \leq C^{\mathrm{in}}
\end{aligned}
$$

Then, as $\epsilon \rightarrow 0$, any subsequence of renormalized collision integrands $\hat{q}_{\epsilon}^{+,-}$is uniformly bounded in $L^{2}\left(b M M_{*} d t d x d v d v_{*} d \sigma\right)$. 
Proof. The elementary inequality (B.8) implies that, for all $t \geq 0$,

$$
\begin{gathered}
\frac{1}{2} \int_{0}^{t} \int_{\mathbb{R}^{3}} \int_{\mathbb{R}^{3} \times \mathbb{R}^{3} \times \mathbb{S}^{2}}\left(\hat{q}_{\epsilon}^{+,-}\right)^{2} b M M_{*} d v d v_{*} d \sigma d x d s \\
\quad=2 \int_{0}^{t} \int_{\mathbb{R}^{3}} \int_{\mathbb{R}^{3} \times \mathbb{R}^{3} \times \mathbb{S}^{2}} \frac{\delta^{2}}{\epsilon^{4}}\left(\sqrt{1+\frac{\epsilon^{2} q_{\epsilon}^{+,-}}{\delta G_{\epsilon}^{+} G_{\epsilon *}^{-}}}-1\right)^{2} f_{\epsilon}^{+} f_{\epsilon *}^{-} b d v d v_{*} d \sigma d x d s \\
\leq \frac{\delta^{2}}{\epsilon^{4}} \int_{0}^{t} \int_{\mathbb{R}^{3}} D\left(f_{\epsilon}^{+}, f_{\epsilon}^{-}\right)(s) d x d s \leq C^{\mathrm{in}}
\end{gathered}
$$

which is the announced result.

The simple Lemma 5.3 provides important information on any subsequence of collision integrands $q_{\epsilon}^{+,-}$. Indeed, a very natural application of this refined a priori estimate follows from decomposing the collision integrands as

$$
q_{\epsilon}^{+,-}=\sqrt{G_{\epsilon}^{+} G_{\epsilon *}^{-}} \hat{q}_{\epsilon}^{+,-}+\frac{\epsilon^{2}}{4 \delta}\left(\hat{q}_{\epsilon}^{+,-}\right)^{2} .
$$

Therefore, at least in the simpler case of the Maxwellian cross-section $b \equiv 1$, we see from Lemma 5.3 that, for any admissible renormalization $\beta(z)$, the renormalized collision integrands $\beta^{\prime}\left(G_{\epsilon}^{+}\right) q_{\epsilon}^{+,-}$are uniformly bounded in $L_{\text {loc }}^{1}\left(d t d x ; L^{1}\left(M M_{*} d v d v_{*} d \sigma\right)\right)$, provided the natural entropy and entropy dissipation bounds are satisfied. Moreover, employing Lemma 5.2 and Egorov's theorem, it is possible to show that

$$
\beta^{\prime}\left(G_{\epsilon}^{+}\right) \sqrt{G_{\epsilon}^{+} G_{\epsilon *}^{-}} \rightarrow \beta^{\prime}(1) \quad \text { in } L_{\mathrm{loc}}^{2}\left(d t d x ; L^{2}\left(M M_{*} d v d v_{*} d \sigma\right)\right) .
$$

In particular, if $q^{+,-}$is a weak limit point in $L^{2}\left(M M_{*} d t d x d v d v_{*} d \sigma\right)$ of a converging subsequence of collision integrands $\hat{q}_{\epsilon}^{+,-}$, then $\beta^{\prime}\left(G_{\epsilon}^{+}\right) q_{\epsilon}^{+,-}$converges - up to extraction of jointly converging subsequences - towards $\beta^{\prime}(1) q^{+,-}$in the weak topology of $L_{\mathrm{loc}}^{1}\left(d t d x ; L^{1}\left(M M_{*} d v d v_{*} d \sigma\right)\right)$.

\subsection{Relaxation towards thermodynamic equilibrium}

In this section, we establish the relaxation of fluctuations towards thermodynamic equilibrium as a consequence of the relative entropy and the entropy dissipation bounds. As we consider fluctuations around a global equilibrium, the linearized collision operator $\mathcal{L}$, defined in $(2.8)$ and $(2.35)$, is expected to play here a fundamental role.

We recall that, for the sake of simplicity, we restrict our attention, in this part of our work, to the case of Maxwellian molecules, that is to constant collision crosssections, say $b \equiv 1$. Nevertheless, up to additional technical difficulties, the results in this section will be extended to general cross-sections in the remaining parts of the present work.

The spectral analysis of the linearized collision operator uses crucially the following decomposition based on a clever change of variables sometimes called "Carleman's collision parametrization", although it goes back to Hilbert 42 (see equation (17) in 42 and the computations therein). We refer to 68 for more details and to 49 for a modern and general treatment of the linearized Boltzmann operator.

Proposition 5.4 (Hilbert's decomposition of $\mathcal{L}$ ). The linearized collision operator $\mathcal{L}$ defined by

$$
\mathcal{L} g=-\frac{1}{M}(Q(M g, M)+Q(M, M g))=\int_{\mathbb{R}^{3} \times \mathbb{S}^{2}}\left(g+g_{*}-g^{\prime}-g_{*}^{\prime}\right) M_{*} d v_{*} d \sigma,
$$


can be decomposed as

$$
\mathcal{L} g=g-\mathcal{K} g
$$

where $\mathcal{K}$ is a compact integral operator on $L^{2}(M d v)$.

As an immediate consequence of the preceding proposition, the operator $\mathcal{L}$ satisfies the Fredholm alternative, as well as some coercivity estimate, which will be used to control the relaxation process. We refer to [49 or 68 for details and justifications of the following proposition, or to the proof of the more general Proposition 5.7 below.

Proposition 5.5 (Coercivity of $\mathcal{L}$ ). The linear collision operator $\mathcal{L}$ is a nonnegative self-adjoint operator on $L^{2}(M d v)$ with nullspace

$$
\operatorname{Ker}(\mathcal{L})=\operatorname{span}\left\{1, v_{1}, v_{2}, v_{3},|v|^{2}\right\}
$$

Moreover, the following coercivity estimate holds : there exists $C>0$ such that, for each $g \in \operatorname{Ker}(\mathcal{L})^{\perp} \subset L^{2}(M d v)$,

$$
\|g\|_{L^{2}(M d v)}^{2} \leq C \int_{\mathbb{R}^{3}} g \mathcal{L} g(v) M(v) d v .
$$

In particular, for any $g \in \operatorname{Ker}(\mathcal{L})^{\perp} \subset L^{2}(M d v)$,

$$
\|g\|_{L^{2}(M d v)} \leq C\|\mathcal{L} g\|_{L^{2}(M d v)} .
$$

We will also need the generalization of the preceding propositions to the linearized collision operator for two species of particles $\mathbf{L}$. In fact, employing the results from 49, we easily obtain the following Hilbert's decomposition for $\mathbf{L}$.

Proposition 5.6 (Hilbert's decomposition of $\mathbf{L}$ ). The linearized collision operator for two species $\mathbf{L}$ defined by

$$
\mathbf{L}\left(\begin{array}{l}
g \\
h
\end{array}\right)=\left(\begin{array}{l}
\mathcal{L} g+\mathcal{L}(g, h) \\
\mathcal{L} h+\mathcal{L}(h, g)
\end{array}\right)
$$

where

$$
\mathcal{L}(g, h)=-\frac{1}{M}(Q(M g, M)+Q(M, M h))=\int_{\mathbb{R}^{3} \times \mathbb{S}^{2}}\left(g+h_{*}-g^{\prime}-h_{*}^{\prime}\right) M_{*} d v_{*} d \sigma
$$

can be decomposed as

$$
\mathbf{L}\left(\begin{array}{l}
g \\
h
\end{array}\right)=2\left(\begin{array}{l}
g \\
h
\end{array}\right)-\mathbf{K}\left(\begin{array}{l}
g \\
h
\end{array}\right),
$$

where $\mathbf{K}$ is a compact integral operator on $L^{2}(M d v)$.

As an immediate consequence of the preceding proposition, the operator $\mathbf{L}$ satisfies the Fredholm alternative, as well as some coercivity estimate, which will be used to control the relaxation process for two species of particles. For the sake of completeness, we provide here a brief justification of the following proposition.

Proposition 5.7 (Coercivity of $\mathbf{L}$ ). The linear collision operator $\mathbf{L}$ is a nonnegative self-adjoint operator on $L^{2}(M d v)$ with nullspace

$$
\operatorname{Ker}(\mathbf{L})=\operatorname{span}\left\{\left(\begin{array}{l}
1 \\
0
\end{array}\right),\left(\begin{array}{l}
0 \\
1
\end{array}\right),\left(\begin{array}{l}
v_{1} \\
v_{1}
\end{array}\right),\left(\begin{array}{l}
v_{2} \\
v_{2}
\end{array}\right),\left(\begin{array}{l}
v_{3} \\
v_{3}
\end{array}\right),\left(\begin{array}{l}
|v|^{2} \\
|v|^{2}
\end{array}\right)\right\} .
$$

Moreover, the following coercivity estimate holds : there exists $C>0$ such that, for $\operatorname{each}\left(\begin{array}{l}g \\ h\end{array}\right) \in \operatorname{Ker}(\mathbf{L})^{\perp} \subset L^{2}(M d v)$,

$$
\left\|\left(\begin{array}{l}
g \\
h
\end{array}\right)\right\|_{L^{2}(M d v)}^{2} \leq C \int_{\mathbb{R}^{3}}\left(\begin{array}{l}
g \\
h
\end{array}\right) \cdot \mathbf{L}\left(\begin{array}{l}
g \\
h
\end{array}\right)(v) M(v) d v .
$$


In particular, for any $\left(\begin{array}{l}g \\ h\end{array}\right) \in \operatorname{Ker}(\mathbf{L})^{\perp} \subset L^{2}(M d v)$,

$$
\left\|\left(\begin{array}{l}
g \\
h
\end{array}\right)\right\|_{L^{2}(M d v)} \leq C\left\|\mathbf{L}\left(\begin{array}{l}
g \\
h
\end{array}\right)\right\|_{L^{2}(M d v)} .
$$

Proof. The non-negativity and the self-adjointness of $\mathbf{L}$ easily follow from a standard use of the collision symmetries by showing that

$$
\begin{aligned}
\int_{\mathbb{R}^{3}} & \left(\begin{array}{l}
g \\
h
\end{array}\right) \cdot \mathbf{L}\left(\frac{\bar{g}}{h}\right)(v) M(v) d v \\
& =\frac{1}{4} \int_{\mathbb{R}^{3} \times \mathbb{R}^{3} \times \mathbb{S}^{2}}\left(g+g_{*}-g^{\prime}-g_{*}^{\prime}\right)\left(\bar{g}+\bar{g}_{*}-\bar{g}^{\prime}-\bar{g}_{*}^{\prime}\right) M M_{*} d v d v_{*} d \sigma \\
& +\frac{1}{4} \int_{\mathbb{R}^{3} \times \mathbb{R}^{3} \times \mathbb{S}^{2}}\left(h+h_{*}-h^{\prime}-h_{*}^{\prime}\right)\left(\bar{h}+\bar{h}_{*}-\bar{h}^{\prime}-\bar{h}_{*}^{\prime}\right) M M_{*} d v d v_{*} d \sigma \\
& +\frac{1}{2} \int_{\mathbb{R}^{3} \times \mathbb{R}^{3} \times \mathbb{S}^{2}}\left(g+h_{*}-g^{\prime}-h_{*}^{\prime}\right)\left(\bar{g}+\bar{h}_{*}-\bar{g}^{\prime}-\bar{h}_{*}^{\prime}\right) M M_{*} d v d v_{*} d \sigma .
\end{aligned}
$$

Next, consider $\left(\begin{array}{l}g \\ h\end{array}\right) \in \operatorname{Ker}(\mathbf{L})$. We deduce from 5.9 that, necessarily, $g=\Pi g$, $h=\Pi h$ and

$$
\int_{\mathbb{R}^{3} \times \mathbb{R}^{3} \times \mathbb{S}^{2}}\left(\Pi(g-h)-(\Pi(g-h))^{\prime}\right)^{2} M M_{*} d v d v_{*} d \sigma=0 .
$$

A simple and direct computation shows then that $h$ and $g$ have the same bulk velocity and temperature, which completes the characterization of the kernel of L. In particular, the orthogonal projection onto the kernel of $\mathbf{L}$ in $L^{2}(M d v)$ is explicitly given by

$$
\mathbb{P}\left(\begin{array}{l}
g \\
h
\end{array}\right)=\left(\begin{array}{c}
\frac{1}{2} \int_{\mathbb{R}^{3}}(g-h) M d v+\Pi \frac{g+h}{2} \\
-\frac{1}{2} \int_{\mathbb{R}^{3}}(g-h) M d v+\Pi \frac{g+h}{2}
\end{array}\right) .
$$

Finally, since $\mathbf{L}$ is positive definite, self-adjoint and satisfies Hilbert's decomposition from Proposition 5.6, we easily obtain, by the spectral theorem for compact self-adjoint operators, writing $\left(\begin{array}{l}g \\ h\end{array}\right)$ in the Hilbert basis of eigenvectors of $\mathbf{L}$, that

$$
\left\|\left(\begin{array}{l}
g \\
h
\end{array}\right)-\mathbb{P}\left(\begin{array}{l}
g \\
h
\end{array}\right)\right\|_{L^{2}(M d v)}^{2} \leq C \int_{\mathbb{R}^{3}}\left(\begin{array}{l}
g \\
h
\end{array}\right) \cdot \mathbf{L}\left(\begin{array}{l}
g \\
h
\end{array}\right) M d v,
$$

which concludes the justification of the proposition.

Finally, we also extend the preceding propositions to the linearized collision operator $\mathfrak{L}$. In fact, employing the results from [49], we easily obtain the following Hilbert's decomposition for $\mathfrak{L}$.

Proposition 5.8 (Hilbert's decomposition of $\mathfrak{L}$ ). The linearized collision operator $\mathfrak{L}$ defined by

$$
\begin{aligned}
\mathfrak{L} g=\mathcal{L}(g,-g) & =-\frac{1}{M}(Q(M g, M)-Q(M, M g)) \\
& =\int_{\mathbb{R}^{3} \times \mathbb{S}^{2}}\left(g-g_{*}-g^{\prime}+g_{*}^{\prime}\right) M_{*} d v_{*} d \sigma,
\end{aligned}
$$

can be decomposed as

$$
\mathfrak{L} g=g-\mathfrak{K} g,
$$

where $\mathfrak{K}$ is a compact integral operator on $L^{2}(M d v)$. 
Note that the definition of $\mathfrak{L}$ above coincides with 2.61).

As an immediate consequence of the preceding proposition, the operator $\mathfrak{L}$ satisfies the Fredholm alternative, as well as some coercivity estimate, which will be used to control the relaxation process for two species of particles. For the sake of completeness, we provide here a brief justification of the following proposition.

Proposition 5.9 (Coercivity of $\mathfrak{L}$ ). The linear collision operator $\mathfrak{L}$ is a nonnegative self-adjoint operator on $L^{2}(M d v)$ with nullspace

$$
\operatorname{Ker}(\mathfrak{L})=\operatorname{span}\{1\} .
$$

Moreover, the following coercivity estimate holds : there exists $C>0$ such that, for each $g \in \operatorname{Ker}(\mathfrak{L})^{\perp} \subset L^{2}(M d v)$,

$$
\|g\|_{L^{2}(M d v)}^{2} \leq C \int_{\mathbb{R}^{3}} g \mathfrak{L} g(v) M(v) d v .
$$

In particular, for any $g \in \operatorname{Ker}(\mathfrak{L})^{\perp} \subset L^{2}(M d v)$,

$$
\|g\|_{L^{2}(M d v)} \leq C\|\mathfrak{L} g\|_{L^{2}(M d v)} .
$$

Proof. The non-negativity and the self-adjointness of $\mathfrak{L}$ easily follow from a standard use of the collision symmetries by showing that

$$
\begin{array}{rl}
\int_{\mathbb{R}^{3}} g & \mathfrak{L} h(v) M(v) d v \\
\quad=\frac{1}{4} \int_{\mathbb{R}^{3} \times \mathbb{R}^{3} \times \mathbb{S}^{2}}\left(g-g_{*}-g^{\prime}+g_{*}^{\prime}\right)\left(h-h_{*}-h^{\prime}+h_{*}^{\prime}\right) M M_{*} d v d v_{*} d \sigma .
\end{array}
$$

Next, consider $g \in \operatorname{Ker}(\mathfrak{L})$. We deduce from 5.10 that, necessarily,

$$
g-g_{*}=g^{\prime}-g_{*}^{\prime},
$$

almost everywhere. Hence, since the change of variable $\sigma \mapsto-\sigma$ merely exchanges $v^{\prime}$ and $v_{*}^{\prime}$, we find, averaging over $\sigma \in \mathbb{S}^{2}$,

$$
g-g_{*}=\frac{1}{\left|\mathbb{S}^{2}\right|} \int_{\mathbb{S}^{2}}\left(g^{\prime}-g_{*}^{\prime}\right) d \sigma=0
$$

for every $v, v_{*} \in \mathbb{R}^{3}$. It follows that $g$ is a constant function.

Finally, since $\mathfrak{L}$ is positive definite, self-adjoint and satisfies Hilbert's decomposition from Proposition 5.8, we easily obtain, by the spectral theorem for compact self-adjoint operators, writing $g$ in the Hilbert basis of eigenvectors of $\mathfrak{L}$, that

$$
\left\|g-\int_{\mathbb{R}^{3}} g_{*} M_{*} d v_{*}\right\|_{L^{2}(M d v)}^{2} \leq C \int_{\mathbb{R}^{3}} g \mathfrak{L} g M d v,
$$

which concludes the justification of the proposition.

It is to be emphasized that, since we are only considering here the case of Maxwellian molecules $b \equiv 1$, the linearized operator $\mathfrak{L}$ can be explicitly rewritten, using that $\int_{\mathbb{S}^{2}}\left(g^{\prime}-g_{*}^{\prime}\right) d \sigma=0$, as

$$
\mathfrak{L} g=\left|\mathbb{S}^{2}\right|\left(g-\int_{\mathbb{R}^{3}} g_{*} M_{*} d v_{*}\right),
$$

which renders the proofs of Propositions 5.8 and 5.9 trivial. However, we chose to provide more robust justifications of both propositions, which work in more general settings of hard and soft potentials, as well. 
5.3.1. Infinitesimal Maxwellians. Using the usual relative entropy and entropy dissipation bounds together with the coercivity of the linearized collision operator, we easily get that each species of particles reaches almost instantaneously the local thermodynamic equilibrium in the fast relaxation limit. More precisely, we have the following lemma.

LEMMA 5.10. Let $f_{\epsilon}(t, x, v)$ be a family of measurable, almost everywhere nonnegative distribution functions such that, for all $t \geq 0$,

$$
\frac{1}{\epsilon^{2}} H\left(f_{\epsilon}\right)(t)+\frac{1}{\epsilon^{4}} \int_{0}^{t} \int_{\mathbb{R}^{3}} D\left(f_{\epsilon}\right)(s) d x d s \leq C^{\mathrm{in}} .
$$

Then, as $\epsilon \rightarrow 0$, any subsequence of renormalized fluctuations $\hat{g}_{\epsilon}$ satisfies the relaxation estimate

$$
\left\|\hat{g}_{\epsilon}-\Pi \hat{g}_{\epsilon}\right\|_{L^{2}(M d v)} \leq O(\epsilon)\left\|\hat{g}_{\epsilon}\right\|_{L^{2}(M d v)}^{2}+O(\epsilon)_{L^{2}(d t d x)}
$$

where $\Pi$ denotes the orthogonal projection on $\operatorname{Ker} \mathcal{L}$ in $L^{2}(M d v)$.

Proof. We start from the elementary decomposition

$$
\mathcal{L} \hat{g}_{\epsilon}=\frac{\epsilon}{2} \mathcal{Q}\left(\hat{g}_{\epsilon}, \hat{g}_{\epsilon}\right)-\frac{2}{\epsilon} \mathcal{Q}\left(\sqrt{G_{\epsilon}}, \sqrt{G_{\epsilon}}\right)
$$

and we estimate each term in the right-hand side separately.

First, since $b \equiv 1$, it is readily seen that the quadratic collision operator is continuous on $L^{2}(M d v)$ :

$$
\begin{aligned}
\left\|\mathcal{Q}\left(\hat{g}_{\epsilon}, \hat{g}_{\epsilon}\right)\right\|_{L^{2}(M d v)} & =\left\|\int_{\mathbb{R}^{3} \times \mathbb{S}^{2}}\left(\hat{g}_{\epsilon}^{\prime} \hat{g}_{\epsilon *}^{\prime}-\hat{g}_{\epsilon} \hat{g}_{\epsilon *}\right) M_{*} d v_{*} d \sigma\right\|_{L^{2}(M d v)} \\
& \leq\left|\mathbb{S}^{2}\right|^{\frac{1}{2}}\left\|\left(\int_{\mathbb{R}^{3} \times \mathbb{S}^{2}}\left(\hat{g}_{\epsilon}^{\prime} \hat{g}_{\epsilon *}^{\prime}-\hat{g}_{\epsilon} \hat{g}_{\epsilon *}\right)^{2} M_{*} d v_{*} d \sigma\right)^{\frac{1}{2}}\right\|_{L^{2}(M d v)} \\
& \leq 2\left|\mathbb{S}^{2}\right|\left\|\hat{g}_{\epsilon}\right\|_{L^{2}(M d v)}^{2},
\end{aligned}
$$

which, when combined with the bound $(5.4)$ from the proof of Lemma 5.2 , yields

$$
\left\|\mathcal{Q}\left(\hat{g}_{\epsilon}, \hat{g}_{\epsilon}\right)\right\|_{L^{1}\left(d x ; L^{2}(M d v)\right)} \leq 8\left|\mathbb{S}^{2}\right| C^{\text {in }}
$$

Furthermore, employing the uniform $L^{2}$-estimate 5.7 from the proof of Lemma 5.3 on the renormalized collision integrands $\hat{q}_{\epsilon}$ and the Cauchy-Schwarz inequality, we deduce that

$$
\begin{aligned}
\int_{0}^{t} \int_{\mathbb{R}^{3}} \int_{\mathbb{R}^{3}} & \left(\frac{1}{\epsilon^{2}} \mathcal{Q}\left(\sqrt{G_{\epsilon}}, \sqrt{G_{\epsilon}}\right)\right)^{2} M d v d x d s \\
& =\frac{1}{4} \int_{0}^{t} \int_{\mathbb{R}^{3}} \int_{\mathbb{R}^{3}}\left(\int_{\mathbb{R}^{3} \times \mathbb{S}^{2}} \hat{q}_{\epsilon} M_{*} d v_{*} d \sigma\right)^{2} M d v d x d s \\
& \leq \frac{\left|\mathbb{S}^{2}\right|}{4} \int_{0}^{t} \int_{\mathbb{R}^{3}} \int_{\mathbb{R}^{3} \times \mathbb{R}^{3} \times \mathbb{S}^{2}}\left(\hat{q}_{\epsilon}\right)^{2} M M_{*} d v d v_{*} d \sigma d x d s \\
& \leq \frac{\left|\mathbb{S}^{2}\right|}{\epsilon^{4}} \int_{0}^{t} \int_{\mathbb{R}^{3}} D\left(f_{\epsilon}\right)(s) d x d s \leq\left|\mathbb{S}^{2}\right| C^{\mathrm{in}}
\end{aligned}
$$



leads to

Therefore, combining 5.12 with the coercivity estimate from Proposition 5.5

$$
\begin{aligned}
\left\|\hat{g}_{\epsilon}-\Pi \hat{g}_{\epsilon}\right\|_{L^{2}(M d v)} & \leq C\left\|\mathcal{L} \hat{g}_{\epsilon}\right\|_{L^{2}(M d v)} \\
& \leq C \epsilon\left(\left\|\mathcal{Q}\left(\hat{g}_{\epsilon}, \hat{g}_{\epsilon}\right)\right\|_{L^{2}(M d v)}+\left\|\frac{1}{\epsilon^{2}} \mathcal{Q}\left(\sqrt{G_{\epsilon}}, \sqrt{G_{\epsilon}}\right)\right\|_{L^{2}(M d v)}\right) \\
& \leq C \epsilon\left(\left\|\hat{g}_{\epsilon}\right\|_{L^{2}(M d v)}^{2}+\left\|\frac{1}{\epsilon^{2}} \mathcal{Q}\left(\sqrt{G_{\epsilon}}, \sqrt{G_{\epsilon}}\right)\right\|_{L^{2}(M d v)}\right) \\
& =C \epsilon\left\|\hat{g}_{\epsilon}\right\|_{L^{2}(M d v)}^{2}+O(\epsilon)_{L^{2}(d t d x)},
\end{aligned}
$$

which concludes the proof of the lemma.

5.3.2. Bulk velocity and temperature. When considering the two species Vlasov-Maxwell-Boltzmann system (4.34), we have an additional relaxation estimate on bulk velocities and temperatures coming from the mixed entropy dissipation.

Lemma 5.11. Let $f_{\epsilon}^{+}(t, x, v)$ and $f_{\epsilon}^{-}(t, x, v)$ be two families of measurable, almost everywhere non-negative distribution functions such that, for all $t \geq 0$,

$$
\begin{aligned}
\frac{1}{\epsilon^{2}} H\left(f_{\epsilon}^{+}\right) & +\frac{1}{\epsilon^{2}} H\left(f_{\epsilon}^{-}\right) \\
& +\frac{1}{\epsilon^{4}} \int_{0}^{t} \int_{\mathbb{R}^{3}}\left(D\left(f_{\epsilon}^{+}\right)+D\left(f_{\epsilon}^{-}\right)+\delta^{2} D\left(f_{\epsilon}^{+}, f_{\epsilon}^{-}\right)\right)(s) d x d s \leq C^{\text {in }} .
\end{aligned}
$$

Then, as $\epsilon \rightarrow 0$, any subsequence of renormalized fluctuations $\hat{g}_{\epsilon}^{ \pm}$satisfies the relaxation estimate

$$
\left\|\left(\begin{array}{c}
\hat{g}_{\epsilon}^{+} \\
\hat{g}_{\epsilon}^{-}
\end{array}\right)-\mathbb{P}\left(\begin{array}{c}
\hat{g}_{\epsilon}^{+} \\
\hat{g}_{\epsilon}^{-}
\end{array}\right)\right\|_{L^{2}(M d v)} \leq O(\epsilon)\left\|\left(\begin{array}{c}
\hat{g}_{\epsilon}^{+} \\
\hat{g}_{\epsilon}^{-}
\end{array}\right)\right\|_{L^{2}(M d v)}^{2}+O\left(\frac{\epsilon}{\delta}\right)_{L_{\mathrm{loc}}^{2}\left(d t ; L^{2}(d x)\right)},
$$

where $\mathbb{P}$ denotes the orthogonal projection on $\operatorname{Ker} \mathbf{L}$ in $L^{2}(M d v)$.

In particular, further considering the densities $\hat{\rho}_{\epsilon}^{ \pm}$, bulk velocities $\hat{u}_{\epsilon}^{ \pm}$and temperatures $\hat{\theta}_{\epsilon}^{ \pm}$respectively associated with the renormalized fluctuations $\hat{g}_{\epsilon}^{ \pm}$, it holds that

(5.14) $\hat{h}_{\epsilon}=\frac{\delta}{\epsilon}\left[\left(\hat{g}_{\epsilon}^{+}-\hat{g}_{\epsilon}^{-}\right)-\hat{n}_{\epsilon}\right] \quad$ is uniformly bounded in $L_{\mathrm{loc}}^{1}\left(d t d x ; L^{2}(M d v)\right)$, where $\hat{n}_{\epsilon}=\hat{\rho}_{\epsilon}^{+}-\hat{\rho}_{\epsilon}^{-}$, and

$$
\begin{gathered}
\hat{j}_{\epsilon}=\frac{\delta}{\epsilon}\left(\hat{u}_{\epsilon}^{+}-\hat{u}_{\epsilon}^{-}\right) \quad \text { and } \quad \hat{w}_{\epsilon}=\frac{\delta}{\epsilon}\left(\hat{\theta}_{\epsilon}^{+}-\hat{\theta}_{\epsilon}^{-}\right) \\
\text {are uniformly bounded in } L_{\mathrm{loc}}^{1}(d t d x) .
\end{gathered}
$$

Finally, one also has the refined relaxation estimate

$$
\begin{aligned}
& \left\|\hat{h}_{\epsilon}-\frac{\delta}{2} \hat{n}_{\epsilon}\left(\hat{g}_{\epsilon}^{ \pm}-\hat{\rho}_{\epsilon}^{ \pm}\right)\right\|_{L^{2}(M d v)} \\
& \quad \leq O(\delta)\left\|\hat{g}_{\epsilon}^{+}-\hat{g}_{\epsilon}^{-}-\hat{n}_{\epsilon}\right\|_{L^{2}(M d v)}\left\|\hat{g}_{\epsilon}^{ \pm}\right\|_{L^{2}(M d v)}+O(1)_{L_{\mathrm{loc}}^{2}\left(d t ; L^{2}(d x)\right)} .
\end{aligned}
$$

ProOF. First, a direct application of Lemma 5.10 yields

$$
\left\|\hat{g}_{\epsilon}^{ \pm}-\Pi \hat{g}_{\epsilon}^{ \pm}\right\|_{L^{2}(M d v)} \leq O(\epsilon)\left\|\hat{g}_{\epsilon}^{ \pm}\right\|_{L^{2}(M d v)}^{2}+O(\epsilon)_{L_{\mathrm{loc}}^{2}\left(d t ; L^{2}(d x)\right)} .
$$

Next, we apply similar arguments from the proof of Lemma 5.10 to the mixed entropy dissipation $D\left(f_{\epsilon}^{+}, f_{\epsilon}^{-}\right)$. Thus, according to the definitions of $\mathcal{L}(g, h)$ and 
$\mathcal{Q}(g, h)$ in 2.35 , we start from the elementary decomposition

$$
\mathcal{L}\left(\hat{g}_{\epsilon}^{ \pm}, \hat{g}_{\epsilon}^{\mp}\right)=\frac{\epsilon}{2} \mathcal{Q}\left(\hat{g}_{\epsilon}^{ \pm}, \hat{g}_{\epsilon}^{\mp}\right)-\frac{2}{\epsilon} \mathcal{Q}\left(\sqrt{G_{\epsilon}^{ \pm}}, \sqrt{G_{\epsilon}^{\mp}}\right)
$$

and we estimate each term in the right-hand side separately.

Since $b \equiv 1$, it is readily seen that the quadratic collision operator is continuous on $L^{2}(M d v)$ :

$$
\begin{aligned}
\left\|\mathcal{Q}\left(\hat{g}_{\epsilon}^{ \pm}, \hat{g}_{\epsilon}^{\mp}\right)\right\|_{L^{2}(M d v)} & =\left\|\int_{\mathbb{R}^{3} \times \mathbb{S}^{2}}\left(\hat{g}_{\epsilon}^{ \pm \prime} \hat{g}_{\epsilon *}^{\mp \prime}-\hat{g}_{\epsilon}^{ \pm} \hat{g}_{\epsilon *}^{\mp}\right) M_{*} d v_{*} d \sigma\right\|_{L^{2}(M d v)} \\
& \leq\left|\mathbb{S}^{2}\right|^{\frac{1}{2}}\left\|\left(\int_{\mathbb{R}^{3} \times \mathbb{S}^{2}}\left(\hat{g}_{\epsilon}^{ \pm \prime} \hat{g}_{\epsilon *}^{\mp \prime}-\hat{g}_{\epsilon}^{ \pm} \hat{g}_{\epsilon *}^{\mp}\right)^{2} M_{*} d v_{*} d \sigma\right)^{\frac{1}{2}}\right\|_{L^{2}(M d v)} \\
& \leq 2\left|\mathbb{S}^{2}\right|\left\|\hat{g}_{\epsilon}^{+}\right\|_{L^{2}(M d v)}\left\|\hat{g}_{\epsilon}^{-}\right\|_{L^{2}(M d v)},
\end{aligned}
$$

which, when combined with the bound $(5.4)$ from the proof of Lemma 5.2, yields

$$
\left\|\mathcal{Q}\left(\hat{g}_{\epsilon}^{ \pm}, \hat{g}_{\epsilon}^{\mp}\right)\right\|_{L^{1}\left(d x ; L^{2}(M d v)\right)} \leq 8\left|\mathbb{S}^{2}\right| C^{\text {in }}
$$

Furthermore, employing the uniform $L^{2}$-estimate (5.7) from the proof of Lemma 5.3 on the renormalized collision integrands $\hat{q}_{\epsilon}^{ \pm}$and the Cauchy-Schwarz inequality, we deduce that

$$
\begin{aligned}
\int_{0}^{t} \int_{\mathbb{R}^{3}} \int_{\mathbb{R}^{3}} & \left(\frac{\delta}{\epsilon^{2}} \mathcal{Q}\left(\sqrt{G_{\epsilon}^{+}}, \sqrt{G_{\epsilon}^{-}}\right)\right)^{2} M d v d x d s \\
& =\frac{1}{4} \int_{0}^{t} \int_{\mathbb{R}^{3}} \int_{\mathbb{R}^{3}}\left(\int_{\mathbb{R}^{3} \times \mathbb{S}^{2}} \hat{q}_{\epsilon}^{+,-} M_{*} d v_{*} d \sigma\right)^{2} M d v d x d s \\
& \leq \frac{\left|\mathbb{S}^{2}\right|}{4} \int_{0}^{t} \int_{\mathbb{R}^{3}} \int_{\mathbb{R}^{3} \times \mathbb{R}^{3} \times \mathbb{S}^{2}}\left(\hat{q}_{\epsilon}^{+,-}\right)^{2} M M_{*} d v d v_{*} d \sigma d x d s \\
& \leq \frac{\left|\mathbb{S}^{2}\right| \delta^{2}}{2 \epsilon^{4}} \int_{0}^{t} \int_{\mathbb{R}^{3}} D\left(f_{\epsilon}^{+}, f_{\epsilon}^{-}\right)(s) d x d s \leq \frac{\left|\mathbb{S}^{2}\right|}{2} C^{\mathrm{in}} .
\end{aligned}
$$

Notice that the same estimate holds on the renormalized collision integrands $\hat{q}_{\epsilon}^{-,+}$, which yields

$$
\begin{aligned}
\int_{0}^{t} \int_{\mathbb{R}^{3}} \int_{\mathbb{R}^{3}} & \left(\frac{\delta}{\epsilon^{2}} \mathcal{Q}\left(\sqrt{G_{\epsilon}^{-}}, \sqrt{G_{\epsilon}^{+}}\right)\right)^{2} M d v d x d s \\
& \leq \frac{\left|\mathbb{S}^{2}\right| \delta^{2}}{2 \epsilon^{4}} \int_{0}^{t} \int_{\mathbb{R}^{3}} D\left(f_{\epsilon}^{+}, f_{\epsilon}^{-}\right)(s) d x d s \leq \frac{\left|\mathbb{S}^{2}\right|}{2} C^{\mathrm{in}}
\end{aligned}
$$


Therefore, combining 5.16 and 5.17 with the coercivity estimate from Proposition 5.7 leads to

$$
\begin{aligned}
& \left\|\left(\begin{array}{c}
\hat{g}_{\epsilon}^{+} \\
\hat{g}_{\epsilon}^{-}
\end{array}\right)-\mathbb{P}\left(\begin{array}{c}
\hat{g}_{\epsilon}^{+} \\
\hat{g}_{\epsilon}^{-}
\end{array}\right)\right\|_{L^{2}(M d v)} \\
& \leq C\left\|\mathbf{L}\left(\begin{array}{c}
\hat{g}_{\epsilon}^{+} \\
\hat{g}_{\epsilon}^{-}
\end{array}\right)\right\|_{L^{2}(M d v)} \leq C\left\|\left(\begin{array}{c}
\mathcal{L} \hat{g}_{\epsilon}^{+} \\
\mathcal{L} \hat{g}_{\epsilon}^{-}
\end{array}\right)\right\|_{L^{2}(M d v)}+C\left\|\left(\begin{array}{c}
\mathcal{L}\left(\hat{g}_{\epsilon}^{+}, \hat{g}_{\epsilon}^{-}\right) \\
\mathcal{L}\left(\hat{g}_{\epsilon}^{-}, \hat{g}_{\epsilon}^{+}\right)
\end{array}\right)\right\|_{L^{2}(M d v)} \\
& \leq C\left\|\left(\begin{array}{c}
\hat{g}_{\epsilon}^{+}-\Pi \hat{g}_{\epsilon}^{+} \\
\hat{g}_{\epsilon}^{-}-\Pi \hat{g}_{\epsilon}^{-}
\end{array}\right)\right\|_{L^{2}(M d v)}+C \epsilon\left\|\left(\begin{array}{c}
\mathcal{Q}\left(\hat{g}_{\epsilon}^{+}, \hat{g}_{\epsilon}^{-}\right) \\
\mathcal{Q}\left(\hat{g}_{\epsilon}^{-}, \hat{g}_{\epsilon}^{+}\right)
\end{array}\right)\right\|_{L^{2}(M d v)} \\
& +C \frac{\epsilon}{\delta}\left\|\frac{\delta}{\epsilon^{2}}\left(\begin{array}{l}
\mathcal{Q}\left(\sqrt{G_{\epsilon}^{+}}, \sqrt{G_{\epsilon}^{-}}\right) \\
\mathcal{Q}\left(\sqrt{G_{\epsilon}^{-}}, \sqrt{G_{\epsilon}^{+}}\right)
\end{array}\right)\right\|_{L^{2}(M d v)} \\
& \leq O(\epsilon)\left\|\left(\begin{array}{l}
\hat{g}_{\epsilon}^{+} \\
\hat{g}_{\epsilon}^{-}
\end{array}\right)\right\|_{L^{2}(M d v)}^{2}+O(\epsilon)_{L_{\mathrm{loc}}^{2}\left(d t ; L^{2}(d x)\right)}+O\left(\frac{\epsilon}{\delta}\right)_{L_{\mathrm{loc}}^{2}\left(d t ; L^{2}(d x)\right)},
\end{aligned}
$$

which concludes the proof of the relaxation estimate $(5.13)$.

Then, in order to deduce the control of $\hat{h}_{\epsilon}, \hat{j}_{\epsilon}$ and $\hat{w}_{\epsilon}$, it suffices to notice that

$$
\left(\begin{array}{c}
1 \\
-1
\end{array}\right) \cdot\left[\left(\begin{array}{c}
\hat{g}_{\epsilon}^{+} \\
\hat{g}_{\epsilon}^{-}
\end{array}\right)-\mathbb{P}\left(\begin{array}{c}
\hat{g}_{\epsilon}^{+} \\
\hat{g}_{\epsilon}^{-}
\end{array}\right)\right]=\left(\hat{g}_{\epsilon}^{+}-\hat{g}_{\epsilon}^{-}\right)-\left(\hat{\rho}_{\epsilon}^{+}-\hat{\rho}_{\epsilon}^{-}\right),
$$

and

$$
\begin{aligned}
\Pi\left[\left(\begin{array}{c}
\hat{g}_{\epsilon}^{+} \\
\hat{g}_{\epsilon}^{-}
\end{array}\right)-\mathbb{P}\left(\begin{array}{c}
\hat{g}_{\epsilon}^{+} \\
\hat{g}_{\epsilon}^{-}
\end{array}\right)\right] & =\left(\begin{array}{l}
\Pi \frac{\hat{g}_{\epsilon}^{+}-\hat{g}_{\epsilon}^{-}}{2}-\frac{1}{2} \int_{\mathbb{R}^{3}}\left(\hat{g}_{\epsilon}^{+}-\hat{g}_{\epsilon}^{-}\right) M d v \\
\Pi \frac{\hat{g}_{\epsilon}^{-}-\hat{g}_{\epsilon}^{+}}{2}-\frac{1}{2} \int_{\mathbb{R}^{3}}\left(\hat{g}_{\epsilon}^{-}-\hat{g}_{\epsilon}^{+}\right) M d v
\end{array}\right) \\
& =\left(\begin{array}{l}
\frac{\hat{u}_{\epsilon}^{+}-\hat{u}_{\epsilon}^{-}}{2} \cdot v+\frac{\hat{\theta}_{\epsilon}^{+}-\hat{\theta}_{\epsilon}^{-}}{2}\left(\frac{|v|^{2}}{2}-\frac{3}{2}\right) \\
\frac{\hat{u}_{\epsilon}^{-}-\hat{u}_{\epsilon}^{+}}{2} \cdot v+\frac{\hat{\theta}_{\epsilon}^{-}-\hat{\theta}_{\epsilon}^{+}}{2}\left(\frac{|v|^{2}}{2}-\frac{3}{2}\right)
\end{array}\right),
\end{aligned}
$$

whence

$$
\left\|\Pi\left[\left(\begin{array}{c}
\hat{g}_{\epsilon}^{+} \\
\hat{g}_{\epsilon}^{-}
\end{array}\right)-\mathbb{P}\left(\begin{array}{l}
\hat{g}_{\epsilon}^{+} \\
\hat{g}_{\epsilon}^{-}
\end{array}\right)\right]\right\|_{L^{2}(M d v)}^{2}=\frac{1}{2}\left(\hat{u}_{\epsilon}^{+}-\hat{u}_{\epsilon}^{-}\right)^{2}+\frac{3}{4}\left(\hat{\theta}_{\epsilon}^{+}-\hat{\theta}_{\epsilon}^{-}\right)^{2} .
$$

There only remains to establish the more precise relaxation estimate 5.15 on $\hat{h}_{\epsilon}$, which is achieved by employing the coercivity of the operator $\mathfrak{L}$. To this end, we use the identities (5.17) to decompose

$$
\begin{aligned}
\mathfrak{L} \hat{h}_{\epsilon} & =\frac{\delta}{\epsilon} \mathfrak{L}\left(\hat{g}_{\epsilon}^{+}-\hat{g}_{\epsilon}^{-}\right) \\
& =\frac{\delta}{2}\left[\mathcal{Q}\left(\hat{g}_{\epsilon}^{+}, \hat{g}_{\epsilon}^{-}\right)-\mathcal{Q}\left(\hat{g}_{\epsilon}^{-}, \hat{g}_{\epsilon}^{+}\right)\right]-\frac{2 \delta}{\epsilon^{2}}\left[\mathcal{Q}\left(\sqrt{G_{\epsilon}^{+}}, \sqrt{G_{\epsilon}^{-}}\right)-\mathcal{Q}\left(\sqrt{G_{\epsilon}^{-}}, \sqrt{G_{\epsilon}^{+}}\right)\right] \\
& =\frac{\delta}{2}\left[\mathcal{Q}\left(\hat{g}_{\epsilon}^{+}-\hat{g}_{\epsilon}^{-}, \hat{g}_{\epsilon}^{ \pm}\right)-\mathcal{Q}\left(\hat{g}_{\epsilon}^{ \pm}, \hat{g}_{\epsilon}^{+}-\hat{g}_{\epsilon}^{-}\right)\right]-\int_{\mathbb{R}^{3} \times \mathbb{S}^{2}}\left(\hat{q}_{\epsilon}^{+,-}-\hat{q}_{\epsilon}^{-,+}\right) M_{*} d v_{*} d \sigma .
\end{aligned}
$$

It follows that

$$
\begin{aligned}
\mathfrak{L}\left(\hat{h}_{\epsilon}-\frac{\delta}{2} \hat{n}_{\epsilon} \hat{g}_{\epsilon}^{ \pm}\right) & =\frac{\epsilon}{2}\left[\mathcal{Q}\left(\hat{h}_{\epsilon}, \hat{g}_{\epsilon}^{ \pm}\right)-\mathcal{Q}\left(\hat{g}_{\epsilon}^{ \pm}, \hat{h}_{\epsilon}\right)\right] \\
& -\int_{\mathbb{R}^{3} \times \mathbb{S}^{2}}\left(\hat{q}_{\epsilon}^{+,-}-\hat{q}_{\epsilon}^{-,+}\right) M_{*} d v_{*} d \sigma .
\end{aligned}
$$


Therefore, repeating the estimates (5.18), (5.19) and (5.20), we find that

$$
\begin{aligned}
\left\|\mathfrak{L}\left(\hat{h}_{\epsilon}-\frac{\delta}{2} \hat{n}_{\epsilon} \hat{g}_{\epsilon}^{ \pm}\right)\right\|_{L^{2}(M d v)} & \leq O(\epsilon)\left\|\hat{h}_{\epsilon}\right\|_{L^{2}(M d v)}\left\|\hat{g}_{\epsilon}^{ \pm}\right\|_{L^{2}(M d v)}+O(1)_{L_{\mathrm{loc}}^{2}\left(d t ; L^{2}(d x)\right)} \\
& =O(\delta)\left\|\hat{g}_{\epsilon}^{+}-\hat{g}_{\epsilon}^{-}-\hat{n}_{\epsilon}\right\|_{L^{2}(M d v)}\left\|\hat{g}_{\epsilon}^{ \pm}\right\|_{L^{2}(M d v)} \\
& +O(1)_{L_{\mathrm{loc}}^{2}\left(d t ; L^{2}(d x)\right)} .
\end{aligned}
$$

Finally, employing the coercivity of $\mathfrak{L}$ from Proposition 5.9 , we easily conclude that 5.15 holds, which concludes the proof of the lemma.

REMARK. Under the hypotheses of the preceding lemma, it is possible to obtain a very explicit identity providing some improved information on the relaxation of $\hat{h}_{\epsilon}$. To this end, we decompose

$$
\begin{aligned}
\int_{\mathbb{R}^{3} \times \mathbb{S}^{2}} & \left(\hat{q}_{\epsilon}^{+,-}-\hat{q}_{\epsilon}^{-,+}\right) M_{*} d v_{*} d \sigma \\
= & \frac{2 \delta}{\epsilon^{2}} \mathcal{Q}\left(\sqrt{G_{\epsilon}^{+}}, \sqrt{G_{\epsilon}^{-}}\right)-\frac{2 \delta}{\epsilon^{2}} \mathcal{Q}\left(\sqrt{G_{\epsilon}^{-}}, \sqrt{G_{\epsilon}^{+}}\right) \\
= & \frac{2 \delta}{\epsilon^{2}} \mathcal{Q}\left(\sqrt{G_{\epsilon}^{+}}-\sqrt{G_{\epsilon}^{-}}-\frac{\epsilon}{2} \frac{\hat{n}_{\epsilon}}{1+\frac{\epsilon}{2} \hat{\rho}_{\epsilon}^{ \pm}} \sqrt{G_{\epsilon}^{ \pm}}, \sqrt{G_{\epsilon}^{ \pm}}\right) \\
& -\frac{2 \delta}{\epsilon^{2}} \mathcal{Q}\left(\sqrt{G_{\epsilon}^{ \pm}}, \sqrt{G_{\epsilon}^{+}}-\sqrt{G_{\epsilon}^{-}}-\frac{\epsilon}{2} \frac{\hat{n}_{\epsilon}}{1+\frac{\epsilon}{2} \hat{\rho}_{\epsilon}^{ \pm}} \sqrt{G_{\epsilon}^{ \pm}}\right) \\
= & \mathcal{Q}\left(\hat{h}_{\epsilon}-\frac{\delta}{2} \hat{n}_{\epsilon} \frac{\hat{g}_{\epsilon}^{ \pm}-\hat{\rho}_{\epsilon}^{ \pm}}{1+\frac{\epsilon}{2} \hat{\rho}_{\epsilon}^{ \pm}}, \sqrt{G_{\epsilon}^{ \pm}}\right)-\mathcal{Q}\left(\sqrt{G_{\epsilon}^{ \pm}}, \hat{h}_{\epsilon}-\frac{\delta}{2} \hat{n}_{\epsilon} \frac{\hat{g}_{\epsilon}^{ \pm}-\hat{\rho}_{\epsilon}^{ \pm}}{1+\frac{\epsilon}{2} \hat{\rho}_{\epsilon}^{ \pm}}\right) .
\end{aligned}
$$

Then, since we are only considering here the Maxwellian cross-section $b \equiv 1$, notice that the gain terms cancel each other out

$$
\mathcal{Q}^{+}\left(\hat{h}_{\epsilon}-\frac{\delta}{2} \hat{n}_{\epsilon} \frac{\hat{g}_{\epsilon}^{ \pm}-\hat{\rho}_{\epsilon}^{ \pm}}{1+\frac{\epsilon}{2} \hat{\rho}_{\epsilon}^{ \pm}}, \sqrt{G_{\epsilon}^{ \pm}}\right)-\mathcal{Q}^{+}\left(\sqrt{G_{\epsilon}^{ \pm}}, \hat{h}_{\epsilon}-\frac{\delta}{2} \hat{n}_{\epsilon} \frac{\hat{g}_{\epsilon}^{ \pm}-\hat{\rho}_{\epsilon}^{ \pm}}{1+\frac{\epsilon}{2} \hat{\rho}_{\epsilon}^{ \pm}}\right)=0,
$$

for the change of variable $\sigma \mapsto-\sigma$ merely exchanges $v^{\prime}$ and $v_{*}^{\prime}$, and that one of the two loss terms vanishes

$$
\mathcal{Q}^{-}\left(\sqrt{G_{\epsilon}^{ \pm}}, \hat{h}_{\epsilon}-\frac{\delta}{2} \hat{n}_{\epsilon} \frac{\hat{g}_{\epsilon}^{ \pm}-\hat{\rho}_{\epsilon}^{ \pm}}{1+\frac{\epsilon}{2} \hat{\rho}_{\epsilon}^{ \pm}}\right)=0 .
$$

Thus, on the whole, we are left with the identity

$$
\begin{aligned}
\left|\mathbb{S}^{2}\right|\left(\left(1+\frac{\epsilon}{2} \hat{\rho}_{\epsilon}^{ \pm}\right) \hat{h}_{\epsilon}-\frac{\delta}{2} \hat{n}_{\epsilon}\left(\hat{g}_{\epsilon}^{ \pm}-\hat{\rho}_{\epsilon}^{ \pm}\right)\right) & =\mathcal{Q}^{-}\left(\hat{h}_{\epsilon}-\frac{\delta}{2} \hat{n}_{\epsilon} \frac{\hat{g}_{\epsilon}^{ \pm}-\hat{\rho}_{\epsilon}^{ \pm}}{1+\frac{\epsilon}{2} \hat{\rho}_{\epsilon}^{ \pm}}, \sqrt{G_{\epsilon}^{ \pm}}\right) \\
& =-\int_{\mathbb{R}^{3} \times \mathbb{S}^{2}}\left(\hat{q}_{\epsilon}^{+,-}-\hat{q}_{\epsilon}^{-,+}\right) M_{*} d v_{*} d \sigma
\end{aligned}
$$

which yields the control, in view of Lemma 5.3 .

$$
\left(1+\frac{\epsilon}{2} \hat{\rho}_{\epsilon}^{ \pm}\right) \hat{h}_{\epsilon}-\frac{\delta}{2} \hat{n}_{\epsilon}\left(\hat{g}_{\epsilon}^{ \pm}-\hat{\rho}_{\epsilon}^{ \pm}\right)=O(1)_{L^{2}(M d t d x d v)} .
$$

In particular, further integrating against $v M d v$ and $\left(\frac{|v|^{2}}{3}-1\right) M d v$, we obtain that

$$
\begin{aligned}
& \left(1+\frac{\epsilon}{2} \hat{\rho}_{\epsilon}^{ \pm}\right) \hat{j}_{\epsilon}-\frac{\delta}{2} \hat{n}_{\epsilon} \hat{u}_{\epsilon}^{ \pm}=O(1)_{L^{2}(d t d x)}, \\
& \left(1+\frac{\epsilon}{2} \hat{\rho}_{\epsilon}^{ \pm}\right) \hat{w}_{\epsilon}-\frac{\delta}{2} \hat{n}_{\epsilon} \hat{\theta}_{\epsilon}^{ \pm}=O(1)_{L^{2}(d t d x)} .
\end{aligned}
$$


These estimates are slightly more precise than (5.15). However, their significance is unclear.

\subsection{Improved integrability in velocity}

Another important consequence of the control on the relaxation is to provide further integrability on the renormalized fluctuations $\hat{g}_{\epsilon}$ and $\hat{g}_{\epsilon}^{ \pm}$with respect to the $v$ variable at infinity. The following result, which was first established as such in 68. (see Lemma 3.2.5 therein) and [38 (see Proposition 3.2 therein), improves Lemma 5.2 and is a direct consequence of Lemma 5.10. It constitutes a significant simplification with respect to earlier works on hydrodynamic limits of the Boltzmann equation, which required convoluted estimates to establish some improved integrability in the $v$ variable.

LEMMA 5.12. Let $f_{\epsilon}(t, x, v)$ be a family of measurable, almost everywhere nonnegative distribution functions such that, for all $t \geq 0$,

$$
\frac{1}{\epsilon^{2}} H\left(f_{\epsilon}\right)(t)+\frac{1}{\epsilon^{4}} \int_{0}^{t} \int_{\mathbb{R}^{3}} D\left(f_{\epsilon}\right)(s) d x d s \leq C^{\mathrm{in}} .
$$

Then, as $\epsilon \rightarrow 0$, any subsequence of renormalized fluctuations $\hat{g}_{\epsilon}$ is uniformly bounded in $L_{\mathrm{loc}}^{2}\left(d t d x ; L^{2}\left(\left(1+|v|^{2}\right) M d v\right)\right)$.

Furthermore, the family $\left|\hat{g}_{\epsilon}\right|^{2}$ is equi-integrable in $v$ (or uniformly integrable in $v$ ) in the sense that, for any $\eta>0$ and every compact subset $K \subset[0, \infty) \times \mathbb{R}^{3} \times \mathbb{R}^{3}$, there exists $\gamma>0$ such that, if $A \subset K$ is a measurable set satisfying

$$
\sup _{(t, x) \in[0, \infty) \times \mathbb{R}^{3}} \int_{\mathbb{R}^{3}} \mathbb{1}_{A}(t, x, v) d v<\gamma,
$$

then

$$
\sup _{\epsilon>0} \int_{A}\left|\hat{g}_{\epsilon}\right|^{2} d t d x d v<\eta .
$$

We also have that, for any $\lambda>0$ and any $1 \leq p<2$, the families $\mathbb{1}_{\left\{\lambda \epsilon\left|\hat{g}_{\epsilon}\right| \leq 1\right\}}\left|\hat{g}_{\epsilon}\right|^{2}$ and $\frac{\left|\hat{g}_{\epsilon}\right|^{2}}{1+\lambda \sqrt{G_{\epsilon}}}$ are uniformly bounded in $L_{\mathrm{loc}}^{1}\left(d t d x ; L^{p}(M d v)\right)$.

Proof. The crucial idea behind these results rests upon decomposing $\hat{g}_{\epsilon}$ according to

$$
\hat{g}_{\epsilon}=\left(\hat{g}_{\epsilon}-\Pi \hat{g}_{\epsilon}\right)+\Pi \hat{g}_{\epsilon},
$$

and then using the control on the relaxation provided by Lemma 5.10 :

$$
\hat{g}_{\epsilon}-\Pi \hat{g}_{\epsilon}=O(\epsilon)_{L_{\mathrm{loc}}^{1}\left(d t d x ; L^{2}(M d v)\right)} .
$$

- First, we establish the uniform control on the high speed tails of $\left|\hat{g}_{\epsilon}\right|^{2}$, i.e. the uniform weighted integrability estimate in $L_{\text {loc }}^{2}\left(d t d x ; L^{2}\left(\left(1+|v|^{2}\right) M d v\right)\right)$. To this end, we start from the decomposition

$$
(1+|v|)^{2}\left|\hat{g}_{\epsilon}\right|^{2}=\hat{g}_{\epsilon}\left[(1+|v|)^{2} \Pi \hat{g}_{\epsilon}\right]+\left[(1+|v|) \hat{g}_{\epsilon}\right]\left[(1+|v|)\left(\hat{g}_{\epsilon}-\Pi \hat{g}_{\epsilon}\right)\right] .
$$

Next, recalling from Lemma 5.2 that

$$
\hat{g}_{\epsilon} \text { is uniformly bounded in } L^{\infty}\left(d t ; L^{2}(M d x d v)\right),
$$

we see, by definition of the hydrodynamic projection $\Pi$, for any $1 \leq p<\infty$, that

$$
(1+|v|)^{2} \Pi \hat{g}_{\epsilon} \text { is uniformly bounded in } L^{\infty}\left(d t ; L^{2}\left(d x ; L^{p}(M d v)\right)\right),
$$

whence, for any $1 \leq r<2$,

(5.24) $\hat{g}_{\epsilon}\left[(1+|v|)^{2} \Pi \hat{g}_{\epsilon}\right]$ is uniformly bounded in $L^{\infty}\left(d t ; L^{1}\left(d x ; L^{r}(M d v)\right)\right)$, which takes care of the first term in right-hand side of 5.22. 
In order to estimate the second term in the right-hand side of 5.22 , we first apply Young's inequality (B.3) with $z=g_{\epsilon}, y=\frac{(1+|v|)^{2}}{\gamma}, \alpha=4 \gamma, \beta=\frac{4 \gamma}{\epsilon}$ and $\gamma>0$, to get, employing the elementary inequality (B.7),

$$
\begin{aligned}
(1+|v|)^{2} \hat{g}_{\epsilon}^{2} & \leq(1+|v|)^{2} \frac{4}{\epsilon^{2}}\left|G_{\epsilon}-1\right|=\frac{4 \gamma}{\epsilon}\left|g_{\epsilon}\right| \frac{(1+|v|)^{2}}{\gamma} \\
& \leq \frac{4 \gamma}{\epsilon^{2}} h\left(\epsilon g_{\epsilon}\right)+\frac{4 \gamma}{\epsilon^{2}} e^{\frac{(1+|v|)^{2}}{\gamma}} .
\end{aligned}
$$

Therefore, setting $\gamma=4$ in 5.25 , we obtain that

$$
\begin{aligned}
\mid(1+|v|) & \hat{g}_{\epsilon}(1+|v|)\left(\hat{g}_{\epsilon}-\Pi \hat{g}_{\epsilon}\right) \mid \\
& \leq \frac{4}{\epsilon} \sqrt{h\left(\epsilon g_{\epsilon}\right)}(1+|v|)\left|\hat{g}_{\epsilon}-\Pi \hat{g}_{\epsilon}\right|+\frac{4}{\epsilon} e^{\frac{(1+|v|)^{2}}{8}}(1+|v|)\left|\hat{g}_{\epsilon}-\Pi \hat{g}_{\epsilon}\right| \\
& \leq \frac{16}{\epsilon^{2}} h\left(\epsilon g_{\epsilon}\right)+\frac{1}{4}(1+|v|)^{2}\left|\hat{g}_{\epsilon}-\Pi \hat{g}_{\epsilon}\right|^{2}+\frac{4}{\epsilon} e^{\frac{(1+|v|)^{2}}{8}}(1+|v|)\left|\hat{g}_{\epsilon}-\Pi \hat{g}_{\epsilon}\right| \\
& \leq \frac{16}{\epsilon^{2}} h\left(\epsilon g_{\epsilon}\right)+\frac{1}{2}(1+|v|)^{2}\left(\left|\hat{g}_{\epsilon}\right|^{2}+\left|\Pi \hat{g}_{\epsilon}\right|^{2}\right)+\frac{4}{\epsilon} e^{\frac{(1+|v|)^{2}}{8}}(1+|v|)\left|\hat{g}_{\epsilon}-\Pi \hat{g}_{\epsilon}\right|,
\end{aligned}
$$

which, by virtue of the uniform entropy bound, the uniform estimate (5.23) and the relaxation estimate 5.11 from Lemma 5.10 , yields

$$
\left|(1+|v|) \hat{g}_{\epsilon}(1+|v|)\left(\hat{g}_{\epsilon}-\Pi \hat{g}_{\epsilon}\right)\right| \leq O(1)_{L_{\mathrm{loc}}^{1}\left(d t d x ; L^{1}(M d v)\right)}+\frac{1}{2}(1+|v|)^{2}\left|\hat{g}_{\epsilon}\right|^{2} .
$$

On the whole, incorporating $(5.24)$ and 5.26 into the decomposition 5.22 , we deduce that

$$
(1+|v|)^{2}\left|\hat{g}_{\epsilon}\right|^{2} \leq O(1)_{L_{\mathrm{loc}}^{1}\left(d t d x ; L^{1}(M d v)\right)}+\frac{1}{2}(1+|v|)^{2}\left|\hat{g}_{\epsilon}\right|^{2} .
$$

Hence, we conclude

$$
(1+|v|)^{2}\left|\hat{g}_{\epsilon}\right|^{2}=O(1)_{L_{\mathrm{loc}}^{1}\left(d t d x ; L^{1}(M d v)\right)},
$$

which is the expected result.

- We establish now the uniform integrability statement of the lemma. To this end, we start from the decomposition, for any large $\lambda>e$,

$$
\left|\hat{g}_{\epsilon}\right|^{2}=\mathbb{1}_{\left\{G_{\epsilon}>\lambda\right\}}\left|\hat{g}_{\epsilon}\right|^{2}+\mathbb{1}_{\left\{G_{\epsilon} \leq \lambda\right\}} \hat{g}_{\epsilon} \Pi \hat{g}_{\epsilon}+\mathbb{1}_{\left\{G_{\epsilon} \leq \lambda\right\}} \hat{g}_{\epsilon}\left(\hat{g}_{\epsilon}-\Pi \hat{g}_{\epsilon}\right) .
$$

Then, we use the relative entropy bound, a pointwise estimate of $h(z)$, for $z>\lambda$, and the elementary inequality (B.7) to control the large tails of $G_{\epsilon}$ as follows

$$
\begin{aligned}
\mathbb{1}_{\left\{G_{\epsilon}>\lambda\right\}}\left|\hat{g}_{\epsilon}\right|^{2} & \leq \frac{4}{\epsilon^{2}} \mathbb{1}_{\left\{G_{\epsilon}>\lambda\right\}}\left|G_{\epsilon}-1\right| \leq \frac{8}{\epsilon^{2}} \mathbb{1}_{\left\{G_{\epsilon}>\lambda\right\}} G_{\epsilon} \\
& \leq \frac{8}{\epsilon^{2}(\log \lambda-1)} \mathbb{1}_{\left\{G_{\epsilon}>\lambda\right\}} G_{\epsilon}\left(\log G_{\epsilon}-1\right) \leq \frac{8}{\epsilon^{2}(\log \lambda-1)} h\left(\epsilon g_{\epsilon}\right) \\
& =O\left(\frac{1}{\log \lambda}\right)_{L^{\infty}\left(d t ; L^{1}(M d x d v)\right)},
\end{aligned}
$$

which takes care of the first term in the right-hand side of (5.27), while the second term is handled by estimate (5.24). As for the remaining term in (5.27), we deduce from the relaxation estimate (5.11) in Lemma 5.10 and from the pointwise estimate $\left|\mathbb{1}_{\left\{G_{\epsilon} \leq \lambda\right\}} \hat{g}_{\epsilon}\right| \leq \frac{2}{\epsilon}(1+\sqrt{\lambda})$, which follows straightforwardly from (B.7), that

$$
\mathbb{1}_{\left\{G_{\epsilon} \leq \lambda\right\}} \hat{g}_{\epsilon}\left(\hat{g}_{\epsilon}-\Pi \hat{g}_{\epsilon}\right)=O(\sqrt{\lambda})_{L_{\mathrm{loc}}^{1}\left(d t d x ; L^{2}(M d v)\right)} .
$$


Thus, on the whole, we have established from the decomposition 5.27 that, for any arbitrarily large $\lambda$ and each $1 \leq r<2$,

$$
\left|\hat{g}_{\epsilon}\right|^{2}=O\left(\frac{1}{\log \lambda}\right)_{L^{\infty}\left(d t ; L^{1}(M d x d v)\right)}+O(\sqrt{\lambda})_{L_{\mathrm{loc}}^{1}\left(d t d x ; L^{r}(M d v)\right)},
$$

which clearly implies that $\left|\hat{g}_{\epsilon}\right|^{2}$ is locally uniformly integrable in $v$.

- The final statement is easily obtained by combining decomposition (5.21) with the bounds

$$
\begin{aligned}
\frac{\hat{g}_{\epsilon}}{1+\lambda \epsilon\left|\hat{g}_{\epsilon}\right|} & =O(1)_{L^{\infty}\left(d t ; L^{2}(M d x d v)\right)}, \\
\frac{\hat{g}_{\epsilon}}{1+\lambda \epsilon\left|\hat{g}_{\epsilon}\right|} & =O\left(\frac{1}{\lambda \epsilon}\right)_{L^{\infty}(d t d x d v)},
\end{aligned}
$$

for any $\lambda>0$, and noticing that $1+\lambda \epsilon\left|\hat{g}_{\epsilon}\right| \leq \max \{2,1+2 \lambda\}\left(1+\lambda \sqrt{G_{\epsilon}}\right)$. We indeed find, for any $1 \leq p<2$, that

$$
\begin{aligned}
\left\|\frac{\hat{g}_{\epsilon}^{2}}{1+\lambda \epsilon\left|\hat{g}_{\epsilon}\right|}\right\|_{L^{p}(M d v)} & =\left\|\left(\hat{g}_{\epsilon}-\Pi \hat{g}_{\epsilon}\right) \frac{\hat{g}_{\epsilon}}{1+\lambda \epsilon\left|\hat{g}_{\epsilon}\right|}+\Pi \hat{g}_{\epsilon} \frac{\hat{g}_{\epsilon}}{1+\lambda \epsilon\left|\hat{g}_{\epsilon}\right|}\right\|_{L^{p}(M d v)} \\
& \leq \frac{1}{\lambda \epsilon}\left\|\hat{g}_{\epsilon}-\Pi \hat{g}_{\epsilon}\right\|_{L^{p}(M d v)}+\left\|\hat{g}_{\epsilon} \Pi \hat{g}_{\epsilon}\right\|_{L^{p}(M d v)} \\
& \leq \frac{C}{\lambda \epsilon}\left\|\hat{g}_{\epsilon}-\Pi \hat{g}_{\epsilon}\right\|_{L^{2}(M d v)}+C\left\|\hat{g}_{\epsilon}\right\|_{L^{2}(M d v)}^{2} \\
& \leq C\left\|\hat{g}_{\epsilon}\right\|_{L^{2}(M d v)}^{2}+\left\|\hat{q}_{\epsilon}\right\|_{L^{2}\left(M M_{*} d v d v_{*} d \sigma\right)} \\
& =O(1)_{L^{\infty}\left(d t ; L^{1}(d x)\right)}+O(1)_{L^{2}(d t d x)},
\end{aligned}
$$

which concludes the proof of the lemma.

In the two species case, the preceding lemma has simple but important consequences on the integrability of the difference of fluctuations, which is the content of the next lemmas.

LEMMA 5.13. Let $f_{\epsilon}^{+}(t, x, v)$ and $f_{\epsilon}^{-}(t, x, v)$ be two families of measurable, almost everywhere non-negative distribution functions such that, for all $t \geq 0$,

$$
\begin{aligned}
\frac{1}{\epsilon^{2}} H\left(f_{\epsilon}^{+}\right) & +\frac{1}{\epsilon^{2}} H\left(f_{\epsilon}^{-}\right) \\
& +\frac{1}{\epsilon^{4}} \int_{0}^{t} \int_{\mathbb{R}^{3}}\left(D\left(f_{\epsilon}^{+}\right)+D\left(f_{\epsilon}^{-}\right)+\delta^{2} D\left(f_{\epsilon}^{+}, f_{\epsilon}^{-}\right)\right)(s) d x d s \leq C^{\text {in }} .
\end{aligned}
$$

Then, as $\epsilon \rightarrow 0$, considering the densities $\rho_{\epsilon}^{ \pm}$, bulk velocities $u_{\epsilon}^{ \pm}$and temperatures $\theta_{\epsilon}^{ \pm}$respectively associated with any subsequence of fluctuations $g_{\epsilon}^{ \pm}$, it holds that

$$
h_{\epsilon}=\frac{\delta}{\epsilon}\left[\left(g_{\epsilon}^{+}-g_{\epsilon}^{-}\right)-n_{\epsilon}\right]
$$

is uniformly bounded in $L_{\mathrm{loc}}^{1}\left(d t d x ; L^{1}\left(\left(1+|v|^{2}\right) M d v\right)\right)$,

where $n_{\epsilon}=\rho_{\epsilon}^{+}-\rho_{\epsilon}^{-}$, and

$$
\begin{gathered}
j_{\epsilon}=\frac{\delta}{\epsilon}\left(u_{\epsilon}^{+}-u_{\epsilon}^{-}\right) \quad \text { and } \quad w_{\epsilon}=\frac{\delta}{\epsilon}\left(\theta_{\epsilon}^{+}-\theta_{\epsilon}^{-}\right) \\
\text {are uniformly bounded in } L_{\mathrm{loc}}^{1}(d t d x) .
\end{gathered}
$$

Proof. According to the decomposition (5.5), it is readily seen that

$$
h_{\epsilon}=\hat{h}_{\epsilon}+\frac{\delta}{4}\left[\left|\hat{g}_{\epsilon}^{+}\right|^{2}-\left|\hat{g}_{\epsilon}^{-}\right|^{2}+\int_{\mathbb{R}^{3}}\left(\left|\hat{g}_{\epsilon}^{+}\right|^{2}-\left|\hat{g}_{\epsilon}^{-}\right|^{2}\right) M d v\right] \text {, }
$$


whence, by virtue of Lemma 5.12 ,

$$
h_{\epsilon}=\hat{h}_{\epsilon}+O(\delta)_{L_{\mathrm{loc}}^{1}\left(d t d x ; L^{1}\left(\left(1+|v|^{2}\right) M d v\right)\right)},
$$

which establishes the uniform bound on $h_{\epsilon}$, thanks to the uniform bound on $\hat{h}_{\epsilon}$ from Lemma 5.11 .

Finally, integrating the above decomposition against $v M d v$ and $\left(\frac{|v|^{2}}{3}-1\right) M d v$ clearly yields

$$
j_{\epsilon}=\hat{j}_{\epsilon}+O(\delta)_{L_{\mathrm{loc}}^{1}(d t d x)} \quad \text { and } \quad w_{\epsilon}=\hat{w}_{\epsilon}+O(\delta)_{L_{\mathrm{loc}}^{1}(d t d x)},
$$

which, employing the uniform bounds on $\hat{j}_{\epsilon}$ and $\hat{w}_{\epsilon}$ from Lemma 5.11 , concludes the justification of the lemma.

LEMmA 5.14. Let $f_{\epsilon}^{+}(t, x, v)$ and $f_{\epsilon}^{-}(t, x, v)$ be two families of measurable, almost everywhere non-negative distribution functions such that, for all $t \geq 0$,

$$
\begin{aligned}
\frac{1}{\epsilon^{2}} H\left(f_{\epsilon}^{+}\right) & +\frac{1}{\epsilon^{2}} H\left(f_{\epsilon}^{-}\right) \\
& +\frac{1}{\epsilon^{4}} \int_{0}^{t} \int_{\mathbb{R}^{3}}\left(D\left(f_{\epsilon}^{+}\right)+D\left(f_{\epsilon}^{-}\right)+\delta^{2} D\left(f_{\epsilon}^{+}, f_{\epsilon}^{-}\right)\right)(s) d x d s \leq C^{\text {in }} .
\end{aligned}
$$

Then, as $\epsilon \rightarrow 0$, in the case of weak interspecies interactions, i.e. when $\delta=$ $o(1)$ and $\frac{\delta}{\epsilon}$ is unbounded, any subsequences of fluctuations $g_{\epsilon}^{ \pm}$and renormalized fluctuations $\hat{g}_{\epsilon}^{ \pm}$satisfy that

$$
h_{\epsilon}=\frac{\delta}{\epsilon}\left[\left(g_{\epsilon}^{+}-g_{\epsilon}^{-}\right)-n_{\epsilon}\right] \quad \text { and } \quad \hat{h}_{\epsilon}=\frac{\delta}{\epsilon}\left[\left(\hat{g}_{\epsilon}^{+}-\hat{g}_{\epsilon}^{-}\right)-\hat{n}_{\epsilon}\right]
$$

are weakly relatively compact in $L_{\mathrm{loc}}^{1}\left(d t d x ; L^{1}\left(\left(1+|v|^{2}\right) M d v\right)\right)$,

and

$$
\begin{gathered}
j_{\epsilon}=\frac{\delta}{\epsilon}\left(u_{\epsilon}^{+}-u_{\epsilon}^{-}\right), \quad w_{\epsilon}=\frac{\delta}{\epsilon}\left(\theta_{\epsilon}^{+}-\theta_{\epsilon}^{-}\right), \\
\hat{j}_{\epsilon}=\frac{\delta}{\epsilon}\left(\hat{u}_{\epsilon}^{+}-\hat{u}_{\epsilon}^{-}\right), \quad \hat{w}_{\epsilon}=\frac{\delta}{\epsilon}\left(\hat{\theta}_{\epsilon}^{+}-\hat{\theta}_{\epsilon}^{-}\right), \\
\text {are weakly relatively compact in } L_{\mathrm{loc}}^{1}(d t d x) .
\end{gathered}
$$

Moreover, as $\epsilon \rightarrow 0$, in the case of strong interspecies interactions, i.e. when $\delta=1$, any subsequences of fluctuations $g_{\epsilon}^{ \pm}$and renormalized fluctuations $\hat{g}_{\epsilon}^{ \pm}$satisfy that

$$
\frac{\hat{h}_{\epsilon}}{1+\left\|\hat{g}_{\epsilon}^{+}-\hat{g}_{\epsilon}^{-}\right\|_{L^{2}(M d v)}} \text { is uniformly bounded in } L_{\mathrm{loc}}^{2}\left(d t ; L^{2}(M d x d v)\right),
$$

and

$$
\begin{gathered}
\frac{\hat{j}_{\epsilon}}{1+\left\|\hat{g}_{\epsilon}^{+}-\hat{g}_{\epsilon}^{-}\right\|_{L^{2}(M d v)}} \quad \text { and } \quad \frac{\hat{w}_{\epsilon}}{1+\left\|\hat{g}_{\epsilon}^{+}-\hat{g}_{\epsilon}^{-}\right\|_{L^{2}(M d v)}} \\
\text { are uniformly bounded in } L_{\mathrm{loc}}^{2}\left(d t ; L^{2}(d x)\right),
\end{gathered}
$$

while

$$
\begin{gathered}
\frac{h_{\epsilon}}{1+\left\|\hat{g}_{\epsilon}^{+}-\hat{g}_{\epsilon}^{-}\right\|_{L^{2}(M d v)}} \text { is } \\
\text { uniformly bounded in } L_{\mathrm{loc}}^{2}\left(d t d x ; L^{1}((1+|v|) M d v)\right) \\
\text { and weakly relatively compact in } L_{\mathrm{loc}}^{1}\left(d t d x ; L^{1}((1+|v|) M d v)\right),
\end{gathered}
$$

and

$$
\frac{j_{\epsilon}}{1+\left\|\hat{g}_{\epsilon}^{+}-\hat{g}_{\epsilon}^{-}\right\|_{L^{2}(M d v)}} \text { is uniformly bounded in } L_{\mathrm{loc}}^{2}(d t d x) \text {. }
$$


Proof. We handle the case $\delta=o(1)$ first.

We have already established the uniform boundedness of $\hat{h}_{\epsilon}, \hat{j}_{\epsilon}$ and $\hat{w}_{\epsilon}$ in Lemma 5.11. while the uniform boundedness of $h_{\epsilon}, j_{\epsilon}$ and $w_{\epsilon}$ comes from Lemma 5.13. Moreover, the tightness in $v$ of $\hat{h}_{\epsilon}$ is easily deduced from the bound (5.14) from Lemma 5.11. Therefore, according to the Dunford-Pettis compactness criterion (see 66 ), it suffices to show that $h_{\epsilon}, j_{\epsilon}, w_{\epsilon}, \hat{h}_{\epsilon}, \hat{j}_{\epsilon}$ and $\hat{w}_{\epsilon}$ are uniformly integrable in all variables and that $h_{\epsilon}$ is tight in $v$.

We deal with $\hat{h}_{\epsilon}, \hat{j}_{\epsilon}$ and $\hat{w}_{\epsilon}$ first. To this end, simply notice Lemma 5.11 provides the control

$$
\left|\hat{j}_{\epsilon}\right|+\left|\hat{w}_{\epsilon}\right| \leq C\left\|\hat{h}_{\epsilon}\right\|_{L^{2}(M d v)} \leq O(\delta)\left\|\left(\begin{array}{c}
\hat{g}_{\epsilon}^{+} \\
\hat{g}_{\epsilon}^{-}
\end{array}\right)\right\|_{L^{2}(M d v)}^{2}+O(1)_{L_{\mathrm{loc}}^{2}\left(d t ; L^{2}(d x)\right)},
$$

whence, by Lemma 5.2 ,

$$
\left|\hat{j}_{\epsilon}\right|+\left|\hat{w}_{\epsilon}\right| \leq C\left\|\hat{h}_{\epsilon}\right\|_{L^{2}(M d v)} \leq O(\delta)_{L^{\infty}\left(d t ; L^{1}(d x)\right)}+O(1)_{L_{\mathrm{loc}}^{2}\left(d t ; L^{2}(d x)\right)},
$$

which establishes the equi-integrability of $\hat{j}_{\epsilon}, \hat{w}_{\epsilon}$ and $\left\|\hat{h}_{\epsilon}\right\|_{L^{2}(M d v)}$ in $t$ and $x$. Furthermore, since $\hat{h}_{\epsilon}$ is clearly equi-integrable in $v$ thanks to the bound (5.14) from Lemma 5.11, a direct application of Lemma 5.2 from [37. yields that $\hat{h}_{\epsilon}$ is equiintegrable in all variables.

Next, we deduce the relative weak compactness of $h_{\epsilon}, j_{\epsilon}$ and $w_{\epsilon}$ from the relative weak compactness of $\hat{h}_{\epsilon}, \hat{j}_{\epsilon}$ and $\hat{w}_{\epsilon}$ employing the decomposition (5.5), which clearly yields

$$
h_{\epsilon}=\hat{h}_{\epsilon}+\frac{\delta}{4}\left[\left|\hat{g}_{\epsilon}^{+}\right|^{2}-\left|\hat{g}_{\epsilon}^{-}\right|^{2}-\int_{\mathbb{R}^{3}}\left(\left|\hat{g}_{\epsilon}^{+}\right|^{2}-\left|\hat{g}_{\epsilon}^{-}\right|^{2}\right) M d v\right] .
$$

Therefore, since $\delta=o(1)$, it is readily seen, by virtue of the uniform integrability of $\hat{j}_{\epsilon}, \hat{w}_{\epsilon}$ and $\hat{h}_{\epsilon}$ and the uniform boundedness of $\hat{g}_{\epsilon}^{ \pm}$in $L_{\mathrm{loc}}^{2}\left(d t d x ; L^{2}\left(\left(1+|v|^{2}\right) M d v\right)\right)$ from Lemma 5.12, that $j_{\epsilon}, w_{\epsilon}$ and $h_{\epsilon}$ are uniformly integrable in all variables, as well, and that $h_{\epsilon}$ is tight in $v$ in $L_{\text {loc }}^{1}\left(d t d x ; L^{1}\left(\left(1+|v|^{2}\right) M d v\right)\right)$.

We turn now to the case $\delta=1$.

It is readily seen that the estimate 5.15 from Lemma 5.11 provides the refined control

$$
\left\|\hat{h}_{\epsilon}\right\|_{L^{2}(M d v)} \leq C\left\|\hat{g}_{\epsilon}^{+}-\hat{g}_{\epsilon}^{-}\right\|_{L^{2}(M d v)}\left\|\hat{g}_{\epsilon}^{ \pm}\right\|_{L^{2}(M d v)}+O(1)_{L_{\mathrm{loc}}^{2}\left(d t ; L^{2}(d x)\right)} .
$$

In particular, in view of the boundedness of $\hat{g}_{\epsilon}^{ \pm}$in $L^{\infty}\left(d t ; L^{2}(M d x d v)\right)$ from Lemma 5.2, it follows that

$$
\frac{\hat{h}_{\epsilon}}{1+\left\|\hat{g}_{\epsilon}^{+}-\hat{g}_{\epsilon}^{-}\right\|_{L^{2}(M d v)}} \quad \text { is uniformly bounded in } L_{\text {loc }}^{2}\left(d t ; L^{2}(M d x d v)\right) \text {, }
$$

and, incidentally, that

$$
\frac{\left|\hat{j}_{\epsilon}\right|+\left|\hat{w}_{\epsilon}\right|}{1+\left\|\hat{g}_{\epsilon}^{+}-\hat{g}_{\epsilon}^{-}\right\|_{L^{2}(M d v)}} \quad \text { is uniformly bounded in } L_{\mathrm{loc}}^{2}\left(d t ; L^{2}(d x)\right) \text {. }
$$

Next, we deduce the relative weak compactness of $h_{\epsilon}$ and the uniform bound on $j_{\epsilon}$ from the uniform bound on $\hat{h}_{\epsilon}$ employing the decomposition (5.5), which clearly yields

$$
h_{\epsilon}=\hat{h}_{\epsilon}+\frac{1}{4}\left[\left(\hat{g}_{\epsilon}^{+}-\hat{g}_{\epsilon}^{-}\right)\left(\hat{g}_{\epsilon}^{+}+\hat{g}_{\epsilon}^{-}\right)-\int_{\mathbb{R}^{3}}\left(\hat{g}_{\epsilon}^{+}-\hat{g}_{\epsilon}^{-}\right)\left(\hat{g}_{\epsilon}^{+}+\hat{g}_{\epsilon}^{-}\right) M d v\right] \text {. }
$$


Therefore, it is readily seen that

$$
\begin{aligned}
\left\|h_{\epsilon}\right\|_{L^{1}((1+|v|) M d v)} & \leq C\left\|\hat{h}_{\epsilon}\right\|_{L^{2}(M d v)} \\
& +C\left\|\hat{g}_{\epsilon}^{+}-\hat{g}_{\epsilon}^{-}\right\|_{L^{2}(M d v)}\left\|\hat{g}_{\epsilon}^{+}+\hat{g}_{\epsilon}^{-}\right\|_{L^{2}\left(\left(1+|v|^{2}\right) M d v\right)},
\end{aligned}
$$

whence

$\frac{h_{\epsilon}}{1+\left\|\hat{g}_{\epsilon}^{+}-\hat{g}_{\epsilon}^{-}\right\|_{L^{2}(M d v)}} \quad$ is uniformly bounded in $L_{\mathrm{loc}}^{2}\left(d t d x ; L^{1}((1+|v|) M d v)\right)$, and, incidentally,

$$
\frac{j_{\epsilon}}{1+\left\|\hat{g}_{\epsilon}^{+}-\hat{g}_{\epsilon}^{-}\right\|_{L^{2}(M d v)}} \quad \text { is uniformly bounded in } L_{\mathrm{loc}}^{2}(d t d x) \text {. }
$$

Now, it is readily seen from the uniform bound on $\hat{h}_{\epsilon}$ in $L_{\text {loc }}^{1}\left(d t d x ; L^{2}(M d v)\right)$ established in Lemma 5.11 and from the equi-integrability in $v$ of the families $\left|\hat{g}_{\epsilon}^{ \pm}\right|^{2}$ established in Lemma 5.12 that the decomposition 5.30 yields that $h_{\epsilon}$ is equiintegrable in $v$, as well. Consequently, a direct application of Lemma 5.2 from [37. yields that the family $\frac{h_{\epsilon}}{1+\left\|\hat{g}_{\epsilon}^{+}-\hat{g}_{\epsilon}^{-}\right\|_{L^{2}(M d v)}}$ is equi-integrable in all variables, which, according to the Dunford-Pettis compactness criterion (see [66 ), implies its weak relative compactness in $L_{\text {loc }}^{1}\left(d t d x ; L^{1}(M d v)\right)$. Finally, further using the uniform bound 5.29 from Lemma 5.13, we deduce that the family $\frac{h_{\epsilon}}{1+\left\|\hat{g}_{\epsilon}^{+}-\hat{g}_{\epsilon}^{-}\right\|_{L^{2}(M d v)}}$ is weakly relatively compact in $L_{\text {loc }}^{1}\left(d t d x ; L^{1}((1+|v|) M d v)\right)$, which concludes the proof of the lemma. 


\section{CHAPTER 6}

\section{Lower order linear constraint equations and energy inequalities}

In the preceding chapter, we have established uniform estimates and controls on the fluctuations and collision integrands by analyzing the relative entropy and entropy dissipation bounds. At this stage, we have now all the necessary tools to derive the asymptotic lower order linear constraint equations and energy inequalities from Theorems 4.4 and 4.5. This first part of the rigorous convergence proofs is therefore very similar for both theorems.

The derivation of higher order and nonlinear constraint equations - in particular, constraints pertaining to Theorem 4.6 - is performed in Chapter 8 and will require more advanced methods and refined properties on the fluctuations. More precisely, strong compactness and nonlinear weak compactness properties of the fluctuations, established later on in Chapter 7, will allow us to obtain the remaining constraint equations such as Ohm's law.

\subsection{Macroscopic constraint equations for one species}

The macroscopic constraint equations are obtained by integrating the limiting kinetic equation against the collision invariants. In the simplest case of a one species plasma, taking limits in the kinetic equation is straightforward once we introduce the suitable renormalization.

Proposition 6.1. Let $\left(f_{\epsilon}, E_{\epsilon}, B_{\epsilon}\right)$ be the sequence of renormalized solutions to the scaled one species Vlasov-Maxwell-Boltzmann system (4.27) considered in Theorem 4.4. In accordance with Lemmas 5.1, 5.2 and 5.3, denote by

$$
\begin{gathered}
g \in L^{\infty}\left(d t ; L^{2}(M d x d v)\right), \quad q \in L^{2}\left(M M_{*} d t d x d v d v_{*} d \sigma\right), \\
\text { and } E, B \in L^{\infty}\left(d t ; L^{2}(d x)\right),
\end{gathered}
$$

any joint limit points of the families $\hat{g}_{\epsilon}$ and $\hat{q}_{\epsilon}$ defined by (5.3) and (5.6), E $E_{\epsilon}$ and $B_{\epsilon}$, respectively.

Then, one has

$$
\int_{\mathbb{R}^{3} \times \mathbb{S}^{2}} q M_{*} d v_{*} d \sigma=\phi: \nabla_{x} u+\psi \cdot \nabla_{x} \theta
$$

where $u$ and $\theta$ are, respectively, the bulk velocity and temperature associated with the limiting fluctuation $g$, and $\phi$ and $\psi$ are the kinetic fluxes defined by 2.12. Furthermore, $\rho, u, \theta$ and $E$ satisfy the following constraints

$$
\operatorname{div} u=0, \quad \nabla_{x}(\rho+\theta)-E=0,
$$

where $\rho$ is the density associated with the limiting fluctuation $g$.

Proof. We start from some square root renormalization of the scaled VlasovBoltzmann equation 4.27). More precisely, we choose the admissible renormalization

$$
\beta(z)=\frac{\sqrt{z+\epsilon^{a}}-1}{\epsilon}
$$


for some given $1<a<4$, which yields, using the decomposition of collision integrands (5.8),

$$
\begin{aligned}
& \left(\epsilon \partial_{t}+v \cdot \nabla_{x}+\epsilon\left(E_{\epsilon}+v \wedge B_{\epsilon}\right) \cdot \nabla_{v}\right) \frac{\sqrt{G_{\epsilon}+\epsilon^{a}}-1}{\epsilon}-E_{\epsilon} \cdot v \frac{G_{\epsilon}}{2 \sqrt{G_{\epsilon}+\epsilon^{a}}} \\
& \quad=\frac{\sqrt{G_{\epsilon}}}{2 \sqrt{G_{\epsilon}+\epsilon^{a}}} \int_{\mathbb{R}^{3} \times \mathbb{S}^{2}} \sqrt{G_{\epsilon *}} \hat{q}_{\epsilon} M_{*} d v_{*} d \sigma+\frac{\epsilon^{2}}{8 \sqrt{G_{\epsilon}+\epsilon^{a}}} \int_{\mathbb{R}^{3} \times \mathbb{S}^{2}} \hat{q}_{\epsilon}^{2} M_{*} d v_{*} d \sigma \\
& \quad \stackrel{\text { def }}{=} Q_{\epsilon}^{1}+Q_{\epsilon}^{2} .
\end{aligned}
$$

Then, thanks to Lemma 5.2 it holds that

$$
\sqrt{G_{\epsilon *}}=1+O(\epsilon)_{L_{\mathrm{loc}}^{2}\left(d t ; L^{2}\left(M_{*} d x d v_{*}\right)\right)},
$$

whence, employing the uniform bound $\hat{q}_{\epsilon} \in L^{2}\left(M M_{*} d t d x d v d v_{*} d \sigma\right)$ from Lemma 5.3 .

$$
\begin{aligned}
Q_{\epsilon}^{1} & =\frac{\sqrt{G_{\epsilon}}}{2 \sqrt{G_{\epsilon}+\epsilon^{a}}} \int_{\mathbb{R}^{3} \times \mathbb{S}^{2}} \hat{q}_{\epsilon} M_{*} d v_{*} d \sigma+O(\epsilon)_{L_{\mathrm{loc}}^{1}\left(d t d x ; L^{2}(M d v)\right)}, \\
Q_{\epsilon}^{2} & =O\left(\epsilon^{2-\frac{a}{2}}\right)_{L^{1}(M d t d x d v)} .
\end{aligned}
$$

Next, since, decomposing according to the tails of $G_{\epsilon}$,

$$
\begin{aligned}
\left|\frac{\sqrt{G_{\epsilon}+\epsilon^{a}}-1}{\epsilon}-\frac{\sqrt{G_{\epsilon}}-1}{\epsilon}\right| & =\frac{\epsilon^{a-1}}{\sqrt{\epsilon^{a}+G_{\epsilon}}+\sqrt{G_{\epsilon}}} \\
& \leq \frac{\epsilon^{a-1}}{\sqrt{\epsilon^{a}+G_{\epsilon}}+\sqrt{G_{\epsilon}}} \mathbb{1}_{\left\{G_{\epsilon}>\frac{1}{2}\right\}}+\epsilon^{\frac{a}{2}-1} \mathbb{1}_{\left\{G_{\epsilon} \leq \frac{1}{2}\right\}} \\
& \leq O\left(\epsilon^{a-1}\right)_{L^{\infty}(d t d x d v)}+\epsilon^{\frac{a}{2}}(2+\sqrt{2})\left|\frac{\sqrt{G_{\epsilon}}-1}{\epsilon}\right|,
\end{aligned}
$$

one proves, by virtue of Lemma 5.2 or Lemma 5.12 , that

(6.5)

$$
\begin{aligned}
& 2 \frac{\sqrt{G_{\epsilon}+\epsilon^{a}}-1}{\epsilon}-\hat{g}_{\epsilon}=O\left(\epsilon^{\frac{a}{2}-1}\right)_{L^{\infty}(d t d x d v)}, \\
& 2 \frac{\sqrt{G_{\epsilon}+\epsilon^{a}}-1}{\epsilon}-\hat{g}_{\epsilon}=O\left(\epsilon^{a-1}\right)_{L^{\infty}(d t d x d v)}+O\left(\epsilon^{\frac{a}{2}}\right)_{L^{\infty}\left(d t ; L^{2}(M d x d v)\right)}, \\
& 2 \frac{\sqrt{G_{\epsilon}+\epsilon^{a}}-1}{\epsilon}-\hat{g}_{\epsilon}=O\left(\epsilon^{a-1}\right)_{L^{\infty}(d t d x d v)}+O\left(\epsilon^{\frac{a}{2}}\right)_{L_{\mathrm{loc}}^{2}\left(d t d x ; L^{2}\left(\left(1+|v|^{2}\right) M d v\right)\right)},
\end{aligned}
$$

and

$$
\begin{aligned}
E_{\epsilon} \cdot v & \frac{G_{\epsilon}}{\sqrt{G_{\epsilon}+\epsilon^{a}}} \\
& =E_{\epsilon} \cdot v\left(1+\epsilon \frac{\sqrt{G_{\epsilon}+\epsilon^{a}}-1}{\epsilon}\right)-E_{\epsilon} \cdot v \frac{\epsilon^{a}}{\sqrt{G_{\epsilon}+\epsilon^{a}}} \\
& =E_{\epsilon} \cdot v+O\left(\epsilon+\epsilon^{\frac{a}{2}+1}\right)_{L^{\infty}\left(d t ; L^{1}(M d x d v)\right)}+O\left(\epsilon^{a}+\epsilon^{\frac{a}{2}}\right)_{L^{\infty}\left(d t ; L^{2}(M d x d v)\right)} .
\end{aligned}
$$

In particular, employing 6.5 to deduce that

$$
\begin{aligned}
\left|\frac{\sqrt{G_{\epsilon}}}{\sqrt{G_{\epsilon}+\epsilon^{a}}}-1\right| & =\frac{\epsilon}{\sqrt{G_{\epsilon}+\epsilon^{a}}}\left|\frac{\sqrt{G_{\epsilon}+\epsilon^{a}}-1}{\epsilon}-\frac{1}{2} \hat{g}_{\epsilon}\right| \\
& \leq \epsilon^{1-\frac{a}{2}}\left|\frac{\sqrt{G_{\epsilon}+\epsilon^{a}}-1}{\epsilon}-\frac{1}{2} \hat{g}_{\epsilon}\right| \\
& \leq O\left(\epsilon^{\frac{a}{2}}\right)_{L^{\infty}(d t d x d v)}+O(\epsilon)_{L_{\mathrm{loc}}^{2}\left(d t d x ; L^{2}\left(\left(1+|v|^{2}\right) M d v\right)\right)},
\end{aligned}
$$


we obtain the following refinement of 6.4

$$
\begin{aligned}
Q_{\epsilon}^{1} & =\frac{1}{2} \int_{\mathbb{R}^{3} \times \mathbb{S}^{2}} \hat{q}_{\epsilon} M_{*} d v_{*} d \sigma+O\left(\epsilon^{\frac{a}{2}}\right)_{L^{2}(M d t d x d v)}+O(\epsilon)_{L_{\mathrm{loc}}^{1}\left(d t d x ; L^{1}((1+|v|) M d v)\right)}, \\
Q_{\epsilon}^{2} & =O\left(\epsilon^{2-\frac{a}{2}}\right)_{L^{1}(M d t d x d v)} .
\end{aligned}
$$

Therefore, taking weak limits in 6.3 leads to

$$
v \cdot \nabla_{x} g-E \cdot v=\int_{\mathbb{R}^{3} \times \mathbb{S}^{2}} q M_{*} d v_{*} d \sigma,
$$

which, together with the fact, according to Lemma 5.10, that $g$ is an infinitesimal Maxwellian, provides that

$$
\begin{aligned}
\int_{\mathbb{R}^{3} \times \mathbb{S}^{2}} q M_{*} d v_{*} d \sigma & =\operatorname{div}\left((\rho+\theta) v+\frac{|v|^{2}}{3} u+\phi u+\theta \psi\right)-E \cdot v \\
& =\left(\phi: \nabla_{x} u+\psi \cdot \nabla_{x} \theta\right)+\left(\nabla_{x}(\rho+\theta)-E\right) \cdot v+\frac{1}{3}(\operatorname{div} u)|v|^{2} .
\end{aligned}
$$

Then, remarking that $q$ inherits the collisional symmetries of $q_{\epsilon}$ and $\hat{q}_{\epsilon}$, we get

$$
\int_{\mathbb{R}^{3} \times \mathbb{R}^{3} \times \mathbb{S}^{2}} q\left(\begin{array}{c}
1 \\
v \\
\frac{|v|^{2}}{2}
\end{array}\right) M M_{*} d v d v_{*} d \sigma=0,
$$

so that, since $\phi(v)$ and $\psi(v)$ are orthogonal to the collisional invariants, the constraints 6.2 hold.

The proof of the proposition is complete.

\subsection{Macroscopic constraint equations for two species, weak interactions}

In the case of a two species plasma, the renormalization process is more complicated because there are two different distributions. Nevertheless, for weak interspecies interactions, i.e. $\delta=o(1)$ and $\frac{\delta}{\epsilon}$ unbounded, we have a result quite similar to the preceding proposition. As for strong interspecies interactions, i.e. $\delta=1$, even the lowest order constraints will require the dealing with nonlinear terms and, therefore, will be handled with more advanced techniques in Chapter 8 (see Propositions 8.3 and 8.4 .

Proposition 6.2. Let $\left(f_{\epsilon}^{ \pm}, E_{\epsilon}, B_{\epsilon}\right)$ be the sequence of renormalized solutions to the scaled two species Vlasov-Maxwell-Boltzmann system (4.34) considered in Theorem 4.5 for weak interspecies interactions, i.e. $\delta=o(1)$ and $\frac{\delta}{\epsilon}$ unbounded. In accordance with Lemmas 5.1, 5.2 and 5.3, denote by

$$
\begin{gathered}
g^{ \pm} \in L^{\infty}\left(d t ; L^{2}(M d x d v)\right), \quad q^{ \pm}, q^{ \pm, \mp} \in L^{2}\left(M M_{*} d t d x d v d v_{*} d \sigma\right), \\
\text { and } E, B \in L^{\infty}\left(d t ; L^{2}(d x)\right),
\end{gathered}
$$

any joint limit points of the families $\hat{g}_{\epsilon}^{ \pm}, \hat{q}_{\epsilon}^{ \pm}$and $\hat{q}_{\epsilon}^{ \pm, \mp}$ defined by (5.3) and (5.6), $E_{\epsilon}$ and $B_{\epsilon}$, respectively.

Then, one has

$$
\int_{\mathbb{R}^{3} \times \mathbb{S}^{2}} q^{ \pm} M_{*} d v_{*} d \sigma=\phi: \nabla_{x} u+\psi \cdot \nabla_{x} \theta,
$$

where $u$ and $\theta$ are, respectively, the bulk velocity and temperature associated with the limiting fluctuations $g^{ \pm}$, and $\phi$ and $\psi$ are the kinetic fluxes defined by 2.12 . Furthermore, $\rho^{ \pm}, u$ and $\theta$ satisfy the following constraints

$$
\operatorname{div} u=0, \quad \nabla_{x}\left(\rho^{ \pm}+\theta\right)=0,
$$


where $\rho^{ \pm}$are the densities respectively associated with the limiting fluctuations $g^{ \pm}$. In particular, the strong Boussinesq relation $\rho^{ \pm}+\theta=0$ holds and, moreover, since $\nabla_{x}\left(\rho^{+}-\rho^{-}\right)=0$, it also holds that $\rho^{+}=\rho^{-}$.

Proof. We start from some square root renormalization of the scaled VlasovBoltzmann equation 4.34). More precisely, we choose, as previously in the proof of Proposition 6.1, the admissible renormalization

$$
\beta(z)=\frac{\sqrt{z+\epsilon^{a}}-1}{\epsilon},
$$

for some given $1<a<4$, which yields, recalling the definitions 5.6 of renormalized collision integrands and using the decomposition of collision integrands (5.8),

$$
\begin{aligned}
\left(\epsilon \partial_{t}+v \cdot \nabla_{x} \pm \delta\left(\epsilon E_{\epsilon}+v \wedge B_{\epsilon}\right)\right. & \left.\cdot \nabla_{v}\right) \frac{\sqrt{G_{\epsilon}^{ \pm}+\epsilon^{a}}-1}{\epsilon} \mp \delta E_{\epsilon} \cdot v \frac{G_{\epsilon}^{ \pm}}{2 \sqrt{G_{\epsilon}^{ \pm}+\epsilon^{a}}} \\
& =\frac{\sqrt{G_{\epsilon}^{ \pm}}}{2 \sqrt{G_{\epsilon}^{ \pm}+\epsilon^{a}}} \int_{\mathbb{R}^{3} \times \mathbb{S}^{2}} \sqrt{G_{\epsilon *}^{ \pm}} \hat{q}_{\epsilon}^{ \pm} M_{*} d v_{*} d \sigma \\
& +\frac{\epsilon^{2}}{8 \sqrt{G_{\epsilon}^{ \pm}+\epsilon^{a}}} \int_{\mathbb{R}^{3} \times \mathbb{S}^{2}}\left(\hat{q}_{\epsilon}^{ \pm}\right)^{2} M_{*} d v_{*} d \sigma \\
& +\frac{\delta \sqrt{G_{\epsilon}^{ \pm}}}{2 \sqrt{G_{\epsilon}^{ \pm}+\epsilon^{a}}} \int_{\mathbb{R}^{3} \times \mathbb{S}^{2}} \sqrt{G_{\epsilon *}^{\mp}} \hat{q}_{\epsilon}^{ \pm, \mp} M_{*} d v_{*} d \sigma \\
& +\frac{\epsilon^{2}}{8 \sqrt{G_{\epsilon}^{ \pm}+\epsilon^{a}}} \int_{\mathbb{R}^{3} \times \mathbb{S}^{2}}\left(\hat{q}_{\epsilon}^{ \pm, \mp}\right)^{2} M_{*} d v_{*} d \sigma .
\end{aligned}
$$

The proof follows then the exact same lines as the proof of Proposition 6.1. In particular, we obtain without any additional difficulty the fact that the right-hand side of 6.9 converges weakly to

$$
\frac{1}{2} \int_{\mathbb{R}^{3} \times \mathbb{S}^{2}} q^{ \pm} M_{*} d v_{*} d \sigma
$$

while the renormalized densities satisfy, following (6.5),

$$
\begin{aligned}
& 2 \frac{\sqrt{G_{\epsilon}^{ \pm}+\epsilon^{a}}-1}{\epsilon}-\hat{g}_{\epsilon}^{ \pm}=O\left(\epsilon^{\frac{a}{2}-1}\right)_{L^{\infty}(d t d x d v)}, \\
& 2 \frac{\sqrt{G_{\epsilon}^{ \pm}+\epsilon^{a}}-1}{\epsilon}-\hat{g}_{\epsilon}^{ \pm}=O\left(\epsilon^{a-1}\right)_{L^{\infty}(d t d x d v)}+O\left(\epsilon^{\frac{a}{2}}\right)_{L^{\infty}\left(d t ; L^{2}(M d x d v)\right)}, \\
& 2 \frac{\sqrt{G_{\epsilon}^{ \pm}+\epsilon^{a}}-1}{\epsilon}-\hat{g}_{\epsilon}^{ \pm}=O\left(\epsilon^{a-1}\right)_{L^{\infty}(d t d x d v)}+O\left(\epsilon^{\frac{a}{2}}\right)_{L_{\mathrm{loc}}^{2}\left(d t d x ; L^{2}\left(\left(1+|v|^{2}\right) M d v\right)\right)} .
\end{aligned}
$$

Next, using the uniform $L^{\infty}\left(d t ; L^{2}(d x)\right)$ bounds on $E_{\epsilon}$ and $B_{\epsilon}$, as well as the $L_{\text {loc }}^{2}\left(d t d x ; L^{2}(M d v)\right)$ bounds on the renormalized fluctuations $\frac{\sqrt{G_{\epsilon}^{ \pm}+\epsilon^{a}}-1}{\epsilon}$ and $\frac{G_{\epsilon}^{ \pm}}{2 \sqrt{G_{\epsilon}^{ \pm}+\epsilon^{a}}}$, we easily obtain that all the terms coming from the Lorentz force in 6.9 vanish in the weak limit :

$$
\pm \delta\left(\epsilon E_{\epsilon}+v \wedge B_{\epsilon}\right) \cdot \nabla_{v} \frac{\sqrt{G_{\epsilon}^{ \pm}+\epsilon^{a}}-1}{\epsilon} \mp \delta E_{\epsilon} \cdot v \frac{G_{\epsilon}^{ \pm}}{2 \sqrt{G_{\epsilon}^{ \pm}+\epsilon^{a}}} \rightarrow 0 .
$$

Therefore, taking weak limits in 6.9 leads to

$$
v \cdot \nabla_{x} g^{ \pm}=\int_{\mathbb{R}^{3} \times \mathbb{S}^{2}} q^{ \pm} M_{*} d v_{*} d \sigma
$$


which, together with the fact, according to Lemmas 5.10 and 5.11 , that $g^{+}$and $g^{-}$are infinitesimal Maxwellians, which differ only by their densities $\rho^{+}$and $\rho^{-}$, provides that

$$
\begin{aligned}
\int_{\mathbb{R}^{3} \times \mathbb{S}^{2}} q^{ \pm} M_{*} d v_{*} d \sigma & =\operatorname{div}\left(\left(\rho^{ \pm}+\theta\right) v+\frac{|v|^{2}}{3} u+\phi u+\theta \psi\right) \\
& =\left(\phi: \nabla_{x} u+\psi \cdot \nabla_{x} \theta\right)+\nabla_{x}\left(\rho^{ \pm}+\theta\right) \cdot v+\frac{1}{3}(\operatorname{div} u)|v|^{2} .
\end{aligned}
$$

Then, remarking that $q^{ \pm}$inherits the collisional symmetries of $q_{\epsilon}^{ \pm}$and $\hat{q}_{\epsilon}^{ \pm}$, we get

$$
\int_{\mathbb{R}^{3} \times \mathbb{R}^{3} \times \mathbb{S}^{2}} q^{ \pm}\left(\begin{array}{c}
1 \\
v \\
\frac{|v|^{2}}{2}
\end{array}\right) M M_{*} d v d v_{*} d \sigma=0
$$

so that, since $\phi(v)$ and $\psi(v)$ are orthogonal to the collisional invariants, the constraints 6.8 hold.

The proof of the proposition is complete.

Proposition 6.3. Let $\left(f_{\epsilon}^{ \pm}, E_{\epsilon}, B_{\epsilon}\right)$ be the sequence of renormalized solutions to the scaled two species Vlasov-Maxwell-Boltzmann system (4.34) considered in Theorem 4.5 for weak interspecies interactions, i.e. $\delta=o(1)$ and $\frac{\delta}{\epsilon}$ unbounded. In accordance with Lemmas 5.1, 5.2, 5.3, 5.13 and 5.14 denote by

$$
\begin{aligned}
g^{ \pm} & \in L^{\infty}\left(d t ; L^{2}(M d x d v)\right), \\
q^{ \pm, \mp} & \in L^{2}\left(M M_{*} d t d x d v d v_{*} d \sigma\right), \\
h & \in L_{\text {loc }}^{1}\left(d t d x ; L^{1}\left(\left(1+|v|^{2}\right) M d v\right)\right),
\end{aligned}
$$

any joint limit points of the families $\hat{g}_{\epsilon}^{ \pm}, \hat{q}_{\epsilon}^{ \pm, \mp}$ and $h_{\epsilon}$ defined by (5.3), 5.6 and 5.29, respectively.

Then, one has $h=j \cdot v+w\left(\frac{|v|^{2}}{2}-\frac{3}{2}\right)$ and

$$
\pm 2 \int_{\mathbb{R}^{3} \times \mathbb{S}^{2}} q^{ \pm, \mp} M_{*} d v_{*} d \sigma=-\mathfrak{L}(h)=-j \cdot \mathfrak{L}(v)-w \mathfrak{L}\left(\frac{|v|^{2}}{2}\right),
$$

where $j$ and $w$ are, respectively, the bulk velocity and temperature associated with the limiting fluctuation $h$, i.e. $j$ is the electric current and $w$ is the internal electric energy.

Proof. We start from the decomposition

$$
h_{\epsilon}=\hat{h}_{\epsilon}+\frac{\delta}{4}\left[\left|\hat{g}_{\epsilon}^{+}\right|^{2}-\left|\hat{g}_{\epsilon}^{-}\right|^{2}-\int_{\mathbb{R}^{3}}\left(\left|\hat{g}_{\epsilon}^{+}\right|^{2}-\left|\hat{g}_{\epsilon}^{-}\right|^{2}\right) M d v\right],
$$

which follows from the decomposition 5.5 of fluctuations. In particular, integrating 6.12 against $v M d v$ and $\left(\frac{|v|^{2}}{3}-1\right) M d v$ yields

$$
\begin{aligned}
j_{\epsilon} & =\hat{j}_{\epsilon}+\frac{\delta}{4} \int_{\mathbb{R}^{3}}\left(\left|\hat{g}_{\epsilon}^{+}\right|^{2}-\left|\hat{g}_{\epsilon}^{-}\right|^{2}\right) v M d v \\
w_{\epsilon} & =\hat{w}_{\epsilon}+\frac{\delta}{4} \int_{\mathbb{R}^{3}}\left(\left|\hat{g}_{\epsilon}^{+}\right|^{2}-\left|\hat{g}_{\epsilon}^{-}\right|^{2}\right)\left(\frac{|v|^{2}}{3}-1\right) M d v .
\end{aligned}
$$

According to Lemma 5.14 , we consider now weakly convergent subsequences

$$
h_{\epsilon} \rightarrow h, \quad \hat{h}_{\epsilon} \rightarrow \hat{h},
$$

in $L_{\text {loc }}^{1}\left(d t d x ; L^{1}\left(\left(1+|v|^{2}\right) M d v\right)\right)$, and

$$
j_{\epsilon} \rightarrow j, \quad w_{\epsilon} \rightarrow w, \quad \hat{j}_{\epsilon} \rightarrow \hat{j}, \quad \hat{w}_{\epsilon} \rightarrow \hat{w}
$$


in $L_{\text {loc }}^{1}(d t d x)$. Clearly, since $\delta=o(1)$, we easily obtain, in view of the uniform $L_{\text {loc }}^{2}\left(d t d x ; L^{2}\left(\left(1+|v|^{2}\right) M d v\right)\right)$ bound on $\hat{g}_{\epsilon}^{ \pm}$provided by Lemma 5.12 passing to the limit in 6.12 , that

$$
h=\hat{h}, \quad j=\hat{j} \quad \text { and } \quad w=\hat{w} .
$$

Furthermore, using the relaxation estimate (5.11) from Lemma 5.10, it holds that

$$
\hat{h}_{\epsilon}-\Pi \hat{h}_{\epsilon}=\frac{\delta}{\epsilon}\left(\hat{g}_{\epsilon}^{+}-\Pi \hat{g}_{\epsilon}^{+}-\hat{g}_{\epsilon}^{-}+\Pi \hat{g}_{\epsilon}^{+}\right)=O(\delta)_{L_{\mathrm{loc}}^{1}\left(d t d x ; L^{2}(M d v)\right)},
$$

whence $\hat{h}=\Pi \hat{h}$, for $\delta$ vanishes asymptotically, and, therefore,

$$
h=\hat{h}=j \cdot v+w\left(\frac{|v|^{2}}{2}-\frac{3}{2}\right) .
$$

Next, it is readily seen that the elementary decompositions

$$
\begin{aligned}
\mathcal{L}\left(\hat{g}_{\epsilon}^{ \pm}, \hat{g}_{\epsilon}^{\mp}\right) & =\frac{\epsilon}{2} \mathcal{Q}\left(\hat{g}_{\epsilon}^{ \pm}, \hat{g}_{\epsilon}^{\mp}\right)-\frac{2}{\epsilon} \mathcal{Q}\left(\sqrt{G_{\epsilon}^{ \pm}}, \sqrt{G_{\epsilon}^{\mp}}\right), \\
\mathcal{L}\left(\hat{g}_{\epsilon}^{ \pm}, \hat{g}_{\epsilon}^{\mp}\right) & = \pm \frac{\epsilon}{2 \delta} \mathfrak{L}\left(\hat{h}_{\epsilon}\right)+\frac{1}{2} \mathcal{L}\left(\hat{g}_{\epsilon}^{+}+\hat{g}_{\epsilon}^{-}\right),
\end{aligned}
$$

yield that

$$
\begin{aligned}
\mathfrak{L}\left(\hat{h}_{\epsilon}\right) & =\mp \frac{\delta}{\epsilon} \mathcal{L}\left(\hat{g}_{\epsilon}^{+}+\hat{g}_{\epsilon}^{-}\right) \pm \delta \mathcal{Q}\left(\hat{g}_{\epsilon}^{ \pm}, \hat{g}_{\epsilon}^{\mp}\right) \mp \frac{4 \delta}{\epsilon^{2}} \mathcal{Q}\left(\sqrt{G_{\epsilon}^{ \pm}}, \sqrt{G_{\epsilon}^{\mp}}\right) \\
& =\mp \delta\left(\mathcal{L}\left(\frac{\hat{g}_{\epsilon}^{+}-\Pi \hat{g}_{\epsilon}^{+}}{\epsilon}\right)+\mathcal{L}\left(\frac{\hat{g}_{\epsilon}^{-}-\Pi \hat{g}_{\epsilon}^{-}}{\epsilon}\right)-\mathcal{Q}\left(\hat{g}_{\epsilon}^{ \pm}, \hat{g}_{\epsilon}^{\mp}\right)\right) \\
& \mp 2 \int_{\mathbb{R}^{3} \times \mathbb{S}^{2}} \hat{q}_{\epsilon}^{ \pm, \mp} M_{*} d v_{*} d \sigma .
\end{aligned}
$$

Therefore, passing to the limit $\epsilon \rightarrow 0$ in 6.14, we find, in view of the control (5.11) from Lemma 5.10 and since the linear and quadratic collision operators are continuous on $L^{2}(M d v)$ (see $(5.18)$ ), that

$$
\mathfrak{L}(h)=\mp 2 \int_{\mathbb{R}^{3} \times \mathbb{S}^{2}} q^{ \pm, \mp} M_{*} d v_{*} d \sigma .
$$

Further employing the infinitesimal Maxwellian expression of $h$ from 6.13, we arrive at

$$
j \cdot \mathfrak{L}(v)+\frac{1}{2} w \mathfrak{L}\left(|v|^{2}\right)=\mp 2 \int_{\mathbb{R}^{3} \times \mathbb{S}^{2}} q^{ \pm, \mp} M_{*} d v_{*} d \sigma,
$$

which concludes the proof of the proposition.

\subsection{Energy inequalities}

In view of the results from Sections 6.1 and 6.2 , we are now able to establish the limiting energy inequalities for one species and for two species in the case of weak interactions only. The limiting energy inequality for strong interactions will require the results from Section 8.2 and, thus, will be treated later on in Section 8.3 .

Proposition 6.4. Let $\left(f_{\epsilon}, E_{\epsilon}, B_{\epsilon}\right)$ be the sequence of renormalized solutions to the scaled one species Vlasov-Maxwell-Boltzmann system 4.27) considered in Theorem 4.4. In accordance with Lemmas 5.1, 5.2 and 5.3, denote by

$$
\begin{gathered}
g \in L^{\infty}\left(d t ; L^{2}(M d x d v)\right), \quad q \in L^{2}\left(M M_{*} d t d x d v d v_{*} d \sigma\right), \\
\text { and } E, B \in L^{\infty}\left(d t ; L^{2}(d x)\right),
\end{gathered}
$$

any joint limit points of the families $\hat{g}_{\epsilon}$ and $\hat{q}_{\epsilon}$ defined by (5.3) and (5.6), $E_{\epsilon}$ and $B_{\epsilon}$, respectively. 
Then, one has the energy inequality, for almost every $t \geq 0$,

$$
\begin{aligned}
\frac{1}{2}\left(\|\rho\|_{L_{x}^{2}}^{2}+\|u\|_{L_{x}^{2}}^{2}\right. & \left.+\frac{3}{2}\|\theta\|_{L_{x}^{2}}^{2}+\|E\|_{L_{x}^{2}}^{2}+\|B\|_{L_{x}^{2}}^{2}\right)(t) \\
& +\int_{0}^{t}\left(\mu\left\|\nabla_{x} u\right\|_{L_{x}^{2}}^{2}+\frac{5}{2} \kappa\left\|\nabla_{x} \theta\right\|_{L_{x}^{2}}^{2}\right)(s) d s \leq C^{\mathrm{in}},
\end{aligned}
$$

where $\rho, u$ and $\theta$ are, respectively, the density, bulk velocity and temperature associated with the limiting fluctuation $g$, and the viscosity $\mu>0$ and thermal conductivity $\kappa>0$ are defined by 2.27.

Proof. First, by the estimate (5.7) from Lemma 5.3 and the weak sequential lower semi-continuity of convex functionals, we find that, for all $t \geq 0$,

$$
\begin{aligned}
\frac{1}{4} \int_{0}^{t} \int_{\mathbb{R}^{3}} \int_{\mathbb{R}^{3} \times \mathbb{R}^{3} \times \mathbb{S}^{2}} & q^{2} M M_{*} d v d v_{*} d \sigma d x d s \\
& \leq \liminf _{\epsilon \rightarrow 0} \frac{1}{4} \int_{0}^{t} \int_{\mathbb{R}^{3}} \int_{\mathbb{R}^{3} \times \mathbb{R}^{3} \times \mathbb{S}^{2}} \hat{q}_{\epsilon}^{2} M M_{*} d v d v_{*} d \sigma d x d s \\
& \leq \liminf _{\epsilon \rightarrow 0} \frac{1}{\epsilon^{4}} \int_{0}^{t} \int_{\mathbb{R}^{3}} D\left(f_{\epsilon}\right)(s) d x d s
\end{aligned}
$$

which, when combined with Lemma 5.1, yields, passing to the limit in the entropy inequality 4.28, for almost every $t \geq 0$,

$$
\begin{aligned}
\frac{1}{2} \int_{\mathbb{R}^{3} \times \mathbb{R}^{3}} g^{2}(t) M d x d v & +\frac{1}{2} \int_{\mathbb{R}^{3}}\left(|E|^{2}+|B|^{2}\right)(t) d x \\
& +\frac{1}{4} \int_{0}^{t} \int_{\mathbb{R}^{3}} \int_{\mathbb{R}^{3} \times \mathbb{R}^{3} \times \mathbb{S}^{2}} q^{2} M M_{*} d v d v_{*} d \sigma d x d s \leq C^{\mathrm{in}}
\end{aligned}
$$

Since, according to Lemma 5.10 the limiting fluctuation $g=\rho+u \cdot v+\theta\left(\frac{|v|^{2}}{2}-\frac{3}{2}\right)$ is an infinitesimal Maxwellian, we easily compute that

$$
\int_{\mathbb{R}^{3}} g^{2} M d v=\rho^{2}+|u|^{2}+\frac{3}{2} \theta^{2},
$$

which implies

$$
\begin{aligned}
\frac{1}{2}\left(\|\rho\|_{L_{x}^{2}}^{2}+\|u\|_{L_{x}^{2}}^{2}\right. & \left.+\frac{3}{2}\|\theta\|_{L_{x}^{2}}^{2}+\|E\|_{L_{x}^{2}}^{2}+\|B\|_{L_{x}^{2}}^{2}\right) \\
& +\frac{1}{4} \int_{0}^{t} \int_{\mathbb{R}^{3}} \int_{\mathbb{R}^{3} \times \mathbb{R}^{3} \times \mathbb{S}^{2}} q^{2} M M_{*} d v d v_{*} d \sigma d x d s \leq C^{\mathrm{in}} .
\end{aligned}
$$

There only remains to evaluate the contribution of the entropy dissipation in 6.15, which will result from a direct application of the following Bessel inequality, established in [9, Lemma 4.7] :

$$
\begin{aligned}
\frac{2}{\mu}\left|\int_{\mathbb{R}^{3} \times \mathbb{R}^{3} \times \mathbb{S}^{2}} q \tilde{\phi} M M_{*} d v d v_{*} d \sigma\right|^{2} & +\frac{8}{5 \kappa}\left|\int_{\mathbb{R}^{3} \times \mathbb{R}^{3} \times \mathbb{S}^{2}} q \tilde{\psi} M M_{*} d v d v_{*} d \sigma\right|^{2} \\
& \leq \int_{\mathbb{R}^{3} \times \mathbb{R}^{3} \times \mathbb{S}^{2}} q^{2} M M_{*} d v d v_{*} d \sigma
\end{aligned}
$$

where $\tilde{\phi}$ and $\tilde{\psi}$ are defined by (2.13).

For the sake of completeness and for later reference, we provide a short justification of (6.16) below. But prior to this, let us conclude the proof of the present proposition. To this end, we employ the identity 6.1 from Proposition 6.1 in combination with the relations 2.26$)$, which we reproduce here for the mere convenience 
of the reader :

$$
\begin{aligned}
\int_{\mathbb{R}^{3}} \phi_{i j} \tilde{\phi}_{k l} M d v & =\mu\left(\delta_{i k} \delta_{j l}+\delta_{i l} \delta_{j k}-\frac{2}{3} \delta_{i j} \delta_{k l}\right), \\
\int_{\mathbb{R}^{3}} \psi_{i} \tilde{\psi}_{j} M d v & =\frac{5}{2} \kappa \delta_{i j},
\end{aligned}
$$

to deduce from the inequality $(6.16)$ that

$$
2 \mu\left|\nabla_{x} u+\nabla_{x}^{t} u-\frac{2}{3}(\operatorname{div} u) \operatorname{Id}\right|^{2}+10 \kappa\left|\nabla_{x} \theta\right|^{2} \leq \int_{\mathbb{R}^{3} \times \mathbb{R}^{3} \times \mathbb{S}^{2}} q^{2} M M_{*} d v d v_{*} d \sigma
$$

whence, thanks to the solenoidal constraint on $u$ established in 6.2 ,

$$
\begin{aligned}
\int_{0}^{t}\left(\mu\left\|\nabla_{x} u\right\|_{L_{x}^{2}}^{2}+\frac{5}{2} \kappa\right. & \left.\left\|\nabla_{x} \theta\right\|_{L_{x}^{2}}^{2}\right)(s) d s \\
& \leq \frac{1}{4} \int_{0}^{t} \int_{\mathbb{R}^{3}} \int_{\mathbb{R}^{3} \times \mathbb{R}^{3} \times \mathbb{S}^{2}} q^{2} M M_{*} d v d v_{*} d \sigma d x d s
\end{aligned}
$$

Combining this with 6.15 concludes the proof of the proposition.

Now, as announced above, we give a short proof of $(6.16)$. To this end, following 9. Lemma 4.7], we recall that, for any traceless symmetric matrix $A \in \mathbb{R}^{3 \times 3}$ and any vector $a \in \mathbb{R}^{3}$, one computes straightforwardly, employing the identities 2.26 . (reproduced above, for convenience) and the collisional symmetries, that

$$
\begin{aligned}
\frac{1}{16} \int_{\mathbb{R}^{3} \times \mathbb{R}^{3} \times \mathbb{S}^{2}} & \left(A:\left(\tilde{\phi}+\tilde{\phi}_{*}-\tilde{\phi}^{\prime}-\tilde{\phi}_{*}^{\prime}\right)+a \cdot\left(\tilde{\psi}+\tilde{\psi}_{*}-\tilde{\psi}^{\prime}-\tilde{\psi}_{*}^{\prime}\right)\right)^{2} M M_{*} d v d v_{*} d \sigma \\
= & \frac{1}{4}(A \otimes A): \int_{\mathbb{R}^{3}}(\phi \otimes \tilde{\phi}) M d v+\frac{1}{4}(a \otimes a): \int_{\mathbb{R}^{3}}(\psi \otimes \tilde{\psi}) M d v \\
& =\frac{1}{2} \mu A: A+\frac{5}{8} \kappa a \cdot a .
\end{aligned}
$$

Therefore, defining, for any $q_{0} \in L^{2}\left(M M_{*} d v d v_{*} d \sigma\right)$, the projection

$$
\bar{q}_{0}=A_{0}: \frac{1}{4}\left(\tilde{\phi}+\tilde{\phi}_{*}-\tilde{\phi}^{\prime}-\tilde{\phi}_{*}^{\prime}\right)+a_{0} \cdot \frac{1}{4}\left(\tilde{\psi}+\tilde{\psi}_{*}-\tilde{\psi}^{\prime}-\tilde{\psi}_{*}^{\prime}\right)
$$

where

$$
\begin{aligned}
& A_{0}=\frac{1}{2 \mu} \int_{\mathbb{R}^{3} \times \mathbb{R}^{3} \times \mathbb{S}^{2}} q_{0}\left(\tilde{\phi}+\tilde{\phi}_{*}-\tilde{\phi}^{\prime}-\tilde{\phi}_{*}^{\prime}\right) M M_{*} d v d v_{*} d \sigma \\
& a_{0}=\frac{2}{5 \kappa} \int_{\mathbb{R}^{3} \times \mathbb{R}^{3} \times \mathbb{S}^{2}} q_{0}\left(\tilde{\psi}+\tilde{\psi}_{*}-\tilde{\psi}^{\prime}-\tilde{\psi}_{*}^{\prime}\right) M M_{*} d v d v_{*} d \sigma
\end{aligned}
$$

we find that

$\int_{\mathbb{R}^{3} \times \mathbb{R}^{3} \times \mathbb{S}^{2}} q_{0} \bar{q}_{0} M M_{*} d v d v_{*} d \sigma=\frac{1}{2} \mu A_{0}: A_{0}+\frac{5}{8} \kappa a_{0} \cdot a_{0}=\int_{\mathbb{R}^{3} \times \mathbb{R}^{3} \times \mathbb{S}^{2}} \bar{q}_{0}^{2} M M_{*} d v d v_{*} d \sigma$.

Hence the Bessel inequality

$$
\begin{aligned}
\frac{1}{2} \mu A_{0}: A_{0}+\frac{5}{8} \kappa a_{0} \cdot a_{0} & =\int_{\mathbb{R}^{3} \times \mathbb{R}^{3} \times \mathbb{S}^{2}} \bar{q}_{0}^{2} M M_{*} d v d v_{*} d \sigma \\
& \leq \int_{\mathbb{R}^{3} \times \mathbb{R}^{3} \times \mathbb{S}^{2}} q_{0}^{2} M M_{*} d v d v_{*} d \sigma .
\end{aligned}
$$


Therefore, setting $q_{0}=q$ in 6.17 , we find, exploiting the collisional symmetries of $q$, that

$$
\begin{aligned}
\frac{2}{\mu} \int_{\mathbb{R}^{3} \times \mathbb{R}^{3} \times \mathbb{S}^{2}} q \tilde{\phi} M M_{*} d v d v_{*} d \sigma: \int_{\mathbb{R}^{3} \times \mathbb{R}^{3} \times \mathbb{S}^{2}} q \tilde{\phi} M M_{*} d v d v_{*} d \sigma \\
+\frac{8}{5 \kappa} \int_{\mathbb{R}^{3} \times \mathbb{R}^{3} \times \mathbb{S}^{2}} q \tilde{\psi} M M_{*} d v d v_{*} d \sigma \cdot \int_{\mathbb{R}^{3} \times \mathbb{R}^{3} \times \mathbb{S}^{2}} q \tilde{\psi} M M_{*} d v d v_{*} d \sigma \\
\leq \int_{\mathbb{R}^{3} \times \mathbb{R}^{3} \times \mathbb{S}^{2}} q^{2} M M_{*} d v d v_{*} d \sigma
\end{aligned}
$$

which concludes the justification of 6.16 .

Proposition 6.5. Let $\left(f_{\epsilon}^{ \pm}, E_{\epsilon}, B_{\epsilon}\right)$ be the sequence of renormalized solutions to the scaled two species Vlasov-Maxwell-Boltzmann system (4.34) considered in Theorem 4.5 for weak interspecies interactions, i.e. $\delta=o(1)$ and $\frac{\delta}{\epsilon}$ unbounded. In accordance with Lemmas 5.1, 5.2, 5.3 and 5.14, denote by

$$
\begin{gathered}
g^{ \pm} \in L^{\infty}\left(d t ; L^{2}(M d x d v)\right), \quad h \in L_{\mathrm{loc}}^{1}\left(d t d x ; L^{1}\left(\left(1+|v|^{2}\right) M d v\right)\right), \\
q^{ \pm}, q^{ \pm, \mp} \in L^{2}\left(M M_{*} d t d x d v d v_{*} d \sigma\right) \quad \text { and } \quad E, B \in L^{\infty}\left(d t ; L^{2}(d x)\right),
\end{gathered}
$$

any joint limit points of the families $\hat{g}_{\epsilon}^{ \pm}, h_{\epsilon}, \hat{q}_{\epsilon}^{ \pm}$and $\hat{q}_{\epsilon}^{ \pm, \mp}$ defined by (5.3), (5.29) and (5.6), $E_{\epsilon}$ and $B_{\epsilon}$, respectively.

Then, one has the energy inequality, for almost every $t \geq 0$,

$$
\begin{aligned}
& \frac{1}{2}\left(2\|u\|_{L_{x}^{2}}^{2}+5\|\theta\|_{L_{x}^{2}}^{2}+\|E\|_{L_{x}^{2}}^{2}+\|B\|_{L_{x}^{2}}^{2}\right)(t) \\
& \quad+\int_{0}^{t}\left(2 \mu\left\|\nabla_{x} u\right\|_{L_{x}^{2}}^{2}+5 \kappa\left\|\nabla_{x} \theta\right\|_{L_{x}^{2}}^{2}+\frac{1}{\sigma}\|j\|_{L_{x}^{2}}^{2}+\frac{1}{2 \lambda}\|w\|_{L_{x}^{2}}^{2}\right)(s) d s \leq C^{\mathrm{in}}
\end{aligned}
$$

where $\rho, u$ and $\theta$ are, respectively, the density, bulk velocity and temperature associated with the limiting fluctuation $g$, while $j$ and $w$ are, respectively, the electric current and the internal electric energy associated with the limiting fluctuation $h$, and, finally, the viscosity $\mu>0$, thermal conductivity $\kappa>0$, electric conductivity $\sigma>0$ and energy conductivity $\lambda>0$ are respectively defined by 2.56, 2.70) and (2.71).

Proof. First, by the estimate (5.7) from Lemma 5.3 and the weak sequential lower semi-continuity of convex functionals, we find that, for all $t \geq 0$,

$$
\begin{aligned}
\frac{1}{4} \int_{0}^{t} \int_{\mathbb{R}^{3}} \int_{\mathbb{R}^{3} \times \mathbb{R}^{3} \times \mathbb{S}^{2}} & \left(q^{ \pm}\right)^{2} M M_{*} d v d v_{*} d \sigma d x d s \\
& \leq \liminf _{\epsilon \rightarrow 0} \frac{1}{4} \int_{0}^{t} \int_{\mathbb{R}^{3}} \int_{\mathbb{R}^{3} \times \mathbb{R}^{3} \times \mathbb{S}^{2}}\left(\hat{q}_{\epsilon}^{ \pm}\right)^{2} M M_{*} d v d v_{*} d \sigma d x d s \\
& \leq \liminf _{\epsilon \rightarrow 0} \frac{1}{\epsilon^{4}} \int_{0}^{t} \int_{\mathbb{R}^{3}} D\left(f_{\epsilon}^{ \pm}\right)(s) d x d s
\end{aligned}
$$

and

$$
\begin{aligned}
\frac{1}{2} \int_{0}^{t} \int_{\mathbb{R}^{3}} \int_{\mathbb{R}^{3} \times \mathbb{R}^{3} \times \mathbb{S}^{2}} & \left(q^{ \pm, \mp}\right)^{2} M M_{*} d v d v_{*} d \sigma d x d s \\
& \leq \liminf _{\epsilon \rightarrow 0} \frac{1}{2} \int_{0}^{t} \int_{\mathbb{R}^{3}} \int_{\mathbb{R}^{3} \times \mathbb{R}^{3} \times \mathbb{S}^{2}}\left(\hat{q}_{\epsilon}^{ \pm, \mp}\right)^{2} M M_{*} d v d v_{*} d \sigma d x d s \\
& \leq \liminf _{\epsilon \rightarrow 0} \frac{\delta^{2}}{\epsilon^{4}} \int_{0}^{t} \int_{\mathbb{R}^{3}} D\left(f_{\epsilon}^{+}, f_{\epsilon}^{-}\right)(s) d x d s
\end{aligned}
$$


which, when combined with Lemma 5.1, yields, passing to the limit in the entropy inequality 4.35, for almost every $t \geq 0$,

$$
\begin{aligned}
& \frac{1}{2} \int_{\mathbb{R}^{3} \times \mathbb{R}^{3}}\left(\left(g^{+}\right)^{2}+\left(g^{-}\right)^{2}\right)(t) M d x d v+\frac{1}{2} \int_{\mathbb{R}^{3}}\left(|E|^{2}+|B|^{2}\right)(t) d x \\
& \quad+\frac{1}{4} \int_{0}^{t} \int_{\mathbb{R}^{3}} \int_{\mathbb{R}^{3} \times \mathbb{R}^{3} \times \mathbb{S}^{2}}\left(\left(q^{+}\right)^{2}+\left(q^{-}\right)^{2}+\left(q^{+,-}\right)^{2}+\left(q^{-,+}\right)^{2}\right) M M_{*} d v d v_{*} d \sigma d x d s \\
& \quad \leq C^{\mathrm{in}} .
\end{aligned}
$$

Since, according to Lemmas 5.10 and 5.11 and Proposition 6.2 the limiting fluctuations $g^{ \pm}=\rho+u \cdot v+\theta\left(\frac{|v|^{2}}{2}-\frac{3}{2}\right)$ are infinitesimal Maxwellians which coincide, we easily compute that, in view of the strong Boussinesq relation $\rho+\theta=0$ following from 6.8 ,

$$
\frac{1}{2} \int_{\mathbb{R}^{3}}\left(\left(g^{+}\right)^{2}+\left(g^{-}\right)^{2}\right) M d v=\rho^{2}+|u|^{2}+\frac{3}{2} \theta^{2}=|u|^{2}+\frac{5}{2} \theta^{2},
$$

which implies

$$
\begin{aligned}
& \left(\|u\|_{L_{x}^{2}}^{2}+\frac{5}{2}\|\theta\|_{L_{x}^{2}}^{2}+\frac{1}{2}\|E\|_{L_{x}^{2}}^{2}+\frac{1}{2}\|B\|_{L_{x}^{2}}^{2}\right) \\
& +\frac{1}{4} \int_{0}^{t} \int_{\mathbb{R}^{3}} \int_{\mathbb{R}^{3} \times \mathbb{R}^{3} \times \mathbb{S}^{2}}\left(\left(q^{+}\right)^{2}+\left(q^{-}\right)^{2}+\left(q^{+,-}\right)^{2}+\left(q^{-,+}\right)^{2}\right) M M_{*} d v d v_{*} d \sigma d x d s \\
& \leq C^{\text {in }} .
\end{aligned}
$$

There only remains to evaluate the contribution of the entropy dissipation in 6.18. To this end, applying the method of proof of Proposition 6.4, based on the Bessel inequality (6.16), with the constraints 6.7 and 6.8 from Proposition 6.2. note that it holds

$$
\begin{aligned}
\int_{0}^{t}\left(\mu\left\|\nabla_{x} u\right\|_{L_{x}^{2}}^{2}+\frac{5}{2} \kappa\right. & \left.\left\|\nabla_{x} \theta\right\|_{L_{x}^{2}}^{2}\right)(s) d s \\
& \leq \frac{1}{4} \int_{0}^{t} \int_{\mathbb{R}^{3}} \int_{\mathbb{R}^{3} \times \mathbb{R}^{3} \times \mathbb{S}^{2}}\left(q^{ \pm}\right)^{2} M M_{*} d v d v_{*} d \sigma d x d s .
\end{aligned}
$$

Next, the contributions of the mixed entropy dissipations $q^{ \pm, \mp}$ will be evaluated through a direct application of the following Bessel inequality :

$$
\begin{aligned}
2 \sigma\left|\int_{\mathbb{R}^{3} \times \mathbb{R}^{3} \times \mathbb{S}^{2}} q^{ \pm, \mp} v M M_{*} d v d v_{*} d \sigma\right|^{2} & +\left.\left.\lambda\left|\int_{\mathbb{R}^{3} \times \mathbb{R}^{3} \times \mathbb{S}^{2}} q^{ \pm, \mp}\right| v\right|^{2} M M_{*} d v d v_{*} d \sigma\right|^{2} \\
& \leq \int_{\mathbb{R}^{3} \times \mathbb{R}^{3} \times \mathbb{S}^{2}}\left(q^{ \pm, \mp}\right)^{2} M M_{*} d v d v_{*} d \sigma
\end{aligned}
$$

For the sake of completeness, we provide a short justification of 6.20 below. But prior to this, let us conclude the proof of the present proposition. To this end, we employ the identity 6.11 from Proposition 6.3 in combination with the relations 2.70 and 2.71 to deduce from the inequality 6.20 that

$$
\frac{2}{\sigma}|j|^{2}+\frac{1}{\lambda}|w|^{2} \leq \int_{\mathbb{R}^{3} \times \mathbb{R}^{3} \times \mathbb{S}^{2}}\left(q^{ \pm, \mp}\right)^{2} M M_{*} d v d v_{*} d \sigma .
$$

Combining this with 6.18 and 6.19 concludes the proof of the proposition.

Now, as announced, we give a short proof of $\sqrt{6.20}$. To this end, for any vector $A \in \mathbb{R}^{3}$ and any scalar $a \in \mathbb{R}$, one computes straightforwardly, employing 
Proposition A.1 and the collisional symmetries, that

$$
\begin{aligned}
\int_{\mathbb{R}^{3} \times \mathbb{R}^{3} \times \mathbb{S}^{2}} & \left(A \cdot\left(v-v_{*}-v^{\prime}+v_{*}^{\prime}\right)+a\left(|v|^{2}-\left|v_{*}\right|^{2}-\left|v^{\prime}\right|^{2}+\left|v_{*}^{\prime}\right|^{2}\right)\right)^{2} M M_{*} d v d v_{*} d \sigma \\
& =4 \int_{\mathbb{R}^{3} \times \mathbb{R}^{3} \times \mathbb{S}^{2}}\left(A \cdot\left(v-v^{\prime}\right)+a\left(|v|^{2}-\left|v^{\prime}\right|^{2}\right)\right)^{2} M M_{*} d v d v_{*} d \sigma \\
& =\frac{8}{\sigma}|A|^{2}+\frac{16}{\lambda} a^{2} .
\end{aligned}
$$

Therefore, defining, for any $q_{0} \in L^{2}\left(M M_{*} d v d v_{*} d \sigma\right)$, the projection

$$
\bar{q}_{0}=A_{0} \cdot\left(v-v_{*}-v^{\prime}+v_{*}^{\prime}\right)+a_{0}\left(|v|^{2}-\left|v_{*}\right|^{2}-\left|v^{\prime}\right|^{2}+\left|v_{*}^{\prime}\right|^{2}\right),
$$

where

$$
\begin{aligned}
& A_{0}=\frac{\sigma}{8} \int_{\mathbb{R}^{3} \times \mathbb{R}^{3} \times \mathbb{S}^{2}} q_{0}\left(v-v_{*}-v^{\prime}+v_{*}^{\prime}\right) M M_{*} d v d v_{*} d \sigma \\
& a_{0}=\frac{\lambda}{16} \int_{\mathbb{R}^{3} \times \mathbb{R}^{3} \times \mathbb{S}^{2}} q_{0}\left(|v|^{2}-\left|v_{*}\right|^{2}-\left|v^{\prime}\right|^{2}+\left|v_{*}^{\prime}\right|^{2}\right) M M_{*} d v d v_{*} d \sigma,
\end{aligned}
$$

we find that

$$
\int_{\mathbb{R}^{3} \times \mathbb{R}^{3} \times \mathbb{S}^{2}} q_{0} \bar{q}_{0} M M_{*} d v d v_{*} d \sigma=\frac{8}{\sigma}\left|A_{0}\right|^{2}+\frac{16}{\lambda} a_{0}^{2}=\int_{\mathbb{R}^{3} \times \mathbb{R}^{3} \times \mathbb{S}^{2}} \bar{q}_{0}^{2} M M_{*} d v d v_{*} d \sigma .
$$

Hence the Bessel inequality

$$
\begin{aligned}
\frac{8}{\sigma}\left|A_{0}\right|^{2}+\frac{16}{\lambda} a_{0}^{2} & =\int_{\mathbb{R}^{3} \times \mathbb{R}^{3} \times \mathbb{S}^{2}} \bar{q}_{0}^{2} M M_{*} d v d v_{*} d \sigma \\
& \leq \int_{\mathbb{R}^{3} \times \mathbb{R}^{3} \times \mathbb{S}^{2}} q_{0}^{2} M M_{*} d v d v_{*} d \sigma
\end{aligned}
$$

Therefore, setting $q_{0}=q^{ \pm, \mp}$ in 6.22 , we find, exploiting the collisional symmetries of $q^{ \pm, \mp}$, that

$$
\begin{aligned}
2 \sigma\left|\int_{\mathbb{R}^{3} \times \mathbb{R}^{3} \times \mathbb{S}^{2}} q^{ \pm, \mp} v M M_{*} d v d v_{*} d \sigma\right|^{2} & +\lambda\left(\int_{\mathbb{R}^{3} \times \mathbb{R}^{3} \times \mathbb{S}^{2}} q^{ \pm, \mp}|v|^{2} M M_{*} d v d v_{*} d \sigma\right)^{2} \\
& \leq \int_{\mathbb{R}^{3} \times \mathbb{R}^{3} \times \mathbb{S}^{2}}\left(q^{ \pm, \mp}\right)^{2} M M_{*} d v d v_{*} d \sigma
\end{aligned}
$$

which concludes the justification of 6.20 .

\subsection{The limiting Maxwell's equations}

Using the uniform $L^{\infty}\left(d t ; L^{2}(d x)\right)$ bounds on the electromagnetic fields $E_{\epsilon}$ and $B_{\epsilon}$, and the controls from Chapter 5 on the fluctuations, we can also take limits in the full Maxwell system for one species and for two species in the case of weak interactions only. Because of the scaling of the light speed, we obtain different kinds of limiting systems in the two regimes to be considered, but there is no particular difficulty here, for everything remains linear.

As for the case of two species with strong interactions, we will not be able to pass to the limit in Maxwell's equations. Indeed, Ampère's equation is nonlinear in this setting, which is a major obstacle to the weak stability of the system. More comments on this issue are provided below. 
In the regime leading to the incompressible quasi-static Navier-Stokes-FourierMaxwell-Poisson system, considered in Section 4.2, we start from

$$
\left\{\begin{aligned}
\epsilon \partial_{t} E_{\epsilon}-\operatorname{rot} B_{\epsilon} & =-\int_{\mathbb{R}^{3}} g_{\epsilon} v M d v, \\
\epsilon \partial_{t} B_{\epsilon}+\operatorname{rot} E_{\epsilon} & =0 \\
\operatorname{div} E_{\epsilon} & =\int_{\mathbb{R}^{3}} g_{\epsilon} M d v, \\
\operatorname{div} B_{\epsilon} & =0 .
\end{aligned}\right.
$$

Then, the weak compactness of the fluctuations from Lemma 5.1, inherited from the scaled entropy inequality (4.28), allows us to consider converging subsequences

$$
\begin{array}{ll}
E_{\epsilon} \stackrel{*}{\rightarrow} E & \text { in } L^{\infty}\left(d t ; L^{2}(d x)\right), \\
B_{\epsilon} \stackrel{*}{\rightarrow} B & \text { in } L^{\infty}\left(d t ; L^{2}(d x)\right), \\
g_{\epsilon} \rightarrow g & \text { in } L_{\mathrm{loc}}^{1}\left(d t d x ; L^{1}\left(\left(1+|v|^{2}\right) M d v\right)\right),
\end{array}
$$

which easily leads to

$$
\operatorname{rot} E=0, \quad \operatorname{div} E=\rho, \quad \operatorname{rot} B=u, \quad \operatorname{div} B=0,
$$

where $\rho$ and $u$ respectively denote the density and bulk velocity associated to the limiting fluctuation $g$. Formally, this limit amounts to discarding the terms involving time derivatives in Maxwell's equations, which accounts for the terminology of "quasi-static approximation" since temporal variations are neglected.

Next, in the regime leading to the two-fluid incompressible Navier-StokesFourier-Maxwell system with (solenoidal) Ohm's law, considered in Section 4.3. we start from

$$
\left\{\begin{aligned}
\partial_{t} E_{\epsilon}-\operatorname{rot} B_{\epsilon} & =-\frac{\delta}{\epsilon} \int_{\mathbb{R}^{3}}\left(g_{\epsilon}^{+}-g_{\epsilon}^{-}\right) v M d v=-\int_{\mathbb{R}^{3}} h_{\epsilon} v M d v \\
\partial_{t} B_{\epsilon}+\operatorname{rot} E_{\epsilon} & =0 \\
\operatorname{div} E_{\epsilon} & =\delta \int_{\mathbb{R}^{3}}\left(g_{\epsilon}^{+}-g_{\epsilon}^{-}\right) M d v \\
\operatorname{div} B_{\epsilon} & =0
\end{aligned}\right.
$$

We consider first the simpler case of weak interspecies collisions, i.e. the case $\delta=$ $o(1)$ and $\frac{\delta}{\epsilon}$ unbounded. The weak compactness of the fluctuations from Lemmas 5.1 and 5.14 , inherited from the scaled entropy inequality (4.35), allows us to consider converging subsequences

$$
\begin{array}{ll}
E_{\epsilon} \stackrel{*}{\rightarrow} E & \text { in } L^{\infty}\left(d t ; L^{2}(d x)\right), \\
B_{\epsilon} \stackrel{*}{\rightarrow} B & \text { in } L^{\infty}\left(d t ; L^{2}(d x)\right), \\
g_{\epsilon}^{ \pm} \rightarrow g^{ \pm} & \text {in } L_{\mathrm{loc}}^{1}\left(d t d x ; L^{1}\left(\left(1+|v|^{2}\right) M d v\right)\right), \\
h_{\epsilon} \rightarrow h & \text { in } L_{\mathrm{loc}}^{1}\left(d t d x ; L^{1}\left(\left(1+|v|^{2}\right) M d v\right)\right),
\end{array}
$$

which easily leads to

$$
\left\{\begin{aligned}
\partial_{t} E-\operatorname{rot} B & =-j, \\
\partial_{t} B+\operatorname{rot} E & =0, \\
\operatorname{div} E & =0, \\
\operatorname{div} B & =0,
\end{aligned}\right.
$$

where $j$ denotes the electric current, that is the bulk velocity associated to the limiting fluctuation $h$. 
Now, we see that in the case of strong interspecies collisions, i.e. $\delta=1$, Lemma 5.14 provides no longer enough compactness on $h_{\epsilon}$ to take weak limits in Ampère's equation. Indeed, in view of Lemma 5.13, it holds, at best, that the $h_{\epsilon}$ 's are uniformly bounded in $L_{\text {loc }}^{1}\left(d t d x ; L^{1}\left(\left(1+|v|^{2}\right) M d v\right)\right)$, but nothing prevents the fluctuations $h_{\epsilon}$ from concentrating on small sets and, therefore, to converge towards a singular measure. Thus, in this asymptotic regime, Ampère's equation will not be satisfied in the sense of distributions but only in a dissipative sense, which will be encoded in the inequality defining the dissipative solutions obtained in Chapter 12 through a generalized relative entropy method.

A closer inspection of Ampère's equation in the limiting system 4.37) (or (2.74) provides some insight on its lack of weak stability in the hydrodynamic limit. Indeed, even though Maxwell's system in (4.37) is linear in the variables $(E, B, n, j)$, the energy inequality associated with 4.37) suggests that the right mathematical variables are rather $(E, B, j-n u, n, u)$, which renders Ampère's equation nonlinear.

Nevertheless, the rest of Maxwell's system remains linear and we can easily pass to the limit in Faraday's equation and Gauss' laws. Indeed, the weak compactness of the fluctuations from Lemma 5.1, inherited from the scaled entropy inequality (4.35), allows us to consider converging subsequences

$$
\begin{array}{ll}
E_{\epsilon} \stackrel{*}{\rightarrow} E & \text { in } L^{\infty}\left(d t ; L^{2}(d x)\right), \\
B_{\epsilon} \stackrel{*}{\rightarrow} B & \text { in } L^{\infty}\left(d t ; L^{2}(d x)\right), \\
g_{\epsilon}^{ \pm} \rightarrow g^{ \pm} & \text {in } L_{\mathrm{loc}}^{1}\left(d t d x ; L^{1}\left(\left(1+|v|^{2}\right) M d v\right)\right),
\end{array}
$$

which easily leads to

$$
\left\{\begin{aligned}
\partial_{t} B+\operatorname{rot} E & =0, \\
\operatorname{div} E & =n, \\
\operatorname{div} B & =0,
\end{aligned}\right.
$$

where $n=\rho^{+}-\rho^{-}$is the electric charge associated with the limiting fluctuations $g^{ \pm}$, i.e. $\rho^{ \pm}$are the macroscopic densities of $g^{ \pm}$. 



\section{CHAPTER 7}

\section{Strong compactness and hypoellipticity}

In Chapter 5, we have established uniform bounds and relaxation estimates on the fluctuations and collision integrands as consequences of the scaled relative entropy inequalities 4.28 and 4.35. This is sufficient to handle linear terms. Thus, in Chapter 6, we exploited these uniform estimates to derive limiting constraint equations and energy inequalities.

In order to go any further in the rigorous derivation of the hydrodynamic limits under study, we need now to obtain precise strong compactness estimates on the fluctuations through a refined understanding of the Vlasov-Boltzmann equations from 4.27 and (4.34). More precisely, in the present chapter, we are going to introduce mathematical tools used to study the dependence in $x$ and $v$ of the families of fluctuations and, then, deduce important strong compactness properties of these fluctuations.

The first and simplest step, performed in Section 7.1 below, consists in understanding the dependence of fluctuations with respect to the velocity variable, which is essentially controlled by the relaxation mechanism. Since these estimates in $v$ are based only on results from functional analysis and on the relative entropy and entropy dissipation bounds, they will hold similarly in both regimes 4.27) and 4.34.

This first step is novel and differs considerably from previous works on hydrodynamic limits of Boltzmann equations with cutoff assumptions in that it shows strong compactness of the fluctuations in velocity, whereas former results only employed weak bounds in $v$, such as the equi-integrability in $v$ from Lemma 5.12. This strong compactness is crucial in order to carry out the next stage of the proof in Section 7.2. Note that strong velocity compactness has also been used in [4] to treat hydrodynamic limits of the Boltzmann equation without any cutoff assumptions. The approach therein heavily relied on the smoothing effect in $v$ peculiar to long-range interactions, though. In fact, the methods developed here can also be used to improve the results from 4 (see Part 3).

The second, more convoluted step, performed in Section 7.2, uses then the hypoellipticity in kinetic transport equations studied in 6] to transfer strong compactness from the velocity variable $v$ to the space variable $x$. Some non-trivial technical care will be required in order to extend the results from [6], which mainly concern the stationary kinetic transport equation, to the non-stationary transport equation with a vanishing time derivative.

Note that this second step also differs substantially from previous works on the subject, for these traditionally relied on classical velocity averaging lemmas to show some strong space compactness of the moments of the fluctuations (not the fluctuations themselves).

It is to be emphasized that the compactness properties for the two species regime 4.34 obtained in Section 7.2.3 below, are substantially weaker than those corresponding to the one species regime 4.27) and derived in Section 7.2.2. Essentially, the two species regime considered here being quite singular, the corresponding fluctuations cannot be shown to enjoy as much equi-integrability as in the one 
species regime, which will lead to significant difficulties in the remainder of our proofs.

The results from the present chapter constitute a crucial and difficult step in the rigorous proofs of hydrodynamic convergence. They will allow us to obtain higher order nonlinear constraint and evolution equations in the coming chapters. Finally, note that the results obtained here are only concerned with the compactness properties of fluctuations in $x$ and $v$, but not in $t$. In fact, there may be oscillations in time and the temporal behavior of fluctuations will be analyzed later on in Chapter 10 .

\subsection{Compactness with respect to $v$}

We have already shown in Section 5.4 how the relaxation process towards statistical equilibrium provides improved integrability in $v$ on the fluctuations. We show now how it further yields dissipative properties in the velocity variable. Loosely speaking, such a dissipation mechanism stems from the fact that the entropy dissipation controls the distance from the solutions to the set of statistical equilibria, which are in general smooth distributions in velocity.

As we consider fluctuations around a global equilibrium, the linearized collision operator will play a fundamental role, just as in Section 5.3 on the relaxation. In order to get strong compactness results, we will further need to control the correctors coming from the nonlinear part of the collision operator. To this end, we recall now the important regularizing effects of the gain term of the Boltzmann collision operator. This property will be crucial in our proof of compactness.

The results presented in Section 7.1.1, below, concern general cross-sections satisfying some integrability assumptions. The properties from Section 7.1.2, however, only concern the Maxwellian collision kernel $b \equiv 1$. The corresponding results for general cross-sections will be discussed in the remaining parts of our work.

7.1.1. Compactness of the gain term. In [51, Lions exhibited the compactifying and regularizing effects of the gain term of the Boltzmann collision operator. The essential result contained therein establishes the regularity of the gain term for a smooth and truncated collision kernel. The precise result from $\mathbf{5 1}$ which is of interest to us is recalled in the following theorem. Variants and refinements of this result were obtained in [12, 75. In particular, a simple argument based on the Fourier transform, due to Bouchut and Desvillettes in [12], also provides a convenient compactness result.

Theorem $7.1([\mathbf{5 1}])$. Let $b(z, \sigma)=b\left(|z|, \frac{z}{|z|} \cdot \sigma\right) \in C_{c}^{\infty}((0, \infty) \times(0, \pi))$ be a smooth compactly supported collision kernel.

Then, there exists a finite $C>0$ such that

$$
\left\|Q^{+}(f, g)\right\|_{H^{1}\left(\mathbb{R}^{3}\right)} \leq C\|f\|_{L^{2}\left(\mathbb{R}^{3}\right)}\|g\|_{L^{1}\left(\mathbb{R}^{3}\right)}
$$

for any $f \in L^{2}\left(\mathbb{R}^{3}\right)$ and $g \in L^{1}\left(\mathbb{R}^{3}\right)$, and

$$
\left\|Q^{+}(f, g)\right\|_{H^{1}\left(\mathbb{R}^{3}\right)} \leq C\|f\|_{L^{1}\left(\mathbb{R}^{3}\right)}\|g\|_{L^{2}\left(\mathbb{R}^{3}\right)}
$$

for any $f \in L^{1}\left(\mathbb{R}^{3}\right)$ and $g \in L^{2}\left(\mathbb{R}^{3}\right)$.

Note that, in the statement of the above theorem, we have carefully avoided the endpoints on the domain of definition of the collision kernel in order to restrict the compact support of $b(z, \sigma)$. More precisely, Lions' result only considers smooth kernels whose support is contained in $\left\{\lambda<|z|<\frac{1}{\lambda},\left|\frac{z}{|z|} \cdot \sigma\right|<1-\lambda\right\}$, for some small $\lambda>0$. This hypothesis is definitely not optimal, but at least some truncation is clearly required in order to obtain the optimal gain of regularity for $Q^{+}(f, g)$. 
For more general collision kernels, it is still possible to obtain some compactness of the gain operator by standard approximation procedures based on convolution inequalities for the gain term $Q^{+}(f, g)$. See for instance 3 for such general convolution inequalities. Here, we will merely use an elementary version of these inequalities which we presently recall for convenience.

Thus, let $1 \leq s \leq p, q \leq r^{\prime} \leq \infty$ be such that

$$
1+\frac{1}{s}=\frac{1}{p}+\frac{1}{q}+\frac{1}{r}
$$

and consider $f \in L^{p}\left(\mathbb{R}^{3}\right), g \in L^{q}\left(\mathbb{R}^{3}\right), \varphi \in L^{s^{\prime}}\left(\mathbb{R}^{3}\right)$ and a general collision kernel $b(z, \sigma) \in L^{r}\left(\mathbb{R}^{3} ; L^{1}\left(\mathbb{S}^{2}\right)\right)$. Then, employing the collision symmetries with Hölder's and Young's inequalities and using the change of variables $v_{*} \mapsto V=v-v_{*}$, we find

$$
\begin{aligned}
\mid \int_{\mathbb{R}^{3}} Q^{+} & (f, g)(v) \varphi(v) d v \mid \\
& \leq \int_{\mathbb{R}^{3} \times \mathbb{R}^{3} \times \mathbb{S}^{2}}\left|f g_{*} \varphi^{\prime}\right| b\left(v-v_{*}, \sigma\right) d v d v_{*} d \sigma \\
& =\int_{\mathbb{R}^{3} \times \mathbb{R}^{3} \times \mathbb{S}^{2}}\left|f(v) g(v-V) \varphi\left(v-\frac{V}{2}+\frac{|V|}{2} \sigma\right)\right| b(V, \sigma) d V d v d \sigma \\
& \leq\|\varphi\|_{L^{s^{\prime}}} \int_{\mathbb{R}^{3}}\|f(v) g(v-V)\|_{L_{v}^{s}} \int_{\mathbb{S}^{2}} b(V, \sigma) d \sigma d V \\
& \leq\|\varphi\|_{L^{s^{\prime}}}\|\| f(v) g(v-V)\left\|_{L_{v}^{s}}\right\|_{L_{V}^{r^{\prime}}}\left\|\int_{\mathbb{S}^{2}} b(V, \sigma) d \sigma\right\|_{L_{V}^{r}} \\
& =\|\varphi\|_{L^{s^{\prime}}}\left\|\int_{\mathbb{R}^{3}}|f(v)|^{s}|g(v-V)|^{s} d v\right\|_{L_{V}^{\frac{r^{\prime}}{s}}}^{\frac{1}{s}}\left\|\int_{\mathbb{S}^{2}} b(V, \sigma) d \sigma\right\|_{L_{V}^{r}} \\
& \leq\|\varphi\|_{L^{s^{\prime}}}\|f\|_{L^{p}}\|g\|_{L^{q}}\left\|\int_{\mathbb{S}^{2}} b(V, \sigma) d \sigma\right\|_{L_{V}^{r}} .
\end{aligned}
$$

Notice that the exact same reasoning can be applied to the loss operator $Q^{-}(f, g)$, so that, considering the supremum over all $\varphi \in L^{s^{\prime}}\left(\mathbb{R}^{3}\right)$, we arrive at the following estimate

$$
\left\|Q^{ \pm}(f, g)\right\|_{L^{s}} \leq\|f\|_{L^{p}}\|g\|_{L^{q}}\left\|\int_{\mathbb{S}^{2}} b(z, \sigma) d \sigma\right\|_{L_{z}^{r}} .
$$

It turns out that it is possible to extend the above inequality to the full range of parameters $1 \leq p, q, r, s \leq \infty$ for the gain term $Q^{+}(f, g)$ only, provided we have a better control on the angular collision kernel. This is consistent with the fact that $Q^{+}(f, g)$ behaves nicely and better than $Q^{-}(f, g)$. Such results can be found in [3].

Thus, combining the regularizing properties from Theorem 7.1 with the convolution inequalities (7.1), we obtain the following convenient proposition.

Proposition 7.2. Let $b(z, \sigma)$ be a cross-section such that

$$
\int_{\mathbb{S}^{2}} b(z, \sigma) d \sigma \in L^{2}\left(\mathbb{R}^{3}, M(z)^{\alpha} d z\right)
$$

for some given $\alpha<\frac{1}{2}$.

Then, the bilinear operator

$$
\begin{aligned}
& L^{2}\left(\mathbb{R}^{3}, M d v\right) \times L^{2}\left(\mathbb{R}^{3}, M d v\right) \quad \longrightarrow \quad L^{2}\left(\mathbb{R}^{3}, M^{1+2 \alpha} d v\right) \\
& (f, g) \quad \longmapsto \quad \mathcal{Q}^{+}(f, g)
\end{aligned}
$$

is locally compact. That is to say, it maps bounded subsets of $L^{2}(M d v) \times L^{2}(M d v)$ into relatively compact subsets of $L_{\mathrm{loc}}^{2}(d v)$. 
Proof. First, it is easy to check that

$$
\begin{aligned}
(M f)^{\prime}(M g)_{*}^{\prime} M^{\alpha-\frac{1}{2}} & =(\sqrt{M} f)^{\prime}(\sqrt{M} g)_{*}^{\prime} M^{\alpha} M_{*}^{\frac{1}{2}} \\
& \leq C(\sqrt{M} f)^{\prime}(\sqrt{M} g)_{*}^{\prime}\left(M M_{*}\right)^{\alpha} \\
& \leq C(\sqrt{M}|f|)^{\prime}(\sqrt{M}|g|)_{*}^{\prime}\left(M\left(v-v_{*}\right)\right)^{\frac{\alpha}{2}},
\end{aligned}
$$

for some $C>0$. Hence, in virtue of the convolution inequality 7.1 , we obtain $(7.2)$

$$
\begin{aligned}
& \left\|\mathcal{Q}^{+}(f, g) M^{\alpha}\right\|_{L^{2}(M d v)} \\
& \quad \leq C\left\|\int_{\mathbb{R}^{3} \times \mathbb{S}^{2}}(\sqrt{M}|f|)^{\prime}(\sqrt{M}|g|)_{*}^{\prime} b\left(v-v_{*}, \sigma\right) M\left(v-v_{*}\right)^{\frac{\alpha}{2}} d v_{*} d \sigma\right\|_{L^{2}(d v)} \\
& \quad \leq C\|f\|_{L^{2}(M d v)}\|g\|_{L^{2}(M d v)}\left\|\int_{\mathbb{S}^{2}} b(z, \sigma) d \sigma\right\|_{L^{2}\left(M^{\alpha} d z\right)},
\end{aligned}
$$

which establishes the boundedness of the quadratic operator.

Next, in order to show the local compactness of the operator, we consider any bounded sequences $\left\{f_{n}\right\}_{n \in \mathbb{N}},\left\{g_{n}\right\}_{n \in \mathbb{N}} \subset L^{2}(M d v)$. Then, defining $\mathcal{Q}_{\lambda}^{+}\left(f_{n}, g_{n}\right)$, for any $\lambda>0$, by simply replacing $b(z, \sigma)$ by some smooth kernel $b_{\lambda}(z, \sigma)=$ $b_{\lambda}\left(|z|, \frac{z}{|z|} \cdot \sigma\right) \in C_{c}^{\infty}((0, \infty) \times(0, \pi))$ such that $0 \leq b_{\lambda} \leq b$ and

$$
\left\|\int_{\mathbb{S}^{2}}\left|b-b_{\lambda}\right|(z, \sigma) d \sigma\right\|_{L^{2}\left(M^{\alpha} d z\right)}<\lambda,
$$

we deduce, thanks to $(7.2)$, that the $\mathcal{Q}^{+}\left(f_{n}, g_{n}\right)$ 's can be uniformly approximated by the $\mathcal{Q}_{\lambda}^{+}\left(f_{n}, g_{n}\right)$ 's in $L^{2}\left(M^{1+2 \alpha} d v\right)$. Since, by Theorem 7.1, the $Q_{\lambda}^{+}\left(M f_{n}, M g_{n}\right)$ 's are relatively compact in $L_{\text {loc }}^{2}(d v)$, we conclude that the original sequence $\left\{Q^{+}\left(M f_{n}, M g_{n}\right)\right\}_{n \in \mathbb{N}}$ is relatively compact in $L_{\text {loc }}^{2}(d v)$, which concludes the justification of the proposition.

7.1.2. Relative entropy, entropy dissipation and strong compactness. Combining the uniform controls from the relative entropy and entropy dissipation with the compactness of the gain term presented in the previous section, we establish now the following result, valid for the Maxwellian collision kernel $b \equiv 1$.

LEMMA 7.3. Let $f_{\epsilon}(t, x, v)$ be a family of measurable, almost everywhere nonnegative distribution functions such that, for all $t \geq 0$,

$$
\frac{1}{\epsilon^{2}} H\left(f_{\epsilon}\right)(t)+\frac{1}{\epsilon^{4}} \int_{0}^{t} \int_{\mathbb{R}^{3}} D\left(f_{\epsilon}\right)(s) d x d s \leq C^{\text {in }} .
$$

Then, as $\epsilon \rightarrow 0$, any subsequence of renormalized fluctuations $\hat{g}_{\epsilon}$ is locally relatively compact in $v$ in $L^{2}(d t d x d v)$ in the sense that, for any $\eta>0$ and every compact subset $K \subset[0, \infty) \times \mathbb{R}^{3} \times \mathbb{R}^{3}$, there exists $\gamma>0$ such that, if $h \in \mathbb{R}^{3}$ satisfies $|h|<\gamma$, then

$$
\sup _{\epsilon>0}\left\|\hat{g}_{\epsilon}(t, x, v+h)-\hat{g}_{\epsilon}(t, x, v)\right\|_{L^{2}(K, d t d x d v)}<\eta .
$$

Proof. Loosely speaking, the present proof can be summarized in three main steps, each corresponding to a decomposition of $\hat{g}_{\epsilon}$. First, we will show how to control the very large values of $\hat{g}_{\epsilon}$, i.e. values larger than $\frac{1}{\epsilon}$, with the entropy bound. This is a rather standard and simple estimate. Second, we obtain the strong compactness in velocity of $\hat{g}_{\epsilon}$ from the entropy dissipation bound and the compactness of the gain term (see Proposition 7.2 making sure that we remain away from vacuum, i.e. away from the values $\int\left(1+\frac{\epsilon}{2} \hat{g}_{\epsilon}\right) M d v \approx 0$, for our estimates 
degenerate in this case. Finally, we deduce the strong compactness near vacuum arguing that the vacuum state $\hat{g}_{\epsilon} \equiv-\frac{2}{\epsilon}$ is actually smooth since it is constant.

Control of very large values. Using the entropy inequality, we first introduce some microscopic truncation of large values. For any fixed small $0<\lambda<\frac{1}{2}$ and any cutoff $\chi(r) \in C_{c}^{\infty}(\mathbb{R})$ such that $\mathbb{1}_{\{|r| \leq 1\}} \leq \chi(r) \leq \mathbb{1}_{\{|r| \leq 2\}}$, we have, since $\epsilon^{2} \hat{g}_{\epsilon}^{2} \leq 4 G_{\epsilon}$ as soon as $\epsilon \hat{g}_{\epsilon} \geq-1$,

$$
\begin{aligned}
\left|\hat{g}_{\epsilon}\left(1-\chi\left(\lambda \epsilon \hat{g}_{\epsilon}\right)\right)\right|^{2} & \leq \hat{g}_{\epsilon}^{2} \mathbb{1}_{\left\{\epsilon \hat{g}_{\epsilon} \geq \frac{1}{\lambda}>2\right\}} \\
& \leq \frac{4}{\epsilon^{2}} G_{\epsilon} \mathbb{1}_{\left\{G_{\epsilon}>\frac{1}{4 \lambda^{2}}\right\}} \\
& \leq \frac{2}{\epsilon^{2}|\log 2 \lambda|} G_{\epsilon} \log G_{\epsilon} \mathbb{1}_{\left\{G_{\epsilon}>\frac{1}{4 \lambda^{2}}\right\}},
\end{aligned}
$$

so that, by the relative entropy bound,

$$
\hat{g}_{\epsilon}\left(1-\chi\left(\lambda \epsilon \hat{g}_{\epsilon}\right)\right)=O\left(\frac{1}{|\log \lambda|^{\frac{1}{2}}}\right)_{L^{\infty}\left(d t ; L^{2}(M d x d v)\right)},
$$

as $\lambda \rightarrow 0$, uniformly in $\epsilon$.

Away from vacuum. We use now Hilbert's decomposition (see Proposition 5.4 for the Maxwellian cross-section $b \equiv 1$ :

$$
\mathcal{L} \hat{g}_{\epsilon}=\hat{g}_{\epsilon}-\mathcal{K} \hat{g}_{\epsilon}
$$

where $\mathcal{K}$ is a compact integral operator on $L^{2}(M d v)$. Then, from the identity

$$
\mathcal{L} \hat{g}_{\epsilon}=\frac{\epsilon}{2} \mathcal{Q}\left(\hat{g}_{\epsilon}, \hat{g}_{\epsilon}\right)-\frac{2}{\epsilon} \mathcal{Q}\left(\sqrt{G_{\epsilon}}, \sqrt{G_{\epsilon}}\right),
$$

we deduce that

$$
\begin{aligned}
\hat{g}_{\epsilon} & =\mathcal{L} \hat{g}_{\epsilon}+\mathcal{K} \hat{g}_{\epsilon} \\
& =\frac{\epsilon}{2} \mathcal{Q}^{+}\left(\hat{g}_{\epsilon}, \hat{g}_{\epsilon}\right)-\frac{\epsilon}{2} \hat{g}_{\epsilon} \int_{\mathbb{R}^{3} \times \mathbb{S}^{2}} \hat{g}_{\epsilon *} M_{*} d v_{*} d \sigma-\epsilon \int_{\mathbb{R}^{3} \times \mathbb{S}^{2}} \hat{q}_{\epsilon} M_{*} d v_{*} d \sigma+\mathcal{K} \hat{g}_{\epsilon},
\end{aligned}
$$

or, equivalently,

$$
\hat{g}_{\epsilon}\left(1+\frac{\epsilon}{2} \int_{\mathbb{R}^{3}} \hat{g}_{\epsilon *} M_{*} d v_{*}\right)=-\epsilon \int_{\mathbb{R}^{3} \times \mathbb{S}^{2}} \hat{q}_{\epsilon} M_{*} d v_{*} d \sigma+\mathcal{K} \hat{g}_{\epsilon}+\frac{\epsilon}{2} \mathcal{Q}^{+}\left(\hat{g}_{\epsilon}, \hat{g}_{\epsilon}\right) .
$$

We are now going to control each term in the right-hand side above separately.

The first term is easily estimated employing the uniform $L^{2}$-estimate from Lemma 5.3 . It yields that

$$
\epsilon \int_{\mathbb{R}^{3} \times \mathbb{S}^{2}} \hat{q}_{\epsilon} M_{*} d v_{*} d \sigma=O(\epsilon)_{L^{2}(M d t d x d v)} .
$$

The second term $\mathcal{K} \hat{g}_{\epsilon}$ satisfies the bound

$$
\left\|\mathcal{K} \hat{g}_{\epsilon}\right\|_{L^{2}(M d v)} \leq C\left\|\hat{g}_{\epsilon}\right\|_{L^{2}(M d v)},
$$

so that, in view of Lemma 5.2 and by the compactness of the operator $\mathcal{K}$,

$$
\mathcal{K} \hat{g}_{\epsilon}=O(1)_{L^{\infty}\left(d t ; L^{2}\left(d x ; \mathcal{C} L^{2}(M d v)\right)\right)},
$$

where we have used the notation $\mathcal{C} L^{2}(M d v)$ to indicate that it is relatively compact with respect to the velocity variable in $L^{\infty}\left(d t ; L^{2}(M d x d v)\right)$. Similarly, the third term $\frac{\epsilon}{2} \mathcal{Q}^{+}\left(\hat{g}_{\epsilon}, \hat{g}_{\epsilon}\right)$ satisfies, in virtue of Proposition 7.2 , the control

$$
\frac{\epsilon}{2} \mathcal{Q}^{+}\left(\hat{g}_{\epsilon}, \hat{g}_{\epsilon}\right)=O(\epsilon)_{L^{\infty}\left(d t ; L^{1}\left(d x ; \mathcal{C} L_{\mathrm{loc}}^{2}(d v)\right)\right)},
$$

where, again, we have used the notation $\mathcal{C} L_{\text {loc }}^{2}(d v)$ to indicate that it is relatively compact with respect to the velocity variable in $L^{\infty}\left(d t ; L^{1}\left(d x ; L_{\text {loc }}^{2}(d v)\right)\right)$. 
On the whole, incorporating the controls $(7.5), 7.6$ and 7.7 into the decomposition (7.4), we have established that

$$
\begin{aligned}
\hat{g}_{\epsilon}\left(1+\frac{\epsilon}{2} \int_{\mathbb{R}^{3}} \hat{g}_{\epsilon *} M_{*} d v_{*}\right) & =O(\epsilon)_{L^{2}(M d t d x d v)} \\
& +O(1)_{L^{\infty}\left(d t ; L^{2}\left(d x ; \mathcal{C} L^{2}(M d v)\right)\right)} \\
& +O(\epsilon)_{L^{\infty}\left(d t ; L^{1}\left(d x ; \mathcal{C} L_{\text {loc }}^{2}(d v)\right)\right)} .
\end{aligned}
$$

Next, since the left-hand side of the above decomposition degenerates close to vacuum, i.e. whenever the density $\frac{\epsilon}{2} \int_{\mathbb{R}^{3}} \hat{g}_{\epsilon *} M_{*} d v_{*}$ is close to -1 , we introduce a macroscopic truncation

$$
\chi_{\epsilon, r}(t, x)=\mathbb{1}_{\left\{1+\frac{\epsilon}{2} \int M(v) \hat{g}_{\epsilon}(t, x, v) d v \geq r\right\}},
$$

for some small $r>0$, thus excluding the domain where this degeneracy is present. It then follows, dividing 7.8 by $1+\frac{\epsilon}{2} \int_{\mathbb{R}^{3}} \hat{g}_{\epsilon *} M_{*} d v_{*}$, that

$$
\begin{aligned}
\chi_{\epsilon, r} \hat{g}_{\epsilon} & =O\left(\frac{\epsilon}{r}\right)_{L^{2}(M d t d x d v)} \\
& +O\left(\frac{1}{r}\right)_{L^{\infty}\left(d t ; L^{2}\left(d x ; \mathcal{C} L^{2}(M d v)\right)\right)} \\
& +O\left(\frac{\epsilon}{r}\right)_{L^{\infty}\left(d t ; L^{1}\left(d x ; \mathcal{C} L_{\mathrm{loc}}^{2}(d v)\right)\right)}
\end{aligned}
$$

Next, for any small $h \in \mathbb{R}^{3}$ and any compact subset $K \subset[0, \infty) \times \mathbb{R}^{3} \times \mathbb{R}^{3}$, it holds that

$$
\begin{aligned}
& \int_{K} \chi_{\epsilon, r}\left|\chi\left(\lambda \epsilon \hat{g}_{\epsilon}\right) \hat{g}_{\epsilon}(t, x, v+h)-\chi\left(\lambda \epsilon \hat{g}_{\epsilon}\right) \hat{g}_{\epsilon}(t, x, v)\right|^{2} d t d x d v \\
& \quad=2 \int_{K} \chi_{\epsilon, r} \chi\left(\lambda \epsilon \hat{g}_{\epsilon}\right) \hat{g}_{\epsilon}(t, x, v)\left[\chi\left(\lambda \epsilon \hat{g}_{\epsilon}\right) \hat{g}_{\epsilon}(t, x, v)-\chi\left(\lambda \epsilon \hat{g}_{\epsilon}\right) \hat{g}_{\epsilon}(t, x, v+h)\right] d t d x d v \\
& \quad \leq C \int_{K} \chi_{\epsilon, r}\left|\chi\left(\lambda \epsilon \hat{g}_{\epsilon}\right) \hat{g}_{\epsilon}(t, x, v)\left(\hat{g}_{\epsilon}(t, x, v+h)-\hat{g}_{\epsilon}(t, x, v)\right)\right| d t d x d v
\end{aligned}
$$

Therefore, since

$$
\begin{aligned}
\left\|\chi\left(\lambda \epsilon \hat{g}_{\epsilon}\right) \hat{g}_{\epsilon}\right\|_{L_{\mathrm{loc}}^{2}(d t d x d v)} & \leq C, \\
\left\|\chi\left(\lambda \epsilon \hat{g}_{\epsilon}\right) \hat{g}_{\epsilon}\right\|_{L^{\infty}(d t d x d v)} & \leq \frac{2}{\lambda \epsilon},
\end{aligned}
$$

we conclude from 7.9 that, for any fixed $0<\lambda, r<1$,

$$
\limsup _{|h| \rightarrow 0} \limsup _{\epsilon \rightarrow 0} \int_{K} \chi_{\epsilon, r}\left|\chi\left(\lambda \epsilon \hat{g}_{\epsilon}\right) \hat{g}_{\epsilon}(t, x, v+h)-\chi\left(\lambda \epsilon \hat{g}_{\epsilon}\right) \hat{g}_{\epsilon}(t, x, v)\right|^{2} d t d x d v=0,
$$

which is the expected relative compactness statement away from vacuum on $\chi_{\epsilon, r} \chi\left(\lambda \epsilon \hat{g}_{\epsilon}\right) \hat{g}_{\epsilon}$. Consequently, combining this result with the control $(7.3)$ on the very large values of $\hat{g}_{\epsilon}$ yields that, for any given small $r>0$,

$$
\chi_{\epsilon, r} \hat{g}_{\epsilon} \text { is relatively compact }
$$

$$
\text { with respect to the velocity variable in } L_{\text {loc }}^{2}(d t d x d v) \text {. }
$$

Near vacuum. It only remains then to get a compactness estimate near vacuum on $\left(1-\chi_{\epsilon, r}\right) \hat{g}_{\epsilon}$. To this end, we simply decompose, for any given small $\lambda>0$,

$$
\chi\left(\lambda \epsilon \hat{g}_{\epsilon}\right) \hat{g}_{\epsilon}=\chi\left(\lambda \epsilon \hat{g}_{\epsilon}\right) \frac{2}{\epsilon}\left(1+\frac{\epsilon}{2} \hat{g}_{\epsilon}\right)+\left(1-\chi\left(\lambda \epsilon \hat{g}_{\epsilon}\right)\right) \frac{2}{\epsilon}-\frac{2}{\epsilon},
$$

and we control each term in the right-hand side above individually. 
Thus, noticing that, for any $0<r \leq \frac{1}{2}$,

$$
\frac{1}{\epsilon}\left(1-\chi_{\epsilon, r}\right)=\frac{1}{\epsilon} \mathbb{1}_{\left\{\frac{2}{\epsilon}<\frac{-\int M \hat{g}_{\epsilon} d v}{1-r}\right\}} \leq \frac{-\int M \hat{g}_{\epsilon} d v}{2(1-r)} \leq\left\|\hat{g}_{\epsilon}\right\|_{L^{2}(M d v)}=O(1)_{L^{\infty}\left(d t ; L^{2}(d x)\right)},
$$

and that, on the support of $\left(1-\chi_{\epsilon, r}\right)$,

$$
\begin{aligned}
\int_{\mathbb{R}^{3}}\left|\chi\left(\lambda \epsilon \hat{g}_{\epsilon}\right)\left(1+\frac{\epsilon}{2} \hat{g}_{\epsilon}\right)\right|^{2} M d v & \leq \int_{\mathbb{R}^{3}} M\left(1+\frac{\epsilon}{2} \hat{g}_{\epsilon}\right) d v\left(1+\frac{1}{\lambda}\right) \\
& \leq r\left(1+\frac{1}{\lambda}\right)=O\left(\frac{r}{\lambda}\right)_{L^{\infty}(d t d x)}
\end{aligned}
$$

we obtain concerning the first term in the right-hand side of 7.11 that

$$
\left(1-\chi_{\epsilon, r}\right) \chi\left(\lambda \epsilon \hat{g}_{\epsilon}\right) \frac{2}{\epsilon}\left(1+\frac{\epsilon}{2} \hat{g}_{\epsilon}\right)=O\left(\sqrt{\frac{r}{\lambda}}\right)_{L^{\infty}\left(d t ; L^{2}(M d x d v)\right)} .
$$

Then, the second term is easily handled through the estimate

$$
\frac{1}{\epsilon}\left(1-\chi\left(\lambda \epsilon \hat{g}_{\epsilon}\right)\right) \leq \frac{1}{\epsilon} \mathbb{1}_{\left\{\left|\lambda \epsilon \hat{g}_{\epsilon}\right|>1\right\}} \leq \lambda\left|\hat{g}_{\epsilon}\right|,
$$

whereby

$$
\left(1-\chi_{\epsilon, r}\right)\left(1-\chi\left(\lambda \epsilon \hat{g}_{\epsilon}\right)\right) \frac{2}{\epsilon}=O(\lambda)_{L^{\infty}\left(d t ; L^{2}(M d x d v)\right)}
$$

The remaining term in the right-hand side of 7.11 is constant, in particular it is smooth, and so there is no need to further control it, so that, on the whole, incorporating 7.12 and 7.13 into 7.11 , we find

$\left(1-\chi_{\epsilon, r}\right) \chi\left(\lambda \epsilon \hat{g}_{\epsilon}\right) \hat{g}_{\epsilon}=O\left(\sqrt{\frac{r}{\lambda}}\right)_{L^{\infty}\left(d t ; L^{2}(M d x d v)\right)}+O(\lambda)_{L^{\infty}\left(d t ; L^{2}(M d x d v)\right)}-\frac{2}{\epsilon}\left(1-\chi_{\epsilon, r}\right)$.

We therefore conclude, for any small $h \in \mathbb{R}^{3}$ and any compact subset $K \subset$ $[0, \infty) \times \mathbb{R}^{3} \times \mathbb{R}^{3}$, that

$$
\begin{array}{r}
\limsup _{|h| \rightarrow 0} \limsup _{\epsilon \rightarrow 0} \int_{K}\left(1-\chi_{\epsilon, r}\right)\left|\chi\left(\lambda \epsilon \hat{g}_{\epsilon}\right) \hat{g}_{\epsilon}(t, x, v+h)-\chi\left(\lambda \epsilon \hat{g}_{\epsilon}\right) \hat{g}_{\epsilon}(t, x, v)\right|^{2} d t d x d v \\
\leq O\left(\frac{r}{\lambda}\right)+O\left(\lambda^{2}\right) .
\end{array}
$$

Conclusion of proof. On the whole, combining the above estimate (7.14) near vacuum with the control 7.3 on the very large values of $\hat{g}_{\epsilon}$ and the compactness statement 7.10 away from vacuum, we finally arrive at the control, for any compact subset $K \subset[0, \infty) \times \mathbb{R}^{3} \times \mathbb{R}^{3}$,

$$
\begin{aligned}
\limsup _{|h| \rightarrow 0} \limsup _{\epsilon \rightarrow 0} \int_{K} \mid \hat{g}_{\epsilon}(t, x, v+h)- & \left.\hat{g}_{\epsilon}(t, x, v)\right|^{2} d t d x d v \\
& \leq O\left(\frac{r}{\lambda}\right)+O\left(\lambda^{2}\right)+O\left(\frac{1}{|\log \lambda|}\right),
\end{aligned}
$$

which, by the arbitrary smallness of $r>0$ and $\lambda>0$, clearly implies that $\hat{g}_{\epsilon}$ is locally relatively compact with respect to the velocity variable in $L^{2}(d t d x d v)$ and thus concludes the proof of the lemma. 


\subsection{Compactness with respect to $x$}

In order to get a refined description of the dependence of the fluctuations $g_{\epsilon}$ and $g_{\epsilon}^{ \pm}$with respect to $x$, we will use the compactness properties of the free transport operator $v \cdot \nabla_{x}$. More precisely, there are two types of mechanisms at play here :

- the transfer of compactness, which expresses the fact that the free transport mixes the spatial and velocity variables and is a consequence of hypoellipticity ;

- the averaging lemma, which predicts some regularizing effect for the averages with respect to $v$, due to the fact that the symbol of the free transport is elliptic on a large microlocal subset.

Of course both mechanisms require that we have a good control on the advection terms $v \cdot \nabla_{x} \hat{g}_{\epsilon}$ and $v \cdot \nabla_{x} \hat{g}_{\epsilon}^{ \pm}$or some similar quantity (since the square root renormalization is singular at the origin, and thus is not admissible ; see proofs of Lemmas 7.8 and 7.10 below). In particular, we see at this point that the situation is quite different in the one species scaling leading to the incompressible quasi-static Navier-Stokes-Fourier-Maxwell-Poisson system, and in the two species scaling leading to the two-fluid incompressible Navier-Stokes-Fourier-Maxwell system with (solenoidal) Ohm's law.

In the first case, at the formal level, it is natural to expect from 4.29 that, up to some suitable renormalization, the advection term $v \cdot \nabla_{x} g_{\epsilon}$ is uniformly bounded. However, for the multi-species model, we see that (4.36 provides, at least formally, some uniform control on the advection terms $v \cdot \nabla_{x} g_{\epsilon}^{ \pm}$, and therefore some strong compactness on the hydrodynamic variables $\rho_{\epsilon}^{ \pm}, u_{\epsilon}^{ \pm}$and $\theta_{\epsilon}^{ \pm}$, but it does not provide any information on $v \cdot \nabla_{x} \frac{\delta}{\epsilon}\left(g_{\epsilon}^{+}-g_{\epsilon}^{-}\right)$which controls the electrodynamic variables $j_{\epsilon}$ and $w_{\epsilon}$.

As for the limiting systems 4.37) and 4.38, we therefore do not expect the electromagnetic terms $j \wedge B$ from the Lorentz force to be weakly stable (unfortunately, compensated compactness methods also fail here; see [5] for some details on this issue). In this case, we will use, later on in Chapter 12 , some weak-strong stability principle instead of a priori estimates. In other words, the dependence with respect to $x$ is partially understood a posteriori, by comparison with the solutions to the limiting systems.

7.2.1. Hypoellipticity and the transfer of compactness. We first explain our global strategy, presenting the main abstract results we will use on the free transport operator.

As mentioned in the introduction of the present chapter, the key idea here is to transfer the compactness with respect to $v$ inherited from the structure of the collision operator (see Lemma 7.3 onto the spatial variable $x$. To this end, we need the following result.

THEOREM $7.4([\mathbf{6}])$. Let the bounded family of functions

$$
\left\{\phi_{\lambda}(t, x, v)\right\}_{\lambda \in \Lambda} \subset L^{p}\left(\mathbb{R}_{t} \times \mathbb{R}_{x}^{3} \times \mathbb{R}_{v}^{3}\right),
$$

for some $1<p<\infty$, be locally relatively compact in $v$ and such that

$$
\left(\partial_{t}+v \cdot \nabla_{x}\right) \phi_{\lambda}=\left(1-\Delta_{t, x}\right)^{\frac{\beta}{2}}\left(1-\Delta_{v}\right)^{\frac{\alpha}{2}} S_{\lambda},
$$

for all $\lambda \in \Lambda$ and for some bounded family

$$
\left\{S_{\lambda}(t, x, v)\right\}_{\lambda \in \Lambda} \subset L^{p}\left(\mathbb{R}_{t} \times \mathbb{R}_{x}^{3} \times \mathbb{R}_{v}^{3}\right),
$$

where $\alpha \geq 0$ and $0 \leq \beta<1$.

Then, $\left\{\phi_{\lambda}(t, x, v)\right\}_{\lambda \in \Lambda}$ is locally relatively compact in $L^{p}\left(\mathbb{R}_{t} \times \mathbb{R}_{x}^{3} \times \mathbb{R}_{v}^{3}\right)$ (in all variables). 
The above result was formulated in [6]. It may also be deduced from the methods of [11] or from the use of standard averaging lemmas from 33 for instance. However, it is to be emphasized that the methods from [6] are more natural and direct.

It turns out that, for the sake of the rigorous derivation of hydrodynamic limits, it is crucial to understand what happens to Theorem 7.4 when $p=1$ (carefully note that this case is not covered by the above theorem). To be precise, Theorem 7.4 will be sufficient to control oscillations but not concentrations. The basic result in this direction is given by the $L^{1}$ mixing lemma obtained by Golse and the second author in 37, which allows to transfer equi-integrability from $v$ to $x$ when the source term of the kinetic transport equation is locally integrable.

The point here is that, because of the electromagnetic force, the source term involves derivatives with respect to $v$ and, therefore, is not locally integrable. An analogous situation has been dealt with by the first author in [4] when considering non-cutoff collision operators, which behave as nonlinear fractional derivatives with respect to $v$. In this singular setting, we are then able to transfer strong compactness, but - to the best of our knowledge - not mere weak compactness, as the results from [37. do not apply. More precisely, we have the following statement.

THEOREM $7.5([\mathbf{6}])$. Let the bounded family of non-negative functions

$$
\left\{\phi_{\lambda}(t, x, v)\right\}_{\lambda \in \Lambda} \subset L^{1}\left(\mathbb{R}_{t} \times \mathbb{R}_{x}^{3} ; L^{r}\left(\mathbb{R}_{v}^{3}\right)\right),
$$

for some $1<r<\infty$, be locally relatively compact in $v$ and such that

$$
\left(\partial_{t}+v \cdot \nabla_{x}\right) \phi_{\lambda}=\left(1-\Delta_{t, x}\right)^{\frac{\beta}{2}}\left(1-\Delta_{v}\right)^{\frac{\alpha}{2}} S_{\lambda},
$$

for all $\lambda \in \Lambda$ and for some bounded family

$$
\left\{S_{\lambda}(t, x, v)\right\}_{\lambda \in \Lambda} \subset L^{1}\left(\mathbb{R}_{t} \times \mathbb{R}_{x}^{3} ; L^{r}\left(\mathbb{R}_{v}^{3}\right)\right),
$$

where $\alpha \geq 0$ and $0 \leq \beta<1$.

Then, $\left\{\phi_{\lambda}(t, x, v)\right\}_{\lambda \in \Lambda}$ is locally relatively compact in $L^{1}\left(\mathbb{R}_{t} \times \mathbb{R}_{x}^{3} \times \mathbb{R}_{v}^{3}\right)$ (in all variables).

The crucial idea behind such hypoelliptic results is that the free transport operator is "invariant" by Fourier transform in $(x, v)$, so that frequencies are transported by the semi-group. The argument relies then on a good interpolation formula which expresses both the transport and the elliptic nature of the transport operator away from the characteristic manifold. Nevertheless, because $L^{1}$ is not a convenient space for Fourier analysis, the proof is quite complex and requires in particular the use of singular integral operators, as well as a characterization of equi-integrability in terms of compactness in weak Hardy spaces. We refer to [6] for a complete discussion of the subject.

Note that, in the problem we consider in this work, the time derivative of the kinetic equations has a factor $\epsilon$, so that we cannot expect to establish temporal strong compactness and the above theorems cannot be applied as such. However, it is possible to get strong compactness with respect to the fast time variable $\frac{t}{\epsilon}$, but this does not provide any information on the slow dynamics. Thus, we reformulate now the preceding theorems in the following lemmas in order that they be directly applicable to our problem.

LEMMA 7.6. Let the bounded family of functions

$$
\left\{\phi_{\epsilon}(t, x, v)\right\}_{\epsilon>0} \subset L^{p}\left(\mathbb{R}_{t} \times \mathbb{R}_{x}^{3} \times \mathbb{R}_{v}^{3}\right),
$$

for some $1<p<\infty$, be locally relatively compact in $v$ and such that

$$
\left(\epsilon \partial_{t}+v \cdot \nabla_{x}\right) \phi_{\epsilon}=\left(1-\Delta_{x}\right)^{\frac{\beta}{2}}\left(1-\Delta_{v}\right)^{\frac{\alpha}{2}} S_{\epsilon},
$$


for all $\epsilon>0$ and for some bounded family

$$
\left\{S_{\epsilon}(t, x, v)\right\}_{\epsilon>0} \subset L^{p}\left(\mathbb{R}_{t} \times \mathbb{R}_{x}^{3} \times \mathbb{R}_{v}^{3}\right),
$$

where $\alpha \geq 0$ and $0 \leq \beta<1$.

Then, $\left\{\phi_{\epsilon}(t, x, v)\right\}_{\epsilon>0}$ is locally relatively compact in $L^{p}\left(\mathbb{R}_{t} \times \mathbb{R}_{x}^{3} \times \mathbb{R}_{v}^{3}\right)$ in $x$ and $v$ (but not necessarily in $t$ ).

Proof. This result is directly deduced from Theorem 7.4 (and its proof). To this end, we define

so that

$$
\begin{aligned}
& \tilde{\phi}_{\epsilon}(t, x, v)=\epsilon^{\frac{1}{p}} \phi_{\epsilon}(\epsilon t, x, v), \\
& \tilde{S}_{\epsilon}(t, x, v)=\epsilon^{\frac{1}{p}} S_{\epsilon}(\epsilon t, x, v),
\end{aligned}
$$

$$
\left(\partial_{t}+v \cdot \nabla_{x}\right) \tilde{\phi}_{\epsilon}=\left(1-\Delta_{x}\right)^{\frac{\beta}{2}}\left(1-\Delta_{v}\right)^{\frac{\alpha}{2}} \tilde{S}_{\epsilon},
$$

and $\tilde{\phi}_{\epsilon}$ and $\tilde{S}_{\epsilon}$ are uniformly bounded in $L^{p}\left(\mathbb{R}_{t} \times \mathbb{R}_{x}^{3} \times \mathbb{R}_{v}^{3}\right)$.

We apply now Theorem 7.4 to the above transport equation to deduce that $\left\{\tilde{\phi}_{\epsilon}\right\}_{\epsilon>0}$ is locally relatively compact in $L^{p}\left(\mathbb{R}_{t} \times \mathbb{R}_{x}^{3} \times \mathbb{R}_{v}^{3}\right)$. In fact, a closer inspection of the proof of this theorem in [6] reveals that, by possibly localizing without loss of generality the above functions in $v$ only, one has the following global estimate :

$$
\lim _{\gamma \rightarrow 0} \sup _{\epsilon>0} \sup _{|k|+|h|+|l|<\gamma}\left\|\tilde{\phi}_{\epsilon}(t+k, x+h, v+l)-\tilde{\phi}_{\epsilon}(t, x, v)\right\|_{L^{p}\left(\mathbb{R}_{t} \times \mathbb{R}_{x}^{3} \times \mathbb{R}_{v}^{3}\right)}=0 .
$$

It follows that

$$
\lim _{\gamma \rightarrow 0} \sup _{\epsilon>0} \sup _{|h|+|l|<\gamma}\left\|\phi_{\epsilon}(t, x+h, v+l)-\phi_{\epsilon}(t, x, v)\right\|_{L^{p}\left(\mathbb{R}_{t} \times \mathbb{R}_{x}^{3} \times \mathbb{R}_{v}^{3}\right)}=0,
$$

which concludes the proof of the lemma.

LEMMA 7.7. Let the bounded family of non-negative functions

$$
\left\{\phi_{\epsilon}(t, x, v)\right\}_{\epsilon>0} \subset L^{1}\left(\mathbb{R}_{t} \times \mathbb{R}_{x}^{3} ; L^{r}\left(\mathbb{R}_{v}^{3}\right)\right),
$$

for some $1<r<\infty$, be locally relatively compact in $v$ and such that

$$
\left(\epsilon \partial_{t}+v \cdot \nabla_{x}\right) \phi_{\epsilon}=\left(1-\Delta_{x}\right)^{\frac{\beta}{2}}\left(1-\Delta_{v}\right)^{\frac{\alpha}{2}} S_{\epsilon},
$$

for all $\epsilon>0$ and for some bounded family

$$
\left\{S_{\epsilon}(t, x, v)\right\}_{\epsilon>0} \subset L^{1}\left(\mathbb{R}_{t} \times \mathbb{R}_{x}^{3} ; L^{r}\left(\mathbb{R}_{v}^{3}\right)\right),
$$

where $\alpha \geq 0$ and $0 \leq \beta<1$. We further assume that, for any compact set $K \subset$ $\mathbb{R}^{3} \times \mathbb{R}^{3}$,

$$
\left.\left\{\int_{K} \phi_{\epsilon}(t, x, v) d x d v\right\}_{\epsilon>0} \text { is equi-integrable (in } t\right) .
$$

Then, $\left\{\phi_{\epsilon}(t, x, v)\right\}_{\epsilon>0}$ is equi-integrable (in all variables) and locally relatively compact in $L^{1}\left(\mathbb{R}_{t} \times \mathbb{R}_{x}^{3} \times \mathbb{R}_{v}^{3}\right)$ in $x$ and $v$ (but not necessarily in $t$ ).

Moreover, if the $\phi_{\epsilon}$ 's are signed (in the sense that the functions may assume both positive and negative values), the conclusion still holds true, i.e. $\left\{\phi_{\epsilon}(t, x, v)\right\}_{\epsilon>0}$ is locally relatively compact in $L^{1}\left(\mathbb{R}_{t} \times \mathbb{R}_{x}^{3} \times \mathbb{R}_{v}^{3}\right)$ in $x$ and $v$ (but not necessarily in $t$ ), provided $\left\{\phi_{\epsilon}(t, x, v)\right\}_{\epsilon>0}$ is equi-integrable (in all variables) a priori.

Proof. When the $\phi_{\epsilon}$ 's are signed and a priori equi-integrable (in all variables), this result is deduced from Theorem 7.5 (and its proof) utilizing the strategy of proof of Lemma 7.6, that is by dilation of the time variable. To this end, we define

$$
\begin{gathered}
\tilde{\phi}_{\epsilon}(t, x, v)=\epsilon \phi_{\epsilon}(\epsilon t, x, v), \\
\tilde{S}_{\epsilon}(t, x, v)=\epsilon S_{\epsilon}(\epsilon t, x, v),
\end{gathered}
$$


so that

$$
\left(\partial_{t}+v \cdot \nabla_{x}\right) \tilde{\phi}_{\epsilon}=\left(1-\Delta_{x}\right)^{\frac{\beta}{2}}\left(1-\Delta_{v}\right)^{\frac{\alpha}{2}} \tilde{S}_{\epsilon},
$$

and $\tilde{\phi}_{\epsilon}$ and $\tilde{S}_{\epsilon}$ are uniformly bounded in $L^{1}\left(\mathbb{R}_{t} \times \mathbb{R}_{x}^{3} ; L^{r}\left(\mathbb{R}_{v}^{3}\right)\right)$.

We apply now Theorem 7.5 to the above transport equation to deduce that $\left\{\tilde{\phi}_{\epsilon}\right\}_{\epsilon>0}$ is locally relatively compact in $L^{1}\left(\mathbb{R}_{t} \times \mathbb{R}_{x}^{3} \times \mathbb{R}_{v}^{3}\right)$. In fact, a closer inspection of the proof of this theorem in [6] reveals that, by possibly localizing without loss of generality the above functions in $v$ only, one has the following global estimate :

$$
\lim _{\gamma \rightarrow 0} \sup _{\epsilon>0} \sup _{|k|+|h|+|l|<\gamma}\left\|\tilde{\phi}_{\epsilon}(t+k, x+h, v+l)-\tilde{\phi}_{\epsilon}(t, x, v)\right\|_{L^{1, \infty}\left(\mathbb{R}_{t} \times \mathbb{R}_{x}^{3} \times \mathbb{R}_{v}^{3}\right)}=0,
$$

where $L^{1, \infty}$ denotes the standard weak Lebesgue space (or Lorentz space). Note that $L^{1, \infty}$ has the same homogeneity as the Lebesgue space $L^{1}$. It follows that

$$
\lim _{\gamma \rightarrow 0} \sup _{\epsilon>0} \sup _{|h|+|l|<\gamma}\left\|\phi_{\epsilon}(t, x+h, v+l)-\phi_{\epsilon}(t, x, v)\right\|_{L^{1, \infty}\left(\mathbb{R}_{t} \times \mathbb{R}_{x}^{3} \times \mathbb{R}_{v}^{3}\right)}=0 .
$$
that

Next, for any compact set $K \subset \mathbb{R}_{t} \times \mathbb{R}_{x}^{3} \times \mathbb{R}_{v}^{3}$ and any large $R>1$, we have

$$
\begin{aligned}
& \left\|\phi_{\epsilon}(t, x+h, v+l)-\phi_{\epsilon}(t, x, v)\right\|_{L^{1}(K)} \\
& \quad=\int_{0}^{\infty}\left|\left\{(t, x, v) \in K:\left|\phi_{\epsilon}(t, x+h, v+l)-\phi_{\epsilon}(t, x, v)\right|>\lambda\right\}\right| d \lambda \\
& \quad \leq \int_{\frac{1}{R}}^{R}\left|\left\{(t, x, v) \in K:\left|\phi_{\epsilon}(t, x+h, v+l)-\phi_{\epsilon}(t, x, v)\right|>\lambda\right\}\right| d \lambda+\frac{|K|}{R} \\
& \quad+\left\|\left(\phi_{\epsilon}(t, x+h, v+l)-\phi_{\epsilon}(t, x, v)\right) \mathbb{1}_{\left\{\left|\phi_{\epsilon}(t, x+h, v+l)-\phi_{\epsilon}(t, x, v)\right|>R\right\}}\right\|_{L^{1}(K)} \\
& \quad \leq 2 \log R\left\|\phi_{\epsilon}(t, x+h, v+l)-\phi_{\epsilon}(t, x, v)\right\|_{L^{1, \infty}(K)}+\frac{|K|}{R} \\
& \quad+\left\|\left(\phi_{\epsilon}(t, x+h, v+l)-\phi_{\epsilon}(t, x, v)\right) \mathbb{1}_{\left\{\left|\phi_{\epsilon}(t, x+h, v+l)-\phi_{\epsilon}(t, x, v)\right|>R\right\}}\right\|_{L^{1}(K)} .
\end{aligned}
$$

Hence, we deduce, provided the $\phi_{\epsilon}$ 's are equi-integrable in all variables and by the arbitrariness of $R>1$, that

$$
\lim _{\gamma \rightarrow 0} \sup _{\epsilon>0} \sup _{|h|+|l|<\gamma}\left\|\phi_{\epsilon}(t, x+h, v+l)-\phi_{\epsilon}(t, x, v)\right\|_{L_{\text {loc }}^{1}\left(\mathbb{R}_{t} \times \mathbb{R}_{x}^{3} \times \mathbb{R}_{v}^{3}\right)}=0,
$$

which concludes the proof of the lemma when the $\phi_{\epsilon}$ 's are signed and a priori equi-integrable.

Therefore, there only remains to establish the equi-integrability of $\left\{\phi_{\epsilon}(t, x, v)\right\}_{\epsilon>0}$ when it is not already known a priori and when each $\phi_{\epsilon}$ is non-negative. However, the preceding strategy based on time-dilations to deduce results from Theorem 7.5 cannot be repeated here, for the notion of equi-integrability does unfortunately not behave suitably under partial dilations. Instead, the proof of Theorem 2.4 from 6 has to be adapted to treat the present setting, which is rather involved. Therefore, in order to provide a self-contained justification based on 6 and for the sake of clarity, we have moved the remainder of the proof of the present lemma to Appendix C.

7.2.2. Compactness of fluctuations for one species. The next step consists in combining the velocity compactness result from Lemma 7.3 with the hypoelliptic transfer of compactness contained in Lemma 7.7 to infer the compactness in $x$ and $v$ (but not in $t$ ) of the fluctuations $\hat{g}_{\epsilon}$ and $\hat{g}_{\epsilon}^{ \pm}$. To this end, we will need to consider the action of the transport operator $\left(\epsilon \partial_{t}+v \cdot \nabla_{x}\right)$ on the fluctuations $\hat{g}_{\epsilon}$, $\hat{g}_{\epsilon}^{ \pm}$(to control oscillations) and their square $\hat{g}_{\epsilon}^{2}, \hat{g}_{\epsilon}^{ \pm 2}$ (to control concentrations), or truncated versions of these fluctuations. 
Again, note that this strategy differs from the methods developed in previous works on hydrodynamic limits, since we do not use classical averaging lemma. Indeed, we prove below that the fluctuations themselves, and not only their moments with respect to $v$, are strongly compact in $x$ and $v$.

Let us first focus on the regime considered in Theorem 4.4 (with one species) leading to the incompressible quasi-static Navier-Stokes-Fourier-Maxwell-Poisson system 4.30. In this case, we have the following lemma.

LEMmA 7.8. Let $\left(f_{\epsilon}, E_{\epsilon}, B_{\epsilon}\right)$ be the sequence of renormalized solutions to the scaled one species Vlasov-Maxwell-Boltzmann system 4.27) considered in Theorem 4.4.

Then, as $\epsilon \rightarrow 0$, any subsequence of renormalized fluctuations $\hat{g}_{\epsilon}$ is locally relatively compact in $(x, v)$ in $L^{2}(d t d x d v)$ in the sense that, for any $\eta>0$ and every compact subset $K \subset[0, \infty) \times \mathbb{R}^{3} \times \mathbb{R}^{3}$, there exists $\gamma>0$ such that, if $h, l \in \mathbb{R}^{3}$ satisfy $|h|+|l|<\gamma$, then

$$
\sup _{\epsilon>0}\left\|\hat{g}_{\epsilon}(t, x+h, v+l)-\hat{g}_{\epsilon}(t, x, v)\right\|_{L^{2}(K, d t d x d v)}<\eta .
$$

In particular, the family $\left|\hat{g}_{\epsilon}\right|^{2}$ is equi-integrable (in all variables $t, x$ and $v$ ).

Proof. The proof of this lemma proceeds with two main steps. The first one establishes the compactness of $\hat{g}_{\epsilon}$ in $x$ and $v$ in $L_{\text {loc }}^{1}$, while the second one shows the equi-integrability of $\hat{g}_{\epsilon}^{2}$ in all variables. The combination of these two steps will eventually allow us to conclude the proof.

An admissible renormalization $\beta_{1}\left(G_{\epsilon}\right)$. We consider first the admissible square root renormalization

$$
\beta_{1}(z)=\frac{\sqrt{z+\epsilon^{a}}-1}{\epsilon},
$$

for some given $1<a<4$. This renormalization is introduced to circumvent the fact that the natural renormalization $2 \frac{\sqrt{z}-1}{\epsilon}$ corresponding to $\hat{g}_{\epsilon}$ is not admissible for Vlasov-Boltzmann equations, for it is singular at $z=0$, i.e. $\left(2 \frac{\sqrt{z}-1}{\epsilon}\right)^{\prime} \rightarrow \infty$ as $z \rightarrow 0$.

In fact, we have already used a similar strategy in the proof of Proposition 6.1. where we showed, as a consequence of the entropy and entropy dissipation bounds, that (see (6.5))

$$
\begin{aligned}
& 2 \frac{\sqrt{G_{\epsilon}+\epsilon^{a}}-1}{\epsilon}-\hat{g}_{\epsilon}=O\left(\epsilon^{\frac{a}{2}-1}\right)_{L^{\infty}(d t d x d v)}, \\
& 2 \frac{\sqrt{G_{\epsilon}+\epsilon^{a}}-1}{\epsilon}-\hat{g}_{\epsilon}=O\left(\epsilon^{a-1}\right)_{L^{\infty}(d t d x d v)}+O\left(\epsilon^{\frac{a}{2}}\right)_{L^{\infty}\left(d t ; L^{2}(M d x d v)\right)}, \\
& 2 \frac{\sqrt{G_{\epsilon}+\epsilon^{a}}-1}{\epsilon}-\hat{g}_{\epsilon}=O\left(\epsilon^{a-1}\right)_{L^{\infty}(d t d x d v)}+O\left(\epsilon^{\frac{a}{2}}\right)_{L_{\mathrm{loc}}^{2}\left(d t d x ; L^{2}\left(\left(1+|v|^{2}\right) M d v\right)\right)},
\end{aligned}
$$

so that strong compactness properties of $\frac{\sqrt{G_{\epsilon}+\epsilon^{a}}-1}{\epsilon}$ in $L_{\mathrm{loc}}^{p}(d t d x d v)$, for any given $1 \leq p \leq 2$, will entail similar properties on $\hat{g}_{\epsilon}$ in the same space and vice versa.

Compactness of $\beta_{1}\left(G_{\epsilon}\right)$ in $v$. In particular, since, in view of Lemma 7.3, the renormalized fluctuations $\hat{g}_{\epsilon}$ are locally relatively compact in $v$ in $L_{\text {loc }}^{2}(d t d x d v)$, the same holds true for any subsequence of $\frac{\sqrt{G_{\epsilon}+\epsilon^{a}}-1}{\epsilon}$ in the sense that, for any $\eta>0$ and every compact subset $K \subset[0, \infty) \times \mathbb{R}^{3} \times \mathbb{R}^{3}$, there exists $\gamma>0$ such that, if $h \in \mathbb{R}^{3}$ satisfies $|h|<\gamma$, then

$$
\sup _{\epsilon>0}\left\|\beta_{1}\left(G_{\epsilon}\right)(t, x, v+h)-\beta_{1}\left(G_{\epsilon}\right)(t, x, v)\right\|_{L^{2}(K, d t d x d v)}<\eta .
$$

Action of the transport operator on $\beta_{1}\left(G_{\epsilon}\right)$. Thus, using $\beta_{1}(z)$ to renormalize the Vlasov-Boltzmann equation in (4.27) and decomposing the collision integrands 
according to 5.8 , we find that (see 6.3 )

$(7.17)$

$$
\begin{aligned}
\left(\epsilon \partial_{t}+v\right. & \left.\cdot \nabla_{x}+\epsilon\left(E_{\epsilon}+v \wedge B_{\epsilon}\right) \cdot \nabla_{v}\right) \frac{\sqrt{G_{\epsilon}+\epsilon^{a}}-1}{\epsilon}-E_{\epsilon} \cdot v \frac{G_{\epsilon}}{2 \sqrt{G_{\epsilon}+\epsilon^{a}}} \\
= & \frac{\sqrt{G_{\epsilon}}}{2 \sqrt{G_{\epsilon}+\epsilon^{a}}} \int_{\mathbb{R}^{3} \times \mathbb{S}^{2}} \sqrt{G_{\epsilon *}} \hat{q}_{\epsilon} M_{*} d v_{*} d \sigma+\frac{\epsilon^{2}}{8 \sqrt{G_{\epsilon}+\epsilon^{a}}} \int_{\mathbb{R}^{3} \times \mathbb{S}^{2}} \hat{q}_{\epsilon}^{2} M_{*} d v_{*} d \sigma .
\end{aligned}
$$

It follows, employing the uniform bounds $\hat{g}_{\epsilon} \in L^{\infty}\left(d t ; L^{2}(M d x d v)\right)$ and $\hat{q}_{\epsilon} \in$ $L^{2}\left(M M_{*} d t d x d v d v_{*} d \sigma\right)$ from Lemmas 5.2 and 5.3 , respectively, that (see 6.4)

$$
\begin{aligned}
\left(\epsilon \partial_{t}+v\right. & \left.\cdot \nabla_{x}\right) \frac{\sqrt{G_{\epsilon}+\epsilon^{a}}-1}{\epsilon} \\
& =\frac{\sqrt{G_{\epsilon}}}{2 \sqrt{G_{\epsilon}+\epsilon^{a}}} E_{\epsilon} \cdot v\left(1+\frac{\epsilon}{2} \hat{g}_{\epsilon}\right)-\epsilon \nabla_{v} \cdot\left(E_{\epsilon}+v \wedge B_{\epsilon}\right) \frac{\sqrt{G_{\epsilon}+\epsilon^{a}}-1}{\epsilon} \\
& +\frac{\sqrt{G_{\epsilon}}}{2 \sqrt{G_{\epsilon}+\epsilon^{a}}} \int_{\mathbb{R}^{3} \times \mathbb{S}^{2}} \hat{q}_{\epsilon} M_{*} d v_{*} d \sigma+\frac{\epsilon \sqrt{G_{\epsilon}}}{4 \sqrt{G_{\epsilon}+\epsilon^{a}}} \int_{\mathbb{R}^{3} \times \mathbb{S}^{2}} \hat{g}_{\epsilon *} \hat{q}_{\epsilon} M_{*} d v_{*} d \sigma \\
& +\frac{\epsilon^{2}}{8 \sqrt{G_{\epsilon}+\epsilon^{a}}} \int_{\mathbb{R}^{3} \times \mathbb{S}^{2}} \hat{q}_{\epsilon}^{2} M_{*} d v_{*} d \sigma \\
& =O(1)_{L_{\mathrm{loc}}^{1}(d t d x d v)}+O(\epsilon)_{L_{\mathrm{loc}}^{1}}\left(d t d x ; W_{\mathrm{loc}}^{-1,1}(d v)\right)
\end{aligned}
$$

Compactness of $\beta_{1}\left(G_{\epsilon}\right)$ in $(x, v)$. On the whole, we have established the compactness in velocity of $\beta_{1}\left(G_{\epsilon}\right)$ in $(7.16)$ and a bound on the transport operator acting on $\beta_{1}\left(G_{\epsilon}\right)$ in 7.18. Therefore, a direct application of Lemma 7.7 yields that $\beta_{1}\left(G_{\epsilon}\right)=\frac{\sqrt{G_{\epsilon}+\epsilon^{a}-1}}{\epsilon}$ is locally relatively compact in $(x, v)$ in $L_{\text {loc }}^{1}(d t d x d v)$.

Combining this result with (7.15), implies that the renormalized fluctuations $\hat{g}_{\epsilon}$ are relatively compact in $(x, v)$ in $L_{\text {loc }}^{1}(d t d x d v)$ as well, in the sense that, for any $\eta>0$ and every compact subset $K \subset[0, \infty) \times \mathbb{R}^{3} \times \mathbb{R}^{3}$, there exists $\gamma>0$ such that, if $h, l \in \mathbb{R}^{3}$ satisfy $|h|+|l|<\gamma$, then

$$
\sup _{\epsilon>0}\left\|\hat{g}_{\epsilon}(t, x+h, v+l)-\hat{g}_{\epsilon}(t, x, v)\right\|_{L^{1}(K, d t d x d v)}<\eta \text {. }
$$

An admissible renormalization $\beta_{2}\left(G_{\epsilon}\right)$. In order to improve this local strong compactness in $x$ and $v$ from $L_{\text {loc }}^{1}$ to $L_{\text {loc }}^{2}$, we only have to show now that $\hat{g}_{\epsilon}^{2}$ is locally equi-integrable in all variables, which will also be seen as a consequence of Lemma 7.7

To this end, we consider now the admissible renormalization

$$
\beta_{2}(z)=\left(\frac{\sqrt{z+\epsilon^{a}}-1}{\epsilon}\right)^{2} \gamma\left(\lambda\left(\sqrt{z+\epsilon^{a}}-1\right)\right)
$$

where $\gamma(z) \in C^{1}(\mathbb{R})$ is a cutoff satisfying $\mathbb{1}_{[-1,1]} \leq \gamma(z) \leq \mathbb{1}_{[-2,2]}$, for some given $1<a<4$ (in fact, we will further restrict the range of $a$ so that necessarily $a=2$ below) and any small enough $\lambda>0$. As before, this renormalization is introduced to circumvent the fact that the natural renormalization $\left(2 \frac{\sqrt{z}-1}{\epsilon}\right)^{2}$ corresponding to $\hat{g}_{\epsilon}^{2}$ is not admissible for Vlasov-Boltzmann equations, for it is singular at $z=0$, i.e. $\left[\left(2 \frac{\sqrt{z}-1}{\epsilon}\right)^{2}\right]^{\prime} \rightarrow-\infty$ as $z \rightarrow 0$, and its growth at infinity is not admissible, i.e. $\left(2 \frac{\sqrt{z}-1}{\epsilon}\right)^{2} \sim z$ as $z \rightarrow \infty$. 
Next, it is readily seen that 7.15 implies that

$$
\begin{aligned}
\left(2 \frac{\sqrt{G_{\epsilon}+\epsilon^{a}}-1}{\epsilon}\right)^{2}-\hat{g}_{\epsilon}^{2} & =2 \hat{g}_{\epsilon}\left(2 \frac{\sqrt{G_{\epsilon}+\epsilon^{a}}-1}{\epsilon}-\hat{g}_{\epsilon}\right)+\left(2 \frac{\sqrt{G_{\epsilon}+\epsilon^{a}}-1}{\epsilon}-\hat{g}_{\epsilon}\right)^{2} \\
& =O\left(\epsilon^{a-1}+\epsilon^{\frac{a}{2}}\right)_{L^{\infty}\left(d t ; L_{\mathrm{loc}}^{1}\left(d x ; L^{1}(M d v)\right)\right)}
\end{aligned}
$$

so that the equi-integrability of $\beta_{2}\left(G_{\epsilon}\right)=\left(\frac{\sqrt{G_{\epsilon}+\epsilon^{a}}-1}{\epsilon}\right)^{2} \gamma\left(\lambda\left(\sqrt{G_{\epsilon}+\epsilon^{a}}-1\right)\right)$ will entail the equi-integrability of $\hat{g}_{\epsilon}^{2} \gamma\left(\lambda\left(\sqrt{G_{\epsilon}+\epsilon^{a}}-1\right)\right)$, which, when combined with the following control on the very large values of fluctuations (see (5.28)) :

$$
\begin{aligned}
(1-\gamma)\left(\lambda\left(\sqrt{G_{\epsilon}+\epsilon^{a}}-1\right)\right)\left|\hat{g}_{\epsilon}\right|^{2} & \leq \mathbb{1}_{\left\{\lambda\left(\sqrt{G_{\epsilon}+\epsilon^{a}}-1\right)>1\right\}}\left|\hat{g}_{\epsilon}\right|^{2} \\
& =O\left(\frac{1}{|\log \lambda|}\right)_{L^{\infty}\left(d t ; L^{1}(M d x d v)\right)},
\end{aligned}
$$

for any small enough $\lambda>0$, will eventually imply the equi-integrability of $\hat{g}_{\epsilon}^{2}$.

Compactness of $\beta_{2}\left(G_{\epsilon}\right)$ in $v$. Furthermore, note that the velocity compactness stated in 7.16 implies a corresponding property for $\beta_{2}\left(G_{\epsilon}\right)$. Indeed, expressing $\beta_{2}(z)=\beta_{1}(z)^{2} \gamma\left(\epsilon \lambda \beta_{1}(z)\right)$ and noticing that, for any $z_{1}, z_{2} \in \mathbb{R}$,

$$
\begin{aligned}
\left|z_{1}^{2} \gamma\left(\epsilon \lambda z_{1}\right)-z_{2}^{2} \gamma\left(\epsilon \lambda z_{2}\right)\right| & =\left|\left(z_{1}-z_{2}\right) z_{1} \gamma\left(\epsilon \lambda z_{1}\right)+z_{2}\left(z_{1} \gamma\left(\epsilon \lambda z_{1}\right)-z_{2} \gamma\left(\epsilon \lambda z_{2}\right)\right)\right| \\
& \leq C\left|z_{1}-z_{2}\right|\left(\left|z_{1}\right|+\left|z_{2}\right|\right)
\end{aligned}
$$

we deduce, for any $h \in \mathbb{R}^{3}$, that

$$
\begin{aligned}
& \left\|\beta_{2}\left(G_{\epsilon}\right)(t, x, v+h)-\beta_{2}\left(G_{\epsilon}\right)(t, x, v)\right\|_{L_{\mathrm{loc}}^{1}(d t d x d v)} \\
& \leq C\left\|\beta_{1}\left(G_{\epsilon}\right)\right\|_{L_{\mathrm{loc}}^{2}(d t d x d v)}\left\|\beta_{1}\left(G_{\epsilon}\right)(t, x, v+h)-\beta_{1}\left(G_{\epsilon}\right)(t, x, v)\right\|_{L_{\mathrm{loc}}^{2}(d t d x d v)} .
\end{aligned}
$$

It then follows from $(7.16)$ that any subsequence of $\beta_{2}\left(G_{\epsilon}\right)$ is locally relatively compact in $v$ in $L^{1}(d t d x d v)$ in the sense that, for any $\eta>0$ and every compact subset $K \subset[0, \infty) \times \mathbb{R}^{3} \times \mathbb{R}^{3}$, there exists $\gamma>0$ such that, if $h \in \mathbb{R}^{3}$ satisfies $|h|<\gamma$, then

$$
\sup _{\epsilon>0}\left\|\beta_{2}\left(G_{\epsilon}\right)(t, x, v+h)-\beta_{2}\left(G_{\epsilon}\right)(t, x, v)\right\|_{L^{1}(K, d t d x d v)}<\eta .
$$

However, in order to use the above velocity compactness of $\beta_{2}\left(G_{\epsilon}\right)$ in Lemma 7.7, we still need to show that $\beta_{2}\left(G_{\epsilon}\right)$ enjoys an improved integrability with respect to the velocity variable, namely that $\beta_{2}\left(G_{\epsilon}\right)$ is locally bounded in $L^{1}\left(d t d x ; L^{r}(d x)\right)$, for some $r>1$. To this end, we introduce the following decomposition

$$
\begin{aligned}
\beta_{2}\left(G_{\epsilon}\right) & =\frac{1}{2} \Pi \hat{g}_{\epsilon} \frac{\sqrt{G_{\epsilon}+\epsilon^{a}}-1}{\epsilon} \gamma\left(\lambda\left(\sqrt{G_{\epsilon}+\epsilon^{a}}-1\right)\right) \\
& +\left(\frac{\sqrt{G_{\epsilon}+\epsilon^{a}}-1}{\epsilon}-\frac{1}{2} \hat{g}_{\epsilon}\right) \frac{\sqrt{G_{\epsilon}+\epsilon^{a}}-1}{\epsilon} \gamma\left(\lambda\left(\sqrt{G_{\epsilon}+\epsilon^{a}}-1\right)\right) \\
& +\frac{1}{2}\left(\hat{g}_{\epsilon}-\Pi \hat{g}_{\epsilon}\right) \frac{\sqrt{G_{\epsilon}+\epsilon^{a}}-1}{\epsilon} \gamma\left(\lambda\left(\sqrt{G_{\epsilon}+\epsilon^{a}}-1\right)\right) .
\end{aligned}
$$

Since $\Pi \hat{g}_{\epsilon}$ belongs to $L^{\infty}\left(d t ; L^{2}\left(d x ; L^{p}(M d v)\right)\right)$, for any $1 \leq p<\infty$, we therefore get, for any $1 \leq r<2$,

$$
\beta_{2}\left(G_{\epsilon}\right) \leq O(1)_{L_{\mathrm{loc}}^{1}\left(d t d x ; L^{r}(M d v)\right)}+\frac{C}{\lambda \epsilon}\left|\frac{\sqrt{G_{\epsilon}+\epsilon^{a}}-1}{\epsilon}-\frac{1}{2} \hat{g}_{\epsilon}\right|+\frac{C}{\lambda \epsilon}\left|\hat{g}_{\epsilon}-\Pi \hat{g}_{\epsilon}\right| .
$$


Then, by 7.15 and by the relaxation estimate (5.11), we obtain, provided $2 \leq a<$ 4 ,

$$
\begin{aligned}
\beta_{2}\left(G_{\epsilon}\right) & \left.=O(1)_{L_{\mathrm{loc}}^{1}\left(d t d x ; L^{r}(M d v)\right)}+O\left(\frac{1}{\lambda}\right)_{L_{\mathrm{loc}}^{1}\left(d t d x ; L^{2}(M d v)\right)}+\frac{\epsilon^{\frac{a-2}{2}}}{\lambda}\right)_{L_{\mathrm{loc}}^{1}\left(d t d x ; L^{2}(M d v)\right)} \\
& +O\left(\frac{1}{\lambda}\right)_{L_{\mathrm{loc}}^{1}\left(d t d x ; L^{r}(M d v)\right)},
\end{aligned}
$$

for any $1 \leq r<2$.

Finally, combining the preceding estimate with the compactness estimate (7.23), we deduce, for any $1 \leq r<2$, that $\beta_{2}\left(G_{\epsilon}\right)$ is locally relatively compact in $v$ in $L^{1}\left(d t d x ; L^{r}(d v)\right)$ in the sense that, for any $\eta>0$ and every compact subset $K \subset[0, \infty) \times \mathbb{R}^{3} \times \mathbb{R}^{3}$, there exists $\gamma>0$ such that, if $h \in \mathbb{R}^{3}$ satisfies $|h|<\gamma$, then

$$
\sup _{\epsilon>0}\left\|\left(\beta_{2}\left(G_{\epsilon}\right)(t, x, v+h)-\beta_{2}\left(G_{\epsilon}\right)(t, x, v)\right) \mathbb{1}_{K}(t, x, v)\right\|_{L^{1}\left(d t d x ; L^{r}(d v)\right)}<\eta .
$$

Action of the transport operator on $\beta_{2}\left(G_{\epsilon}\right)$. Thus, using $\beta_{2}(z)$ to renormalize the Vlasov-Boltzmann equation in 4.27), decomposing the collision integrands according to (5.8) and writing for convenience

$$
\Gamma(z)=z\left(2 \gamma(\lambda \epsilon z)+\lambda \epsilon z \gamma^{\prime}(\lambda \epsilon z)\right),
$$

so that $\beta_{2}^{\prime}(z)=\beta_{1}^{\prime}(z) \Gamma\left(\beta_{1}(z)\right)$, we have now that (note that this renormalization procedure amounts to multiplying 7.17 by $\left.\Gamma\left(\beta_{1}\left(G_{\epsilon}\right)\right)\right)$

$$
\begin{aligned}
& \left(\epsilon \partial_{t}+v \cdot \nabla_{x}+\epsilon\left(E_{\epsilon}+v \wedge B_{\epsilon}\right) \cdot \nabla_{v}\right) \beta_{2}\left(G_{\epsilon}\right)-E_{\epsilon} \cdot v \frac{G_{\epsilon} \Gamma\left(\beta_{1}\left(G_{\epsilon}\right)\right)}{2 \sqrt{G_{\epsilon}+\epsilon^{a}}} \\
& \quad=\frac{\sqrt{G_{\epsilon}} \Gamma\left(\beta_{1}\left(G_{\epsilon}\right)\right)}{2 \sqrt{G_{\epsilon}+\epsilon^{a}}} \int_{\mathbb{R}^{3} \times \mathbb{S}^{2}} \sqrt{G_{\epsilon *}} \hat{q}_{\epsilon} M_{*} d v_{*} d \sigma+\frac{\epsilon^{2} \Gamma\left(\beta_{1}\left(G_{\epsilon}\right)\right)}{8 \sqrt{G_{\epsilon}+\epsilon^{a}}} \int_{\mathbb{R}^{3} \times \mathbb{S}^{2}} \hat{q}_{\epsilon}^{2} M_{*} d v_{*} d \sigma .
\end{aligned}
$$

It follows, employing the uniform bounds $\hat{g}_{\epsilon} \in L^{\infty}\left(d t ; L^{2}(M d x d v)\right)$ and $\hat{q}_{\epsilon} \in$ $L^{2}\left(M M_{*} d t d x d v d v_{*} d \sigma\right)$ from Lemmas 5.2 and 5.3 , respectively, and the direct estimates

that, provided $1<a \leq 2$,

$$
\begin{aligned}
\left|\Gamma\left(\beta_{1}\left(G_{\epsilon}\right)\right)\right| & \leq C\left|\beta_{1}\left(G_{\epsilon}\right)\right|=O(1)_{L_{\mathrm{loc}}^{2}(d t d x d v)}, \\
\Gamma\left(\beta_{1}\left(G_{\epsilon}\right)\right) & =O\left(\frac{1}{\lambda \epsilon}\right)_{L^{\infty}(d t d x d v)}, \\
\left|\beta_{2}\left(G_{\epsilon}\right)\right| & \leq \frac{C}{\lambda \epsilon}\left|\beta_{1}\left(G_{\epsilon}\right)\right|=O\left(\frac{1}{\lambda \epsilon}\right)_{L_{\mathrm{loc}}^{2}(d t d x d v)},
\end{aligned}
$$

$$
\begin{aligned}
\left(\epsilon \partial_{t}+v\right. & \left.\cdot \nabla_{x}\right) \beta_{2}\left(G_{\epsilon}\right) \\
& =\frac{\sqrt{G_{\epsilon}} \Gamma\left(\beta_{1}\left(G_{\epsilon}\right)\right)}{2 \sqrt{G_{\epsilon}+\epsilon^{a}}} E_{\epsilon} \cdot v\left(1+\frac{\epsilon}{2} \hat{g}_{\epsilon}\right) \\
& -\epsilon \nabla_{v} \cdot\left[\left(E_{\epsilon}+v \wedge B_{\epsilon}\right) \beta_{2}\left(G_{\epsilon}\right)\right] \\
& +\frac{\sqrt{G_{\epsilon}} \Gamma\left(\beta_{1}\left(G_{\epsilon}\right)\right)}{2 \sqrt{G_{\epsilon}+\epsilon^{a}}} \int_{\mathbb{R}^{3} \times \mathbb{S}^{2}} \hat{q}_{\epsilon} M_{*} d v_{*} d \sigma \\
& +\frac{\epsilon \sqrt{G_{\epsilon}} \Gamma\left(\beta_{1}\left(G_{\epsilon}\right)\right)}{4 \sqrt{G_{\epsilon}+\epsilon^{a}}} \int_{\mathbb{R}^{3} \times \mathbb{S}^{2}} \hat{g}_{\epsilon *} \hat{q}_{\epsilon} M_{*} d v_{*} d \sigma+\frac{\epsilon^{2} \Gamma\left(\beta_{1}\left(G_{\epsilon}\right)\right)}{8 \sqrt{G_{\epsilon}+\epsilon^{a}}} \int_{\mathbb{R}^{3} \times \mathbb{S}^{2}} \hat{q}_{\epsilon}^{2} M_{*} d v_{*} d \sigma \\
& =O\left(\frac{1}{\lambda}\right)_{L_{\mathrm{loc}}^{1}\left(d t d x ; W_{\mathrm{loc}}^{-1,1}(d v)\right)} .
\end{aligned}
$$


Equi-integrability of $\beta_{2}\left(G_{\epsilon}\right)$ in $(t, x, v)$. On the whole, we have established the compactness in velocity of $\beta_{2}\left(G_{\epsilon}\right)$ in $(7.24)$ and a bound on the transport operator acting on $\beta_{2}\left(G_{\epsilon}\right)$ in 7.25$)$. Therefore, further noticing that $\beta_{2}\left(G_{\epsilon}\right)$ is non-negative and, recalling $\hat{g}_{\epsilon}^{2} \in L^{\infty}\left(d t ; L^{1}(M d x d v)\right)$ and the error estimate $(7.20)$, that the family $\int_{K} \beta_{2}\left(G_{\epsilon}\right) d x d v$ is equi-integrable, for any compact set $K \subset \mathbb{R}^{3} \times \mathbb{R}^{3}$, a direct application of Lemma 7.7 yields that $\beta_{2}\left(G_{\epsilon}\right)=\left(\frac{\sqrt{G_{\epsilon}+\epsilon^{a}}-1}{\epsilon}\right)^{2} \gamma\left(\lambda\left(\sqrt{G_{\epsilon}+\epsilon^{a}}-1\right)\right)$ is equi-integrable in all variables $(t, x, v)$.

Combining this result with 7.20 and 7.21 , implies that the renormalized fluctuations $\hat{g}_{\epsilon}^{2}$ are equi-integrable in all variables $(t, x, v)$ as well.

Finally, further combining the equi-integrability of $\hat{g}_{\epsilon}^{2}$ with the local strong compactness estimate 7.19 , we deduce that the renormalized fluctuations $\hat{g}_{\epsilon}$ are relatively compact in $(x, v)$ in $L_{\text {loc }}^{2}(d t d x d v)$, in the sense that, for any $\eta>0$ and every compact subset $K \subset[0, \infty) \times \mathbb{R}^{3} \times \mathbb{R}^{3}$, there exists $\gamma>0$ such that, if $h, l \in \mathbb{R}^{3}$ satisfy $|h|+|l|<\gamma$, then

$$
\sup _{\epsilon>0}\left\|\hat{g}_{\epsilon}(t, x+h, v+l)-\hat{g}_{\epsilon}(t, x, v)\right\|_{L^{2}(K, d t d x d v)}<\eta,
$$

which concludes the proof of the lemma.

Immediate consequences of the preceding strong compactness lemma are :

- the relative compactness in $(x, v)$ in $L^{1}(d t d x d v)$ of any subsequence of renormalized fluctuations $\hat{g}_{\epsilon}^{2}$ in the sense that, for any $\eta>0$ and every compact subset $K \subset[0, \infty) \times \mathbb{R}^{3} \times \mathbb{R}^{3}$, there exists $\gamma>0$ such that, if $h, l \in \mathbb{R}^{3}$ satisfy $|h|+|l|<\gamma$, then

$$
\sup _{\epsilon>0}\left\|\hat{g}_{\epsilon}^{2}(t, x+h, v+l)-\hat{g}_{\epsilon}^{2}(t, x, v)\right\|_{L^{1}(K, d t d x d v)}<\eta .
$$

- the nonlinear weak compactness property, for any $p<2$,

$$
\left(1+|v|^{p}\right) \hat{g}_{\epsilon}^{2} \text { is weakly relatively compact in } L_{\mathrm{loc}}^{1}\left(d t d x ; L^{1}(M d v)\right),
$$

which follows from the Dunford-Pettis compactness criterion (see 66 ) by deducing the equi-integrability of $\hat{g}_{\epsilon}^{2}$ from Lemma 7.8 and the tightness of $\left(1+|v|^{p}\right) \hat{g}_{\epsilon}^{2}$ from Lemma 5.12 .

- the strong spatial compactness of the moments $\int_{\mathbb{R}^{3}} \hat{g}_{\epsilon} \varphi(v) M d v$ in $L_{\mathrm{loc}}^{2}(d t d x)$, for any $\varphi(v) \in L^{2}\left(\left(1+|v|^{2}\right)^{-1} M d v\right)$, in particular

$$
\begin{aligned}
& \lim _{|h| \rightarrow 0} \sup _{\epsilon>0}\left\|\hat{\rho}_{\epsilon}(t, x+h)-\hat{\rho}_{\epsilon}(t, x)\right\|_{L_{\mathrm{loc}}^{2}(d t d x)}=0, \\
& \lim _{|h| \rightarrow 0} \sup _{\epsilon>0}\left\|\hat{u}_{\epsilon}(t, x+h)-\hat{u}_{\epsilon}(t, x)\right\|_{L_{\mathrm{loc}}^{2}(d t d x)}=0, \\
& \lim _{|h| \rightarrow 0} \sup _{\epsilon>0}\left\|\hat{\theta}_{\epsilon}(t, x+h)-\hat{\theta}_{\epsilon}(t, x)\right\|_{L_{\mathrm{loc}}^{2}(d t d x)}=0 .
\end{aligned}
$$

The next lemma is also a direct consequence of the strong compactness properties from the preceding lemma and concerns a refinement of the relaxation estimate 5.11 to $L_{\mathrm{loc}}^{2}(d t d x d v)$.

LEMMA 7.9. Let $\left(f_{\epsilon}, E_{\epsilon}, B_{\epsilon}\right)$ be the sequence of renormalized solutions to the scaled one species Vlasov-Maxwell-Boltzmann system 4.27) considered in Theorem 4.4.

Then, as $\epsilon \rightarrow 0$, any subsequence of renormalized fluctuations $\hat{g}_{\epsilon}$ satisfies the relaxation estimate

$$
\hat{g}_{\epsilon}-\Pi \hat{g}_{\epsilon} \rightarrow 0 \quad \text { in } L_{\mathrm{loc}}^{2}\left(d t d x ; L^{2}(M d v)\right) .
$$


Proof. On the one hand, we already know from Lemma 5.10 that

$$
\hat{g}_{\epsilon}-\Pi \hat{g}_{\epsilon}=O(\epsilon)_{L_{\mathrm{loc}}^{1}\left(d t d x ; L^{2}(M d v)\right)} .
$$

On the other hand, the uniform integrability in all variables of $\left|\hat{g}_{\epsilon}\right|^{2}$ from Lemma 7.8 and the tightness in $v$ of $\left|\hat{g}_{\epsilon}\right|^{2} M$ implied by Lemma 5.12 shows that

$$
\int_{\mathbb{R}^{3}}\left|\Pi \hat{g}_{\epsilon}\right|^{2} M d v \leq C \int_{\mathbb{R}^{3}}\left|\hat{g}_{\epsilon}\right|^{2} M d v \quad \text { is uniformly integrable in } t \text { and } x .
$$

Therefore, we deduce that

$$
\left\|\hat{g}_{\epsilon}-\Pi \hat{g}_{\epsilon}\right\|_{L^{2}(M d v)}^{2} \quad \text { is uniformly integrable in } t \text { and } x \text {. }
$$

Then, decomposing, for any large $\lambda>0$,

$$
\hat{g}_{\epsilon}-\Pi \hat{g}_{\epsilon}=\left(\hat{g}_{\epsilon}-\Pi \hat{g}_{\epsilon}\right) \mathbb{1}_{\left\{\left\|\hat{g}_{\epsilon}-\Pi \hat{g}_{\epsilon}\right\|_{L^{2}(M d v)} \leq \lambda\right\}}+\left(\hat{g}_{\epsilon}-\Pi \hat{g}_{\epsilon}\right) \mathbb{1}_{\left\{\left\|\hat{g}_{\epsilon}-\Pi \hat{g}_{\epsilon}\right\|_{L^{2}(M d v)}>\lambda\right\}},
$$

we find that

$$
\begin{aligned}
\left\|\hat{g}_{\epsilon}-\Pi \hat{g}_{\epsilon}\right\|_{L_{\mathrm{loc}}^{2}\left(d t d x ; L^{2}(M d v)\right)} & \leq \sqrt{\lambda}\left\|\hat{g}_{\epsilon}-\Pi \hat{g}_{\epsilon}\right\|_{L_{\mathrm{loc}}^{1}\left(d t d x ; L^{2}(M d v)\right)}^{\frac{1}{2}} \\
& +\left\|\left(\hat{g}_{\epsilon}-\Pi \hat{g}_{\epsilon}\right) \mathbb{1}_{\left\{\left\|\hat{g}_{\epsilon}-\Pi \hat{g}_{\epsilon}\right\|_{L^{2}(M d v)}>\lambda\right\}}\right\|_{L_{\mathrm{loc}}^{2}\left(d t d x ; L^{2}(M d v)\right)}
\end{aligned}
$$

whence, by virtue of 7.29 ,

$$
\begin{aligned}
\limsup _{\epsilon \rightarrow 0} & \left\|\hat{g}_{\epsilon}-\Pi \hat{g}_{\epsilon}\right\|_{L_{\mathrm{loc}}^{2}\left(d t d x ; L^{2}(M d v)\right)} \\
& \leq \sup _{\epsilon>0}\left\|\left(\hat{g}_{\epsilon}-\Pi \hat{g}_{\epsilon}\right) \mathbb{1}_{\left\{\left\|\hat{g}_{\epsilon}-\Pi \hat{g}_{\epsilon}\right\|_{L^{2}(M d v)}>\lambda\right\}}\right\|_{L_{\mathrm{loc}}^{2}\left(d t d x ; L^{2}(M d v)\right)} .
\end{aligned}
$$

Finally, thanks to the uniform integrability 7.30 and by the arbitrariness of $\lambda$, we infer

$$
\lim _{\epsilon \rightarrow 0}\left\|\hat{g}_{\epsilon}-\Pi \hat{g}_{\epsilon}\right\|_{L_{\mathrm{loc}}^{2}\left(d t d x ; L^{2}(M d v)\right)}=0,
$$

which concludes the proof of the lemma.

7.2.3. Compactness of fluctuations for two species. We move on now to the study of strong compactness properties of the fluctuations considered in Theorems 4.5 and 4.6 leading to the two-fluid incompressible Navier-Stokes-FourierMaxwell systems with Ohm's laws 4.37) and 4.38).

Unlike the estimates from Chapter 5 infered from entropy and entropy dissipation bounds, here, we cannot deduce results for the two species case from results for the one species case. In fact, the regimes considered in Theorems 4.5 and 4.6 are much more singular than the regime studied in Theorem 4.4 and, as a result, the compactness properties asserted in Lemma 7.8 may not hold in the two species case.

It is to be emphasized that this lack of compactness is one the main drawbacks and difficulties preventing the improvement of Theorems 4.5 and 4.6 to a weak compactness result similar to Theorem 4.4. Recall, however, that such an improvement is not to be expected so readily since the limiting systems 4.37 and 4.38 are not stable under weak convergence in the energy space and, in particular, are not known to have global weak solutions (see corresponding discussion in Section 3.2.

Thus, in the two species case, we only have the following weaker strong compactness result.

LEMMA 7.10. Let $\left(f_{\epsilon}^{ \pm}, E_{\epsilon}, B_{\epsilon}\right)$ be the sequence of renormalized solutions to the scaled two species Vlasov-Maxwell-Boltzmann system 4.34 considered in Theorems 4.5 and 4.6 .

Then, as $\epsilon \rightarrow 0$, any subsequence of renormalized fluctuations $\hat{g}_{\epsilon}^{ \pm}$is locally relatively compact in $(x, v)$ in $L^{p}(d t d x d v)$, for any $1 \leq p<2$, in the sense that, 
for any $\eta>0$ and every compact subset $K \subset[0, \infty) \times \mathbb{R}^{3} \times \mathbb{R}^{3}$, there exists $\gamma>0$ such that, if $h, l \in \mathbb{R}^{3}$ satisfy $|h|+|l|<\gamma$, then

$$
\sup _{\epsilon>0}\left\|\hat{g}_{\epsilon}^{ \pm}(t, x+h, v+l)-\hat{g}_{\epsilon}^{ \pm}(t, x, v)\right\|_{L^{p}(K, d t d x d v)}<\eta .
$$

Furthermore, for any $\lambda>0$, the families $\left|\hat{g}_{\epsilon}^{ \pm}\right|^{2} \mathbb{1}_{\left\{\delta \lambda\left|\hat{g}_{\epsilon}^{ \pm}\right| \leq 1\right\}}$ are equi-integrable (in all variables $t, x$ and $v$ ).

REMARK. We do not know whether the families $\left|\hat{g}_{\epsilon}^{ \pm}\right|^{2}$ are equi-integrable (in all variables $t, x$ and $v$ ) or not.

Proof. The method of proof of this lemma is similar to the strategy used in the proof of Lemma 7.8 .

An admissible renormalization $\beta_{1}\left(G_{\epsilon}^{ \pm}\right)$. As in the proof of Lemma 7.8 , we consider first the admissible square root renormalization

$$
\beta_{1}(z)=\frac{\sqrt{z+\epsilon^{a}}-1}{\epsilon},
$$

for some given $1<a<4$.

Similarly to 7.15 , as a consequence of the entropy and entropy dissipation bounds, we have now that

$$
\begin{aligned}
& 2 \frac{\sqrt{G_{\epsilon}^{ \pm}+\epsilon^{a}}-1}{\epsilon}-\hat{g}_{\epsilon}^{ \pm}=O\left(\epsilon^{\frac{a}{2}-1}\right)_{L^{\infty}(d t d x d v)}, \\
& 2 \frac{\sqrt{G_{\epsilon}^{ \pm}+\epsilon^{a}}-1}{\epsilon}-\hat{g}_{\epsilon}^{ \pm}=O\left(\epsilon^{a-1}\right)_{L^{\infty}(d t d x d v)}+O\left(\epsilon^{\frac{a}{2}}\right)_{L^{\infty}\left(d t ; L^{2}(M d x d v)\right)}, \\
& 2 \frac{\sqrt{G_{\epsilon}^{ \pm}+\epsilon^{a}}-1}{\epsilon}-\hat{g}_{\epsilon}^{ \pm}=O\left(\epsilon^{a-1}\right)_{L^{\infty}(d t d x d v)}+O\left(\epsilon^{\frac{a}{2}}\right)_{L_{\mathrm{loc}}^{2}\left(d t d x ; L^{2}\left(\left(1+|v|^{2}\right) M d v\right)\right)},
\end{aligned}
$$

so that strong compactness properties of $\frac{\sqrt{G_{\epsilon}+\epsilon^{a}}-1}{\epsilon}$ in $L_{\mathrm{loc}}^{p}(d t d x d v)$, for any given $1 \leq p \leq 2$, will entail similar properties on $\hat{g}_{\epsilon}^{ \pm}$in the same space and vice versa.

Compactness of $\beta_{1}\left(G_{\epsilon}^{ \pm}\right)$in $v$. In particular, since, in view of Lemma 7.3 , the renormalized fluctuations $\hat{g}_{\epsilon}^{ \pm}$are locally relatively compact in $v$ in $L_{\text {loc }}^{2}(d t d x d v)$, the same holds true for any subsequence of $\frac{\sqrt{G_{\epsilon}^{ \pm}+\epsilon^{a}}-1}{\epsilon}$ in the sense that, for any $\eta>0$ and every compact subset $K \subset[0, \infty) \times \mathbb{R}^{\epsilon} \times \mathbb{R}^{3}$, there exists $\gamma>0$ such that, if $h \in \mathbb{R}^{3}$ satisfies $|h|<\gamma$, then

$$
\sup _{\epsilon>0}\left\|\beta_{1}\left(G_{\epsilon}^{ \pm}\right)(t, x, v+h)-\beta_{1}\left(G_{\epsilon}^{ \pm}\right)(t, x, v)\right\|_{L^{2}(K, d t d x d v)}<\eta .
$$

Action of the transport operator on $\beta_{1}\left(G_{\epsilon}^{ \pm}\right)$. Thus, using $\beta_{1}(z)$ to renormalize the Vlasov-Boltzmann equations in (4.34) and decomposing the collision integrands according to 5.8 , we find that (see $(6.9)$ )

$$
\begin{aligned}
\left(\epsilon \partial_{t}+v \cdot \nabla_{x} \pm \delta\left(\epsilon E_{\epsilon}+v \wedge B_{\epsilon}\right)\right. & \left.\cdot \nabla_{v}\right) \frac{\sqrt{G_{\epsilon}^{ \pm}+\epsilon^{a}}-1}{\epsilon} \mp \delta E_{\epsilon} \cdot v \frac{G_{\epsilon}^{ \pm}}{2 \sqrt{G_{\epsilon}^{ \pm}+\epsilon^{a}}} \\
& =\frac{\sqrt{G_{\epsilon}^{ \pm}}}{2 \sqrt{G_{\epsilon}^{ \pm}+\epsilon^{a}}} \int_{\mathbb{R}^{3} \times \mathbb{S}^{2}} \sqrt{G_{\epsilon *}^{ \pm}} \hat{q}_{\epsilon}^{ \pm} M_{*} d v_{*} d \sigma \\
& +\frac{\epsilon^{2}}{8 \sqrt{G_{\epsilon}^{ \pm}+\epsilon^{a}}} \int_{\mathbb{R}^{3} \times \mathbb{S}^{2}}\left(\hat{q}_{\epsilon}^{ \pm}\right)^{2} M_{*} d v_{*} d \sigma \\
& +\frac{\delta \sqrt{G_{\epsilon}^{ \pm}}}{2 \sqrt{G_{\epsilon}^{ \pm}+\epsilon^{a}}} \int_{\mathbb{R}^{3} \times \mathbb{S}^{2}} \sqrt{G_{\epsilon *}^{\mp}} \hat{q}_{\epsilon}^{ \pm, \mp} M_{*} d v_{*} d \sigma \\
& +\frac{\epsilon^{2}}{8 \sqrt{G_{\epsilon}^{ \pm}+\epsilon^{a}}} \int_{\mathbb{R}^{3} \times \mathbb{S}^{2}}\left(\hat{q}_{\epsilon}^{ \pm, \mp}\right)^{2} M_{*} d v_{*} d \sigma .
\end{aligned}
$$


It follows, employing the uniform bounds $\hat{g}_{\epsilon}^{ \pm} \in L^{\infty}\left(d t ; L^{2}(M d x d v)\right)$ and $\hat{q}_{\epsilon}^{ \pm}, \hat{q}_{\epsilon}^{ \pm, \mp} \in$ $L^{2}\left(M M_{*} d t d x d v d v_{*} d \sigma\right)$ from Lemmas 5.2 and 5.3 , respectively, that

$$
\begin{aligned}
\left(\epsilon \partial_{t}+v\right. & \left.\cdot \nabla_{x}\right) \frac{\sqrt{G_{\epsilon}^{ \pm}+\epsilon^{a}}-1}{\epsilon} \\
& = \pm \frac{\delta \sqrt{G_{\epsilon}^{ \pm}}}{2 \sqrt{G_{\epsilon}^{ \pm}+\epsilon^{a}}} E_{\epsilon} \cdot v\left(1+\frac{\epsilon}{2} \hat{g}_{\epsilon}\right) \mp \delta \nabla_{v} \cdot\left(\epsilon E_{\epsilon}+v \wedge B_{\epsilon}\right) \frac{\sqrt{G_{\epsilon}^{ \pm}+\epsilon^{a}}-1}{\epsilon} \\
& +\frac{\sqrt{G_{\epsilon}^{ \pm}}}{2 \sqrt{G_{\epsilon}^{ \pm}+\epsilon^{a}}} \int_{\mathbb{R}^{3} \times \mathbb{S}^{2}} \hat{q}_{\epsilon}^{ \pm} M_{*} d v_{*} d \sigma+\frac{\epsilon \sqrt{G_{\epsilon}^{ \pm}}}{4 \sqrt{G_{\epsilon}^{ \pm}+\epsilon^{a}}} \int_{\mathbb{R}^{3} \times \mathbb{S}^{2}} \hat{g}_{\epsilon *}^{ \pm} \hat{q}_{\epsilon}^{ \pm} M_{*} d v_{*} d \sigma \\
& +\frac{\epsilon^{2}}{8 \sqrt{G_{\epsilon}^{ \pm}+\epsilon^{a}}} \int_{\mathbb{R}^{3} \times \mathbb{S}^{2}}\left(\hat{q}_{\epsilon}^{ \pm}\right)^{2} M_{*} d v_{*} d \sigma \\
& +\frac{\delta \sqrt{G_{\epsilon}^{ \pm}}}{2 \sqrt{G_{\epsilon}^{ \pm}+\epsilon^{a}}} \int_{\mathbb{R}^{3} \times \mathbb{S}^{2}} \hat{q}_{\epsilon}^{ \pm, \mp} M_{*} d v_{*} d \sigma+\frac{\delta \epsilon \sqrt{G_{\epsilon}^{ \pm}}}{4 \sqrt{G_{\epsilon}^{ \pm}+\epsilon^{a}}} \int_{\mathbb{R}^{3} \times \mathbb{S}^{2}} \hat{g}_{\epsilon *}^{\mp} \hat{q}_{\epsilon}^{ \pm, \mp} M_{*} d v_{*} d \sigma \\
& +\frac{\epsilon^{2}}{8 \sqrt{G_{\epsilon}^{ \pm}+\epsilon^{a}}} \int_{\mathbb{R}^{3} \times \mathbb{S}^{2}}\left(\hat{q}_{\epsilon}^{ \pm, \mp}\right)^{2} M_{*} d v_{*} d \sigma \\
& =O(1)_{L_{\mathrm{loc}}^{1}(d t d x d v)}+O(\delta)_{L_{\mathrm{loc}}^{1}\left(d t d x ; W_{\mathrm{loc}}^{-1,1}(d v)\right)}
\end{aligned}
$$

Compactness of $\beta_{1}\left(G_{\epsilon}^{ \pm}\right)$in $(x, v)$. On the whole, we have established the compactness in velocity of $\beta_{1}\left(G_{\epsilon}^{ \pm}\right)$in $(7.32$ and a bound on the transport operator acting on $\beta_{1}\left(G_{\epsilon}^{ \pm}\right)$in 7.33$)$. Therefore, a direct application of Lemma 7.7 yields that $\beta_{1}\left(G_{\epsilon}^{ \pm}\right)=\frac{\sqrt{G_{\epsilon}^{ \pm}+\epsilon^{a}-1}}{\epsilon}$ is locally relatively compact in $(x, v)$ in $L_{\text {loc }}^{1}(d t d x d v)$.

Combining this result with (7.31), implies that the renormalized fluctuations $\hat{g}_{\epsilon}^{ \pm}$are relatively compact in $(x, v)$ in $L_{\text {loc }}^{1}(d t d x d v)$ as well. Moreover, since the families $\hat{g}_{\epsilon}^{ \pm}$are uniformly bounded in $L^{\infty}\left(d t ; L^{2}(M d x d v)\right)$, we easily deduce that the renormalized fluctuations $\hat{g}_{\epsilon}^{ \pm}$are relatively compact in $(x, v)$ in $L_{\text {loc }}^{p}(d t d x d v)$, for any $1 \leq p<2$, in the sense that, for any $\eta>0$ and every compact subset $K \subset[0, \infty) \times \mathbb{R}^{3} \times \mathbb{R}^{3}$, there exists $\gamma>0$ such that, if $h, l \in \mathbb{R}^{3}$ satisfy $|h|+|l|<\gamma$, then

$$
\sup _{\epsilon>0}\left\|\hat{g}_{\epsilon}^{ \pm}(t, x+h, v+l)-\hat{g}_{\epsilon}^{ \pm}(t, x, v)\right\|_{L^{p}(K, d t d x d v)}<\eta,
$$

which concludes the proof of the first part of the lemma.

An admissible renormalization $\beta_{2}\left(G_{\epsilon}^{ \pm}\right)$. We proceed now to showing that, for any $\lambda>0$, the families $\left|\hat{g}_{\epsilon}^{ \pm}\right|^{2} \mathbb{1}_{\left\{\delta \lambda\left|\hat{g}_{\epsilon}^{ \pm}\right| \leq 1\right\}}$ are equi-integrable (in all variables $t, x$ and $v$ ), which will also be seen as a consequence of Lemma 7.7 .

To this end, we consider now the admissible renormalization

$$
\beta_{2}(z)=\left(\frac{\sqrt{z+\epsilon^{a}}-1}{\epsilon}\right)^{2} \gamma\left(\delta \lambda\left(\frac{\sqrt{z+\epsilon^{a}}-1}{\epsilon}\right)\right),
$$

where $\gamma(z) \in C^{1}(\mathbb{R})$ is a cutoff satisfying $\mathbb{1}_{[-1,1]} \leq \gamma(z) \leq \mathbb{1}_{[-2,2]}$, for some given $1<a<4$ (in fact, we will further restrict the range of $a$ so that necessarily $a=2$ below) and any small enough $\lambda>0$.

Next, expressing $\beta_{2}(z)=\beta_{1}(z)^{2} \gamma\left(\delta \lambda \beta_{1}(z)\right)$ and using a slight variant of 7.22$)$, it is readily seen that 7.31 implies that

$$
\begin{aligned}
\left|4 \beta_{2}\left(G_{\epsilon}^{ \pm}\right)-\hat{g}_{\epsilon}^{ \pm 2} \gamma\left(\frac{\delta \lambda}{2} \hat{g}_{\epsilon}^{ \pm}\right)\right| & \leq C\left|2 \beta_{1}\left(G_{\epsilon}^{ \pm}\right)-\hat{g}_{\epsilon}^{ \pm}\right|\left(\left|\beta_{1}\left(G_{\epsilon}^{ \pm}\right)\right|+\left|\hat{g}_{\epsilon}^{ \pm}\right|\right) \\
& =O\left(\epsilon^{a-1}+\epsilon^{\frac{a}{2}}\right)_{L^{\infty}\left(d t ; L_{\mathrm{loc}}^{1}\left(d x ; L^{1}(M d v)\right)\right)}
\end{aligned}
$$


so that the equi-integrability of $\beta_{2}\left(G_{\epsilon}^{ \pm}\right)=\left(\frac{\sqrt{G_{\epsilon}^{ \pm}+\epsilon^{a}}-1}{\epsilon}\right)^{2} \gamma\left(\delta \lambda\left(\frac{\sqrt{G_{\epsilon}^{ \pm}+\epsilon^{a}}-1}{\epsilon}\right)\right)$ will clearly entail the equi-integrability of $\left|\hat{g}_{\epsilon}^{ \pm}\right|^{2} \mathbb{1}_{\left\{\delta \lambda\left|\hat{g}_{\epsilon}^{ \pm}\right| \leq 1\right\}}$, for any $\lambda>0$.

Compactness of $\beta_{2}\left(G_{\epsilon}^{ \pm}\right)$in $v$. Furthermore, note that the velocity compactness stated in 7.32 implies a corresponding property for $\beta_{2}\left(G_{\epsilon}^{ \pm}\right)$. Indeed, using a slight modification of 7.22 again, we deduce, for any $h \in \mathbb{R}^{3}$, that

$$
\begin{aligned}
& \left\|\beta_{2}\left(G_{\epsilon}^{ \pm}\right)(t, x, v+h)-\beta_{2}\left(G_{\epsilon}^{ \pm}\right)(t, x, v)\right\|_{L_{\mathrm{loc}}^{1}(d t d x d v)} \\
& \leq C\left\|\beta_{1}\left(G_{\epsilon}^{ \pm}\right)\right\|_{L_{\mathrm{loc}}^{2}(d t d x d v)}\left\|\beta_{1}\left(G_{\epsilon}^{ \pm}\right)(t, x, v+h)-\beta_{1}\left(G_{\epsilon}^{ \pm}\right)(t, x, v)\right\|_{L_{\mathrm{loc}}^{2}(d t d x d v)} .
\end{aligned}
$$

It then follows from 7.32 that any subsequence of $\beta_{2}\left(G_{\epsilon}^{ \pm}\right)$is locally relatively compact in $v$ in $L^{1}(d t d x d v)$ in the sense that, for any $\eta>0$ and every compact subset $K \subset[0, \infty) \times \mathbb{R}^{3} \times \mathbb{R}^{3}$, there exists $\gamma>0$ such that, if $h \in \mathbb{R}^{3}$ satisfies $|h|<\gamma$, then

$$
\sup _{\epsilon>0}\left\|\beta_{2}\left(G_{\epsilon}^{ \pm}\right)(t, x, v+h)-\beta_{2}\left(G_{\epsilon}^{ \pm}\right)(t, x, v)\right\|_{L^{1}(K, d t d x d v)}<\eta .
$$

However, in order to use the above velocity compactness of $\beta_{2}\left(G_{\epsilon}^{ \pm}\right)$in Lemma 7.7, we still need to show that $\beta_{2}\left(G_{\epsilon}^{ \pm}\right)$enjoys an improved integrability with respect to the velocity variable. To this end, we introduce the following decomposition

$$
\begin{aligned}
\beta_{2}\left(G_{\epsilon}^{ \pm}\right) & =\frac{1}{2} \Pi \hat{g}_{\epsilon}^{ \pm} \frac{\sqrt{G_{\epsilon}^{ \pm}+\epsilon^{a}}-1}{\epsilon} \gamma\left(\delta \lambda\left(\frac{\sqrt{G_{\epsilon}^{ \pm}+\epsilon^{a}}-1}{\epsilon}\right)\right) \\
& +\left(\frac{\sqrt{G_{\epsilon}^{ \pm}+\epsilon^{a}}-1}{\epsilon}-\frac{1}{2} \hat{g}_{\epsilon}^{ \pm}\right) \frac{\sqrt{G_{\epsilon}^{ \pm}+\epsilon^{a}}-1}{\epsilon} \gamma\left(\delta \lambda\left(\frac{\sqrt{G_{\epsilon}^{ \pm}+\epsilon^{a}}-1}{\epsilon}\right)\right) \\
& +\frac{1}{2}\left(\hat{g}_{\epsilon}^{ \pm}-\Pi \hat{g}_{\epsilon}^{ \pm}\right) \frac{\sqrt{G_{\epsilon}^{ \pm}+\epsilon^{a}}-1}{\epsilon} \gamma\left(\delta \lambda\left(\frac{\sqrt{G_{\epsilon}^{ \pm}+\epsilon^{a}}-1}{\epsilon}\right)\right) .
\end{aligned}
$$

Since $\Pi \hat{g}_{\epsilon}^{ \pm}$belongs to $L^{\infty}\left(d t ; L^{2}\left(d x ; L^{p}(M d v)\right)\right)$, for any $1 \leq p<\infty$, we therefore get, for any $1 \leq r<2$,

$$
\beta_{2}\left(G_{\epsilon}^{ \pm}\right) \leq O(1)_{L_{\mathrm{loc}}^{1}\left(d t d x ; L^{r}(M d v)\right)}+\frac{C}{\lambda \delta}\left|\frac{\sqrt{G_{\epsilon}^{ \pm}+\epsilon^{a}}-1}{\epsilon}-\frac{1}{2} \hat{g}_{\epsilon}^{ \pm}\right|+\frac{C}{\lambda \delta}\left|\hat{g}_{\epsilon}^{ \pm}-\Pi \hat{g}_{\epsilon}^{ \pm}\right| .
$$

Then, by 7.31 and by the relaxation estimate 5.11 , we obtain, provided $2 \leq a<$ 4 ,

for any $1 \leq r<2$.

$$
\begin{aligned}
\beta_{2}\left(G_{\epsilon}^{ \pm}\right) & \left.=O(1)_{L_{\mathrm{loc}}^{1}\left(d t d x ; L^{r}(M d v)\right)}+O\left(\frac{\epsilon}{\lambda \delta}\right)_{L_{\mathrm{loc}}^{1}\left(d t d x ; L^{2}(M d v)\right)}+\frac{\epsilon^{\frac{a}{2}}}{\lambda \delta}\right)_{L_{\mathrm{loc}}^{1}\left(d t d x ; L^{2}(M d v)\right)}+ \\
& =O\left(\frac{1}{\lambda}\right)_{L_{\mathrm{loc}}^{1}\left(d t d x ; L^{r}(M d v)\right)},
\end{aligned}
$$

Finally, combining the preceding estimate with the compactness estimate (7.35), we deduce, for any $1 \leq r<2$, that $\beta_{2}\left(G_{\epsilon}^{ \pm}\right)$is locally relatively compact in $v$ in $L^{1}\left(d t d x ; L^{r}(d v)\right)$ in the sense that, for any $\eta>0$ and every compact subset $K \subset[0, \infty) \times \mathbb{R}^{3} \times \mathbb{R}^{3}$, there exists $\gamma>0$ such that, if $h \in \mathbb{R}^{3}$ satisfies $|h|<\gamma$, then

$$
\sup _{\epsilon>0}\left\|\left(\beta_{2}\left(G_{\epsilon}^{ \pm}\right)(t, x, v+h)-\beta_{2}\left(G_{\epsilon}^{ \pm}\right)(t, x, v)\right) \mathbb{1}_{K}(t, x, v)\right\|_{L^{1}\left(d t d x ; L^{r}(d v)\right)}<\eta .
$$

Action of the transport operator on $\beta_{2}\left(G_{\epsilon}^{ \pm}\right)$. Thus, using $\beta_{2}(z)$ to renormalize the Vlasov-Boltzmann equations in (4.34), decomposing the collision integrands 
according to 5.8 and writing for convenience

$$
\Gamma(z)=z\left(2 \gamma(\lambda \delta z)+\lambda \delta z \gamma^{\prime}(\lambda \delta z)\right),
$$

so that $\beta_{2}^{\prime}(z)=\beta_{1}^{\prime}(z) \Gamma\left(\beta_{1}(z)\right)$, we have now that

$$
\begin{aligned}
& \left(\epsilon \partial_{t}+v \cdot \nabla_{x} \pm \delta\left(\epsilon E_{\epsilon}+v \wedge B_{\epsilon}\right) \cdot \nabla_{v}\right) \beta_{2}\left(G_{\epsilon}^{ \pm}\right) \mp \delta E_{\epsilon} \cdot v \frac{G_{\epsilon}^{ \pm} \Gamma\left(\beta_{1}\left(G_{\epsilon}^{ \pm}\right)\right)}{2 \sqrt{G_{\epsilon}^{ \pm}+\epsilon^{a}}} \\
& =\frac{\sqrt{G_{\epsilon}^{ \pm}} \Gamma\left(\beta_{1}\left(G_{\epsilon}^{ \pm}\right)\right)}{2 \sqrt{G_{\epsilon}^{ \pm}+\epsilon^{a}}} \int_{\mathbb{R}^{3} \times \mathbb{S}^{2}} \sqrt{G_{\epsilon *}^{ \pm}} \hat{q}_{\epsilon}^{ \pm} M_{*} d v_{*} d \sigma \\
& +\frac{\epsilon^{2} \Gamma\left(\beta_{1}\left(G_{\epsilon}^{ \pm}\right)\right)}{8 \sqrt{G_{\epsilon}^{ \pm}+\epsilon^{a}}} \int_{\mathbb{R}^{3} \times \mathbb{S}^{2}}\left(\hat{q}_{\epsilon}^{ \pm}\right)^{2} M_{*} d v_{*} d \sigma \\
& +\frac{\delta \sqrt{G_{\epsilon}^{ \pm}} \Gamma\left(\beta_{1}\left(G_{\epsilon}^{ \pm}\right)\right)}{2 \sqrt{G_{\epsilon}^{ \pm}+\epsilon^{a}}} \int_{\mathbb{R}^{3} \times \mathbb{S}^{2}} \sqrt{G_{\epsilon *}^{\mp}} \hat{q}_{\epsilon}^{ \pm, \mp} M_{*} d v_{*} d \sigma \\
& +\frac{\epsilon^{2} \Gamma\left(\beta_{1}\left(G_{\epsilon}^{ \pm}\right)\right)}{8 \sqrt{G_{\epsilon}^{ \pm}+\epsilon^{a}}} \int_{\mathbb{R}^{3} \times \mathbb{S}^{2}}\left(\hat{q}_{\epsilon}^{ \pm, \mp}\right)^{2} M_{*} d v_{*} d \sigma .
\end{aligned}
$$

It follows, employing the uniform bounds $\hat{g}_{\epsilon}^{ \pm} \in L^{\infty}\left(d t ; L^{2}(M d x d v)\right)$ and $\hat{q}_{\epsilon}^{ \pm}, \hat{q}_{\epsilon}^{ \pm, \mp} \in$ $L^{2}\left(M M_{*} d t d x d v d v_{*} d \sigma\right)$ from Lemmas 5.2 and 5.3 , respectively, and the direct estimates

$$
\begin{aligned}
\left|\Gamma\left(\beta_{1}\left(G_{\epsilon}^{ \pm}\right)\right)\right| & \leq C\left|\beta_{1}\left(G_{\epsilon}^{ \pm}\right)\right|=O(1)_{L_{\mathrm{loc}}^{2}(d t d x d v)}, \\
\Gamma\left(\beta_{1}\left(G_{\epsilon}^{ \pm}\right)\right) & =O\left(\frac{1}{\lambda \delta}\right)_{L^{\infty}(d t d x d v)}, \\
\left|\beta_{2}\left(G_{\epsilon}^{ \pm}\right)\right| & \leq \frac{C}{\lambda \delta}\left|\beta_{1}\left(G_{\epsilon}^{ \pm}\right)\right|=O\left(\frac{1}{\lambda \delta}\right)_{L_{\mathrm{loc}}^{2}(d t d x d v)},
\end{aligned}
$$

that, provided $1<a \leq 2$,

(7.37)

$$
\begin{aligned}
&\left(\epsilon \partial_{t}+v\right.\left.\cdot \nabla_{x}\right) \beta_{2}\left(G_{\epsilon}^{ \pm}\right) \\
&= \pm \frac{\delta \sqrt{G_{\epsilon}^{ \pm}} \Gamma\left(\beta_{1}\left(G_{\epsilon}^{ \pm}\right)\right)}{2 \sqrt{G_{\epsilon}^{ \pm}+\epsilon^{a}}} E_{\epsilon} \cdot v\left(1+\frac{\epsilon}{2} \hat{g}_{\epsilon}\right) \mp \delta \nabla_{v} \cdot\left[\left(\epsilon E_{\epsilon}+v \wedge B_{\epsilon}\right) \beta_{2}\left(G_{\epsilon}^{ \pm}\right)\right] \\
&+\frac{\sqrt{G_{\epsilon}^{ \pm}} \Gamma\left(\beta_{1}\left(G_{\epsilon}^{ \pm}\right)\right)}{2 \sqrt{G_{\epsilon}^{ \pm}+\epsilon^{a}}} \int_{\mathbb{R}^{3} \times \mathbb{S}^{2}} \hat{q}_{\epsilon}^{ \pm} M_{*} d v_{*} d \sigma \\
&+\frac{\epsilon \sqrt{G_{\epsilon}^{ \pm}} \Gamma\left(\beta_{1}\left(G_{\epsilon}^{ \pm}\right)\right)}{4 \sqrt{G_{\epsilon}^{ \pm}+\epsilon^{a}}} \int_{\mathbb{R}^{3} \times \mathbb{S}^{2}} \hat{g}_{\epsilon *}^{ \pm} \hat{q}_{\epsilon}^{ \pm} M_{*} d v_{*} d \sigma \\
&+\frac{\epsilon^{2} \Gamma\left(\beta_{1}\left(G_{\epsilon}^{ \pm}\right)\right)}{8 \sqrt{G_{\epsilon}^{ \pm}+\epsilon^{a}}} \int_{\mathbb{R}^{3} \times \mathbb{S}^{2}}\left(\hat{q}_{\epsilon}^{ \pm}\right)^{2} M_{*} d v_{*} d \sigma \\
&+\frac{\delta \sqrt{G_{\epsilon}^{ \pm}} \Gamma\left(\beta_{1}\left(G_{\epsilon}^{ \pm}\right)\right)}{2 \sqrt{G_{\epsilon}^{ \pm}+\epsilon^{a}}} \int_{\mathbb{R}^{3} \times \mathbb{S}^{2}} \hat{q}_{\epsilon}^{ \pm, \mp} M_{*} d v_{*} d \sigma \\
&+\frac{\delta \epsilon \sqrt{G_{\epsilon}^{ \pm}} \Gamma\left(\beta_{1}\left(G_{\epsilon}^{ \pm}\right)\right)}{4 \sqrt{G_{\epsilon}^{ \pm}+\epsilon^{a}}} \int_{\mathbb{R}^{3} \times \mathbb{S}^{2}} \hat{g}_{\epsilon *}^{\mp} \hat{q}_{\epsilon}^{ \pm, \mp} M_{*} d v_{*} d \sigma \\
&+ \frac{\epsilon^{2} \Gamma\left(\beta_{1}\left(G_{\epsilon}^{ \pm}\right)\right)}{8 \sqrt{G_{\epsilon}^{ \pm}+\epsilon^{a}}} \int_{\mathbb{R}^{3} \times \mathbb{S}^{2}}\left(\hat{q}_{\epsilon}^{ \pm, \mp}\right)^{2} M_{*} d v_{*} d \sigma \\
&= O\left(\frac{1}{\lambda}\right) \\
& L_{\mathrm{loc}}^{ \pm}\left(d t d x ; W_{\mathrm{loc}}^{-1,1}(d v)\right)
\end{aligned}
$$


Note here that the critical term in 7.37 preventing a better control on the transport acting on $\beta_{2}\left(G_{\epsilon}^{ \pm}\right)$and, thereby, on the concentrations of $\left|\hat{g}_{\epsilon}^{ \pm}\right|^{2}$, is precisely

$$
\mp \delta \nabla_{v} \cdot\left(v \wedge B_{\epsilon}\right) \beta_{2}\left(G_{\epsilon}^{ \pm}\right) .
$$

Equi-integrability of $\beta_{2}\left(G_{\epsilon}^{ \pm}\right)$in $(t, x, v)$. On the whole, we have established the compactness in velocity of $\beta_{2}\left(G_{\epsilon}^{ \pm}\right)$in $(7.36)$ and a bound on the transport operator acting on $\beta_{2}\left(G_{\epsilon}^{ \pm}\right)$in $(7.37)$. Therefore, further noticing that $\beta_{2}\left(G_{\epsilon}^{ \pm}\right)$is non-negative and, recalling $\hat{g}_{\epsilon}^{ \pm 2} \in L^{\infty}\left(d t ; L^{1}(M d x d v)\right)$ and the error estimate $(7.34)$, that the family $\int_{K} \beta_{2}\left(G_{\epsilon}^{ \pm}\right) d x d v$ is equi-integrable, for any compact set $K \subset \mathbb{R}^{3} \times \mathbb{R}^{3}$, a direct application of Lemma 7.7 yields that $\beta_{2}\left(G_{\epsilon}^{ \pm}\right)=\left(\frac{\sqrt{G_{\epsilon}^{ \pm}+\epsilon^{a}}-1}{\epsilon}\right)^{2} \gamma\left(\delta \lambda\left(\frac{\sqrt{G_{\epsilon}^{ \pm}+\epsilon^{a}}-1}{\epsilon}\right)\right)$ is equi-integrable in all variables $(t, x, v)$.

Finally, combining this result with 7.34), implies that the renormalized fluctuations $\left|\hat{g}_{\epsilon}^{ \pm}\right|^{2} \mathbb{1}_{\left\{\delta \lambda\left|\hat{g}_{\epsilon}^{ \pm}\right| \leq 1\right\}}$, for any $\lambda>0$, are equi-integrable in all variables $(t, x, v)$ as well, which concludes the proof of the lemma.

REMARK. Note that the estimates for one species 7.26, 7.27) and 7.28, which were deduced directly from Lemma 7.8, are no longer valid for two species in the settings of weak or strong interactions considered here.

Notice, however, that the control (5.28) on the very large values of fluctuations holds in all cases, for it is a mere consequence of the entropy bound only. Thus, on the one hand, estimate 5.28 implies, for any $\lambda_{1}>0$ small enough, that

$$
\left|\hat{g}_{\epsilon}^{ \pm}\right|^{2} \mathbb{1}_{\left\{\epsilon \lambda_{1}\left|\hat{g}_{\epsilon}^{ \pm}\right|>1\right\}}=O\left(\frac{1}{\left|\log \lambda_{1}\right|}\right)_{L^{\infty}\left(d t ; L^{1}(M d x d v)\right)},
$$

while, on the other hand, we showed in Lemma 7.10 by controlling the action of the transport operator on the fluctuations that, for any $\lambda_{2}>0$,

$$
\left|\hat{g}_{\epsilon}^{ \pm}\right|^{2} \mathbb{1}_{\left\{\delta \lambda_{2}\left|\hat{g}_{\epsilon}^{ \pm}\right| \leq 1\right\}} \quad \text { is equi-integrable (in all variables } t, x \text { and } v \text { ). }
$$

In order to establish the equi-integrability of $\left|\hat{g}_{\epsilon}^{ \pm}\right|^{2}$, there would therefore only remain to control the quantity

$$
\left|\hat{g}_{\epsilon}^{ \pm}\right|^{2} \mathbb{1}_{\left\{\frac{1}{\delta \lambda_{2}}<\left|\hat{g}_{\epsilon}^{ \pm}\right| \leq \frac{1}{\epsilon \lambda_{1}}\right\}}
$$

by showing that it is equi-integrable or uniformly small in $L_{\mathrm{loc}}^{1}(d t d x d v)$ as $\lambda_{2} \rightarrow 0$. But nothing seems to imply such a control. At least, we do not know how to prove it.

The compactness results stated in Lemma 7.10 are valid in both regimes of weak and strong interspecies interactions. However, it is to be emphasized that the equi-integrability of $\left|\hat{g}_{\epsilon}^{ \pm}\right|^{2} \mathbb{1}_{\left\{\delta \lambda\left|\hat{g}_{\epsilon}^{ \pm}\right| \leq 1\right\}}$ contained therein definitely becomes a weaker compactness property as the parameter $\delta$ converges slower to zero. In other words, the more singular the regime, the weaker the compactness. In particular, in the extreme case of strong interspecies interactions considered in Theorem 4.6, i.e. in the case $\delta=1$, the above equi-integrability statement is void, for $\left|\hat{g}_{\epsilon}^{ \pm}\right|^{2} \mathbb{1}_{\left\{\delta \lambda\left|\hat{g}_{\epsilon}^{ \pm}\right| \leq 1\right\}}$ is then uniformly bounded pointwise by $\lambda^{-2}$.

On the other hand, the more singular the regime, the stronger the bounds on the fluctuations provided by the entropy dissipation. This fact is epitomized by Lemmas 5.13 and 5.14 , where it is apparent that the fluctuations $g_{\epsilon}^{+}-g_{\epsilon}^{-}-n_{\epsilon}$ and $\hat{g}_{\epsilon}^{+}-\hat{g}_{\epsilon}^{-}-\hat{n}_{\epsilon}$ vanish faster as the parameter $\delta$ converges slower to zero. From this perspective, the extreme case of strong interspecies interactions, i.e. the case 
$\delta=1$, enjoys better convergence properties than other less singular settings. In particular, employing the electrodynamic continuity equation from 4.34

$$
\partial_{t} n_{\epsilon}+\frac{1}{\delta} \nabla_{x} \cdot j_{\epsilon}=0
$$

it is possible to deduce some strong compactness of $n_{\epsilon}$ in both $t$ and $x$ when $\delta=1$. This fails whenever $\delta=o(1)$. This crucial compactness will then allow us to consider the renormalized convergence of $h_{\epsilon}$ and $\hat{h}_{\epsilon}$, which is the content of the following lemma.

LEMMA 7.11. Let $\left(f_{\epsilon}^{ \pm}, E_{\epsilon}, B_{\epsilon}\right)$ be the sequence of renormalized solutions to the scaled two species Vlasov-Maxwell-Boltzmann system 4.34 considered in Theorem 4.6 for strong interspecies interactions, i.e. in the case $\delta=1$.

Then, as $\epsilon \rightarrow 0$, any subsequence of renormalized fluctuations $\hat{g}_{\epsilon}^{ \pm}$satisfies that $\hat{n}_{\epsilon}$ is relatively compact in $L_{\mathrm{loc}}^{p}(d t d x)$, for any $1 \leq p<2$, and that $\hat{g}_{\epsilon}^{+}-\hat{g}_{\epsilon}^{-}$is relatively compact in $L_{\mathrm{loc}}^{p}\left(d t d x ; L^{2}(M d v)\right)$, for any $1 \leq p<2$. In particular, $\left\|\hat{g}_{\epsilon}^{+}-\hat{g}_{\epsilon}^{-}\right\|_{L^{2}(M d v)}-\left|\hat{n}_{\epsilon}\right| \rightarrow 0$ in $L_{\mathrm{loc}}^{p}(d t d x)$, for any $1 \leq p<2$.

Furthermore, let $n \in L^{\infty}\left(d t ; L^{2}(d x)\right)$ be a limit point of $\hat{n}_{\epsilon}$ and, according to Lemma 5.14, let $H \in L_{\text {loc }}^{1}\left(d t d x ; L^{1}((1+|v|) M d v)\right)$ and $\hat{H} \in L_{\text {loc }}^{2}\left(d t d x ; L^{2}(M d v)\right)$ be limit points of $\frac{h_{\epsilon}}{1+\left\|\hat{g}_{\epsilon}^{+}-\hat{g}_{\epsilon}^{-}\right\|_{L^{2}(M d v)}}$ and $\frac{\hat{h}_{\epsilon}}{1+\left\|\hat{g}_{\epsilon}^{+}-\hat{g}_{\epsilon}^{-}\right\|_{L^{2}(M d v)}}$, respectively.

Then, there exist $h \in L_{\mathrm{loc}}^{1}\left(d t d x ; L^{1}\left(\left(1+|v|^{2}\right) M d v\right)\right)$ and $\hat{h} \in L_{\mathrm{loc}}^{1}\left(d t d x ; L^{2}(M d v)\right)$ such that

$$
H=\frac{h}{1+|n|} \quad \text { and } \quad \hat{H}=\frac{\hat{h}}{1+|n|} .
$$

Proof. It is readily seen from Lemma 7.10, that the family $\hat{n}_{\epsilon}$ is locally relatively compact in $x$ in $L_{\text {loc }}^{p}(d t d x)$. Therefore, according to the decomposition (5.5), so is $n_{\epsilon}$ in $L_{\mathrm{loc}}^{1}(d t d x)$.

Furthermore, taking the divergence of Ampère's equation in 4.34 yields the continuity equation

$$
\partial_{t} n_{\epsilon}+\operatorname{div} j_{\epsilon}=0
$$

which, since $j_{\epsilon}$ is uniformly bounded in $L_{\text {loc }}^{1}(d t d x)$ by Lemma 5.13 yields some temporal regularity on $n_{\epsilon}$ allowing us to establish, invoking a classical compactness result by Aubin and Lions [8, 50. (see also [71] for a sharp compactness criterion), that the family $n_{\epsilon}$ is strongly relatively compact in all variables in $L_{\text {loc }}^{1}(d t d x)$. Employing the decomposition (5.5), again, we deduce that $\hat{n}_{\epsilon}$ is strongly relatively compact in all variables in $L_{\mathrm{loc}}^{1}(d t d x)$.

Then, by virtue of the uniform bounds on $\hat{g}_{\epsilon}^{ \pm}$from Lemma 5.2, which clearly imply that $\hat{n}_{\epsilon}$ is uniformly bounded in $L^{\infty}\left(d t ; L^{2}(d x)\right)$, we conclude, by interpolation, that $\hat{n}_{\epsilon}$ is strongly relatively compact in all variables in $L_{\text {loc }}^{p}(d t d x)$, for any $1 \leq p<2$.

Finally, we decompose

$$
\hat{g}_{\epsilon}^{+}-\hat{g}_{\epsilon}^{-}=\left(\hat{g}_{\epsilon}^{+}-\hat{g}_{\epsilon}^{-}-\hat{n}_{\epsilon}\right)+\hat{n}_{\epsilon}=\epsilon \hat{h}_{\epsilon}+\hat{n}_{\epsilon},
$$

to deduce, using the bound (5.14) on $\hat{h}_{\epsilon}$ from Lemma 5.11 that $\hat{g}_{\epsilon}^{+}-\hat{g}_{\epsilon}^{-}$is relatively strongly compact in $L_{\text {loc }}^{1}\left(d t d x ; L^{2}(M d v)\right)$ and that

$$
\left\|\hat{g}_{\epsilon}^{+}-\hat{g}_{\epsilon}^{-}\right\|_{L^{2}(M d v)}-\left|\hat{n}_{\epsilon}\right| \rightarrow 0 \quad \text { in } L_{\mathrm{loc}}^{1}(d t d x) .
$$

Then, again, by virtue of the uniform bounds on $\hat{g}_{\epsilon}^{ \pm}$from Lemma 5.2 , which clearly imply that $\left\|\hat{g}_{\epsilon}^{+}-\hat{g}_{\epsilon}^{-}\right\|_{L^{2}(M d v)}$ is uniformly bounded in $L^{\infty}\left(d t ; L^{2}(d x)\right)$, we conclude, 
by interpolation, that $\hat{g}_{\epsilon}^{+}-\hat{g}_{\epsilon}^{-}$is strongly relatively compact in all variables in $L_{\text {loc }}^{p}\left(d t d x ; L^{2}(M d v)\right)$ and that

$$
\left\|\hat{g}_{\epsilon}^{+}-\hat{g}_{\epsilon}^{-}\right\|_{L^{2}(M d v)}-\left|\hat{n}_{\epsilon}\right| \rightarrow 0 \quad \text { in } L_{\text {loc }}^{p}(d t d x),
$$

for any $1 \leq p<2$.

There only remains to characterize the weak limits of $\frac{h_{\epsilon}}{1+\left\|\hat{g}_{\epsilon}^{+}-\hat{g}_{\epsilon}^{-}\right\|_{L^{2}(M d v)}}$ and $\frac{\hat{h}_{\epsilon}}{1+\left\|\hat{g}_{\epsilon}^{+}-\hat{g}_{\epsilon}^{-}\right\|_{L^{2}(M d v)}}$. To this end, we first assume, up to extraction of subsequences, that $\left\|\hat{g}_{\epsilon}^{+}-\hat{g}_{\epsilon}^{-}\right\|_{L^{2}(M d v)}$ converges almost everywhere to $|n|$. Therefore, by the weak compactness results from Lemma 5.14 and the Product Limit Theorem (see 9 , Appendix B] and [68, Appendix A]), we obtain that, for every $\lambda>0$,

$$
\begin{aligned}
\frac{h_{\epsilon}}{1+\lambda\left\|\hat{g}_{\epsilon}^{+}-\hat{g}_{\epsilon}^{-}\right\|_{L^{2}(M d v)}} & =\frac{1+\left\|\hat{g}_{\epsilon}^{+}-\hat{g}_{\epsilon}^{-}\right\|_{L^{2}(M d v)}}{1+\lambda\left\|\hat{g}_{\epsilon}^{+}-\hat{g}_{\epsilon}^{-}\right\|_{L^{2}(M d v)}} \frac{h_{\epsilon}}{1+\left\|\hat{g}_{\epsilon}^{+}-\hat{g}_{\epsilon}^{-}\right\|_{L^{2}(M d v)}} \\
& \rightarrow \frac{1+|n|}{1+\lambda|n|} H \quad \text { in } w-L_{\mathrm{loc}}^{1}\left(d t d x ; w-L^{1}((1+|v|) M d v)\right),
\end{aligned}
$$

and, similarly,

$$
\begin{aligned}
\frac{\hat{h}_{\epsilon}}{1+\lambda\left\|\hat{g}_{\epsilon}^{+}-\hat{g}_{\epsilon}^{-}\right\|_{L^{2}(M d v)}} & =\frac{1+\left\|\hat{g}_{\epsilon}^{+}-\hat{g}_{\epsilon}^{-}\right\|_{L^{2}(M d v)}}{1+\lambda\left\|\hat{g}_{\epsilon}^{+}-\hat{g}_{\epsilon}^{-}\right\|_{L^{2}(M d v)}} \frac{\hat{h}_{\epsilon}}{1+\left\|\hat{g}_{\epsilon}^{+}-\hat{g}_{\epsilon}^{-}\right\|_{L^{2}(M d v)}} \\
& \rightarrow \frac{1+|n|}{1+\lambda|n|} \hat{H} \quad \text { in } w-L_{\text {loc }}^{2}\left(d t d x ; w-L^{2}(M d v)\right) .
\end{aligned}
$$

Therefore, for any $\varphi(v) \in C_{c}^{\infty}\left(\mathbb{R}^{3}\right)$ such that $|\varphi(v)| \leq\left(1+|v|^{2}\right)$, we find

$$
\left\|\frac{1+|n|}{1+\lambda|n|} H \varphi\right\|_{L_{\mathrm{loc}}^{1}\left(d t d x ; L^{1}(M d v)\right)} \leq \liminf _{\epsilon \rightarrow 0}\left\|h_{\epsilon}\right\|_{L_{\mathrm{loc}}^{1}\left(d t d x ; L^{1}\left(\left(1+|v|^{2}\right) M d v\right)\right)},
$$

and

$$
\left\|\frac{1+|n|}{1+\lambda|n|} \hat{H}\right\|_{L_{\mathrm{loc}}^{1}\left(d t d x ; L^{2}(M d v)\right)} \leq \liminf _{\epsilon \rightarrow 0}\left\|\hat{h}_{\epsilon}\right\|_{L_{\mathrm{loc}}^{1}\left(d t d x ; L^{2}(M d v)\right)},
$$

so that, in view of the bound on $h_{\epsilon}$ from Lemma 5.13 and the bound on $\hat{h}_{\epsilon}$ from Lemma 5.11 and by the arbitrariness of $\lambda$ and $\varphi$, we conclude

$$
(1+|n|) H \in L_{\mathrm{loc}}^{1}\left(d t d x ; L^{1}\left(\left(1+|v|^{2}\right) M d v\right)\right),
$$

and

$$
(1+|n|) \hat{H} \in L_{\text {loc }}^{1}\left(d t d x ; L^{2}(M d v)\right) .
$$

The justification of the lemma is complete. 


\section{CHAPTER 8}

\section{Higher order and nonlinear constraint equations}

In Chapter 6, using weak compactness methods, we have derived lower order linear macroscopic constraint equations for one species and for two species in a weak interactions regime. For the one species case considered in Theorem 4.4 . this is sufficient to obtain all constraint equations contained in the limiting system 4.30. As for the two species case considered in Theorems 4.5 and 4.6, the corresponding limiting systems (4.38) and 4.37), respectively, contain higher order constraint equations (appearing as singular perturbations of the equations of motion) and nonlinear constraint equations, namely the (solenoidal) Ohm's law and the internal electric energy constraint, which cannot be deduced solely from the weak compactness bounds established in Chapter 5. We address now these singular limits employing the strong compactness bounds obtained in Chapter 7 .

\subsection{Macroscopic constraint equations for two species, weak interactions}

As seen in Section 6.2 (see 6.10) in the proof of Proposition 6.2), it is possible to derive limiting kinetic equations of the type

$$
v \cdot \nabla_{x} g^{ \pm}=\int_{\mathbb{R}^{3} \times \mathbb{S}^{2}} q^{ \pm} M_{*} d v_{*} d \sigma
$$

from 4.34 when $\delta=o(1)$.

What we intend to do next is to take advantage of the symmetries of the collision integrands $q^{ \pm}$and $q^{ \pm, \mp}$ and of the strong compactness bounds from Chapter 7 to go one order further and, thus, to derive a singular limit in the regime considered in Theorem 4.5. This singular limit is precisely the content of Proposition 8.1. which will eventually yield the solenoidal Ohm's law and internal electric energy constraint from 4.38 in Proposition 8.2 below. Of course, since we are considering renormalized fluctuations, we do not expect that the integrals in $v$ of the right-hand sides of the Vlasov-Boltzmann equations in (4.34) against collision invariants are zero, but they should converge to zero as $\epsilon \rightarrow 0$ provided that we choose some appropriate renormalization which is sufficiently close to the identity. To estimate the ensuing conservation defects, we will also need to truncate large velocities.

Note that, even if conservation laws were known to hold for renormalized solutions of (4.34), we would have to introduce similar truncations of large tails and large velocities in order to control uniformly the flux and acceleration terms.

The main result in this section concerning the derivation of higher order nonlinear constraint equations in the regime considered in Theorem 4.5 is contained in the following proposition.

Proposition 8.1. Let $\left(f_{\epsilon}^{ \pm}, E_{\epsilon}, B_{\epsilon}\right)$ be the sequence of renormalized solutions to the scaled two species Vlasov-Maxwell-Boltzmann system (4.34) considered in Theorem 4.5 for weak interspecies interactions, i.e. $\delta=o(1)$ and $\frac{\delta}{\epsilon}$ unbounded. In 
accordance with Lemmas 5.1, 5.2 and 5.3, denote by

$$
\begin{gathered}
g^{ \pm} \in L^{\infty}\left(d t ; L^{2}(M d x d v)\right), \quad q^{ \pm, \mp} \in L^{2}\left(M M_{*} d t d x d v d v_{*} d \sigma\right), \\
\text { and } E, B \in L^{\infty}\left(d t ; L^{2}(d x)\right),
\end{gathered}
$$

any joint limit points of the families $\hat{g}_{\epsilon}^{ \pm}$and $\hat{q}_{\epsilon}^{ \pm, \mp}$ defined by (5.3) and (5.6), $E_{\epsilon}$ and $B_{\epsilon}$, respectively.

Then, one has

$$
\begin{aligned}
\pm \int_{\mathbb{R}^{3} \times \mathbb{R}^{3} \times \mathbb{S}^{2}} q^{ \pm, \mp} v M M_{*} d v d v_{*} d \sigma & =\nabla_{x} \bar{p}-(E+u \wedge B) \\
\int_{\mathbb{R}^{3} \times \mathbb{R}^{3} \times \mathbb{S}^{2}} q^{ \pm, \mp}\left(\frac{|v|^{2}}{2}-\frac{5}{2}\right) M M_{*} d v d v_{*} d \sigma & =0
\end{aligned}
$$

where $u$ is the bulk velocity associated with the limiting fluctuations $g^{ \pm}$and $\bar{p} \in$ $L_{\mathrm{loc}}^{1}(d t d x)$ is a pressure.

The proof of Proposition 8.1 is lengthy and contains several steps. Therefore, for the sake of clarity, it is deferred to Section 8.1.1, below.

As a direct consequence of the previous proposition, we derive in the next result the solenoidal Ohm's law and the internal electric energy constraint from 4.38).

Proposition 8.2. Let $\left(f_{\epsilon}^{ \pm}, E_{\epsilon}, B_{\epsilon}\right)$ be the sequence of renormalized solutions to the scaled two species Vlasov-Maxwell-Boltzmann system (4.34) considered in Theorem 4.5 for weak interspecies interactions, i.e. $\delta=o(1)$ and $\frac{\delta}{\epsilon}$ unbounded. In accordance with Lemmas 5.1, 5.2. 5.13 and 5.14 denote by

$$
\begin{gathered}
g^{ \pm} \in L^{\infty}\left(d t ; L^{2}(M d x d v)\right), \quad h \in L_{\mathrm{loc}}^{1}\left(d t d x ; L^{1}\left(\left(1+|v|^{2}\right) M d v\right)\right), \\
\text { and } E, B \in L^{\infty}\left(d t ; L^{2}(d x)\right),
\end{gathered}
$$

any joint limit points of the families $\hat{g}_{\epsilon}^{ \pm}$and $h_{\epsilon}$ defined by (5.3) and $(5.29), E_{\epsilon}$ and $B_{\epsilon}$, respectively.

Then, one has

$$
j=\sigma\left(-\nabla_{x} \bar{p}+E+u \wedge B\right) \quad \text { and } \quad w=0,
$$

where $u$ is the bulk velocity associated with the limiting fluctuations $g^{ \pm}, j$ and $w$ are, respectively, the electric current and the internal electric energy associated with the limiting fluctuation $h, \bar{p} \in L_{\mathrm{loc}}^{1}(d t d x)$ is a pressure and the electric conductivity $\sigma>0$ is defined by 2.70 .

Proof. By Proposition 8.1, we have that

$$
\begin{aligned}
\pm \int_{\mathbb{R}^{3} \times \mathbb{R}^{3} \times \mathbb{S}^{2}} q^{ \pm, \mp} v M M_{*} d v d v_{*} d \sigma & =\nabla_{x} \bar{p}-(E+u \wedge B), \\
\int_{\mathbb{R}^{3} \times \mathbb{R}^{3} \times \mathbb{S}^{2}} q^{ \pm, \mp}\left(\frac{|v|^{2}}{2}-\frac{5}{2}\right) M M_{*} d v d v_{*} d \sigma & =0 .
\end{aligned}
$$

Then, further incorporating identity 6.11 from Proposition 6.3 into the above relations yields that

$$
\begin{gathered}
E+u \wedge B-\frac{1}{2} \int_{\mathbb{R}^{3}} j \cdot \mathfrak{L}(v) v M d v-\frac{1}{2} \int_{\mathbb{R}^{3}} w \mathfrak{L}\left(\frac{|v|^{2}}{2}\right) v M d v=\nabla_{x} \bar{p}, \\
\int_{\mathbb{R}^{3}} j \cdot \mathfrak{L}(v)\left(\frac{|v|^{2}}{2}-\frac{5}{2}\right) M d v+\int_{\mathbb{R}^{3}} w \mathfrak{L}\left(\frac{|v|^{2}}{2}\right)\left(\frac{|v|^{2}}{2}-\frac{5}{2}\right) M d v=0 .
\end{gathered}
$$

Finally, since, by symmetry considerations, $\int_{\mathbb{R}^{3}} \mathfrak{L}(v)\left(\frac{|v|^{2}}{2}-\frac{5}{2}\right) M d v=\int_{\mathbb{R}^{3}} \mathfrak{L}\left(\frac{|v|^{2}}{2}\right) v M d v=$ 0 and $\int_{\mathbb{R}^{3}} \mathfrak{L}\left(v_{i}\right) v_{j} M d v=0$, if $i \neq j$, we compute that

$$
E+u \wedge B-\frac{1}{\sigma} j=\nabla_{x} \bar{p} \quad \text { and } \quad \frac{1}{\lambda} w=0,
$$


where $\sigma>0$ and $\lambda>0$ are defined in 2.70) and 2.71), respectively, which concludes the proof of the proposition.

8.1.1. Proof of Proposition 8.1. Here, we analyze the equations 4.36), which have to be renormalized, at a higher order. This becomes more complicated than the previous asymptotic analysis of $\sqrt{6.9}$ in the proof of Proposition 6.2 . because we do not have enough strong compactness to take limits in the nonlinear terms

$$
\pm\left(v \wedge B_{\epsilon}\right) \cdot \nabla_{v} \frac{\sqrt{G_{\epsilon}^{ \pm}+\epsilon^{a}}-1}{\epsilon},
$$

therein. More precisely, we are not able to control the concentrations of $\left|\hat{g}_{\epsilon}^{ \pm}\right|^{2}$ (see Lemma 7.10.

We will therefore consider a stronger renormalization of the equation for the fluctuations of density and exploit the equi-integrability from Lemma 7.10 , however weak it may be.

8.1.1.1. An admissible renormalization. We introduce the admissible renormalization $\Gamma_{\lambda}(z)$ defined by

$$
\Gamma_{\lambda}(z)-1=(z-1) \gamma\left(\lambda \delta \frac{z-1}{\epsilon}\right)
$$

where $\frac{\epsilon}{\delta} \leq \lambda \leq 1$ is small and $\gamma \in C^{1}(\mathbb{R})$ satisfies that

$$
\mathbb{1}_{[-1,1]}(z) \leq \gamma(z) \leq \mathbb{1}_{[-2,2]}(z), \quad \text { for all } z \in \mathbb{R} .
$$

Without distinguishing, for simplicity, the notation for cations and anions, we denote $\gamma_{\epsilon}^{\lambda}$ for $\gamma\left(\lambda \delta g_{\epsilon}^{ \pm}\right)$and $\hat{\gamma}_{\epsilon}^{\lambda}$ for $\Gamma_{\lambda}^{\prime}\left(G_{\epsilon}^{ \pm}\right)$. Thus, renormalizing the VlasovBoltzmann equation from 4.34 with respect to $\Gamma_{\lambda}(z)$ yields

$$
\begin{aligned}
\left(\frac{\epsilon}{\delta} \partial_{t}+\frac{1}{\delta} v \cdot \nabla_{x} \pm\left(\epsilon E_{\epsilon}+v \wedge B_{\epsilon}\right) \cdot \nabla_{v}\right) & g_{\epsilon}^{ \pm} \gamma_{\epsilon}^{\lambda} \mp E_{\epsilon} \cdot v G_{\epsilon}^{ \pm} \hat{\gamma}_{\epsilon}^{\lambda} \\
& =\frac{1}{\delta \epsilon^{2}} \hat{\gamma}_{\epsilon}^{\lambda} \mathcal{Q}\left(G_{\epsilon}^{ \pm}, G_{\epsilon}^{ \pm}\right)+\frac{\delta}{\epsilon^{2}} \hat{\gamma}_{\epsilon}^{\lambda} \mathcal{Q}\left(G_{\epsilon}^{ \pm}, G_{\epsilon}^{\mp}\right)
\end{aligned}
$$

Notice here that there are two singular terms in the equations above, namely $\frac{1}{\delta} v \cdot \nabla_{x} g_{\epsilon}^{ \pm} \gamma_{\epsilon}^{\lambda}$ and the first term in the right-hand side (as shown below, the second term in the right-hand side is not singular). Therefore, in order to annihilate asymptotically these singular expressions, we integrate now the above equations against $\varphi(v) \chi\left(\frac{|v|^{2}}{K_{\delta}}\right) M d v$, with $K_{\delta}=K|\log \delta|$, for some large $K>0$ to be fixed later on, for any collision invariant $\varphi(v)$ and some smooth compactly supported truncation $\chi \in C_{c}^{\infty}([0, \infty))$ satisfying $\mathbb{1}_{[0,1]} \leq \chi \leq \mathbb{1}_{[0,2]}$, which leads to

$$
\begin{aligned}
\frac{\epsilon}{\delta} \partial_{t} \int_{\mathbb{R}^{3}} g_{\epsilon}^{ \pm} \gamma_{\epsilon}^{\lambda} \varphi \chi\left(\frac{|v|^{2}}{K_{\delta}}\right) M d v+\frac{1}{\delta} \nabla_{x} \cdot \int_{\mathbb{R}^{3}} g_{\epsilon}^{ \pm} \gamma_{\epsilon}^{\lambda} \varphi \chi\left(\frac{|v|^{2}}{K_{\delta}}\right) v M d v \\
\mp \int_{\mathbb{R}^{3}} g_{\epsilon}^{ \pm} \gamma_{\epsilon}^{\lambda}\left(\epsilon E_{\epsilon}+v \wedge B_{\epsilon}\right) \cdot \nabla_{v}\left(\varphi \chi\left(\frac{|v|^{2}}{K_{\delta}}\right) M\right) d v \\
\mp E_{\epsilon} \cdot \int_{\mathbb{R}^{3}}\left(1+\epsilon g_{\epsilon}^{ \pm}\right) \hat{\gamma}_{\epsilon}^{\lambda} \varphi \chi\left(\frac{|v|^{2}}{K_{\delta}}\right) v M d v \\
=\frac{1}{\delta \epsilon^{2}} \int_{\mathbb{R}^{3}} \hat{\gamma}_{\epsilon}^{\lambda} \mathcal{Q}\left(G_{\epsilon}^{ \pm}, G_{\epsilon}^{ \pm}\right) \varphi \chi\left(\frac{|v|^{2}}{K_{\delta}}\right) M d v \\
+\frac{\delta}{\epsilon^{2}} \int_{\mathbb{R}^{3}} \hat{\gamma}_{\epsilon}^{\lambda} \mathcal{Q}\left(G_{\epsilon}^{ \pm}, G_{\epsilon}^{\mp}\right) \varphi \chi\left(\frac{|v|^{2}}{K_{\delta}}\right) M d v
\end{aligned}
$$


8.1.1.2. Convergence of collision integrals. Let us focus on the right-hand side of 8.3 first. One has

$$
\begin{aligned}
\frac{\delta}{\epsilon^{2}} \int_{\mathbb{R}^{3}} \hat{\gamma}_{\epsilon}^{\lambda} \mathcal{Q} & \left(G_{\epsilon}^{ \pm}, G_{\epsilon}^{\mp}\right) \varphi \chi\left(\frac{|v|^{2}}{K_{\delta}}\right) M d v \\
& =\int_{\mathbb{R}^{3} \times \mathbb{R}^{3} \times \mathbb{S}^{2}} \hat{\gamma}_{\epsilon}^{\lambda} \sqrt{G_{\epsilon}^{ \pm} G_{\epsilon}^{\mp}} \hat{q}_{\epsilon}^{ \pm, \mp} \varphi \chi\left(\frac{|v|^{2}}{K_{\delta}}\right) M M_{*} d v d v_{*} d \sigma \\
& +\frac{\epsilon^{2}}{4 \delta} \int_{\mathbb{R}^{3} \times \mathbb{R}^{3} \times \mathbb{S}^{2}} \hat{\gamma}_{\epsilon}^{\lambda}\left(\hat{q}_{\epsilon}^{ \pm, \mp}\right)^{2} \varphi \chi\left(\frac{|v|^{2}}{K_{\delta}}\right) M M_{*} d v d v_{*} d \sigma .
\end{aligned}
$$

Since $\frac{\epsilon}{\delta}$ vanishes, $\varphi \chi\left(\frac{|v|^{2}}{K_{\delta}}\right)$ is bounded pointwise by a constant multiple of $|\log \delta|$ and the collision integrands $\hat{q}_{\epsilon}^{ \pm, \mp}$ are uniformly bounded in $L^{2}\left(M M_{*} d t d x d v d v_{*} d \sigma\right)$, according to Lemma 5.3, we find that the second term from the right-hand side of 8.4 vanishes in $L^{1}(d t d x)$. Further utilizing that, thanks to Lemma 5.2 .

$$
\begin{aligned}
& \sqrt{G_{\epsilon}^{ \pm}}=1+O(\epsilon)_{L_{\mathrm{loc}}^{2}\left(d t ; L^{2}(M d x d v)\right)}, \\
& \sqrt{G_{\epsilon *}^{ \pm}}=1+O(\epsilon)_{L_{\mathrm{loc}}^{2}\left(d t ; L^{2}\left(M_{*} d x d v_{*}\right)\right)},
\end{aligned}
$$

and that $\hat{\gamma}_{\epsilon}^{\lambda} \sqrt{G_{\epsilon}^{ \pm}}$is uniformly bounded pointwise, it is readily seen that the weak limit of the first term of the right-hand side of 8.4 coincides with the weak limit of

$$
\int_{\mathbb{R}^{3} \times \mathbb{R}^{3} \times \mathbb{S}^{2}} \hat{\gamma}_{\epsilon}^{\lambda} \hat{q}_{\epsilon}^{ \pm, \mp} \varphi \chi\left(\frac{|v|^{2}}{K_{\delta}}\right) M M_{*} d v d v_{*} d \sigma
$$

which, since $\hat{\gamma}_{\epsilon}^{\lambda} \varphi \chi\left(\frac{|v|^{2}}{K_{\epsilon}}\right)$ is dominated by $|\varphi|$ and converges almost everywhere to $\varphi$, is easily shown to converge weakly in $L^{2}(d t d x)$ towards

$$
\int_{\mathbb{R}^{3} \times \mathbb{R}^{3} \times \mathbb{S}^{2}} q^{ \pm, \mp} \varphi M M_{*} d v d v_{*} d \sigma
$$

Thus, so far, we have established that the second term in the right-hand side of (8.3) satisfies

$$
\begin{gathered}
\frac{\delta}{\epsilon^{2}} \int_{\mathbb{R}^{3}} \hat{\gamma}_{\epsilon}^{\lambda} \mathcal{Q}\left(G_{\epsilon}^{ \pm}, G_{\epsilon}^{\mp}\right) \varphi \chi\left(\frac{|v|^{2}}{K_{\delta}}\right) M d v \rightarrow \int_{\mathbb{R}^{3} \times \mathbb{R}^{3} \times \mathbb{S}^{2}} q^{ \pm, \mp} \varphi M M_{*} d v d v_{*} d \sigma \\
\operatorname{in~} L_{\mathrm{loc}}^{1}(d t d x) .
\end{gathered}
$$

The first term of the right-hand side of 8.3 is more singular and, therefore, harder to control. One has, in this case, taking advantage of collisional symmetries, 
that

$$
\begin{aligned}
\frac{1}{\delta \epsilon^{2}} \int_{\mathbb{R}^{3}} \hat{\gamma}_{\epsilon}^{\lambda} \mathcal{Q} & \left(G_{\epsilon}^{ \pm}, G_{\epsilon}^{ \pm}\right) \varphi \chi\left(\frac{|v|^{2}}{K_{\delta}}\right) M d v \\
& =\frac{\epsilon^{2}}{4 \delta} \int_{\mathbb{R}^{3} \times \mathbb{R}^{3} \times \mathbb{S}^{2}} \hat{\gamma}_{\epsilon}^{\lambda} \varphi \chi\left(\frac{|v|^{2}}{K_{\delta}}\right)\left(\hat{q}_{\epsilon}^{ \pm}\right)^{2} M M_{*} d v d v_{*} d \sigma \\
& -\frac{1}{\delta} \int_{\mathbb{R}^{3} \times \mathbb{R}^{3} \times \mathbb{S}^{2}} \hat{\gamma}_{\epsilon}^{\lambda} \varphi\left(1-\chi\left(\frac{|v|^{2}}{K_{\delta}}\right)\right) \hat{q}_{\epsilon}^{ \pm} \sqrt{G_{\epsilon}^{ \pm} G_{\epsilon *}^{ \pm}} M M_{*} d v d v_{*} d \sigma \\
& +\frac{1}{\delta} \int_{\mathbb{R}^{3} \times \mathbb{R}^{3} \times \mathbb{S}^{2}} \hat{\gamma}_{\epsilon}^{\lambda}\left(1-\hat{\gamma}_{\epsilon *}^{\lambda}\right) \varphi \hat{q}_{\epsilon}^{ \pm} \sqrt{G_{\epsilon}^{ \pm} G_{\epsilon *}^{ \pm}} M M_{*} d v d v_{*} d \sigma \\
& +\frac{1}{\delta} \int_{\mathbb{R}^{3} \times \mathbb{R}^{3} \times \mathbb{S}^{2}} \hat{\gamma}_{\epsilon}^{\lambda} \hat{\gamma}_{\epsilon *}^{\lambda}\left(1-\hat{\gamma}_{\epsilon}^{\lambda \prime} \hat{\gamma}_{\epsilon *}^{\lambda \prime}\right) \varphi \hat{q}_{\epsilon}^{ \pm} \sqrt{G_{\epsilon}^{ \pm} G_{\epsilon *}^{ \pm}} M M_{*} d v d v_{*} d \sigma \\
& -\frac{\epsilon^{2}}{4 \delta} \int_{\mathbb{R}^{3} \times \mathbb{R}^{3} \times \mathbb{S}^{2}} \hat{\gamma}_{\epsilon}^{\lambda} \hat{\gamma}_{\epsilon *}^{\lambda} \hat{\gamma}_{\epsilon}^{\lambda \prime} \hat{\gamma}_{\epsilon *}^{\lambda \prime} \varphi\left(\hat{q}_{\epsilon}^{ \pm}\right)^{2} M M_{*} d v d v_{*} d \sigma \\
& \text { def } D_{\epsilon}^{1}(\varphi)+D_{\epsilon}^{2}(\varphi)+D_{\epsilon}^{3}(\varphi)+D_{\epsilon}^{4}(\varphi)+D_{\epsilon}^{5}(\varphi),
\end{aligned}
$$

where we have used that $\varphi$ is a collision invariant to symmetrize the last term.

Now, we control each term $D_{\epsilon}^{i}(\varphi), i=1, \ldots, 5$, separately.

- The vanishing of the first term $D_{\epsilon}^{1}(\varphi)$, for any function $\varphi(v)$ growing at most quadratically at infinity, easily follows, using Lemma 5.3, from the estimate

$$
\begin{aligned}
\left\|D_{\epsilon}^{1}(\varphi)\right\|_{L^{1}(d t d x)} & \leq \frac{\epsilon^{2}}{4 \delta}\left\|\hat{q}_{\epsilon}^{ \pm}\right\|_{L^{2}\left(M M_{*} d t d x d v d v_{*} d \sigma\right)}^{2}\left\|\hat{\gamma}_{\epsilon}^{\lambda}\right\|_{L^{\infty}}\left\|\chi\left(\frac{|v|^{2}}{K_{\delta}}\right) \varphi\right\|_{L^{\infty}} \\
& \leq C \frac{\epsilon^{2}}{\delta} K_{\delta}=C K \frac{\epsilon^{2}}{\delta}|\log \delta| .
\end{aligned}
$$

- The second term $D_{\epsilon}^{2}(\varphi)$ is controlled by the following estimate on the tails of Gaussian distributions : for any $p \in \mathbb{R}$, as $R \rightarrow \infty$,

$$
\int_{\left\{|v|^{2}>R\right\}}|v|^{p} M(v) d v \sim \sqrt{\frac{2}{\pi}} R^{\frac{p+1}{2}} e^{-\frac{R}{2}},
$$

in the sense that the quotient of both sides converges to 1 as $R \rightarrow \infty$, which is easily established by applying Bernoulli-l'Hospital's rule.

We have indeed

$$
\begin{aligned}
& \left|D_{\epsilon}^{2}(\varphi)\right| \\
& \leq \frac{1}{\delta}\left\|\hat{q}_{\epsilon}^{ \pm}\right\|_{L^{2}\left(M M_{*} d v d v_{*} d \sigma\right)}\left\|\hat{\gamma}_{\epsilon}^{\lambda} \varphi \mathbb{1}_{\left\{|v|^{2} \geq K_{\delta}\right\}} \sqrt{G_{\epsilon}^{ \pm} G_{\epsilon *}^{ \pm}}\right\|_{L^{2}\left(M M_{*} d v d v_{*} d \sigma\right)} \\
& \leq \frac{C}{\delta}\left\|\hat{q}_{\epsilon}^{ \pm}\right\|_{L^{2}\left(M M_{*} d v d v_{*} d \sigma\right)}\left\|\hat{\gamma}_{\epsilon}^{\lambda} \sqrt{G_{\epsilon}^{ \pm}}\right\|_{L^{\infty}}\left\|\sqrt{G_{\epsilon}^{ \pm}}\right\|_{L^{2}(M d v)}\left\|\mathbb{1}_{\left\{|v|^{2} \geq K_{\delta}\right\}} \varphi\right\|_{L^{2}(M d v)} .
\end{aligned}
$$

Thus, using the bound from Lemma 5.3 , the pointwise boundedness of $\Gamma_{\lambda}^{\prime}(z) \sqrt{z}$ and the Gaussian decay estimate 8.8 , we get, for all $\varphi(v)$ growing at most quadratically at infinity,

$$
D_{\epsilon}^{2}(\varphi)=O\left(\delta^{\frac{K}{4}-1}|\log \delta|^{\frac{5}{4}}\right)_{L_{\mathrm{loc}}^{1}(d t d x)}
$$

which tends to zero as soon as $K>4$. 
- The last term $D_{\epsilon}^{5}(\varphi)$ is mastered using the same tools. For high energies, i.e. when $|v|^{2} \geq K|\log \delta|$, we obtain

$$
\begin{aligned}
& D_{\epsilon}^{5>}(\varphi) \\
& \stackrel{\text { def }}{=} \frac{\epsilon^{2}}{4 \delta} \int_{\mathbb{R}^{3} \times \mathbb{R}^{3} \times \mathbb{S}^{2}} \hat{\gamma}_{\epsilon}^{\lambda} \hat{\gamma}_{\epsilon *}^{\lambda} \hat{\gamma}_{\epsilon}^{\lambda \prime} \hat{\gamma}_{\epsilon *}^{\lambda \prime} \varphi \mathbb{1}_{\left\{|v|^{2} \geq K_{\delta}\right\}}\left(\hat{q}_{\epsilon}^{ \pm}\right)^{2} M M_{*} d v d v_{*} d \sigma \\
& \leq \frac{1}{\delta}\left\|\hat{\gamma}_{\epsilon}^{\lambda} \sqrt{G_{\epsilon}^{ \pm}}\right\|_{L^{\infty}}^{2}\left\|\hat{\gamma}_{\epsilon}^{\lambda}\right\|_{L^{\infty}}^{2}\left\|\hat{q}_{\epsilon}^{ \pm}\right\|_{L^{2}\left(M M_{*} d v d v_{*} d \sigma\right)}\left\|\varphi \mathbb{1}_{\left\{|v|^{2} \geq K_{\delta}\right\}}\right\|_{L^{2}\left(M M_{*} d v d v_{*} d \sigma\right)},
\end{aligned}
$$

so that, using the estimate (8.8) on the tails of Gaussian distributions and the bound on $\hat{q}_{\epsilon}^{ \pm}$from Lemma 5.3

$$
D_{\epsilon}^{5>}(\varphi)=O\left(\delta^{\frac{K}{4}-1}|\log \delta|^{\frac{5}{4}}\right)_{L^{2}(d t d x)}
$$

which tends to zero as soon as $K>4$.

For moderate energies, i.e. when $|v|^{2}<K|\log \delta|$, we easily find

$$
\begin{aligned}
D_{\epsilon}^{5<}(\varphi) & \stackrel{\text { def }}{=} \frac{\epsilon^{2}}{4 \delta} \int_{\mathbb{R}^{3} \times \mathbb{R}^{3} \times \mathbb{S}^{2}} \hat{\gamma}_{\epsilon}^{\lambda} \hat{\gamma}_{\epsilon *}^{\lambda} \hat{\gamma}_{\epsilon}^{\lambda \prime} \hat{\gamma}_{\epsilon *}^{\lambda \prime} \varphi \mathbb{1}_{\left\{|v|^{2}<K_{\delta}\right\}}\left(\hat{q}_{\epsilon}^{ \pm}\right)^{2} M M_{*} d v d v_{*} d \sigma \\
& \leq C K \frac{\epsilon^{2}}{\delta}|\log \delta|\left\|\hat{q}_{\epsilon}^{ \pm}\right\|_{L^{2}\left(M M_{*} d v d v_{*} d \sigma\right)}^{2},
\end{aligned}
$$

so that the entropy dissipation bound from Lemma 5.3 provides

$$
D_{\epsilon}^{5<}(\varphi)=O\left(\frac{\epsilon^{2}}{\delta}|\log \delta|\right)_{L^{1}(d t d x)} .
$$

- The handling of $D_{\epsilon}^{3}(\varphi)$ accounts for the introduction of the small parameter $\lambda$. First, one has, by the Cauchy-Schwarz inequality,

$$
\begin{aligned}
& \left|D_{\epsilon}^{3}(\varphi)\right| \\
& \leq \frac{1}{\delta}\left\|\hat{q}_{\epsilon}^{ \pm}\right\|_{L^{2}\left(M M_{*} d v d v_{*} d \sigma\right)}\left\|\hat{\gamma}_{\epsilon}^{\lambda}\left(1-\hat{\gamma}_{\epsilon *}^{\lambda}\right) \varphi \sqrt{G_{\epsilon}^{ \pm} G_{\epsilon *}^{ \pm}}\right\|_{L^{2}\left(M M_{*} d v d v_{*} d \sigma\right)} \\
& \leq C\left\|\hat{q}_{\epsilon}^{ \pm}\right\|_{L^{2}\left(M M_{*} d v d v_{*} d \sigma\right)}\left\|\hat{\gamma}_{\epsilon}^{\lambda} \sqrt{G_{\epsilon}^{ \pm}}\right\|_{L^{\infty}}\left\|\frac{1}{\delta}\left(1-\hat{\gamma}_{\epsilon}^{\lambda}\right) \sqrt{G_{\epsilon}^{ \pm}}\right\|_{L^{2}(M d v)}\|\varphi\|_{L^{2}(M d v)}\|\varphi\|_{L^{2}(M d v)}\left\|\hat{q}_{\epsilon}\right\|_{L^{2}\left(M M_{*} d v d v_{*} d \sigma\right)}\left\|\hat{\gamma}_{\epsilon}^{\lambda} \sqrt{G_{\epsilon}^{ \pm}}\right\|_{L^{\infty}}\left\|\frac{1}{\delta}\left(1-\hat{\gamma}_{\epsilon}^{\lambda}\right)\right\|_{L^{2}(M d v)}\left\|\hat{\gamma}_{\epsilon}^{\lambda} \sqrt{G_{\epsilon}^{ \pm}}\right\|_{L^{\infty}}\left\|\hat{g}_{\epsilon}^{ \pm}\right\|_{L^{2}(M d v)}\|\varphi\|_{L^{2}(M d v)} .
\end{aligned}
$$

Moreover, in view of the hypotheses on $\gamma(z)$, the support of $\Gamma_{\lambda}^{\prime}(z)-1=\gamma\left(\lambda \delta \frac{z-1}{\epsilon}\right)-$ $1+\lambda \delta \frac{z-1}{\epsilon} \gamma^{\prime}\left(\lambda \delta \frac{z-1}{\epsilon}\right)$ is clearly restricted to $\lambda \delta \frac{|z-1|}{\epsilon} \in[1, \infty)$, so that, employing the decomposition (5.5),

$$
\begin{aligned}
\frac{1}{\delta}\left|1-\hat{\gamma}_{\epsilon}^{\lambda}\right| & =\frac{1}{\delta}\left|1-\hat{\gamma}_{\epsilon}^{\lambda}\right| \mathbb{1}_{\left\{\left|\epsilon \hat{g}_{\epsilon}^{ \pm}\right| \leq 1\right\}}+\frac{1}{\delta}\left|1-\hat{\gamma}_{\epsilon}^{\lambda}\right| \mathbb{1}_{\left\{\left|\epsilon \hat{g}_{\epsilon}^{ \pm}\right|>1\right\}} \\
& \leq \lambda\left|1-\hat{\gamma}_{\epsilon}^{\lambda}\right|\left|g_{\epsilon}^{ \pm}\right| \mathbb{1}_{\left\{\left|\epsilon \hat{g}_{\epsilon}^{ \pm}\right| \leq 1\right\}}+\frac{\epsilon}{\delta}\left|1-\hat{\gamma}_{\epsilon}^{\lambda}\right|\left|\hat{g}_{\epsilon}^{ \pm}\right| \mathbb{1}_{\left\{\left|\epsilon \hat{g}_{\epsilon}^{ \pm}\right|>1\right\}} \\
& \leq C \lambda\left|\hat{g}_{\epsilon}^{ \pm}+\frac{\epsilon}{4} \hat{g}_{\epsilon}^{ \pm 2}\right| \mathbb{1}_{\left\{\left|\epsilon \hat{g}_{\epsilon}^{ \pm}\right| \leq 1\right\}}+C \frac{\epsilon}{\delta}\left|\hat{g}_{\epsilon}^{ \pm}\right| \\
& \leq C\left(\lambda+\frac{\epsilon}{\delta}\right)\left|\hat{g}_{\epsilon}^{ \pm}\right| .
\end{aligned}
$$

Therefore, thanks to the bound on $\hat{q}_{\epsilon}$ from Lemma 5.3 , we infer

$$
\left\|D_{\epsilon}^{3}(\varphi)\right\|_{L_{\mathrm{loc}}^{1}(d t d x)} \leq C\left(\lambda+\frac{\epsilon}{\delta}\right)\left\|\hat{g}_{\epsilon}^{ \pm}\right\|_{L_{\mathrm{loc}}^{2}\left(d t d x ; L^{2}(M d v)\right)} .
$$


Thus, we conclude that

$$
\left\|D_{\epsilon}^{3}(\varphi)\right\|_{L_{\mathrm{loc}}^{1}(d t d x)} \leq C \lambda
$$

- A similar argument provides the convergence of the remaining term $D_{\epsilon}^{4}(\varphi)$. Thus, one has by the Cauchy-Schwarz inequality, for any $2<p<\infty$,

$$
\begin{aligned}
& \left|D_{\epsilon}^{4}(\varphi)\right| \\
& \leq \frac{1}{\delta}\left\|\hat{q}_{\epsilon}^{ \pm}\right\|_{L^{2}\left(M M_{*} d v d v_{*} d \sigma\right)}\left\|\hat{\gamma}_{\epsilon}^{\lambda} \hat{\gamma}_{\epsilon *}^{\lambda}\left(1-\hat{\gamma}_{\epsilon}^{\lambda \prime} \hat{\gamma}_{\epsilon *}^{\lambda \prime}\right) \varphi \sqrt{G_{\epsilon}^{ \pm} G_{\epsilon *}^{ \pm}}\right\|_{L^{2}\left(M M_{*} d v d v_{*} d \sigma\right)} \\
& \leq C\left\|\hat{q}_{\epsilon}^{ \pm}\right\|_{L^{2}\left(M M_{*} d v d v_{*} d \sigma\right)}\left\|\hat{\gamma}_{\epsilon}^{\lambda} \sqrt{G_{\epsilon}^{ \pm}}\right\|_{L^{\infty}}^{2}\left\|\frac{1}{\delta}\left(1-\hat{\gamma}_{\epsilon}^{\lambda \prime} \hat{\gamma}_{\epsilon *}^{\lambda \prime}\right) \varphi\right\|_{L^{2}\left(M M_{*} d v d v_{*} d \sigma\right)} \\
& \leq C_{p}\left\|\hat{q}_{\epsilon}^{ \pm}\right\|_{L^{2}\left(M M_{*} d v d v_{*} d \sigma\right)}\left\|\frac{1}{\delta}\left(1-\hat{\gamma}_{\epsilon}^{\lambda}\right)\right\|_{L^{p}(M d v)} .
\end{aligned}
$$

Therefore, thanks to the bound on $\hat{q}_{\epsilon}^{ \pm}$from Lemma 5.3 . we infer, for any $2<p<\infty$,

$$
\left\|D_{\epsilon}^{4}(\varphi)\right\|_{L_{\mathrm{loc}}^{1}(d t d x)} \leq C\left\|\frac{1}{\delta}\left(1-\hat{\gamma}_{\epsilon}^{\lambda}\right)\right\|_{L_{\mathrm{loc}}^{2}\left(d t d x ; L^{p}(M d v)\right)} .
$$
that

Next, using estimate 5.11 from Lemma 5.10 and the bound $(8.12$, we find

$$
\begin{aligned}
\frac{1}{\delta^{2}} & \left|1-\hat{\gamma}_{\epsilon}^{\lambda}\right|^{2} \\
& \leq C\left(\lambda+\frac{\epsilon}{\delta}\right)\left|\hat{g}_{\epsilon}^{ \pm}\right| \frac{1}{\delta}\left|1-\hat{\gamma}_{\epsilon}^{\lambda}\right| \\
& \leq C\left(\lambda+\frac{\epsilon}{\delta}\right)\left(\left|\Pi \hat{g}_{\epsilon}^{ \pm}\right|+\left|\hat{g}_{\epsilon}^{ \pm}-\Pi \hat{g}_{\epsilon}^{ \pm}\right|\right) \frac{1}{\delta}\left|1-\hat{\gamma}_{\epsilon}^{\lambda}\right| \\
& \leq C\left(\lambda+\frac{\epsilon}{\delta}\right)^{2}\left|\Pi \hat{g}_{\epsilon}^{ \pm}\right|\left|\hat{g}_{\epsilon}^{ \pm}\right|+C\left(\lambda+\frac{\epsilon}{\delta}\right) \frac{1}{\delta}\left|\hat{g}_{\epsilon}^{ \pm}-\Pi \hat{g}_{\epsilon}^{ \pm}\right| \\
& \leq O\left(\left(\lambda+\frac{\epsilon}{\delta}\right)^{2}\right)_{L_{\mathrm{loc}}^{1}\left(d t d x ; L^{r}(M d v)\right)}+O\left(\left(\lambda+\frac{\epsilon}{\delta}\right) \frac{\epsilon}{\delta}\right)_{L_{\mathrm{loc}}^{1}\left(d t d x ; L^{2}(M d v)\right)}
\end{aligned}
$$

for any $1 \leq r<2$.

Then, we end up with

$$
\left\|D_{\epsilon}^{4}(\varphi)\right\|_{L_{\text {loc }}^{1}(d t d x)} \leq C \lambda .
$$

Finally, incorporating (8.7), 8.9), 8.10, 8 8.11), 8.13) and (8.14) into 8.6), we have shown that the first term in the right-hand side of $(8.3)$ is uniformly bounded in $L_{\text {loc }}^{1}(d t d x)$ and satisfies, for any $\lambda>0$,

$$
\limsup _{\epsilon \rightarrow 0}\left\|\frac{1}{\delta \epsilon^{2}} \int_{\mathbb{R}^{3}} \hat{\gamma}_{\epsilon}^{\lambda} \mathcal{Q}\left(G_{\epsilon}^{ \pm}, G_{\epsilon}^{ \pm}\right) \varphi \chi\left(\frac{|v|^{2}}{K_{\delta}}\right) M d v\right\|_{L_{\mathrm{loc}}^{1}(d t d x)} \leq C \lambda .
$$

In particular, combining 8.5 and 8.15), it follows from the Banach-Alaoglu theorem, up to further extraction of subsequences, that the right-hand side of 8.3 converges in the weak-* topology of Radon measures $\mathcal{M}_{\text {loc }}\left([0, \infty) \times \mathbb{R}^{3}\right)$ towards

$$
\int_{\mathbb{R}^{3} \times \mathbb{R}^{3} \times \mathbb{S}^{2}} q^{ \pm, \mp} \varphi M M_{*} d v d v_{*} d \sigma+Q^{\lambda}(\varphi)
$$

where the Radon measure $Q^{\lambda}(\varphi) \in \mathcal{M}_{\text {loc }}\left([0, \infty) \times \mathbb{R}^{3}\right)$ satisfies the control

$$
\left\|Q^{\lambda}(\varphi)\right\|_{\mathcal{M}_{\mathrm{loc}}\left([0, \infty) \times \mathbb{R}^{3}\right)} \leq C \lambda .
$$


8.1.1.3. Decomposition of flux terms. Next, we treat the convergence in 8.3 of the flux terms

$$
\frac{1}{\delta} \nabla_{x} \cdot \int_{\mathbb{R}^{3}} g_{\epsilon}^{ \pm} \gamma_{\epsilon}^{\lambda} \varphi \chi\left(\frac{|v|^{2}}{K_{\delta}}\right) v M d v
$$

To this end, we use the decomposition (5.5) to write

$$
g_{\epsilon}^{ \pm}=\hat{g}_{\epsilon}^{ \pm}+\frac{\epsilon}{4}\left(\hat{g}_{\epsilon}^{ \pm}\right)^{2}=\Pi \hat{g}_{\epsilon}^{ \pm}+\left(\hat{g}_{\epsilon}^{ \pm}-\Pi \hat{g}_{\epsilon}^{ \pm}\right)+\frac{\epsilon}{4}\left(\hat{g}_{\epsilon}^{ \pm}\right)^{2},
$$

where $\Pi$ is the orthogonal projection on $\operatorname{Ker} \mathcal{L}$ in $L^{2}(M d v)$, which yields the decomposition of flux terms

$$
\begin{aligned}
\frac{1}{\delta} \nabla_{x} \cdot \int_{\mathbb{R}^{3}} g_{\epsilon}^{ \pm} \gamma_{\epsilon}^{\lambda} \varphi \chi\left(\frac{|v|^{2}}{K_{\delta}}\right) v M d v & =\frac{\epsilon}{4 \delta} \nabla_{x} \cdot \int_{\mathbb{R}^{3}}\left(\hat{g}_{\epsilon}^{ \pm}\right)^{2} \gamma_{\epsilon}^{\lambda} \varphi \chi\left(\frac{|v|^{2}}{K_{\delta}}\right) v M d v \\
& +\frac{1}{\delta} \nabla_{x} \cdot \int_{\mathbb{R}^{3}}\left(\hat{g}_{\epsilon}^{ \pm}-\Pi \hat{g}_{\epsilon}^{ \pm}\right) \gamma_{\epsilon}^{\lambda} \varphi \chi\left(\frac{|v|^{2}}{K_{\delta}}\right) v M d v \\
& +\frac{1}{\delta} \nabla_{x} \cdot \int_{\mathbb{R}^{3}} \Pi \hat{g}_{\epsilon}^{ \pm} \varphi\left(\chi\left(\frac{|v|^{2}}{K_{\delta}}\right) \gamma_{\epsilon}^{\lambda}-1\right) v M d v \\
& +\frac{1}{\delta} \nabla_{x} \cdot \int_{\mathbb{R}^{3}} \Pi \hat{g}_{\epsilon}^{ \pm} \varphi v M d v \\
& \stackrel{\operatorname{def}}{=} F_{\epsilon}^{1}(\varphi)+F_{\epsilon}^{2}(\varphi)+F_{\epsilon}^{3}(\varphi)+F_{\epsilon}^{4}(\varphi) .
\end{aligned}
$$

Then, from the condition on the support of $\gamma$ and since $\left|\hat{g}_{\epsilon}^{ \pm}\right|=\left|\frac{g_{\epsilon}^{ \pm}}{1+\frac{\epsilon}{4} \hat{g}_{\epsilon}^{ \pm}}\right| \leq 2\left|g_{\epsilon}^{ \pm}\right|$, we deduce that, on the support of $\gamma_{\epsilon}^{\lambda}$,

$$
\left|\hat{g}_{\epsilon}^{ \pm}\right| \leq 2\left|g_{\epsilon}^{ \pm}\right| \leq \frac{4}{\lambda \delta} \leq \frac{4}{\epsilon}
$$

Therefore, by Lemma 5.12 , for any $1 \leq p<2$, we have that $\left(\hat{g}_{\epsilon}^{ \pm}\right)^{2} \gamma_{\epsilon}^{\lambda}$ is uniformly bounded in $L_{\text {loc }}^{1}\left(d t d x ; L^{p}(M d v)\right)$. Hence, we conclude that

$$
\left\|F_{\epsilon}^{1}(\varphi)\right\|_{W_{\mathrm{loc}}^{-1,1}(d t d x)} \leq C \frac{\epsilon}{\delta}\left\|\left(\hat{g}_{\epsilon}^{ \pm}\right)^{2} \gamma_{\epsilon}^{\lambda}\right\|_{L_{\mathrm{loc}}^{1}\left(d t d x ; L^{p}(M d v)\right)}\|v \varphi\|_{L^{p^{\prime}}(M d v)} \leq C \frac{\epsilon}{\delta} .
$$

Moreover, by 5.11, we easily get

$$
\left\|F_{\epsilon}^{2}(\varphi)\right\|_{W_{\mathrm{loc}}^{-1,1}(d t d x)} \leq \frac{C}{\delta}\left\|\hat{g}_{\epsilon}^{ \pm}-\Pi \hat{g}_{\epsilon}^{ \pm}\right\|_{L_{\mathrm{loc}}^{1}\left(d t d x ; L^{2}(M d v)\right)}\|\gamma\|_{\infty}\|v \varphi\|_{L^{2}(M d v)} \leq C \frac{\epsilon}{\delta},
$$

which handles the second term.

Further note that, by definition of the projection $\Pi$, we have, for any $2<p<\infty$,

$$
\left\|\Pi \hat{g}_{\epsilon}^{ \pm}\right\|_{L^{\infty}\left(d t ; L^{2}\left(d x ; L^{p}(M d v)\right)\right)} \leq C_{p}\left\|\hat{g}_{\epsilon}^{ \pm}\right\|_{L^{\infty}\left(d t ; L^{2}(M d x d v)\right)},
$$

whence

$$
\begin{aligned}
\left\|F_{\epsilon}^{3}(\varphi)\right\|_{W_{\mathrm{loc}}^{-1,1}(d t d x)} & \leq C_{p}\left\|\hat{g}_{\epsilon}^{ \pm}\right\|_{L^{\infty}\left(d t ; L^{2}(M d x d v)\right)}\left\|\frac{1-\gamma_{\epsilon}^{\lambda}}{\delta}\right\|_{L_{\mathrm{loc}}^{2}\left(d t d x ; L^{2}(M d v)\right)}\|v \varphi\|_{L^{q}(M d v)} \\
& +C \frac{1}{\delta}\left\|\hat{g}_{\epsilon}^{ \pm}\right\|_{L^{\infty}\left(d t ; L^{2}(M d x d v)\right)}\left\|v \varphi\left(1-\chi\left(\frac{|v|^{2}}{K_{\delta}}\right)\right)\right\|_{L^{2}(M d v)},
\end{aligned}
$$

with $\frac{1}{q}=\frac{1}{2}-\frac{1}{p}$. Then, using estimate 8.12 (with $\gamma_{\epsilon}^{\lambda}$ instead of $\hat{\gamma}_{\epsilon}^{\lambda}$ ) and the control of Gaussian tails 8.8 to respectively bound the first and second terms in the right-hand side above, we infer that

$$
\left\|F_{\epsilon}^{3}(\varphi)\right\|_{W_{\mathrm{loc}}^{-1,1}(d t d x)} \leq C \lambda+C \delta^{\frac{K}{4}-1}|\log \delta|^{\frac{7}{4}},
$$


which is small provided that $K>4$. Therefore, up to further extraction of subsequences, we deduce that

$$
F_{\epsilon}^{3}(\varphi) \rightarrow \nabla_{x} \cdot R^{\lambda}(\varphi) \text { in the sense of distributions, }
$$

where the Radon measure $R^{\lambda}(\varphi) \in \mathcal{M}_{\text {loc }}\left([0, \infty) \times \mathbb{R}^{3}\right)$ satisfies the control

$$
\left\|R^{\lambda}(\varphi)\right\|_{\mathcal{M}_{\mathrm{loc}}\left([0, \infty) \times \mathbb{R}^{3}\right)} \leq C \lambda
$$

The form of the last remaining flux term $F_{\epsilon}^{4}(\varphi)$ depends on the collision invariant $\varphi$ :

- If $\varphi(v)=v$, we get, using that $\phi(v)=v \otimes v-\frac{|v|^{2}}{3}$ Id is orthogonal to the collision invariants,

$$
\begin{aligned}
F_{\epsilon}^{4}(\varphi) & =\frac{1}{\delta} \nabla_{x} \cdot \int_{\mathbb{R}^{3}} \Pi \hat{g}_{\epsilon}^{ \pm} v \otimes v M d v \\
& =\frac{1}{3 \delta} \nabla_{x} \int_{\mathbb{R}^{3}} \Pi \hat{g}_{\epsilon}^{ \pm}|v|^{2} M d v \\
& =\frac{1}{\delta} \nabla_{x}\left(\hat{\rho}_{\epsilon}^{ \pm}+\hat{\theta}_{\epsilon}^{ \pm}\right),
\end{aligned}
$$

where $\hat{\rho}_{\epsilon}^{ \pm}$and $\hat{\theta}_{\epsilon}^{ \pm}$denote the densities and temperatures respectively associated with the renormalized fluctuations $\hat{g}_{\epsilon}^{ \pm}$. Thus, this term takes the form of a gradient and will therefore vanish upon integrating it against divergence free vector fields as required by the theory of weak solutions of Leray.

- If $\varphi(v)=\frac{|v|^{2}}{2}-\frac{5}{2}$, we obtain

$$
F_{\epsilon}^{4}(\varphi)=\frac{1}{\delta} \nabla_{x} \cdot \int_{\mathbb{R}^{3}} \Pi \hat{g}_{\epsilon}^{ \pm}\left(\frac{|v|^{2}}{2}-\frac{5}{2}\right) v M d v=0
$$

for $\psi(v)=\left(\frac{|v|^{2}}{2}-\frac{5}{2}\right) v$ is orthogonal to the collision invariants.

Thus, on the whole incorporating 8.19, 8.20, 8.21), 8.23 and 8.24 into (8.18), we conclude that the flux terms satisfy the following convergences in the sense of distributions :

$$
\begin{gathered}
P\left(\frac{1}{\delta} \nabla_{x} \cdot \int_{\mathbb{R}^{3}} g_{\epsilon}^{ \pm} \gamma_{\epsilon}^{\lambda} \chi\left(\frac{|v|^{2}}{K_{\delta}}\right) v \otimes v M d v\right) \rightarrow P\left(\nabla_{x} \cdot R^{\lambda}(v)\right), \\
\frac{1}{\delta} \nabla_{x} \cdot \int_{\mathbb{R}^{3}} g_{\epsilon}^{ \pm} \gamma_{\epsilon}^{\lambda} \chi\left(\frac{|v|^{2}}{K_{\delta}}\right)\left(\frac{|v|^{2}}{2}-\frac{5}{2}\right) v M d v \rightarrow \nabla_{x} \cdot R^{\lambda}\left(\frac{|v|^{2}}{2}-\frac{5}{2}\right),
\end{gathered}
$$

where $P$ denotes the Leray projector onto solenoidal vector fields. 
8.1.1.4. Decomposition of acceleration terms. It only remains to deal with the terms involving the electromagnetic field in 8.3 , which we decompose as $(8.26)$

$$
\begin{aligned}
& \int_{\mathbb{R}^{3}} g_{\epsilon}^{ \pm} \gamma_{\epsilon}^{\lambda}\left(\epsilon E_{\epsilon}+v \wedge B_{\epsilon}\right) \cdot \nabla_{v}\left(\varphi \chi\left(\frac{|v|^{2}}{K_{\delta}}\right) M\right) d v \\
& +E_{\epsilon} \cdot \int_{\mathbb{R}^{3}}\left(1+\epsilon g_{\epsilon}^{ \pm}\right) \hat{\gamma}_{\epsilon}^{\lambda} \varphi \chi\left(\frac{|v|^{2}}{K_{\delta}}\right) v M d v \\
& =\left[\epsilon E_{\epsilon} \cdot \int_{\mathbb{R}^{3}} g_{\epsilon}^{ \pm} \gamma_{\epsilon}^{\lambda} \nabla_{v}\left(\varphi \chi\left(\frac{|v|^{2}}{K_{\delta}}\right) M\right) d v+\epsilon E_{\epsilon} \cdot \int_{\mathbb{R}^{3}} g_{\epsilon}^{ \pm} \hat{\gamma}_{\epsilon}^{\lambda} \varphi \chi\left(\frac{|v|^{2}}{K_{\delta}}\right) v M d v\right] \\
& +\left[E_{\epsilon} \cdot \int_{\mathbb{R}^{3}} \varphi v M d v+E_{\epsilon} \cdot \int_{\mathbb{R}^{3}}\left(\chi\left(\frac{|v|^{2}}{K_{\delta}}\right) \hat{\gamma}_{\epsilon}^{\lambda}-1\right) \varphi v M d v\right] \\
& -\left[B_{\epsilon} \cdot \int_{\mathbb{R}^{3}} g_{\epsilon}^{ \pm} \gamma_{\epsilon}^{\lambda} v \wedge \nabla_{v} \varphi M d v+B_{\epsilon} \cdot \int_{\mathbb{R}^{3}} g_{\epsilon}^{ \pm} \gamma_{\epsilon}^{\lambda} v \wedge\left(\nabla_{v} \varphi\right)\left(\chi\left(\frac{|v|^{2}}{K_{\delta}}\right)-1\right) M d v\right] \\
& \stackrel{\text { def }}{=} A_{\epsilon}^{1}(\varphi)+A_{\epsilon}^{2}(\varphi)-A_{\epsilon}^{3}(\varphi) .
\end{aligned}
$$

From the condition on the support of $\gamma$ and since $\left|\hat{g}_{\epsilon}^{ \pm}\right|=\left|\frac{g_{\epsilon}^{ \pm}}{1+\frac{\epsilon}{4} \hat{g}_{\epsilon}^{ \pm}}\right| \leq 2\left|g_{\epsilon}^{ \pm}\right|$, we clearly have that

$$
\begin{aligned}
& \left\|A_{\epsilon}^{1}(\varphi)\right\|_{L^{\infty}\left(d t ; L^{1}(d x)\right)} \\
& \leq C \epsilon\left\|E_{\epsilon}\right\|_{L^{\infty}\left(d t ; L^{2}(d x)\right)}\left\|g_{\epsilon}^{ \pm} \mathbb{1}_{\left\{\left|\lambda \delta g_{\epsilon}^{ \pm}\right| \leq 2\right\}}\right\|_{L^{\infty}\left(d t ; L^{2}(M d x d v)\right)}\left\|\varphi \chi\left(\frac{|v|^{2}}{K_{\delta}}\right) v\right\|_{H^{1}(M d v)} \leq C \epsilon\left\|\hat{g}_{\epsilon}^{ \pm}\right\|_{L^{\infty}\left(d t ; L^{2}(M d x d v)\right)}, \\
& \leq C \epsilon\left\|\left(\hat{g}_{\epsilon}^{ \pm}+\frac{\epsilon}{4} \hat{g}_{\epsilon}^{ \pm 2}\right) \mathbb{1}_{\left\{\left|\lambda \delta \hat{g}_{\epsilon}^{ \pm}\right| \leq 4\right\}}\right\|_{L^{\infty}\left(d t ; L^{2}(M d x d v)\right)} \leq
\end{aligned}
$$

which handles the first acceleration term.

Then, in order to deal with the second acceleration term, we estimate first, using 8.12 and the control of Gaussian tails 8.8, that

$$
\begin{aligned}
& \left\|\int_{\mathbb{R}^{3}}\left(\chi\left(\frac{|v|^{2}}{K_{\delta}}\right) \hat{\gamma}_{\epsilon}^{\lambda}-1\right) \varphi v M d v\right\|_{L_{\mathrm{loc}}^{2}(d t d x)} \\
& \leq\left\|\int_{\mathbb{R}^{3}}\left(\hat{\gamma}_{\epsilon}^{\lambda}-1\right) \varphi v M d v\right\|_{L_{\mathrm{loc}}^{2}(d t d x)}+\left\|\int_{\mathbb{R}^{3}}\left(\chi\left(\frac{|v|^{2}}{K_{\delta}}\right)-1\right) \varphi v M d v\right\|_{L_{\mathrm{loc}}^{2}(d t d x)} \\
& \leq C \lambda \delta\left\|\hat{g}_{\epsilon}^{ \pm}\right\|_{L_{\mathrm{loc}}^{2}\left(d t d x ; L^{2}(M d v)\right)}+C \delta^{\frac{K}{2}}|\log \delta|^{2}
\end{aligned}
$$

whence, as $\epsilon \rightarrow 0$,

$$
A_{\epsilon}^{2}(\varphi) \stackrel{*}{\rightarrow} E \cdot \int_{\mathbb{R}^{3}} \varphi v M d v \quad \text { in } L^{\infty}\left(d t ; L^{2}(d x)\right)
$$

As for the remaining term $A_{\epsilon}^{3}(\varphi)$, note first, using (8.8) again, that

$$
\begin{aligned}
& \left\|B_{\epsilon} \cdot \int_{\mathbb{R}^{3}} g_{\epsilon}^{ \pm} \gamma_{\epsilon}^{\lambda} v \wedge\left(\nabla_{v} \varphi\right)\left(\chi\left(\frac{|v|^{2}}{K_{\delta}}\right)-1\right) M d v\right\|_{L^{\infty}\left(d t ; L^{2}(d x)\right)} \\
& \leq \frac{C}{\lambda \delta}\left\|B_{\epsilon}\right\|_{L^{\infty}\left(d t ; L^{2}(d x)\right)} \int_{\mathbb{R}^{3}}\left(\chi\left(\frac{|v|^{2}}{K_{\delta}}\right)-1\right)|v|^{2} M d v \leq \frac{C}{\lambda} \delta^{\frac{K}{2}-1}|\log \delta|^{\frac{3}{2}},
\end{aligned}
$$


which is small as soon as $K>2$. Moreover, in view of (5.5), we find

$$
\begin{aligned}
\| B_{\epsilon} \cdot \int_{\mathbb{R}^{3}}\left(g_{\epsilon}^{ \pm}-\hat{g}_{\epsilon}^{ \pm}\right) & \gamma_{\epsilon}^{\lambda} v \wedge \nabla_{v} \varphi M d v \|_{L_{\mathrm{loc}}^{1}(d t d x)} \\
& =\left\|B_{\epsilon} \cdot \int_{\mathbb{R}^{3}} \frac{\epsilon}{4} \hat{g}_{\epsilon}^{ \pm 2} \gamma_{\epsilon}^{\lambda} v \wedge \nabla_{v} \varphi M d v\right\|_{L_{\mathrm{loc}}^{1}(d t d x)} \\
& \leq C \frac{\epsilon}{\lambda \delta}\left\|B_{\epsilon}\right\|_{L_{\mathrm{loc}}^{2}(d t d x)}\left\|\hat{g}_{\epsilon}^{ \pm}\right\|_{L_{\mathrm{loc}}^{2}\left(d t d x ; L^{2}(M d v)\right)},
\end{aligned}
$$

so that, on the whole, the weak limit of $A_{\epsilon}^{3}(\varphi)$ will coincide with the weak limit of

$$
B_{\epsilon} \cdot \int_{\mathbb{R}^{3}} \hat{g}_{\epsilon}^{ \pm} \gamma_{\epsilon}^{\lambda} v \wedge \nabla_{v} \varphi M d v .
$$

In order to take the weak limit of the preceding term, notice, in view of Lemma 7.10 through a straighforward application of the mean value theorem to the function $z \gamma\left(\lambda \delta\left(z+\frac{\epsilon}{4} z^{2}\right)\right)$, that $\hat{g}_{\epsilon}^{ \pm} \gamma_{\epsilon}^{\lambda}=\hat{g}_{\epsilon}^{ \pm} \gamma\left(\lambda \delta\left(\hat{g}_{\epsilon}^{ \pm}+\frac{\epsilon}{4} \hat{g}_{\epsilon}^{ \pm 2}\right)\right)$ is locally relatively compact in $(x, v)$ in $L^{p}(d t d x d v)$, for any $1 \leq p<2$. In fact, Lemma 7.10 further implies that $\left|\hat{g}_{\epsilon}^{ \pm} \gamma_{\epsilon}^{\lambda}\right|^{2} \leq\left|\hat{g}_{\epsilon}^{ \pm}\right|^{2} \mathbb{1}_{\left\{\lambda \delta\left|\hat{g}_{\epsilon}^{ \pm}\right| \leq 4\right\}}$ is equi-integrable. Therefore, we conclude that $\hat{g}_{\epsilon}^{ \pm} \gamma_{\epsilon}^{\lambda}$ is locally relatively compact in $(x, v)$ in $L^{2}(d t d x d v)$. In particular, for any fixed $\lambda>0$, it is possible to approximate $\hat{g}_{\epsilon}^{ \pm} \gamma_{\epsilon}^{\lambda}$, uniformly in $\epsilon>0$, in $L_{\text {loc }}^{2}(d t d x d v)$ by its regularized version $\left(\hat{g}_{\epsilon}^{ \pm} \gamma_{\epsilon}^{\lambda}\right) *_{x, v} \chi_{a}$, where $a>0$ and $\chi_{a}(x, v)=\frac{1}{a^{6}} \chi\left(\frac{x}{a}, \frac{v}{a}\right)$ is an approximate identity, with $\chi \in C_{c}^{\infty}\left(\mathbb{R}^{3} \times \mathbb{R}^{3}\right)$ such that $\int_{\mathbb{R}^{3} \times \mathbb{R}^{3}} \chi(x, v) d x d v=1$.

We use now compensated compactness in the following form. From the Faraday equation in 4.34, we deduce that

$$
\partial_{t} B_{\epsilon} \in L^{\infty}\left(d t ; H^{-1}(d x)\right)
$$

so that $B_{\epsilon}$ enjoys some strong compactness with respect to the time variable. We then deduce, up to extraction of subsequences, that

$$
B_{\epsilon}\left(\hat{g}_{\epsilon}^{ \pm} \gamma_{\epsilon}^{\lambda}\right) *_{x, v} \chi_{a} \rightarrow B g^{ \pm} *_{x, v} \chi_{a}
$$

where $g^{ \pm}$is the weak limit of $\hat{g}_{\epsilon}^{ \pm} \gamma_{\epsilon}^{\lambda}$, which coincides with the weak limits of $\hat{g}_{\epsilon}^{ \pm}$and $g_{\epsilon}^{ \pm}$(note that $\gamma_{\epsilon}^{\lambda} \rightarrow 1$ almost everywhere). Incidentally, by the uniformity of the approximation of $\hat{g}_{\epsilon}^{ \pm} \gamma_{\epsilon}^{\lambda}$ by $\left(\hat{g}_{\epsilon}^{ \pm} \gamma_{\epsilon}^{\lambda}\right) *_{x, v} \chi_{a}$ in $L_{\mathrm{loc}}^{2}(d t d x d v)$, we infer that

$$
B_{\epsilon} \hat{g}_{\epsilon}^{ \pm} \gamma_{\epsilon}^{\lambda} \rightarrow B g^{ \pm}
$$

in $L_{\text {loc }}^{1}(d t d x d v)$, whence

$$
A_{\epsilon}^{3}(\varphi) \rightarrow B \cdot \int_{\mathbb{R}^{3}} g^{ \pm} v \wedge \nabla_{v} \varphi M d v \quad \text { in } L_{\mathrm{loc}}^{1}(d t d x) .
$$
that

Thus, incorporating 8.27), 8.28 and 8.29 into 8.26 , we finally conclude

$$
\begin{aligned}
\int_{\mathbb{R}^{3}} g_{\epsilon}^{ \pm} \gamma_{\epsilon}^{\lambda}\left(\epsilon E_{\epsilon}+v \wedge B_{\epsilon}\right) \cdot \nabla_{v} & \left(\varphi \chi\left(\frac{|v|^{2}}{K_{\delta}}\right) M\right) d v \\
& +E_{\epsilon} \cdot \int_{\mathbb{R}^{3}}\left(1+\epsilon g_{\epsilon}^{ \pm}\right) \hat{\gamma}_{\epsilon}^{\lambda} \varphi \chi\left(\frac{|v|^{2}}{K_{\delta}}\right) v M d v \\
& \rightarrow E \cdot \int_{\mathbb{R}^{3}} \varphi v M d v-B \cdot \int_{\mathbb{R}^{3}} g^{ \pm} v \wedge \nabla_{v} \varphi M d v
\end{aligned}
$$

in $L_{\mathrm{loc}}^{1}(d t d x)$. 
8.1.1.5. Convergence. We are now in a position to pass to the limit in (8.3). To this end, note first that, since $\frac{\epsilon}{\delta} \rightarrow 0$ and $g_{\epsilon}^{ \pm}$is uniformly bounded in $L_{\text {loc }}^{1}\left(d t d x ; L^{1}\left(\left(1+|v|^{2}\right) M d v\right)\right)$ by Lemma 5.1, the density term

$$
\frac{\epsilon}{\delta} \partial_{t} \int_{\mathbb{R}^{3}} g_{\epsilon}^{ \pm} \gamma_{\epsilon}^{\lambda} \varphi \chi\left(\frac{|v|^{2}}{K_{\delta}}\right) M d v
$$

vanishes as $\epsilon \rightarrow 0$ and thus brings no contribution to the weak limit. Therefore, according to the weak limits of conservation defects 8.16), flux terms 8.25 and acceleration terms 8.30), we conclude, letting $\epsilon \rightarrow 0$ in (8.3) in the sense of distributions for the collision invariants $\varphi=v$ and $\varphi=\frac{|v|^{2}}{2}-\frac{5}{2}$, that

$$
\begin{aligned}
& P\left(\nabla_{x} \cdot R^{\lambda}(v) \mp(E+u \wedge B)\right) \\
&=P\left(\int_{\mathbb{R}^{3} \times \mathbb{R}^{3} \times \mathbb{S}^{2}} q^{ \pm, \mp} v M M_{*} d v d v_{*} d \sigma+Q^{\lambda}(v)\right) \\
& \nabla_{x} \cdot R^{\lambda}\left(\frac{|v|^{2}}{2}-\frac{5}{2}\right) \\
& \quad=\int_{\mathbb{R}^{3} \times \mathbb{R}^{3} \times \mathbb{S}^{2}} q^{ \pm, \mp}\left(\frac{|v|^{2}}{2}-\frac{5}{2}\right) M M_{*} d v d v_{*} d \sigma+Q^{\lambda}\left(\frac{|v|^{2}}{2}-\frac{5}{2}\right),
\end{aligned}
$$

where $u$ denotes the bulk velocity associated with the limiting fluctuations $g^{ \pm}$(recall that, according to Lemmas 5.10 and 5.11, $g^{+}$and $g^{-}$are infinitesimal Maxwellians which differ only by their densities).

Next, in view of the bounds 8.17) and 8.22 on the Radon measures $Q^{\lambda}$ and $R^{\lambda}$, respectively, we deduce, by the arbitrariness of $\lambda>0$, that

$$
\begin{aligned}
P\left(E+u \wedge B \pm \int_{\mathbb{R}^{3} \times \mathbb{R}^{3} \times \mathbb{S}^{2}} q^{ \pm, \mp} v M M_{*} d v d v_{*} d \sigma\right) & =0, \\
\int_{\mathbb{R}^{3} \times \mathbb{R}^{3} \times \mathbb{S}^{2}} q^{ \pm, \mp}\left(\frac{|v|^{2}}{2}-\frac{5}{2}\right) M M_{*} d v d v_{*} d \sigma & =0,
\end{aligned}
$$

which concludes the proof of Proposition 8.1

\subsection{Macroscopic constraint equations for two species, strong interactions}

We move on now to the regime of strong interspecies interactions considered in Theorem 4.6. In this setting, the derivation of even the simplest macroscopic constraint equations (such as the incompressibility and Boussinesq contraints) involves the handling of nonlinear terms. Indeed, the limiting kinetic equation 8.1) for weak interspecies interactions (obtained in Chapter 6 with weak compactness methods) corresponds now, for strong interspecies interactions, to the nonlinear equation

$$
\left(v \cdot \nabla_{x} \pm(v \wedge B) \cdot \nabla_{v}\right) g^{ \pm} \mp E \cdot v=\int_{\mathbb{R}^{3} \times \mathbb{S}^{2}}\left(q^{ \pm}+q^{ \pm, \mp}\right) M_{*} d v_{*} d \sigma
$$

which is obtained in the proof of Proposition 8.3. below (see (8.37) ), and requires the compactness properties established in Chapter 7.

The next result fully characterizes the limiting kinetic equations in the regime of strong interactions.

Proposition 8.3. Let $\left(f_{\epsilon}^{ \pm}, E_{\epsilon}, B_{\epsilon}\right)$ be the sequence of renormalized solutions to the scaled two species Vlasov-Maxwell-Boltzmann system (4.34) considered in Theorem 4.6 for strong interspecies interactions, i.e. $\delta=1$. In accordance with Lemmas 5.1, 5.2 and 5.3, denote by

$$
g^{ \pm} \in L^{\infty}\left(d t ; L^{2}(M d x d v)\right) \quad \text { and } \quad q^{ \pm}, q^{ \pm, \mp} \in L^{2}\left(M M_{*} d t d x d v d v_{*} d \sigma\right)
$$


any joint limit points of the families $\hat{g}_{\epsilon}^{ \pm}, \hat{q}_{\epsilon}^{ \pm}$and $\hat{q}_{\epsilon}^{ \pm, \mp}$ defined by (5.3) and (5.6), respectively, and by

$$
E, B \in L^{\infty}\left(d t ; L^{2}(d x)\right)
$$

any joint limit points of the families $E_{\epsilon}$ and $B_{\epsilon}$, respectively.

Then, one has

$$
\frac{1}{2} \int_{\mathbb{R}^{3} \times \mathbb{S}^{2}}\left(q^{+}+q^{-}+q^{+,-}+q^{-,+}\right) M_{*} d v_{*} d \sigma=\phi: \nabla_{x} u+\psi \cdot \nabla_{x} \theta
$$

and

$$
\begin{aligned}
\frac{1}{2} \int_{\mathbb{R}^{3} \times \mathbb{S}^{2}}\left(q^{+}-q^{-}+q^{+,-}-q^{-,+}\right) & M_{*} d v_{*} d \sigma \\
& =\left(\frac{1}{2} \nabla_{x}\left(\rho^{+}-\rho^{-}\right)-(E+u \wedge B)\right) \cdot v,
\end{aligned}
$$

where $\rho^{ \pm}, u$ and $\theta$ are, respectively, the densities, bulk velocity and temperature associated with the limiting fluctuations $g^{ \pm}$, and $\phi$ and $\psi$ are the kinetic fluxes defined by (2.12). Furthermore, $\rho^{ \pm}$, $u$ and $\theta$ satisfy the following constraints

$$
\operatorname{div} u=0, \quad \nabla_{x}\left(\frac{\rho^{+}+\rho^{-}}{2}+\theta\right)=0 .
$$

In particular, the strong Boussinesq relation $\frac{\rho^{+}+\rho^{-}}{2}+\theta=0$ holds.

Proof. The case $\delta=1$ is more complicated because we do not have enough strong compactness to take limits in the nonlinear terms

$$
\pm\left(v \wedge B_{\epsilon}\right) \cdot \nabla_{v} \frac{\sqrt{G_{\epsilon}^{ \pm}+\epsilon^{a}}-1}{\epsilon} .
$$

More precisely, we are not able to control the concentrations of $\left|\hat{g}_{\epsilon}^{ \pm}\right|^{2}$ (see Lemma 7.10 .

The idea is therefore to consider a stronger renormalization of the equation for the fluctuations of density. To this end, we introduce the admissible renormalization $\Gamma_{\lambda}(z)$ defined by

$$
\Gamma_{\lambda}(z)-1=(z-1) \gamma\left(\lambda \frac{z-1}{\epsilon}\right),
$$

where $\lambda>0$ is small and $\gamma \in C^{1}(\mathbb{R})$ satisfies that

$$
\mathbb{1}_{[-1,1]}(z) \leq \gamma(z) \leq \mathbb{1}_{[-2,2]}(z), \quad \text { for all } z \in \mathbb{R} .
$$

Without distinguishing, for simplicity, the notation for cations and anions, we denote $\gamma_{\epsilon}^{\lambda}$ for $\gamma\left(\lambda g_{\epsilon}^{ \pm}\right)$and $\hat{\gamma}_{\epsilon}^{\lambda}$ for $\Gamma_{\lambda}^{\prime}\left(G_{\epsilon}^{ \pm}\right)$. Thus, renormalizing the Vlasov-Boltzmann equation from (4.34) with respect to $\Gamma_{\lambda}(z)$ yields

$$
\begin{aligned}
\left(\epsilon \partial_{t}\right. & \left.+v \cdot \nabla_{x} \pm\left(\epsilon E_{\epsilon}+v \wedge B_{\epsilon}\right) \cdot \nabla_{v}\right) g_{\epsilon}^{ \pm} \gamma_{\epsilon}^{\lambda} \mp E_{\epsilon} \cdot v G_{\epsilon}^{ \pm} \hat{\gamma}_{\epsilon}^{\lambda} \\
& =\hat{\gamma}_{\epsilon}^{\lambda} \sqrt{G_{\epsilon}^{ \pm}} \int_{\mathbb{R}^{3} \times \mathbb{S}^{2}} \sqrt{G_{\epsilon *}^{ \pm}} \hat{q}_{\epsilon}^{ \pm} M_{*} d v_{*} d \sigma+\frac{\epsilon^{2}}{4} \hat{\gamma}_{\epsilon}^{\lambda} \int_{\mathbb{R}^{3} \times \mathbb{S}^{2}}\left(\hat{q}_{\epsilon}^{ \pm}\right)^{2} M_{*} d v_{*} d \sigma \\
& +\hat{\gamma}_{\epsilon}^{\lambda} \sqrt{G_{\epsilon}^{ \pm}} \int_{\mathbb{R}^{3} \times \mathbb{S}^{2}} \sqrt{G_{\epsilon *}^{\mp}} \hat{q}_{\epsilon}^{ \pm, \mp} M_{*} d v_{*} d \sigma+\frac{\epsilon^{2}}{4} \hat{\gamma}_{\epsilon}^{\lambda} \int_{\mathbb{R}^{3} \times \mathbb{S}^{2}}\left(\hat{q}_{\epsilon}^{ \pm, \mp}\right)^{2} M_{*} d v_{*} d \sigma
\end{aligned}
$$

Next, employing a strategy similar to the proof of Proposition 6.1, in particular, since $\hat{\gamma}_{\epsilon}^{\lambda} \sqrt{G_{\epsilon}^{ \pm}}$is uniformly bounded pointwise, utilizing that, thanks to Lemma 5.2

$$
\begin{aligned}
& \sqrt{G_{\epsilon}^{ \pm}}=1+O(\epsilon)_{L_{\mathrm{loc}}^{2}\left(d t ; L^{2}(M d x d v)\right)}, \\
& \sqrt{G_{\epsilon *}^{ \pm}}=1+O(\epsilon)_{L_{\mathrm{loc}}^{2}\left(d t ; L^{2}\left(M_{*} d x d v_{*}\right)\right)},
\end{aligned}
$$


and that, thanks to Lemma 5.3 the collision integrands $\hat{q}_{\epsilon}^{ \pm}$and $\hat{q}_{\epsilon}^{ \pm, \mp}$ are uniformly bounded in $L^{2}\left(M M_{*} d t d x d v d v_{*} d \sigma\right)$, we see that the weak limit of the right-hand side of 8.34 coincides with the weak limit of

$$
\int_{\mathbb{R}^{3} \times \mathbb{S}^{2}}\left(\hat{q}_{\epsilon}^{ \pm}+\hat{q}_{\epsilon}^{ \pm, \mp}\right) M_{*} d v_{*} d \sigma+Q_{\epsilon}^{\lambda},
$$

where we denote the remainder

$$
Q_{\epsilon}^{\lambda}=\left[\hat{\gamma}_{\epsilon}^{\lambda}-1\right] \int_{\mathbb{R}^{3} \times \mathbb{S}^{2}}\left(\hat{q}_{\epsilon}^{ \pm}+\hat{q}_{\epsilon}^{ \pm, \mp}\right) M_{*} d v_{*} d \sigma .
$$

Then, since $\left|\Gamma_{\lambda}^{\prime}(z)-1\right| \leq C_{r}\left|\lambda \frac{z-1}{\epsilon}\right|^{\frac{1}{r}}$, for any given $1 \leq r \leq \infty$ and for every $z \geq 0$, it holds that, employing the uniform bounds from Lemmas 5.1 and 5.3 , for any $2 \leq r \leq \infty$,

$$
\begin{aligned}
\left|Q_{\epsilon}^{\lambda}\right| & \leq C \lambda^{\frac{1}{r}}\left|g_{\epsilon}^{ \pm}\right|^{\frac{1}{r}} \int_{\mathbb{R}^{3} \times \mathbb{S}^{2}}\left(\hat{q}_{\epsilon}^{ \pm}+\hat{q}_{\epsilon}^{ \pm, \mp}\right) M_{*} d v_{*} d \sigma \\
& \leq C \lambda^{\frac{1}{r}}\left|g_{\epsilon}^{ \pm}\right|^{\frac{1}{r}}\left(\int_{\mathbb{R}^{3} \times \mathbb{S}^{2}}\left(\hat{q}_{\epsilon}^{ \pm}+\hat{q}_{\epsilon}^{ \pm, \mp}\right)^{2} M_{*} d v_{*} d \sigma\right)^{\frac{1}{2}} \\
& =O\left(\lambda^{\frac{1}{r}}\right)_{L_{\mathrm{loc}}^{\frac{2 r}{2+r}}(d t d x d v)} .
\end{aligned}
$$

Moreover, when $r=2$, it is readily seen, in view of the weak relative compactness of $g_{\epsilon}^{ \pm}$in $L_{\text {loc }}^{1}(d t d x d v)$ established in Lemma 5.1 and employing the DunfordPettis compactness criterion (see [66]), that $Q_{\epsilon}^{\lambda}$ is weakly relatively compact in $L_{\text {loc }}^{1}(d t d x d v)$, as well.

Therefore, up to extraction of a further subsequence as $\epsilon \rightarrow 0$, we may assume that $Q_{\epsilon}^{\lambda}$ converges weakly in $L_{\mathrm{loc}}^{\frac{2 r}{2+r}}(d t d x d v)$ to some $Q^{\lambda} \in L_{\mathrm{loc}}^{\frac{2 r}{2+r}}(d t d x d v)$, for any $2 \leq r \leq \infty$, whose magnitude is at most of order $\lambda^{\frac{1}{r}}$. On the whole, we have evaluated that the right-hand side of 8.34 converges weakly towards

$$
\begin{aligned}
\int_{\mathbb{R}^{3} \times \mathbb{S}^{2}} & \left(q^{ \pm}+q^{ \pm, \mp}\right) M_{*} d v_{*} d \sigma+Q^{\lambda} \\
& =\int_{\mathbb{R}^{3} \times \mathbb{S}^{2}}\left(q^{ \pm}+q^{ \pm, \mp}\right) M_{*} d v_{*} d \sigma+O\left(\lambda^{\frac{1}{r}}\right)_{L_{\mathrm{loc}}^{2+r}}(d t d x d v)
\end{aligned}
$$

As for the left-hand side of (8.34), we first have that

$$
E_{\epsilon} \cdot v G_{\epsilon}^{ \pm} \hat{\gamma}_{\epsilon}^{\lambda}=E_{\epsilon} \cdot v+E_{\epsilon} \cdot v\left(\hat{\gamma}_{\epsilon}^{\lambda}-1\right)+\epsilon E_{\epsilon} \cdot v g_{\epsilon}^{ \pm} \hat{\gamma}_{\epsilon}^{\lambda} \text {. }
$$

Therefore, since $g_{\epsilon}^{ \pm} \hat{\gamma}_{\epsilon}^{\lambda}$ is uniformly bounded pointwise and $\left|\Gamma_{\lambda}^{\prime}(z)-1\right| \leq C_{r}\left|\lambda \frac{z-1}{\epsilon}\right|^{\frac{1}{r}}$, for any given $1 \leq r \leq \infty$ and for every $z \geq 0$, we find, for any $2 \leq r \leq \infty$,

$$
E_{\epsilon} \cdot v G_{\epsilon}^{ \pm} \hat{\gamma}_{\epsilon}^{\lambda}=E_{\epsilon} \cdot v+O\left(\lambda^{\frac{1}{r}}\right)_{L_{\mathrm{loc}}^{\frac{2 r}{2+r}}(d t d x d v)}+O\left(\frac{\epsilon}{\lambda}\right)_{L_{\mathrm{loc}}^{2}(d t d x d v)},
$$

so that the expression from 8.35 converges weakly, for any $2 \leq r \leq \infty$, towards

$$
E \cdot v+O\left(\lambda^{\frac{1}{r}}\right)_{L_{\mathrm{loc}}^{\frac{2 r}{2+r}}(d t d x d v)} .
$$

Next, since $g_{\epsilon}^{ \pm}$is weakly relatively compact in $L_{\text {loc }}^{1}(d t d x d v)$, by Lemma 5.1 it holds that $g_{\epsilon}^{ \pm}\left(1-\gamma_{\epsilon}^{\lambda}\right)$ is uniformly small in $L_{\text {loc }}^{1}(d t d x d v)$, when $\lambda>0$ is small. In particular, we deduce that the family $g_{\epsilon}^{ \pm} \gamma_{\epsilon}^{\lambda}$, which is weakly relatively compact in $L_{\text {loc }}^{p}(d t d x d v)$, for any $1 \leq p<\infty$, converges weakly towards some $g^{ \pm, \lambda} \in$ $L_{\text {loc }}^{p}(d t d x d v)$ such that

$$
g^{ \pm, \lambda}=g^{ \pm}+o(1)_{L_{\mathrm{loc}}^{1}(d t d x d v)}
$$


as $\lambda \rightarrow 0$. In fact, we claim that this can be improved to

$$
g^{ \pm, \lambda}=g^{ \pm}+o(1)_{L_{\mathrm{loc}}^{2}(d t d x d v)}
$$

as $\lambda \rightarrow 0$. Indeed, up to extraction of subsequences, denoting by $r^{ \pm} \in L_{\mathrm{loc}}^{2}(d t d x d v)$ the weak limit of $\left|g_{\epsilon}^{ \pm}\right|$in $L_{\text {loc }}^{1}(d t d x d v)$, which coincides, in view of (5.5), with the weak limit of $\left|\hat{g}_{\epsilon}^{ \pm}\right|$in $L_{\text {loc }}^{2}(d t d x d v)$, it clearly holds that, for any non-negative $\varphi \in C_{c}^{\infty}\left([0, \infty) \times \mathbb{R}^{3} \times \mathbb{R}^{3}\right)$,

$$
\begin{aligned}
\int_{[0, \infty) \times \mathbb{R}^{3} \times \mathbb{R}^{3}}\left|g^{ \pm}-g^{ \pm, \lambda}\right| \varphi d t d x d v \\
\leq \liminf _{\epsilon \rightarrow 0} \int_{[0, \infty) \times \mathbb{R}^{3} \times \mathbb{R}^{3}}\left|g_{\epsilon}^{ \pm}\left(1-\gamma_{\epsilon}^{\lambda}\right)\right| \varphi d t d x d v \\
\leq \liminf _{\epsilon \rightarrow 0} \int_{[0, \infty) \times \mathbb{R}^{3} \times \mathbb{R}^{3}}\left|g_{\epsilon}^{ \pm}\right| \varphi d t d x d v \\
=\int_{[0, \infty) \times \mathbb{R}^{3} \times \mathbb{R}^{3}} r^{ \pm} \varphi d t d x d v
\end{aligned}
$$

whence $\left|g^{ \pm}-g^{ \pm, \lambda}\right|^{2}$ is dominated by the integrable function $\left(r^{ \pm}\right)^{2}$. It then follows from a direct application of Lebesgue's dominated convergence theorem that 8.36 holds.

At last, we deal with the convergence of the problematic nonlinear term $B_{\epsilon} g_{\epsilon}^{ \pm} \gamma_{\epsilon}^{\lambda}$ from 8.34). To this end, note first, according to (5.5) and Lemma 7.10, for any given $\lambda>0$, that the family $g_{\epsilon}^{ \pm} \gamma_{\epsilon}^{\lambda}$ is locally relatively compact in $(x, v)$ in $L^{2}(d t d x d v)$. In particular, for any fixed $\lambda>0$, it is possible to approximate $g_{\epsilon}^{ \pm} \gamma_{\epsilon}^{\lambda}$, uniformly in $\epsilon>0$, in $L_{\text {loc }}^{2}(d t d x d v)$ by its regularized version $\left(g_{\epsilon}^{ \pm} \gamma_{\epsilon}^{\lambda}\right) *_{x, v} \chi_{a}$, where $a>0$ and $\chi_{a}(x, v)=\frac{1}{a^{6}} \chi\left(\frac{x}{a}, \frac{v}{a}\right)$ is an approximate identity, with $\chi \in C_{c}^{\infty}\left(\mathbb{R}^{3} \times \mathbb{R}^{3}\right)$ such that $\int_{\mathbb{R}^{3} \times \mathbb{R}^{3}} \chi(x, v) d x d v=1$.

We use now compensated compactness in the following form. From the Faraday equation in 4.34, we deduce that

$$
\partial_{t} B_{\epsilon} \in L^{\infty}\left(d t ; H^{-1}(d x)\right),
$$

so that $B_{\epsilon}$ enjoys some strong compactness with respect to the time variable. We then deduce, up to extraction of subsequences, that

$$
B_{\epsilon}\left(g_{\epsilon}^{ \pm} \gamma_{\epsilon}^{\lambda}\right) *_{x, v} \chi_{a} \rightarrow B g^{ \pm, \lambda} *_{x, v} \chi_{a},
$$

and, incidentally, by the uniformity of the approximation of $g_{\epsilon}^{ \pm} \gamma_{\epsilon}^{\lambda}$ by $\left(g_{\epsilon}^{ \pm} \gamma_{\epsilon}^{\lambda}\right) *_{x, v} \chi_{a}$ in $L_{\mathrm{loc}}^{2}(d t d x d v)$, that

$$
B_{\epsilon} g_{\epsilon}^{ \pm} \gamma_{\epsilon}^{\lambda} \rightarrow B g^{ \pm, \lambda}
$$

in $L_{\text {loc }}^{1}(d t d x d v)$.

We may now take weak limits in (8.34) to infer that, for any given $2 \leq r \leq \infty$,

$$
\begin{aligned}
&\left(v \cdot \nabla_{x} \pm\right.\left.(v \wedge B) \cdot \nabla_{v}\right) g^{ \pm, \lambda} \mp E \cdot v \\
&=\int_{\mathbb{R}^{3} \times \mathbb{S}^{2}}\left(q^{ \pm}+q^{ \pm, \mp}\right) M_{*} d v_{*} d \sigma+O\left(\lambda^{\frac{1}{r}}\right)_{L_{\mathrm{loc}}^{\frac{2 r}{2+r}}(d t d x d v)} .
\end{aligned}
$$

Finally, in view of 8.36 , letting $\lambda \rightarrow 0$, we arrive at

$$
\left(v \cdot \nabla_{x} \pm(v \wedge B) \cdot \nabla_{v}\right) g^{ \pm} \mp E \cdot v=\int_{\mathbb{R}^{3} \times \mathbb{S}^{2}}\left(q^{ \pm}+q^{ \pm, \mp}\right) M_{*} d v_{*} d \sigma,
$$

which, together with the fact, according to Lemmas 5.10 and 5.11 , that $g^{+}$and $g^{-}$are infinitesimal Maxwellians, which differ only by their densities $\rho^{+}$and $\rho^{-}$, 
provides that

$$
\begin{aligned}
\int_{\mathbb{R}^{3} \times \mathbb{S}^{2}} & \left(q^{ \pm}+q^{ \pm, \mp}\right) M_{*} d v_{*} d \sigma \\
& =\operatorname{div}\left(\left(\rho^{ \pm}+\theta\right) v+\frac{|v|^{2}}{3} u+\phi u+\theta \psi\right) \mp(E+u \wedge B) \cdot v \\
& =\left(\phi: \nabla_{x} u+\psi \cdot \nabla_{x} \theta\right)+\left(\nabla_{x}\left(\rho^{ \pm}+\theta\right) \mp(E+u \wedge B)\right) \cdot v+\frac{1}{3}(\operatorname{div} u)|v|^{2} .
\end{aligned}
$$

Equivalently, we find that

$$
\begin{aligned}
\frac{1}{2} \int_{\mathbb{R}^{3} \times \mathbb{S}^{2}} & \left(q^{+}+q^{-}+q^{+,-}+q^{-,+}\right) M_{*} d v_{*} d \sigma \\
& =\left(\phi: \nabla_{x} u+\psi \cdot \nabla_{x} \theta\right)+\nabla_{x}\left(\frac{\rho^{+}+\rho^{-}}{2}+\theta\right) \cdot v+\frac{1}{3}(\operatorname{div} u)|v|^{2}
\end{aligned}
$$

and

$$
\begin{aligned}
\frac{1}{2} \int_{\mathbb{R}^{3} \times \mathbb{S}^{2}}\left(q^{+}-q^{-}+q^{+,-}-q^{-,+}\right) & M_{*} d v_{*} d \sigma \\
& =\left(\frac{1}{2} \nabla_{x}\left(\rho^{+}-\rho^{-}\right)-(E+u \wedge B)\right) \cdot v .
\end{aligned}
$$

Then, remarking that $q^{ \pm}$and $q^{ \pm, \mp}$ inherit the collisional symmetries of $q_{\epsilon}^{ \pm}$, $q_{\epsilon}^{ \pm, \mp}, \hat{q}_{\epsilon}^{ \pm}$and $\hat{q}_{\epsilon}^{ \pm, \mp}$, we get

$$
\int_{\mathbb{R}^{3} \times \mathbb{R}^{3} \times \mathbb{S}^{2}}\left(q^{+}+q^{-}+q^{+,-}+q^{-,+}\right)\left(\begin{array}{c}
1 \\
v \\
\frac{|v|^{2}}{2}
\end{array}\right) M M_{*} d v d v_{*} d \sigma=0,
$$

so that, since $\phi(v)$ and $\psi(v)$ are orthogonal to the collisional invariants, the constraints 8.33 hold.

The proof of the proposition is now complete.

The next proposition further characterizes the limiting collision integrands.

Proposition 8.4. Let $\left(f_{\epsilon}^{ \pm}, E_{\epsilon}, B_{\epsilon}\right)$ be the sequence of renormalized solutions to the scaled two species Vlasov-Maxwell-Boltzmann system (4.34) considered in Theorem 4.6 for strong interspecies interactions, i.e. $\delta=1$. In accordance with Lemmas 5.1, 5.2, 5.3, 5.13, 5.14 and 7.11 denote by

$$
\begin{aligned}
g^{ \pm} & \in L^{\infty}\left(d t ; L^{2}(M d x d v)\right), \\
q^{ \pm, \mp} & \in L^{2}\left(M M_{*} d t d x d v d v_{*} d \sigma\right), \\
h & \in L_{\text {loc }}^{1}\left(d t d x ; L^{1}\left(\left(1+|v|^{2}\right) M d v\right)\right),
\end{aligned}
$$

any joint limit points of the families $\hat{g}_{\epsilon}^{ \pm}, \hat{q}_{\epsilon}^{ \pm, \mp}$ and $h_{\epsilon}$ defined by (5.3), (5.6) and (5.29, respectively.

Then, one has

$$
\begin{gathered}
\int_{\mathbb{R}^{3} \times \mathbb{S}^{2}}\left(q^{+}-q^{-}\right) M_{*} d v_{*} d \sigma=-\mathcal{L}(h), \\
\int_{\mathbb{R}^{3} \times \mathbb{S}^{2}}\left(q^{+,-}-q^{-,+}\right) M_{*} d v_{*} d \sigma=n u \cdot \mathfrak{L}(v)+n \theta \mathfrak{L}\left(\frac{|v|^{2}}{2}\right)-\mathfrak{L}(h),
\end{gathered}
$$

$$
q^{+}+q^{-}-q^{+,-}-q^{-,+}=0,
$$

where $n=\rho^{+}-\rho^{-}, u$ and $\theta$ are, respectively, the charge density, bulk velocity and temperature associated with the limiting fluctuations $g^{ \pm}$. 
Proof. We start from the decomposition

$$
\begin{aligned}
h_{\epsilon} & =\hat{h}_{\epsilon}+\frac{1}{4}\left[\left|\hat{g}_{\epsilon}^{+}\right|^{2}-\left|\hat{g}_{\epsilon}^{-}\right|^{2}-\int_{\mathbb{R}^{3}}\left(\left|\hat{g}_{\epsilon}^{+}\right|^{2}-\left|\hat{g}_{\epsilon}^{-}\right|^{2}\right) M d v\right] \\
& =\hat{h}_{\epsilon}+\frac{1}{4}\left[\left(\hat{g}_{\epsilon}^{+}-\hat{g}_{\epsilon}^{-}-\hat{n}_{\epsilon}\right)\left(\hat{g}_{\epsilon}^{+}+\hat{g}_{\epsilon}^{-}\right)+\hat{n}_{\epsilon}\left(\hat{g}_{\epsilon}^{+}+\hat{g}_{\epsilon}^{-}\right)\right] \\
& -\frac{1}{4}\left[\int_{\mathbb{R}^{3}}\left(\hat{g}_{\epsilon}^{+}-\hat{g}_{\epsilon}^{-}-\hat{n}_{\epsilon}\right)\left(\hat{g}_{\epsilon}^{+}+\hat{g}_{\epsilon}^{-}\right) M d v+\hat{n}_{\epsilon}\left(\hat{\rho}_{\epsilon}^{+}+\hat{\rho}_{\epsilon}^{-}\right)\right],
\end{aligned}
$$

which follows from the decomposition (5.5) of fluctuations. In order to apply the compactness results from Lemmas 5.14 and 7.11 , we consider the following renormalization of the above decomposition :

$$
\begin{aligned}
\frac{h_{\epsilon}}{R_{\epsilon}}=\frac{\hat{h}_{\epsilon}}{R_{\epsilon}}+\frac{1}{4}\left[\epsilon \frac{\hat{h}_{\epsilon}}{R_{\epsilon}}\left(\hat{g}_{\epsilon}^{+}+\hat{g}_{\epsilon}^{-}\right)+\frac{\hat{n}_{\epsilon}}{R_{\epsilon}}\left(\hat{g}_{\epsilon}^{+}+\hat{g}_{\epsilon}^{-}\right)\right] \\
-\frac{1}{4}\left[\epsilon \int_{\mathbb{R}^{3}} \frac{\hat{h}_{\epsilon}}{R_{\epsilon}}\left(\hat{g}_{\epsilon}^{+}+\hat{g}_{\epsilon}^{-}\right) M d v+\frac{\hat{n}_{\epsilon}}{R_{\epsilon}}\left(\hat{\rho}_{\epsilon}^{+}+\hat{\rho}_{\epsilon}^{-}\right)\right],
\end{aligned}
$$

where we have written $R_{\epsilon}=1+\left\|\hat{g}_{\epsilon}^{+}-\hat{g}_{\epsilon}^{-}\right\|_{L^{2}(M d v)}$, for convenience.

Then, according to Lemmas 5.14 and 7.11 , we have now weakly convergent subsequences

$$
\frac{h_{\epsilon}}{R_{\epsilon}} \rightarrow \frac{h}{1+|n|} \quad \text { in } w-L_{\mathrm{loc}}^{1}\left(d t d x ; w-L^{1}(M d v)\right),
$$

where $h \in L_{\text {loc }}^{1}\left(d t d x ; L^{1}\left(\left(1+|v|^{2}\right) M d v\right)\right)$, and

$$
\frac{\hat{h}_{\epsilon}}{R_{\epsilon}} \rightarrow \frac{\hat{h}}{1+|n|} \quad \text { in } w-L_{\mathrm{loc}}^{2}\left(d t d x ; w-L^{2}(M d v)\right),
$$

where $\hat{h} \in L_{\text {loc }}^{1}\left(d t d x ; L^{2}(M d v)\right)$. Hence, taking weak limits in 8.42, we find, further utilizing the strong convergence $\frac{\hat{n}_{\epsilon}}{R_{\epsilon}} \rightarrow \frac{n}{1+|n|}$ in $L_{\mathrm{loc}}^{2}(d t d x)$ from Lemma 7.11, that

$$
h=\hat{h}+\frac{1}{2} n\left(u \cdot v+\theta\left(\frac{|v|^{2}}{2}-\frac{3}{2}\right)\right) .
$$

Next, it is readily seen that the elementary decompositions

$$
\begin{aligned}
\mathcal{L}\left(\hat{g}_{\epsilon}^{ \pm}\right) & =\frac{\epsilon}{2} \mathcal{Q}\left(\hat{g}_{\epsilon}^{ \pm}, \hat{g}_{\epsilon}^{ \pm}\right)-\frac{2}{\epsilon} \mathcal{Q}\left(\sqrt{G_{\epsilon}^{ \pm}}, \sqrt{G_{\epsilon}^{ \pm}}\right), \\
\mathcal{L}\left(\hat{g}_{\epsilon}^{ \pm}, \hat{g}_{\epsilon}^{\mp}\right) & =\frac{\epsilon}{2} \mathcal{Q}\left(\hat{g}_{\epsilon}^{ \pm}, \hat{g}_{\epsilon}^{\mp}\right)-\frac{2}{\epsilon} \mathcal{Q}\left(\sqrt{G_{\epsilon}^{ \pm}}, \sqrt{G_{\epsilon}^{\mp}}\right),
\end{aligned}
$$

yield that

$$
\begin{aligned}
\mathcal{L}\left(\hat{h}_{\epsilon}\right) & =\frac{1}{2}\left[\mathcal{Q}\left(\hat{g}_{\epsilon}^{+}, \hat{g}_{\epsilon}^{+}\right)-\mathcal{Q}\left(\hat{g}_{\epsilon}^{-}, \hat{g}_{\epsilon}^{-}\right)\right] \\
& -\frac{2}{\epsilon^{2}}\left[\mathcal{Q}\left(\sqrt{G_{\epsilon}^{+}}, \sqrt{G_{\epsilon}^{+}}\right)-\mathcal{Q}\left(\sqrt{G_{\epsilon}^{-}}, \sqrt{G_{\epsilon}^{-}}\right)\right] \\
& =\frac{1}{2}\left[\mathcal{Q}\left(\hat{g}_{\epsilon}^{+}-\hat{g}_{\epsilon}^{-}-\hat{n}_{\epsilon}, \hat{g}_{\epsilon}^{+}\right)+\mathcal{Q}\left(\hat{g}_{\epsilon}^{-}, \hat{g}_{\epsilon}^{+}-\hat{g}_{\epsilon}^{-}-\hat{n}_{\epsilon}\right)\right] \\
& +\frac{1}{2} \hat{n}_{\epsilon}\left[\mathcal{Q}\left(1, \hat{g}_{\epsilon}^{+}\right)+\mathcal{Q}\left(\hat{g}_{\epsilon}^{-}, 1\right)\right]-\int_{\mathbb{R}^{3} \times \mathbb{S}^{2}}\left(\hat{q}_{\epsilon}^{+}-\hat{q}_{\epsilon}^{-}\right) M_{*} d v_{*} d \sigma
\end{aligned}
$$


and

$$
\begin{aligned}
\mathfrak{L}\left(\hat{h}_{\epsilon}\right) & =\frac{1}{2}\left[\mathcal{Q}\left(\hat{g}_{\epsilon}^{+}, \hat{g}_{\epsilon}^{-}\right)-\mathcal{Q}\left(\hat{g}_{\epsilon}^{-}, \hat{g}_{\epsilon}^{+}\right)\right] \\
& -\frac{2}{\epsilon^{2}}\left[\mathcal{Q}\left(\sqrt{G_{\epsilon}^{+}}, \sqrt{G_{\epsilon}^{-}}\right)-\mathcal{Q}\left(\sqrt{G_{\epsilon}^{-}}, \sqrt{G_{\epsilon}^{+}}\right)\right] \\
& =\frac{1}{2}\left[\mathcal{Q}\left(\hat{g}_{\epsilon}^{+}-\hat{g}_{\epsilon}^{-}-\hat{n}_{\epsilon}, \hat{g}_{\epsilon}^{-}\right)-\mathcal{Q}\left(\hat{g}_{\epsilon}^{-}, \hat{g}_{\epsilon}^{+}-\hat{g}_{\epsilon}^{-}-\hat{n}_{\epsilon}\right)\right] \\
& +\frac{1}{2} \hat{n}_{\epsilon}\left[\mathcal{Q}\left(1, \hat{g}_{\epsilon}^{-}\right)-\mathcal{Q}\left(\hat{g}_{\epsilon}^{-}, 1\right)\right]-\int_{\mathbb{R}^{3} \times \mathbb{S}^{2}}\left(\hat{q}_{\epsilon}^{+,-}-\hat{q}_{\epsilon}^{-,+}\right) M_{*} d v_{*} d \sigma .
\end{aligned}
$$

We also have the simple decomposition

$$
\begin{aligned}
\hat{q}_{\epsilon}^{+}+\hat{q}_{\epsilon}^{-}-\hat{q}_{\epsilon}^{+,-}-\hat{q}_{\epsilon}^{-,+} & =\frac{1}{2}\left(\hat{g}_{\epsilon}^{+}-\hat{g}_{\epsilon}^{-}\right)^{\prime}\left(\hat{g}_{\epsilon}^{+}-\hat{g}_{\epsilon}^{-}\right)_{*}^{\prime}-\frac{1}{2}\left(\hat{g}_{\epsilon}^{+}-\hat{g}_{\epsilon}^{-}\right)\left(\hat{g}_{\epsilon}^{+}-\hat{g}_{\epsilon}^{-}\right)_{*} \\
& =\frac{\epsilon}{2}\left(\hat{g}_{\epsilon}^{+}-\hat{g}_{\epsilon}^{-}\right)^{\prime} \hat{h}_{\epsilon *}^{\prime}-\frac{\epsilon}{2}\left(\hat{g}_{\epsilon}^{+}-\hat{g}_{\epsilon}^{-}\right) \hat{h}_{\epsilon *}+\frac{\epsilon}{2} \hat{h}_{\epsilon}^{\prime} \hat{n}_{\epsilon}-\frac{\epsilon}{2} \hat{h}_{\epsilon} \hat{n}_{\epsilon} .
\end{aligned}
$$

As previously, we renormalize the above identities into

$$
\begin{aligned}
\mathcal{L}\left(\frac{\hat{h}_{\epsilon}}{R_{\epsilon}}\right) & =\frac{\epsilon}{2}\left[\mathcal{Q}\left(\frac{\hat{h}_{\epsilon}}{R_{\epsilon}}, \hat{g}_{\epsilon}^{+}\right)+\mathcal{Q}\left(\hat{g}_{\epsilon}^{-}, \frac{\hat{h}_{\epsilon}}{R_{\epsilon}}\right)\right] \\
& +\frac{\hat{n}_{\epsilon}}{2 R_{\epsilon}}\left[\mathcal{Q}\left(1, \hat{g}_{\epsilon}^{+}\right)+\mathcal{Q}\left(\hat{g}_{\epsilon}^{-}, 1\right)\right]-\frac{1}{R_{\epsilon}} \int_{\mathbb{R}^{3} \times \mathbb{S}^{2}}\left(\hat{q}_{\epsilon}^{+}-\hat{q}_{\epsilon}^{-}\right) M_{*} d v_{*} d \sigma, \\
\mathfrak{L}\left(\frac{\hat{h}_{\epsilon}}{R_{\epsilon}}\right) & =\frac{\epsilon}{2}\left[\mathcal{Q}\left(\frac{\hat{h}_{\epsilon}}{R_{\epsilon}}, \hat{g}_{\epsilon}^{-}\right)-\mathcal{Q}\left(\hat{g}_{\epsilon}^{-}, \frac{\hat{h}_{\epsilon}}{R_{\epsilon}}\right)\right] \\
& +\frac{\hat{n}_{\epsilon}}{2 R_{\epsilon}}\left[\mathcal{Q}\left(1, \hat{g}_{\epsilon}^{-}\right)-\mathcal{Q}\left(\hat{g}_{\epsilon}^{-}, 1\right)\right]-\frac{1}{R_{\epsilon}} \int_{\mathbb{R}^{3} \times \mathbb{S}^{2}}\left(\hat{q}_{\epsilon}^{+,-}-\hat{q}_{\epsilon}^{-,+}\right) M_{*} d v_{*} d \sigma,
\end{aligned}
$$

and

$$
\begin{aligned}
\frac{1}{R_{\epsilon}}\left(\hat{q}_{\epsilon}^{+}+\hat{q}_{\epsilon}^{-}-\hat{q}_{\epsilon}^{+,-}-\hat{q}_{\epsilon}^{-,+}\right) & =\frac{\epsilon}{2}\left(\hat{g}_{\epsilon}^{+}-\hat{g}_{\epsilon}^{-}\right)^{\prime}\left(\frac{\hat{h}_{\epsilon}}{R_{\epsilon}}\right)_{*}^{\prime}-\frac{\epsilon}{2}\left(\hat{g}_{\epsilon}^{+}-\hat{g}_{\epsilon}^{-}\right)\left(\frac{\hat{h}_{\epsilon}}{R_{\epsilon}}\right)_{*} \\
& +\frac{\epsilon}{2}\left(\frac{\hat{h}_{\epsilon}}{R_{\epsilon}}\right)^{\prime} \hat{n}_{\epsilon}-\frac{\epsilon}{2} \frac{\hat{h}_{\epsilon}}{R_{\epsilon}} \hat{n}_{\epsilon} .
\end{aligned}
$$

Finally, taking weak limits, we find, utilizing again the strong convergence from Lemma 7.11, that

$$
\begin{aligned}
\mathcal{L}(\hat{h}) & =-\int_{\mathbb{R}^{3} \times \mathbb{S}^{2}}\left(q^{+}-q^{-}\right) M_{*} d v_{*} d \sigma \\
\mathfrak{L}(\hat{h}) & =\frac{1}{2} n u \cdot[\mathcal{Q}(1, v)-\mathcal{Q}(v, 1)]+\frac{1}{4} n \theta\left[\mathcal{Q}\left(1,|v|^{2}\right)-\mathcal{Q}\left(|v|^{2}, 1\right)\right] \\
& -\int_{\mathbb{R}^{3} \times \mathbb{S}^{2}}\left(q^{+,-}-q^{-,+}\right) M_{*} d v_{*} d \sigma \\
& =\frac{1}{2} n u \cdot \mathfrak{L}(v)+\frac{1}{4} n \theta \mathfrak{L}\left(|v|^{2}\right)-\int_{\mathbb{R}^{3} \times \mathbb{S}^{2}}\left(q^{+,-}-q^{-,+}\right) M_{*} d v_{*} d \sigma,
\end{aligned}
$$

and

which yields, in view of 8.43 , that

$$
\mathcal{L}(h)=-\int_{\mathbb{R}^{3} \times \mathbb{S}^{2}}\left(q^{+}-q^{-}\right) M_{*} d v_{*} d \sigma,
$$

and

$$
\mathfrak{L}(h)=n u \cdot \mathfrak{L}(v)+\frac{1}{2} n \theta \mathfrak{L}\left(|v|^{2}\right)-\int_{\mathbb{R}^{3} \times \mathbb{S}^{2}}\left(q^{+,-}-q^{-,+}\right) M_{*} d v_{*} d \sigma,
$$


and concludes the proof of the proposition.

As a direct consequence of the previous propositions, we derive in the next result Ohm's law and the internal electric energy constraint from 4.37).

Proposition 8.5. Let $\left(f_{\epsilon}^{ \pm}, E_{\epsilon}, B_{\epsilon}\right)$ be the sequence of renormalized solutions to the scaled two species Vlasov-Maxwell-Boltzmann system (4.34) considered in Theorem 4.6 for strong interspecies interactions, i.e. $\delta=1$. In accordance with Lemmas 5.1. 5.2, 5.13, 5.14 and 7.11 denote by

$$
\begin{gathered}
g^{ \pm} \in L^{\infty}\left(d t ; L^{2}(M d x d v)\right), \quad h \in L_{\mathrm{loc}}^{1}\left(d t d x ; L^{1}\left(\left(1+|v|^{2}\right) M d v\right)\right) \\
\text { and } E, B \in L^{\infty}\left(d t ; L^{2}(d x)\right),
\end{gathered}
$$

any joint limit points of the families $\hat{g}_{\epsilon}^{ \pm}$and $h_{\epsilon}$ defined by (5.3) and (5.29), $E_{\epsilon}$ and $B_{\epsilon}$, respectively.

Then, one has

$$
j-n u=\sigma\left(-\frac{1}{2} \nabla_{x}\left(\rho^{+}-\rho^{-}\right)+E+u \wedge B\right) \quad \text { and } \quad w=n \theta
$$

where $\rho^{ \pm}, u$ and $\theta$ are, respectively, the densities, bulk velocity and temperature associated with the limiting fluctuations $g^{ \pm}, j$ and $w$ are, respectively, the electric current and the internal electric energy associated with the limiting fluctuation $h$ and the electric conductivity $\sigma>0$ is defined by 2.65.

Proof. By Proposition 8.3 , we have that

$$
\begin{aligned}
\int_{\mathbb{R}^{3} \times \mathbb{R}^{3} \times \mathbb{S}^{2}}\left(q^{+}-q^{-}+q^{+,-}-q^{-,+}\right) & \tilde{\Phi} M M_{*} d v d v_{*} d \sigma \\
& =\sigma\left(\frac{1}{2} \nabla_{x}\left(\rho^{+}-\rho^{-}\right)-(E+u \wedge B)\right) \\
\int_{\mathbb{R}^{3} \times \mathbb{R}^{3} \times \mathbb{S}^{2}}\left(q^{+}-q^{-}+q^{+,-}-q^{-,+}\right) & \tilde{\Psi} M M_{*} d v d v_{*} d \sigma \\
& =0
\end{aligned}
$$

where we have used the identity (2.64) and $\tilde{\Phi}$ and $\tilde{\Psi}$ are defined by (2.63). Then, further incorporating identities (8.38) and (8.39) from Proposition 8.4 into the above relations yields that

$$
\begin{aligned}
\int_{\mathbb{R}^{3}}\left(n u \cdot \mathfrak{L}(v)+n \theta \mathfrak{L}\left(\frac{|v|^{2}}{2}\right)-(\mathcal{L}+\mathfrak{L})(h)\right) \tilde{\Phi} M d v & \\
= & \sigma\left(\frac{1}{2} \nabla_{x}\left(\rho^{+}-\rho^{-}\right)-(E+u \wedge B)\right), \\
\int_{\mathbb{R}^{3}}\left(n u \cdot \mathfrak{L}(v)+n \theta \mathfrak{L}\left(\frac{|v|^{2}}{2}\right)-(\mathcal{L}+\mathfrak{L})(h)\right) \tilde{\Psi} M d v & =0 .
\end{aligned}
$$


Finally, using 2.63 and the self-adjointness of $\mathcal{L}+\mathfrak{L}$, we deduce that

$$
\begin{aligned}
n u-j & =\int_{\mathbb{R}^{3}}(n u \cdot \Phi+n \theta \Psi-h) \Phi M d v \\
& =\int_{\mathbb{R}^{3}}(\mathcal{L}+\mathfrak{L})(n u \cdot \Phi+n \theta \Psi-h) \tilde{\Phi} M d v \\
& =\int_{\mathbb{R}^{3}}\left(n u \cdot \mathfrak{L}(v)+n \theta \mathfrak{L}\left(\frac{|v|^{2}}{2}\right)-(\mathcal{L}+\mathfrak{L})(h)\right) \tilde{\Phi} M d v \\
& =\sigma\left(\frac{1}{2} \nabla_{x}\left(\rho^{+}-\rho^{-}\right)-(E+u \wedge B)\right), \\
\frac{3}{2}(n \theta-w) & =\int_{\mathbb{R}^{3}}(n u \cdot \Phi+n \theta \Psi-h) \Psi M d v \\
& =\int_{\mathbb{R}^{3}}(\mathcal{L}+\mathfrak{L})(n u \cdot \Phi+n \theta \Psi-h) \tilde{\Psi} M d v \\
& =\int_{\mathbb{R}^{3}}\left(n u \cdot \mathfrak{L}(v)+n \theta \mathfrak{L}\left(\frac{|v|^{2}}{2}\right)-(\mathcal{L}+\mathfrak{L})(h)\right) \tilde{\Psi} M d v \\
& =0,
\end{aligned}
$$

which concludes the proof of the proposition.

\subsection{Energy inequalities}

In view of the results from Section 8.2 , we are now able to establish the limiting energy inequality for two species in the case of strong interactions.

Proposition 8.6. Let $\left(f_{\epsilon}^{ \pm}, E_{\epsilon}, B_{\epsilon}\right)$ be the sequence of renormalized solutions to the scaled two species Vlasov-Maxwell-Boltzmann system (4.34) considered in Theorem 4.6 for strong interspecies interactions, i.e. $\delta=1$. In accordance with Lemmas 5.1, 5.2, 5.3 and 7.11, denote by

$$
\begin{gathered}
g^{ \pm} \in L^{\infty}\left(d t ; L^{2}(M d x d v)\right), \quad h \in L_{\text {loc }}^{1}\left(d t d x ; L^{1}\left(\left(1+|v|^{2}\right) M d v\right)\right) \\
\text { and } \quad q^{ \pm}, q^{ \pm, \mp} \in L^{2}\left(M M_{*} d t d x d v d v_{*} d \sigma\right)
\end{gathered}
$$

any joint limit points of the families $\hat{g}_{\epsilon}^{ \pm}, h_{\epsilon}, \hat{q}_{\epsilon}^{ \pm}$and $\hat{q}_{\epsilon}^{ \pm, \mp}$ defined by (5.3), (5.29) and (5.6), respectively, and by

$$
E, B \in L^{\infty}\left(d t ; L^{2}(d x)\right)
$$

any joint limit points of the families $E_{\epsilon}$ and $B_{\epsilon}$, respectively.

Then, one has the energy inequality, for almost every $t \geq 0$,

$$
\begin{aligned}
& \frac{1}{2}\left(\frac{1}{2}\|n\|_{L_{x}^{2}}^{2}+2\|u\|_{L_{x}^{2}}^{2}+5\|\theta\|_{L_{x}^{2}}^{2}+\|E\|_{L_{x}^{2}}^{2}+\|B\|_{L_{x}^{2}}^{2}\right)(t) \\
& +\int_{0}^{t}\left(2 \mu\left\|\nabla_{x} u\right\|_{L_{x}^{2}}^{2}+5 \kappa\left\|\nabla_{x} \theta\right\|_{L_{x}^{2}}^{2}+\frac{1}{\sigma}\|j-n u\|_{L_{x}^{2}}^{2}+\frac{9}{8 \lambda}\|w-n \theta\|_{L_{x}^{2}}^{2}\right)(s) d s \\
& \leq C^{\text {in }}
\end{aligned}
$$

where $\rho^{ \pm}, u$ and $\theta$ are, respectively, the densities, bulk velocity and temperature associated with the limiting fluctuations $g^{ \pm}$and the charge density is given by $n=$ $\rho^{+}-\rho^{-}$, while $j$ and $w$ are, respectively, the electric current and the internal electric energy associated with the limiting fluctuation $h$, and, finally, the viscosity $\mu>0$, thermal conductivity $\kappa>0$, electric conductivity $\sigma>0$ and energy conductivity $\lambda>0$ are respectively defined by 2.56, 2.65 and 2.66. 
Proof. First, by the estimate (5.7) from Lemma 5.3 and the weak sequential lower semi-continuity of convex functionals, we find that, for all $t \geq 0$,

$$
\begin{aligned}
\frac{1}{4} \int_{0}^{t} \int_{\mathbb{R}^{3}} \int_{\mathbb{R}^{3} \times \mathbb{R}^{3} \times \mathbb{S}^{2}} & \left(q^{ \pm}\right)^{2} M M_{*} d v d v_{*} d \sigma d x d s \\
& \leq \liminf _{\epsilon \rightarrow 0} \frac{1}{4} \int_{0}^{t} \int_{\mathbb{R}^{3}} \int_{\mathbb{R}^{3} \times \mathbb{R}^{3} \times \mathbb{S}^{2}}\left(\hat{q}_{\epsilon}^{ \pm}\right)^{2} M M_{*} d v d v_{*} d \sigma d x d s \\
& \leq \liminf _{\epsilon \rightarrow 0} \frac{1}{\epsilon^{4}} \int_{0}^{t} \int_{\mathbb{R}^{3}} D\left(f_{\epsilon}^{ \pm}\right)(s) d x d s,
\end{aligned}
$$

and

$$
\begin{aligned}
\frac{1}{2} \int_{0}^{t} \int_{\mathbb{R}^{3}} \int_{\mathbb{R}^{3} \times \mathbb{R}^{3} \times \mathbb{S}^{2}} & \left(q^{ \pm, \mp}\right)^{2} M M_{*} d v d v_{*} d \sigma d x d s \\
& \leq \liminf _{\epsilon \rightarrow 0} \frac{1}{2} \int_{0}^{t} \int_{\mathbb{R}^{3}} \int_{\mathbb{R}^{3} \times \mathbb{R}^{3} \times \mathbb{S}^{2}}\left(\hat{q}_{\epsilon}^{ \pm, \mp}\right)^{2} M M_{*} d v d v_{*} d \sigma d x d s \\
& \leq \liminf _{\epsilon \rightarrow 0} \frac{1}{\epsilon^{4}} \int_{0}^{t} \int_{\mathbb{R}^{3}} D\left(f_{\epsilon}^{+}, f_{\epsilon}^{-}\right)(s) d x d s
\end{aligned}
$$

which, when combined with Lemma 5.1, yields, passing to the limit in the entropy inequality 4.35, for almost every $t \geq 0$,

$$
\begin{aligned}
& \frac{1}{2} \int_{\mathbb{R}^{3} \times \mathbb{R}^{3}}\left(\left(g^{+}\right)^{2}+\left(g^{-}\right)^{2}\right)(t) M d x d v+\frac{1}{2} \int_{\mathbb{R}^{3}}\left(|E|^{2}+|B|^{2}\right)(t) d x \\
& \quad+\frac{1}{4} \int_{0}^{t} \int_{\mathbb{R}^{3}} \int_{\mathbb{R}^{3} \times \mathbb{R}^{3} \times \mathbb{S}^{2}}\left(\left(q^{+}\right)^{2}+\left(q^{-}\right)^{2}+\left(q^{+,-}\right)^{2}+\left(q^{-,+}\right)^{2}\right) M M_{*} d v d v_{*} d \sigma d x d s \\
& \quad \leq C^{\mathrm{in}}
\end{aligned}
$$

Since, according to Lemmas 5.10 and 5.11 the limiting fluctuations $g^{ \pm}=\rho^{ \pm}+u$. $v+\theta\left(\frac{|v|^{2}}{2}-\frac{3}{2}\right)$ are infinitesimal Maxwellians which differ only by their densities $\rho^{+}$and $\rho^{-}$, we easily compute that, in view of the strong Boussinesq relation $\frac{\rho^{+}+\rho^{-}}{2}+\theta=0$ following from 8.33 ,

$$
\begin{aligned}
\frac{1}{2} \int_{\mathbb{R}^{3}}\left(\left(g^{+}\right)^{2}+\left(g^{-}\right)^{2}\right) M d v & =\frac{\left(\rho^{+}\right)^{2}+\left(\rho^{-}\right)^{2}}{2}+|u|^{2}+\frac{3}{2} \theta^{2} \\
& =\frac{1}{4} n^{2}+|u|^{2}+\frac{5}{2} \theta^{2},
\end{aligned}
$$

where $n=\rho^{+}-\rho^{-}$, which implies

$$
\begin{aligned}
& \left(\frac{1}{4}\|n\|_{L_{x}^{2}}^{2}+\|u\|_{L_{x}^{2}}^{2}+\frac{5}{2}\|\theta\|_{L_{x}^{2}}^{2}+\frac{1}{2}\|E\|_{L_{x}^{2}}^{2}+\frac{1}{2}\|B\|_{L_{x}^{2}}^{2}\right) \\
& +\frac{1}{16} \int_{0}^{t} \int_{\mathbb{R}^{3}} \int_{\mathbb{R}^{3} \times \mathbb{R}^{3} \times \mathbb{S}^{2}}\left(q^{+}+q^{+,-}+q^{-}+q^{-,+}\right)^{2} M M_{*} d v d v_{*} d \sigma d x d s \\
& +\frac{1}{8} \int_{0}^{t} \int_{\mathbb{R}^{3}} \int_{\mathbb{R}^{3} \times \mathbb{R}^{3} \times \mathbb{S}^{2}}\left(\left(q^{+}-q^{-}\right)^{2}+\left(q^{+,-}-q^{-,+}\right)^{2}\right) M M_{*} d v d v_{*} d \sigma d x d s \\
& \leq C^{\mathrm{in}}
\end{aligned}
$$

where we have used the identity 8.40 .

There only remains to evaluate the contribution of the entropy dissipation in 8.46). To this end, applying the method of proof of Proposition 6.4 based on the Bessel inequality (6.16) (where $\mu$ and $\kappa$ are now defined by 2.56 with $\delta=1$ 
instead of (2.27), which introduces a factor 2 in (6.16), with the constraints 8.31 and 8.33 from Proposition 8.3, note that it holds

$$
\begin{aligned}
& \int_{0}^{t}\left(2 \mu\left\|\nabla_{x} u\right\|_{L_{x}^{2}}^{2}+5 \kappa\left\|\nabla_{x} \theta\right\|_{L_{x}^{2}}^{2}\right)(s) d s \\
& \quad \leq \frac{1}{16} \int_{0}^{t} \int_{\mathbb{R}^{3}} \int_{\mathbb{R}^{3} \times \mathbb{R}^{3} \times \mathbb{S}^{2}}\left(q^{+}+q^{+,-}+q^{-}+q^{-,+}\right)^{2} M M_{*} d v d v_{*} d \sigma d x d s
\end{aligned}
$$

Next, the remaining contributions in the entropy dissipation will be evaluated through a direct application of the following Bessel inequality :

$$
\begin{aligned}
& \frac{8}{\sigma}\left|\int_{\mathbb{R}^{3} \times \mathbb{R}^{3} \times \mathbb{S}^{2}}\left(q^{+}+q^{+,-}-q^{-}-q^{-,+}\right) \tilde{\Phi} M M_{*} d v d v_{*} d \sigma\right|^{2} \\
& \quad+\frac{4}{\lambda}\left(\int_{\mathbb{R}^{3} \times \mathbb{R}^{3} \times \mathbb{S}^{2}}\left(q^{+}+q^{+,-}-q^{-}-q^{-,+}\right) \tilde{\Psi} M M_{*} d v d v_{*} d \sigma\right)^{2} \\
& \quad \leq \int_{\mathbb{R}^{3} \times \mathbb{R}^{3} \times \mathbb{S}^{2}}\left(\left(q^{+}-q^{-}\right)^{2}+\left(q^{+,-}-q^{-,+}\right)^{2}\right) M M_{*} d v d v_{*} d \sigma
\end{aligned}
$$

where $\tilde{\Phi}$ and $\tilde{\Psi}$ are defined by 2.63 .

For the sake of completeness, we provide a short justification of (8.48) below. But prior to this, let us conclude the proof of the present proposition. To this end, we employ the identities 8.38 and 8.39 from Proposition 8.4 in combination with the relations 2.63) and the self-adjointness of $\mathcal{L}+\mathfrak{L}$ to deduce from the inequality (8.48) that

$$
\begin{aligned}
\frac{8}{\sigma}|j-n u|^{2}+ & \frac{9}{\lambda}(w-n \theta)^{2} \\
& \leq \int_{\mathbb{R}^{3} \times \mathbb{R}^{3} \times \mathbb{S}^{2}}\left(\left(q^{+}-q^{-}\right)^{2}+\left(q^{+,-}-q^{-,+}\right)^{2}\right) M M_{*} d v d v_{*} d \sigma .
\end{aligned}
$$

Combining this with 8.46 and 8.47 concludes the proof of the proposition.

Now, as announced above, we give a short proof of 8.48 . To this end, for any vector $A \in \mathbb{R}^{3}$ and any scalar $a \in \mathbb{R}$, one computes straightforwardly, employing the identities (2.64) and (2.66), and the collisional symmetries, that

$$
\begin{aligned}
& \int_{\mathbb{R}^{3} \times \mathbb{R}^{3} \times \mathbb{S}^{2}}\left|\left(\begin{array}{c}
A \cdot\left(\tilde{\Phi}+\tilde{\Phi}_{*}-\tilde{\Phi}^{\prime}-\tilde{\Phi}_{*}^{\prime}\right)+a\left(\tilde{\Psi}+\tilde{\Psi}_{*}-\tilde{\Psi}^{\prime}-\tilde{\Psi}_{*}^{\prime}\right) \\
A \cdot\left(\tilde{\Phi}-\tilde{\Phi}_{*}-\tilde{\Phi}^{\prime}+\tilde{\Phi}_{*}^{\prime}\right)+a\left(\tilde{\Psi}-\tilde{\Psi}_{*}-\tilde{\Psi}^{\prime}+\tilde{\Psi}_{*}^{\prime}\right)
\end{array}\right)\right|^{2} M M_{*} d v d v_{*} d \sigma \\
& =\int_{\mathbb{R}^{3} \times \mathbb{R}^{3} \times \mathbb{S}^{2}}\left(\left|A \cdot\left(\tilde{\Phi}+\tilde{\Phi}_{*}-\tilde{\Phi}^{\prime}-\tilde{\Phi}_{*}^{\prime}\right)\right|^{2}+\left|A \cdot\left(\tilde{\Phi}-\tilde{\Phi}_{*}-\tilde{\Phi}^{\prime}+\tilde{\Phi}_{*}^{\prime}\right)\right|^{2}\right. \\
& \left.+\left|a\left(\tilde{\Psi}+\tilde{\Psi}_{*}-\tilde{\Psi}^{\prime}-\tilde{\Psi}_{*}^{\prime}\right)\right|^{2}+\left|a\left(\tilde{\Psi}-\tilde{\Psi}_{*}-\tilde{\Psi}^{\prime}+\tilde{\Psi}_{*}^{\prime}\right)\right|^{2}\right) M M_{*} d v d v_{*} d \sigma \\
& =4(A \otimes A): \int_{\mathbb{R}^{3}}(\Phi \otimes \tilde{\Phi}) M d v+4 a^{2} \int_{\mathbb{R}^{3}}(\Psi \tilde{\Psi}) M d v \\
& =2 \sigma A \cdot A+4 \lambda a^{2} .
\end{aligned}
$$

Therefore, defining, for any $q_{0}, q_{1} \in L^{2}\left(M M_{*} d v d v_{*} d \sigma\right)$, the projection

$$
\left(\begin{array}{c}
\bar{q}_{0} \\
\bar{q}_{1}
\end{array}\right)=\left(\begin{array}{l}
A_{0} \cdot\left(\begin{array}{l}
\tilde{\Phi}+\tilde{\Phi}_{*}-\tilde{\Phi}^{\prime}-\tilde{\Phi}_{*}^{\prime} \\
A_{0} \cdot\left(\tilde{\Phi}-\tilde{\Phi}_{*}-\tilde{\Phi}^{\prime}+\tilde{\Phi}_{*}^{\prime}\right.
\end{array}\right)+a_{0}\left(\tilde{\Psi}+\tilde{\Psi}_{*}-\tilde{\Psi}_{*}^{\prime}-\tilde{\Psi}_{*}^{\prime}-\tilde{\Psi}^{\prime}+\tilde{\Psi}_{*}^{\prime}\right.
\end{array}\right)
$$


where

$$
\begin{aligned}
A_{0} & =\frac{1}{2 \sigma} \int_{\mathbb{R}^{3} \times \mathbb{R}^{3} \times \mathbb{S}^{2}} q_{0}\left(\tilde{\Phi}+\tilde{\Phi}_{*}-\tilde{\Phi}^{\prime}-\tilde{\Phi}_{*}^{\prime}\right) M M_{*} d v d v_{*} d \sigma \\
& +\frac{1}{2 \sigma} \int_{\mathbb{R}^{3} \times \mathbb{R}^{3} \times \mathbb{S}^{2}} q_{1}\left(\tilde{\Phi}-\tilde{\Phi}_{*}-\tilde{\Phi}^{\prime}+\tilde{\Phi}_{*}^{\prime}\right) M M_{*} d v d v_{*} d \sigma, \\
a_{0} & =\frac{1}{4 \lambda} \int_{\mathbb{R}^{3} \times \mathbb{R}^{3} \times \mathbb{S}^{2}} q_{0}\left(\tilde{\Psi}+\tilde{\Psi}_{*}-\tilde{\Psi}^{\prime}-\tilde{\Psi}_{*}^{\prime}\right) M M_{*} d v d v_{*} d \sigma \\
& +\frac{1}{4 \lambda} \int_{\mathbb{R}^{3} \times \mathbb{R}^{3} \times \mathbb{S}^{2}} q_{1}\left(\tilde{\Psi}-\tilde{\Psi}_{*}-\tilde{\Psi}^{\prime}+\tilde{\Psi}_{*}^{\prime}\right) M M_{*} d v d v_{*} d \sigma,
\end{aligned}
$$

we find that

$$
\begin{aligned}
\int_{\mathbb{R}^{3} \times \mathbb{R}^{3} \times \mathbb{S}^{2}}\left(\begin{array}{c}
q_{0} \\
q_{1}
\end{array}\right) \cdot\left(\begin{array}{c}
\bar{q}_{0} \\
\bar{q}_{1}
\end{array}\right) M M_{*} d v d v_{*} d \sigma & =2 \sigma A_{0} \cdot A_{0}+4 \lambda a_{0}^{2} \\
& =\int_{\mathbb{R}^{3} \times \mathbb{R}^{3} \times \mathbb{S}^{2}}\left|\left(\begin{array}{c}
\bar{q}_{0} \\
\bar{q}_{1}
\end{array}\right)\right|^{2} M M_{*} d v d v_{*} d \sigma .
\end{aligned}
$$

Hence the Bessel inequality

$$
\begin{aligned}
2 \sigma A_{0} \cdot A_{0}+4 \lambda a_{0}^{2} & =\int_{\mathbb{R}^{3} \times \mathbb{R}^{3} \times \mathbb{S}^{2}}\left|\left(\begin{array}{c}
\bar{q}_{0} \\
\bar{q}_{1}
\end{array}\right)\right|^{2} M M_{*} d v d v_{*} d \sigma \\
& \leq \int_{\mathbb{R}^{3} \times \mathbb{R}^{3} \times \mathbb{S}^{2}}\left|\left(\begin{array}{c}
q_{0} \\
q_{1}
\end{array}\right)\right|^{2} M M_{*} d v d v_{*} d \sigma .
\end{aligned}
$$

Therefore, setting $q_{0}=q^{+}-q^{-}$and $q_{1}=q^{+,-}-q^{-,+}$in 8.50, we find, exploiting the collisional symmetries of $q^{ \pm}$and $q^{ \pm, \mp}$, that

$$
\begin{aligned}
& \frac{8}{\sigma}\left|\int_{\mathbb{R}^{3} \times \mathbb{R}^{3} \times \mathbb{S}^{2}}\left(q^{+}+q^{+,-}-q^{-}-q^{-,+}\right) \tilde{\Phi} M M_{*} d v d v_{*} d \sigma\right|^{2} \\
& \quad+\frac{4}{\lambda}\left(\int_{\mathbb{R}^{3} \times \mathbb{R}^{3} \times \mathbb{S}^{2}}\left(q^{+}+q^{+,-}-q^{-}-q^{-,+}\right) \tilde{\Psi} M M_{*} d v d v_{*} d \sigma\right)^{2} \\
& \quad \leq \int_{\mathbb{R}^{3} \times \mathbb{R}^{3} \times \mathbb{S}^{2}}\left(\left(q^{+}-q^{-}\right)^{2}+\left(q^{+,-}-q^{-,+}\right)^{2}\right) M M_{*} d v d v_{*} d \sigma,
\end{aligned}
$$

which concludes the justification of 8.48 . 



\section{CHAPTER 9}

\section{Approximate macroscopic equations}

The most difficult part of the asymptotic analysis consists in deriving the evolution equations for the bulk velocity and temperature insofar as they involve a singular limit and nonlinear advection terms. In particular, we expect the situation to be very different according to the asymptotic regime from Theorems 4.4, 4.5 and 4.6 under consideration. Indeed, the corresponding limiting systems, (4.30) and 4.38, respectively, do not enjoy the same stability properties : as explained in Chapter 3, the incompressible quasi-static Navier-Stokes-Fourier-Maxwell-Poisson system 4.30 is weakly stable in the energy space, which is not the case for the twofluid incompressible Navier-Stokes-Fourier-Maxwell system with solenoidal Ohm's law 4.38.

Before focusing on this question of stability, we will first investigate the consistency of the electro-magneto-hydrodynamic approximation. For renormalized solutions (even though we cannot prove their existence, see Section 4.1), it is not known that conservation laws are satisfied.

We therefore have to prove that approximate conservation laws hold and control their conservation defects. However, the uniform bounds established in Chapter 5 are not sufficient to do so :

- In the regime of Theorem 4.4 leading to the incompressible quasi-static Navier-Stokes-Fourier-Maxwell-Poisson system (4.30), we will also use the nonlinear weak compactness contained in Lemma 7.8 .

- In the more singular regimes of Theorems 4.5 and 4.6 leading to the twofluid incompressible Navier-Stokes-Fourier-Maxwell systems with (solenoidal) Ohm's laws 4.37) and 4.38), we are not able to establish such an a priori nonlinear control (compare Lemma 7.8 to the weaker nonlinear compactness statement of Lemma 7.10). The idea is therefore to use a modulated energy (or relative entropy) argument, in the same spirit as the weakstrong stability results of Chapter 3 .

In order to simplify the presentation, we will first detail the decompositions and convergence proof in the one species case of Theorem 4.4, thus enlightening the points where the equi-integrability from Lemma 7.8 is required. We will then explain how to adapt these parts of the proof to the more singular regimes of Theorems 4.5 and 4.6 .

\subsection{Approximate conservation of mass, momentum and energy for one species}

In fact, in Chapter 8, we have already treated a similar singular limit (of lower order, though) in the regime of weak interactions for two species, which led to the derivation of the solenoidal Ohm's law and internal electric energy constraint (see Proposition 8.2). Here, in order to deduce the limiting evolution equations for one species, we are confronted with an even more singular limit and face similar difficulties, which we briefly recall now. 
We have seen in Section 6.1 that it is possible to derive limiting kinetic equations of the type

$$
v \cdot \nabla_{x} g-E \cdot v=\int_{\mathbb{R}^{3} \times \mathbb{S}^{2}} q M_{*} d v_{*} d \sigma,
$$

from 4.27 (see 6.6 in the proof of Proposition 6.1). Here, we intend to take advantage of the symmetries of the collision integrand $q$ to go one order further and, thus, to derive a singular limit. Of course, since we are considering renormalized fluctuations, we do not expect that the integrals in $v$ of the right-hand side of the Vlasov-Boltzmann equation in 4.27) against collision invariants are zero, but they should converge to zero as $\epsilon \rightarrow 0$ provided that we choose some appropriate renormalization which is sufficiently close to the identity. To estimate the ensuing conservation defects, we will also need to truncate large velocities. The precise construction is detailed below and will be essentially the same, later on in Section 9.2, for approximate conservation laws of mass, momentum and energy associated with 4.34.

Note that, even if conservation laws were known to hold for renormalized solutions of 4.27 and 4.34, we would have to introduce similar truncations of large tails and large velocities in order to control uniformly the flux and acceleration terms.

Thus, similarly to the proof of Proposition 8.1. we start from the VlasovBoltzmann equation from 4.27 renormalized with the admissible nonlinearity $\Gamma(z)$ defined by

$$
\Gamma(z)-1=(z-1) \gamma(z),
$$

where $\gamma \in C^{1}([0, \infty) ; \mathbb{R})$ satisfies the following assumptions, for some given $C>0$ :

$$
\begin{aligned}
\gamma(z) & \equiv 1, & & \text { for all } z \in[0,2], \\
\gamma(z) & \rightarrow 0, & & \text { as } z \rightarrow \infty \\
\left|\gamma^{\prime}(z)\right| & \leq \frac{C}{(1+z)^{\frac{3}{2}}}, & & \text { for all } z \in[0, \infty) .
\end{aligned}
$$

Note that necessarily $|\gamma(z)| \leq \frac{2 C}{(1+z)^{\frac{1}{2}}}$.

With the notation $\gamma_{\epsilon}$ for $\gamma\left(G_{\epsilon}\right)$ and $\hat{\gamma}_{\epsilon}$ for $\Gamma^{\prime}\left(G_{\epsilon}\right)$, the scaled Vlasov-Boltzmann equation in (4.27) renormalized relatively to the Maxwellian $M$ with the admissible nonlinearity $\overline{\Gamma(z)}$ reads

$$
\begin{aligned}
\partial_{t}\left(g_{\epsilon} \gamma_{\epsilon}\right)+\frac{1}{\epsilon} v \cdot \nabla_{x}\left(g_{\epsilon} \gamma_{\epsilon}\right)+\left(E_{\epsilon}+v \wedge B_{\epsilon}\right) \cdot \nabla_{v}\left(g_{\epsilon} \gamma_{\epsilon}\right) & -\frac{1}{\epsilon} E_{\epsilon} \cdot v G_{\epsilon} \hat{\gamma}_{\epsilon} \\
& =\frac{1}{\epsilon^{3}} \hat{\gamma}_{\epsilon} \mathcal{Q}\left(G_{\epsilon}, G_{\epsilon}\right)
\end{aligned}
$$

We also introduce a truncation of large velocities $\chi\left(\frac{|v|^{2}}{K_{\epsilon}}\right)$, with $K_{\epsilon}=K|\log \epsilon|$, for some large $K>0$ to be fixed later on, and $\chi \in C_{c}^{\infty}([0, \infty))$ a smooth compactly supported function such that $\mathbb{1}_{[0,1]} \leq \chi \leq \mathbb{1}_{[0,2]}$.

Thus, multiplying each side of the above equation by $\varphi(v) \chi\left(\frac{|v|^{2}}{K_{\epsilon}}\right)$, where $\varphi$ is a collision invariant, and averaging with respect to $M d v$ leads to the approximate conservation laws

$$
\partial_{t} \int_{\mathbb{R}^{3}} g_{\epsilon} \gamma_{\epsilon} \varphi \chi\left(\frac{|v|^{2}}{K_{\epsilon}}\right) M d v+\nabla_{x} \cdot F_{\epsilon}(\varphi)=A_{\epsilon}(\varphi)+D_{\epsilon}(\varphi),
$$

with the notations

$$
F_{\epsilon}(\varphi)=\frac{1}{\epsilon} \int_{\mathbb{R}^{3}} g_{\epsilon} \gamma_{\epsilon} \varphi \chi\left(\frac{|v|^{2}}{K_{\epsilon}}\right) v M d v
$$


for the fluxes,

$$
\begin{aligned}
A_{\epsilon}(\varphi) & =\frac{1}{\epsilon} E_{\epsilon} \cdot \int_{\mathbb{R}^{3}}\left(1+\epsilon g_{\epsilon}\right) \hat{\gamma}_{\epsilon} \varphi \chi\left(\frac{|v|^{2}}{K_{\epsilon}}\right) v M d v \\
& +\int_{\mathbb{R}^{3}} g_{\epsilon} \gamma_{\epsilon}\left(E_{\epsilon}+v \wedge B_{\epsilon}\right) \cdot \nabla_{v}\left(\varphi \chi\left(\frac{|v|^{2}}{K_{\epsilon}}\right) M\right) d v \\
& =\frac{1}{\epsilon} E_{\epsilon} \cdot \int_{\mathbb{R}^{3}} \hat{\gamma}_{\epsilon} \varphi \chi\left(\frac{|v|^{2}}{K_{\epsilon}}\right) v M d v+E_{\epsilon} \cdot \int_{\mathbb{R}^{3}} g_{\epsilon} \hat{\gamma}_{\epsilon} \varphi \chi\left(\frac{|v|^{2}}{K_{\epsilon}}\right) v M d v \\
& +E_{\epsilon} \cdot \int_{\mathbb{R}^{3}} g_{\epsilon} \gamma_{\epsilon} \varphi\left(\frac{2}{K_{\epsilon}} \chi^{\prime}\left(\frac{|v|^{2}}{K_{\epsilon}}\right)-\chi\left(\frac{|v|^{2}}{K_{\epsilon}}\right)\right) v M d v \\
& +\int_{\mathbb{R}^{3}} g_{\epsilon} \gamma_{\epsilon}\left(E_{\epsilon}+v \wedge B_{\epsilon}\right) \cdot\left(\nabla_{v} \varphi\right) \chi\left(\frac{|v|^{2}}{K_{\epsilon}}\right) M d v,
\end{aligned}
$$

for the acceleration terms, and

$$
D_{\epsilon}(\varphi)=\frac{1}{\epsilon^{3}} \int_{\mathbb{R}^{3}} \hat{\gamma}_{\epsilon} \mathcal{Q}\left(G_{\epsilon}, G_{\epsilon}\right) \varphi \chi\left(\frac{|v|^{2}}{K_{\epsilon}}\right) M d v,
$$

for the corresponding conservation defects.

By describing the asymptotic behavior of $F_{\epsilon}(\varphi), A_{\epsilon}(\varphi)$ and $D_{\epsilon}(\varphi)$, we will prove the following consistency result (compare with the formal macroscopic conservation laws 2.14).

Proposition 9.1. Let $\left(f_{\epsilon}, E_{\epsilon}, B_{\epsilon}\right)$ be the sequence of renormalized solutions to the scaled one species Vlasov-Maxwell-Boltzmann system (4.27) considered in Theorem 4.4 and denote by $\tilde{\rho}_{\epsilon}, \tilde{u}_{\epsilon}$ and $\tilde{\theta}_{\epsilon}$ the density, bulk velocity and temperature associated with the renormalized fluctuations $g_{\epsilon} \gamma_{\epsilon} \chi\left(\frac{|v|^{2}}{K_{\epsilon}}\right)$.

Then, one has the approximate conservation laws

$$
\begin{aligned}
\partial_{t} \tilde{\rho}_{\epsilon}+\frac{1}{\epsilon} \nabla_{x} \cdot \tilde{u}_{\epsilon} & =R_{\epsilon, 1}, \\
\partial_{t} \tilde{u}_{\epsilon}+\nabla_{x} \cdot\left(\tilde{u}_{\epsilon} \otimes \tilde{u}_{\epsilon}-\frac{\left|\tilde{u}_{\epsilon}\right|^{2}}{3} \mathrm{Id}\right. & \left.-\int_{\mathbb{R}^{3} \times \mathbb{R}^{3} \times \mathbb{S}^{2}} \hat{q}_{\epsilon} \tilde{\phi} M M_{*} d v d v_{*} d \sigma\right) \\
& =-\frac{1}{\epsilon} \nabla_{x}\left(\tilde{\rho}_{\epsilon}+\tilde{\theta}_{\epsilon}\right)+\frac{1}{\epsilon} E_{\epsilon}+\tilde{\rho}_{\epsilon} E_{\epsilon}+\tilde{u}_{\epsilon} \wedge B_{\epsilon}+R_{\epsilon, 2}, \\
\partial_{t}\left(\frac{3}{2} \tilde{\theta}_{\epsilon}-\tilde{\rho}_{\epsilon}\right)+\nabla_{x} \cdot\left(\frac{5}{2} \tilde{u}_{\epsilon} \tilde{\theta}_{\epsilon}\right. & \left.-\int_{\mathbb{R}^{3} \times \mathbb{R}^{3} \times \mathbb{S}^{2}} \hat{q}_{\epsilon} \tilde{\psi} M M_{*} d v d v_{*} d \sigma\right) \\
& =\tilde{u}_{\epsilon} \cdot E_{\epsilon}+R_{\epsilon, 3},
\end{aligned}
$$

where $\tilde{\phi}$ and $\tilde{\psi}$ are defined by $(2.12)$ and $(2.13)$, and the remainders $R_{\epsilon, i}, i=1,2,3$, converge to 0 in $L_{\mathrm{loc}}^{1}\left(d t ; W_{\mathrm{loc}}^{-1,1}(d x)\right)$.

The proof of Proposition 9.1 consists in three steps respectively devoted to the study of conservation defects, fluxes and acceleration terms in (9.3). It does not present any particular difficulty and relies on refined decompositions of the different terms in the same spirit as the proof of Proposition 8.2 .

For the sake of clarity, these three steps are respectively detailed in Sections 9.1.1, 9.1.2 and 9.1.3, below.

More precisely, Proposition 9.1 will clearly follow from the combination of the approximate conservation laws $(9.3)$ with Lemma 9.2 , which handles the vanishing of conservation defects $D_{\epsilon}(\varphi)$, for any collision invariant $\varphi$, Lemma 9.3 , which establishes the asymptotic behavior of the fluxes $F_{\epsilon}(v)$ and $F_{\epsilon}\left(\frac{|v|^{2}}{2}-\frac{5}{2}\right)$, and Lemma 
9.4 which characterizes the acceleration terms $A_{\epsilon}(1), A_{\epsilon}(v)$ and $A_{\epsilon}\left(\frac{|v|^{2}}{2}-\frac{5}{2}\right)$ as $\epsilon \rightarrow 0$.

In order to easily extend, later on in Section 9.2, the arguments from the present section to the case of two species, we are going to carefully keep track of and emphasize the different points where the equi-integrability property from Lemma 7.8 is used.

9.1.1. Conservation defects. The first step of the proof is to establish the vanishing of conservation defects.

Lemma 9.2. The conservation defects defined by (9.6) converge to zero. More precisely, for any collision invariant $\varphi$,

$$
D_{\epsilon}(\varphi) \rightarrow 0 \text { in } L_{\mathrm{loc}}^{1}(d t d x) \text { as } \epsilon \rightarrow 0 .
$$

Proof. Following the strategy of proof of Proposition 8.1 we introduce a convenient decomposition of $D_{\epsilon}(\varphi)$, for any collision invariant $\varphi$, and then estimate the different terms using the uniform bounds from Lemmas 5.2 and 5.3 (provided by the relative entropy and entropy dissipation), the relaxation estimate (5.11) from Lemma 5.10, as well as the equi-integrability coming from Lemma 7.8

Thus, using (5.8), we decompose $D_{\epsilon}(\varphi)$, taking advantage of collisional symmetries :

$$
\begin{aligned}
D_{\epsilon}(\varphi) & =\frac{\epsilon}{4} \int_{\mathbb{R}^{3} \times \mathbb{R}^{3} \times \mathbb{S}^{2}} \hat{\gamma}_{\epsilon} \varphi \chi\left(\frac{|v|^{2}}{K_{\epsilon}}\right) \hat{q}_{\epsilon}^{2} M M_{*} d v d v_{*} d \sigma \\
& -\frac{1}{\epsilon} \int_{\mathbb{R}^{3} \times \mathbb{R}^{3} \times \mathbb{S}^{2}} \hat{\gamma}_{\epsilon} \varphi\left(1-\chi\left(\frac{|v|^{2}}{K_{\epsilon}}\right)\right) \hat{q}_{\epsilon} \sqrt{G_{\epsilon} G_{\epsilon *}} M M_{*} d v d v_{*} d \sigma \\
& +\frac{1}{\epsilon} \int_{\mathbb{R}^{3} \times \mathbb{R}^{3} \times \mathbb{S}^{2}} \hat{\gamma}_{\epsilon}\left(1-\hat{\gamma}_{\epsilon *}\right) \varphi \hat{q}_{\epsilon} \sqrt{G_{\epsilon} G_{\epsilon *}} M M_{*} d v d v_{*} d \sigma \\
& +\frac{1}{\epsilon} \int_{\mathbb{R}^{3} \times \mathbb{R}^{3} \times \mathbb{S}^{2}} \hat{\gamma}_{\epsilon} \hat{\gamma}_{\epsilon *}\left(1-\hat{\gamma}_{\epsilon}^{\prime} \hat{\gamma}_{\epsilon *}^{\prime}\right) \varphi \hat{q}_{\epsilon} \sqrt{G_{\epsilon} G_{\epsilon *}} M M_{*} d v d v_{*} d \sigma \\
& -\frac{\epsilon}{4} \int_{\mathbb{R}^{3} \times \mathbb{R}^{3} \times \mathbb{S}^{2}} \hat{\gamma}_{\epsilon} \hat{\gamma}_{\epsilon *} \hat{\gamma}_{\epsilon}^{\prime} \hat{\gamma}_{\epsilon *}^{\prime} \varphi \hat{q}_{\epsilon}^{2} M M_{*} d v d v_{*} d \sigma \\
& \stackrel{\text { def }}{=} D_{\epsilon}^{1}(\varphi)+D_{\epsilon}^{2}(\varphi)+D_{\epsilon}^{3}(\varphi)+D_{\epsilon}^{4}(\varphi)+D_{\epsilon}^{5}(\varphi),
\end{aligned}
$$

where we have used that $\varphi$ is a collision invariant to symmetrize the last term.

Now, we show that each term $D_{\epsilon}^{i}(\varphi), i=1, \ldots, 5$, vanishes separately.

- The vanishing of the first term $D_{\epsilon}^{1}(\varphi)$, for any function $\varphi(v)$ growing at most quadratically at infinity, easily follows, using Lemma 5.3, from the estimate

$$
\begin{aligned}
\left\|D_{\epsilon}^{1}(\varphi)\right\|_{L^{1}(d t d x)} & \leq \frac{\epsilon}{4}\left\|\hat{q}_{\epsilon}\right\|_{L^{2}\left(M M_{*} d t d x d v d v_{*} d \sigma\right)}^{2}\left\|\hat{\gamma}_{\epsilon}\right\|_{L^{\infty}}\left\|\chi\left(\frac{|v|^{2}}{K_{\epsilon}}\right) \varphi\right\|_{L^{\infty}} \\
& \leq C \epsilon K_{\epsilon}=C K \epsilon|\log \epsilon| .
\end{aligned}
$$

- The second term $D_{\epsilon}^{2}(\varphi)$ is controlled by estimate 8.8 on the tails of Gaussian distributions. Using the bound from Lemma 5.3 and the pointwise boundedness of $\Gamma^{\prime}(z) \sqrt{z}$, we get indeed, for all $\varphi(v)$ growing at most quadratically at infinity,

$$
\begin{aligned}
\left\|D_{\epsilon}^{2}(\varphi)\right\|_{L_{\mathrm{loc}}^{1}(d t d x)} & \leq \frac{C}{\epsilon}\left\|\hat{q}_{\epsilon}\right\|_{L^{2}\left(M M_{*} d t d x d v d v_{*} d \sigma\right)}\left\|\hat{\gamma}_{\epsilon} \sqrt{G_{\epsilon}}\right\|_{L^{\infty}} \\
& \times\left\|\sqrt{G_{\epsilon}}\right\|_{L_{\mathrm{loc}}^{2}\left(d t d x ; L^{2}(M d v)\right)}\left\|\mathbb{1}_{\left\{|v|^{2} \geq K_{\epsilon}\right\}} \varphi\right\|_{L^{2}(M d v)} \\
& \leq C \epsilon^{\frac{K}{4}-1}|\log \epsilon|^{\frac{5}{4}},
\end{aligned}
$$

which tends to zero as soon as $K>4$. 
- The last term $D_{\epsilon}^{5}(\varphi)$ is mastered using the same tools. For high energies, i.e. when $|v|^{2} \geq K|\log \epsilon|$, we obtain

$$
\begin{aligned}
& D_{\epsilon}^{5>}(\varphi) \\
& \stackrel{\text { def }}{=} \frac{\epsilon}{4} \int_{\mathbb{R}^{3} \times \mathbb{R}^{3} \times \mathbb{S}^{2}} \hat{\gamma}_{\epsilon} \hat{\gamma}_{\epsilon *} \hat{\gamma}_{\epsilon}^{\prime} \hat{\gamma}_{\epsilon *}^{\prime} \varphi \mathbb{1}_{\left\{|v|^{2} \geq K_{\epsilon}\right\}} \hat{q}_{\epsilon}^{2} M M_{*} d v d v_{*} d \sigma \\
& \leq \frac{1}{\epsilon}\left\|\hat{\gamma}_{\epsilon} \sqrt{G_{\epsilon}}\right\|_{L^{\infty}}^{2}\left\|\hat{\gamma}_{\epsilon}\right\|_{L^{\infty}}^{2}\left\|\hat{q}_{\epsilon}\right\|_{L^{2}\left(M M_{*} d v d v_{*} d \sigma\right)}\left\|\varphi \mathbb{1}_{\left\{|v|^{2} \geq K_{\epsilon}\right\}}\right\|_{L^{2}\left(M M_{*} d v d v_{*} d \sigma\right)},
\end{aligned}
$$

so that, using the estimate 8.8 on the tails of Gaussian distributions and the bound on $\hat{q}_{\epsilon}$ from Lemma 5.3 .

$$
D_{\epsilon}^{5>}(\varphi)=O\left(\epsilon^{\frac{K}{4}-1}|\log \epsilon|^{\frac{5}{4}}\right)_{L^{2}(d t d x)}
$$

which tends to zero as soon as $K>4$.

For moderate energies, i.e. when $|v|^{2}<K|\log \epsilon|$, we easily find

$$
\begin{aligned}
D_{\epsilon}^{5<}(\varphi) & \stackrel{\text { def }}{=} \frac{\epsilon}{4} \int_{\mathbb{R}^{3} \times \mathbb{R}^{3} \times \mathbb{S}^{2}} \hat{\gamma}_{\epsilon} \hat{\gamma}_{\epsilon *} \hat{\gamma}_{\epsilon}^{\prime} \hat{\gamma}_{\epsilon *}^{\prime} \varphi \mathbb{1}_{\left\{|v|^{2}<K_{\epsilon}\right\}} \hat{q}_{\epsilon}^{2} M M_{*} d v d v_{*} d \sigma \\
& \leq C K \epsilon|\log \epsilon|\left\|\hat{q}_{\epsilon}\right\|_{L^{2}\left(M M_{*} d v d v_{*} d \sigma\right)}^{2},
\end{aligned}
$$

so that the entropy dissipation bound from Lemma 5.3 provides

$$
D_{\epsilon}^{5<}(\varphi)=O(\epsilon|\log \epsilon|)_{L^{1}(d t d x)} .
$$

- The handling of $D_{\epsilon}^{3}(\varphi)$ requires the equi-integrability coming from Lemma 7.8 , First, one has, by the Cauchy-Schwarz inequality,

$$
\begin{aligned}
& \left|D_{\epsilon}^{3}(\varphi)\right| \\
& \leq \frac{1}{\epsilon}\left\|\hat{q}_{\epsilon}\right\|_{L^{2}\left(M M_{*} d v d v_{*} d \sigma\right)}\left\|\hat{\gamma}_{\epsilon}\left(1-\hat{\gamma}_{\epsilon *}\right) \varphi \sqrt{G_{\epsilon} G_{\epsilon *}}\right\|_{L^{2}\left(M M_{*} d v d v_{*} d \sigma\right)} \\
& \leq C\left\|\hat{q}_{\epsilon}\right\|_{L^{2}\left(M M_{*} d v d v_{*} d \sigma\right)}\left\|\hat{\gamma}_{\epsilon} \sqrt{G_{\epsilon}}\right\|_{L^{\infty}}\left\|\frac{1}{\epsilon}\left(1-\hat{\gamma}_{\epsilon}\right) \sqrt{G_{\epsilon}}\right\|_{L^{2}(M d v)}\|\varphi\|_{L^{2}(M d v)} \\
& \leq C\left\|\hat{q}_{\epsilon}\right\|_{L^{2}\left(M M_{*} d v d v_{*} d \sigma\right)}\left\|\hat{\gamma}_{\epsilon} \sqrt{G_{\epsilon}}\right\|_{L^{\infty}}\left\|\frac{1}{\epsilon}\left(1-\hat{\gamma}_{\epsilon}\right)\right\|_{L^{2}(M d v)}\|\varphi\|_{L^{2}(M d v)}\left\|\hat{\gamma}_{\epsilon} \sqrt{G_{\epsilon}}\right\|_{L^{\infty}}\left\|\left(1-\hat{\gamma}_{\epsilon}\right) \hat{g}_{\epsilon}\right\|_{L^{2}(M d v)}\|\varphi\|_{L^{2}(M d v)} .
\end{aligned}
$$

As the support of $\Gamma^{\prime}(z)-1=\gamma(z)-1+(z-1) \gamma^{\prime}(z)$ is a subset of $[2, \infty)$ and since $G_{\epsilon} \geq 2$ implies that $\epsilon \hat{g}_{\epsilon} \geq 2(\sqrt{2}-1)$, we infer

$$
\left\|D_{\epsilon}^{3}(\varphi)\right\|_{L_{\mathrm{loc}}^{1}(d t d x)} \leq C\left\|\left(1-\hat{\gamma}_{\epsilon}\right) \hat{g}_{\epsilon}\right\|_{L_{\mathrm{loc}}^{2}\left(d t d x ; L^{2}(M d v)\right)} .
$$

Next, from the equi-integrability of $\hat{g}_{\epsilon}^{2}$ (see Lemma 7.8 ) and the fact that $1-\hat{\gamma}_{\epsilon}$ is uniformly bounded in $L^{\infty}$ and converges almost everywhere to zero (possibly up to extraction of a subsequence), we deduce by the Product Limit Theorem that

$$
\left(1-\hat{\gamma}_{\epsilon}\right) \hat{g}_{\epsilon} \rightarrow 0 \text { in } L_{\text {loc }}^{2}\left(d t d x ; L^{2}(M d v)\right)
$$

Thus, we conclude that

$$
D_{\epsilon}^{3}(\varphi) \rightarrow 0 \text { in } L_{\text {loc }}^{1}(d t d x)
$$


- A similar argument provides the convergence of the remaining term $D_{\epsilon}^{4}(\varphi)$. Thus, one has by the Cauchy-Schwarz inequality, for any $2<p<\infty$,

$$
\begin{aligned}
& \left|D_{\epsilon}^{4}(\varphi)\right| \\
& \leq \frac{1}{\epsilon}\left\|\hat{q}_{\epsilon}\right\|_{L^{2}\left(M M_{*} d v d v_{*} d \sigma\right)}\left\|\hat{\gamma}_{\epsilon} \hat{\gamma}_{\epsilon *}\left(1-\hat{\gamma}_{\epsilon}^{\prime} \hat{\gamma}_{\epsilon *}^{\prime}\right) \varphi \sqrt{G_{\epsilon} G_{\epsilon *}}\right\|_{L^{2}\left(M M_{*} d v d v_{*} d \sigma\right)} \\
& \leq C\left\|\hat{q}_{\epsilon}\right\|_{L^{2}\left(M M_{*} d v d v_{*} d \sigma\right)}\left\|\hat{\gamma}_{\epsilon} \sqrt{G_{\epsilon}}\right\|_{L^{\infty}}^{2}\left\|\frac{1}{\epsilon}\left(1-\hat{\gamma}_{\epsilon}^{\prime} \hat{\gamma}_{\epsilon *}^{\prime}\right) \varphi\right\|_{L^{2}\left(M M_{*} d v d v_{*} d \sigma\right)} \\
& \leq C_{p}\left\|\hat{q}_{\epsilon}\right\|_{L^{2}\left(M M_{*} d v d v_{*} d \sigma\right)}\left\|\frac{1}{\epsilon}\left(1-\hat{\gamma}_{\epsilon}\right)\right\|_{L^{p}(M d v)} .
\end{aligned}
$$

Therefore, thanks to the bound on $\hat{q}_{\epsilon}$ from Lemma 5.3. we infer, for any $2<p<\infty$,

$$
\left\|D_{\epsilon}^{4}(\varphi)\right\|_{L_{\mathrm{loc}}^{1}(d t d x)} \leq C\left\|\frac{1}{\epsilon}\left(1-\hat{\gamma}_{\epsilon}\right)\right\|_{L_{\mathrm{loc}}^{2}\left(d t d x ; L^{p}(M d v)\right)} .
$$

Next, the hypotheses 9.1 on $\gamma(z)$ imply that

$$
\left|\frac{1}{\epsilon}\left(1-\hat{\gamma}_{\epsilon}\right)\right| \leq \frac{1}{2(\sqrt{2}-1)}\left|1-\hat{\gamma}_{\epsilon}\right|\left|\hat{g}_{\epsilon}\right| \leq \frac{1}{2(\sqrt{2}-1)}\left|1-\hat{\gamma}_{\epsilon}\right|\left(\left|\Pi \hat{g}_{\epsilon}\right|+\left|\hat{g}_{\epsilon}-\Pi \hat{g}_{\epsilon}\right|\right),
$$

whence

$$
\begin{aligned}
\left|\frac{1}{\epsilon^{2}}\left(1-\hat{\gamma}_{\epsilon}\right)\right| & \leq \frac{1}{2(\sqrt{2}-1) \epsilon}\left|1-\hat{\gamma}_{\epsilon}\right|\left(\left|\Pi \hat{g}_{\epsilon}\right|+\left|\hat{g}_{\epsilon}-\Pi \hat{g}_{\epsilon}\right|\right) \\
& \leq \frac{1}{4(\sqrt{2}-1)^{2}}\left|1-\hat{\gamma}_{\epsilon}\right|\left|\hat{g}_{\epsilon} \Pi \hat{g}_{\epsilon}\right|+\frac{1}{2(\sqrt{2}-1)}\left|1-\hat{\gamma}_{\epsilon}\right| \frac{1}{\epsilon}\left|\hat{g}_{\epsilon}-\Pi \hat{g}_{\epsilon}\right|,
\end{aligned}
$$

which, with the relaxation estimate 5.11 from Lemma 5.10, shows that, for all $1 \leq r<2$,

$$
\frac{1}{\epsilon^{2}}\left(1-\hat{\gamma}_{\epsilon}\right)=O(1)_{L_{\mathrm{loc}}^{1}\left(d t d x ; L^{r}(M d v)\right)} .
$$

Therefore, for every $2 \leq p<4$,

$$
\frac{1}{\epsilon}\left(1-\hat{\gamma}_{\epsilon}\right)=O(1)_{L_{\mathrm{loc}}^{2}\left(d t d x ; L^{p}(M d v)\right)} .
$$

Moreover, from the equi-integrability of $\hat{g}_{\epsilon}^{2}$ and the fact that $1-\hat{\gamma}_{\epsilon}$ is uniformly bounded in $L^{\infty}$ and converges almost everywhere to zero (possibly up to extraction of a subsequence), we deduce by the Product Limit Theorem that

$$
\frac{1}{\epsilon}\left(1-\hat{\gamma}_{\epsilon}\right) \rightarrow 0 \text { in } L_{\text {loc }}^{2}\left(d t d x ; L^{2}(M d v)\right) .
$$

Therefore, by interpolation, we obtain that, for every $2 \leq p<4$,

$$
\frac{1}{\epsilon}\left(1-\hat{\gamma}_{\epsilon}\right) \rightarrow 0 \text { in } L_{\text {loc }}^{2}\left(d t d x ; L^{p}(M d v)\right) .
$$

Thus, we conclude that

$$
D_{\epsilon}^{4}(\varphi) \rightarrow 0 \text { in } L_{\mathrm{loc}}^{1}(d t d x) .
$$

On the whole, we have shown that each term from (9.7) vanishes as $\epsilon \rightarrow 0$ in $L_{\text {loc }}^{1}(d t d x)$, which leads to the expected convergence and concludes the proof of the lemma. 
9.1.2. Decomposition of flux terms. We characterize now the asymptotic behavior of the flux terms.

LEMma 9.3. The flux terms defined by 9.4 satisfy

$$
\begin{array}{r}
F_{\epsilon}(v)-\frac{1}{\epsilon}\left(\tilde{\rho}_{\epsilon}+\tilde{\theta}_{\epsilon}\right) \mathrm{Id}-\left(\tilde{u}_{\epsilon} \otimes \tilde{u}_{\epsilon}-\frac{\left|\tilde{u}_{\epsilon}\right|^{2}}{3} \mathrm{Id}\right)+\int_{\mathbb{R}^{3} \times \mathbb{R}^{3} \times \mathbb{S}^{2}} \hat{q_{\epsilon}} \tilde{\phi} M M_{*} d v d v_{*} d \sigma \rightarrow 0, \\
F_{\epsilon}\left(\frac{|v|^{2}}{2}-\frac{5}{2}\right)-\frac{5}{2} \tilde{u}_{\epsilon} \tilde{\theta}_{\epsilon}+\int_{\mathbb{R}^{3} \times \mathbb{R}^{3} \times \mathbb{S}^{2}} \hat{q}_{\epsilon} \tilde{\psi} M M_{*} d v d v_{*} d \sigma \rightarrow 0,
\end{array}
$$

in $L_{\mathrm{loc}}^{1}(d t d x)$ as $\epsilon \rightarrow 0$, where $\tilde{\phi}, \tilde{\psi} \in L^{2}(M d v)$ are the kinetic momentum and energy fluxes defined by 2.12 and 2.13.

Proof. In order to characterize the asymptotic behavior of fluxes, we use, following the strategy of proof of Proposition 8.1. the linearized version of the Chapman-Enskog decomposition

$$
\hat{g}_{\epsilon}=\Pi \hat{g}_{\epsilon}+\left(\hat{g}_{\epsilon}-\Pi \hat{g}_{\epsilon}\right),
$$

where $\Pi$ is the orthogonal projection onto $\operatorname{Ker} \mathcal{L}$ in $L^{2}(M d v)$ and $\hat{g}_{\epsilon}$ is the renormalized fluctuation. Note, however, that we need here a more refined decomposition than the one used in the proof of Proposition 8.1 as we consider now a more singular limit.

- Notice that, modulo the diagonal term in the momentum flux

$$
\frac{1}{\epsilon} \int_{\mathbb{R}^{3}} g_{\epsilon} \gamma_{\epsilon} \chi\left(\frac{|v|^{2}}{K_{\epsilon}}\right) \frac{|v|^{2}}{3} M d v=\frac{1}{\epsilon}\left(\tilde{\rho}_{\epsilon}+\tilde{\theta}_{\epsilon}\right),
$$

the flux terms have the following structure

$$
\tilde{F}_{\epsilon}(\zeta)=\frac{1}{\epsilon} \int_{\mathbb{R}^{3}} g_{\epsilon} \gamma_{\epsilon} \zeta \chi\left(\frac{|v|^{2}}{K_{\epsilon}}\right) M d v,
$$

where $\zeta \in \operatorname{Ker}(\mathcal{L})^{\perp} \subset L^{2}(M d v)$. Indeed, it is readily seen that the kinetic fluxes $\phi(v)$ and $\psi(v)$, defined by 2.12, are orthogonal to collision invariants.

Furthermore, using the identity (5.5), the fluxes can be rewritten in the following form

$$
\begin{aligned}
\tilde{F}_{\epsilon}(\zeta) & =\frac{1}{4} \int_{\mathbb{R}^{3}} \hat{g}_{\epsilon}^{2} \gamma_{\epsilon} \zeta \chi\left(\frac{|v|^{2}}{K_{\epsilon}}\right) M d v+\frac{1}{\epsilon} \int_{\mathbb{R}^{3}} \hat{g}_{\epsilon} \gamma_{\epsilon} \zeta \chi\left(\frac{|v|^{2}}{K_{\epsilon}}\right) M d v \\
& =\frac{1}{4} \int_{\mathbb{R}^{3}}\left(\Pi \hat{g}_{\epsilon}\right)^{2} \zeta M d v+\frac{1}{\epsilon} \int_{\mathbb{R}^{3}} \hat{g}_{\epsilon} \zeta M d v \\
& +\frac{1}{4} \int_{\mathbb{R}^{3}}\left(\hat{g}_{\epsilon}^{2}-\left(\Pi \hat{g}_{\epsilon}\right)^{2}\right) \gamma_{\epsilon} \chi\left(\frac{|v|^{2}}{K_{\epsilon}}\right) \zeta M d v \\
& +\frac{1}{4} \int_{\mathbb{R}^{3}}\left(\gamma_{\epsilon} \chi\left(\frac{|v|^{2}}{K_{\epsilon}}\right)-1\right)\left(\Pi \hat{g}_{\epsilon}\right)^{2} \zeta M d v \\
& +\frac{1}{\epsilon} \int_{\mathbb{R}^{3}} \hat{g}_{\epsilon}\left(\gamma_{\epsilon} \chi\left(\frac{|v|^{2}}{K_{\epsilon}}\right)-1\right) \zeta M d v \\
& \stackrel{\text { def }}{=} \frac{1}{4} \int_{\mathbb{R}^{3}}\left(\Pi \hat{g}_{\epsilon}\right)^{2} \zeta M d v+\frac{1}{\epsilon} \int_{\mathbb{R}^{3}} \hat{g}_{\epsilon} \zeta M d v+F_{\epsilon}^{1}(\zeta)+F_{\epsilon}^{2}(\zeta)+F_{\epsilon}^{3}(\zeta) .
\end{aligned}
$$

Now, by 5.4, (5.11) and Lemma 7.8, the remainder terms $F_{\epsilon}^{1}(\zeta), F_{\epsilon}^{2}(\zeta)$ and $F_{\epsilon}^{3}(\zeta)$ will all be shown below to converge to 0 in $L_{\text {loc }}^{1}(d t d x)$ as $\epsilon \rightarrow 0$. Furthermore, explicit computations will identify the asymptotic behavior of the first term in the above right-hand side. However, there still remains to handle the second term in the right-hand side above, for the limit of this singular expression is not apparent yet (even formally). It is precisely for this term that we have to employ the crucial fact that $\zeta$ belongs to $\operatorname{Ker}(\mathcal{L})^{\perp}$, as we now explain. 
Indeed, note first that the properties of the linearized Boltzmann operator $\mathcal{L}$ stated in Propositions 5.4 and 5.5 combined with the Fredholm alternative imply that $\mathcal{L}$ is self-adjoint and Fredholm of index zero on $L^{2}(M d v)$. Therefore, its range is exactly the orthogonal complement of its kernel. It follows that any $\zeta \in$ $\operatorname{Ker}(\mathcal{L})^{\perp} \subset L^{2}(M d v)$ belongs to the range of $\mathcal{L}$ and, thus, that there is an inverse $\tilde{\zeta} \in L^{2}(M d v)$ such that

$$
\zeta=\mathcal{L} \tilde{\zeta}
$$

uniquely determined by the fact that it is orthogonal to the kernel of $\mathcal{L}$ (i.e. to the collision invariants).

Then, making use of the simple identity

$$
\mathcal{L} \hat{g}_{\epsilon}=\frac{\epsilon}{2} \mathcal{Q}\left(\hat{g}_{\epsilon}, \hat{g}_{\epsilon}\right)-\epsilon \int_{\mathbb{R}^{3} \times \mathbb{S}^{2}} \hat{q}_{\epsilon} M_{*} d v_{*} d \sigma
$$

one has therefore

(9.14)

$$
\begin{aligned}
\frac{1}{\epsilon} \int_{\mathbb{R}^{3}} \hat{g}_{\epsilon} \zeta M d v & =\frac{1}{\epsilon} \int_{\mathbb{R}^{3}} \hat{g}_{\epsilon} \mathcal{L} \tilde{\zeta} M d v=\frac{1}{\epsilon} \int_{\mathbb{R}^{3}} \mathcal{L} \hat{g}_{\epsilon} \tilde{\zeta} M d v \\
& =\frac{1}{2} \int_{\mathbb{R}^{3}} \mathcal{Q}\left(\hat{g}_{\epsilon}, \hat{g}_{\epsilon}\right) \tilde{\zeta} M d v-\int_{\mathbb{R}^{3} \times \mathbb{R}^{3} \times \mathbb{S}^{2}} \hat{q}_{\epsilon} \tilde{\zeta} M M_{*} d v d v_{*} d \sigma \\
& =\frac{1}{2} \int_{\mathbb{R}^{3}} \mathcal{Q}\left(\Pi \hat{g}_{\epsilon}, \Pi \hat{g}_{\epsilon}\right) \tilde{\zeta} M d v-\int_{\mathbb{R}^{3} \times \mathbb{R}^{3} \times \mathbb{S}^{2}} \hat{q}_{\epsilon} \tilde{\zeta} M M_{*} d v d v_{*} d \sigma+F_{\epsilon}^{4}(\zeta),
\end{aligned}
$$

where

$$
F_{\epsilon}^{4}(\zeta) \stackrel{\text { def }}{=} \frac{1}{4} \int_{\mathbb{R}^{3}} \mathcal{Q}\left(\hat{g}_{\epsilon}-\Pi \hat{g}_{\epsilon}, \hat{g}_{\epsilon}+\Pi \hat{g}_{\epsilon}\right) \tilde{\zeta} M d v+\frac{1}{4} \int_{\mathbb{R}^{3}} \mathcal{Q}\left(\hat{g}_{\epsilon}+\Pi \hat{g}_{\epsilon}, \hat{g}_{\epsilon}-\Pi \hat{g}_{\epsilon}\right) \tilde{\zeta} M d v .
$$

Now, combining 9.13 with 9.14 and using the identity

$$
\mathcal{Q}\left(\Pi \hat{g}_{\epsilon}, \Pi \hat{g}_{\epsilon}\right)=\frac{1}{2} \mathcal{L}\left(\left(\Pi \hat{g}_{\epsilon}\right)^{2}\right),
$$

which straightforwardly follows from the following computation, valid for any collision invariant $\varphi$,

$$
\varphi^{\prime} \varphi_{*}^{\prime}-\varphi \varphi_{*}=\frac{1}{2} \underbrace{\left(\left(\varphi^{\prime}+\varphi_{*}^{\prime}\right)^{2}-\left(\varphi+\varphi_{*}\right)^{2}\right)}_{=0}+\frac{1}{2}\left(\varphi^{2}+\varphi_{*}^{2}-\varphi^{\prime 2}-\varphi_{*}^{\prime 2}\right),
$$

we deduce that

$$
\begin{aligned}
\tilde{F}_{\epsilon}(\zeta)-\frac{1}{2} \int_{\mathbb{R}^{3}} & \left(\Pi \hat{g}_{\epsilon}\right)^{2} \zeta M d v+\int_{\mathbb{R}^{3} \times \mathbb{R}^{3} \times \mathbb{S}^{2}} \hat{q}_{\epsilon} \tilde{\zeta} M M_{*} d v d v_{*} d \sigma \\
& =\tilde{F}_{\epsilon}(\zeta)-\frac{1}{4} \int_{\mathbb{R}^{3}}\left(\Pi \hat{g}_{\epsilon}\right)^{2} \zeta M d v \\
& -\frac{1}{4} \int_{\mathbb{R}^{3}} \mathcal{L}\left(\left(\Pi \hat{g}_{\epsilon}\right)^{2}\right) \tilde{\zeta} M d v+\int_{\mathbb{R}^{3} \times \mathbb{R}^{3} \times \mathbb{S}^{2}} \hat{q}_{\epsilon} \tilde{\zeta} M M_{*} d v d v_{*} d \sigma \\
& =F_{\epsilon}^{1}(\zeta)+F_{\epsilon}^{2}(\zeta)+F_{\epsilon}^{3}(\zeta)+F_{\epsilon}^{4}(\zeta) .
\end{aligned}
$$

Explicit computations show that the advection terms can be conveniently expressed with the moments of $\Pi \hat{g}_{\epsilon}$ (which are equal, by definition, to those of $\hat{g}_{\epsilon}$ ). 
Indeed, decomposing

$$
\begin{aligned}
\left(\Pi \hat{g}_{\epsilon}\right)^{2} & =\underbrace{\left(\hat{\rho}_{\epsilon}+\hat{u}_{\epsilon} \cdot v+\hat{\theta}_{\epsilon}\left(\frac{|v|^{2}}{2}-\frac{3}{2}\right)\right)^{2}}_{\in \operatorname{Ker} \mathcal{L}} \\
& =\underbrace{\hat{\rho}_{\epsilon}^{2}-3 \hat{\rho}_{\epsilon} \hat{\theta}_{\epsilon}-\frac{3}{2} \hat{\theta}_{\epsilon}^{2}+2\left(\hat{\rho}_{\epsilon}+\hat{\theta}_{\epsilon}\right) \hat{u}_{\epsilon} \cdot v+\left(\frac{\left|\hat{u}_{\epsilon}\right|^{2}}{3}+\hat{\rho}_{\epsilon} \hat{\theta}_{\epsilon}+\hat{\theta}_{\epsilon}^{2}\right)|v|^{2}}_{\perp \operatorname{Ker} \mathcal{L}} \\
& +\underbrace{\hat{u}^{2}}_{\hat{u}_{\epsilon} \otimes \hat{u}_{\epsilon}: \phi+2 \hat{\theta}_{\epsilon} \hat{u}_{\epsilon} \cdot \psi+\hat{\theta}_{\epsilon}^{2}\left(\frac{|v|^{4}}{4}-\frac{5|v|^{2}}{2}+\frac{15}{4}\right)},
\end{aligned}
$$

where $\phi$ and $\psi$ are defined in 2.12 and $\hat{\rho}_{\epsilon}, \hat{u}_{\epsilon}$ and $\hat{\theta}_{\epsilon}$ are, respectively, the density, bulk velocity and temperature associated with $\hat{g}_{\epsilon}$, we find that

$$
\begin{aligned}
& \frac{1}{2} \int_{\mathbb{R}^{3}}\left(\Pi \hat{g}_{\epsilon}\right)^{2} \phi M d v=\hat{u}_{\epsilon} \otimes \hat{u}_{\epsilon}-\frac{\left|\hat{u}_{\epsilon}\right|^{2}}{3} \mathrm{Id}, \\
& \frac{1}{2} \int_{\mathbb{R}^{3}}\left(\Pi \hat{g}_{\epsilon}\right)^{2} \psi M d v=\frac{5}{2} \hat{u}_{\epsilon} \hat{\theta}_{\epsilon} .
\end{aligned}
$$

In particular, it follows from $(9.15)$ that

$$
\begin{aligned}
\tilde{F}_{\epsilon}(\phi)-\left(\hat{u}_{\epsilon} \otimes \hat{u}_{\epsilon}-\frac{\left|\hat{u}_{\epsilon}\right|^{2}}{3} \mathrm{Id}\right)+\int_{\mathbb{R}^{3} \times \mathbb{R}^{3} \times \mathbb{S}^{2}} \hat{q}_{\epsilon} \tilde{\phi} M M_{*} d v d v_{*} d \sigma & \\
& =F_{\epsilon}^{1}(\phi)+F_{\epsilon}^{2}(\phi)+F_{\epsilon}^{3}(\phi)+F_{\epsilon}^{4}(\phi), \\
\tilde{F}_{\epsilon}(\psi)-\frac{5}{2} \hat{u}_{\epsilon} \hat{\theta}_{\epsilon}+\int_{\mathbb{R}^{3} \times \mathbb{R}^{3} \times \mathbb{S}^{2}} \hat{q}_{\epsilon} \tilde{\psi} M M_{*} d v d v_{*} d \sigma & =F_{\epsilon}^{1}(\psi)+F_{\epsilon}^{2}(\psi)+F_{\epsilon}^{3}(\psi)+F_{\epsilon}^{4}(\psi) .
\end{aligned}
$$

Next, writing

$$
g_{\epsilon} \gamma_{\epsilon} \chi\left(\frac{|v|^{2}}{K_{\epsilon}}\right)-\hat{g}_{\epsilon}=\frac{1}{2} \hat{g}_{\epsilon}\left(\gamma_{\epsilon} \chi\left(\frac{|v|^{2}}{K_{\epsilon}}\right)\left(\sqrt{G_{\epsilon}}+1\right)-2\right),
$$

using the equi-integrability of $\hat{g}_{\epsilon}^{2}$ from Lemma 7.8 , the fact that the second factor $\gamma_{\epsilon} \chi\left(\frac{|v|^{2}}{K_{\epsilon}}\right)\left(\sqrt{G_{\epsilon}}+1\right)-2$ is uniformly bounded in $L^{\infty}$ and converges almost everywhere to 0 , observe that, by the Product Limit Theorem,

$$
g_{\epsilon} \gamma_{\epsilon} \chi\left(\frac{|v|^{2}}{K_{\epsilon}}\right)-\hat{g}_{\epsilon} \rightarrow 0 \text { in } L_{\mathrm{loc}}^{2}\left(d t d x ; L^{2}(M d v)\right) .
$$

In particular

(9.19) $\tilde{\rho}_{\epsilon}-\hat{\rho}_{\epsilon} \rightarrow 0, \quad \tilde{u}_{\epsilon}-\hat{u}_{\epsilon} \rightarrow 0 \quad$ and $\quad \tilde{\theta}_{\epsilon}-\hat{\theta}_{\epsilon} \rightarrow 0 \quad$ in $L_{\text {loc }}^{2}(d t d x)$ as $\epsilon \rightarrow 0$.

Therefore, on the whole, combining 9.17 with 9.19 , we see that proving Lemma 9.3 comes down to establishing the vanishing of the four remainder terms $F_{\epsilon}^{1}(\zeta), F_{\epsilon}^{2}(\zeta), F_{\epsilon}^{3}(\zeta)$ and $F_{\epsilon}^{4}(\zeta)$, for any $\zeta=O\left(|v|^{3}\right)$ as $|v| \rightarrow \infty$.

- The first term

$$
F_{\epsilon}^{1}(\zeta)=\frac{1}{4} \int_{\mathbb{R}^{3}}\left(\hat{g}_{\epsilon}^{2}-\left(\Pi \hat{g}_{\epsilon}\right)^{2}\right) \gamma_{\epsilon} \chi\left(\frac{|v|^{2}}{K_{\epsilon}}\right) \zeta M d v,
$$

requires a careful treatment because of the growth of $\zeta(v)=O\left(|v|^{3}\right)$ for large velocities. By the Cauchy-Schwarz inequality, it holds that

$$
\begin{aligned}
\left\|F_{\epsilon}^{1}(\zeta)\right\|_{L_{\mathrm{loc}}^{1}(d t d x)} & \leq\left\|\left(\hat{g}_{\epsilon}+\Pi \hat{g}_{\epsilon}\right) \gamma_{\epsilon} \chi\left(\frac{|v|^{2}}{K_{\epsilon}}\right) \zeta\right\|_{L_{\mathrm{loc}}^{2}\left(d t d x ; L^{2}(M d v)\right)} \\
& \times\left\|\hat{g}_{\epsilon}-\Pi \hat{g}_{\epsilon}\right\|_{L_{\mathrm{loc}}^{2}\left(d t d x ; L^{2}(M d v)\right)} .
\end{aligned}
$$


We already know from Lemma 7.9 that

$$
\left\|\hat{g}_{\epsilon}-\Pi \hat{g}_{\epsilon}\right\|_{L_{\mathrm{loc}}^{2}\left(d t d x ; L^{2}(M d v)\right)} \rightarrow 0 \quad \text { as } \epsilon \rightarrow 0 .
$$

It remains then to bound the first term in the right-hand side of $(9.20)$ by obtaining a suitable control of large velocities. This follows from Lemma 5.12 and the definition of $\Pi$, which yields, for all $2 \leq p<4$,

$$
\left|\left(\hat{g}_{\epsilon}+\Pi \hat{g}_{\epsilon}\right) \gamma_{\epsilon}\right| \leq \frac{C\left|\hat{g}_{\epsilon}\right|}{1+\sqrt{G_{\epsilon}}}+C\left|\Pi \hat{g}_{\epsilon}\right|=O(1)_{L_{\mathrm{loc}}^{2}\left(d t d x ; L^{p}(M d v)\right)} .
$$

Hence, incorporating this last estimate in 9.20 leads to, in view of 9.21 ,

$$
F_{\epsilon}^{1}(\zeta) \rightarrow 0 \quad \text { in } L_{\mathrm{loc}}^{1}(d t d x) \text { as } \epsilon \rightarrow 0 .
$$

- The term

$$
F_{\epsilon}^{2}(\zeta)=\frac{1}{4} \int_{\mathbb{R}^{3}}\left(\gamma_{\epsilon} \chi\left(\frac{|v|^{2}}{K_{\epsilon}}\right)-1\right)\left(\Pi \hat{g}_{\epsilon}\right)^{2} \zeta M d v,
$$

is easily disposed of, using the equi-integrability of $\hat{g}_{\epsilon}^{2}$ from Lemma 7.8 which implies in particular that

$$
\left(\Pi \hat{g}_{\epsilon}\right)^{2}\left(1+|v|^{p}\right) M \text { is uniformly integrable on }[0, T] \times K \times \mathbb{R}^{3},
$$

for each $T>0$, each compact $K \subset \mathbb{R}^{3}$ and each $p \in \mathbb{R}$. Indeed, by the Product Limit Theorem, as $\left(\gamma_{\epsilon} \chi\left(\frac{|v|^{2}}{K_{\epsilon}}\right)-1\right)$ is bounded in $L^{\infty}$ and converges almost everywhere to zero, we obtain, for any $p \in \mathbb{R}$,

$$
\left(\Pi \hat{g}_{\epsilon}\right)^{2}\left(\gamma_{\epsilon} \chi\left(\frac{|v|^{2}}{K_{\epsilon}}\right)-1\right) \rightarrow 0 \quad \text { in } L_{\mathrm{loc}}^{1}\left(d t d x ; L^{1}\left(\left(1+|v|^{p}\right) M d v\right)\right) .
$$

In particular, it follows that

$$
F_{\epsilon}^{2}(\zeta) \rightarrow 0 \text { in } L_{\mathrm{loc}}^{1}(d t d x) \text { as } \epsilon \rightarrow 0 .
$$

- In order to get the convergence of

$$
F_{\epsilon}^{3}(\zeta)=\frac{1}{\epsilon} \int_{\mathbb{R}^{3}} \hat{g}_{\epsilon}\left(\gamma_{\epsilon} \chi\left(\frac{|v|^{2}}{K_{\epsilon}}\right)-1\right) \zeta M d v,
$$

we use both the estimate (8.8) on the tails of Gaussian distributions and the convergence 9.12 previously obtained in the proof of Lemma 9.2

Since $\zeta^{2}(v)=O\left(|v|^{6}\right)$ as $|v| \rightarrow \infty$, one has first, by (8.8), that

$$
\begin{aligned}
& \left\|\frac{1}{\epsilon} \int_{\mathbb{R}^{3}} \hat{g}_{\epsilon} \gamma_{\epsilon}\left(\chi\left(\frac{|v|^{2}}{K_{\epsilon}}\right)-1\right) \zeta M d v\right\|_{L^{\infty}\left(d t ; L^{2}(d x)\right)} \\
& \leq \frac{1}{\epsilon}\left\|\gamma_{\epsilon}\right\|_{L^{\infty}}\left\|\hat{g}_{\epsilon}\right\|_{L^{\infty}\left(d t ; L^{2}(M d x d v)\right)}\left(\int_{\mathbb{R}^{3}} \mathbb{1}_{\left\{|v|^{2}>K_{\epsilon}\right\}} \zeta^{2} M d v\right)^{1 / 2} \leq C \epsilon^{\frac{K}{4}-1}|\log \epsilon|^{\frac{7}{4}},
\end{aligned}
$$

which vanishes as soon as $K>4$. Furthermore, by 9.12 , we find

$$
\begin{aligned}
& \left\|\frac{1}{\epsilon} \int_{\mathbb{R}^{3}} \hat{g}_{\epsilon}\left(\gamma_{\epsilon}-1\right) \zeta M d v\right\|_{L_{\mathrm{loc}}^{2}\left(d t ; L_{\mathrm{loc}}^{1}(d x)\right)} \\
& \leq\left\|\zeta \frac{\gamma_{\epsilon}-1}{\epsilon}\right\|_{L_{\mathrm{loc}}^{2}\left(d t d x ; L^{2}(M d v)\right)}\left\|\hat{g}_{\epsilon}\right\|_{L^{\infty}\left(d t ; L^{2}(M d x d v)\right)}=o(1) .
\end{aligned}
$$

Thus, combining the preceding estimates yields

$$
F_{\epsilon}^{3}(\zeta) \rightarrow 0 \text { in } L_{\mathrm{loc}}^{1}(d t d x) \text { as } \epsilon \rightarrow 0 .
$$

- Finally, the continuity of $\mathcal{Q}$

$$
\|\mathcal{Q}(g, g)\|_{L^{2}(M d v)} \leq C\|g\|_{L^{2}(M d v)}^{2},
$$


easily implies that

$$
\begin{aligned}
\left\|F_{\epsilon}^{4}(\zeta)\right\|_{L_{\mathrm{loc}}^{1}(d t d x)} & \leq C\|\tilde{\zeta}\|_{L^{2}(M d v)}\left\|\hat{g}_{\epsilon}-\Pi \hat{g}_{\epsilon}\right\|_{L_{\mathrm{loc}}^{2}\left(d t d x ; L^{2}(M d v)\right)} \\
& \times\left\|\hat{g}_{\epsilon}+\Pi \hat{g}_{\epsilon}\right\|_{L_{\mathrm{loc}}^{2}\left(d t d x ; L^{2}(M d v)\right)} \\
& \leq C\|\tilde{\zeta}\|_{L^{2}(M d v)}\left\|\hat{g}_{\epsilon}-\Pi \hat{g}_{\epsilon}\right\|_{L_{\mathrm{loc}}^{2}\left(d t d x ; L^{2}(M d v)\right)} \\
& \times\left\|\hat{g}_{\epsilon}\right\|_{L_{\mathrm{loc}}^{2}\left(d t d x ; L^{2}(M d v)\right)},
\end{aligned}
$$

whence, in view of 9.21,

$$
F_{\epsilon}^{4}(\zeta) \rightarrow 0 \quad \text { in } L_{\mathrm{loc}}^{1}(d t d x) \text { as } \epsilon \rightarrow 0 .
$$

On the whole, combining estimates $9.23,(9.25),(9.28)$ and 9.30 leads to the expected vanishing of flux remainders which concludes the proof of the lemma.

9.1.3. Decomposition of acceleration terms. It only remains to deal with the acceleration terms.

Lemma 9.4. The acceleration terms defined by 9.5 satisfy

$$
A_{\epsilon}(1) \rightarrow 0,
$$

$$
\begin{array}{r}
A_{\epsilon}(v)-\frac{1}{\epsilon} E_{\epsilon}-\left(\tilde{\rho}_{\epsilon} E_{\epsilon}+\tilde{u}_{\epsilon} \wedge B_{\epsilon}\right) \rightarrow 0, \\
A_{\epsilon}\left(\frac{|v|^{2}}{2}-\frac{5}{2}\right)-\tilde{u}_{\epsilon} \cdot E_{\epsilon} \rightarrow 0,
\end{array}
$$

in $L_{\mathrm{loc}}^{1}(d t d x)$ as $\epsilon \rightarrow 0$.

Proof. By definition of the acceleration terms, one has the decomposition (9.31)

$$
\begin{aligned}
A_{\epsilon}(\varphi)-\frac{1}{\epsilon} E_{\epsilon} \cdot \int_{\mathbb{R}^{3}} \varphi v M d v-\int_{\mathbb{R}^{3}} g_{\epsilon} \gamma_{\epsilon}\left(E_{\epsilon}+v \wedge B_{\epsilon}\right) & \cdot\left(\nabla_{v} \varphi\right) \chi\left(\frac{|v|^{2}}{K_{\epsilon}}\right) M d v \\
& =A_{\epsilon}^{1}(\varphi)+A_{\epsilon}^{2}(\varphi)+A_{\epsilon}^{3}(\varphi),
\end{aligned}
$$

with

$$
\begin{aligned}
& A_{\epsilon}^{1}(\varphi)=-\frac{1}{\epsilon} E_{\epsilon} \cdot \int_{\mathbb{R}^{3}} \varphi(v)(1-\chi)\left(\frac{|v|^{2}}{K_{\epsilon}}\right) v M d v, \\
& A_{\epsilon}^{2}(\varphi)=E_{\epsilon} \cdot \int_{\mathbb{R}^{3}}\left(g_{\epsilon} \hat{\gamma}_{\epsilon}-\frac{1-\hat{\gamma}_{\epsilon}}{\epsilon}-g_{\epsilon} \gamma_{\epsilon}\right) \varphi(v) \chi\left(\frac{|v|^{2}}{K_{\epsilon}}\right) v M d v, \\
& A_{\epsilon}^{3}(\varphi)=\frac{2}{K_{\epsilon}} E_{\epsilon} \cdot \int_{\mathbb{R}^{3}} g_{\epsilon} \gamma_{\epsilon} \varphi(v) \chi^{\prime}\left(\frac{|v|^{2}}{K_{\epsilon}}\right) v M d v .
\end{aligned}
$$

As previously, describing the convergence requires a careful treatment.

- By the Gaussian decay estimate (8.8) and the uniform $L^{2}$ bound on $E_{\epsilon}$ inherited from the entropy inequality [4.28), we get, for all $v \varphi(v)=O\left(|v|^{3}\right)$ as $|v| \rightarrow \infty$,

$$
A_{\epsilon}^{1}(\varphi)=O\left(\epsilon^{\frac{K}{2}-1}|\log \epsilon|^{2}\right)_{L_{\mathrm{loc}}^{2}(d t d x)},
$$

which tends to 0 as soon as $K>2$.

- For the second term, recalling $\hat{\gamma}_{\epsilon}=\gamma_{\epsilon}+\epsilon g_{\epsilon} \gamma^{\prime}\left(G_{\epsilon}\right)$ and writing $g_{\epsilon}=\frac{1}{2} \hat{g}_{\epsilon}\left(1+\sqrt{G_{\epsilon}}\right)$, an easy computation provides

$$
\begin{aligned}
A_{\epsilon}^{2}(\varphi) & =E_{\epsilon} \cdot \int_{\mathbb{R}^{3}}\left(\epsilon g_{\epsilon}^{2} \gamma^{\prime}\left(G_{\epsilon}\right)-\frac{1-\hat{\gamma}_{\epsilon}}{\epsilon}\right) \varphi(v) \chi\left(\frac{|v|^{2}}{K_{\epsilon}}\right) v M d v \\
& =E_{\epsilon} \cdot \int_{\mathbb{R}^{3}}\left(\frac{1}{2} \hat{g}_{\epsilon}\left(1+\sqrt{G_{\epsilon}}\right)\left(G_{\epsilon}-1\right) \gamma^{\prime}\left(G_{\epsilon}\right)-\frac{1-\hat{\gamma}_{\epsilon}}{\epsilon}\right) \varphi(v) \chi\left(\frac{|v|^{2}}{K_{\epsilon}}\right) v M d v .
\end{aligned}
$$


By 9.12 , we have

$$
\int_{\mathbb{R}^{3}} \frac{1-\hat{\gamma}_{\epsilon}}{\epsilon} \varphi(v) \chi\left(\frac{|v|^{2}}{K_{\epsilon}}\right) v M d v \rightarrow 0 \text { in } L_{\mathrm{loc}}^{2}(d t d x) .
$$

Similarly, from the equi-integrability of $\hat{g}_{\epsilon}^{2}$ (see Lemma 7.8) and the fact that, by the hypotheses (9.1) on $\gamma(z),\left(1+\sqrt{G_{\epsilon}}\right)\left(G_{\epsilon}-1\right) \gamma^{\prime}\left(G_{\epsilon}\right)$ is uniformly bounded in $L^{\infty}$ and converges almost everywhere to zero (possibly up to extraction of a subsequence), we deduce by the Product Limit Theorem that

$$
\hat{g}_{\epsilon}\left(1+\sqrt{G_{\epsilon}}\right)\left(G_{\epsilon}-1\right) \gamma^{\prime}\left(G_{\epsilon}\right) \rightarrow 0 \text { in } L_{\mathrm{loc}}^{2}\left(d t d x ; L^{2}(M d v)\right) .
$$

Therefore, it follows that

$$
\int_{\mathbb{R}^{3}}\left(\frac{1}{2} \hat{g}_{\epsilon}\left(1+\sqrt{G_{\epsilon}}\right)\left(G_{\epsilon}-1\right) \gamma^{\prime}\left(G_{\epsilon}\right)-\frac{1-\hat{\gamma}_{\epsilon}}{\epsilon}\right) \varphi(v) \chi\left(\frac{|v|^{2}}{K_{\epsilon}}\right) v M d v \rightarrow 0,
$$

in $L_{\text {loc }}^{2}(d t d x)$, which, when combined with the uniform $L^{2}$ bound on $E_{\epsilon}$, implies that

$$
A_{\epsilon}^{2}(\varphi) \rightarrow 0 \text { in } L_{\mathrm{loc}}^{1}(d t d x) .
$$

- The last remainder term is easy to control. From the uniform $L^{2}$ estimates on $E_{\epsilon}$ and $\left|g_{\epsilon} \gamma_{\epsilon}\right|=\frac{1}{2}\left|\hat{g}_{\epsilon}\left(1+\sqrt{G_{\epsilon}}\right) \gamma\left(G_{\epsilon}\right)\right| \leq C\left|\hat{g}_{\epsilon}\right|$, and the fact that

$$
\frac{1}{K_{\epsilon}^{2}} \int_{\mathbb{R}^{3}} \varphi^{2} \chi^{\prime}\left(\frac{|v|^{2}}{K_{\epsilon}}\right)^{2}|v|^{2} M d v \rightarrow 0
$$

we deduce that

$$
A_{\epsilon}^{3}(\varphi) \rightarrow 0 \text { in } L_{\mathrm{loc}}^{1}(d t d x)
$$

Thus, on the whole, incorporating the convergences of remainder terms 9.32 , (9.34) and 9.35 into the decomposition 9.31) and performing direct computations of $\int_{\mathbb{R}^{3}} \varphi v M d v$ and $\int_{\mathbb{R}^{3}} g_{\epsilon} \gamma_{\epsilon}\left(E_{\epsilon}+v \wedge B_{\epsilon}\right) \cdot\left(\nabla_{v} \varphi\right) \chi\left(\frac{|v|^{2}}{K_{\epsilon}}\right) M d v$ leads then to the expected convergences and concludes the proof of the lemma.

\subsection{Approximate conservation of mass, momentum and energy for two species}

In a way very similar to the one species case from Section 9.1, we can write approximate conservation laws for the two species Vlasov-Maxwell-Boltzmann system (4.34). However, there are two main differences. The first one is that we do not expect the momentum and energy of each species to be conserved separately, for the mixed collision operators in 4.34 do not vanish (even formally) when integrated against collision invariants (except constants) unless they are added together. The second one is that the perturbation in 4.34 is more singular so that we do not expect to be able to establish a weak compactness statement such as Lemma 7.8: the remainders will therefore be controlled by a modulated entropy, which will yield the convergence of remainders to zero at the very end of the proof using Grönwall's lemma (see Chapter 12).

Having in mind to establish some loop estimates with Grönwall's lemma, which are characteristic of modulated energy (or relative entropy) methods, we impose now some bound from below on the renormalizations. More precisely, we consider here an admissible nonlinearity $\Gamma(z)$ defined by

$$
\Gamma(z)-1=(z-1) \gamma(z),
$$


where $\gamma \in C^{1}([0, \infty) ; \mathbb{R})$ satisfies the following assumptions, for some given $C_{1}, C_{2}>$ 0 :

$$
\begin{array}{rlrl}
\gamma(z) & \equiv 1, & \text { for all } z \in[0,2], \\
\gamma(z) \leq 1, & \text { for all } z \in[0, \infty), \\
\gamma(z) & \rightarrow 0, & \text { as } z \rightarrow \infty, \\
\gamma(z) \geq \frac{C_{1}}{(1+z)^{\frac{1}{2}}}, & \text { for all } z \in[0, \infty), \\
\left|\gamma^{\prime}(z)\right| \leq \frac{C_{2}}{(1+z)^{\frac{3}{2}}}, & \text { for all } z \in[0, \infty) .
\end{array}
$$

The above hypotheses on $\Gamma(z)$ are clearly more restrictive than the corresponding assumptions 9.1 in the one species case. Note that necessarily $|\gamma(z)| \leq \frac{2 C_{2}}{(1+z)^{\frac{1}{2}}}$ and

$$
C_{1}(\sqrt{z}-1)^{2} \leq(z-1)^{2} \gamma(z)^{2}=(\Gamma(z)-1)^{2} .
$$

With the notation $\gamma_{\epsilon}^{ \pm}$for $\gamma\left(G_{\epsilon}^{ \pm}\right)$and $\hat{\gamma}_{\epsilon}^{ \pm}\left(G_{\epsilon}\right)$ for $\Gamma^{\prime}\left(G_{\epsilon}^{ \pm}\right)$, the scaled VlasovBoltzmann equation in 4.34 renormalized relatively to the Maxwellian $M$ with the admissible nonlinearity $\Gamma(z)$ reads

$$
\begin{array}{r}
\partial_{t}\left(g_{\epsilon}^{ \pm} \gamma_{\epsilon}^{ \pm}\right)+\frac{1}{\epsilon} v \cdot \nabla_{x}\left(g_{\epsilon}^{ \pm} \gamma_{\epsilon}^{ \pm}\right) \pm \frac{\delta}{\epsilon}\left(\epsilon E_{\epsilon}+v \wedge B_{\epsilon}\right) \cdot \nabla_{v}\left(g_{\epsilon}^{ \pm} \gamma_{\epsilon}^{ \pm}\right) \mp \frac{\delta}{\epsilon} E_{\epsilon} \cdot v G_{\epsilon}^{ \pm} \hat{\gamma}_{\epsilon}^{ \pm} \\
=\frac{1}{\epsilon^{3}} \hat{\gamma}_{\epsilon}^{ \pm} \mathcal{Q}\left(G_{\epsilon}^{ \pm}, G_{\epsilon}^{ \pm}\right)+\frac{\delta^{2}}{\epsilon^{3}} \hat{\gamma}_{\epsilon}^{ \pm} \mathcal{Q}\left(G_{\epsilon}^{ \pm}, G_{\epsilon}^{\mp}\right) .
\end{array}
$$

Following the strategy of Section 9.1, we also introduce a truncation of large velocities $\chi\left(\frac{|v|^{2}}{K_{\epsilon}}\right)$, with $K_{\epsilon}=K|\log \epsilon|$, for some large $K>0$ to be fixed later on, and $\chi \in C_{c}^{\infty}([0, \infty))$ a smooth compactly supported function such that $\mathbb{1}_{[0,1]} \leq$ $\chi \leq \mathbb{1}_{[0,2]}$.

Thus, multiplying each side of the above equation by $\varphi(v) \chi\left(\frac{|v|^{2}}{K_{\epsilon}}\right)$, where $\varphi$ is a collision invariant, and averaging with respect to $M d v$ leads to the moment equations

$$
\partial_{t} \int_{\mathbb{R}^{3}} g_{\epsilon}^{ \pm} \gamma_{\epsilon}^{ \pm} \varphi \chi\left(\frac{|v|^{2}}{K_{\epsilon}}\right) M d v+\nabla_{x} \cdot F_{\epsilon}^{ \pm}(\varphi)= \pm A_{\epsilon}^{ \pm}(\varphi)+D_{\epsilon}^{ \pm}(\varphi)+\Delta_{\epsilon}^{ \pm}(\varphi),
$$

with the notations

$$
F_{\epsilon}^{ \pm}(\varphi)=\frac{1}{\epsilon} \int_{\mathbb{R}^{3}} g_{\epsilon}^{ \pm} \gamma_{\epsilon}^{ \pm} \varphi \chi\left(\frac{|v|^{2}}{K_{\epsilon}}\right) v M d v
$$

for the fluxes,

$$
\begin{aligned}
A_{\epsilon}^{ \pm}(\varphi) & =\frac{\delta}{\epsilon} E_{\epsilon} \cdot \int_{\mathbb{R}^{3}}\left(1+\epsilon g_{\epsilon}^{ \pm}\right) \hat{\gamma}_{\epsilon}^{ \pm} \varphi \chi\left(\frac{|v|^{2}}{K_{\epsilon}}\right) v M d v \\
& +\frac{\delta}{\epsilon} \int_{\mathbb{R}^{3}} g_{\epsilon}^{ \pm} \gamma_{\epsilon}^{ \pm}\left(\epsilon E_{\epsilon}+v \wedge B_{\epsilon}\right) \cdot \nabla_{v}\left(\varphi \chi\left(\frac{|v|^{2}}{K_{\epsilon}}\right) M\right) d v \\
& =\frac{\delta}{\epsilon} E_{\epsilon} \cdot \int_{\mathbb{R}^{3}} \hat{\gamma}_{\epsilon}^{ \pm} \varphi \chi\left(\frac{|v|^{2}}{K_{\epsilon}}\right) v M d v+\delta E_{\epsilon} \cdot \int_{\mathbb{R}^{3}} g_{\epsilon}^{ \pm} \hat{\gamma}_{\epsilon}^{ \pm} \varphi \chi\left(\frac{|v|^{2}}{K_{\epsilon}}\right) v M d v \\
& +\delta E_{\epsilon} \cdot \int_{\mathbb{R}^{3}} g_{\epsilon}^{ \pm} \gamma_{\epsilon}^{ \pm} \varphi\left(\frac{2}{K_{\epsilon}} \chi^{\prime}\left(\frac{|v|^{2}}{K_{\epsilon}}\right)-\chi\left(\frac{|v|^{2}}{K_{\epsilon}}\right)\right) v M d v \\
& +\frac{\delta}{\epsilon} \int_{\mathbb{R}^{3}} g_{\epsilon}^{ \pm} \gamma_{\epsilon}^{ \pm}\left(\epsilon E_{\epsilon}+v \wedge B_{\epsilon}\right) \cdot\left(\nabla_{v} \varphi\right) \chi\left(\frac{|v|^{2}}{K_{\epsilon}}\right) M d v
\end{aligned}
$$


for the acceleration terms, and

$$
\begin{aligned}
& D_{\epsilon}^{ \pm}(\varphi)=\frac{1}{\epsilon^{3}} \int_{\mathbb{R}^{3}} \hat{\gamma}_{\epsilon}^{ \pm} \mathcal{Q}\left(G_{\epsilon}^{ \pm}, G_{\epsilon}^{ \pm}\right) \varphi \chi\left(\frac{|v|^{2}}{K_{\epsilon}}\right) M d v, \\
& \Delta_{\epsilon}^{ \pm}(\varphi)=\frac{\delta^{2}}{\epsilon^{3}} \int_{\mathbb{R}^{3}} \hat{\gamma}_{\epsilon}^{ \pm} \mathcal{Q}\left(G_{\epsilon}^{ \pm}, G_{\epsilon}^{\mp}\right) \varphi \chi\left(\frac{|v|^{2}}{K_{\epsilon}}\right) M d v,
\end{aligned}
$$

for the corresponding conservation defects.

By describing the asymptotic behavior of $F_{\epsilon}^{ \pm}(\varphi), A_{\epsilon}^{ \pm}(\varphi), D_{\epsilon}^{ \pm}(\varphi)$ and $\Delta_{\epsilon}^{ \pm}(\varphi)$, we will prove the following consistency result (compare with the formal macroscopic conservation laws (2.46) and (2.69) by setting $\alpha=\delta \epsilon, \beta=\delta$ and $\gamma=1$ therein).

Proposition 9.5. Let $\left(f_{\epsilon}^{ \pm}, E_{\epsilon}, B_{\epsilon}\right)$ be the sequence of renormalized solutions to the scaled two species Vlasov-Maxwell-Boltzmann system (4.34) considered in Theorem 4.5 for weak interspecies interactions, i.e. $\delta=o(1)$ and $\frac{\delta}{\epsilon}$ unbounded, or in Theorem 4.6 for strong interspecies interactions, i.e. $\delta=1$, and denote by $\tilde{\rho}_{\epsilon}^{ \pm}, \tilde{u}_{\epsilon}^{ \pm}$ and $\tilde{\theta}_{\epsilon}^{ \pm}$the density, bulk velocity and temperature associated with the renormalized fluctuations $g_{\epsilon}^{ \pm} \gamma_{\epsilon}^{ \pm} \chi\left(\frac{|v|^{2}}{K_{\epsilon}}\right)$. Further define the hydrodynamic variables

$$
\tilde{\rho}_{\epsilon}=\frac{\tilde{\rho}_{\epsilon}^{+}+\tilde{\rho}_{\epsilon}^{-}}{2}, \quad \tilde{u}_{\epsilon}=\frac{\tilde{u}_{\epsilon}^{+}+\tilde{u}_{\epsilon}^{-}}{2}, \quad \tilde{\theta}_{\epsilon}=\frac{\tilde{\theta}_{\epsilon}^{+}+\tilde{\theta}_{\epsilon}^{-}}{2},
$$

and electrodynamic variables

$$
\tilde{n}_{\epsilon}=\tilde{\rho}_{\epsilon}^{+}-\tilde{\rho}_{\epsilon}^{-}, \quad \tilde{j}_{\epsilon}=\frac{\delta}{\epsilon}\left(\tilde{u}_{\epsilon}^{+}-\tilde{u}_{\epsilon}^{-}\right), \quad \tilde{w}_{\epsilon}=\frac{\delta}{\epsilon}\left(\tilde{\theta}_{\epsilon}^{+}-\tilde{\theta}_{\epsilon}^{-}\right) .
$$

Then, one has the approximate hydrodynamic conservation laws

$$
\begin{gathered}
\partial_{t} \tilde{\rho}_{\epsilon}+\frac{1}{\epsilon} \nabla_{x} \cdot \tilde{u}_{\epsilon}=R_{\epsilon, 1}, \\
\partial_{t} \tilde{u}_{\epsilon}+\nabla_{x} \cdot\left(\tilde{u}_{\epsilon} \otimes \tilde{u}_{\epsilon}-\frac{\left|\tilde{u}_{\epsilon}\right|^{2}}{3} \mathrm{Id}\right. \\
\left.-\int_{\mathbb{R}^{3} \times \mathbb{R}^{3} \times \mathbb{S}^{2}} \frac{\hat{q}_{\epsilon}^{+}+\hat{q}_{\epsilon}^{-}}{2} \tilde{\phi} M M_{*} d v d v_{*} d \sigma\right) \\
=-\frac{1}{\epsilon} \nabla_{x}\left(\tilde{\rho}_{\epsilon}+\tilde{\theta}_{\epsilon}\right)+\frac{1}{2}\left(\delta \tilde{n}_{\epsilon} E_{\epsilon}+\tilde{j}_{\epsilon} \wedge B_{\epsilon}\right)+R_{\epsilon, 2}, \\
\partial_{t}\left(\frac{3}{2} \tilde{\theta}_{\epsilon}-\tilde{\rho}_{\epsilon}\right)+\nabla_{x} \cdot\left(\frac{5}{2} \tilde{u}_{\epsilon} \tilde{\theta}_{\epsilon}-\int_{\mathbb{R}^{3} \times \mathbb{R}^{3} \times \mathbb{S}^{2}} \frac{\hat{q}_{\epsilon}^{+}+\hat{q}_{\epsilon}^{-}}{2} \tilde{\psi} M M_{*} d v d v_{*} d \sigma\right) \\
=R_{\epsilon, 3},
\end{gathered}
$$

where:

- $\tilde{\phi}$ and $\tilde{\psi}$ are defined by 2.12 and (2.13),

- and the remainders $R_{\epsilon, i}, i=1,2,3$, satisfy

$$
\begin{aligned}
& \left\|R_{\epsilon, i}\right\|_{W_{\mathrm{loc}}^{-1,1}(d x)} \\
& \leq C \delta\left\|E_{\epsilon}-\bar{E}\right\|_{L^{2}(d x)}\left\|\left(g_{\epsilon}^{+} \gamma_{\epsilon}^{+} \chi\left(\frac{|v|^{2}}{K_{\epsilon}}\right)-\bar{g}^{+}, g_{\epsilon}^{-} \gamma_{\epsilon}^{-} \chi\left(\frac{|v|^{2}}{K_{\epsilon}}\right)-\bar{g}^{-}\right)\right\|_{L^{2}(M d x d v)} \\
& +C\left\|\left(g_{\epsilon}^{+} \gamma_{\epsilon}^{+} \chi\left(\frac{|v|^{2}}{K_{\epsilon}}\right)-\bar{g}^{+}, g_{\epsilon}^{-} \gamma_{\epsilon}^{-} \chi\left(\frac{|v|^{2}}{K_{\epsilon}}\right)-\bar{g}^{-}\right)\right\|_{L^{2}(M d x d v)}^{2} \\
& +C\left\|\left(\hat{q}_{\epsilon}^{+}-\bar{q}^{+}, \hat{q}_{\epsilon}^{-}-\bar{q}^{-}, \hat{q}_{\epsilon}^{+,-}-\bar{q}^{+,-}, \hat{q}_{\epsilon}^{-,+}-\bar{q}^{-,+}\right)\right\|_{L^{2}\left(M M_{*} d x d v d v_{*} d \sigma\right)} \\
& \times\left\|\left(g_{\epsilon}^{+} \gamma_{\epsilon}^{+} \chi\left(\frac{|v|^{2}}{K_{\epsilon}}\right)-\bar{g}^{+}, g_{\epsilon}^{-} \gamma_{\epsilon}^{-} \chi\left(\frac{|v|^{2}}{K_{\epsilon}}\right)-\bar{g}^{-}\right)\right\|_{L^{2}(M d x d v)} \\
& +o(1)_{L_{\mathrm{loc}}^{1}(d t)},
\end{aligned}
$$


for any two given infinitesimal Maxwellians, which differ only by their densities,

$$
\bar{g}^{ \pm}=\bar{\rho}^{ \pm}+\bar{u} \cdot v+\bar{\theta}\left(\frac{|v|^{2}}{2}-\frac{3}{2}\right),
$$

with $\bar{\rho}^{ \pm}, \bar{u}, \bar{\theta} \in L^{\infty}(d t d x) \cap L^{\infty}\left(d t ; L^{2}(d x)\right)$, any collision integrands $\bar{q}^{ \pm}, \bar{q}^{ \pm, \mp} \in$ $L^{\infty}\left(d t d x ; L^{2}\left(M M_{*} d v d v_{*} d \sigma\right)\right) \cap L^{2}\left(M M_{*} d t d x d v d v_{*} d \sigma\right)$ and any electric field $\bar{E} \in L^{\infty}(d t d x) \cap L^{\infty}\left(d t ; L^{2}(d x)\right)$.

One also has the approximate electrodynamic conservation laws

$$
\begin{aligned}
\partial_{t} \tilde{n}_{\epsilon}+\frac{1}{\delta} \nabla_{x} \cdot \tilde{j}_{\epsilon} & =R_{\epsilon, 4}, \\
\frac{\epsilon^{2}}{\delta^{2}} \partial_{t} \tilde{j}_{\epsilon}+\frac{1}{\delta} \nabla_{x}\left(\tilde{n}_{\epsilon}+\frac{\epsilon}{\delta} \tilde{w}_{\epsilon}\right) & =2\left(E_{\epsilon}+\tilde{u}_{\epsilon} \wedge B_{\epsilon}\right) \\
& +\int_{\mathbb{R}^{3} \times \mathbb{R}^{3} \times \mathbb{S}^{2}}\left(\hat{q}_{\epsilon}^{+,-}-\hat{q}_{\epsilon}^{-,+}\right) v M M_{*} d v d v_{*} d \sigma+R_{\epsilon, 5}, \\
\partial_{t}\left(\frac{3}{2} \frac{\epsilon^{2}}{\delta^{2}} \tilde{w}_{\epsilon}-\frac{\epsilon}{\delta} \tilde{n}_{\epsilon}\right) & =\int_{\mathbb{R}^{3} \times \mathbb{R}^{3} \times \mathbb{S}^{2}}\left(\hat{q}_{\epsilon}^{+,-}-\hat{q}_{\epsilon}^{-,+}\right)\left(\frac{|v|^{2}}{2}-\frac{5}{2}\right) M M_{*} d v d v_{*} d \sigma \\
& +R_{\epsilon, 6},
\end{aligned}
$$

where:

- the remainder $R_{\epsilon, 4}$ also satisfies 9.43,

- and the remainders $R_{\epsilon, i}, i=5,6$, converge to 0 in $L_{\mathrm{loc}}^{1}\left(d t ; W_{\mathrm{loc}}^{-1,1}(d x)\right)$.

Just like in the proof of proposition 9.1 the proof of Proposition 9.5 consists in three steps respectively devoted to the study of conservation defects, fluxes and acceleration terms in 9.39 .

For the sake of clarity, these three steps are respectively detailed in Sections 9.2.1, 9.2.2 and 9.2.3, below.

More precisely, Proposition 9.5 will clearly follow from the combination of the approximate conservation laws $\overline{9.39}$ with Lemma 9.11, which handles the conservation defects $D_{\epsilon}^{ \pm}(\varphi)$ and $\Delta_{\epsilon}^{ \pm}(\varphi)$, for any collision invariant $\varphi$, Lemma 9.12, which establishes the asymptotic behavior of the fluxes $F_{\epsilon}^{ \pm}(v)$ and $F_{\epsilon}^{ \pm}\left(\frac{|v|^{2}}{2}-\frac{5}{2}\right)$, Lemma 9.13 which characterizes the acceleration terms $A_{\epsilon}^{ \pm}(1), A_{\epsilon}^{ \pm}(v)$ and $A_{\epsilon}^{ \pm}\left(\frac{|v|^{2}}{2}-\frac{5}{2}\right)$ as $\epsilon \rightarrow 0$, and with the following simple estimates of nonlinear terms :

$$
\begin{aligned}
& \left|\frac{1}{2}\left(\tilde{u}_{\epsilon}^{+} \otimes \tilde{u}_{\epsilon}^{+}+\tilde{u}_{\epsilon}^{-} \otimes \tilde{u}_{\epsilon}^{-}\right)-\tilde{u}_{\epsilon} \otimes \tilde{u}_{\epsilon}\right| \\
& =\frac{1}{4} \mid\left(\tilde{u}_{\epsilon}^{+}-\bar{u}\right) \otimes\left(\tilde{u}_{\epsilon}^{+}-\bar{u}\right)+\left(\tilde{u}_{\epsilon}^{-}-\bar{u}\right) \otimes\left(\tilde{u}_{\epsilon}^{-}-\bar{u}\right) \\
& -\left(\tilde{u}_{\epsilon}^{+}-\bar{u}\right) \otimes\left(\tilde{u}_{\epsilon}^{-}-\bar{u}\right)-\left(\tilde{u}_{\epsilon}^{-}-\bar{u}\right) \otimes\left(\tilde{u}_{\epsilon}^{+}-\bar{u}\right) \mid \\
& \leq C\left\|\left(g_{\epsilon}^{+} \gamma_{\epsilon}^{+} \chi\left(\frac{|v|^{2}}{K_{\epsilon}}\right)-\bar{g}^{+}, g_{\epsilon}^{-} \gamma_{\epsilon}^{-} \chi\left(\frac{|v|^{2}}{K_{\epsilon}}\right)-\bar{g}^{-}\right)\right\|_{L^{2}(M d v)}^{2}, \\
& \left|\frac{1}{2}\left(\tilde{u}_{\epsilon}^{+} \tilde{\theta}_{\epsilon}^{+}+\tilde{u}_{\epsilon}^{-} \tilde{\theta}_{\epsilon}^{-}\right)-\tilde{u}_{\epsilon} \tilde{\theta}_{\epsilon}\right| \\
& =\frac{1}{4} \mid\left(\tilde{u}_{\epsilon}^{+}-\bar{u}\right)\left(\tilde{\theta}_{\epsilon}^{+}-\bar{\theta}\right)+\left(\tilde{u}_{\epsilon}^{-}-\bar{u}\right)\left(\tilde{\theta}_{\epsilon}^{-}-\bar{\theta}\right) \\
& -\left(\tilde{u}_{\epsilon}^{+}-\bar{u}\right)\left(\tilde{\theta}_{\epsilon}^{-}-\bar{\theta}\right)-\left(\tilde{u}_{\epsilon}^{-}-\bar{u}\right)\left(\tilde{\theta}_{\epsilon}^{+}-\bar{\theta}\right) \mid \\
& \leq C\left\|\left(g_{\epsilon}^{+} \gamma_{\epsilon}^{+} \chi\left(\frac{|v|^{2}}{K_{\epsilon}}\right)-\bar{g}^{+}, g_{\epsilon}^{-} \gamma_{\epsilon}^{-} \chi\left(\frac{|v|^{2}}{K_{\epsilon}}\right)-\bar{g}^{-}\right)\right\|_{L^{2}(M d v)}^{2}
\end{aligned}
$$


As it turns out, the macroscopic conservation laws provided by Proposition 9.5 will not be sufficient to complete the renormalized relative entropy method in Chapter 12 , for the renormalized electric current $\tilde{j}_{\epsilon}$ in the approximate conservation of momentum of Proposition 9.5 is not controlled by the entropy dissipation. This difficulty will be bypassed by expressing the Lorentz force with the Poynting vector $E_{\epsilon} \wedge B_{\epsilon}$ (as performed in Section 4.1.4), which will consequently require the handling of the defect measures $m_{\epsilon}$ and $a_{\epsilon}$, introduced in Section 4.1.4. stemming from the terms $\int_{\mathbb{R}^{3}}\left(f_{\epsilon}^{+}+f_{\epsilon}^{-}\right) v \otimes v d v$ and $\left(\begin{array}{c}E_{\epsilon} \\ B_{\epsilon}\end{array}\right) \otimes\left(\begin{array}{c}E_{\epsilon} \\ B_{\epsilon}\end{array}\right)$, respectively. Fortunately, the defects $m_{\epsilon}$ and $a_{\epsilon}$ are naturally controlled by the scaled entropy inequality (4.35).

The following proposition appropriately provides an alternate approximate conservation of momentum law based on the Poynting vector, which will be crucial for the renormalized relative entropy method detailed in Chapter 12 . For convenience, the proof of this proposition is deferred to Section 9.2 .4 below.

Proposition 9.6. Let $\left(f_{\epsilon}^{ \pm}, E_{\epsilon}, B_{\epsilon}\right)$ be the sequence of renormalized solutions to the scaled two species Vlasov-Maxwell-Boltzmann system (4.34) considered in Theorem 4.5 for weak interspecies interactions, i.e. $\delta=o(1)$ and $\frac{\delta}{\epsilon}$ unbounded, or in Theorem 4.6 for strong interspecies interactions, i.e. $\delta=1$, and denote by $\tilde{\rho}_{\epsilon}^{ \pm}, \tilde{u}_{\epsilon}^{ \pm}$ and $\tilde{\theta}_{\epsilon}^{ \pm}$the density, bulk velocity and temperature associated with the renormalized fluctuations $g_{\epsilon}^{ \pm} \gamma_{\epsilon}^{ \pm} \chi\left(\frac{|v|^{2}}{K_{\epsilon}}\right)$. Further define the hydrodynamic variables

$$
\tilde{\rho}_{\epsilon}=\frac{\tilde{\rho}_{\epsilon}^{+}+\tilde{\rho}_{\epsilon}^{-}}{2}, \quad \tilde{u}_{\epsilon}=\frac{\tilde{u}_{\epsilon}^{+}+\tilde{u}_{\epsilon}^{-}}{2}, \quad \tilde{\theta}_{\epsilon}=\frac{\tilde{\theta}_{\epsilon}^{+}+\tilde{\theta}_{\epsilon}^{-}}{2} .
$$

Then, one has the approximate conservation of momentum law

$$
\begin{aligned}
& \partial_{t}\left(\tilde{u}_{\epsilon}+\frac{1}{2} E_{\epsilon} \wedge B_{\epsilon}+\frac{1}{2}\left(\begin{array}{c}
a_{\epsilon 26}-a_{\epsilon 35} \\
a_{\epsilon 34}-a_{\epsilon 16} \\
a_{\epsilon 15}-a_{\epsilon 24}
\end{array}\right)\right) \\
& \quad+\nabla_{x} \cdot\left(\tilde{u}_{\epsilon} \otimes \tilde{u}_{\epsilon}-\frac{\left|\tilde{u}_{\epsilon}\right|^{2}}{3} \mathrm{Id}+\frac{1}{2 \epsilon^{2}} m_{\epsilon}-\int_{\mathbb{R}^{3} \times \mathbb{R}^{3} \times \mathbb{S}^{2}} \frac{\hat{q}_{\epsilon}^{+}+\hat{q}_{\epsilon}^{-}}{2} \tilde{\phi} M M_{*} d v d v_{*} d \sigma\right) \\
& \quad-\frac{1}{2} \nabla_{x} \cdot\left(E_{\epsilon} \otimes E_{\epsilon}+e_{\epsilon}+B_{\epsilon} \otimes B_{\epsilon}+b_{\epsilon}\right)+\nabla_{x}\left(\frac{\left|E_{\epsilon}\right|^{2}+\left|B_{\epsilon}\right|^{2}+\operatorname{Tr} a_{\epsilon}}{4}\right) \\
& \quad=-\frac{1}{\epsilon} \nabla_{x}\left(\tilde{\rho}_{\epsilon}+\tilde{\theta}_{\epsilon}\right)+\partial_{t}\left(o(1)_{L^{\infty}\left(d t ; L_{\mathrm{loc}}^{1}(d x)\right)}\right)+R_{\epsilon, 7},
\end{aligned}
$$

where :

- $\tilde{\phi}$ is defined by 2.12 and 2.13,

- the remainder $R_{\epsilon, 7}$ satisfies

$$
\begin{aligned}
\left\|R_{\epsilon, 7}\right\|_{W_{\mathrm{loc}}^{-1,1}(d x)} & \leq C_{1} \int_{\mathbb{R}^{3} \times \mathbb{R}^{3}}\left(\frac{1}{\epsilon^{2}} h\left(\epsilon g_{\epsilon}^{+}\right)-\frac{1}{2}\left(g_{\epsilon}^{+} \gamma_{\epsilon}^{+} \chi\left(\frac{|v|^{2}}{K_{\epsilon}}\right)\right)^{2}\right) M d x d v \\
& +C_{1} \int_{\mathbb{R}^{3} \times \mathbb{R}^{3}}\left(\frac{1}{\epsilon^{2}} h\left(\epsilon g_{\epsilon}^{-}\right)-\frac{1}{2}\left(g_{\epsilon}^{-} \gamma_{\epsilon}^{-} \chi\left(\frac{|v|^{2}}{K_{\epsilon}}\right)\right)^{2}\right) M d x d v \\
& +C_{2}\left\|\left(g_{\epsilon}^{+} \gamma_{\epsilon}^{+} \chi\left(\frac{|v|^{2}}{K_{\epsilon}}\right)-\bar{g}^{+}, g_{\epsilon}^{-} \gamma_{\epsilon}^{-} \chi\left(\frac{|v|^{2}}{K_{\epsilon}}\right)-\bar{g}^{-}\right)\right\|_{L^{2}(M d x d v)}^{2} \\
& +o(1)_{L_{\mathrm{loc}}^{1}(d t)},
\end{aligned}
$$

for any two given infinitesimal Maxwellians, which differ only by their densities,

$$
\bar{g}^{ \pm}=\bar{\rho}^{ \pm}+\bar{u} \cdot v+\bar{\theta}\left(\frac{|v|^{2}}{2}-\frac{3}{2}\right)
$$


with $\bar{\rho}^{ \pm}, \bar{u}, \bar{\theta} \in L^{\infty}(d t d x) \cap L^{\infty}\left(d t ; L^{2}(d x)\right)$,

- and the symmetric positive definite matrix measures $m_{\epsilon}$ and $a_{\epsilon}$ are the defects introduced in Section 4.1.4 stemming from the terms $\int_{\mathbb{R}^{3}}\left(f_{\epsilon}^{+}+f_{\epsilon}^{-}\right) v \otimes$ $v d v$ and $\left(\begin{array}{l}E_{\epsilon} \\ B_{\epsilon}\end{array}\right) \otimes\left(\begin{array}{l}E_{\epsilon} \\ B_{\epsilon}\end{array}\right)$, respectively, with the notation $e_{\epsilon}=\left(a_{\epsilon i j}\right)_{1 \leq i, j \leq 3}$ and $b_{\epsilon}=\left(a_{\epsilon(i+3)(j+3)}\right)_{1 \leq i, j \leq 3}$.

In the limit $\epsilon \rightarrow 0$ and for well-prepared initial data, we expect that $\left\|g_{\epsilon}^{ \pm} \gamma_{\epsilon}^{ \pm} \chi\left(\frac{|v|^{2}}{K_{\epsilon}}\right)-\bar{g}^{ \pm}\right\|_{L^{2}(M d v)}^{2}$ should converge strongly to zero for a suitable choice of $\bar{g}^{ \pm}$. Propositions 9.5 and 9.6 provide then the expected consistency.

A close inspection of $(9.2)$ and $(9.38)$ shows that the main specificities of the two species case handled here, by comparison with the one species case treated in Section 9.1, are the following :

- Mixed collision terms do not have all the usual microscopic symmetries, so that we cannot expect macroscopic momentum and energy conservation to hold for each species separately. In other words, there is an exchange of momentum and energy (but not mass) between cations and anions. Symmetries and conservation laws are retrieved by considering the total momentum and total energy.

- The magnetic force is stronger, so that its contribution to the acceleration terms has to be studied carefully.

- The assumptions 9.36 on the renormalization $\Gamma(z)$ are more restrictive than (9.1). Whereas (9.1) permitted us to consider a uniformly bounded renormalization if necessary, (9.36) requires $\Gamma(z)$ to behave like $\sqrt{z}$ for large values of $z$. Thus, we can no longer have an $L^{\infty}$ bound on the renormalized fluctuations. However, it still holds true that $\sqrt{G_{\epsilon}^{ \pm}} \gamma_{\epsilon}^{ \pm}$and $\sqrt{G_{\epsilon}^{ \pm}} \hat{\gamma}_{\epsilon}^{ \pm}$are uniformly bounded pointwise, which is the only property of $\Gamma(z)$ that we have actually used in Section 9.1

The precise usefulness of hypotheses (9.36) will become apparent in the proof of Lemma 9.7 below, where the growth properties of $\Gamma$ are employed to compare $\hat{g}_{\epsilon}^{ \pm}$with $g_{\epsilon}^{ \pm} \gamma_{\epsilon}$. (Note that this is an instance of the importance of having a theory of renormalized solutions valid for square root renormalizations.) It would be possible to consider here more general renormalizations by working with auxiliary renormalizations when controlling the remainders in Proposition 9.5. However, this would only add useless technical cumbersomeness to the estimates, which we prefer to avoid by imposing the more restrictive assumptions $(9.36)$ on $\Gamma$.

- The equi-integrability of $\left|\hat{g}_{\epsilon}\right|^{2}$ stated in Lemma 7.8 is no longer valid here (only Lemma 7.10 holds here) and we have to substitute compactness estimates by the consistency estimates provided by Lemmas 9.7 and 9.8 below.

To be precise, in Section 9.1 the equi-integrability of $\left|\hat{g}_{\epsilon}\right|^{2}$ has been used to control $D_{\epsilon}^{3}$ and $D_{\epsilon}^{4}$ in the conservation defects, $F_{\epsilon}^{1}, F_{\epsilon}^{2}, F_{\epsilon}^{3}$ and $F_{\epsilon}^{4}$ in the fluxes, as well as $A_{\epsilon}^{2}$ in the acceleration terms.

In order to circumvent this lack of compactness, we need to understand how to substitute the convergences $9.9,99.12,9.18,9.21,9.24$ and 9.33 by bounds which will be absorbed through appropriate loop estimates later on (using Grönwall's lemma). This is precisely the goal of the following lemmas, whose technical proofs are postponed to Section 9.2.5 below, for clarity. 
LEMma 9.7. For any $2 \leq p<4$ and $1 \leq q<\infty$, and denoting, for convenience,

$$
\left[g_{\epsilon}^{ \pm} \gamma_{\epsilon}^{ \pm}-\bar{g}^{ \pm}\right]=\left\|g_{\epsilon}^{ \pm} \gamma_{\epsilon}^{ \pm}-\bar{g}^{ \pm}\right\|_{L^{2}(M d v)}+o(1)_{L_{\mathrm{loc}}^{2}(d t d x)},
$$

one has the following consistency estimates

$$
\begin{aligned}
&\left\|\mathbb{1}_{\left\{G_{\epsilon}^{ \pm} \geq 2\right\}} \hat{g}_{\epsilon}^{ \pm}\right\|_{L^{2}(M d v)} \leq C\left[g_{\epsilon}^{ \pm} \gamma_{\epsilon}^{ \pm}-\bar{g}^{ \pm}\right], \\
& \| g_{\epsilon}^{ \pm} \gamma_{\epsilon}^{ \pm} \chi\left(\frac{|v|^{2}}{K_{\epsilon}}\right)-\hat{g}_{\epsilon}^{ \pm} \|_{L^{2}(M d v)} \leq C\left[g_{\epsilon}^{ \pm} \gamma_{\epsilon}^{ \pm}-\bar{g}^{ \pm}\right], \\
&\left\|g_{\epsilon}^{ \pm} \gamma_{\epsilon}^{ \pm}-\hat{g}_{\epsilon}^{ \pm}\right\|_{L^{2}(M d v)} \leq C\left[g_{\epsilon}^{ \pm} \gamma_{\epsilon}^{ \pm}-\bar{g}^{ \pm}\right], \\
&\left\|\hat{g}_{\epsilon}^{ \pm}-\Pi \hat{g}_{\epsilon}^{ \pm}\right\|_{L^{2}(M d v)} \leq C\left[g_{\epsilon}^{ \pm} \gamma_{\epsilon}^{ \pm}-\bar{g}^{ \pm}\right], \\
&\left\|\left(\Pi \hat{g}_{\epsilon}^{ \pm}\right)^{2}\left(\gamma_{\epsilon}^{ \pm} \chi\left(\frac{|v|^{2}}{K_{\epsilon}}\right)-1\right)\right\|_{L^{q}(M d v)} \leq C\left[g_{\epsilon}^{ \pm} \gamma_{\epsilon}^{ \pm}-\bar{g}^{ \pm}\right]^{2}, \\
& \| \frac{1}{\epsilon} \mathbb{1}_{\left\{G_{\epsilon}^{ \pm} \geq 2\right\}} \leq C\left[g_{\epsilon}^{ \pm} \gamma_{\epsilon}^{ \pm}-\bar{g}^{ \pm}\right] .
\end{aligned}
$$

LEMmA 9.8. For any $2 \leq p<4$ and $1 \leq q<2$, one has the following consistency estimates

$$
\begin{aligned}
&\left\|\mathbb{1}_{\left\{G_{\epsilon}^{ \pm} \geq 2\right\}} \hat{g}_{\epsilon}^{ \pm}\right\|_{L^{2}(M d v)}=o(1)_{L_{\mathrm{loc}}^{q}(d t d x)}, \\
& \| g_{\epsilon}^{ \pm} \gamma_{\epsilon}^{ \pm} \chi\left(\frac{|v|^{2}}{K_{\epsilon}}\right)-\hat{g}_{\epsilon}^{ \pm} \|_{L^{2}(M d v)}=o(1)_{L_{\mathrm{loc}}^{q}(d t d x)}, \\
&\left\|g_{\epsilon}^{ \pm} \gamma_{\epsilon}^{ \pm}-\hat{g}_{\epsilon}^{ \pm}\right\|_{L^{2}(M d v)}=o(1)_{L_{\mathrm{loc}}^{q}(d t d x)}, \\
&\left\|\hat{g}_{\epsilon}^{ \pm}-\Pi \hat{g}_{\epsilon}^{ \pm}\right\|_{L^{2}(M d v)}=o(1)_{L_{\mathrm{loc}}^{q}(d t d x)}, \\
&\left\|\frac{1}{\epsilon} \mathbb{1}_{\left\{G_{\epsilon}^{ \pm} \geq 2\right\}}\right\|_{L^{p}(M d v)}=o(1)_{L_{\mathrm{loc}}^{q}(d t d x)}
\end{aligned}
$$

The following lemma provides a refinement, displaying improved velocity integrability, of the bound 9.45 from Lemma 9.7. It is based on the method of proof of Lemma 5.12 and is crucial in the demonstration of Proposition 9.6 .

LEMMA 9.9. One has the following consistency estimates

$$
\begin{aligned}
\left\|\mathbb{1}_{\left\{G_{\epsilon}^{ \pm} \geq 2\right\}} \hat{g}_{\epsilon}^{ \pm}\right\|_{L^{2}\left((1+|v|)^{2} M d v\right)}^{2} & \leq C_{1} \int_{\mathbb{R}^{3}}\left(\frac{1}{\epsilon^{2}} h\left(\epsilon g_{\epsilon}^{ \pm}\right)-\frac{1}{2}\left(g_{\epsilon}^{ \pm} \gamma_{\epsilon}^{ \pm}\right)^{2}\right) M d v \\
& +C_{2}\left\|g_{\epsilon}^{ \pm} \gamma_{\epsilon}^{ \pm}-\bar{g}^{ \pm}\right\|_{L^{2}(M d v)}^{2}+o(1)_{L_{\mathrm{loc}}^{1}(d t d x)},
\end{aligned}
$$

and

$$
\begin{aligned}
&\left\|\mathbb{1}_{\left\{G_{\epsilon}^{ \pm} \geq 2\right\}} \hat{g}_{\epsilon}^{ \pm}\right\|_{L_{\mathrm{loc}}^{2}\left(d x ; L^{2}\left((1+|v|)^{2} M d v\right)\right)}^{2} \\
& \quad \leq C_{1} \int_{\mathbb{R}^{3} \times \mathbb{R}^{3}}\left(\frac{1}{\epsilon^{2}} h\left(\epsilon g_{\epsilon}^{ \pm}\right)-\frac{1}{2}\left(g_{\epsilon}^{ \pm} \gamma_{\epsilon}^{ \pm} \chi\left(\frac{|v|^{2}}{K_{\epsilon}}\right)\right)^{2}\right) M d x d v \\
& \quad+C_{2}\left\|g_{\epsilon}^{ \pm} \gamma_{\epsilon}^{ \pm} \chi\left(\frac{|v|^{2}}{K_{\epsilon}}\right)-\bar{g}^{ \pm}\right\|_{L^{2}(M d x d v)}^{2}+o(1)_{L_{\mathrm{loc}}^{1}(d t)} .
\end{aligned}
$$

The next result comprises yet another important consistency estimate following from the preceding lemma. This estimate is not used in the present chapter, we only record it here for later reference in the proof of Theorem 4.6 in Chapter 12 for strong interspecies interactions. 
LEMMA 9.10. One has the following consistency estimates

$$
\begin{aligned}
\left\|\left(\hat{g}_{\epsilon}^{+}-\hat{g}_{\epsilon}^{-}-\hat{n}_{\epsilon}\right) \hat{g}_{\epsilon}^{ \pm}\right\|_{L^{1}\left((1+|v|)^{2} M d v\right)} & \leq C_{1} \int_{\mathbb{R}^{3}}\left(\frac{1}{\epsilon^{2}} h\left(\epsilon g_{\epsilon}^{ \pm}\right)-\frac{1}{2}\left(g_{\epsilon}^{ \pm} \gamma_{\epsilon}^{ \pm}\right)^{2}\right) M d v \\
& +C_{2}\left\|g_{\epsilon}^{ \pm} \gamma_{\epsilon}^{ \pm}-\bar{g}^{ \pm}\right\|_{L^{2}(M d v)}^{2}+o(1)_{L_{\mathrm{loc}}^{1}(d t d x)},
\end{aligned}
$$

and

$$
\begin{aligned}
& \left\|\left(\hat{g}_{\epsilon}^{+}-\hat{g}_{\epsilon}^{-}-\hat{n}_{\epsilon}\right) \hat{g}_{\epsilon}^{ \pm}\right\|_{L_{\mathrm{loc}}^{1}\left(d x ; L^{1}\left((1+|v|)^{2} M d v\right)\right)} \\
& \quad \leq C_{1} \int_{\mathbb{R}^{3} \times \mathbb{R}^{3}}\left(\frac{1}{\epsilon^{2}} h\left(\epsilon g_{\epsilon}^{ \pm}\right)-\frac{1}{2}\left(g_{\epsilon}^{ \pm} \gamma_{\epsilon}^{ \pm} \chi\left(\frac{|v|^{2}}{K_{\epsilon}}\right)\right)^{2}\right) M d x d v \\
& \quad+C_{2}\left\|g_{\epsilon}^{ \pm} \gamma_{\epsilon}^{ \pm} \chi\left(\frac{|v|^{2}}{K_{\epsilon}}\right)-\bar{g}^{ \pm}\right\|_{L^{2}(M d x d v)}^{2}+o(1)_{L_{\mathrm{loc}}^{1}(d t)}
\end{aligned}
$$

where $\hat{n}_{\epsilon}$ is the charge density associated with $\hat{g}_{\epsilon}^{ \pm}$.

9.2.1. Conservation defects. The first step of the proof of Proposition 9.5 is to establish the control of conservation defects.

Lemma 9.11. The conservation defects defined by $(9.42)$ satisfy the controls, for any collision invariant $\varphi$,

$$
\begin{aligned}
\left|D_{\epsilon}^{ \pm}(\varphi)\right| & \leq C\left\|\hat{q}_{\epsilon}^{ \pm}-\bar{q}^{ \pm}\right\|_{L^{2}\left(M M_{*} d v d v_{*} d \sigma\right)}\left\|g_{\epsilon}^{ \pm} \gamma_{\epsilon}^{ \pm}-\bar{g}^{ \pm}\right\|_{L^{2}(M d v)} \\
& +o(1)_{L_{\mathrm{loc}}^{1}(d t d x)}, \\
\left|\Delta_{\epsilon}^{+}(\varphi)+\Delta_{\epsilon}^{-}(\varphi)\right| & \leq C \delta\left\|\left(\hat{q}_{\epsilon}^{+,-}-\bar{q}^{+,-}, \hat{q}_{\epsilon}^{-,+}-\bar{q}^{-,+}\right)\right\|_{L^{2}\left(M M_{*} d v d v_{*} d \sigma\right)} \\
& \times\left\|\left(g_{\epsilon}^{+} \gamma_{\epsilon}^{+}-\bar{g}^{+}, g_{\epsilon}^{-} \gamma_{\epsilon}^{-}-\bar{g}^{-}\right)\right\|_{L^{2}(M d v)}+o(1)_{L_{\mathrm{loc}}^{1}(d t d x)}, \\
\left|\Delta_{\epsilon}^{ \pm}(1)\right| & \leq C \delta\left\|\hat{q}_{\epsilon}^{ \pm, \mp}-\bar{q}^{ \pm, \mp}\right\|_{L^{2}\left(M M_{*} d v d v_{*} d \sigma\right)} \\
& \times\left\|\left(g_{\epsilon}^{+} \gamma_{\epsilon}^{+}-\bar{g}^{+}, g_{\epsilon}^{-} \gamma_{\epsilon}^{-}-\bar{g}^{-}\right)\right\|_{L^{2}(M d v)}+o(1)_{L_{\mathrm{loc}}^{1}(d t d x)}, \\
\frac{\epsilon}{\delta} \Delta_{\epsilon}^{ \pm}(\varphi) & =\int_{\mathbb{R}^{3} \times \mathbb{R}^{3} \times \mathbb{S}^{2}} \hat{q}_{\epsilon}^{ \pm, \mp} \varphi M M_{*} d v d v_{*} d \sigma+o(1)_{L_{\mathrm{loc}}^{1}(d t d x)} .
\end{aligned}
$$

Proof. We follow the proof of Lemma 9.2 in the one species case. Thus, we first note that $D_{\epsilon}^{ \pm}(\varphi)$ can be decomposed exactly as in $(9.7)$, which yields

$$
\begin{aligned}
D_{\epsilon}^{ \pm}(\varphi) & =\frac{\epsilon}{4} \int_{\mathbb{R}^{3} \times \mathbb{R}^{3} \times \mathbb{S}^{2}} \hat{\gamma}_{\epsilon}^{ \pm} \varphi \chi\left(\frac{|v|^{2}}{K_{\epsilon}}\right) \hat{q}_{\epsilon}^{ \pm 2} M M_{*} d v d v_{*} d \sigma \\
& -\frac{1}{\epsilon} \int_{\mathbb{R}^{3} \times \mathbb{R}^{3} \times \mathbb{S}^{2}} \hat{\gamma}_{\epsilon}^{ \pm} \varphi\left(1-\chi\left(\frac{|v|^{2}}{K_{\epsilon}}\right)\right) \hat{q}_{\epsilon}^{ \pm} \sqrt{G_{\epsilon}^{ \pm} G_{\epsilon *}^{ \pm}} M M_{*} d v d v_{*} d \sigma \\
& +\frac{1}{\epsilon} \int_{\mathbb{R}^{3} \times \mathbb{R}^{3} \times \mathbb{S}^{2}} \hat{\gamma}_{\epsilon}^{ \pm}\left(1-\hat{\gamma}_{\epsilon *}^{ \pm}\right) \varphi \hat{q}_{\epsilon}^{ \pm} \sqrt{G_{\epsilon}^{ \pm} G_{\epsilon *}^{ \pm}} M M_{*} d v d v_{*} d \sigma \\
& +\frac{1}{\epsilon} \int_{\mathbb{R}^{3} \times \mathbb{R}^{3} \times \mathbb{S}^{2}} \hat{\gamma}_{\epsilon}^{ \pm} \hat{\gamma}_{\epsilon *}^{ \pm}\left(1-\hat{\gamma}_{\epsilon}^{ \pm \prime} \hat{\gamma}_{\epsilon *}^{ \pm \prime}\right) \varphi \hat{q}_{\epsilon}^{ \pm} \sqrt{G_{\epsilon}^{ \pm} G_{\epsilon *}^{ \pm}} M M_{*} d v d v_{*} d \sigma \\
& -\frac{\epsilon}{4} \int_{\mathbb{R}^{3} \times \mathbb{R}^{3} \times \mathbb{S}^{2}} \hat{\gamma}_{\epsilon}^{ \pm} \hat{\gamma}_{\epsilon *}^{ \pm} \hat{\gamma}_{\epsilon}^{ \pm \prime} \hat{\gamma}_{\epsilon *}^{ \pm \prime} \varphi \hat{q}_{\epsilon}^{ \pm 2} M M_{*} d v d v_{*} d \sigma \\
& \stackrel{\text { def }}{=} D_{\epsilon}^{ \pm 1}(\varphi)+D_{\epsilon}^{ \pm 2}(\varphi)+D_{\epsilon}^{ \pm 3}(\varphi)+D_{\epsilon}^{ \pm 4}(\varphi)+D_{\epsilon}^{ \pm 5}(\varphi)
\end{aligned}
$$

where we have used that $\varphi$ is a collision invariant to symmetrize the last term.

Then, we estimate the defects $D_{\epsilon}^{ \pm 1}(\varphi), D_{\epsilon}^{ \pm 2}(\varphi)$ and $D_{\epsilon}^{ \pm 5}(\varphi)$ exactly as $D_{\epsilon}^{1}(\varphi)$, $D_{\epsilon}^{2}(\varphi)$ and $D_{\epsilon}^{5}(\varphi)$ in the one species case. Indeed, the control of these terms only depends on the bounds provided by the relative entropy and entropy dissipation 
through Lemmas 5.2 and 5.3 and, therefore, holds in both the one species and two species cases. Thus, we have that

$$
D_{\epsilon}^{ \pm 1}(\varphi), D_{\epsilon}^{ \pm 2}(\varphi), D_{\epsilon}^{ \pm 5}(\varphi) \rightarrow 0 \text { in } L_{\text {loc }}^{1}(d t d x) \text { as } \epsilon \rightarrow 0
$$

The remaining terms cannot be handled as in Lemma 9.2 and do not necessarily vanish, because of the lack of equi-integrability of $\left|\hat{g}_{\epsilon}^{ \pm}\right|^{2}$. Note, however, that the estimates $(9.8)$ and $(9.10)$ can be reproduced here without difficulty, which yields, for any $2<p<\infty$,

$$
\begin{aligned}
\left|D_{\epsilon}^{ \pm 3}(\varphi)\right| & \leq C\left\|\hat{q}_{\epsilon}^{ \pm}\right\|_{L^{2}\left(M M_{*} d v d v_{*} d \sigma\right)}\left\|\left(1-\hat{\gamma}_{\epsilon}^{ \pm}\right) \hat{g}_{\epsilon}^{ \pm}\right\|_{L^{2}(M d v)} \\
& \leq C\left\|\hat{q}_{\epsilon}^{ \pm}-\bar{q}^{ \pm}\right\|_{L^{2}\left(M M_{*} d v d v_{*} d \sigma\right)}\left\|\left(1-\hat{\gamma}_{\epsilon}^{ \pm}\right) \hat{g}_{\epsilon}^{ \pm}\right\|_{L^{2}(M d v)} \\
& +C\left\|\bar{q}^{ \pm}\right\|_{L^{\infty}\left(d t d x ; L^{2}\left(M M_{*} d v d v_{*} d \sigma\right)\right)}\left\|\left(1-\hat{\gamma}_{\epsilon}^{ \pm}\right) \hat{g}_{\epsilon}^{ \pm}\right\|_{L^{2}(M d v)}, \\
\left|D_{\epsilon}^{ \pm 4}(\varphi)\right| & \leq C\left\|\hat{q}_{\epsilon}^{ \pm}\right\|_{L^{2}\left(M M_{*} d v d v_{*} d \sigma\right)}\left\|\frac{1}{\epsilon}\left(1-\hat{\gamma}_{\epsilon}^{ \pm}\right)\right\|_{L^{p}(M d v)} \\
& \leq C\left\|\hat{q}_{\epsilon}^{ \pm}-\bar{q}^{ \pm}\right\|_{L^{2}\left(M M_{*} d v d v_{*} d \sigma\right)}\left\|\frac{1}{\epsilon}\left(1-\hat{\gamma}_{\epsilon}^{ \pm}\right)\right\|_{L^{p}(M d v)}\left\|\frac{1}{\epsilon}\left(1-\hat{\gamma}_{\epsilon}^{ \pm}\right)\right\|_{L^{p}(M d v)} \\
& +C\left\|\bar{q}^{ \pm}\right\|_{L^{\infty}\left(d t d x ; L^{2}\left(M M_{*} d v d v_{*} d \sigma\right)\right)} .
\end{aligned}
$$

Then, instead of using the convergences $(9.9)$ and $\sqrt{9.12}$ (which are not valid here), we employ the pairs of controls (9.45)-(9.51) and (9.50)-(9.55), respectively, provided by Lemmas 9.7 and 9.8 , which yields

$$
\begin{aligned}
& \left|D_{\epsilon}^{ \pm 3}(\varphi)\right| \leq C\left\|\hat{q}_{\epsilon}^{ \pm}-\bar{q}^{ \pm}\right\|_{L^{2}\left(M M_{*} d v d v_{*} d \sigma\right)}\left\|g_{\epsilon}^{ \pm} \gamma_{\epsilon}^{ \pm}-\bar{g}^{ \pm}\right\|_{L^{2}(M d v)}+o(1)_{L_{\mathrm{loc}}^{1}(d t d x)}, \\
& \left|D_{\epsilon}^{ \pm 4}(\varphi)\right| \leq C\left\|\hat{q}_{\epsilon}^{ \pm}-\bar{q}^{ \pm}\right\|_{L^{2}\left(M M_{*} d v d v_{*} d \sigma\right)}\left\|g_{\epsilon}^{ \pm} \gamma_{\epsilon}^{ \pm}-\bar{g}^{ \pm}\right\|_{L^{2}(M d v)}+o(1)_{L_{\mathrm{loc}}^{1}(d t d x)}
\end{aligned}
$$

On the whole, combining the preceding estimates clearly concludes the proof of the control of $D_{\epsilon}^{ \pm}(\varphi)$.

We turn to the analysis of the mixed terms $\Delta_{\epsilon}^{ \pm}(\varphi)$, which are handled in a very similar fashion. Let us just recall that we do not expect the conservation of momentum and energy to hold for each species separately, so that only the total mixed conservation defects $\Delta_{\epsilon}^{+}(\varphi)+\Delta_{\epsilon}^{-}(\varphi)$ are expected to vanish in the limit, in general.

First, we decompose

$$
\Delta_{\epsilon}^{+}(\varphi)+\Delta_{\epsilon}^{-}(\varphi)=\Delta_{\epsilon}^{1}(\varphi)+\Delta_{\epsilon}^{2}(\varphi)+\Delta_{\epsilon}^{3}(\varphi)+\Delta_{\epsilon}^{4}(\varphi)+\Delta_{\epsilon}^{5}(\varphi)
$$


where we define

$$
\begin{aligned}
& \Delta_{\epsilon}^{1}(\varphi)=\frac{\epsilon}{4} \int_{\mathbb{R}^{3} \times \mathbb{R}^{3} \times \mathbb{S}^{2}} \hat{\gamma}_{\epsilon}^{+} \varphi \chi\left(\frac{|v|^{2}}{K_{\epsilon}}\right)\left(\hat{q}_{\epsilon}^{+,-}\right)^{2} M M_{*} d v d v_{*} d \sigma \\
& +\frac{\epsilon}{4} \int_{\mathbb{R}^{3} \times \mathbb{R}^{3} \times \mathbb{S}^{2}} \hat{\gamma}_{\epsilon}^{-} \varphi \chi\left(\frac{|v|^{2}}{K_{\epsilon}}\right)\left(\hat{q}_{\epsilon}^{-,+}\right)^{2} M M_{*} d v d v_{*} d \sigma, \\
& \Delta_{\epsilon}^{2}(\varphi)=-\frac{\delta}{\epsilon} \int_{\mathbb{R}^{3} \times \mathbb{R}^{3} \times \mathbb{S}^{2}} \hat{\gamma}_{\epsilon}^{+} \varphi\left(1-\chi\left(\frac{|v|^{2}}{K_{\epsilon}}\right)\right) \hat{q}_{\epsilon}^{+,-} \sqrt{G_{\epsilon}^{+} G_{\epsilon *}^{-}} M M_{*} d v d v_{*} d \sigma \\
& -\frac{\delta}{\epsilon} \int_{\mathbb{R}^{3} \times \mathbb{R}^{3} \times \mathbb{S}^{2}} \hat{\gamma}_{\epsilon}^{-} \varphi\left(1-\chi\left(\frac{|v|^{2}}{K_{\epsilon}}\right)\right) \hat{q}_{\epsilon}^{-,+} \sqrt{G_{\epsilon}^{-} G_{\epsilon *}^{+}} M M_{*} d v d v_{*} d \sigma, \\
& \Delta_{\epsilon}^{3}(\varphi)=\frac{\delta}{\epsilon} \int_{\mathbb{R}^{3} \times \mathbb{R}^{3} \times \mathbb{S}^{2}} \hat{\gamma}_{\epsilon}^{+}\left(1-\hat{\gamma}_{\epsilon *}^{-}\right) \varphi \hat{q}_{\epsilon}^{+,-} \sqrt{G_{\epsilon}^{+} G_{\epsilon *}^{-}} M M_{*} d v d v_{*} d \sigma \\
& +\frac{\delta}{\epsilon} \int_{\mathbb{R}^{3} \times \mathbb{R}^{3} \times \mathbb{S}^{2}} \hat{\gamma}_{\epsilon}^{-}\left(1-\hat{\gamma}_{\epsilon *}^{+}\right) \varphi \hat{q}_{\epsilon}^{-,+} \sqrt{G_{\epsilon}^{-} G_{\epsilon *}^{+}} M M_{*} d v d v_{*} d \sigma, \\
& \Delta_{\epsilon}^{4}(\varphi)=\frac{\delta}{\epsilon} \int_{\mathbb{R}^{3} \times \mathbb{R}^{3} \times \mathbb{S}^{2}} \hat{\gamma}_{\epsilon}^{+} \hat{\gamma}_{\epsilon *}^{-}\left(1-\hat{\gamma}_{\epsilon}^{+\prime} \hat{\gamma}_{\epsilon *}^{-\prime}\right) \varphi \hat{q}_{\epsilon}^{+,-} \sqrt{G_{\epsilon}^{+} G_{\epsilon *}^{-}} M M_{*} d v d v_{*} d \sigma \\
& +\frac{\delta}{\epsilon} \int_{\mathbb{R}^{3} \times \mathbb{R}^{3} \times \mathbb{S}^{2}} \hat{\gamma}_{\epsilon}^{-} \hat{\gamma}_{\epsilon *}^{+}\left(1-\hat{\gamma}_{\epsilon}^{-\prime} \hat{\gamma}_{\epsilon *}^{+\prime}\right) \varphi \hat{q}_{\epsilon}^{-,+} \sqrt{G_{\epsilon}^{-} G_{\epsilon *}^{+}} M M_{*} d v d v_{*} d \sigma, \\
& \Delta_{\epsilon}^{5}(\varphi)=-\frac{\epsilon}{4} \int_{\mathbb{R}^{3} \times \mathbb{R}^{3} \times \mathbb{S}^{2}} \hat{\gamma}_{\epsilon}^{+} \hat{\gamma}_{\epsilon *}^{-} \hat{\gamma}_{\epsilon}^{+\prime} \hat{\gamma}_{\epsilon *}^{-\prime} \varphi\left(\hat{q}_{\epsilon}^{+,--}\right)^{2} M M_{*} d v d v_{*} d \sigma \\
& -\frac{\epsilon}{4} \int_{\mathbb{R}^{3} \times \mathbb{R}^{3} \times \mathbb{S}^{2}} \hat{\gamma}_{\epsilon}^{-} \hat{\gamma}_{\epsilon *}^{+} \hat{\gamma}_{\epsilon}^{-\prime} \hat{\gamma}_{\epsilon *}^{+\prime} \varphi\left(\hat{q}_{\epsilon}^{-,+}\right)^{2} M M_{*} d v d v_{*} d \sigma
\end{aligned}
$$

Note that we use the fact that $\varphi$ is a collision invariant, i.e. that $\varphi+\varphi_{*}=\varphi^{\prime}+\varphi_{*}^{\prime}$, only to symmetrize $\Delta_{\epsilon}^{5}(\varphi)$ :

$$
\begin{aligned}
\Delta_{\epsilon}^{5}(\varphi) & =\frac{\delta}{\epsilon} \int_{\mathbb{R}^{3} \times \mathbb{R}^{3} \times \mathbb{S}^{2}} \hat{\gamma}_{\epsilon}^{+} \hat{\gamma}_{\epsilon *}^{-} \hat{\gamma}_{\epsilon}^{+\prime} \hat{\gamma}_{\epsilon *}^{-\prime} \varphi \hat{q}_{\epsilon}^{+,-} \sqrt{G_{\epsilon}^{+} G_{\epsilon *}^{-}} M M_{*} d v d v_{*} d \sigma \\
& +\frac{\delta}{\epsilon} \int_{\mathbb{R}^{3} \times \mathbb{R}^{3} \times \mathbb{S}^{2}} \hat{\gamma}_{\epsilon}^{-} \hat{\gamma}_{\epsilon *}^{+} \hat{\gamma}_{\epsilon}^{-\prime} \hat{\gamma}_{\epsilon *}^{+\prime} \varphi \hat{q}_{\epsilon}^{-,}+\sqrt{G_{\epsilon}^{-} G_{\epsilon *}^{+}} M M_{*} d v d v_{*} d \sigma \\
& =\frac{\delta}{\epsilon} \int_{\mathbb{R}^{3} \times \mathbb{R}^{3} \times \mathbb{S}^{2}} \hat{\gamma}_{\epsilon}^{+} \hat{\gamma}_{\epsilon *}^{-} \hat{\gamma}_{\epsilon}^{+\prime} \hat{\gamma}_{\epsilon *}^{-\prime}\left(\varphi+\varphi_{*}\right) \hat{q}_{\epsilon}^{+,-} \sqrt{G_{\epsilon}^{+} G_{\epsilon *}^{-}} M M_{*} d v d v_{*} d \sigma \\
& =\frac{\delta}{2 \epsilon} \int_{\mathbb{R}^{3} \times \mathbb{R}^{3} \times \mathbb{S}^{2}} \hat{\gamma}_{\epsilon}^{+} \hat{\gamma}_{\epsilon *}^{-} \hat{\gamma}_{\epsilon}^{+\prime} \hat{\gamma}_{\epsilon *}^{-\prime}\left(\varphi+\varphi_{*}\right) \hat{q}_{\epsilon}^{+,-} \\
& \times\left(\sqrt{G_{\epsilon}^{+} G_{\epsilon *}^{-}}-\sqrt{G_{\epsilon}^{+\prime} G_{\epsilon *}^{-\prime}}\right) M M_{*} d v d v_{*} d \sigma \\
& =-\frac{\epsilon}{4} \int_{\mathbb{R}^{3} \times \mathbb{R}^{3} \times \mathbb{S}^{2}} \hat{\gamma}_{\epsilon}^{+} \hat{\gamma}_{\epsilon *}^{-} \hat{\gamma}_{\epsilon}^{+\prime} \hat{\gamma}_{\epsilon *}^{-\prime}\left(\varphi+\varphi_{*}\right)\left(\hat{q}_{\epsilon}^{+,-}\right)^{2} M M_{*} d v d v_{*} d \sigma .
\end{aligned}
$$

This is precisely the point where we need to consider the sum of the mixed collision integrands over both species. Note that, if $\varphi \equiv 1$, then we have $\varphi=\varphi_{*}$, so that the conservation defects can be dealt with separately.

Anyway, the terms in (9.57) are all similar to those in (9.56). We even have an additional factor $\delta$ in the terms $\Delta_{\epsilon}^{2}(\varphi), \Delta_{\epsilon}^{3}(\varphi)$ and $\Delta_{\epsilon}^{4}(\varphi)$. Therefore, with the exact same arguments used to treat the conservation defects $D_{\epsilon}^{ \pm}(\varphi)$, we conclude the proof of the controls over $\Delta_{\epsilon}^{+}(\varphi)+\Delta_{\epsilon}^{-}(\varphi)$ and $\Delta_{\epsilon}^{ \pm}(1)$. 
Finally, in order to derive the control of $\frac{\epsilon}{\delta} \Delta_{\epsilon}^{ \pm}(\varphi)$, we consider the simple decomposition, writing $\sqrt{G_{* \epsilon}^{\mp}}=1+\frac{\epsilon}{2} \hat{g}_{* \epsilon}^{\mp}$,

$$
\begin{aligned}
\frac{\epsilon}{\delta} \Delta_{\epsilon}^{ \pm}(\varphi) & -\int_{\mathbb{R}^{3} \times \mathbb{R}^{3} \times \mathbb{S}^{2}} \hat{q}_{\epsilon}^{ \pm, \mp} \varphi M M_{*} d v d v_{*} d \sigma \\
& =\frac{\epsilon}{2} \int_{\mathbb{R}^{3} \times \mathbb{R}^{3} \times \mathbb{S}^{2}} \hat{\gamma}_{\epsilon}^{ \pm} \sqrt{G_{\epsilon}^{ \pm}} \chi\left(\frac{|v|^{2}}{K_{\epsilon}}\right) \hat{g}_{\epsilon *}^{\mp} \hat{q}_{\epsilon}^{ \pm, \mp} \varphi M M_{*} d v d v_{*} d \sigma \\
& +\int_{\mathbb{R}^{3} \times \mathbb{R}^{3} \times \mathbb{S}^{2}}\left(\hat{\gamma}_{\epsilon}^{ \pm} \sqrt{G_{\epsilon}^{ \pm}} \chi\left(\frac{|v|^{2}}{K_{\epsilon}}\right)-1\right) \hat{q}_{\epsilon}^{ \pm, \mp} \varphi M M_{*} d v d v_{*} d \sigma \\
& +\frac{\epsilon^{2}}{4 \delta} \int_{\mathbb{R}^{3} \times \mathbb{R}^{3} \times \mathbb{S}^{2}} \hat{\gamma}_{\epsilon}^{ \pm}\left(\hat{q}_{\epsilon}^{ \pm, \mp}\right)^{2} \varphi \chi\left(\frac{|v|^{2}}{K_{\epsilon}}\right) M M_{*} d v d v_{*} d \sigma
\end{aligned}
$$

Then, since $\frac{\epsilon}{\delta}$ vanishes, $\varphi \chi\left(\frac{|v|^{2}}{K_{\epsilon}}\right)$ is bounded pointwise by a constant multiple of $|\log \epsilon|$, the renormalized fluctuations $\hat{g}_{\epsilon *}^{\mp}$ are uniformly bounded in $L^{\infty}\left(d t ; L^{2}\left(M_{*} d x d v_{*}\right)\right)$ and the collision integrands $\hat{q}_{\epsilon}^{ \pm, \mp}$ are uniformly bounded in $L^{2}\left(M M_{*} d t d x d v d v_{*} d \sigma\right)$, we find that the first and third terms from the right-hand side above vanish in $L_{\text {loc }}^{1}(d t d x)$.

Further noticing that $\left(\hat{\gamma}_{\epsilon}^{ \pm} \sqrt{G_{\epsilon}^{ \pm}} \chi\left(\frac{|v|^{2}}{K_{\epsilon}}\right)-1\right) \varphi$ is dominated by $|\varphi|$ and converges almost everywhere to 0 , it is easily shown that the second term in the above right-hand side vanishes in $L_{\text {loc }}^{1}(d t d x)$, as well.

The proof of the lemma is now complete.

9.2.2. Decomposition of flux terms. We characterize now the asymptotic behavior of the flux terms.

LEMmA 9.12. The flux terms defined by 9.40 satisfy

$$
\begin{aligned}
& \left|F_{\epsilon}^{ \pm}(v)-\frac{1}{\epsilon}\left(\tilde{\rho}_{\epsilon}^{ \pm}+\tilde{\theta}_{\epsilon}^{ \pm}\right) \mathrm{Id}-\tilde{u}_{\epsilon}^{ \pm} \otimes \tilde{u}_{\epsilon}^{ \pm}+\frac{\left|\tilde{u}_{\epsilon}^{ \pm}\right|^{2}}{3} \mathrm{Id}+\int_{\mathbb{R}^{3} \times \mathbb{R}^{3} \times \mathbb{S}^{2}} \hat{q}_{\epsilon}^{ \pm} \tilde{\phi} M M_{*} d v d v_{*} d \sigma\right| \\
& \leq C\left\|g_{\epsilon}^{ \pm} \gamma_{\epsilon}^{ \pm}-\bar{g}^{ \pm}\right\|_{L^{2}(M d v)}^{2}+o(1)_{L_{\mathrm{loc}}^{1}(d t d x)},
\end{aligned}
$$

and

$$
\begin{aligned}
& \left|F_{\epsilon}^{ \pm}\left(\frac{|v|^{2}}{2}-\frac{5}{2}\right)-\frac{5}{2} \tilde{u}_{\epsilon}^{ \pm} \tilde{\theta}_{\epsilon}^{ \pm}+\int_{\mathbb{R}^{3} \times \mathbb{R}^{3} \times \mathbb{S}^{2}} \hat{q}_{\epsilon}^{ \pm} \tilde{\psi} M M_{*} d v d v_{*} d \sigma\right| \\
& \leq C\left\|g_{\epsilon}^{ \pm} \gamma_{\epsilon}^{ \pm}-\bar{g}^{ \pm}\right\|_{L^{2}(M d v)}^{2}+o(1)_{L_{\mathrm{loc}}^{1}(d t d x)},
\end{aligned}
$$

where $\tilde{\phi}, \tilde{\psi} \in L^{2}(M d v)$ are the kinetic momentum and energy fluxes defined by 2.12 and 2.13 .

Proof. Flux terms are strictly identical to those handled in Lemma 9.3 for the one species case, so that we can reproduce essentially the same arguments.

Thus, we notice first that, modulo the diagonal term in the momentum flux

$$
\frac{1}{\epsilon} \int_{\mathbb{R}^{3}} g_{\epsilon}^{ \pm} \gamma_{\epsilon}^{ \pm} \chi\left(\frac{|v|^{2}}{K_{\epsilon}}\right) \frac{|v|^{2}}{3} M d v=\frac{1}{\epsilon}\left(\tilde{\rho}_{\epsilon}^{ \pm}+\tilde{\theta}_{\epsilon}^{ \pm}\right),
$$

the flux terms have the following structure

$$
\tilde{F}_{\epsilon}^{ \pm}(\zeta)=\frac{1}{\epsilon} \int_{\mathbb{R}^{3}} g_{\epsilon}^{ \pm} \gamma_{\epsilon}^{ \pm} \zeta \chi\left(\frac{|v|^{2}}{K_{\epsilon}}\right) M d v,
$$

where $\zeta \in \operatorname{Ker}(\mathcal{L})^{\perp} \subset L^{2}(M d v)$. Indeed, it is readily seen that the kinetic fluxes $\phi(v)$ and $\psi(v)$, defined by 2.12 , are orthogonal to collision invariants. 

find

Then, reproducing the decomposition 9.15 from the proof of Lemma 9.3 , we

$$
\begin{aligned}
\tilde{F}_{\epsilon}^{ \pm}(\zeta)-\frac{1}{2} \int_{\mathbb{R}^{3}}\left(\Pi \hat{g}_{\epsilon}^{ \pm}\right)^{2} \zeta M d v & +\int_{\mathbb{R}^{3} \times \mathbb{R}^{3} \times \mathbb{S}^{2}} \hat{q}_{\epsilon}^{ \pm} \tilde{\zeta} M M_{*} d v d v_{*} d \sigma \\
& =F_{\epsilon}^{ \pm 1}(\zeta)+F_{\epsilon}^{ \pm 2}(\zeta)+F_{\epsilon}^{ \pm 3}(\zeta)+F_{\epsilon}^{ \pm 4}(\zeta)
\end{aligned}
$$

where $\zeta=\mathcal{L} \tilde{\zeta}$ and

$$
\begin{aligned}
F_{\epsilon}^{ \pm 1}(\zeta) & =\frac{1}{4} \int_{\mathbb{R}^{3}}\left(\hat{g}_{\epsilon}^{ \pm 2}-\left(\Pi \hat{g}_{\epsilon}^{ \pm}\right)^{2}\right) \gamma_{\epsilon}^{ \pm} \chi\left(\frac{|v|^{2}}{K_{\epsilon}}\right) \zeta M d v \\
F_{\epsilon}^{ \pm 2}(\zeta) & =\frac{1}{4} \int_{\mathbb{R}^{3}}\left(\gamma_{\epsilon}^{ \pm} \chi\left(\frac{|v|^{2}}{K_{\epsilon}}\right)-1\right)\left(\Pi \hat{g}_{\epsilon}^{ \pm}\right)^{2} \zeta M d v \\
F_{\epsilon}^{ \pm 3}(\zeta) & =\frac{1}{\epsilon} \int_{\mathbb{R}^{3}} \hat{g}_{\epsilon}^{ \pm}\left(\gamma_{\epsilon}^{ \pm} \chi\left(\frac{|v|^{2}}{K_{\epsilon}}\right)-1\right) \zeta M d v \\
F_{\epsilon}^{ \pm 4}(\zeta) & =\frac{1}{4} \int_{\mathbb{R}^{3}} \mathcal{Q}\left(\hat{g}_{\epsilon}^{ \pm}-\Pi \hat{g}_{\epsilon}^{ \pm}, \hat{g}_{\epsilon}^{ \pm}+\Pi \hat{g}_{\epsilon}^{ \pm}\right) \tilde{\zeta} M d v \\
& +\frac{1}{4} \int_{\mathbb{R}^{3}} \mathcal{Q}\left(\hat{g}_{\epsilon}^{ \pm}+\Pi \hat{g}_{\epsilon}^{ \pm}, \hat{g}_{\epsilon}^{ \pm}-\Pi \hat{g}_{\epsilon}^{ \pm}\right) \tilde{\zeta} M d v
\end{aligned}
$$

The remainder terms $F_{\epsilon}^{ \pm 1}(\zeta), F_{\epsilon}^{ \pm 2}(\zeta), F_{\epsilon}^{ \pm 3}(\zeta)$ and $F_{\epsilon}^{ \pm 4}(\zeta)$ cannot be handled here as in Lemma 9.3 and do not necessarily vanish, because of the lack of equiintegrability of $\left|\hat{g}_{\epsilon}^{ \pm}\right|^{2}$. Note, however, that the estimates $(9.20),(9.22),(9.26),(9.27)$ and $(9.29$ can be reproduced here without difficulty, which yields, for any $2<p<4$ and $1<q<\infty$,

$$
\begin{aligned}
\left|F_{\epsilon}^{ \pm 1}(\zeta)\right| & \leq C\left(\left\|\hat{g}_{\epsilon}^{ \pm}\right\|_{L^{2}(M d v)}+\left\|\hat{q}_{\epsilon}^{ \pm}\right\|_{L^{2}\left(M M_{*} d v d v_{*} d \sigma\right)}^{\frac{1}{2}}\right)\left\|\hat{g}_{\epsilon}^{ \pm}-\Pi \hat{g}_{\epsilon}^{ \pm}\right\|_{L^{2}(M d v)} \\
& \leq C\left\|\hat{g}_{\epsilon}^{ \pm}-\bar{g}^{ \pm}\right\|_{L^{2}(M d v)}\left\|\hat{g}_{\epsilon}^{ \pm}-\Pi \hat{g}_{\epsilon}^{ \pm}\right\|_{L^{2}(M d v)} \\
& +C\left(\left\|\bar{g}^{ \pm}\right\|_{L^{2}(M d v)}+\left\|\hat{q}_{\epsilon}^{ \pm}\right\|_{L^{2}\left(M M_{*} d v d v_{*} d \sigma\right)}^{\frac{1}{2}}\right)\left\|\hat{g}_{\epsilon}^{ \pm}-\Pi \hat{g}_{\epsilon}^{ \pm}\right\|_{L^{2}(M d v)}, \\
\left|F_{\epsilon}^{ \pm 2}(\zeta)\right| & \leq C\left\|\left(\Pi \hat{g}_{\epsilon}^{ \pm}\right)^{2}\left(\gamma_{\epsilon}^{ \pm} \chi\left(\frac{|v|^{2}}{K_{\epsilon}}\right)-1\right)\right\|_{L^{q}(M d v)}, \\
\left|F_{\epsilon}^{ \pm 3}(\zeta)\right| & \leq C\left\|\hat{g}_{\epsilon}^{ \pm}\right\|_{L^{2}(M d v)}\left\|\frac{1-\gamma_{\epsilon}^{ \pm}}{\epsilon}\right\|_{L^{p}(M d v)}+C \epsilon^{\frac{K}{4}-1}|\log \epsilon|^{\frac{7}{4}}\left\|\hat{g}_{\epsilon}^{ \pm}\right\|_{L^{2}(M d v)} \\
& \leq C\left\|\hat{g}_{\epsilon}^{ \pm}-\bar{g}^{ \pm}\right\|_{L^{2}(M d v)}\left\|\frac{1-\gamma_{\epsilon}^{ \pm}}{\epsilon}\right\|_{L^{p}(M d v)} \| 1-\gamma_{\epsilon}^{ \pm} \\
& +C\left\|\bar{g}^{ \pm}\right\|_{L^{2}(M d v)}\left\|\frac{1}{\epsilon}+C \epsilon_{L^{p}(M d v)}^{\frac{K}{4}-1}|\log \epsilon|^{\frac{7}{4}}\right\| \hat{g}_{\epsilon}^{ \pm} \|_{L^{2}(M d v)} \\
\left|F_{\epsilon}^{ \pm 4}(\zeta)\right| & \leq C\left\|\hat{g}_{\epsilon}^{ \pm}\right\|_{L^{2}(M d v)}\left\|\hat{g}_{\epsilon}^{ \pm}-\Pi \hat{g}_{\epsilon}^{ \pm}\right\|_{L^{2}(M d v)} \\
& \leq C\left\|\hat{g}_{\epsilon}^{ \pm}-\bar{g}^{ \pm}\right\|_{L^{2}(M d v)}\left\|\hat{g}_{\epsilon}^{ \pm}-\Pi \hat{g}_{\epsilon}^{ \pm}\right\|_{L^{2}(M d v)} \\
& +C\left\|\bar{g}^{ \pm}\right\|_{L^{2}(M d v)}\left\|\hat{g}_{\epsilon}^{ \pm}-\Pi \hat{g}_{\epsilon}^{ \pm}\right\|_{L^{2}(M d v)} .
\end{aligned}
$$

Then, instead of using the convergences 9.12), (9.21) and 9.24) (which are not valid here), we employ the combinations of controls $(9.50)-(9.55),(9.48)-(9.54)$ and 
9.49, respectively, provided by Lemmas 9.7 and 9.8 which yields

$$
\begin{aligned}
& \left|F_{\epsilon}^{ \pm 1}(\zeta)\right| \leq C\left\|\hat{g}_{\epsilon}^{ \pm}-\bar{g}^{ \pm}\right\|_{L^{2}(M d v)}\left\|g_{\epsilon}^{ \pm} \gamma_{\epsilon}^{ \pm}-\bar{g}^{ \pm}\right\|_{L^{2}(M d v)}+o(1)_{L_{\mathrm{loc}}^{1}(d t d x)} \\
& \left|F_{\epsilon}^{ \pm 2}(\zeta)\right| \leq C\left\|g_{\epsilon}^{ \pm} \gamma_{\epsilon}^{ \pm}-\bar{g}^{ \pm}\right\|_{L^{2}(M d v)}^{2}+o(1)_{L_{\mathrm{loc}}^{1}(d t d x)}, \\
& \left|F_{\epsilon}^{ \pm 3}(\zeta)\right| \leq C\left\|\hat{g}_{\epsilon}^{ \pm}-\bar{g}^{ \pm}\right\|_{L^{2}(M d v)}\left\|g_{\epsilon}^{ \pm} \gamma_{\epsilon}^{ \pm}-\bar{g}^{ \pm}\right\|_{L^{2}(M d v)}+o(1)_{L_{\mathrm{loc}}^{1}(d t d x)} \\
& \left|F_{\epsilon}^{ \pm 4}(\zeta)\right| \leq C\left\|\hat{g}_{\epsilon}^{ \pm}-\bar{g}^{ \pm}\right\|_{L^{2}(M d v)}\left\|g_{\epsilon}^{ \pm} \gamma_{\epsilon}^{ \pm}-\bar{g}^{ \pm}\right\|_{L^{2}(M d v)}+o(1)_{L_{\mathrm{loc}}^{1}(d t d x)}
\end{aligned}
$$

On the whole, using (9.47) and then incorporating the preceding estimates into (9.58, we obtain

$$
\begin{array}{r}
\left|\tilde{F}_{\epsilon}^{ \pm}(\zeta)-\frac{1}{2} \int_{\mathbb{R}^{3}}\left(\Pi \hat{g}_{\epsilon}^{ \pm}\right)^{2} \zeta M d v+\int_{\mathbb{R}^{3} \times \mathbb{R}^{3} \times \mathbb{S}^{2}} \hat{q}_{\epsilon}^{ \pm} \tilde{\zeta} M M_{*} d v d v_{*} d \sigma\right| \\
\leq C\left\|g_{\epsilon}^{ \pm} \gamma_{\epsilon}^{ \pm}-\bar{g}^{ \pm}\right\|_{L^{2}(M d v)}^{2}+o(1)_{L_{\text {loc }}^{1}(d t d x)},
\end{array}
$$

which, when further combined with the direct computation (9.16), yields

$$
\begin{array}{r}
\left|\tilde{F}_{\epsilon}^{ \pm}(\phi)-\left(\hat{u}_{\epsilon}^{ \pm} \otimes \hat{u}_{\epsilon}^{ \pm}-\frac{\left|\hat{u}_{\epsilon}^{ \pm}\right|^{2}}{3} \mathrm{Id}\right)+\int_{\mathbb{R}^{3} \times \mathbb{R}^{3} \times \mathbb{S}^{2}} \hat{q}_{\epsilon}^{ \pm} \tilde{\phi} M M_{*} d v d v_{*} d \sigma\right| \\
\leq C\left\|g_{\epsilon}^{ \pm} \gamma_{\epsilon}^{ \pm}-\bar{g}^{ \pm}\right\|_{L^{2}(M d v)}^{2}+o(1)_{L_{\mathrm{loc}}^{1}(d t d x)}, \\
\left|\tilde{F}_{\epsilon}^{ \pm}(\psi)-\frac{5}{2} \hat{u}_{\epsilon}^{ \pm} \hat{\theta}_{\epsilon}^{ \pm}+\int_{\mathbb{R}^{3} \times \mathbb{R}^{3} \times \mathbb{S}^{2}} \hat{q}_{\epsilon}^{ \pm} \tilde{\psi} M M_{*} d v d v_{*} d \sigma\right| \\
\leq C\left\|g_{\epsilon}^{ \pm} \gamma_{\epsilon}^{ \pm}-\bar{g}^{ \pm}\right\|_{L^{2}(M d v)}^{2}+o(1)_{L_{\mathrm{loc}}^{1}(d t d x)},
\end{array}
$$

where $\hat{u}_{\epsilon}^{ \pm}$and $\hat{\theta}_{\epsilon}^{ \pm}$are, respectively, the bulk velocity and temperature associated with $\hat{g}_{\epsilon}^{ \pm}$.

Finally, employing 9.46 , 9.47) and $(9.52$, we easily obtain that

$$
\begin{aligned}
& \left|\left(\tilde{u}_{\epsilon}^{ \pm} \otimes \tilde{u}_{\epsilon}^{ \pm}-\frac{\left|\tilde{u}_{\epsilon}^{ \pm}\right|^{2}}{3} \mathrm{Id}\right)-\left(\hat{u}_{\epsilon}^{ \pm} \otimes \hat{u}_{\epsilon}^{ \pm}-\frac{\left|\hat{u}_{\epsilon}^{ \pm}\right|^{2}}{3} \mathrm{Id}\right)\right| \\
& \leq C\left|\tilde{u}_{\epsilon}^{ \pm}-\hat{u}_{\epsilon}^{ \pm}\right|\left|\tilde{u}_{\epsilon}^{ \pm}-\bar{u}\right|+C\left|\hat{u}_{\epsilon}^{ \pm}-\bar{u}\right|\left|\tilde{u}_{\epsilon}^{ \pm}-\hat{u}_{\epsilon}^{ \pm}\right|+C\left|\tilde{u}_{\epsilon}^{ \pm}-\hat{u}_{\epsilon}^{ \pm}\right||\bar{u}| \\
& \leq C\left\|g_{\epsilon}^{ \pm} \gamma_{\epsilon}^{ \pm}-\bar{g}^{ \pm}\right\|_{L^{2}(M d v)}^{2}+o(1)_{L_{\mathrm{loc}}^{1}(d t d x)}, \\
& \left|\frac{5}{2} \tilde{u}_{\epsilon}^{ \pm} \tilde{\theta}_{\epsilon}^{ \pm}-\frac{5}{2} \hat{u}_{\epsilon}^{ \pm} \hat{\theta}_{\epsilon}^{ \pm}\right| \\
& \leq C\left|\tilde{u}_{\epsilon}^{ \pm}-\hat{u}_{\epsilon}^{ \pm}\right|\left|\tilde{\theta}_{\epsilon}^{ \pm}-\bar{\theta}\right|+C\left|\hat{u}_{\epsilon}^{ \pm}-\bar{u}\right|\left|\tilde{\theta}_{\epsilon}^{ \pm}-\hat{\theta}_{\epsilon}^{ \pm}\right| \\
& +C\left|\tilde{u}_{\epsilon}^{ \pm}-\hat{u}_{\epsilon}^{ \pm}\right||\bar{\theta}|+C|\bar{u}|\left|\tilde{\theta}_{\epsilon}^{ \pm}-\hat{\theta}_{\epsilon}^{ \pm}\right| \\
& \leq C\left\|g_{\epsilon}^{ \pm} \gamma_{\epsilon}^{ \pm}-\bar{g}^{ \pm}\right\|_{L^{2}(M d v)}^{2}+o(1)_{L_{\mathrm{loc}}^{1}(d t d x)} \text {. }
\end{aligned}
$$

Combining the preceding estimates concludes the proof of the lemma.

9.2.3. Decomposition of acceleration terms. It only remains to deal with the acceleration terms. 
LEMma 9.13. The acceleration terms defined by (9.41) satisfy

$$
\begin{aligned}
\left|A_{\epsilon}^{ \pm}(1)\right| & \leq C \delta\left|E_{\epsilon}-\bar{E}\right|\left\|g_{\epsilon}^{ \pm} \gamma_{\epsilon}^{ \pm}-\bar{g}^{ \pm}\right\|_{L^{2}(M d v)} \\
& +o(1)_{L_{\mathrm{loc}}^{1}(d t d x)}, \\
\left|A_{\epsilon}^{ \pm}(v)-\frac{\delta}{\epsilon} E_{\epsilon}-\delta \tilde{\rho}_{\epsilon}^{ \pm} E_{\epsilon}-\frac{\delta}{\epsilon} \tilde{u}_{\epsilon}^{ \pm} \wedge B_{\epsilon}\right| & \leq C \delta\left|E_{\epsilon}-\bar{E}\right|\left\|g_{\epsilon}^{ \pm} \gamma_{\epsilon}^{ \pm}-\bar{g}^{ \pm}\right\|_{L^{2}(M d v)} \\
& +o(1)_{L_{\mathrm{loc}}^{1}(d t d x)}, \\
\left|A_{\epsilon}^{ \pm}\left(\frac{|v|^{2}}{2}-\frac{5}{2}\right)-\delta \tilde{u}_{\epsilon}^{ \pm} \cdot E_{\epsilon}\right| & \leq C \delta\left|E_{\epsilon}-\bar{E}\right|\left\|g_{\epsilon}^{ \pm} \gamma_{\epsilon}^{ \pm}-\bar{g}^{ \pm}\right\|_{L^{2}(M d v)} \\
& +o(1)_{L_{\mathrm{loc}}^{1}(d t d x)} .
\end{aligned}
$$

Proof. We follow a strategy similar to the proof of Lemma 9.4 in the one species case.

$$
\begin{aligned}
& A_{\epsilon}^{ \pm}(\varphi)-\frac{\delta}{\epsilon} E_{\epsilon} \cdot \int_{\mathbb{R}^{3}} \varphi v M d v-\frac{\delta}{\epsilon} \int_{\mathbb{R}^{3}} g_{\epsilon}^{ \pm} \gamma_{\epsilon}^{ \pm}\left(\epsilon E_{\epsilon}+v \wedge B_{\epsilon}\right) \cdot\left(\nabla_{v} \varphi\right) \chi\left(\frac{|v|^{2}}{K_{\epsilon}}\right) M d v \\
&=A_{\epsilon}^{ \pm 1}(\varphi)+A_{\epsilon}^{ \pm 2}(\varphi)+A_{\epsilon}^{ \pm 3}(\varphi)
\end{aligned}
$$

with

$$
\begin{aligned}
& A_{\epsilon}^{ \pm 1}(\varphi)=-\frac{\delta}{\epsilon} E_{\epsilon} \cdot \int_{\mathbb{R}^{3}} \varphi(v)(1-\chi)\left(\frac{|v|^{2}}{K_{\epsilon}}\right) v M d v \\
& A_{\epsilon}^{ \pm 2}(\varphi)=\delta E_{\epsilon} \cdot \int_{\mathbb{R}^{3}}\left(g_{\epsilon}^{ \pm} \hat{\gamma}_{\epsilon}^{ \pm}-\frac{1-\hat{\gamma}_{\epsilon}^{ \pm}}{\epsilon}-g_{\epsilon}^{ \pm} \gamma_{\epsilon}^{ \pm}\right) \varphi(v) \chi\left(\frac{|v|^{2}}{K_{\epsilon}}\right) v M d v \\
& A_{\epsilon}^{ \pm 3}(\varphi)=\frac{2 \delta}{K_{\epsilon}} E_{\epsilon} \cdot \int_{\mathbb{R}^{3}} g_{\epsilon}^{ \pm} \gamma_{\epsilon}^{ \pm} \varphi(v) \chi^{\prime}\left(\frac{|v|^{2}}{K_{\epsilon}}\right) v M d v
\end{aligned}
$$

Then, we estimate the remainders $A_{\epsilon}^{ \pm 1}(\varphi)$ and $A_{\epsilon}^{ \pm 3}(\varphi)$ exactly as $A_{\epsilon}^{1}(\varphi)$ and $A_{\epsilon}^{3}(\varphi)$ in the one species case. It yields that

$$
A_{\epsilon}^{ \pm 1}(\varphi), A_{\epsilon}^{ \pm 3}(\varphi) \rightarrow 0 \text { in } L_{\text {loc }}^{1}(d t d x) \text { as } \epsilon \rightarrow 0
$$

The remaining term $A_{\epsilon}^{ \pm 2}(\varphi)$ cannot be handled as in Lemma 9.4 . Note, however, that, writing $g_{\epsilon}^{ \pm}=\frac{1}{2} \hat{g}_{\epsilon}^{ \pm}\left(1+\sqrt{G_{\epsilon}^{ \pm}}\right)$, an easy computation provides

$$
\begin{aligned}
A_{\epsilon}^{ \pm 2}(\varphi) & =\delta E_{\epsilon} \cdot \int_{\mathbb{R}^{3}}\left(\epsilon g_{\epsilon}^{ \pm 2} \gamma^{\prime}\left(G_{\epsilon}^{ \pm}\right)-\frac{1-\hat{\gamma}_{\epsilon}^{ \pm}}{\epsilon}\right) \varphi(v) \chi\left(\frac{|v|^{2}}{K_{\epsilon}}\right) v M d v \\
& =\delta E_{\epsilon} \cdot \int_{\mathbb{R}^{3}} \frac{1}{2} \hat{g}_{\epsilon}^{ \pm}\left(1+\sqrt{G_{\epsilon}^{ \pm}}\right)\left(G_{\epsilon}^{ \pm}-1\right) \gamma^{\prime}\left(G_{\epsilon}^{ \pm}\right) \varphi(v) \chi\left(\frac{|v|^{2}}{K_{\epsilon}}\right) v M d v \\
& -\delta E_{\epsilon} \cdot \int_{\mathbb{R}^{3}} \frac{1-\hat{\gamma}_{\epsilon}^{ \pm}}{\epsilon} \varphi(v) \chi\left(\frac{|v|^{2}}{K_{\epsilon}}\right) v M d v
\end{aligned}
$$

Then, simply noticing, in view of the hypotheses 9.36 on the renormalization, that $(1+\sqrt{z})(z-1) \gamma^{\prime}(z)$ is bounded pointwise and supported on values $z \geq 2$, we deduce

$$
\begin{aligned}
\left|A_{\epsilon}^{ \pm 2}(\varphi)\right| & \leq C \delta\left|E_{\epsilon}\right|\left\|\hat{g}_{\epsilon}^{ \pm}\left(1+\sqrt{G_{\epsilon}^{ \pm}}\right)\left(G_{\epsilon}^{ \pm}-1\right) \gamma^{\prime}\left(G_{\epsilon}^{ \pm}\right)\right\|_{L^{2}(M d v)} \\
& +C \delta\left|E_{\epsilon}\right|\left\|\frac{1-\hat{\gamma}_{\epsilon}^{ \pm}}{\epsilon}\right\| \|_{L^{2}(M d v)} \\
& \leq C \delta\left|E_{\epsilon}\right|\left\|_{\left\{G_{\epsilon}^{ \pm} \geq 2\right\}} \hat{g}_{\epsilon}^{ \pm}\right\|_{L^{2}(M d v)} \\
& \leq C \delta\left|E_{\epsilon}-\bar{E}\right|\left\|_{\left\{G_{\epsilon}^{ \pm} \geq 2\right\}} \hat{g}_{\epsilon}^{ \pm}\right\|_{L^{2}(M d v)}+C \delta|\bar{E}|\left\|\mathbb{1}_{\left\{G_{\epsilon}^{ \pm} \geq 2\right\}} \hat{g}_{\epsilon}^{ \pm}\right\|_{L^{2}(M d v)},
\end{aligned}
$$


so that we easily obtain from 9.45 in Lemma 9.7 and 9.51 in Lemma 9.8 that

$$
\left|A_{\epsilon}^{ \pm 2}(\varphi)\right| \leq C \delta\left|E_{\epsilon}-\bar{E}\right|\left\|g_{\epsilon}^{ \pm} \gamma_{\epsilon}^{ \pm}-\bar{g}^{ \pm}\right\|_{L^{2}(M d v)}+o(1)_{L_{\mathrm{loc}}^{1}(d t d x)} .
$$

Finally, incorporating the preceding remainder estimates into 9.59 and performing direct computations of $\int_{\mathbb{R}^{3}} \varphi v M d v$ and $\int_{\mathbb{R}^{3}} g_{\epsilon}^{ \pm} \gamma_{\epsilon}^{ \pm}\left(\epsilon E_{\epsilon}+v \wedge B_{\epsilon}\right) \cdot\left(\nabla_{v} \varphi\right) \chi\left(\frac{|v|^{2}}{K_{\epsilon}}\right) M d v$ leads then to the expected controls of acceleration terms and concludes the proof of the lemma.

9.2.4. Proof of Proposition 9.6. We justify here the validity of the approximate conservation of momentum law provided by Proposition 9.6

Proof of Proposition 9.6. According to Section 4.1.4, renormalized solutions of the two species Vlasov-Maxwell-Boltzmann system (4.34) satisfy the conservation of momentum

$$
\begin{aligned}
& \partial_{t}\left(\int_{\mathbb{R}^{3}}\left(g_{\epsilon}^{+}+g_{\epsilon}^{-}\right) v M d v+E_{\epsilon} \wedge B_{\epsilon}+\left(\begin{array}{c}
a_{\epsilon 26}-a_{\epsilon 35} \\
a_{\epsilon 34}-a_{\epsilon 16} \\
a_{\epsilon 15}-a_{\epsilon 24}
\end{array}\right)\right) \\
& \quad+\nabla_{x} \cdot\left(\frac{1}{\epsilon} \int_{\mathbb{R}^{3}}\left(g_{\epsilon}^{+}+g_{\epsilon}^{-}\right) v \otimes v M d v+\frac{1}{\epsilon^{2}} m_{\epsilon}-E_{\epsilon} \otimes E_{\epsilon}-e_{\epsilon}-B_{\epsilon} \otimes B_{\epsilon}-b_{\epsilon}\right) \\
& \quad+\nabla_{x}\left(\frac{\left|E_{\epsilon}\right|^{2}+\left|B_{\epsilon}\right|^{2}+\operatorname{Tr} a_{\epsilon}}{2}\right)=0 .
\end{aligned}
$$

$$
\begin{aligned}
g_{\epsilon}^{ \pm} & =g_{\epsilon}^{ \pm} \gamma_{\epsilon}^{ \pm} \chi\left(\frac{|v|^{2}}{K_{\epsilon}}\right)+g_{\epsilon}^{ \pm} \gamma_{\epsilon}^{ \pm}\left(1-\chi\left(\frac{|v|^{2}}{K_{\epsilon}}\right)\right)+g_{\epsilon}^{ \pm}\left(1-\gamma_{\epsilon}^{ \pm}\right) \\
& =g_{\epsilon}^{ \pm} \gamma_{\epsilon}^{ \pm} \chi\left(\frac{|v|^{2}}{K_{\epsilon}}\right)+g_{\epsilon}^{ \pm} \gamma_{\epsilon}^{ \pm}\left(1-\chi\left(\frac{|v|^{2}}{K_{\epsilon}}\right)\right)+\hat{g}_{\epsilon}^{ \pm}\left(1-\gamma_{\epsilon}^{ \pm}\right)+\frac{\epsilon}{4} \hat{g}_{\epsilon}^{ \pm 2}\left(1-\gamma_{\epsilon}^{ \pm}\right) .
\end{aligned}
$$

Then, using that $g_{\epsilon}^{ \pm} \gamma_{\epsilon}^{ \pm}$is dominated by $\left|\hat{g}_{\epsilon}^{ \pm}\right|$with the uniform bounds from Lemma 5.2 and the control of Gaussian tails 8.8, it holds that, for any $p \in \mathbb{R}$,

$$
\begin{aligned}
\int_{\mathbb{R}^{3}}\left|g_{\epsilon}^{ \pm} \gamma_{\epsilon}^{ \pm}\left(1-\chi\left(\frac{|v|^{2}}{K_{\epsilon}}\right)\right)\right||v|^{\frac{p}{2}} M d v & \leq C \int_{\left\{|v|^{2} \geq K_{\epsilon}\right\}}\left|\hat{g}_{\epsilon}^{ \pm}\right||v|^{\frac{p}{2}} M d v \\
& \leq C\left\|\hat{g}_{\epsilon}^{ \pm}\right\|_{L^{2}(M d v)}\left(\int_{\left\{|v|^{2} \geq K_{\epsilon}\right\}}|v|^{p} M d v\right)^{\frac{1}{2}} \\
& \leq C(K|\log \epsilon|)^{\frac{p+1}{4}} \epsilon^{\frac{K}{4}}\left\|\hat{g}_{\epsilon}^{ \pm}\right\|_{L^{2}(M d v)} \\
& =o(\epsilon)_{L^{\infty}\left(d t ; L^{2}(d x)\right)}
\end{aligned}
$$

as soon as $K>4$.

Moreover, since $G_{\epsilon}^{ \pm} \geq 2$ implies $\epsilon \hat{g}_{\epsilon}^{ \pm} \geq 2(\sqrt{2}-1)$, whence

$$
\left|g_{\epsilon}^{ \pm}\right|=\left|\hat{g}_{\epsilon}^{ \pm}+\frac{\epsilon}{4} \hat{g}_{\epsilon}^{ \pm 2}\right| \leq C \epsilon \hat{g}_{\epsilon}^{ \pm 2},
$$

we find, employing the uniform bounds from Lemmas 5.1 and 5.2 , that

$$
\begin{aligned}
\int_{\mathbb{R}^{3}}\left|g_{\epsilon}^{ \pm}\left(1-\gamma_{\epsilon}^{ \pm}\right)\right|(1+|v|) M d v & \leq C \epsilon^{\frac{1}{2}} \int_{\mathbb{R}^{3}}\left|g_{\epsilon}^{ \pm}\right|^{\frac{1}{2}}\left|\hat{g}_{\epsilon}^{ \pm}\right|(1+|v|) M d v \\
& \leq C \epsilon^{\frac{1}{2}}\left\|g_{\epsilon}^{ \pm}\right\|_{L^{1}\left(\left(1+|v|^{2}\right) M d v\right)}^{\frac{1}{2}}\left\|\hat{g}_{\epsilon}^{ \pm}\right\|_{L^{2}(M d v)} \\
& =o(1)_{L^{\infty}\left(d t ; L_{\mathrm{loc}}^{1}(d x)\right)} .
\end{aligned}
$$


Alternately, using Lemma 5.12, we obtain

$$
\begin{aligned}
\int_{\mathbb{R}^{3}}\left|\hat{g}_{\epsilon}^{ \pm}\left(1-\gamma_{\epsilon}^{ \pm}\right)+\frac{\epsilon}{4} \hat{g}_{\epsilon}^{ \pm 2}\left(1-\gamma_{\epsilon}^{ \pm}\right)\right|(1+|v|) M d v & \leq C \epsilon\left\|\hat{g}_{\epsilon}^{ \pm}\right\|_{L^{2}((1+|v|) M d v)}^{2} \\
& =o(1)_{L_{\mathrm{loc}}^{1}}(d t d x) .
\end{aligned}
$$

If, instead of Lemma 5.12 , one applies Lemma 9.9 then one finds that

$$
\begin{aligned}
& \frac{1}{\epsilon}\left\|\int_{\mathbb{R}^{3}}\left|\hat{g}_{\epsilon}^{ \pm}\left(1-\gamma_{\epsilon}^{ \pm}\right)+\frac{\epsilon}{4} \hat{g}_{\epsilon}^{ \pm 2}\left(1-\gamma_{\epsilon}^{ \pm}\right)\right|\left(1+|v|^{2}\right) M d v\right\|_{L_{\mathrm{loc}}^{1}(d x)} \\
& \leq C\left\|\mathbb{1}_{\left\{G_{\epsilon}^{ \pm} \geq 2\right\}} \hat{g}_{\epsilon}^{ \pm}\right\|_{L_{\mathrm{loc}}^{2}\left(d x ; L^{2}\left(\left(1+|v|^{2}\right) M d v\right)\right)}^{2} \\
& \leq C_{1} \int_{\mathbb{R}^{3} \times \mathbb{R}^{3}}\left(\frac{1}{\epsilon^{2}} h\left(\epsilon g_{\epsilon}^{ \pm}\right)-\frac{1}{2}\left(g_{\epsilon}^{ \pm} \gamma_{\epsilon}^{ \pm} \chi\left(\frac{|v|^{2}}{K_{\epsilon}}\right)\right)^{2}\right) M d x d v \\
& +C_{2}\left\|g_{\epsilon}^{ \pm} \gamma_{\epsilon}^{ \pm} \chi\left(\frac{|v|^{2}}{K_{\epsilon}}\right)-\bar{g}^{ \pm}\right\|_{L^{2}(M d x d v)}^{2}+o(1)_{L_{\mathrm{loc}}^{1}(d t)} .
\end{aligned}
$$

Thus, combining the preceding estimates with the decomposition 9.61, we arrive at

$$
\int_{\mathbb{R}^{3}} g_{\epsilon}^{ \pm} \varphi(v) M d v=\int_{\mathbb{R}^{3}} g_{\epsilon}^{ \pm} \gamma_{\epsilon}^{ \pm} \chi\left(\frac{|v|^{2}}{K_{\epsilon}}\right) \varphi(v) M d v+o(1)_{L^{\infty}\left(d t ; L_{\mathrm{loc}}^{1}(d x)\right)}
$$

for all $\varphi(v)$ such that $\frac{\varphi(v)}{1+|v|} \in L^{\infty}(d v)$, and

$$
\begin{aligned}
& \frac{1}{\epsilon}\left\|\int_{\mathbb{R}^{3}}\left(g_{\epsilon}^{ \pm}-g_{\epsilon}^{ \pm} \gamma_{\epsilon}^{ \pm} \chi\left(\frac{|v|^{2}}{K_{\epsilon}}\right)\right) \varphi(v) M d v\right\|_{L_{\mathrm{loc}}^{1}(d x)} \\
& \quad \leq C_{1} \int_{\mathbb{R}^{3} \times \mathbb{R}^{3}}\left(\frac{1}{\epsilon^{2}} h\left(\epsilon g_{\epsilon}^{ \pm}\right)-\frac{1}{2}\left(g_{\epsilon}^{ \pm} \gamma_{\epsilon}^{ \pm} \chi\left(\frac{|v|^{2}}{K_{\epsilon}}\right)\right)^{2}\right) M d x d v \\
& \quad+C_{2}\left\|g_{\epsilon}^{ \pm} \gamma_{\epsilon}^{ \pm} \chi\left(\frac{|v|^{2}}{K_{\epsilon}}\right)-\bar{g}^{ \pm}\right\|_{L^{2}(M d x d v)}^{2}+o(1)_{L_{\mathrm{loc}}^{1}(d t)},
\end{aligned}
$$

for all $\varphi(v)$ such that $\frac{\varphi(v)}{1+|v|^{2}} \in L^{\infty}(d v)$, which, when incorporated into 9.60, , yields the approximate conservation law

$$
\begin{aligned}
& \partial_{t}\left(\int_{\mathbb{R}^{3}}\left(g_{\epsilon}^{+} \gamma_{\epsilon}^{+}+g_{\epsilon}^{-} \gamma_{\epsilon}^{-}\right) \chi\left(\frac{|v|^{2}}{K_{\epsilon}}\right) v M d v+E_{\epsilon} \wedge B_{\epsilon}+\left(\begin{array}{c}
a_{\epsilon 26}-a_{\epsilon 35} \\
a_{\epsilon 34}-a_{\epsilon 16} \\
a_{\epsilon 15}-a_{\epsilon 24}
\end{array}\right)\right) \\
& \quad+\nabla_{x} \cdot\left(\frac{1}{\epsilon} \int_{\mathbb{R}^{3}}\left(g_{\epsilon}^{+} \gamma_{\epsilon}^{+}+g_{\epsilon}^{-} \gamma_{\epsilon}^{-}\right) \chi\left(\frac{|v|^{2}}{K_{\epsilon}}\right) v \otimes v M d v+\frac{1}{\epsilon^{2}} m_{\epsilon}\right) \\
& \quad-\nabla_{x} \cdot\left(E_{\epsilon} \otimes E_{\epsilon}+e_{\epsilon}+B_{\epsilon} \otimes B_{\epsilon}+b_{\epsilon}\right)+\nabla_{x}\left(\frac{\left|E_{\epsilon}\right|^{2}+\left|B_{\epsilon}\right|^{2}+\operatorname{Tr} a_{\epsilon}}{2}\right) \\
& \quad=\partial_{t}\left(o(1)_{L^{\infty}\left(d t ; L_{\mathrm{loc}}^{1}(d x)\right)}\right)+\widetilde{R}_{\epsilon},
\end{aligned}
$$


where the remainder $\widetilde{R}_{\epsilon}$ satisfies

$(9.63)$

$$
\begin{aligned}
\left\|\widetilde{R}_{\epsilon}\right\|_{W_{\mathrm{loc}}^{-1,1}(d x)} & \leq C_{1} \int_{\mathbb{R}^{3} \times \mathbb{R}^{3}}\left(\frac{1}{\epsilon^{2}} h\left(\epsilon g_{\epsilon}^{+}\right)-\frac{1}{2}\left(g_{\epsilon}^{+} \gamma_{\epsilon}^{+} \chi\left(\frac{|v|^{2}}{K_{\epsilon}}\right)\right)^{2}\right) M d x d v \\
& +C_{1} \int_{\mathbb{R}^{3} \times \mathbb{R}^{3}}\left(\frac{1}{\epsilon^{2}} h\left(\epsilon g_{\epsilon}^{-}\right)-\frac{1}{2}\left(g_{\epsilon}^{-} \gamma_{\epsilon}^{-} \chi\left(\frac{|v|^{2}}{K_{\epsilon}}\right)\right)^{2}\right) M d x d v \\
& +C_{2}\left\|\left(g_{\epsilon}^{+} \gamma_{\epsilon}^{+} \chi\left(\frac{|v|^{2}}{K_{\epsilon}}\right)-\bar{g}^{+}, g_{\epsilon}^{-} \gamma_{\epsilon}^{-} \chi\left(\frac{|v|^{2}}{K_{\epsilon}}\right)-\bar{g}^{-}\right)\right\|_{L^{2}(M d x d v)}^{2} \\
& +o(1)_{L_{\mathrm{loc}}^{1}(d t)} .
\end{aligned}
$$

Then, expressing the flux terms above with Lemma 9.12 , we find that

$$
\begin{aligned}
& \partial_{t}\left(2 \tilde{u}_{\epsilon}+E_{\epsilon} \wedge B_{\epsilon}+\left(\begin{array}{l}
a_{\epsilon 26}-a_{\epsilon 35} \\
a_{\epsilon 34}-a_{\epsilon 16} \\
a_{\epsilon 15}-a_{\epsilon 24}
\end{array}\right)\right) \\
& \quad+\nabla_{x} \cdot\left(\tilde{u}_{\epsilon}^{+} \otimes \tilde{u}_{\epsilon}^{+}+\tilde{u}_{\epsilon}^{-} \otimes \tilde{u}_{\epsilon}^{-}-\frac{\left|\tilde{u}_{\epsilon}^{+}\right|^{2}+\left|\tilde{u}_{\epsilon}^{-}\right|^{2}}{3} \mathrm{Id}+\frac{1}{\epsilon^{2}} m_{\epsilon}\right) \\
& \quad-\nabla_{x} \cdot\left(\int_{\mathbb{R}^{3} \times \mathbb{R}^{3} \times \mathbb{S}^{2}}\left(\hat{q}_{\epsilon}^{+}+\hat{q}_{\epsilon}^{-}\right) \tilde{\phi} M M_{*} d v d v_{*} d \sigma\right) \\
& \quad-\nabla_{x} \cdot\left(E_{\epsilon} \otimes E_{\epsilon}+e_{\epsilon}+B_{\epsilon} \otimes B_{\epsilon}+b_{\epsilon}\right)+\nabla_{x}\left(\frac{\left|E_{\epsilon}\right|^{2}+\left|B_{\epsilon}\right|^{2}+\operatorname{Tr} a_{\epsilon}}{2}\right) \\
& \quad=-\frac{2}{\epsilon} \nabla_{x}\left(\tilde{\rho}_{\epsilon}+\tilde{\theta}_{\epsilon}\right)+\partial_{t}\left(o(1)_{L^{\infty}\left(d t ; L_{\mathrm{loc}}^{1}(d x)\right)}\right)+\bar{R}_{\epsilon},
\end{aligned}
$$

where the remainder $\bar{R}_{\epsilon}$ also satisfies 9.63 . sition.

Finally, an application of the estimate $(9.44)$ concludes the proof of the propo-

9.2.5. Proofs of Lemmas 9.7, 9.8, 9.9 and 9.10, At last, we provide a complete justification of Lemmas $9.7,9.8,9.9$ and 9.10.

Proof of Lemma 9.7. This lemma hinges upon the simple fact that the renormalization $\Gamma(z)$ enjoys the suitable bound from below $(9.37)$. In terms of the renormalized fluctuations, this bound implies that

$$
\frac{1}{C}\left|\hat{g}_{\epsilon}^{ \pm}\right| \leq\left|g_{\epsilon}^{ \pm} \gamma_{\epsilon}^{ \pm}\right| \leq C\left|\hat{g}_{\epsilon}^{ \pm}\right|,
$$

for some $C>1$, which will be used repeatedly throughout the present proof.

- In order to establish the first bound (9.45), notice that, since $G_{\epsilon}^{ \pm} \geq 2$ implies $\epsilon \hat{g}_{\epsilon}^{ \pm} \geq 2(\sqrt{2}-1)$

$$
\begin{aligned}
\left|\mathbb{1}_{\left\{G_{\epsilon}^{ \pm} \geq 2\right\}} \hat{g}_{\epsilon}^{ \pm}\right| & \leq C\left|\mathbb{1}_{\left\{G_{\epsilon}^{ \pm} \geq 2\right\}} \gamma_{\epsilon}^{ \pm} g_{\epsilon}^{ \pm}\right| \\
& \leq C\left|g_{\epsilon}^{ \pm} \gamma_{\epsilon}^{ \pm}-\bar{g}^{ \pm}\right|+C\left|\mathbb{1}_{\left\{G_{\epsilon}^{ \pm} \geq 2\right\}} \bar{g}^{ \pm}\right| \\
& \leq C\left|g_{\epsilon}^{ \pm} \gamma_{\epsilon}^{ \pm}-\bar{g}^{ \pm}\right|+C \epsilon\left|\hat{g}_{\epsilon}^{ \pm}\right|\left|\bar{g}^{ \pm}\right|,
\end{aligned}
$$

whence

$$
\begin{aligned}
\left\|\mathbb{1}_{\left\{G_{\epsilon}^{ \pm} \geq 2\right\}} \hat{g}_{\epsilon}^{ \pm} \chi\left(\frac{|v|^{2}}{K_{\epsilon}}\right)\right\|_{L^{2}(M d v)} & \leq C\left\|g_{\epsilon}^{ \pm} \gamma_{\epsilon}^{ \pm}-\bar{g}^{ \pm}\right\|_{L^{2}(M d v)} \\
& +C \epsilon K_{\epsilon}\left\|\hat{g}_{\epsilon}^{ \pm}\right\|_{L^{2}(M d v)}\left\|\frac{\bar{g}^{ \pm}}{1+|v|^{2}}\right\|_{L^{\infty}(d t d x d v)} .
\end{aligned}
$$


Moreover, it is readily seen that

$$
\left\|\mathbb{1}_{\left\{G_{\epsilon}^{ \pm} \geq 2\right\}} \hat{g}_{\epsilon}^{ \pm}\left(1-\chi\left(\frac{|v|^{2}}{K_{\epsilon}}\right)\right)\right\|_{L^{2}(M d v)} \leq \frac{C}{K_{\epsilon}^{\frac{1}{2}}}\left\|\hat{g}_{\epsilon}^{ \pm}\right\|_{L^{2}\left(\left(1+|v|^{2}\right) M d v\right)} .
$$

Therefore, it follows that, combining the preceding estimates and considering the uniform bounds from Lemmas 5.2 and 5.12 .

$$
\begin{aligned}
\left\|\mathbb{1}_{\left\{G_{\epsilon}^{ \pm} \geq 2\right\}} \hat{g}_{\epsilon}^{ \pm}\right\|_{L^{2}(M d v)} & \leq C\left\|g_{\epsilon}^{ \pm} \gamma_{\epsilon}^{ \pm}-\bar{g}^{ \pm}\right\|_{L^{2}(M d v)} \\
& +O(\epsilon|\log \epsilon|)_{L^{\infty}\left(d t ; L^{2}(d x)\right)}+O\left(\frac{1}{|\log \epsilon|^{\frac{1}{2}}}\right)_{L_{\mathrm{loc}}^{2}(d t d x)},
\end{aligned}
$$

which concludes the proof of $(9.45)$.

- To deduce the second bound (9.46), we decompose, writing $g_{\epsilon}^{ \pm}=\hat{g}_{\epsilon}^{ \pm}\left(1+\frac{\epsilon}{4} \hat{g}_{\epsilon}^{ \pm}\right)$ and using that $\left(1+\frac{\epsilon}{4} \hat{g}_{\epsilon}^{ \pm}\right) \gamma_{\epsilon}^{ \pm}$is uniformly bounded pointwise,

$$
\begin{aligned}
\left|g_{\epsilon}^{ \pm} \gamma_{\epsilon}^{ \pm} \chi\left(\frac{|v|^{2}}{K_{\epsilon}}\right)-\hat{g}_{\epsilon}^{ \pm}\right| & =\left|\hat{g}_{\epsilon}^{ \pm}\left(\left(1+\frac{\epsilon}{4} \hat{g}_{\epsilon}^{ \pm}\right) \gamma_{\epsilon}^{ \pm} \chi\left(\frac{|v|^{2}}{K_{\epsilon}}\right)-1\right)\right| \\
& \leq C\left|g_{\epsilon}^{ \pm} \gamma_{\epsilon}^{ \pm}\left(\left(1+\frac{\epsilon}{4} \hat{g}_{\epsilon}^{ \pm}\right) \gamma_{\epsilon}^{ \pm} \chi\left(\frac{|v|^{2}}{K_{\epsilon}}\right)-1\right)\right| \\
& \leq C\left|g_{\epsilon}^{ \pm} \gamma_{\epsilon}^{ \pm}-\bar{g}^{ \pm}\right| \\
& +C\left|\bar{g}^{ \pm}\left(\left(1+\frac{\epsilon}{4} \hat{g}_{\epsilon}^{ \pm}\right) \gamma_{\epsilon}^{ \pm} \chi\left(\frac{|v|^{2}}{K_{\epsilon}}\right)-1\right)\right| \\
& \leq C\left|g_{\epsilon}^{ \pm} \gamma_{\epsilon}^{ \pm}-\bar{g}^{ \pm}\right|+C\left|\bar{g}^{ \pm}\left(1-\chi\left(\frac{|v|^{2}}{K_{\epsilon}}\right)\right)\right| \\
& +C \epsilon\left|\bar{g}^{ \pm} \hat{g}_{\epsilon}^{ \pm} \gamma_{\epsilon}^{ \pm} \chi\left(\frac{|v|^{2}}{K_{\epsilon}}\right)\right|+C\left|\bar{g}^{ \pm}\left(\gamma_{\epsilon}^{ \pm}-1\right) \chi\left(\frac{|v|^{2}}{K_{\epsilon}}\right)\right|
\end{aligned}
$$

which implies

$$
\begin{aligned}
\| g_{\epsilon}^{ \pm} \gamma_{\epsilon}^{ \pm} \chi & \left(\frac{|v|^{2}}{K_{\epsilon}}\right)-\hat{g}_{\epsilon}^{ \pm} \|_{L^{2}(M d v)} \\
& \leq C\left\|g_{\epsilon}^{ \pm} \gamma_{\epsilon}^{ \pm}-\bar{g}^{ \pm}\right\|_{L^{2}(M d v)}+C \epsilon K_{\epsilon}\left\|\hat{g}_{\epsilon}^{ \pm}\right\|_{L^{2}(M d v)}\left\|\frac{\bar{g}^{ \pm}}{1+|v|^{2}}\right\|_{L^{\infty}(d t d x d v)} \\
& +C\left\|\left(1+|v|^{2}\right)\left(1-\chi\left(\frac{|v|^{2}}{K_{\epsilon}}\right)\right)\right\|_{L^{2}(M d v)}\left\|\frac{\bar{g}^{ \pm}}{1+|v|^{2}}\right\|_{L^{\infty}(d t d x d v)} .
\end{aligned}
$$

Then, employing the control of Gaussian tails 8.8 and the uniform bound from Lemma 5.2, we infer that

$$
\begin{aligned}
& \left\|g_{\epsilon}^{ \pm} \gamma_{\epsilon}^{ \pm} \chi\left(\frac{|v|^{2}}{K_{\epsilon}}\right)-\hat{g}_{\epsilon}^{ \pm}\right\|_{L^{2}(M d v)} \\
& \quad \leq C\left\|g_{\epsilon}^{ \pm} \gamma_{\epsilon}^{ \pm}-\bar{g}^{ \pm}\right\|_{L^{2}(M d v)}+O(\epsilon|\log \epsilon|)_{L^{\infty}\left(d t ; L^{2}(d x)\right)}+C \epsilon^{\frac{K}{4}}|\log \epsilon|^{\frac{5}{4}},
\end{aligned}
$$

which establishes $(9.46)$.

- The third bound (9.47) easily follows from the estimate

$$
\begin{aligned}
& \left\|g_{\epsilon}^{ \pm} \gamma_{\epsilon}^{ \pm}-\hat{g}_{\epsilon}^{ \pm}\right\|_{L^{2}(M d v)} \\
& \leq\left\|g_{\epsilon}^{ \pm} \gamma_{\epsilon}^{ \pm} \chi\left(\frac{|v|^{2}}{K_{\epsilon}}\right)-\hat{g}_{\epsilon}^{ \pm}\right\|_{L^{2}(M d v)}+\left\|\hat{g}_{\epsilon}^{ \pm}\left(1-\chi\left(\frac{|v|^{2}}{K_{\epsilon}}\right)\right)\right\|_{L^{2}(M d v)} \\
& \leq\left\|g_{\epsilon}^{ \pm} \gamma_{\epsilon}^{ \pm} \chi\left(\frac{|v|^{2}}{K_{\epsilon}}\right)-\hat{g}_{\epsilon}^{ \pm}\right\|_{L^{2}(M d v)}+\frac{C}{K_{\epsilon}^{\frac{1}{2}}}\left\|\hat{g}_{\epsilon}^{ \pm}\right\|_{L^{2}\left(\left(1+|v|^{2}\right) M d v\right)},
\end{aligned}
$$


which, when combined with the second bound $(9.46)$, concludes its justification.

- The justification of (9.48) is simple. Since $\Pi \bar{g}^{ \pm}=\bar{g}^{ \pm}$, we easily estimate

$$
\begin{aligned}
\left\|\hat{g}_{\epsilon}^{ \pm}-\Pi \hat{g}_{\epsilon}^{ \pm}\right\|_{L^{2}(M d v)} & \leq C\left\|\hat{g}_{\epsilon}^{ \pm}-\bar{g}^{ \pm}\right\|_{L^{2}(M d v)}+C\left\|\Pi \bar{g}^{ \pm}-\Pi \hat{g}_{\epsilon}^{ \pm}\right\|_{L^{2}(M d v)} \\
& \leq C\left\|\hat{g}_{\epsilon}^{ \pm}-\bar{g}^{ \pm}\right\|_{L^{2}(M d v)} .
\end{aligned}
$$

Therefore, the bound $(9.48)$ is obtained by combining the preceding control with (9.47).

- We focus now on 9.49 . We first easily find that

$$
\begin{aligned}
& \left\|\left(\Pi \hat{g}_{\epsilon}^{ \pm}\right)^{2}\left(\gamma_{\epsilon}^{ \pm} \chi\left(\frac{|v|^{2}}{K_{\epsilon}}\right)-1\right)\right\|_{L^{q}(M d v)} \\
& \leq C\left\|\Pi\left(\hat{g}_{\epsilon}^{ \pm}-\bar{g}^{ \pm}\right)\right\|_{L^{2 q}(M d v)}^{2}+C\left\|\left(\bar{g}^{ \pm}\right)^{2}\left(\gamma_{\epsilon}^{ \pm} \chi\left(\frac{|v|^{2}}{K_{\epsilon}}\right)-1\right)\right\|_{L^{q}(M d v)} \\
& \leq C\left\|\hat{g}_{\epsilon}^{ \pm}-\bar{g}^{ \pm}\right\|_{L^{2}(M d v)}^{2} \\
& +C\left\|\frac{\bar{g}^{ \pm}}{1+|v|^{2}}\right\|_{L^{\infty}(d t d x d v)}^{2}\left\|\left(1+|v|^{4}\right) \chi\left(\frac{|v|^{2}}{K_{\epsilon}}\right)\left(\gamma_{\epsilon}^{ \pm}-1\right)\right\|_{L^{q}(M d v)} \\
& +C\left\|\frac{\bar{g}^{ \pm}}{1+|v|^{2}}\right\|_{L^{\infty}(d t d x d v)}^{2}\left\|\left(1+|v|^{4}\right)\left(\chi\left(\frac{|v|^{2}}{K_{\epsilon}}\right)-1\right)\right\|_{L^{q}(M d v)} .
\end{aligned}
$$

Therefore, utilizing the control of Gaussian tails 8.8 and the fact that $G_{\epsilon}^{ \pm} \geq 2$ on the support of $\gamma_{\epsilon}^{ \pm}-1$, we deduce that

$$
\begin{aligned}
& \left\|\left(\Pi \hat{g}_{\epsilon}^{ \pm}\right)^{2}\left(\gamma_{\epsilon}^{ \pm} \chi\left(\frac{|v|^{2}}{K_{\epsilon}}\right)-1\right)\right\|_{L^{q}(M d v)} \\
& \leq C\left\|\hat{g}_{\epsilon}^{ \pm}-\bar{g}^{ \pm}\right\|_{L^{2}(M d v)}^{2}+C K_{\epsilon}^{2} \epsilon^{\frac{2}{q}}\left\|\hat{g}_{\epsilon}^{ \pm}\right\|_{L^{2}(M d v)}^{\frac{2}{q}}+C|\log \epsilon|^{2+\frac{1}{2 q}} \frac{K}{2 q}^{\frac{K}{2 q}},
\end{aligned}
$$

which, when combined with (9.47), concludes the proof of 9.49).

- Next, we establish the last bound (9.50). Note first that the case $p=2$ is easily deduced from $\left(9.45\right.$, using again that $G_{\epsilon}^{ \pm} \geq 2$ implies $\epsilon \hat{g}_{\epsilon}^{ \pm} \geq 2(\sqrt{2}-1)$. Thus, the difficulty here lies in obtaining a gain of velocity integrability.

To this end, we introduce the macroscopic truncation

$$
\chi_{\epsilon}^{ \pm}=\mathbb{1}_{\left\{\epsilon\left\|g_{\epsilon}^{ \pm} \gamma_{\epsilon}^{ \pm}-\bar{g}^{ \pm}\right\|_{L^{2}(M d v)} \leq 1\right\}} .
$$

Then, we have

$$
\left(1-\chi_{\epsilon}^{ \pm}\right)\left\|\frac{1}{\epsilon} \mathbb{1}_{\left\{G_{\epsilon}^{ \pm} \geq 2\right\}}\right\|_{L^{p}(M d v)} \leq C\left\|g_{\epsilon}^{ \pm} \gamma_{\epsilon}-\bar{g}^{ \pm}\right\|_{L^{2}(M d v)} .
$$

Moreover, controlling Gaussian tails with 8.8 , it clearly holds that

$$
\chi_{\epsilon}^{ \pm}\left\|\frac{1}{\epsilon} \mathbb{1}_{\left\{G_{\epsilon}^{ \pm} \geq 2\right\}}\left(1-\chi\left(\frac{|v|^{2}}{K_{\epsilon}}\right)\right)\right\|_{L^{p}(M d v)} \leq C \epsilon^{\frac{K}{2 p}-1}|\log \epsilon|^{\frac{1}{2 p}},
$$

which is small as soon as $K \geq 8>2 p$, so that we only have to control the size of $\frac{1}{\epsilon} \mathbb{1}_{\left\{G_{\epsilon}^{ \pm} \geq 2\right\}} \chi\left(\frac{|v|^{2}}{K_{\epsilon}}\right)$ on the support of $\chi_{\epsilon}^{ \pm}$.

Thus, employing the decomposition

$$
\frac{1}{\epsilon} \mathbb{1}_{\left\{G_{\epsilon}^{ \pm} \geq 2\right\}} \leq \frac{3}{2} \mathbb{1}_{\left\{G_{\epsilon}^{ \pm} \geq 2\right\}}\left|\hat{g}_{\epsilon}^{ \pm}\right| \leq \frac{3}{2} \mathbb{1}_{\left\{G_{\epsilon}^{ \pm} \geq 2\right\}}\left(\left|\Pi \hat{g}_{\epsilon}^{ \pm}\right|+\left|\hat{g}_{\epsilon}^{ \pm}-\Pi \hat{g}_{\epsilon}^{ \pm}\right|\right),
$$


we find, for any $1<r<2$, the interpolation estimate

$$
\begin{aligned}
& \frac{1}{\epsilon^{2}} \mathbb{1}_{\left\{G_{\epsilon}^{ \pm} \geq 2\right\}} \chi\left(\frac{|v|^{2}}{K_{\epsilon}}\right) \\
& \leq \frac{C}{\epsilon^{2-\frac{2}{r}}} \mathbb{1}_{\left\{G_{\epsilon}^{ \pm} \geq 2\right\}} \chi\left(\frac{|v|^{2}}{K_{\epsilon}}\right)\left(\left|\Pi \hat{g}_{\epsilon}^{ \pm}\right|+\left|\hat{g}_{\epsilon}^{ \pm}-\Pi \hat{g}_{\epsilon}^{ \pm}\right|\right)^{\frac{2}{r}} \\
& \leq C \mathbb{1}_{\left\{G_{\epsilon}^{ \pm} \geq 2\right\}}\left|\hat{g}_{\epsilon}^{ \pm}\right|^{2-\frac{2}{r}}\left|\Pi \hat{g}_{\epsilon}^{ \pm}\right|^{\frac{2}{r}} \chi\left(\frac{|v|^{2}}{K_{\epsilon}}\right)+\frac{C}{\epsilon^{2-\frac{2}{r}}}\left|\hat{g}_{\epsilon}^{ \pm}-\Pi \hat{g}_{\epsilon}^{ \pm}\right|^{\frac{2}{r}} \\
& \leq C \mathbb{1}_{\left\{G_{\epsilon}^{ \pm} \geq 2\right\}}\left|\hat{g}_{\epsilon}^{ \pm}\right|^{2-\frac{2}{r}}\left|\Pi\left(\hat{g}_{\epsilon}^{ \pm}-\bar{g}^{ \pm}\right)\right|^{\frac{2}{r}} \\
& +C \mathbb{1}_{\left\{G_{\epsilon}^{ \pm} \geq 2\right\}}\left|\hat{g}_{\epsilon}^{ \pm}\right|^{2-\frac{2}{r}}\left|\bar{g}^{ \pm}\right|^{\frac{2}{r}} \chi\left(\frac{|v|^{2}}{K_{\epsilon}}\right)+\frac{C}{\epsilon^{2-\frac{2}{r}}}\left|\hat{g}_{\epsilon}^{ \pm}-\Pi \hat{g}_{\epsilon}^{ \pm}\right|^{\frac{2}{r}} \\
& \leq C \mathbb{1}_{\left\{G_{\epsilon}^{ \pm} \geq 2\right\}}\left|\hat{g}_{\epsilon}^{ \pm}\right|^{2-\frac{2}{r}}\left|\Pi\left(\hat{g}_{\epsilon}^{ \pm}-\bar{g}^{ \pm}\right)\right|^{\frac{2}{r}} \\
& +C K_{\epsilon}^{\frac{2}{r}} \epsilon^{\frac{4}{r}-2}\left|\hat{g}_{\epsilon}^{ \pm}\right|^{\frac{2}{r}}\left\|\frac{\bar{g}^{ \pm}}{1+|v|^{2}}\right\|_{L^{\infty}(d t d x d v)}^{\frac{2}{r}}+\frac{C}{\epsilon^{2-\frac{2}{r}}}\left|\hat{g}_{\epsilon}^{ \pm}-\Pi \hat{g}_{\epsilon}^{ \pm}\right|^{\frac{2}{r}} .
\end{aligned}
$$

Therefore, combining the preceding estimate with the relaxation 5.11 from Lemma 5.10 , we deduce that

$$
\begin{aligned}
& \left\|\frac{1}{\epsilon^{2}} \mathbb{1}_{\left\{G_{\epsilon}^{ \pm} \geq 2\right\}} \chi\left(\frac{|v|^{2}}{K_{\epsilon}}\right)\right\|_{L^{r}(M d v)} \\
& \leq C\left\|\mathbb{1}_{\left\{G_{\epsilon}^{ \pm} \geq 2\right\}} \hat{g}_{\epsilon}^{ \pm}\right\|_{L^{2}(M d v)}^{2-\frac{2}{r}}\left\|\Pi\left(\hat{g}_{\epsilon}^{ \pm}-\bar{g}^{ \pm}\right)\right\|_{L^{\frac{2}{2-r}}(M d v)}^{\frac{2}{r}} \\
& +C|\log \epsilon|^{\frac{2}{r}} \epsilon^{\frac{4}{r}-2}\left\|\hat{g}_{\epsilon}^{ \pm}\right\|_{L^{2}(M d v)}^{\frac{2}{r}}+\frac{C}{\epsilon^{2-\frac{2}{r}}}\left\|\hat{g}_{\epsilon}^{ \pm}-\Pi \hat{g}_{\epsilon}^{ \pm}\right\|_{L^{2}(M d v)}^{\frac{2}{r}} \\
& \leq C\left\|\mathbb{1}_{\left\{G_{\epsilon}^{ \pm} \geq 2\right\}} \hat{g}_{\epsilon}^{ \pm}\right\|_{L^{2}(M d v)}^{2-\frac{2}{r}}\left\|\hat{g}_{\epsilon}^{ \pm}-\bar{g}^{ \pm}\right\|_{L^{2}}^{\frac{2}{r}}(M d v) \\
& +C|\log \epsilon|^{\frac{2}{r}} \epsilon^{\frac{4}{r}-2}\left\|\hat{g}_{\epsilon}^{ \pm}\right\|_{L^{2}(M d v)}^{\frac{2}{r}}+\frac{C}{\epsilon^{2-\frac{2}{r}}}\left(\epsilon\left\|\hat{g}_{\epsilon}^{ \pm}\right\|_{L^{2}(M d v)}^{2}+O(\epsilon)_{L^{2}(d t d x)}\right)^{\frac{2}{r}} \\
& \leq C\left\|\mathbb{1}_{\left\{G_{\epsilon}^{ \pm} \geq 2\right\}} \hat{g}_{\epsilon}^{ \pm}\right\|_{L^{2}(M d v)}^{2}+C\left\|\hat{g}_{\epsilon}^{ \pm}-\bar{g}^{ \pm}\right\|_{L^{2}(M d v)}^{2} \\
& +C|\log \epsilon|^{\frac{2}{r}} \epsilon^{\frac{4}{r}-2}\left\|\hat{g}_{\epsilon}^{ \pm}\right\|_{L^{2}(M d v)}^{\frac{2}{r}}+C \epsilon^{\frac{4}{r}-2}\left\|g_{\epsilon}^{ \pm} \gamma_{\epsilon}^{ \pm}\right\|_{L^{2}(M d v)}^{\frac{4}{r}}+O\left(\epsilon^{\frac{4}{r}-2}\right) \\
& L^{r}(d t d x) \\
& \leq C\left\|\mathbb{1}_{\left\{G_{\epsilon}^{ \pm} \geq 2\right\}} \hat{g}_{\epsilon}^{ \pm}\right\|_{L^{2}(M d v)}^{2}+C\left\|\hat{g}_{\epsilon}^{ \pm}-\bar{g}^{ \pm}\right\|_{L^{2}(M d v)}^{2} \\
& +C|\log \epsilon|^{\frac{2}{r}} \epsilon^{\frac{4}{r}-2}\left\|\hat{g}_{\epsilon}^{ \pm}\right\|_{L^{2}(M d v)}^{\frac{2}{r}}+C \epsilon^{\frac{4}{r}-2}\left\|g_{\epsilon}^{ \pm} \gamma_{\epsilon}^{ \pm}-\bar{g}^{ \pm}\right\|_{L^{2}(M d v)}^{\frac{4}{r}} \\
& +C \epsilon^{\frac{4}{r}-2}\left\|\bar{g}^{ \pm}\right\|_{L^{2}(M d v)}^{\frac{4}{r}}+O\left(\epsilon^{\frac{4}{r}-2}\right) \\
& L^{r}(d t d x)
\end{aligned}
$$

Note that these controls do not yield vanishing remainders in the endpoint case $r=2$, which explains the use of the interpolation parameter $1<r<2$.

Then, recalling that the preceding estimate only needs to be performed on the support of $\chi_{\epsilon}^{ \pm}$and denoting $p=2 r$, we infer

$$
\begin{aligned}
& \chi_{\epsilon}^{ \pm}\left\|\frac{1}{\epsilon} \mathbb{1}_{\left\{G_{\epsilon}^{ \pm} \geq 2\right\}} \chi\left(\frac{|v|^{2}}{K_{\epsilon}}\right)\right\|_{L^{p}(M d v)} \\
& \leq C\left\|\mathbb{1}_{\left\{G_{\epsilon}^{ \pm} \geq 2\right\}} \hat{g}_{\epsilon}^{ \pm}\right\|_{L^{2}(M d v)}+C\left\|\hat{g}_{\epsilon}^{ \pm}-\bar{g}^{ \pm}\right\|_{L^{2}(M d v)}+C\left\|g_{\epsilon}^{ \pm} \gamma_{\epsilon}^{ \pm}-\bar{g}^{ \pm}\right\|_{L^{2}(M d v)} \\
& +C|\log \epsilon|^{\frac{2}{p}} \epsilon^{\frac{4}{p}-1}\left\|\hat{g}_{\epsilon}^{ \pm}\right\|_{L^{2}(M d v)}^{\frac{2}{p}}+C \epsilon^{\frac{4}{p}-1}\left\|\bar{g}^{ \pm}\right\|_{L^{2}(M d v)}^{\frac{4}{p}}+O\left(\epsilon^{\frac{4}{p}-1}\right)_{L^{p}(d t d x)}
\end{aligned}
$$


which, when combined with the bounds 9.45 and 9.47 , concludes the proof of (9.50).

The proof of the lemma is now complete.

Proof of Lemma 9.8. This lemma is a simple consequence of the relaxation estimate provided by Lemma 5.10 :

$$
\hat{g}_{\epsilon}^{ \pm}-\Pi \hat{g}_{\epsilon}^{ \pm}=O(\epsilon)_{L_{\mathrm{loc}}^{1}\left(d t d x ; L^{2}(M d v)\right)} .
$$

- In order to establish the first bound (9.51), notice that, since $G_{\epsilon}^{ \pm} \geq 2$ implies $\epsilon \hat{g}_{\epsilon}^{ \pm} \geq 2(\sqrt{2}-1)$

$$
\begin{aligned}
\left|\mathbb{1}_{\left\{G_{\epsilon}^{ \pm} \geq 2\right\}} \hat{g}_{\epsilon}^{ \pm}\right| & \leq\left|\hat{g}_{\epsilon}^{ \pm}-\Pi \hat{g}_{\epsilon}^{ \pm}\right|+\left|\mathbb{1}_{\left\{G_{\epsilon}^{ \pm} \geq 2\right\}} \Pi \hat{g}_{\epsilon}^{ \pm}\right| \\
& \leq\left|\hat{g}_{\epsilon}^{ \pm}-\Pi \hat{g}_{\epsilon}^{ \pm}\right|+C\left|\mathbb{1}_{\left\{G_{\epsilon}^{ \pm} \geq 2\right\}}\left(1+|v|^{2}\right)\right|\left\|\hat{g}_{\epsilon}^{ \pm}\right\|_{L^{2}(M d v)},
\end{aligned}
$$

whence

$$
\left\|\mathbb{1}_{\left\{G_{\epsilon}^{ \pm} \geq 2\right\}} \hat{g}_{\epsilon}^{ \pm} \chi\left(\frac{|v|^{2}}{K_{\epsilon}}\right)\right\|_{L^{2}(M d v)} \leq\left\|\hat{g}_{\epsilon}^{ \pm}-\Pi \hat{g}_{\epsilon}^{ \pm}\right\|_{L^{2}(M d v)}+C \epsilon K_{\epsilon}\left\|\hat{g}_{\epsilon}^{ \pm}\right\|_{L^{2}(M d v)}^{2} .
$$

Moreover, it is readily seen that

$$
\left\|\mathbb{1}_{\left\{G_{\epsilon}^{ \pm} \geq 2\right\}} \hat{g}_{\epsilon}^{ \pm}\left(1-\chi\left(\frac{|v|^{2}}{K_{\epsilon}}\right)\right)\right\|_{L^{2}(M d v)} \leq \frac{C}{K_{\epsilon}^{\frac{1}{2}}}\left\|\hat{g}_{\epsilon}^{ \pm}\right\|_{L^{2}\left(\left(1+|v|^{2}\right) M d v\right)} .
$$

Therefore, it follows that, combining the preceding estimates and considering the uniform bounds from Lemmas 5.2 and 5.12 .

$$
\left\|\mathbb{1}_{\left\{G_{\epsilon}^{ \pm} \geq 2\right\}} \hat{g}_{\epsilon}^{ \pm}\right\|_{L^{2}(M d v)}=o(1)_{L_{\mathrm{loc}}^{1}(d t d x)},
$$

which concludes the proof of (9.51) by interpolation.

- To deduce the second bound (9.52), we decompose, writing $g_{\epsilon}^{ \pm}=\hat{g}_{\epsilon}^{ \pm}\left(1+\frac{\epsilon}{4} \hat{g}_{\epsilon}^{ \pm}\right)$ and using that $\left(1+\frac{\epsilon}{4} \hat{g}_{\epsilon}^{ \pm}\right) \gamma_{\epsilon}^{ \pm}$is uniformly bounded pointwise,

$$
\begin{aligned}
\left|g_{\epsilon}^{ \pm} \gamma_{\epsilon}^{ \pm} \chi\left(\frac{|v|^{2}}{K_{\epsilon}}\right)-\hat{g}_{\epsilon}^{ \pm}\right| & =\left|\hat{g}_{\epsilon}^{ \pm}\left(\left(1+\frac{\epsilon}{4} \hat{g}_{\epsilon}^{ \pm}\right) \gamma_{\epsilon}^{ \pm} \chi\left(\frac{|v|^{2}}{K_{\epsilon}}\right)-1\right)\right| \\
& \leq C\left|\hat{g}_{\epsilon}^{ \pm}-\Pi \hat{g}_{\epsilon}^{ \pm}\right|+C\left|\Pi \hat{g}_{\epsilon}^{ \pm}\left(\left(1+\frac{\epsilon}{4} \hat{g}_{\epsilon}^{ \pm}\right) \gamma_{\epsilon}^{ \pm} \chi\left(\frac{|v|^{2}}{K_{\epsilon}}\right)-1\right)\right| \\
& \leq C\left|\hat{g}_{\epsilon}^{ \pm}-\Pi \hat{g}_{\epsilon}^{ \pm}\right|+C\left|\Pi \hat{g}_{\epsilon}^{ \pm}\left(1-\chi\left(\frac{|v|^{2}}{K_{\epsilon}}\right)\right)\right| \\
& +C \epsilon\left|\Pi \hat{g}_{\epsilon}^{ \pm} \hat{g}_{\epsilon}^{ \pm} \gamma_{\epsilon}^{ \pm} \chi\left(\frac{|v|^{2}}{K_{\epsilon}}\right)\right|+C\left|\Pi \hat{g}_{\epsilon}^{ \pm}\left(\gamma_{\epsilon}^{ \pm}-1\right) \chi\left(\frac{|v|^{2}}{K_{\epsilon}}\right)\right|
\end{aligned}
$$

which implies, since $\left\|\frac{\Pi \hat{g}_{\epsilon}}{1+|v|^{2}}\right\|_{L^{\infty}(M d v)} \leq C\left\|\hat{g}_{\epsilon}\right\|_{L^{2}(M d v)}$,

$$
\begin{aligned}
\| g_{\epsilon}^{ \pm} \gamma_{\epsilon}^{ \pm} & \chi\left(\frac{|v|^{2}}{K_{\epsilon}}\right)-\hat{g}_{\epsilon}^{ \pm} \|_{L^{2}(M d v)} \\
& \leq C\left\|\hat{g}_{\epsilon}^{ \pm}-\Pi \hat{g}_{\epsilon}^{ \pm}\right\|_{L^{2}(M d v)}+C \epsilon K_{\epsilon}\left\|\hat{g}_{\epsilon}^{ \pm}\right\|_{L^{2}(M d v)}^{2} \\
& +C\left\|\left(1+|v|^{2}\right)\left(1-\chi\left(\frac{|v|^{2}}{K_{\epsilon}}\right)\right)\right\|_{L^{2}(M d v)}\left\|\hat{g}_{\epsilon}^{ \pm}\right\|_{L^{2}(M d v)} .
\end{aligned}
$$


Then, employing the control of Gaussian tails 8.8 and the uniform bound from Lemma 5.2 , we infer that

$$
\begin{aligned}
\left\|g_{\epsilon}^{ \pm} \gamma_{\epsilon}^{ \pm} \chi\left(\frac{|v|^{2}}{K_{\epsilon}}\right)-\hat{g}_{\epsilon}^{ \pm}\right\|_{L^{2}(M d v)} & \leq C\left\|\hat{g}_{\epsilon}^{ \pm}-\Pi \hat{g}_{\epsilon}^{ \pm}\right\|_{L^{2}(M d v)} \\
& +O(\epsilon|\log \epsilon|)_{L^{\infty}\left(d t ; L^{1}(d x)\right)} \\
& +O\left(\epsilon^{\frac{K}{4}}|\log \epsilon|^{\frac{5}{4}}\right)_{L^{\infty}\left(d t ; L^{2}(d x)\right)}
\end{aligned}
$$

which, with an interpolation argument, establishes (9.52).

- The third bound $(9.53)$ easily follows from the estimate

$$
\begin{aligned}
& \left\|g_{\epsilon}^{ \pm} \gamma_{\epsilon}^{ \pm}-\hat{g}_{\epsilon}^{ \pm}\right\|_{L^{2}(M d v)} \\
& \leq\left\|g_{\epsilon}^{ \pm} \gamma_{\epsilon}^{ \pm} \chi\left(\frac{|v|^{2}}{K_{\epsilon}}\right)-\hat{g}_{\epsilon}^{ \pm}\right\|_{L^{2}(M d v)}+\left\|\hat{g}_{\epsilon}^{ \pm}\left(1-\chi\left(\frac{|v|^{2}}{K_{\epsilon}}\right)\right)\right\|_{L^{2}(M d v)} \\
& \leq\left\|g_{\epsilon}^{ \pm} \gamma_{\epsilon}^{ \pm} \chi\left(\frac{|v|^{2}}{K_{\epsilon}}\right)-\hat{g}_{\epsilon}^{ \pm}\right\|_{L^{2}(M d v)}+\frac{C}{K_{\epsilon}^{\frac{1}{2}}}\left\|\hat{g}_{\epsilon}^{ \pm}\right\|_{L^{2}\left(\left(1+|v|^{2}\right) M d v\right)},
\end{aligned}
$$

which, when combined with the second bound $(9.52)$, concludes its justification.

- Next, simply notice that the fourth bound (9.54) is a reformulation of Lemma 5.10 with an interpolation argument, which we have incorporated here for mere convenience.

- Finally, we easily establish the last bound (9.55). To this end, note first that the case $p=2$ is easily deduced from (9.51), using again that $G_{\epsilon}^{ \pm} \geq 2$ implies $\epsilon \hat{g}_{\epsilon}^{ \pm} \geq 2(\sqrt{2}-1)$. Furthermore, repeating the estimate leading to the bound (9.11) yields here that, for every $2 \leq p<4$,

$$
\frac{1}{\epsilon} \mathbb{1}_{\left\{G_{\epsilon}^{ \pm} \geq 2\right\}}=O(1)_{L_{\mathrm{loc}}^{2}\left(d t d x ; L^{p}(M d v)\right)}
$$

Therefore, the bound 9.55 is obtained by interpolation.

The proof of the lemma is thus complete.

Proof of Lemma 9.9. First, we utilize 5.25, with some fixed $\gamma>4$ therein to be determined later on, to estimate

$$
\begin{aligned}
(1+|v|)^{2}\left|\hat{g}_{\epsilon}^{ \pm}\right|^{2} & \leq \frac{2 \gamma^{\frac{1}{2}}}{\epsilon} \sqrt{h\left(\epsilon g_{\epsilon}^{ \pm}\right)}(1+|v|)\left|\hat{g}_{\epsilon}^{ \pm}\right|+\frac{2 \gamma^{\frac{1}{2}}}{\epsilon} e^{\frac{(1+|v|)^{2}}{2 \gamma}}(1+|v|)\left|\hat{g}_{\epsilon}^{ \pm}\right| \\
& \leq \frac{2 \gamma}{\epsilon^{2}} h\left(\epsilon g_{\epsilon}^{ \pm}\right)+\frac{1}{2}(1+|v|)^{2}\left|\hat{g}_{\epsilon}^{ \pm}\right|^{2}+\frac{2 \gamma^{\frac{1}{2}}}{\epsilon} e^{\frac{(1+|v|)^{2}}{2 \gamma}}(1+|v|)\left|\hat{g}_{\epsilon}^{ \pm}\right|
\end{aligned}
$$

whence

$$
(1+|v|)^{2}\left|\hat{g}_{\epsilon}^{ \pm}\right|^{2} \leq \frac{4 \gamma}{\epsilon^{2}} h\left(\epsilon g_{\epsilon}^{ \pm}\right)+\frac{4 \gamma^{\frac{1}{2}}}{\epsilon} e^{\frac{(1+|v|)^{2}}{2 \gamma}}(1+|v|)\left|\hat{g}_{\epsilon}^{ \pm}\right|
$$


It follows that, for any $2<p<4$,

$$
\begin{aligned}
& \left\|\mathbb{1}_{\left\{G_{\epsilon}^{ \pm} \geq 2\right\}} \hat{g}_{\epsilon}^{ \pm}\right\|_{L^{2}\left((1+|v|)^{2} M d v\right)}^{2} \\
& \leq \frac{4 \gamma}{\epsilon^{2}} \int_{\mathbb{R}^{3}} \mathbb{1}_{\left\{G_{\epsilon}^{ \pm} \geq 2\right\}} h\left(\epsilon g_{\epsilon}^{ \pm}\right) M d v \\
& +2\left\|\mathbb{1}_{\left\{G_{\epsilon}^{ \pm} \geq 2\right\}} \hat{g}_{\epsilon}^{ \pm}\right\|_{L^{2}(M d v)}^{2}+2\left\|\frac{\gamma^{\frac{1}{2}}}{\epsilon} \mathbb{1}_{\left\{G_{\epsilon}^{ \pm} \geq 2\right\}} e^{\frac{(1+|v|)^{2}}{2 \gamma}}(1+|v|)\right\|_{L^{2}(M d v)}^{2} \\
& \leq \frac{4 \gamma}{\epsilon^{2}} \int_{\mathbb{R}^{3}} \mathbb{1}_{\left\{G_{\epsilon}^{ \pm} \geq 2\right\}} h\left(\epsilon g_{\epsilon}^{ \pm}\right) M d v+2\left\|\mathbb{1}_{\left\{G_{\epsilon}^{ \pm} \geq 2\right\}} \hat{g}_{\epsilon}^{ \pm}\right\|_{L^{2}(M d v)}^{2} \\
& +2 \gamma\left\|\frac{1}{\epsilon} \mathbb{1}_{\left\{G_{\epsilon}^{ \pm} \geq 2\right\}}\right\|_{L^{p}(M d v)}^{2}\left\|e^{\frac{(1+|v|)^{2}}{2 \gamma}}(1+|v|)\right\|_{L^{\frac{2 p}{p-2}}(M d v)}^{2} .
\end{aligned}
$$

Here, we need to set the parameter $\gamma$ so large that $\frac{p}{p-2}<\frac{\gamma}{2}$ in order to yield a finite constant in the last term above.

Then, further using that $h(z)=\frac{1}{2}(z \gamma(1+z))^{2}+O\left(z^{3}\right)$, we deduce

$$
\begin{aligned}
& \left\|\mathbb{1}_{\left\{G_{\epsilon}^{ \pm} \geq 2\right\}} \hat{g}_{\epsilon}^{ \pm}\right\|_{L^{2}\left((1+|v|)^{2} M d v\right)}^{2} \\
& \leq \frac{C_{1}}{\epsilon^{2}} \int_{\mathbb{R}^{3}} \mathbb{1}_{\left\{G_{\epsilon}^{ \pm} \geq 2\right\}}\left(h\left(\epsilon g_{\epsilon}^{ \pm}\right)-\frac{\epsilon^{2}}{2}\left(g_{\epsilon}^{ \pm} \gamma_{\epsilon}^{ \pm}\right)^{2}\right) M d v \\
& +C_{2}\left\|\mathbb{1}_{\left\{G_{\epsilon}^{ \pm} \geq 2\right\}} \hat{g}_{\epsilon}^{ \pm}\right\|_{L^{2}(M d v)}^{2}+C_{2}\left\|\frac{1}{\epsilon} \mathbb{1}_{\left\{G_{\epsilon}^{ \pm} \geq 2\right\}}\right\|_{L^{p}(M d v)}^{2} \\
& \leq C_{1} \int_{\mathbb{R}^{3}}\left(\frac{1}{\epsilon^{2}} h\left(\epsilon g_{\epsilon}^{ \pm}\right)-\frac{1}{2}\left(g_{\epsilon}^{ \pm} \gamma_{\epsilon}^{ \pm}\right)^{2}\right) M d v \\
& +C_{2}\left\|_{\left\{G_{\epsilon}^{ \pm} \geq 2\right\}} \hat{g}_{\epsilon}^{ \pm}\right\|_{L^{2}(M d v)}^{2}+C_{2}\left\|\frac{1}{\epsilon} \mathbb{1}_{\left\{G_{\epsilon}^{ \pm} \geq 2\right\}}\right\|_{L^{p}(M d v)}^{2} \\
& +\frac{C_{2}}{\epsilon^{2}} \int_{\mathbb{R}^{3}} \mathbb{1}_{\left\{G_{\epsilon}^{ \pm}<2\right\}} h\left(\epsilon g_{\epsilon}^{ \pm}\right)-\frac{\epsilon^{2}}{2}\left(g_{\epsilon}^{ \pm} \gamma_{\epsilon}^{ \pm}\right)^{2} \mid M d v \\
& \leq C_{1} \int_{\mathbb{R}^{3}}\left(\frac{1}{\epsilon^{2}} h\left(\epsilon g_{\epsilon}^{ \pm}\right)-\frac{1}{2}\left(g_{\epsilon}^{ \pm} \gamma_{\epsilon}^{ \pm}\right)^{2}\right) M d v \\
& +C_{2}\left\|\mathbb{1}_{\left\{G_{\epsilon}^{ \pm} \geq 2\right\}} \hat{g}_{\epsilon}^{ \pm}\right\|_{L^{2}(M d v)}^{2}+C_{2}\left\|\frac{1}{\epsilon} \mathbb{1}_{\left\{G_{\epsilon}^{ \pm} \geq 2\right\}}\right\|_{L^{p}(M d v)}^{2} \\
& +C_{2} \int_{\mathbb{R}^{3}} \mathbb{1}_{\left\{\left|\epsilon g_{\epsilon}^{ \pm}\right|<1\right\}}\left|\epsilon g_{\epsilon}^{ \pm}\right|\left|g_{\epsilon}^{ \pm} \gamma_{\epsilon}^{ \pm}\right|^{2} M d v, \\
&
\end{aligned}
$$

where, $C_{1}>0$ and $C_{2}>0$ denote diverse constants which only depend on fixed parameters and which we do not distiguinsh for simplicity.

Finally, combining the preceding estimate with the bounds $(9.45)$ and $(9.50)$, modulating the last term $\left|g_{\epsilon}^{ \pm} \gamma_{\epsilon}^{ \pm}\right|^{2}=\left|g_{\epsilon}^{ \pm} \gamma_{\epsilon}^{ \pm}-\bar{g}^{ \pm}\right|^{2}+2 g_{\epsilon}^{ \pm} \gamma_{\epsilon}^{ \pm} \bar{g}^{ \pm}-\left|\bar{g}^{ \pm}\right|^{2}$, and using that $\mathbb{1}_{\left\{\left|\epsilon g_{\epsilon}^{ \pm}\right|<1\right\}}\left|\epsilon g_{\epsilon}^{ \pm}\right|$is bounded pointwise and converges almost everywhere to zero (possibly up to extraction of subsequences) with the Product Limit Theorem, we deduce the first estimate of the lemma.

The remaining estimate requires some care, for the function $h(z)-\frac{1}{2}(z \gamma(1+z))^{2}$ can take negative values. Integrating locally in $x$ the previous bound $(9.64)$, we 
first observe that

$$
\begin{aligned}
& \left\|\mathbb{1}_{\left\{G_{\epsilon}^{ \pm} \geq 2\right\}} \hat{g}_{\epsilon}^{ \pm}\right\|_{L_{\mathrm{loc}}^{2}\left(d x ; L^{2}\left((1+|v|)^{2} M d v\right)\right)}^{2} \\
& \leq \frac{C_{1}}{\epsilon^{2}} \int_{\mathbb{R}^{3} \times \mathbb{R}^{3}} \mathbb{1}\left\{h\left(\epsilon g_{\epsilon}^{ \pm}\right) \geq \frac{\epsilon^{2}}{2}\left(g_{\epsilon}^{ \pm} \gamma_{\epsilon}^{ \pm} \chi\left(\frac{|v|^{2}}{K_{\epsilon}}\right)\right)^{2}\right\} \\
& \times\left(h\left(\epsilon g_{\epsilon}^{ \pm}\right)-\frac{\epsilon^{2}}{2}\left(g_{\epsilon}^{ \pm} \gamma_{\epsilon}^{ \pm} \chi\left(\frac{|v|^{2}}{K_{\epsilon}}\right)\right)^{2}\right) M d x d v \\
& +C_{2}\left\|\mathbb{1}_{\left\{G_{\epsilon}^{ \pm} \geq 2\right\}} \hat{g}_{\epsilon}^{ \pm}\right\|_{L_{\mathrm{loc}}^{2}\left(d x ; L^{2}(M d v)\right)}^{2}+C_{2}\left\|\frac{1}{\epsilon} \mathbb{1}_{\left\{G_{\epsilon}^{ \pm} \geq 2\right\}}\right\|_{L_{\mathrm{loc}}^{2}\left(d x ; L^{p}(M d v)\right)}^{2} \\
& \leq C_{1} \int_{\mathbb{R}^{3} \times \mathbb{R}^{3}}\left(\frac{1}{\epsilon^{2}} h\left(\epsilon g_{\epsilon}^{ \pm}\right)-\frac{1}{2}\left(g_{\epsilon}^{ \pm} \gamma_{\epsilon}^{ \pm} \chi\left(\frac{|v|^{2}}{K_{\epsilon}}\right)\right)^{2}\right) M d x d v \\
& +C_{2}\left\|\mathbb{1}_{\left\{G_{\epsilon}^{ \pm} \geq 2\right\}} \hat{g}_{\epsilon}^{ \pm}\right\|_{L_{\mathrm{loc}}^{2}\left(d x ; L^{2}(M d v)\right)}^{2}+C_{2}\left\|\frac{1}{\epsilon} \mathbb{1}_{\left\{G_{\epsilon}^{ \pm} \geq 2\right\}}\right\|_{L_{\mathrm{loc}}^{2}\left(d x ; L^{p}(M d v)\right)}^{2}
\end{aligned}
$$

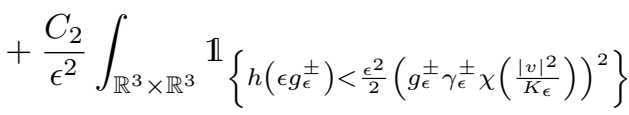

$$
\begin{aligned}
& \times\left(\frac{\epsilon^{2}}{2}\left(g_{\epsilon}^{ \pm} \gamma_{\epsilon}^{ \pm} \chi\left(\frac{|v|^{2}}{K_{\epsilon}}\right)\right)^{2}-h\left(\epsilon g_{\epsilon}^{ \pm}\right)\right) M d x d v \text {, }
\end{aligned}
$$

whence

$$
\begin{aligned}
& \left\|\mathbb{1}_{\left\{G_{\epsilon}^{ \pm} \geq 2\right\}} \hat{g}_{\epsilon}^{ \pm}\right\|_{L_{\mathrm{loc}}^{2}\left(d x ; L^{2}\left((1+|v|)^{2} M d v\right)\right)}^{2} \\
& \leq C_{1} \int_{\mathbb{R}^{3} \times \mathbb{R}^{3}}\left(\frac{1}{\epsilon^{2}} h\left(\epsilon g_{\epsilon}^{ \pm}\right)-\frac{1}{2}\left(g_{\epsilon}^{ \pm} \gamma_{\epsilon}^{ \pm} \chi\left(\frac{|v|^{2}}{K_{\epsilon}}\right)\right)^{2}\right) M d x d v \\
& +C_{2}\left\|\mathbb{1}_{\left\{G_{\epsilon}^{ \pm} \geq 2\right\}} \hat{g}_{\epsilon}^{ \pm}\right\|_{L_{\mathrm{loc}}^{2}\left(d x ; L^{2}(M d v)\right)}^{2}+C_{2}\left\|\frac{1}{\epsilon} \mathbb{1}_{\left\{G_{\epsilon}^{ \pm} \geq 2\right\}}\right\|_{L_{\mathrm{loc}}^{2}\left(d x ; L^{p}(M d v)\right)}^{2} \\
& +\frac{C_{2}}{\epsilon^{2}} \int_{\mathbb{R}^{3} \times \mathbb{R}^{3}} \mathbb{1}_{\left\{h\left(\epsilon g_{\epsilon}^{ \pm}\right)<\frac{\epsilon^{2}}{2}\left(g_{\epsilon}^{ \pm} \gamma_{\epsilon}^{ \pm}\right)^{2}\right\}}\left(\frac{\epsilon^{2}}{2}\left(g_{\epsilon}^{ \pm} \gamma_{\epsilon}^{ \pm}\right)^{2}-h\left(\epsilon g_{\epsilon}^{ \pm}\right)\right) \chi\left(\frac{|v|^{2}}{K_{\epsilon}}\right)^{2} M d x d v .
\end{aligned}
$$

Next, considering $N>0$ so large that $h(z)<\frac{1}{2}(z \gamma(1+z))^{2}$ implies $|z| \leq N$, for any $z \in[-1, \infty)$, which is always possible in view of the assumptions $(9.36)$ on $\gamma(z)$, and using that $h(z)=\frac{1}{2}(z \gamma(1+z))^{2}+O\left(z^{3}\right)$ again, we infer that

$$
\begin{aligned}
& \left\|\mathbb{1}_{\left\{G_{\epsilon}^{ \pm} \geq 2\right\}} \hat{g}_{\epsilon}^{ \pm}\right\|_{L_{\mathrm{loc}}^{2}\left(d x ; L^{2}\left((1+|v|)^{2} M d v\right)\right)}^{2} \\
& \leq C_{1} \int_{\mathbb{R}^{3} \times \mathbb{R}^{3}}\left(\frac{1}{\epsilon^{2}} h\left(\epsilon g_{\epsilon}^{ \pm}\right)-\frac{1}{2}\left(g_{\epsilon}^{ \pm} \gamma_{\epsilon}^{ \pm} \chi\left(\frac{|v|^{2}}{K_{\epsilon}}\right)\right)^{2}\right) M d x d v \\
& +C_{2}\left\|\mathbb{1}_{\left\{G_{\epsilon}^{ \pm} \geq 2\right\}} \hat{g}_{\epsilon}^{ \pm}\right\|_{L_{\mathrm{loc}}^{2}\left(d x ; L^{2}(M d v)\right)}^{2}+C_{2}\left\|\frac{1}{\epsilon} \mathbb{1}_{\left\{G_{\epsilon}^{ \pm} \geq 2\right\}}\right\|_{L_{\mathrm{loc}}^{2}\left(d x ; L^{p}(M d v)\right)}^{2} \\
& +C_{2} \int_{\mathbb{R}^{3} \times \mathbb{R}^{3}} \mathbb{1}_{\left\{\left|\epsilon g_{\epsilon}^{ \pm}\right| \leq N\right\}}\left|\epsilon g_{\epsilon}^{ \pm}\right|\left|g_{\epsilon}^{ \pm} \gamma_{\epsilon}^{ \pm} \chi\left(\frac{|v|^{2}}{K_{\epsilon}}\right)\right|^{2} M d x d v .
\end{aligned}
$$

Then, as before, combining the preceding estimate with the bounds 9.45 and 9.50, and modulating the last term $\frac{1}{2}\left|g_{\epsilon}^{ \pm} \gamma_{\epsilon}^{ \pm} \chi\left(\frac{|v|^{2}}{K_{\epsilon}}\right)\right|^{2} \leq\left|g_{\epsilon}^{ \pm} \gamma_{\epsilon}^{ \pm} \chi\left(\frac{|v|^{2}}{K_{\epsilon}}\right)-\bar{g}^{ \pm}\right|^{2}+$ 
$\left|\bar{g}^{ \pm}\right|^{2}$, we arrive at

$$
\begin{aligned}
& \left\|\mathbb{1}_{\left\{G_{\epsilon}^{ \pm} \geq 2\right\}} \hat{g}_{\epsilon}^{ \pm}\right\|_{L_{\mathrm{loc}}^{2}\left(d x ; L^{2}\left((1+|v|)^{2} M d v\right)\right)}^{2} \\
& \leq C_{1} \int_{\mathbb{R}^{3} \times \mathbb{R}^{3}}\left(\frac{1}{\epsilon^{2}} h\left(\epsilon g_{\epsilon}^{ \pm}\right)-\frac{1}{2}\left(g_{\epsilon}^{ \pm} \gamma_{\epsilon}^{ \pm} \chi\left(\frac{|v|^{2}}{K_{\epsilon}}\right)\right)^{2}\right) M d x d v \\
& +C_{2}\left\|g_{\epsilon}^{ \pm} \gamma_{\epsilon}^{ \pm} \chi\left(\frac{|v|^{2}}{K_{\epsilon}}\right)-\bar{g}^{ \pm}\right\|_{L^{2}(M d x d v)}^{2}+o(1)_{L_{\mathrm{loc}}^{1}(d t)} \\
& +C_{2} \int_{\mathbb{R}^{3} \times \mathbb{R}^{3}} \mathbb{1}_{\left\{\left|\epsilon g_{\epsilon}^{ \pm}\right| \leq N\right\}}\left|\epsilon g_{\epsilon}^{ \pm}\right|\left|\bar{g}^{ \pm}\right|^{2} M d x d v .
\end{aligned}
$$

Finally, since $\bar{g}^{ \pm}$belongs to $L^{\infty}\left(d t ; L^{2}(M d x d v)\right)$ and $\mathbb{1}_{\left\{\left|\epsilon g_{\epsilon}^{ \pm}\right|<1\right\}}\left|\epsilon g_{\epsilon}^{ \pm}\right|$is bounded pointwise and converges almost everywhere to zero, we deduce, through a straightforward application of Egorov's theorem, that the last term above vanishes locally in $L^{1}(d t)$, which concludes the proof of the lemma.

Proof of Lemma 9.10, We begin by estimating, using the relaxation estimate (5.14) from Lemma 5.11 .

$$
\begin{aligned}
& \left\|\left(\hat{g}_{\epsilon}^{+}-\hat{g}_{\epsilon}^{-}-\hat{n}_{\epsilon}\right) \hat{g}_{\epsilon}^{ \pm} \mathbb{1}_{\left\{G_{\epsilon}^{ \pm} \geq 2\right\}}\right\|_{L^{1}\left((1+|v|)^{2} M d v\right)} \\
& \leq\left\|\hat{g}_{\epsilon}^{ \pm} \mathbb{1}_{\left\{G_{\epsilon}^{ \pm} \geq 2\right\}}\right\|_{L^{2}\left((1+|v|)^{2} M d v\right)}^{2}+\frac{1}{4}\left\|\left(\hat{g}_{\epsilon}^{+}-\hat{g}_{\epsilon}^{-}-\hat{n}_{\epsilon}\right)\right\|_{L^{2}\left((1+|v|)^{2} M d v\right)}^{2} \\
& \leq\left\|\hat{g}_{\epsilon}^{ \pm} \mathbb{1}_{\left\{G_{\epsilon}^{ \pm} \geq 2\right\}}\right\|_{L^{2}\left((1+|v|)^{2} M d v\right)}^{2}+\frac{1}{4}\left\|\left(\hat{g}_{\epsilon}^{+}-\hat{g}_{\epsilon}^{-}-\hat{n}_{\epsilon}\right)\left(\left|\hat{g}_{\epsilon}^{+}\right|+\left|\hat{g}_{\epsilon}^{-}\right|\right)\right\|_{L^{1}\left((1+|v|)^{2} M d v\right)} \\
& +\frac{1}{4}\left\|\hat{g}_{\epsilon}^{+}-\hat{g}_{\epsilon}^{-}-\hat{n}_{\epsilon}\right\|_{L^{2}(M d v)}\left\|\hat{n}_{\epsilon}\right\|_{L^{2}\left((1+|v|)^{4} M d v\right)} \\
& \leq\left\|\hat{g}_{\epsilon}^{ \pm} \mathbb{1}_{\left\{G_{\epsilon}^{ \pm} \geq 2\right\}}\right\|_{L^{2}\left((1+|v|)^{2} M d v\right)}^{2}+\frac{1}{4}\left\|\left(\hat{g}_{\epsilon}^{+}-\hat{g}_{\epsilon}^{-}-\hat{n}_{\epsilon}\right)\left(\left|\hat{g}_{\epsilon}^{+}\right|+\left|\hat{g}_{\epsilon}^{-}\right|\right)\right\|_{L^{1}\left((1+|v|)^{2} M d v\right)} \\
& +C\left\|\hat{g}_{\epsilon}^{+}-\hat{g}_{\epsilon}^{-}-\hat{n}_{\epsilon}\right\|_{L^{2}(M d v)}\left\|\hat{g}_{\epsilon}^{+}-\hat{g}_{\epsilon}^{-}\right\| L_{L^{2}(M d v)} \\
& \leq\left\|\hat{g}_{\epsilon}^{ \pm} \mathbb{1}_{\left\{G_{\epsilon}^{ \pm} \geq 2\right\}}\right\|_{L^{2}\left((1+|v|)^{2} M d v\right)}^{2}+\frac{1}{4} \sum_{ \pm}\left\|\left(\hat{g}_{\epsilon}^{+}-\hat{g}_{\epsilon}^{-}-\hat{n}_{\epsilon}\right) \hat{g}_{\epsilon}^{ \pm}\right\|_{L^{1}\left((1+|v|)^{2} M d v\right)} \\
& +C \sum_{ \pm}\left\|\hat{g}_{\epsilon}^{ \pm}-\bar{g}^{ \pm}\right\|_{L^{2}(M d v)}^{2}+o(1)_{L_{1 \mathrm{oc}}^{1}(d t d x)} .
\end{aligned}
$$

It follows that

$$
\begin{aligned}
& \sum_{ \pm}\left\|\left(\hat{g}_{\epsilon}^{+}-\hat{g}_{\epsilon}^{-}-\hat{n}_{\epsilon}\right) \hat{g}_{\epsilon}^{ \pm}\right\|_{L^{1}\left((1+|v|)^{2} M d v\right)} \\
& \quad \leq \sum_{ \pm}\left\|\left(\hat{g}_{\epsilon}^{+}-\hat{g}_{\epsilon}^{-}-\hat{n}_{\epsilon}\right) \hat{g}_{\epsilon}^{ \pm} \mathbb{1}_{\left\{G_{\epsilon}^{ \pm}<2\right\}}\right\|_{L^{1}\left((1+|v|)^{2} M d v\right)} \\
& \quad+\sum_{ \pm}\left\|\left(\hat{g}_{\epsilon}^{+}-\hat{g}_{\epsilon}^{-}-\hat{n}_{\epsilon}\right) \hat{g}_{\epsilon}^{ \pm} \mathbb{1}_{\left\{G_{\epsilon}^{ \pm} \geq 2\right\}}\right\|_{L^{1}\left((1+|v|)^{2} M d v\right)} \\
& \quad \leq \sum_{ \pm}\left\|\left(\hat{g}_{\epsilon}^{+}-\hat{g}_{\epsilon}^{-}-\hat{n}_{\epsilon}\right) \hat{g}_{\epsilon}^{ \pm} \mathbb{1}_{\left\{G_{\epsilon}^{ \pm}<2\right\}}\right\|_{L^{1}\left((1+|v|)^{2} M d v\right)} \\
& \quad+\sum_{ \pm}\left\|\hat{g}_{\epsilon}^{ \pm} \mathbb{1}_{\left\{G_{\epsilon}^{ \pm} \geq 2\right\}}\right\|_{L^{2}\left((1+|v|)^{2} M d v\right)}+\frac{1}{2} \sum_{ \pm}\left\|\left(\hat{g}_{\epsilon}^{+}-\hat{g}_{\epsilon}^{-}-\hat{n}_{\epsilon}\right) \hat{g}_{\epsilon}^{ \pm}\right\|_{L^{1}\left((1+|v|)^{2} M d v\right)} \\
& \quad+C \sum_{ \pm}\left\|\hat{g}_{\epsilon}^{ \pm}-\bar{g}^{ \pm}\right\|_{L^{2}(M d v)}^{2}+o(1)_{L_{\mathrm{loc}}^{1}(d t d x)},
\end{aligned}
$$


whence

$$
\begin{aligned}
& \sum_{ \pm}\left\|\left(\hat{g}_{\epsilon}^{+}-\hat{g}_{\epsilon}^{-}-\hat{n}_{\epsilon}\right) \hat{g}_{\epsilon}^{ \pm}\right\|_{L^{1}\left((1+|v|)^{2} M d v\right)} \\
& \leq 2 \sum_{ \pm}\left\|\left(\hat{g}_{\epsilon}^{+}-\hat{g}_{\epsilon}^{-}-\hat{n}_{\epsilon}\right) \hat{g}_{\epsilon}^{ \pm} \mathbb{1}_{\left\{G_{\epsilon}^{ \pm}<2\right\}}\right\|_{L^{1}\left((1+|v|)^{2} M d v\right)} \\
& +2 \sum_{ \pm}\left\|\hat{g}_{\epsilon}^{ \pm} \mathbb{1}_{\left\{G_{\epsilon}^{ \pm} \geq 2\right\}}\right\|_{L^{2}\left((1+|v|)^{2} M d v\right)}^{2}+C \sum_{ \pm}\left\|\hat{g}_{\epsilon}^{ \pm}-\bar{g}^{ \pm}\right\|_{L^{2}(M d v)}^{2}+o(1)_{L_{\mathrm{loc}}^{1}(d t d x)} .
\end{aligned}
$$

Thus, in view of the estimates 9.46 and 9.47 from Lemma 9.7 and utilizing Lemma 9.9. we deduce that, in order to conclude the proof of the lemma, it is sufficient to establish that

$$
\begin{aligned}
\left\|\left(\hat{g}_{\epsilon}^{+}-\hat{g}_{\epsilon}^{-}-\hat{n}_{\epsilon}\right) \hat{g}_{\epsilon}^{ \pm} \mathbb{1}_{\left\{G_{\epsilon}^{ \pm}<2\right\}}\right\|_{L^{1}\left((1+|v|)^{2} M d v\right)} & \leq C \sum_{ \pm}\left\|\hat{g}_{\epsilon}^{ \pm}-\bar{g}^{ \pm}\right\|_{L^{2}(M d v)}^{2} \\
& +o(1)_{L_{\mathrm{loc}}^{1}(d t d x)} .
\end{aligned}
$$

To this end, employing the estimate 5 from Lemma 5.11, we first obtain that

$$
\begin{aligned}
& \left\|\left(\hat{g}_{\epsilon}^{+}-\hat{g}_{\epsilon}^{-}-\hat{n}_{\epsilon}\right) \hat{g}_{\epsilon}^{ \pm} \mathbb{1}_{\left\{G_{\epsilon}^{ \pm}<2\right\}}\right\|_{L^{1}\left((1+|v|)^{2} M d v\right)} \\
& \leq\left\|\left(\hat{g}_{\epsilon}^{+}-\hat{g}_{\epsilon}^{-}-\hat{n}_{\epsilon}-\frac{\epsilon}{2} \hat{n}_{\epsilon}\left(\hat{g}_{\epsilon}^{ \pm}-\hat{\rho}_{\epsilon}^{ \pm}\right)\right) \hat{g}_{\epsilon}^{ \pm} \mathbb{1}_{\left\{G_{\epsilon}^{ \pm}<2\right\}}\right\|_{L^{1}\left((1+|v|)^{2} M d v\right)} \\
& +\frac{1}{2}\left|\hat{n}_{\epsilon}\right|\left\|\left(\hat{g}_{\epsilon}^{ \pm}-\hat{\rho}_{\epsilon}^{ \pm}\right) \epsilon \hat{g}_{\epsilon}^{ \pm} \mathbb{1}_{\left\{G_{\epsilon}^{ \pm}<2\right\}}\right\|_{L^{1}\left((1+|v|)^{2} M d v\right)} \\
& \leq\left\|\hat{g}_{\epsilon}^{+}-\hat{g}_{\epsilon}^{-}-\hat{n}_{\epsilon}-\frac{\epsilon}{2} \hat{n}_{\epsilon}\left(\hat{g}_{\epsilon}^{ \pm}-\hat{\rho}_{\epsilon}^{ \pm}\right)\right\|_{L^{2}(M d v)}\left\|\hat{g}_{\epsilon}^{ \pm} \mathbb{1}_{\left\{G_{\epsilon}^{ \pm}<2\right\}}\right\|_{L^{2}\left((1+|v|)^{4} M d v\right)} \\
& +C\left(\sum_{ \pm}\left\|\hat{g}_{\epsilon}^{ \pm}\right\|_{L^{2}(M d v)}^{2}\right)\left\|\epsilon \hat{g}_{\epsilon}^{ \pm} \mathbb{1}_{\left\{G_{\epsilon}^{ \pm}<2\right\}}\right\|_{L^{2}\left((1+|v|)^{4} M d v\right)} \\
& \leq C\left(\sum_{ \pm}\left\|\hat{g}_{\epsilon}^{ \pm}\right\|_{L^{2}(M d v)}^{2}\right)\left\|\epsilon \hat{g}_{\epsilon}^{ \pm} \mathbb{1}_{\left\{G_{\epsilon}^{ \pm}<2\right\}}\right\|_{L^{2}\left((1+|v|)^{4} M d v\right)}+o(1)_{L_{\mathrm{loc}}^{1}(d t d x)} .
\end{aligned}
$$

Then, noticing that, in view of Lemma 5.12

$$
\begin{aligned}
& \left\|\epsilon \hat{g}_{\epsilon}^{ \pm} \mathbb{1}_{\left\{G_{\epsilon}^{ \pm}<2\right\}}\right\|_{L^{2}\left((1+|v|)^{4} M d v\right)}=O(1)_{L^{\infty}(d t d x)}, \\
& \left\|\epsilon \hat{g}_{\epsilon}^{ \pm} \mathbb{1}_{\left\{G_{\epsilon}^{ \pm}<2\right\}}\right\|_{L^{2}\left((1+|v|)^{4} M d v\right)}=O(\epsilon)_{L_{\mathrm{loc}}^{2}(d t d x)},
\end{aligned}
$$

we conclude that 9.65 holds, which completes the proof of the lemma. 



\section{CHAPTER 10}

\section{Acoustic and electromagnetic waves}

In Chapter 5, we conducted a rather extensive study of the scaled relative entropy and entropy dissipation bounds. These yielded controls on the fluctuations in all variables $t, x$ and $v$ in appropriate function spaces and, thus, allowed us to establish essential weak compactness estimates on the fluctuations. Moreover, relaxation estimates were also obtained therein, showing that fluctuations remain close to their hydrodynamic projection, which implied improved controls in the $v$ variable.

Then, in Chapter 7, we showed that the control of the behavior of fluctuations in $v$ could be improved to strong compactness estimates in $v$, which could then be transfered - exploiting the hypoelliptic phenomenon in kinetic transport equations to the $x$ variable to deduce strong compactness estimates in both $x$ and $v$.

Thus, we know so far that there are no oscillations in $x$ and $v$ in the fluctuations as the Knudsen number tends to zero. Note, however, that nothing is claimed about the control of oscillations in the $t$ variable in the fluctuations and the control of oscillations in $t$ and $x$ in the electromagnetic fields.

In fact, because of the scaling of the transport operator $\epsilon \partial_{t}+v \cdot \nabla_{x}$, we do not expect to obtain additional regularity or compactness with respect to time on the fluctuations : the natural variable is indeed the fast time $\frac{t}{\epsilon}$ (see discussion in Section 7.2.1). We are however interested in the slow macroscopic dynamics. Since there is nothing to prevent an oscillatory behavior in $t$, we need to further describe the dependence of fluctuations with respect to time and filter the fast oscillations.

There may also be persistence of fast oscillations in both $t$ and $x$ in the electromagnetic fields (and electrodynamic macroscopic variables, such as the electric current), which we do not expect to control due to the hyperbolic nature of Maxwell's equations.

It turns out that oscillations in fluctuations and electromagnetic fields are sometimes coupled. We will therefore need to treat and filter them simultaneously.

In the context of the viscous incompressible hydrodynamic limit of the Boltzmann equation, the filtering of acoustic waves was first understood by Lions and Masmoudi in 55.

In the present chapter, we are going to focus exclusively on the one species setting treated in Theorem 4.4, i.e. on the regime leading to the incompressible quasi-static Navier-Stokes-Fourier-Maxwell-Poisson system. The proof of this result is based on weak compactness methods which require the handling of possible time and electromagnetic oscillations. In this case, the available strong compactness with respect to spatial variables is good enough and we are actually able to get here a rough description of oscillations, which will be sufficient to derive the weak stability and convergence of the Vlasov-Maxwell-Boltzmann system 4.27) as $\epsilon \rightarrow 0$.

As for Theorems 4.5 and 4.6 concerning the two species setting, i.e. in the regime leading to the two-fluid incompressible Navier-Stokes-Fourier-Maxwell system with (solenoidal) Ohm's law, the previous filtering method cannot be applied, and - as 
already mentioned - there is no asymptotic weak stability of the Vlasov-MaxwellBoltzmann system 4.34 (nor existence of weak solutions to the corresponding limiting model). In order to bypass this difficulty, the idea in this setting is then to compare the actual solutions of the scaled Vlasov-Maxwell-Boltzmann system to some approximate solutions (known a priori to be regular in $t$ and $x$ ) capturing the fast oscillations. This method of proof, detailed in Chapter 12 later on, is the so-called renormalized modulated entropy method, which is only performed in this work in the case of well-prepared initial data, for the sake of simplicity. The oscillations are therefore automatically filtered out by the method and we do not need to further describe the time dependence of fluctuations. Of course, the case of ill-prepared initial data for two species is interesting and should be addressed. Nevertheless, this issue only seems to present difficulties somewhat similar to those encountered in the handling of initial data in the asymptotic problems considered in 65. and 68, Chapter 5], for instance.

\subsection{Formal filtering of oscillations}

Now, as mentionned above, let us focus exclusively, for the remainder of the present chapter, on the regime of Theorem 4.4 (with one species of particles only) leading to the incompressible quasi-static Navier-Stokes-Fourier-Maxwell-Poisson system 4.30.

On the one hand, going back to the corresponding formal analysis from Chapter 2 . we expect that the fast time oscillations are governed by the following singular linear system given by 2.16 and 2.21 :

$$
\partial_{t}\left(\begin{array}{c}
\rho_{\epsilon} \\
u_{\epsilon} \\
\sqrt{\frac{3}{2}} \theta_{\epsilon} \\
E_{\epsilon} \\
B_{\epsilon}
\end{array}\right)+\frac{1}{\epsilon} W\left(\begin{array}{c}
\rho_{\epsilon} \\
u_{\epsilon} \\
\sqrt{\frac{3}{2}} \theta_{\epsilon} \\
E_{\epsilon} \\
B_{\epsilon}
\end{array}\right)=O(1)
$$

where the antisymmetric differential operator $W: L^{2}(d x) \rightarrow H^{-1}(d x)$ - the wave operator - is defined by

$$
W=\left(\begin{array}{ccccc}
0 & \operatorname{div} & 0 & 0 & 0 \\
\nabla_{x} & 0 & \sqrt{\frac{2}{3}} \nabla_{x} & - \text { Id } & 0 \\
0 & \sqrt{\frac{2}{3}} \operatorname{div} & 0 & 0 & 0 \\
0 & \text { Id } & 0 & 0 & -\operatorname{rot} \\
0 & 0 & 0 & \text { rot } & 0
\end{array}\right) .
$$

On the other hand, looking back at the formal macroscopic nonlinear system 2.24 , we see that, in order to derive the limiting system 4.30, we will eventually need to pass to the limit in the nonlinear terms

$$
P\left(\nabla_{x} \cdot\left(u_{\epsilon} \otimes u_{\epsilon}\right)-\rho_{\epsilon} E_{\epsilon}-u_{\epsilon} \wedge B_{\epsilon}\right) \quad \text { and } \quad \frac{5}{2} \nabla_{x} \cdot\left(u_{\epsilon} \theta_{\epsilon}\right)-u_{\epsilon} \cdot E_{\epsilon},
$$

where $P: L^{2}(d x) \rightarrow L^{2}(d x)$ denotes the Leray projector onto solenoidal vector fields, and establish their weak stability. Since there are oscillations, this will only be possible if one can show that the linear structure 10.1) is somehow "compatible" with the quadratic forms defined by 10.3 .

We explain now why such a "compatibility" between the structures of (10.1) and $(10.3)$ is to be expected, at least formally. First, we decompose the nonlinear 
terms 10.3 into

$$
\begin{aligned}
P & \left(\nabla_{x} \cdot\left(u_{\epsilon} \otimes u_{\epsilon}\right)-\rho_{\epsilon} E_{\epsilon}-u_{\epsilon} \wedge B_{\epsilon}\right) \\
& =P\left(u_{\epsilon} \operatorname{div} u_{\epsilon}+\frac{1}{2} \nabla_{x}\left|u_{\epsilon}\right|^{2}-u_{\epsilon} \wedge \operatorname{rot} u_{\epsilon}-\rho_{\epsilon} E_{\epsilon}-u_{\epsilon} \wedge B_{\epsilon}\right) \\
& =P\left(u_{\epsilon}\left(\epsilon \partial_{t} \rho_{\epsilon}+\operatorname{div} u_{\epsilon}\right)+\rho_{\epsilon}\left(\epsilon \partial_{t} u_{\epsilon}+\nabla_{x}\left(\rho_{\epsilon}+\theta_{\epsilon}\right)-E_{\epsilon}\right)\right) \\
& -P\left(\rho_{\epsilon} \nabla_{x}\left(\rho_{\epsilon}+\theta_{\epsilon}\right)+u_{\epsilon} \wedge\left(\operatorname{rot} u_{\epsilon}+B_{\epsilon}\right)+\epsilon \partial_{t}\left(\rho_{\epsilon} u_{\epsilon}\right)\right) \\
& =P\left(u_{\epsilon}\left(\epsilon \partial_{t} \rho_{\epsilon}+\operatorname{div} u_{\epsilon}\right)+\rho_{\epsilon}\left(\epsilon \partial_{t} u_{\epsilon}+\nabla_{x}\left(\rho_{\epsilon}+\theta_{\epsilon}\right)-E_{\epsilon}\right)\right) \\
& -P\left(\frac{2 \rho_{\epsilon}-3 \theta_{\epsilon}}{5} \nabla_{x}\left(\rho_{\epsilon}+\theta_{\epsilon}\right)+\frac{3}{10} \nabla_{x}\left(\rho_{\epsilon}+\theta_{\epsilon}\right)^{2}\right) \\
& -P\left(u_{\epsilon} \wedge\left(\operatorname{rot} u_{\epsilon}+B_{\epsilon}\right)+\epsilon \partial_{t}\left(\rho_{\epsilon} u_{\epsilon}\right)\right) \\
& =P\left(u_{\epsilon}\left(\epsilon \partial_{t} \rho_{\epsilon}+\operatorname{div} u_{\epsilon}\right)+\rho_{\epsilon}\left(\epsilon \partial_{t} u_{\epsilon}+\nabla_{x}\left(\rho_{\epsilon}+\theta_{\epsilon}\right)-E_{\epsilon}\right)\right) \\
& -P\left(\frac{2 \rho_{\epsilon}-3 \theta_{\epsilon}}{5} \nabla_{x}\left(\rho_{\epsilon}+\theta_{\epsilon}\right)+u_{\epsilon} \wedge\left(\operatorname{rot} u_{\epsilon}+B_{\epsilon}\right)+\epsilon \partial_{t}\left(\rho_{\epsilon} u_{\epsilon}\right)\right),
\end{aligned}
$$

where we used that $P \frac{1}{2} \nabla_{x}\left|u_{\epsilon}\right|^{2}=P \frac{3}{10} \nabla_{x}\left(\rho_{\epsilon}+\theta_{\epsilon}\right)^{2}=0$, and

$$
\begin{aligned}
\frac{5}{2} & \nabla_{x} \cdot\left(u_{\epsilon} \theta_{\epsilon}\right)-u_{\epsilon} \cdot E_{\epsilon} \\
& =\frac{5}{2} \theta_{\epsilon} \operatorname{div} u_{\epsilon}+\frac{5}{2} u_{\epsilon} \cdot \nabla_{x} \theta_{\epsilon}-u_{\epsilon} \cdot E_{\epsilon} \\
& =\frac{5}{2} \theta_{\epsilon}\left(\frac{3}{2} \epsilon \partial_{t} \theta_{\epsilon}+\operatorname{div} u_{\epsilon}\right)+u_{\epsilon} \cdot\left(\epsilon \partial_{t} u_{\epsilon}+\nabla_{x}\left(\rho_{\epsilon}+\theta_{\epsilon}\right)-E_{\epsilon}\right) \\
& +u_{\epsilon} \cdot \nabla_{x}\left(\frac{3}{2} \theta_{\epsilon}-\rho_{\epsilon}\right)-\frac{15}{8} \epsilon \partial_{t} \theta_{\epsilon}^{2}-\frac{1}{2} \epsilon \partial_{t} u_{\epsilon}^{2}
\end{aligned}
$$

which formally implies, using the first three equations from 10.1, that

$$
\begin{aligned}
P\left(\nabla_{x} \cdot\left(u_{\epsilon} \otimes u_{\epsilon}\right)-\rho_{\epsilon} E_{\epsilon}-u_{\epsilon}\right. & \left.\wedge B_{\epsilon}\right) \\
& =-P\left(\frac{2 \rho_{\epsilon}-3 \theta_{\epsilon}}{5} \nabla_{x}\left(\rho_{\epsilon}+\theta_{\epsilon}\right)+u_{\epsilon} \wedge\left(\operatorname{rot} u_{\epsilon}+B_{\epsilon}\right)\right)+O(\epsilon), \\
\frac{5}{2} \nabla_{x} \cdot\left(u_{\epsilon} \theta_{\epsilon}\right)-u_{\epsilon} \cdot E_{\epsilon} & =u_{\epsilon} \cdot \nabla_{x}\left(\frac{3}{2} \theta_{\epsilon}-\rho_{\epsilon}\right)+O(\epsilon) .
\end{aligned}
$$

Thus, this decomposition is sufficient to deduce the weak stability of the nonlinear terms 10.3 provided the oscillating part of $\left(\rho_{\epsilon}, u_{\epsilon}, \sqrt{\frac{3}{2}} \theta_{\epsilon}, E_{\epsilon}, B_{\epsilon}\right)$ can be restricted to the constraints $3 \theta_{\epsilon}-2 \rho_{\epsilon}=0$ and $\operatorname{rot} u_{\epsilon}+B_{\epsilon}=0$, i.e. provided we can find a decomposition

$$
\left(\begin{array}{c}
\rho_{\epsilon} \\
u_{\epsilon} \\
\sqrt{\frac{3}{2}} \theta_{\epsilon} \\
E_{\epsilon} \\
B_{\epsilon}
\end{array}\right)=\left(\begin{array}{c}
\bar{\rho}_{\epsilon} \\
\bar{u}_{\epsilon} \\
\sqrt{\frac{3}{2}} \bar{\theta}_{\epsilon} \\
\bar{E}_{\epsilon} \\
\bar{B}_{\epsilon}
\end{array}\right)+\left(\begin{array}{c}
\tilde{\rho}_{\epsilon} \\
\tilde{u}_{\epsilon} \\
\sqrt{\frac{3}{2}} \tilde{\theta}_{\epsilon} \\
\tilde{E}_{\epsilon} \\
\tilde{B}_{\epsilon}
\end{array}\right)
$$

such that $\left(\bar{\rho}_{\epsilon}, \bar{u}_{\epsilon}, \sqrt{\frac{3}{2}} \bar{\theta}_{\epsilon}, \bar{E}_{\epsilon}, \bar{B}_{\epsilon}\right)$ is relatively compact in the strong topology of $L_{\text {loc }}^{2}(d t d x)$, whereas $\left(\tilde{\rho}_{\epsilon}, \tilde{u}_{\epsilon}, \sqrt{\frac{3}{2}} \tilde{\theta}_{\epsilon}, \tilde{E}_{\epsilon}, \tilde{B}_{\epsilon}\right) \rightarrow 0$ in $L_{\text {loc }}^{2}(d t d x)$, with $3 \tilde{\theta}_{\epsilon}-2 \tilde{\rho}_{\epsilon}=0$ and $\operatorname{rot} \tilde{u}_{\epsilon}+\tilde{B}_{\epsilon}=0$. 
In order to obtain such a decomposition, it is very natural to orthogonally project $\left(\rho_{\epsilon}, u_{\epsilon}, \sqrt{\frac{3}{2}} \theta_{\epsilon}, E_{\epsilon}, B_{\epsilon}\right)$ onto the kernel of $W$

$$
\operatorname{Ker} W=\left\{\left(\rho, u, \sqrt{\frac{3}{2}} \theta, E, B\right) \in L^{2}(d x): E=\nabla_{x}(\rho+\theta) \text { and } u=\operatorname{rot} B\right\},
$$

and on its orthogonal complement

$$
\operatorname{Ker} W^{\perp}=\left\{\left(\rho, u, \sqrt{\frac{3}{2}} \theta, E, B\right) \in L^{2}(d x): \frac{3}{2} \theta=\rho=\operatorname{div} E \text { and } B=-\operatorname{rot} u\right\} .
$$

More precisely, we define

$$
\left(\begin{array}{c}
\bar{\rho}_{\epsilon} \\
\bar{u}_{\epsilon} \\
\sqrt{\frac{3}{2}} \bar{\theta}_{\epsilon} \\
\bar{E}_{\epsilon} \\
\bar{B}_{\epsilon}
\end{array}\right)=P_{W}\left(\begin{array}{c}
\rho \\
u \\
\sqrt{\frac{3}{2}} \theta \\
E \\
B
\end{array}\right)=\left(\begin{array}{c}
\frac{3}{3-5 \Delta_{x}}(\rho-\operatorname{div} E)+\frac{\Delta_{x}}{3-5 \Delta_{x}}(3 \theta-2 \rho) \\
\frac{\operatorname{rot}}{1-\Delta_{x}}(\operatorname{rot} u+B) \\
\frac{2}{3-5 \Delta_{x}} \sqrt{\frac{3}{2}}(\rho-\operatorname{div} E)+\frac{1-\Delta_{x}}{3-5 \Delta_{x}} \sqrt{\frac{3}{2}}(3 \theta-2 \rho) \\
\frac{5}{3-5 \Delta_{x}} \nabla_{x}(\rho-\operatorname{div} E)+\frac{1}{3-5 \Delta_{x}} \nabla_{x}(3 \theta-2 \rho) \\
\frac{1}{1-\Delta_{x}}\left(\operatorname{rot} u+B-\nabla_{x} \operatorname{div} B\right)
\end{array}\right),
$$

where $P_{W}: L^{2}(d x) \rightarrow L^{2}(d x)$ is the orthogonal projection onto Ker $W$, and

$$
\left(\begin{array}{c}
\tilde{\rho}_{\epsilon} \\
\tilde{u}_{\epsilon} \\
\sqrt{\frac{3}{2}} \tilde{\theta}_{\epsilon} \\
\tilde{E}_{\epsilon} \\
\tilde{B}_{\epsilon}
\end{array}\right)=P_{W}^{\perp}\left(\begin{array}{c}
\rho \\
u \\
\sqrt{\frac{3}{2}} \theta \\
E \\
B
\end{array}\right)=\left(\begin{array}{c}
\frac{3 \operatorname{div}}{3-5 \Delta_{x}}\left(E-\nabla_{x}(\rho+\theta)\right) \\
\frac{1}{1-\Delta_{x}}\left(u-\operatorname{rot} B-\nabla_{x} \operatorname{div} u\right) \\
\frac{2 \operatorname{div}}{3-5 \Delta_{x}} \sqrt{\frac{3}{2}}\left(E-\nabla_{x}(\rho+\theta)\right) \\
\frac{5 \operatorname{rot} \operatorname{rot}}{3-5 \Delta_{x}} E+\frac{3}{3-5 \Delta_{x}}\left(E-\nabla_{x}(\rho+\theta)\right) \\
\frac{\operatorname{rot}}{1-\Delta_{x}}(\operatorname{rot} B-u)
\end{array}\right),
$$

where $P_{W}^{\perp}: L^{2}(d x) \rightarrow L^{2}(d x)$ is the orthogonal projection onto Ker $W^{\perp}$. Note that these projections can also be computed explicitly using basic linear algebra in Fourier variables.

Then, recalling that $W$ is antisymmetric so that its range is orthogonal to its kernel, it holds that $P_{W} W=0$, whence

$$
\partial_{t}\left(\begin{array}{c}
\bar{\rho}_{\epsilon} \\
\bar{u}_{\epsilon} \\
\sqrt{\frac{3}{2}} \bar{\theta}_{\epsilon} \\
\bar{E}_{\epsilon} \\
\bar{B}_{\epsilon}
\end{array}\right)=O(1)
$$

which implies that $\left(\bar{\rho}_{\epsilon}, \bar{u}_{\epsilon}, \sqrt{\frac{3}{2}} \bar{\theta}_{\epsilon}, \bar{E}_{\epsilon}, \bar{B}_{\epsilon}\right)$ is expected to be compact in $t$. Moreover, since $\left(\rho_{\epsilon}, u_{\epsilon}, \sqrt{\frac{3}{2}} \theta_{\epsilon}, E_{\epsilon}, B_{\epsilon}\right)$ belongs to $\operatorname{Ker} W$ in the limit $\epsilon \rightarrow 0$, it is naturally expected that $\left(\tilde{\rho}_{\epsilon}, \tilde{u}_{\epsilon}, \sqrt{\frac{3}{2}} \tilde{\theta}_{\epsilon}, \tilde{E}_{\epsilon}, \tilde{B}_{\epsilon}\right) \rightarrow 0$. Finally, the constraints $3 \tilde{\theta}_{\epsilon}-2 \tilde{\rho}_{\epsilon}=0$ and $\operatorname{rot} \tilde{u}_{\epsilon}+\tilde{B}_{\epsilon}=0$ are implied by the fact that $\left(\tilde{\rho}_{\epsilon}, \tilde{u}_{\epsilon}, \sqrt{\frac{3}{2}} \tilde{\theta}_{\epsilon}, \tilde{E}_{\epsilon}, \tilde{B}_{\epsilon}\right)$ belongs to $\operatorname{Ker} W^{\perp}$.

On the whole, we have shown the formal existence of a decomposition 10.6 , which explains why the nonlinear terms 10.3 are expected to be weakly stable as $\epsilon \rightarrow 0$, at least formally.

Generally speaking, such methods are called "compensated compactness" (following Murat and Tartar [63, 64, 73.), for they consist in compensating the lack of strong compactness in the quadratic terms 10.3 by carefully studying the linear structure 10.1 of oscillations. 


\subsection{Rigorous filtering of oscillations}

This chapter aims at rendering the preceding developments rigorous. Thus, the main result concerning the filtering of acoustic and electromagnetic waves in the nonlinear terms through the method of compensated compactness is described in the following proposition.

Proposition 10.1. Let $\left(f_{\epsilon}, E_{\epsilon}, B_{\epsilon}\right)$ be the sequence of renormalized solutions to the scaled one species Vlasov-Maxwell-Boltzmann system (4.27) considered in Theorem 4.4 and denote by $\tilde{\rho}_{\epsilon}, \tilde{u}_{\epsilon}$ and $\tilde{\theta}_{\epsilon}$ the density, bulk velocity and temperature associated with the renormalized fluctuations $g_{\epsilon} \gamma_{\epsilon} \chi\left(\frac{|v|^{2}}{K_{\epsilon}}\right)$ employed in Proposition 9.1. In accordance with Lemma 5.2, denote by

$$
\rho, u, \theta, E, B \in L^{\infty}\left(d t ; L^{2}(d x)\right),
$$

any joint limit points of the families $\tilde{\rho}_{\epsilon}, \tilde{u}_{\epsilon}, \tilde{\theta}_{\epsilon}, E_{\epsilon}$ and $B_{\epsilon}$, respectively.

Then, as $\epsilon \rightarrow 0$, one has the weak stability of nonlinear terms

$$
\begin{gathered}
P\left(\nabla_{x} \cdot\left(\tilde{u}_{\epsilon} \otimes \tilde{u}_{\epsilon}\right)-\tilde{\rho}_{\epsilon} E_{\epsilon}-\tilde{u}_{\epsilon} \wedge B_{\epsilon}\right) \rightarrow P\left(\nabla_{x} \cdot(u \otimes u)-\rho E-u \wedge B\right), \\
\frac{5}{2} \nabla_{x} \cdot\left(\tilde{u}_{\epsilon} \tilde{\theta}_{\epsilon}\right)-\tilde{u}_{\epsilon} \cdot E_{\epsilon} \rightarrow \frac{5}{2} \nabla_{x} \cdot(u \theta)-u \cdot E,
\end{gathered}
$$

in the sense of distributions (where we only consider smooth compactly supported solenoidal test functions).

Proof. First of all, it is to be emplasized that compactness in $x$ is not an issue at all here, for none of the nonlinear terms in (10.7) involves a product of the electromagnetic fields $E_{\epsilon}$ and $B_{\epsilon}$ only.

Indeed, from the strong compactness 7.28 obtained in Chapter 7

$$
\begin{aligned}
& \lim _{|h| \rightarrow 0} \sup _{\epsilon>0}\left\|\hat{\rho}_{\epsilon}(t, x+h)-\hat{\rho}_{\epsilon}(t, x)\right\|_{L_{\mathrm{loc}}^{2}(d t d x)}=0, \\
& \lim _{|h| \rightarrow 0} \sup _{\epsilon>0}\left\|\hat{u}_{\epsilon}(t, x+h)-\hat{u}_{\epsilon}(t, x)\right\|_{L_{\mathrm{loc}}^{2}(d t d x)}=0, \\
& \lim _{|h| \rightarrow 0} \sup _{\epsilon>0}\left\|\hat{\theta}_{\epsilon}(t, x+h)-\hat{\theta}_{\epsilon}(t, x)\right\|_{L_{\mathrm{loc}}^{2}(d t d x)}=0,
\end{aligned}
$$

where $\hat{\rho}_{\epsilon}, \hat{u}_{\epsilon}$ and $\hat{\theta}_{\epsilon}$ respectively denote the density, bulk velocity and temperature of the renormalized fluctuations $\hat{g}_{\epsilon}$ defined by (5.3), and the comparison (9.19) between $\hat{g}_{\epsilon}$ and $g_{\epsilon} \gamma_{\epsilon} \chi\left(\frac{|v|^{2}}{K_{\epsilon}}\right)$ established in Chapter 9

$$
\tilde{\rho}_{\epsilon}-\hat{\rho}_{\epsilon} \rightarrow 0, \quad \tilde{u}_{\epsilon}-\hat{u}_{\epsilon} \rightarrow 0 \quad \text { and } \quad \tilde{\theta}_{\epsilon}-\hat{\theta}_{\epsilon} \rightarrow 0 \quad \text { in } L_{\text {loc }}^{2}(d t d x) \text { as } \epsilon \rightarrow 0,
$$

we deduce that the following local spatial compactness property holds :

$$
\begin{aligned}
& \lim _{|h| \rightarrow 0} \sup _{\epsilon>0}\left\|\tilde{\rho}_{\epsilon}(t, x+h)-\tilde{\rho}_{\epsilon}(t, x)\right\|_{L_{\mathrm{loc}}^{2}(d t d x)}=0, \\
& \lim _{|h| \rightarrow 0} \sup _{\epsilon>0}\left\|\tilde{u}_{\epsilon}(t, x+h)-\tilde{u}_{\epsilon}(t, x)\right\|_{L_{\mathrm{loc}}^{2}(d t d x)}=0, \\
& \lim _{|h| \rightarrow 0} \sup _{\epsilon>0}\left\|\tilde{\theta}_{\epsilon}(t, x+h)-\tilde{\theta}_{\epsilon}(t, x)\right\|_{L_{\mathrm{loc}}^{2}(d t d x)}=0 .
\end{aligned}
$$

In particular, denoting by $\tilde{\rho}_{\epsilon}^{\delta}, \tilde{u}_{\epsilon}^{\delta}, \tilde{\theta}_{\epsilon}^{\delta}, \rho^{\delta}, u^{\delta}$ and $\theta^{\delta}$ the respective spatial convolutions of $\tilde{\rho}_{\epsilon}, \tilde{u}_{\epsilon}, \tilde{\theta}_{\epsilon}, \rho, u$ and $\theta$ with a smooth compactly supported mollifier $\frac{1}{\delta^{3}} \chi\left(\frac{x}{\delta}\right)$, where $\delta>0$ and $\chi \in C_{c}^{\infty}\left(\mathbb{R}^{3}\right)$, with $\int_{\mathbb{R}^{3}} \chi(x) d x=1$, we see that it is possible to replace each $\tilde{\rho}_{\epsilon}, \tilde{u}_{\epsilon}$ and $\tilde{\theta}_{\epsilon}$ in 10.7 by $\tilde{\rho}_{\epsilon}^{\delta}, \tilde{u}_{\epsilon}^{\delta}$ and $\tilde{\theta}_{\epsilon}^{\delta}$, respectively, producing remainders that are uniformly small in $L_{\mathrm{loc}}^{1}\left(d t ; W_{\mathrm{loc}}^{-1,1}(d x)\right)$ as $\delta \rightarrow 0$. This 
reduces 10.7 to showing the nonlinear convergence

$$
\begin{gathered}
P\left(\nabla_{x} \cdot\left(\tilde{u}_{\epsilon}^{\delta} \otimes \tilde{u}_{\epsilon}^{\delta}\right)-\tilde{\rho}_{\epsilon}^{\delta} E_{\epsilon}-\tilde{u}_{\epsilon}^{\delta} \wedge B_{\epsilon}\right) \rightarrow P\left(\nabla_{x} \cdot\left(u^{\delta} \otimes u^{\delta}\right)-\rho^{\delta} E-u^{\delta} \wedge B\right), \\
\frac{5}{2} \nabla_{x} \cdot\left(\tilde{u}_{\epsilon}^{\delta} \tilde{\theta}_{\epsilon}^{\delta}\right)-\tilde{u}_{\epsilon}^{\delta} \cdot E_{\epsilon} \longrightarrow \frac{5}{2} \nabla_{x} \cdot\left(u^{\delta} \theta^{\delta}\right)-u^{\delta} \cdot E,
\end{gathered}
$$

in the sense of distributions.

Then, denoting by $E_{\epsilon}^{\delta}, B_{\epsilon}^{\delta}, E^{\delta}$ and $B^{\delta}$ the respective spatial convolutions of $E_{\epsilon}, B_{\epsilon}, E$ and $B$ with the mollifier $\frac{1}{\delta^{3}} \chi\left(\frac{x}{\delta}\right)$, we notice, since we are only seeking to establish a convergence in the sence of distributions, that we may also replace $E_{\epsilon}$ and $B_{\epsilon}$ by $E_{\epsilon}^{\delta}$ and $B_{\epsilon}^{\delta}$, respectively, thus further reducing the proof of the present proposition to establishing the nonlinear convergence, for any fixed $\delta>0$,

$$
\begin{gathered}
P\left(\nabla_{x} \cdot\left(\tilde{u}_{\epsilon}^{\delta} \otimes \tilde{u}_{\epsilon}^{\delta}\right)-\tilde{\rho}_{\epsilon}^{\delta} E_{\epsilon}^{\delta}-\tilde{u}_{\epsilon}^{\delta} \wedge B_{\epsilon}^{\delta}\right) \rightarrow P\left(\nabla_{x} \cdot\left(u^{\delta} \otimes u^{\delta}\right)-\rho^{\delta} E^{\delta}-u^{\delta} \wedge B^{\delta}\right), \\
\frac{5}{2} \nabla_{x} \cdot\left(\tilde{u}_{\epsilon}^{\delta} \tilde{\theta}_{\epsilon}^{\delta}\right)-\tilde{u}_{\epsilon}^{\delta} \cdot E_{\epsilon}^{\delta} \rightarrow \frac{5}{2} \nabla_{x} \cdot\left(u^{\delta} \theta^{\delta}\right)-u^{\delta} \cdot E^{\delta},
\end{gathered}
$$

in the sense of distributions.

Now, according to Proposition 9.1, coupling the linear part of the macroscopic equations derived therein with Maxwell's equations, one has the following acousticelectromagnetic wave system, for any fixed $\delta>0$ :

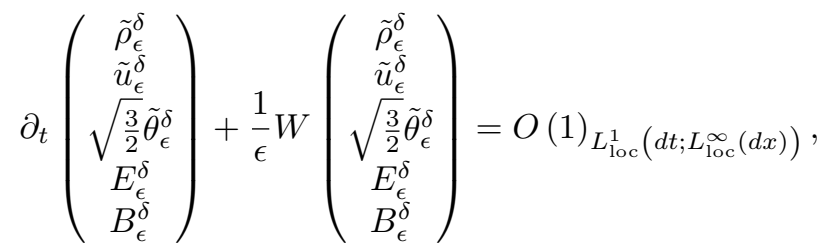

where the wave operator $W$ is defined in 10.2 . In particular, it holds that

$$
\begin{aligned}
\partial_{t}\left(2 \tilde{\rho}_{\epsilon}^{\delta}-3 \tilde{\theta}_{\epsilon}^{\delta}\right) & =O(1)_{L_{\mathrm{loc}}^{1}(d t d x)}, \\
\partial_{t}\left(\operatorname{rot} \tilde{u}_{\epsilon}^{\delta}+B_{\epsilon}^{\delta}\right) & =O(1)_{L_{\mathrm{loc}}^{1}(d t d x)},
\end{aligned}
$$

whence $2 \tilde{\rho}_{\epsilon}^{\delta}-3 \tilde{\theta}_{\epsilon}^{\delta}$ and $\operatorname{rot} \tilde{u}_{\epsilon}^{\delta}+B_{\epsilon}^{\delta}$ are relatively compact in the strong topology of $L_{\text {loc }}^{2}(d t d x)$ (in both variables $t$ and $x$ ). It follows that

$$
\begin{aligned}
2 \tilde{\rho}_{\epsilon}^{\delta}-3 \tilde{\theta}_{\epsilon}^{\delta} & \rightarrow 2 \rho^{\delta}-3 \theta^{\delta}, \\
\operatorname{rot} \tilde{u}_{\epsilon}^{\delta}+B_{\epsilon}^{\delta} & \rightarrow \operatorname{rot} u^{\delta}+B^{\delta},
\end{aligned}
$$

in $L_{\text {loc }}^{2}(d t d x)$.

Next, we reproduce here rigorously the formal identities 10.4) and (10.5), which yields (for fixed $\epsilon$, notice that $\tilde{\rho}_{\epsilon}^{\delta}, \tilde{u}_{\epsilon}^{\delta}$ and $\tilde{\theta}_{\epsilon}^{\delta}$ are now differentiable once in $t$ with a derivative lying in $L_{\mathrm{loc}}^{1}\left(d t ; L_{\mathrm{loc}}^{\infty}(d x)\right)$, due to 10.8$)$ )

$$
\begin{aligned}
P & \left(\nabla_{x} \cdot\left(\tilde{u}_{\epsilon}^{\delta} \otimes \tilde{u}_{\epsilon}^{\delta}\right)-\tilde{\rho}_{\epsilon}^{\delta} E_{\epsilon}^{\delta}-\tilde{u}_{\epsilon}^{\delta} \wedge B_{\epsilon}^{\delta}\right) \\
& =P\left(\tilde{u}_{\epsilon}^{\delta}\left(\epsilon \partial_{t} \tilde{\rho}_{\epsilon}^{\delta}+\operatorname{div} \tilde{u}_{\epsilon}^{\delta}\right)+\tilde{\rho}_{\epsilon}^{\delta}\left(\epsilon \partial_{t} \tilde{u}_{\epsilon}^{\delta}+\nabla_{x}\left(\tilde{\rho}_{\epsilon}^{\delta}+\tilde{\theta}_{\epsilon}^{\delta}\right)-E_{\epsilon}^{\delta}\right)\right) \\
& -P\left(\frac{2 \tilde{\rho}_{\epsilon}^{\delta}-3 \tilde{\theta}_{\epsilon}^{\delta}}{5} \nabla_{x}\left(\tilde{\rho}_{\epsilon}^{\delta}+\tilde{\theta}_{\epsilon}^{\delta}\right)+\tilde{u}_{\epsilon}^{\delta} \wedge\left(\operatorname{rot} \tilde{u}_{\epsilon}^{\delta}+B_{\epsilon}^{\delta}\right)+\epsilon \partial_{t}\left(\tilde{\rho}_{\epsilon}^{\delta} \tilde{u}_{\epsilon}^{\delta}\right)\right),
\end{aligned}
$$

and

$$
\begin{aligned}
& \frac{5}{2} \nabla_{x} \cdot\left(\tilde{u}_{\epsilon}^{\delta} \tilde{\theta}_{\epsilon}^{\delta}\right)-\tilde{u}_{\epsilon}^{\delta} \cdot E_{\epsilon}^{\delta} \\
& \quad=\frac{5}{2} \tilde{\theta}_{\epsilon}^{\delta}\left(\frac{3}{2} \epsilon \partial_{t} \tilde{\theta}_{\epsilon}^{\delta}+\operatorname{div} \tilde{u}_{\epsilon}^{\delta}\right)+\tilde{u}_{\epsilon}^{\delta} \cdot\left(\epsilon \partial_{t} \tilde{u}_{\epsilon}^{\delta}+\nabla_{x}\left(\tilde{\rho}_{\epsilon}^{\delta}+\tilde{\theta}_{\epsilon}^{\delta}\right)-E_{\epsilon}^{\delta}\right) \\
& \quad+\tilde{u}_{\epsilon}^{\delta} \cdot \nabla_{x}\left(\frac{3}{2} \tilde{\theta}_{\epsilon}^{\delta}-\tilde{\rho}_{\epsilon}^{\delta}\right)-\frac{15}{8} \epsilon \partial_{t}\left(\tilde{\theta}_{\epsilon}^{\delta}\right)^{2}-\frac{1}{2} \epsilon \partial_{t}\left|\tilde{u}_{\epsilon}^{\delta}\right|^{2} .
\end{aligned}
$$


Consequently, since 10.8 implies that, for fixed $\delta>0$,

$$
\begin{aligned}
\epsilon \partial_{t} \tilde{\rho}_{\epsilon}^{\delta}+\operatorname{div} \tilde{u}_{\epsilon}^{\delta} & =O(\epsilon)_{L_{\mathrm{loc}}^{1}\left(d t ; L_{\mathrm{loc}}^{\infty}(d x)\right)}, \\
\epsilon \partial_{t} \tilde{u}_{\epsilon}^{\delta}+\nabla_{x}\left(\tilde{\rho}_{\epsilon}^{\delta}+\tilde{\theta}_{\epsilon}^{\delta}\right)-E_{\epsilon}^{\delta} & =O(\epsilon)_{L_{\mathrm{loc}}^{1}\left(d t ; L_{\mathrm{loc}}^{\infty}(d x)\right)}, \\
\frac{3}{2} \epsilon \partial_{t} \tilde{\theta}_{\epsilon}^{\delta}+\operatorname{div} \tilde{u}_{\epsilon}^{\delta} & =O(\epsilon)_{L_{\mathrm{loc}}^{1}\left(d t ; L_{\mathrm{loc}}^{\infty}(d x)\right)},
\end{aligned}
$$

we deduce, in view of the strong convergences 10.9 , that

$$
\begin{aligned}
P\left(\nabla_{x} \cdot\left(\tilde{u}_{\epsilon}^{\delta} \otimes \tilde{u}_{\epsilon}^{\delta}\right)-\tilde{\rho}_{\epsilon}^{\delta} E_{\epsilon}^{\delta}\right. & \left.-\tilde{u}_{\epsilon}^{\delta} \wedge B_{\epsilon}^{\delta}\right) \\
& \rightarrow-P\left(\frac{2 \rho^{\delta}-3 \theta^{\delta}}{5} \nabla_{x}\left(\rho^{\delta}+\theta^{\delta}\right)+u^{\delta} \wedge\left(\operatorname{rot} u^{\delta}+B^{\delta}\right)\right), \\
\frac{5}{2} \nabla_{x} \cdot\left(\tilde{u}_{\epsilon}^{\delta} \tilde{\theta}_{\epsilon}^{\delta}\right)-\tilde{u}_{\epsilon}^{\delta} \cdot E_{\epsilon}^{\delta} & \rightarrow u^{\delta} \cdot \nabla_{x}\left(\frac{3}{2} \theta^{\delta}-\rho^{\delta}\right),
\end{aligned}
$$

in the sense of distributions. Finally, using from Proposition 6.1 that $\operatorname{div} u^{\delta}=0$ and $E^{\delta}=\nabla_{x}\left(\rho^{\delta}+\theta^{\delta}\right)$ to deduce

$$
\begin{aligned}
\frac{2 \rho^{\delta}-3 \theta^{\delta}}{5} \nabla_{x}\left(\rho^{\delta}+\theta^{\delta}\right) & =\rho^{\delta} \nabla_{x}\left(\rho^{\delta}+\theta^{\delta}\right)-\frac{3}{10} \nabla_{x}\left(\rho^{\delta}+\theta^{\delta}\right)^{2} \\
& =\rho^{\delta} E^{\delta}-\frac{3}{10} \nabla_{x}\left(\rho^{\delta}+\theta^{\delta}\right)^{2}, \\
u^{\delta} \wedge \operatorname{rot} u^{\delta} & =-\nabla_{x} \cdot\left(u^{\delta} \otimes u^{\delta}\right)+u^{\delta} \operatorname{div} u^{\delta}+\frac{1}{2} \nabla_{x}\left|u^{\delta}\right|^{2} \\
& =-\nabla_{x} \cdot\left(u^{\delta} \otimes u^{\delta}\right)+\frac{1}{2} \nabla_{x}\left|u^{\delta}\right|^{2}, \\
u^{\delta} \cdot \nabla_{x}\left(\frac{3}{2} \theta^{\delta}-\rho^{\delta}\right) & =\frac{5}{2} \nabla_{x}\left(u^{\delta} \theta^{\delta}\right)-u^{\delta} \cdot \nabla_{x}\left(\rho^{\delta}+\theta^{\delta}\right)-\frac{5}{2} \theta^{\delta} \operatorname{div} u^{\delta} \\
& =\frac{5}{2} \nabla_{x} \cdot\left(u^{\delta} \theta^{\delta}\right)-u^{\delta} \cdot E^{\delta},
\end{aligned}
$$

concludes the proof of the proposition. 



\section{CHAPTER 11}

\section{Grad's moment method}

We are now in a position to proceed to the demonstration of Theorem 4.4 . Generally speaking, the formal approach to this proof follows the method of Grad from [39, 40, which consists in studying the moments of Boltzmann equations as the densities remain close to statistical equilibrium through the formal Hilbert's expansions from 42. In our fully rigorous setting, since we are considering renormalized solutions of the Vlasov-Maxwell-Boltzmann system 4.27) (which, we recall, are not known to exist in general), our method of proof proceeds through the asymptotic analysis of renormalized moments satisfying approximate macroscopic conservation laws leading to the incompressible quasi-static Navier-Stokes-FourierMaxwell-Poisson system 4.30.

We insist on the fact that the result we are about to establish holds globally in time and does not require any additional assumption on the initial data, neither on the initial velocity profile, nor on the initial thermodynamic fields, nor on the corresponding solution to the limiting system.

\subsection{Proof of Theorem 4.4}

Most of the difficult steps of this proof have been performed in the preceding chapters. We therefore only have to appropriately gather previous results together.

11.1.1. Weak convergence of fluctuations, collision integrands and electromagnetic fields. Thus, we are considering here a family of renormalized solutions $\left(f_{\epsilon}, E_{\epsilon}, B_{\epsilon}\right)$ to the scaled one species Vlasov-Maxwell-Boltzmann system 4.27 satisfying the scaled entropy inequality 4.28.

According to Lemmas 5.1 and 5.2 , the corresponding families of fluctuations $g_{\epsilon}$ and renormalized fluctuations $\hat{g}_{\epsilon}$ are weakly compact in $L_{\text {loc }}^{1}\left(d t d x ; L^{1}\left(\left(1+|v|^{2}\right) M d v\right)\right)$ and $L_{\mathrm{loc}}^{2}\left(d t ; L^{2}(M d x d v)\right)$, respectively, while, in view of Lemma 5.3 the corresponding collision integrands $\hat{q}_{\epsilon}$ are weakly compact in $L^{2}\left(M M_{*} d t d x d v d v_{*} d \sigma\right)$. Thus, using Lemma 5.1 again and the decomposition (5.5), we know that there exist $g \in L^{\infty}\left(d t ; L^{2}(M d x d v)\right),(E, B) \in L^{\infty}\left(d t ; L^{2}(d x)\right)$ and $q \in L^{2}\left(M M_{*} d t d x d v d v_{*} d \sigma\right)$, such that, up to extraction of subsequences,

$$
\begin{array}{cl}
g_{\epsilon} \rightarrow g & \text { in } L_{\text {loc }}^{1}\left(d t d x ; L^{1}\left(\left(1+|v|^{2}\right) M d v\right)\right), \\
\hat{g}_{\epsilon} \stackrel{*}{\rightarrow} \quad & \text { in } L^{\infty}\left(d t ; L^{2}(M d x d v)\right), \\
\left(E_{\epsilon}, B_{\epsilon}\right) \stackrel{*}{\rightarrow}(E, B) & \text { in } L^{\infty}\left(d t ; L^{2}(d x)\right), \\
\hat{q}_{\epsilon} \rightarrow q & \text { in } L^{2}\left(M M_{*} d t d x d v d v_{*} d \sigma\right),
\end{array}
$$

as $\epsilon \rightarrow 0$. Therefore, one also has the weak convergence of the density $\rho_{\epsilon}$, bulk velocity $u_{\epsilon}$ and temperature $\theta_{\epsilon}$ corresponding to $g_{\epsilon}$ :

$$
\rho_{\epsilon} \rightarrow \rho, \quad u_{\epsilon} \rightarrow u \text { and } \quad \theta_{\epsilon} \rightarrow \theta \quad \text { in } L_{\mathrm{loc}}^{1}(d t d x) \text { as } \epsilon \rightarrow 0,
$$


where $\rho, u, \theta \in L^{\infty}\left(d t ; L^{2}(d x)\right)$ are, respectively, the density, bulk velocity and temperature corresponding to $g$. In fact, Lemma 5.10 implies that

$$
g=\Pi g=\rho+u \cdot v+\theta\left(\frac{|v|^{2}}{2}-\frac{3}{2}\right) .
$$

Based on the uniform initial bound 4.31), a very slight modification of Lemma 5.1 allows us to deduce similar weak compactness properties on the initial data. Thus, the initial fluctuations $g_{\epsilon}^{\text {in }}$ are weakly relatively compact in $L_{\text {loc }}^{1}\left(d x ; L^{1}\left(\left(1+|v|^{2}\right) M d v\right)\right)$ and so, up to further extraction of subsequences, we may also assume that there are $g_{0}^{\text {in }} \in L^{2}(M d x d v)$ and $\left(E_{0}^{\text {in }}, B_{0}^{\text {in }}\right) \in L^{2}(d x)$, such that, up to extraction of subsequences,

$$
\begin{aligned}
g_{\epsilon}^{\text {in }} \rightarrow g_{0}^{\text {in }} & \text { in } L_{\mathrm{loc}}^{1}\left(d x ; L^{1}\left(\left(1+|v|^{2}\right) M d v\right)\right), \\
\left(E_{\epsilon}^{\text {in }}, B_{\epsilon}^{\text {in }}\right) \rightarrow\left(E_{0}^{\text {in }}, B_{0}^{\text {in }}\right) & \text { in } L^{2}(d x),
\end{aligned}
$$

as $\epsilon \rightarrow 0$. Therefore, one also has the weak convergence of the initial density $\rho_{\epsilon}^{\text {in }}$, bulk velocity $u_{\epsilon}^{\text {in }}$ and temperature $\theta_{\epsilon}^{\text {in }}$ corresponding to $g_{\epsilon}^{\text {in }}$ :

$$
\rho_{\epsilon}^{\text {in }} \rightarrow \rho_{0}^{\text {in }}, \quad u_{\epsilon}^{\text {in }} \rightarrow u_{0}^{\text {in }} \quad \text { and } \quad \theta_{\epsilon}^{\text {in }} \rightarrow \theta_{0}^{\text {in }} \quad \text { in } L_{\text {loc }}^{1}(d x) \text { as } \epsilon \rightarrow 0,
$$

where $\rho_{0}^{\text {in }}, u_{0}^{\text {in }}, \theta_{0}^{\text {in }} \in L^{2}(d x)$ are, respectively, the initial density, bulk velocity and temperature corresponding to $g_{0}^{\text {in }}$. Note that the infinitesimal Maxwellian form (11.1) does not necessarily hold for the initial data $g_{0}^{\text {in }}$.

11.1.2. Constraint equations, Maxwell's system and energy inequality. In view of Proposition 6.1, we already know that the limiting thermodynamic fields $\rho, u$ and $\theta$ satisfy the incompressibility and Boussinesq relations

$$
\operatorname{div} u=0, \quad \nabla_{x}(\rho+\theta)-E=0 .
$$

Furthermore, the discussion in Section 6.4 shows that the limiting electromagnetic field satisfies the electrostatic approximation of Maxwell's equations :

$$
\operatorname{rot} E=0, \quad \operatorname{div} E=\rho, \quad \operatorname{rot} B=u, \quad \operatorname{div} B=0 .
$$

By passing to the weak limit in the initial Gauss' laws 4.32 , one also has initially that

$$
\operatorname{div} E_{0}^{\text {in }}=\rho_{0}^{\text {in }}, \quad \operatorname{div} B_{0}^{\text {in }}=0 .
$$

As for the energy bound, Proposition 6.4 states that, for almost every $t \geq 0$,

$$
\begin{aligned}
\frac{1}{2}\left(\|\rho\|_{L_{x}^{2}}^{2}+\|u\|_{L_{x}^{2}}^{2}\right. & \left.+\frac{3}{2}\|\theta\|_{L_{x}^{2}}^{2}+\|E\|_{L_{x}^{2}}^{2}+\|B\|_{L_{x}^{2}}^{2}\right)(t) \\
& +\int_{0}^{t}\left(\mu\left\|\nabla_{x} u\right\|_{L_{x}^{2}}^{2}+\frac{5}{2} \kappa\left\|\nabla_{x} \theta\right\|_{L_{x}^{2}}^{2}\right)(s) d s \leq C^{\mathrm{in}},
\end{aligned}
$$

where the viscosity $\mu>0$ and thermal conductivity $\kappa>0$ are defined by $(2.27)$. In particular, it holds that

$$
\begin{aligned}
(\rho, u, \theta, B) & \in L^{\infty}\left([0, \infty), d t ; L^{2}\left(\mathbb{R}^{3}, d x\right)\right), \\
(u, \theta) & \in L^{2}\left([0, \infty), d t ; \dot{H}^{1}\left(\mathbb{R}^{3}, d x\right)\right) .
\end{aligned}
$$

This energy bound can be improved to the actual energy inequality $(3.2)$ provided some well-preparedness of the initial data is assumed. This is discussed in the few remarks following the statement of Theorem 4.4 . 
11.1.3. Evolution equations. We move on now to the rigorous derivation of the asymptotic macroscopic evolution equations.

We know from Chapter 9 that some approximate macroscopic evolution equations, which look like the Navier-Stokes-Fourier system with electromagnetic forces, are satisfied up to a remainder which is small in some distribution space. More precisely, according to Proposition 9.1, defining the macroscopic variables $\tilde{\rho}_{\epsilon}, \tilde{u}_{\epsilon}$ and $\tilde{\theta}_{\epsilon}$ as the density, bulk velocity and temperature, respectively, corresponding to the renormalized fluctuations $g_{\epsilon} \gamma_{\epsilon} \chi\left(\frac{|v|^{2}}{K_{\epsilon}}\right)$ used therein, it holds that

$$
\left\{\begin{array}{c}
\partial_{t} \tilde{\rho}_{\epsilon}+\frac{1}{\epsilon} \nabla_{x} \cdot \tilde{u}_{\epsilon}=R_{\epsilon, 1} \\
\partial_{t} \tilde{u}_{\epsilon}+\nabla_{x} \cdot\left(\tilde{u}_{\epsilon} \otimes \tilde{u}_{\epsilon}-\frac{\left|\tilde{u}_{\epsilon}\right|^{2}}{3} \mathrm{Id}-\int_{\mathbb{R}^{3} \times \mathbb{R}^{3} \times \mathbb{S}^{2}} \hat{q}_{\epsilon} \tilde{\phi} M M_{*} d v d v_{*} d \sigma\right) \\
=-\frac{1}{\epsilon} \nabla_{x}\left(\tilde{\rho}_{\epsilon}+\tilde{\theta}_{\epsilon}\right)+\frac{1}{\epsilon} E_{\epsilon}+\tilde{\rho}_{\epsilon} E_{\epsilon}+\tilde{u}_{\epsilon} \wedge B_{\epsilon}+R_{\epsilon, 2} \\
\partial_{t}\left(\frac{3}{2} \tilde{\theta}_{\epsilon}-\tilde{\rho}_{\epsilon}\right)+\nabla_{x} \cdot\left(\frac{5}{2} \tilde{u}_{\epsilon} \tilde{\theta}_{\epsilon}-\int_{\mathbb{R}^{3} \times \mathbb{R}^{3} \times \mathbb{S}^{2}} \hat{q}_{\epsilon} \tilde{\psi} M M_{*} d v d v_{*} d \sigma\right) \\
=\tilde{u}_{\epsilon} \cdot E_{\epsilon}+R_{\epsilon, 3},
\end{array}\right.
$$

where $\tilde{\phi}$ and $\tilde{\psi}$ are defined by 2.12 and 2.13 , and the remainders $R_{\epsilon, i}, i=1,2,3$, converge to 0 in $L_{\text {loc }}^{1}\left(d t ; W_{\text {loc }}^{-1,1}(d x)\right)$.

Since, up to further extraction of subsequences, $\gamma_{\epsilon} \chi\left(\frac{|v|^{2}}{K_{\epsilon}}\right)$ converges almost everywhere towards $1, g_{\epsilon}$ is weakly compact in $L_{\text {loc }}^{1}\left(d t d x ; L^{1}\left(\left(1+|v|^{2}\right) M d v\right)\right)$ and $g_{\epsilon} \gamma_{\epsilon}$ is uniformly bounded in $L^{\infty}\left(d t ; L^{2}(M d x d v)\right)$, we deduce, by the Product Limit Theorem, that

$$
g_{\epsilon} \gamma_{\epsilon} \chi\left(\frac{|v|^{2}}{K_{\epsilon}}\right) \stackrel{*}{\rightarrow} g \text { in } L^{\infty}\left(d t ; L^{2}(M d x d v)\right) .
$$

In particular, one also has the convergence of the renormalized moments

$$
\tilde{\rho}_{\epsilon} \stackrel{*}{\rightarrow} \rho, \quad \tilde{u}_{\epsilon} \stackrel{*}{\rightarrow} u \quad \text { and } \quad \tilde{\theta}_{\epsilon} \stackrel{*}{\rightarrow} \theta \quad \text { in } L^{\infty}\left(d t ; L^{2}(d x)\right),
$$

and the same argument yields the convergence of the initial renormalized moments

$$
\tilde{\rho}_{\epsilon}^{\text {in }} \rightarrow \rho_{0}^{\text {in }}, \quad \tilde{u}_{\epsilon}^{\text {in }} \rightarrow u_{0}^{\text {in }} \quad \text { and } \quad \tilde{\theta}_{\epsilon}^{\text {in }} \rightarrow \theta_{0}^{\text {in }} \quad \text { in } L^{2}(d x)
$$

where $\tilde{\rho}_{\epsilon}^{\text {in }}=\tilde{\rho}_{\epsilon}(t=0), \tilde{u}_{\epsilon}^{\text {in }}=\tilde{u}_{\epsilon}(t=0)$ and $\tilde{\theta}_{\epsilon}^{\text {in }}=\tilde{\theta}_{\epsilon}(t=0)$.

Next we consider the magnetic potentials $A_{\epsilon}, A \in L^{\infty}\left(d t ; \dot{H}^{1}(d x)\right)$ and $A_{\epsilon}^{\text {in }}, A_{0}^{\text {in }} \in$ $\dot{H}^{1}(d x)$ in the Coulomb gauge defined by $A_{\epsilon}=\frac{\operatorname{rot}}{-\Delta_{x}} B_{\epsilon}, \quad A=\frac{\operatorname{rot}}{-\Delta_{x}} B \quad$ and $\quad A_{\epsilon}^{\text {in }}=\frac{\operatorname{rot}}{-\Delta_{x}} B_{\epsilon}^{\text {in }}, \quad A_{0}^{\text {in }}=\frac{\text { rot }}{-\Delta_{x}} B_{0}^{\text {in }}$, so that

$$
\begin{aligned}
B_{\epsilon} & =\operatorname{rot} A_{\epsilon}, & \operatorname{div} A_{\epsilon} & =0, \\
B & =\operatorname{rot} A, & \operatorname{div} A & =0, \\
B_{\epsilon}^{\text {in }} & =\operatorname{rot} A_{\epsilon}^{\text {in }}, & \operatorname{div} A_{\epsilon}^{\text {in }} & =0, \\
B_{0}^{\text {in }} & =\operatorname{rot} A_{\epsilon}^{\text {in }}, & \operatorname{div} A_{0}^{\text {in }} & =0 .
\end{aligned}
$$

Faraday's equation from 4.27 can then be recast as

$$
\epsilon \partial_{t} A_{\epsilon}+\frac{\nabla_{x} \operatorname{div}}{-\Delta_{x}} E_{\epsilon}+E_{\epsilon}=0
$$

Further note that

$$
A_{\epsilon} \rightarrow A \quad \text { and } \quad A_{\epsilon}^{\text {in }} \rightarrow A_{0}^{\text {in }}
$$


in the sense of distributions.

Now, incorporating the preceding relation 11.5 into the evolution equation for $\tilde{u}_{\epsilon}$ in (11.4), we obtain the following system of evolution equations :

$$
\begin{aligned}
\partial_{t}\left(\tilde{u}_{\epsilon}+A_{\epsilon}\right)+\nabla_{x} \cdot\left(\tilde{u}_{\epsilon} \otimes\right. & \left.\tilde{u}_{\epsilon}-\frac{\left|\tilde{u}_{\epsilon}\right|^{2}}{3} \mathrm{Id}-\int_{\mathbb{R}^{3} \times \mathbb{R}^{3} \times \mathbb{S}^{2}} \hat{q}_{\epsilon} \tilde{\phi} M M_{*} d v d v_{*} d \sigma\right) \\
= & -\frac{1}{\epsilon} \nabla_{x}\left(\tilde{\rho}_{\epsilon}+\tilde{\theta}_{\epsilon}+\frac{\operatorname{div}}{-\Delta_{x}} E_{\epsilon}\right)+\tilde{\rho}_{\epsilon} E_{\epsilon}+\tilde{u}_{\epsilon} \wedge B_{\epsilon}+R_{\epsilon, 2}, \\
\partial_{t}\left(\frac{3}{2} \tilde{\theta}_{\epsilon}-\tilde{\rho}_{\epsilon}\right)+ & \nabla_{x} \cdot\left(\frac{5}{2} \tilde{u}_{\epsilon} \tilde{\theta}_{\epsilon}-\int_{\mathbb{R}^{3} \times \mathbb{R}^{3} \times \mathbb{S}^{2}} \hat{q}_{\epsilon} \tilde{\psi} M M_{*} d v d v_{*} d \sigma\right) \\
& =\tilde{u}_{\epsilon} \cdot E_{\epsilon}+R_{\epsilon, 3},
\end{aligned}
$$

whose weak formulation is given by

$$
\begin{gathered}
-\int_{\mathbb{R}^{3}}\left(\tilde{u}_{\epsilon}^{\text {in }}+A_{\epsilon}^{\text {in }}\right) \cdot \varphi(t=0) d x-\int_{[0, \infty) \times \mathbb{R}^{3}}\left(\tilde{u}_{\epsilon}+A_{\epsilon}\right) \cdot \partial_{t} \varphi d t d x \\
-\int_{[0, \infty) \times \mathbb{R}^{3}}\left(\tilde{u}_{\epsilon} \otimes \tilde{u}_{\epsilon}-\int_{\mathbb{R}^{3} \times \mathbb{R}^{3} \times \mathbb{S}^{2}} \hat{q}_{\epsilon} \tilde{\phi} M M_{*} d v d v_{*} d \sigma\right): \nabla_{x} \varphi d t d x \\
=\int_{[0, \infty) \times \mathbb{R}^{3}}\left(\tilde{\rho}_{\epsilon} E_{\epsilon}+\tilde{u}_{\epsilon} \wedge B_{\epsilon}\right) \cdot \varphi d t d x+o(1), \\
-\int_{\mathbb{R}^{3}}\left(\frac{3}{2} \tilde{\theta}_{\epsilon}^{\text {in }}-\tilde{\rho}_{\epsilon}^{\text {in }}\right) \chi(t=0) d x-\int_{[0, \infty) \times \mathbb{R}^{3}}\left(\frac{3}{2} \tilde{\theta}_{\epsilon}-\tilde{\rho}_{\epsilon}\right) \partial_{t} \chi d t d x \\
-\int_{[0, \infty) \times \mathbb{R}^{3}}\left(\frac{5}{2} \tilde{u}_{\epsilon} \tilde{\theta}_{\epsilon}-\int_{\mathbb{R}^{3} \times \mathbb{R}^{3} \times \mathbb{S}^{2}} \hat{q}_{\epsilon} M M_{*} d v d v_{*} d \sigma\right) \cdot \nabla_{x} \chi d t d x \\
=\int_{[0, \infty) \times \mathbb{R}^{3}} \tilde{u}_{\epsilon} \cdot E_{\epsilon} \chi d t d x+o(1),
\end{gathered}
$$

where $\varphi(t, x) \in C_{c}^{\infty}\left([0, \infty) \times \mathbb{R}^{3} ; \mathbb{R}^{3}\right)$ and $\chi(t, x) \in C_{c}^{\infty}\left([0, \infty) \times \mathbb{R}^{3} ; \mathbb{R}\right)$ are test functions such that $\operatorname{div} \varphi=0$.

By the weak stability result stated in Proposition 10.1, we can then pass to limit $\epsilon \rightarrow 0$ in the above weak formulation, thus yielding the following asymptotic system :

$$
\begin{gathered}
-\int_{\mathbb{R}^{3}}\left(u_{0}^{\text {in }}+A_{0}^{\text {in }}\right) \cdot \varphi(t=0) d x-\int_{[0, \infty) \times \mathbb{R}^{3}}(u+A) \cdot \partial_{t} \varphi d t d x \\
-\int_{[0, \infty) \times \mathbb{R}^{3}}\left(u \otimes u-\int_{\mathbb{R}^{3} \times \mathbb{R}^{3} \times \mathbb{S}^{2}} q \tilde{\phi} M M_{*} d v d v_{*} d \sigma\right): \nabla_{x} \varphi d t d x \\
=\int_{[0, \infty) \times \mathbb{R}^{3}}(\rho E+u \wedge B) \cdot \varphi d t d x \\
-\int_{\mathbb{R}^{3}}\left(\frac{3}{2} \theta_{0}^{\text {in }}-\rho_{0}^{\text {in }}\right) \chi(t=0) d x-\int_{[0, \infty) \times \mathbb{R}^{3}}\left(\frac{3}{2} \theta-\rho\right) \partial_{t} \chi d t d x \\
-\int_{[0, \infty) \times \mathbb{R}^{3}}\left(\frac{5}{2} u \theta-\int_{\mathbb{R}^{3} \times \mathbb{R}^{3} \times \mathbb{S}^{2}} q \tilde{\psi} M M_{*} d v d v_{*} d \sigma\right) \cdot \nabla_{x} \chi d t d x \\
=\int_{[0, \infty) \times \mathbb{R}^{3}} u \cdot E \chi d t d x
\end{gathered}
$$


which is precisely the weak formulation of the system

(11.7)

$$
\begin{aligned}
\partial_{t}(u+A)+\nabla_{x} \cdot\left(u \otimes u-\int_{\mathbb{R}^{3} \times \mathbb{R}^{3} \times \mathbb{S}^{2}} q \tilde{\phi} M M_{*} d v d v_{*} d \sigma\right) & =-\nabla_{x} p+\rho E+u \wedge B, \\
\partial_{t}\left(\frac{3}{2} \theta-\rho\right)+\nabla_{x} \cdot\left(\frac{5}{2} u \theta-\int_{\mathbb{R}^{3} \times \mathbb{R}^{3} \times \mathbb{S}^{2}} q \tilde{\psi} M M_{*} d v d v_{*} d \sigma\right) & =u \cdot E
\end{aligned}
$$

with initial data

$$
(u+A)(t=0)=u_{0}^{\text {in }}+A_{0}^{\text {in }} \quad \text { and } \quad\left(\frac{3}{2} \theta-\rho\right)(t=0)=\frac{3}{2} \theta_{0}^{\text {in }}-\rho_{0}^{\text {in }} .
$$

By Proposition 6.1, we can further identify the diffusion terms involving the limiting collision integrand $q$. Indeed, utilizing identity (6.1) with formulas 2.26), we obtain

$$
\begin{aligned}
\int_{\mathbb{R}^{3} \times \mathbb{R}^{3} \times \mathbb{S}^{2}} q \tilde{\phi} M M_{*} d v d v_{*} d \sigma & =\int_{\mathbb{R}^{3}} \phi: \nabla_{x} u \tilde{\phi} M d v=\mu\left(\nabla_{x} u+\nabla_{x}^{t} u-\frac{2}{3} \operatorname{div} u \operatorname{Id}\right), \\
\int_{\mathbb{R}^{3} \times \mathbb{R}^{3} \times \mathbb{S}^{2}} q \tilde{\psi} M M_{*} d v d v_{*} d \sigma & =\int_{\mathbb{R}^{3}} \psi \cdot \nabla_{x} \theta \tilde{\psi} M d v=\frac{5}{2} \kappa \nabla_{x} \theta,
\end{aligned}
$$

where the constants $\mu, \kappa>0$ are defined in 2.27) and $\phi, \psi$ are the kinetic fluxes defined by 2.12 . Incorporating the above relations into 11.7 and recalling that $u$ is a solenoidal vector field, we finally find the evolution system

$$
\begin{aligned}
\partial_{t}(u+A)+\nabla_{x} \cdot(u \otimes u)-\mu \Delta_{x} u & =-\nabla_{x} p+\rho E+u \wedge B, \\
\partial_{t}\left(\frac{3}{2} \theta-\rho\right)+\nabla_{x} \cdot\left(\frac{5}{2} u \theta\right)-\frac{5}{2} \kappa \Delta_{x} \theta & =u \cdot E .
\end{aligned}
$$

Then, defining the adjusted electric field by

$$
\tilde{E}=P \tilde{E}+P^{\perp} \tilde{E}=-\partial_{t} A+\nabla_{x}(\rho+\theta),
$$

the above evolutions system, when combined with the constraint equations 11.2 and 11.3 , can be recast as

$$
\left\{\begin{array}{rlrl}
\partial_{t} u+u \cdot \nabla_{x} u-\mu \Delta_{x} u & =-\nabla_{x} p+\tilde{E}+\rho \nabla_{x} \theta+u \wedge B, \\
\partial_{t}\left(\frac{3}{2} \theta-\rho\right)+u \cdot \nabla_{x}\left(\frac{3}{2} \theta-\rho\right)-\frac{5}{2} \kappa \Delta_{x} \theta & =0, & \operatorname{div} u & =0, \\
\operatorname{rot} B & =u, & \Delta_{x}(\rho+\theta) & =\rho, \\
\partial_{t} B+\operatorname{rot} \tilde{E} & =0, & \operatorname{div} \tilde{E}=\rho, & \operatorname{div} B=0,
\end{array}\right.
$$

which is precisely the incompressible quasi-static Navier-Stokes-Fourier-MaxwellPoisson system 4.30.

11.1.4. Temporal continuity, initial data and conclusion of proof. There only remains to establish the weak temporal continuity of the observables :

$$
(\rho, u, \theta, B) \in C\left([0, \infty) ; w-L^{2}\left(\mathbb{R}^{3}, d x\right)\right),
$$

and to identify their respective initial data.

For the moment, we only know from the weak formulation 11.6 that, for any solenoidal $\varphi(x) \in C_{c}^{\infty}\left(\mathbb{R}^{3} ; \mathbb{R}^{3}\right)$ and $\chi(x) \in C_{c}^{\infty}\left(\mathbb{R}^{3} ; \mathbb{R}\right)$,

$$
\begin{array}{r}
\int_{\mathbb{R}^{3}}(u+A)(t, x) \varphi(x) d x \in C([0, \infty) ; \mathbb{R}), \\
\int_{\mathbb{R}^{3}}\left(\frac{3}{2} \theta-\rho\right)(t, x) \chi(x) d x \in C([0, \infty) ; \mathbb{R}),
\end{array}
$$


and

$$
\begin{aligned}
(u+A)(0, x) & =\left(u_{0}^{\text {in }}+A_{0}^{\text {in }}\right)(x), \\
\left(\frac{3}{2} \theta-\rho\right)(0, x) & =\left(\frac{3}{2} \theta_{0}^{\text {in }}-\rho_{0}^{\text {in }}\right)(x) .
\end{aligned}
$$

Notice, replacing the test function $\varphi$ by $\operatorname{rot} \varphi$, that one also has

$$
\int_{\mathbb{R}^{3}}(\operatorname{rot} u+B)(t, x) \varphi(x) d x \in C([0, \infty) ; \mathbb{R}),
$$

for any $\varphi(x) \in C_{c}^{\infty}\left(\mathbb{R}^{3} ; \mathbb{R}^{3}\right)$, and

$$
(\operatorname{rot} u+B)(0, x)=\left(\operatorname{rot} u_{0}^{\text {in }}+B_{0}^{\text {in }}\right)(x) .
$$

In particular, a straightforward density argument yields that

$$
\operatorname{rot} u+B, \frac{3}{2} \theta-\rho \in C\left([0, \infty) ; w-L^{2}\left(\mathbb{R}^{3}, d x\right)\right) \text {. }
$$

Finally, using the relations $\operatorname{rot} B=u$ and $\Delta_{x}(\rho+\theta)=\rho$, it is easy to express each observable $\rho, u, \theta$ and $B$ in terms of $\operatorname{rot} u+B$ and $\frac{3}{2} \theta-\rho$, only :

$$
\begin{aligned}
\rho & =\frac{3 \Delta_{x}(\rho+\theta)-5 \Delta_{x} \rho}{3-5 \Delta_{x}}=\frac{2 \Delta_{x}}{3-5 \Delta_{x}}\left(\frac{3}{2} \theta-\rho\right), \\
u & =\frac{\operatorname{rot} B-\Delta_{x} u}{1-\Delta_{x}}=\frac{\operatorname{rot}}{1-\Delta_{x}}(\operatorname{rot} u+B), \\
\theta & =\frac{1-\Delta_{x}}{\Delta_{x}} \rho=\frac{2-2 \Delta_{x}}{3-5 \Delta_{x}}\left(\frac{3}{2} \theta-\rho\right), \\
B & =\frac{\operatorname{rot}}{-\Delta_{x}} u=\frac{1}{1-\Delta_{x}}(\operatorname{rot} u+B) .
\end{aligned}
$$

It follows that 11.8 holds true and that the initial data is provided by

$$
\begin{array}{ll}
\rho(t=0)=\frac{\Delta_{x}}{3-5 \Delta_{x}}\left(3 \theta_{0}^{\text {in }}-2 \rho_{0}^{\text {in }}\right), & u(t=0)=\frac{\operatorname{rot}}{1-\Delta_{x}}\left(\operatorname{rot} u_{0}^{\text {in }}+B_{0}^{\text {in }}\right), \\
\theta(t=0)=\frac{1-\Delta_{x}}{3-5 \Delta_{x}}\left(3 \theta_{0}^{\text {in }}-2 \rho_{0}^{\text {in }}\right), & B(t=0)=\frac{1}{1-\Delta_{x}}\left(\operatorname{rot} u_{0}^{\text {in }}+B_{0}^{\text {in }}\right),
\end{array}
$$

which, at last, concludes the proof of Theorem 4.4 


\section{CHAPTER 12}

\section{The renormalized relative entropy method}

We are now going to investigate the more singular asymptotics leading to the two-fluid incompressible Navier-Stokes-Fourier-Maxwell systems with (solenoidal) Ohm's law. As explained in Section 3.2, the limiting models obtained in these regimes are not weakly stable and, thus, are not known to have global solutions (except under suitable regularity and smallness assumptions on the initial data).

However, from the physical point of view, these asymptotic regimes are important insofar as they justify Ohm's laws, which are fundamental in plasma physics.

From the mathematical point of view, the Navier-Stokes-Fourier-Maxwell systems obtained in the limit share many features with the three-dimensional incompressible Euler equations. Proving some convergence results requires then methods which are different from the weak compactness techniques used in the proof of Theorem 4.4 in Chapter 11 and which are typically based on weak-strong stability principles and dissipative solutions (see Section 3.2.3). The main novelty here, compared to the convergence results from the Boltzmann equation to the incompressible Euler equations (see [68, Chapter 5]), is to use renormalization techniques together with the relative entropy method.

\subsection{The relative entropy method : old and new}

The principle of the relative entropy method is to compare the distribution with its formal asymptotics in some appropriate metrics :

- The idea of using the relative entropy $H\left(f_{\epsilon}^{ \pm} \mid M\right)$ to build such metrics goes back to Yau [76] in the framework of the asymptotic study of GinzburgLandau's equation, then to Golse [13. for the hydrodynamic limits of the Boltzmann equation. The important points of this method are the fact that the scaled relative entropy is a Lyapunov functional for the Boltzmann equation and that it controls the size of the fluctuations.

- An approximate solution is obtained by formal expansions (the so-called Hilbert expansions ; see 42]), which consist in seeking a formal solution to the scaled system in the form

$$
f_{\mathrm{app}}^{ \pm}=M\left(1+\epsilon g_{0}^{ \pm}+\epsilon^{2} g_{1}^{ \pm}+\ldots\right) .
$$

Note that the successive approximations $g_{n}^{ \pm}$should depend a priori both on macroscopic variables $t, x$ and on fast variables $\frac{t}{\epsilon}, \frac{t}{\epsilon^{2}}, \ldots, \frac{x}{\epsilon}, \frac{x}{\epsilon^{2}}, \ldots$

For well-prepared initial data, that is for data satisfying some profile condition (thermodynamic equilibrium) as well as macroscopic linear constraints (incompressibility and Boussinesq relations, for instance), there is neither kinetic initial layer nor fast oscillating waves, so that $g_{0}^{ \pm}$reduces actually to the solution of the limiting system. Note that, in the cases considered here, the nonlinear constraints (Ohm's laws) have a different status : solutions of the limiting models are well-defined even though these constraints are not defined initially. This is similar to the existence theory for parabolic equations with initial data which are not in the domain of the diffusion operator. 
- The core of the proof consists then in getting some stability inequality for the scaled modulated entropy

$\sum_{ \pm} \frac{1}{\epsilon^{2}} H\left(f_{\epsilon}^{ \pm} \mid f_{\mathrm{app}}^{ \pm}\right)=\sum_{ \pm} \frac{1}{\epsilon^{2}} \int_{\mathbb{R}^{3} \times \mathbb{R}^{3}}\left(f_{\epsilon}^{ \pm} \log \frac{f_{\epsilon}^{ \pm}}{f_{\mathrm{app}}^{ \pm}}-f_{\epsilon}^{ \pm}+f_{\mathrm{app}}^{ \pm}\right) d x d v$

which measures in some sense the distance between the fluctuations $g_{\epsilon}^{ \pm}$ and their expected limits $g_{0}^{ \pm}$. The convergence relies then on some technical computations and Grönwall's lemma.

The stability inequality we expect to obtain should be reminiscent of the inequality defining the corresponding dissipative solutions of the limiting systems (see Section 3.2.3. Thus, it should be based solely on the decay of the entropy and on local conservation laws. In particular, there is no need for a priori strong compactness : nonlinear terms should be controlled by a loop estimate using Grönwall's lemma.

Unfortunately, this simple strategy fails, in general :

- even for weak solutions in the sense of distributions (not renormalized), provided they exist, we have no control on large velocities ;

- for renormalized solutions in the sense of DiPerna and Lions, provided they exist, local conservation laws are not known to hold.

The main novelty here is to use renormalization techniques combined with the relative entropy method. More precisely, we will not use the usual modulated entropy inequality for renormalized solutions to the kinetic equations. Rather, we will modulate a renormalized version of the entropy inequality, which requires much less a priori information on the solutions.

\subsection{Proof of Theorem 4.5 on weak interactions}

Several steps of this demonstration have been performed in the preceding chapters. We therefore begin our proof by appropriately gathering previous results together.

12.2.1. Weak convergence of fluctuations, collision integrands and electromagnetic fields. Thus, we are considering here a family of renormalized solutions $\left(f_{\epsilon}^{ \pm}, E_{\epsilon}, B_{\epsilon}\right)$ to the scaled two species Vlasov-Maxwell-Boltzmann system (4.34, in the regime of weak interspecies interactions, i.e. $\delta=o(1)$ and $\frac{\delta}{\epsilon}$ is unbounded, satisfying the scaled entropy inequality 4.35 .

According to Lemmas 5.1 and 5.2 , the corresponding families of fluctuations $g_{\epsilon}^{ \pm}$ and renormalized fluctuations $\hat{g}_{\epsilon}^{ \pm}$are weakly compact in $L_{\text {loc }}^{1}\left(d t d x ; L^{1}\left(\left(1+|v|^{2}\right) M d v\right)\right)$ and $L_{\mathrm{loc}}^{2}\left(d t ; L^{2}(M d x d v)\right)$, respectively, while, in view of Lemma 5.3 the corresponding collision integrands $\hat{q}_{\epsilon}^{ \pm}$and $\hat{q}_{\epsilon}^{ \pm, \mp}$ are weakly compact in $L^{2}\left(\overline{M M_{*}} d t d x d v d v_{*} d \sigma\right)$. Thus, using Lemma 5.1 again and the decomposition (5.5), we know that there exist $g^{ \pm} \in L^{\infty}\left(d t ; L^{2}(M d x d v)\right),(E, B) \in L^{\infty}\left(d t ; L^{2}(d x)\right)$ and $q^{ \pm}, q^{ \pm, \mp} \in L^{2}\left(M M_{*} d t d x d v d v_{*} d \sigma\right)$, such that, up to extraction of subsequences,

$$
\begin{array}{cl}
g_{\epsilon}^{ \pm} \rightarrow g^{ \pm} & \text {in } L_{\mathrm{loc}}^{1}\left(d t d x ; L^{1}\left(\left(1+|v|^{2}\right) M d v\right)\right), \\
\hat{g}_{\epsilon}^{ \pm} \stackrel{*}{\rightarrow} g^{ \pm} & \text {in } L^{\infty}\left(d t ; L^{2}(M d x d v)\right), \\
\left(E_{\epsilon}, B_{\epsilon}\right) \stackrel{*}{\rightarrow}(E, B) & \text { in } L^{\infty}\left(d t ; L^{2}(d x)\right), \\
\hat{q}_{\epsilon}^{ \pm} \rightarrow q^{ \pm} & \text {in } L^{2}\left(M M_{*} d t d x d v d v_{*} d \sigma\right), \\
\hat{q}_{\epsilon}^{ \pm, \mp} \rightarrow q^{ \pm, \mp} & \text { in } L^{2}\left(M M_{*} d t d x d v d v_{*} d \sigma\right),
\end{array}
$$


as $\epsilon \rightarrow 0$. Therefore, one also has the weak convergence of the densities $\rho_{\epsilon}^{ \pm}$, bulk velocities $u_{\epsilon}^{ \pm}$and temperatures $\theta_{\epsilon}^{ \pm}$corresponding to $g_{\epsilon}^{ \pm}$:

$$
\rho_{\epsilon}^{ \pm} \rightarrow \rho^{ \pm}, \quad u_{\epsilon}^{ \pm} \rightarrow u^{ \pm} \quad \text { and } \quad \theta_{\epsilon}^{ \pm} \rightarrow \theta^{ \pm} \quad \text { in } L_{\mathrm{loc}}^{1}(d t d x) \text { as } \epsilon \rightarrow 0,
$$

where $\rho^{ \pm}, u^{ \pm}, \theta^{ \pm} \in L^{\infty}\left(d t ; L^{2}(d x)\right)$ are, respectively, the densities, bulk velocities and temperatures corresponding to $g^{ \pm}$. In fact, Lemma 5.10 implies that

$$
g^{ \pm}=\Pi g^{ \pm}=\rho^{ \pm}+u^{ \pm} \cdot v+\theta^{ \pm}\left(\frac{|v|^{2}}{2}-\frac{3}{2}\right) .
$$

Next, we further introduce the scaled fluctuations

$$
h_{\epsilon}=\frac{\delta}{\epsilon}\left[\left(g_{\epsilon}^{+}-g_{\epsilon}^{-}\right)-n_{\epsilon}\right],
$$

where $n_{\epsilon}=\rho_{\epsilon}^{+}-\rho_{\epsilon}^{-}$is the charge density, and the electrodynamic variables

$$
j_{\epsilon}=\frac{\delta}{\epsilon}\left(u_{\epsilon}^{+}-u_{\epsilon}^{-}\right), \quad w_{\epsilon}=\frac{\delta}{\epsilon}\left(\theta_{\epsilon}^{+}-\theta_{\epsilon}^{-}\right),
$$

which are precisely the bulk velocity and temperature associated with the scaled fluctuations $h_{\epsilon}$. In view of Lemma 5.13, the electric current $j_{\epsilon}$ and the internal electric energy $w_{\epsilon}$ are uniformly bounded in $L_{\text {loc }}^{1}(d t d x)$, which necessarily implies, letting $\frac{\epsilon}{\delta} \rightarrow 0$, that $u^{+}=u^{-}$and $\theta^{+}=\theta^{-}$. Furthermore, Proposition 6.2 asserts that $\rho^{+}=\rho^{-}$, as well. Therefore, we appropriately rename the limiting macroscopic variables

$$
\rho=\rho^{+}=\rho^{-}, \quad u=u^{+}=u^{-}, \quad \theta=\theta^{+}=\theta^{-},
$$

and the limiting fluctuation

$$
g=g^{+}=g^{-}=\rho+u \cdot v+\theta\left(\frac{|v|^{2}}{2}-\frac{3}{2}\right) .
$$

Now, according to Lemma 5.14 , it holds that $h_{\epsilon}$ is weakly compact in $L_{\text {loc }}^{1}\left(d t d x ; L^{1}\left(\left(1+|v|^{2}\right) M d v\right)\right)$ and that $j_{\epsilon}$ and $w_{\epsilon}$ are weakly compact in $L_{\text {loc }}^{1}(d t d x)$, so that, up to extraction of subsequences, there are $h \in L_{\text {loc }}^{1}\left(d t d x ; L^{1}\left(\left(1+|v|^{2}\right) M d v\right)\right)$ and $j, w \in L_{\text {loc }}^{1}(d t d x)$ such that

$$
\begin{array}{ll}
h_{\epsilon} \rightarrow h & \text { in } L_{\mathrm{loc}}^{1}\left(d t d x ; L^{1}\left(\left(1+|v|^{2}\right) M d v\right)\right), \\
j_{\epsilon} \rightarrow j & \text { in } L_{\mathrm{loc}}^{1}(d t d x), \\
w_{\epsilon} \rightarrow w & \text { in } L_{\mathrm{loc}}^{1}(d t d x),
\end{array}
$$

as $\epsilon \rightarrow 0$. Moreover, by Proposition 6.3, one has the infinitesimal Maxwellian form

$$
h=j \cdot v+w\left(\frac{|v|^{2}}{2}-\frac{3}{2}\right) .
$$

12.2.2. Constraint equations, Maxwell's system and energy inequality. In view of Proposition 6.2, we already know that the limiting thermodynamic fields $\rho, u$ and $\theta$ satisfy the incompressibility and Boussinesq relations

$$
\operatorname{div} u=0, \quad \rho+\theta=0 .
$$

Moreover, the discussion in Section 6.4 shows that the limiting electromagnetic field satisfies the following form of Maxwell's equations :

$$
\left\{\begin{aligned}
\partial_{t} E-\operatorname{rot} B & =-j \\
\partial_{t} B+\operatorname{rot} E & =0 \\
\operatorname{div} E & =0 \\
\operatorname{div} B & =0
\end{aligned}\right.
$$

Note that, taking the divergence of the Ampère equation above, necessarily $\operatorname{div} j=$ 0 . 
Finally, Proposition 8.2 further establishes that the electrodynamic variables $j$ and $w$ satisfy the solenoidal Ohm's law and the internal electric energy equilibrium relation

$$
j=\sigma\left(-\nabla_{x} \bar{p}+E+u \wedge B\right), \quad w=0,
$$

where the electric conductivity $\sigma>0$ is defined by 2.70 and the pressure gradient $-\nabla_{x} \bar{p}$ is the Lagrange multiplier associated with the solenoidal constraint $\operatorname{div} j=0$.

As for the energy bound, Proposition 6.5 states that, for almost every $t \geq 0$,

$$
\begin{aligned}
& \frac{1}{2}\left(2\|u\|_{L_{x}^{2}}^{2}+5\|\theta\|_{L_{x}^{2}}^{2}+\|E\|_{L_{x}^{2}}^{2}+\|B\|_{L_{x}^{2}}^{2}\right)(t) \\
& \quad+\int_{0}^{t}\left(2 \mu\left\|\nabla_{x} u\right\|_{L_{x}^{2}}^{2}+5 \kappa\left\|\nabla_{x} \theta\right\|_{L_{x}^{2}}^{2}+\frac{1}{\sigma}\|j\|_{L_{x}^{2}}^{2}\right)(s) d s \leq C^{\mathrm{in}},
\end{aligned}
$$

where the viscosity $\mu>0$, thermal conductivity $\kappa>0$ and electric conductivity $\sigma>0$ are respectively defined by 2.56 and 2.70 . In particular, it holds that

$$
\begin{aligned}
(u, \theta, E, B) & \in L^{\infty}\left([0, \infty), d t ; L^{2}\left(\mathbb{R}^{3}, d x\right)\right), \\
(u, \theta) & \in L^{2}\left([0, \infty), d t ; \dot{H}^{1}\left(\mathbb{R}^{3}, d x\right)\right), \\
j & \in L^{2}\left([0, \infty) \times \mathbb{R}^{3}, d t d x\right) .
\end{aligned}
$$

This energy bound can be improved to the actual energy inequality

$$
\begin{aligned}
& \frac{1}{2}\left(2\|u\|_{L_{x}^{2}}^{2}+5\|\theta\|_{L_{x}^{2}}^{2}+\|E\|_{L_{x}^{2}}^{2}+\|B\|_{L_{x}^{2}}^{2}\right)(t) \\
& \quad+\int_{0}^{t}\left(2 \mu\left\|\nabla_{x} u\right\|_{L_{x}^{2}}^{2}+5 \kappa\left\|\nabla_{x} \theta\right\|_{L_{x}^{2}}^{2}+\frac{1}{\sigma}\|j\|_{L_{x}^{2}}^{2}\right)(s) d s \\
& \leq \frac{1}{2}\left(2\left\|u^{\mathrm{in}}\right\|_{L_{x}^{2}}^{2}+5\left\|\theta^{\mathrm{in}}\right\|_{L_{x}^{2}}^{2}+\left\|E^{\mathrm{in}}\right\|_{L_{x}^{2}}^{2}+\left\|B^{\mathrm{in}}\right\|_{L_{x}^{2}}^{2}\right),
\end{aligned}
$$

using the well-preparedness of the initial data 4.41).

12.2.3. The renormalized modulated entropy inequality. We move on now to the rigorous derivation of a stability inequality encoding the asymptotic macroscopic evolution equations for $u$ and $\theta$ in the spirit of the weak-strong stability inequalities used in Section 3.2.3 to define dissipative solutions for some NavierStokes-Maxwell systems. Recall that, as explained therein, such systems are in general not known to display weak stability so that their weak solutions in the energy space are not known to exist.

To this end, as in Section 9.2 , we define the renormalized fluctuations $g_{\epsilon}^{ \pm} \gamma_{\epsilon}^{ \pm} \chi\left(\frac{|v|^{2}}{K_{\epsilon}}\right)$, with $K_{\epsilon}=K|\log \epsilon|$, for some large $K>0$, and $\chi \in C_{c}^{\infty}([0, \infty))$ a smooth compactly supported function such that $\mathbb{1}_{[0,1]} \leq \chi \leq \mathbb{1}_{[0,2]}$, and where $\gamma_{\epsilon}^{ \pm}=\gamma\left(G_{\epsilon}^{ \pm}\right)$for some renormalization $\gamma \in C^{1}([0, \infty) ; \mathbb{R})$ satisfying $(9.36)$.

Since, up to further extraction of subsequences, $\gamma_{\epsilon}^{ \pm} \chi\left(\frac{|v|^{2}}{K_{\epsilon}}\right)$ converges almost everywhere towards $1, g_{\epsilon}^{ \pm}$is weakly compact in $L_{\text {loc }}^{1}\left(d t d x ; L^{1}\left(\left(1+|v|^{2}\right) M d v\right)\right)$ and $g_{\epsilon}^{ \pm} \gamma_{\epsilon}^{ \pm}$is uniformly bounded in $L^{\infty}\left(d t ; L^{2}(M d x d v)\right)$, we deduce, by the Product Limit Theorem, that

$$
g_{\epsilon}^{ \pm} \gamma_{\epsilon}^{ \pm} \chi\left(\frac{|v|^{2}}{K_{\epsilon}}\right) \stackrel{*}{\rightarrow} g \quad \text { in } L^{\infty}\left(d t ; L^{2}(M d x d v)\right)
$$

Therefore, one has the weak convergence of the densities $\tilde{\rho}_{\epsilon}^{ \pm}$, bulk velocities $\tilde{u}_{\epsilon}^{ \pm}$and temperatures $\tilde{\theta}_{\epsilon}^{ \pm}$corresponding to $g_{\epsilon}^{ \pm} \gamma_{\epsilon}^{ \pm} \chi\left(\frac{|v|^{2}}{K_{\epsilon}}\right)$ :

$$
\tilde{\rho}_{\epsilon}^{ \pm} \stackrel{*}{\rightarrow} \rho, \quad \tilde{u}_{\epsilon}^{ \pm} \stackrel{*}{\rightarrow} u \quad \text { and } \quad \tilde{\theta}_{\epsilon}^{ \pm} \stackrel{*}{\rightarrow} \theta \quad \text { in } L^{\infty}\left(d t ; L^{2}(d x)\right) \text { as } \epsilon \rightarrow 0 .
$$


In particular, the hydrodynamic variables $\tilde{\rho}_{\epsilon}=\frac{\tilde{\rho}_{\epsilon}^{+}+\tilde{\rho}_{\epsilon}^{-}}{2}, \tilde{u}_{\epsilon}=\frac{\tilde{u}_{\epsilon}^{+}+\tilde{u}_{\epsilon}^{-}}{2}$ and $\tilde{\theta}_{\epsilon}=\frac{\tilde{\theta}_{\epsilon}^{+}+\tilde{\theta}_{\epsilon}^{-}}{2}$ also obviously verify

$$
\tilde{\rho}_{\epsilon} \stackrel{*}{\rightarrow} \rho, \quad \tilde{u}_{\epsilon} \stackrel{*}{\rightarrow} u \quad \text { and } \quad \tilde{\theta}_{\epsilon} \stackrel{*}{\rightarrow} \theta \quad \text { in } L^{\infty}\left(d t ; L^{2}(d x)\right) \text { as } \epsilon \rightarrow 0 .
$$

It follows that, since $u$ is solenoidal,

$$
P^{\perp} \tilde{u}_{\epsilon} \stackrel{*}{\rightarrow} 0 \quad \text { in } L^{\infty}\left(d t ; L^{2}(d x)\right) \text { as } \epsilon \rightarrow 0,
$$

and, in view of the limiting Boussinesq relation,

$$
\tilde{\rho}_{\epsilon}+\tilde{\theta}_{\epsilon} \stackrel{*}{\rightarrow} 0 \quad \text { in } L^{\infty}\left(d t ; L^{2}(d x)\right) \text { as } \epsilon \rightarrow 0 .
$$

We establish now the convergence of the electric current $\tilde{j}_{\epsilon}=\frac{\delta}{\epsilon}\left(\tilde{u}_{\epsilon}^{+}-\tilde{u}_{\epsilon}^{-}\right)$. Since $\epsilon \hat{g}_{\epsilon}^{ \pm} \geq 2(\sqrt{2}-1)$ on the support of $1-\gamma_{\epsilon}^{ \pm}$, we easily estimate, using the uniform bound from Lemma 5.12 , that

$$
\begin{aligned}
\left|\frac{\delta}{\epsilon} g_{\epsilon}^{ \pm}\left(1-\gamma_{\epsilon}^{ \pm}\right)\right| & =\left|\frac{\delta}{\epsilon}\left(\hat{g}_{\epsilon}^{ \pm}+\frac{\epsilon}{4} \hat{g}_{\epsilon}^{ \pm 2}\right)\left(1-\gamma_{\epsilon}^{ \pm}\right)\right| \\
& \leq\left|\frac{\delta}{\epsilon} \hat{g}_{\epsilon}^{ \pm}\left(1-\gamma_{\epsilon}^{ \pm}\right)\right|+\frac{\delta}{4} \hat{g}_{\epsilon}^{ \pm 2} \\
& \leq C \delta \hat{g}_{\epsilon}^{ \pm 2}=O(\delta)_{L_{\text {loc }}^{1}\left(d t d x ; L^{1}\left(\left(1+|v|^{2}\right) M d v\right)\right)},
\end{aligned}
$$

whereas, using the Gaussian decay 8.8 , we also obtain, provided $K>4$,

$$
\begin{aligned}
\left|\frac{\delta}{\epsilon} g_{\epsilon}^{ \pm}\left(1-\chi\left(\frac{|v|^{2}}{K_{\epsilon}}\right)\right)\right| & =\left|\frac{\delta}{\epsilon}\left(\hat{g}_{\epsilon}^{ \pm}+\frac{\epsilon}{4} \hat{g}_{\epsilon}^{ \pm 2}\right)\left(1-\chi\left(\frac{|v|^{2}}{K_{\epsilon}}\right)\right)\right| \\
& \leq\left|\frac{\delta}{\epsilon} \hat{g}_{\epsilon}^{ \pm}\left(1-\chi\left(\frac{|v|^{2}}{K_{\epsilon}}\right)\right)\right|+\frac{\delta}{4} \hat{g}_{\epsilon}^{ \pm 2} \\
& \leq C \frac{\delta}{\epsilon^{2}}\left(1-\chi\left(\frac{|v|^{2}}{K_{\epsilon}}\right)\right)^{2}+C \delta \hat{g}_{\epsilon}^{ \pm 2} \\
& =O(\delta)_{L_{\mathrm{loc}}^{1}\left(d t d x ; L^{1}\left(\left(1+|v|^{2}\right) M d v\right)\right) .}
\end{aligned}
$$

Thus, we infer that

$$
\frac{\delta}{\epsilon}\left(\tilde{u}_{\epsilon}^{ \pm}-u_{\epsilon}^{ \pm}\right) \rightarrow 0 \quad \text { in } L_{\mathrm{loc}}^{1}(d t d x) \text { as } \epsilon \rightarrow 0,
$$

whence

$$
\tilde{j}_{\epsilon} \rightarrow j \quad \text { in } L_{\text {loc }}^{1}(d t d x) \text { as } \epsilon \rightarrow 0 \text {. }
$$

Now, the $L^{2}(M d x d v)$ norm of $g_{\epsilon}^{ \pm} \gamma_{\epsilon}^{ \pm} \chi\left(\frac{|v|^{2}}{K_{\epsilon}}\right)$ is not a Lyapunov functional but it is nevertheless controlled by the relative entropy

$$
\frac{1}{2}\left\|g_{\epsilon}^{ \pm} \gamma_{\epsilon}^{ \pm} \chi\left(\frac{|v|^{2}}{K_{\epsilon}}\right)\right\|_{L^{2}(M d x d v)}^{2} \leq \frac{C}{\epsilon^{2}} H\left(f_{\epsilon}^{ \pm}\right),
$$

for some $C>1$, and therefore by the initial data 4.39). One may therefore try, in a preliminary attempt to show an asymptotic stability inequality, to modulate the approximate energy associated with $g_{\epsilon}^{ \pm} \gamma_{\epsilon}^{ \pm} \chi\left(\frac{|v|^{2}}{K_{\epsilon}}\right)$, i.e. its $L^{2}(M d x d v)$ norm, by introducing a test function $\bar{g}$ in infinitesimal Maxwellian form :

$$
\bar{g}=\bar{u} \cdot v+\bar{\theta}\left(\frac{|v|^{2}}{2}-\frac{5}{2}\right)
$$

where

$$
\bar{u}(t, x), \bar{\theta}(t, x) \in C_{c}^{\infty}\left([0, \infty) \times \mathbb{R}^{3}\right) \quad \text { with } \operatorname{div} \bar{u}=0,
$$


and then establishing a stability inequality for the modulated energies

$$
\frac{1}{2}\left\|g_{\epsilon}^{ \pm} \gamma_{\epsilon}^{ \pm} \chi\left(\frac{|v|^{2}}{K_{\epsilon}}\right)-\bar{g}\right\|_{L^{2}(M d x d v)}^{2} .
$$

Notice that it holds, utilizing the elementary identity $a^{2}+\frac{3}{2} b^{2}=\frac{3}{5}(a+b)^{2}+$ $\frac{5}{2}\left(\frac{3 b-2 a}{5}\right)^{2}$, for any $a, b \in \mathbb{R}$,

$$
\begin{aligned}
& \left\|g_{\epsilon}^{ \pm} \gamma_{\epsilon}^{ \pm} \chi\left(\frac{|v|^{2}}{K_{\epsilon}}\right)-\bar{g}\right\|_{L^{2}(M d x d v)}^{2} \\
& \geq\left\|\Pi\left(g_{\epsilon}^{ \pm} \gamma_{\epsilon}^{ \pm} \chi\left(\frac{|v|^{2}}{K_{\epsilon}}\right)\right)-\bar{g}\right\|_{L^{2}(M d x d v)}^{2} \\
& =\left(\left\|\tilde{\rho}_{\epsilon}^{ \pm}+\bar{\theta}\right\|_{L^{2}(d x)}^{2}+\left\|\tilde{u}_{\epsilon}^{ \pm}-\bar{u}\right\|_{L^{2}(d x)}^{2}+\frac{3}{2}\left\|\tilde{\theta}_{\epsilon}^{ \pm}-\bar{\theta}\right\|_{L^{2}(d x)}^{2}\right) \\
& =\left(\frac{3}{5}\left\|\tilde{\rho}_{\epsilon}^{ \pm}+\tilde{\theta}_{\epsilon}^{ \pm}\right\|_{L^{2}(d x)}^{2}+\left\|\tilde{u}_{\epsilon}^{ \pm}-\bar{u}\right\|_{L^{2}(d x)}^{2}+\frac{5}{2}\left\|\frac{3 \tilde{\theta}_{\epsilon}^{ \pm}-2 \tilde{\rho}_{\epsilon}^{ \pm}}{5}-\bar{\theta}\right\|_{L^{2}(d x)}^{2}\right) .
\end{aligned}
$$

It turns out that this approach is not quite suitable for our purpose because, even though, for any $0 \leq t_{1}<t_{2}$ (see the proof of Lemma 5.1),

$$
\int_{t_{1}}^{t_{2}} \frac{1}{2}\|g\|_{L^{2}(M d x d v)}^{2} d t \leq \liminf _{\epsilon \rightarrow 0} \int_{t_{1}}^{t_{2}} \frac{1}{\epsilon^{2}} H\left(f_{\epsilon}^{ \pm}\right) d t
$$

it is not possible to set $C=1$ in 12.7 . Indeed, the first term in the polynomial expansion of the function $h(z)=(1+z) \log (1+z)-z$ defining the entropy is $\frac{1}{2} z^{2}$, but the second term is $-\frac{1}{6} z^{3}$ and may be negative.

Some entropy (or energy) is therefore lost by considering the modulated energies 12.8). These considerations lead us to introduce a more precise modulated functional in replacement of $(12.8)$ capturing more information on the fluctuations. To be precise, instead of 12.8$)$, we consider now the renormalized modulated entropies

$$
\frac{1}{\epsilon^{2}} H\left(f_{\epsilon}^{ \pm}\right)-\int_{\mathbb{R}^{3} \times \mathbb{R}^{3}} g_{\epsilon}^{ \pm} \gamma_{\epsilon}^{ \pm} \chi\left(\frac{|v|^{2}}{K_{\epsilon}}\right) \bar{g} M d x d v+\frac{1}{2}\|\bar{g}\|_{L^{2}(M d x d v)}^{2} .
$$

Note that the above functional may be negative for fixed $\epsilon>0$. However, in view of $(12.9)$, it recovers asymptotically a non-negative quantity, which is precisely the asymptotic modulated energy :

(12.11)

$$
\begin{aligned}
& \int_{t_{1}}^{t_{2}} \frac{1}{2}\left(\|u-\bar{u}\|_{L^{2}(d x)}^{2}+\frac{5}{2}\|\theta-\bar{\theta}\|_{L^{2}(d x)}^{2}\right) d t \\
& =\int_{t_{1}}^{t_{2}} \frac{1}{2}\|g-\bar{g}\|_{L^{2}(M d x d v)}^{2} d t \\
& \leq \liminf _{\epsilon \rightarrow 0} \int_{t_{1}}^{t_{2}}\left(\frac{1}{\epsilon^{2}} H\left(f_{\epsilon}^{ \pm}\right)-\int_{\mathbb{R}^{3} \times \mathbb{R}^{3}} g_{\epsilon}^{ \pm} \gamma_{\epsilon}^{ \pm} \chi\left(\frac{|v|^{2}}{K_{\epsilon}}\right) \bar{g} M d x d v+\frac{1}{2}\|\bar{g}\|_{L^{2}(M d x d v)}^{2}\right) d t
\end{aligned}
$$

for all $0 \leq t_{1}<t_{2}$.

The first term in 12.10$)$ is precisely the entropy of $f_{\epsilon}^{ \pm}$and will be controlled by the scaled entropy inequality 4.35 , whereas the last term in 12.10 only involves smooth quantities and will therefore be controlled directly. As for the middle term in the modulated entropy 12.10 , its time derivative will involve the approximate macroscopic conservation laws for $g_{\epsilon}^{ \pm} \gamma_{\epsilon}^{ \pm} \chi\left(\frac{|v|^{2}}{K_{\epsilon}}\right)$. 
Recall that a major difficulty in the relative entropy methods developed for the hydrodynamic limit of the Boltzmann equation towards the incompressible Euler equations (see 67, 68, 69]) pertains to the handling of large velocities. Here, large velocities are no longer a problem, for we deal now with conservations laws of renormalized fluctuations $g_{\epsilon}^{ \pm} \gamma_{\epsilon}^{ \pm} \chi\left(\frac{|v|^{2}}{K_{\epsilon}}\right)$ whose defects are well controlled. Thus, the present method is more robust than the usual relative entropy method which cannot deal with fluctuations of temperature.

Furthermore, thanks to the flatness assumption $(9.36)$ on $\gamma(z)$ near $z=1$, the conservation defects are expected to vanish in the limit (at least formally). In fact, as shown in Section 9.2 they can be estimated in terms of the modulated entropy and entropy dissipation. The convergence will then be obtained through a loop estimate based on an appropriate use of Grönwall's lemma.

Now, in order to establish the renormalized modulated entropy inequality leading to the convergence stated in Theorem 4.5. we introduce further test functions

$$
\bar{E}(t, x), \bar{B}(t, x), \bar{j}(t, x) \in C_{c}^{\infty}\left([0, \infty) \times \mathbb{R}^{3}\right) \quad \text { with } \operatorname{div} \bar{E}=\operatorname{div} \bar{B}=\operatorname{div} \bar{j}=0,
$$

and we define the renormalized modulated entropy

$$
\begin{aligned}
\delta \mathcal{H}_{\epsilon}(t) & =\frac{1}{\epsilon^{2}} H\left(f_{\epsilon}^{+}\right)+\frac{1}{\epsilon^{2}} H\left(f_{\epsilon}^{-}\right) \\
& -\int_{\mathbb{R}^{3} \times \mathbb{R}^{3}}\left(g_{\epsilon}^{+} \gamma_{\epsilon}^{+}+g_{\epsilon}^{-} \gamma_{\epsilon}^{-}\right) \chi\left(\frac{|v|^{2}}{K_{\epsilon}}\right) \bar{g} M d x d v+\|\bar{g}\|_{L^{2}(M d x d v)}^{2} \\
& +\frac{1}{2}\left\|E_{\epsilon}-\bar{E}\right\|_{L^{2}(d x)}^{2}+\frac{1}{2}\left\|B_{\epsilon}-\bar{B}\right\|_{L^{2}(d x)}^{2}+\frac{1}{2} \int_{\mathbb{R}^{3}}\left(\frac{1}{\epsilon^{2}} \operatorname{Tr} m_{\epsilon}+\operatorname{Tr} a_{\epsilon}\right) d x \\
& -\int_{\mathbb{R}^{3}}\left(\left(E_{\epsilon}-\bar{E}\right) \wedge\left(B_{\epsilon}-\bar{B}\right)+\left(\begin{array}{c}
a_{\epsilon 26}-a_{\epsilon 35} \\
a_{\epsilon 34}-a_{\epsilon 16} \\
a_{\epsilon 15}-a_{\epsilon 24}
\end{array}\right)\right) \cdot \bar{u} d x,
\end{aligned}
$$

where the matrix measures $m_{\epsilon}$ and $a_{\epsilon}$ are the defects introduced in Section 4.1.4 and controlled by the scaled entropy inequality 4.35).

We also define the renormalized modulated energy

$$
\begin{aligned}
\delta \mathcal{E}_{\epsilon}(t) & =\frac{1}{2}\left\|g_{\epsilon}^{+} \gamma_{\epsilon}^{+} \chi\left(\frac{|v|^{2}}{K_{\epsilon}}\right)-\bar{g}\right\|_{L^{2}(M d x d v)}^{2}+\frac{1}{2}\left\|g_{\epsilon}^{-} \gamma_{\epsilon}^{-} \chi\left(\frac{|v|^{2}}{K_{\epsilon}}\right)-\bar{g}\right\|_{L^{2}(M d x d v)}^{2} \\
& +\frac{1}{2}\left\|E_{\epsilon}-\bar{E}\right\|_{L^{2}(d x)}^{2}+\frac{1}{2}\left\|B_{\epsilon}-\bar{B}\right\|_{L^{2}(d x)}^{2}+\frac{1}{2} \int_{\mathbb{R}^{3}}\left(\frac{1}{\epsilon^{2}} \operatorname{Tr} m_{\epsilon}+\operatorname{Tr} a_{\epsilon}\right) d x \\
& -\int_{\mathbb{R}^{3}}\left(\left(E_{\epsilon}-\bar{E}\right) \wedge\left(B_{\epsilon}-\bar{B}\right)+\left(\begin{array}{c}
a_{\epsilon 26}-a_{\epsilon 35} \\
a_{\epsilon 34}-a_{\epsilon 16} \\
a_{\epsilon 15}-a_{\epsilon 24}
\end{array}\right)\right) \cdot \bar{u} d x,
\end{aligned}
$$

which is asymptotically equivalent to $\delta \mathcal{H}_{\epsilon}(t)$, at least formally. Note that $\delta \mathcal{H}_{\epsilon}(t)$ controls more accurately the large values of the fluctuations $g_{\epsilon}^{ \pm}$than $\delta \mathcal{E}_{\epsilon}(t)$. Lemma 12.1 below shows how the modulated entropy $\delta \mathcal{H}_{\epsilon}(t)$ controls the modulated energy $\delta \mathcal{E}_{\epsilon}(t)$.

Finally, we introduce the renormalized modulated entropy dissipation

$$
\begin{aligned}
\delta \mathcal{D}_{\epsilon}(t) & =\frac{1}{4}\left\|\hat{q}_{\epsilon}^{+}-\bar{q}^{+}\right\|_{L^{2}\left(M M_{*} d x d v d v_{*} d \sigma\right)}^{2}+\frac{1}{4}\left\|\hat{q}_{\epsilon}^{-}-\bar{q}^{-}\right\|_{L^{2}\left(M M_{*} d x d v d v_{*} d \sigma\right)}^{2} \\
& +\frac{1}{4}\left\|\hat{q}_{\epsilon}^{+,-}-\bar{q}^{+,-}\right\|_{L^{2}\left(M M_{*} d x d v d v_{*} d \sigma\right)}^{2}+\frac{1}{4}\left\|\hat{q}_{\epsilon}^{-,+}-\bar{q}^{-,+}\right\|_{L^{2}\left(M M_{*} d x d v d v_{*} d \sigma\right)}^{2},
\end{aligned}
$$


where

$$
\begin{aligned}
\bar{q}^{ \pm} & =\nabla_{x} \bar{u}:\left(\tilde{\phi}+\tilde{\phi}_{*}-\tilde{\phi}^{\prime}-\tilde{\phi}_{*}^{\prime}\right)+\nabla_{x} \bar{\theta} \cdot\left(\tilde{\psi}+\tilde{\psi}_{*}-\tilde{\psi}^{\prime}-\tilde{\psi}_{*}^{\prime}\right), \\
\bar{q}^{ \pm, \mp} & =\mp \frac{1}{2} \bar{j} \cdot\left(v-v_{*}-v^{\prime}+v_{*}^{\prime}\right),
\end{aligned}
$$

so that

$$
\begin{aligned}
\int_{\mathbb{R}^{3} \times \mathbb{S}^{2}} \bar{q}^{ \pm} M_{*} d v_{*} d \sigma & =\nabla_{x} \bar{u}: \mathcal{L} \tilde{\phi}+\nabla_{x} \bar{\theta} \cdot \mathcal{L} \tilde{\psi}=\nabla_{x} \bar{u}: \phi+\nabla_{x} \bar{\theta} \cdot \psi, \\
\int_{\mathbb{R}^{3} \times \mathbb{S}^{2}} \bar{q}^{ \pm, \mp} M_{*} d v_{*} d \sigma & =\mp \frac{1}{2} \bar{j} \cdot \mathfrak{L}(v),
\end{aligned}
$$

with $\phi, \psi, \tilde{\phi}$ and $\tilde{\psi}$ defined by 2.12 and 2.13 .

Then, assuming from now on that $\|\bar{u}\|_{L^{\infty}(d t d x)}<1$ and using the lower weak sequential semi-continuity of the entropies 12.11) and of the electromagnetic energy (3.24) together with Lemma 4.3 , we find that, for all $0 \leq t_{1}<t_{2}$,

$$
\int_{t_{1}}^{t_{2}} \delta \mathcal{E}(t) d t \leq \liminf _{\epsilon \rightarrow 0} \min \left\{\int_{t_{1}}^{t_{2}} \delta \mathcal{H}_{\epsilon}(t) d t, \int_{t_{1}}^{t_{2}} \delta \mathcal{E}_{\epsilon}(t) d t\right\},
$$

where

$$
\begin{aligned}
\delta \mathcal{E}(t) & =\|g-\bar{g}\|_{L^{2}(M d x d v)}^{2}+\frac{1}{2}\|E-\bar{E}\|_{L^{2}(d x)}^{2}+\frac{1}{2}\|B-\bar{B}\|_{L^{2}(d x)}^{2} \\
& -\int_{\mathbb{R}^{3}}\left(\left(E_{\epsilon}-\bar{E}\right) \wedge\left(B_{\epsilon}-\bar{B}\right)\right) \cdot \bar{u} d x \\
& =\|u-\bar{u}\|_{L^{2}(d x)}^{2}+\frac{5}{2}\|\theta-\bar{\theta}\|_{L^{2}(d x)}^{2}+\frac{1}{2}\|E-\bar{E}\|_{L^{2}(d x)}^{2}+\frac{1}{2}\|B-\bar{B}\|_{L^{2}(d x)}^{2} \\
& -\int_{\mathbb{R}^{3}}\left(\left(E_{\epsilon}-\bar{E}\right) \wedge\left(B_{\epsilon}-\bar{B}\right)\right) \cdot \bar{u} d x,
\end{aligned}
$$

while, repeating mutatis mutandis the computations leading to 6.19 and 6.21 in the proof of Proposition 6.5. we obtain, for all $0 \leq t_{1}<t_{2}$,

$$
\int_{t_{1}}^{t_{2}} \delta \mathcal{D}(t) d t \leq \liminf _{\epsilon \rightarrow 0} \int_{t_{1}}^{t_{2}} \delta \mathcal{D}_{\epsilon}(t) d t
$$

where

$$
\begin{aligned}
\delta \mathcal{D}(t) & =2 \mu\left\|\nabla_{x}(u-\bar{u})\right\|_{L_{x}^{2}}^{2}+5 \kappa\left\|\nabla_{x}(\theta-\bar{\theta})\right\|_{L_{x}^{2}}^{2}+\frac{1}{\sigma}\|j-\bar{j}\|_{L_{x}^{2}}^{2} \\
& \leq \frac{1}{4}\left\|q^{+}-\bar{q}^{+}\right\|_{L^{2}\left(M M_{*} d x d v d v_{*} d \sigma\right)}^{2}+\frac{1}{4}\left\|q^{-}-\bar{q}^{-}\right\|_{L^{2}\left(M M_{*} d x d v d v_{*} d \sigma\right)}^{2} \\
& +\frac{1}{4}\left\|q^{+,-}-\bar{q}^{+,-}\right\|_{L^{2}\left(M M_{*} d x d v d v_{*} d \sigma\right)}^{2}+\frac{1}{4}\left\|q^{-,+}-\bar{q}^{-,+}\right\|_{L^{2}\left(M M_{*} d x d v d v_{*} d \sigma\right)}^{2} .
\end{aligned}
$$

The following lemma shows how the modulated entropy $\delta \mathcal{H}_{\epsilon}$ controls the modulated energy $\delta \mathcal{E}_{\epsilon}$ up to a small remainder.

LEMMA 12.1. It holds that

$$
\delta \mathcal{E}_{\epsilon}(t) \leq C \delta \mathcal{H}_{\epsilon}(t)+o(1)_{L^{\infty}(d t)}
$$

for some fixed constant $C>1$.

Proof. Recall first, in view of the hypotheses on the renormalization (9.36), that

$$
\frac{1}{C}\left|\hat{g}_{\epsilon}^{ \pm}\right| \leq\left|g_{\epsilon}^{ \pm} \gamma_{\epsilon}^{ \pm}\right| \leq C\left|\hat{g}_{\epsilon}^{ \pm}\right|
$$


for some $C>1$, and that the elementary inequality $\mathrm{B} .5$ implies

$$
\frac{1}{4} \hat{g}_{\epsilon}^{ \pm 2} \leq \frac{1}{\epsilon^{2}} h\left(\epsilon g_{\epsilon}^{ \pm}\right)
$$

We proceed then with the straightforward estimate, where $C_{0}>1$ is a large constant and $\lambda>0$ is a small parameter to be determined later on,

$$
\begin{aligned}
& \frac{1}{2}\left(g_{\epsilon}^{ \pm} \gamma_{\epsilon}^{ \pm} \chi\left(\frac{|v|^{2}}{K_{\epsilon}}\right)-\bar{g}\right)^{2} \mathbb{1}_{\left\{\left|\epsilon g_{\epsilon}^{ \pm} \gamma_{\epsilon}^{ \pm}\right|>\lambda\right\}} \\
& \quad \leq\left(\frac{C_{0}}{\epsilon^{2}} h\left(\epsilon g_{\epsilon}^{ \pm}\right)-g_{\epsilon}^{ \pm} \gamma_{\epsilon}^{ \pm} \chi\left(\frac{|v|^{2}}{K_{\epsilon}}\right) \bar{g}+\frac{1}{2} \bar{g}^{2}\right) \mathbb{1}_{\left\{\left|\epsilon g_{\epsilon}^{ \pm} \gamma_{\epsilon}^{ \pm}\right|>\lambda\right\}} \\
& \quad \leq C_{0}\left(\frac{1}{\epsilon^{2}} h\left(\epsilon g_{\epsilon}^{ \pm}\right)-g_{\epsilon}^{ \pm} \gamma_{\epsilon}^{ \pm} \chi\left(\frac{|v|^{2}}{K_{\epsilon}}\right) \bar{g}+\frac{1}{2} \bar{g}^{2}\right) \mathbb{1}_{\left\{\left|\epsilon g_{\epsilon}^{ \pm} \gamma_{\epsilon}^{ \pm}\right|>\lambda\right\}} \\
& \quad+\left(C_{0}-1\right) g_{\epsilon}^{ \pm} \gamma_{\epsilon}^{ \pm} \chi\left(\frac{|v|^{2}}{K_{\epsilon}}\right) \bar{g} \mathbb{1}_{\left\{\left|\epsilon g_{\epsilon}^{ \pm} \gamma_{\epsilon}^{ \pm}\right|>\lambda\right\}} \\
& \quad \leq C_{0}\left(\frac{1}{\epsilon^{2}} h\left(\epsilon g_{\epsilon}^{ \pm}\right)-g_{\epsilon}^{ \pm} \gamma_{\epsilon}^{ \pm} \chi\left(\frac{|v|^{2}}{K_{\epsilon}}\right) \bar{g}+\frac{1}{2} \bar{g}^{2}\right) \mathbb{1}_{\left\{\left|\epsilon g_{\epsilon}^{ \pm} \gamma_{\epsilon}^{ \pm}\right|>\lambda\right\}}+\frac{C K \epsilon|\log \epsilon|}{\lambda} \hat{g}_{\epsilon}^{ \pm 2} .
\end{aligned}
$$

Furthermore, utilizing the simple inequality (B.6), we deduce that

$$
\begin{aligned}
& \frac{1}{2}\left(g_{\epsilon}^{ \pm} \gamma_{\epsilon}^{ \pm} \chi\left(\frac{|v|^{2}}{K_{\epsilon}}\right)-\bar{g}\right)^{2} \mathbb{1}_{\left\{\left|\epsilon g_{\epsilon}^{ \pm} \gamma_{\epsilon}^{ \pm}\right| \leq \lambda\right\}} \\
& \quad \leq\left(\frac{1}{\epsilon^{2}} h\left(\epsilon g_{\epsilon}^{ \pm} \gamma_{\epsilon}^{ \pm} \chi\left(\frac{|v|^{2}}{K_{\epsilon}}\right)\right)-g_{\epsilon}^{ \pm} \gamma_{\epsilon}^{ \pm} \chi\left(\frac{|v|^{2}}{K_{\epsilon}}\right) \bar{g}+\frac{1}{2} \bar{g}^{2}\right) \mathbb{1}_{\left\{\left|\epsilon g_{\epsilon}^{ \pm} \gamma_{\epsilon}^{ \pm}\right| \leq \lambda\right\}} \\
& \quad+\frac{\epsilon}{6}\left(g_{\epsilon}^{ \pm} \gamma_{\epsilon}^{ \pm} \chi\left(\frac{|v|^{2}}{K_{\epsilon}}\right)\right)^{3} \mathbb{1}_{\left\{\left|\epsilon g_{\epsilon}^{ \pm} \gamma_{\epsilon}^{ \pm}\right| \leq \lambda\right\}} \\
& \quad \leq\left(\frac{1}{\epsilon^{2}} h\left(\epsilon g_{\epsilon}^{ \pm}\right)-g_{\epsilon}^{ \pm} \gamma_{\epsilon}^{ \pm} \chi\left(\frac{|v|^{2}}{K_{\epsilon}}\right) \bar{g}+\frac{1}{2} \bar{g}^{2}\right) \mathbb{1}_{\left\{\left|\epsilon g_{\epsilon}^{ \pm} \gamma_{\epsilon}^{ \pm}\right| \leq \lambda\right\}} \\
& \quad+\frac{1}{6}\left(g_{\epsilon}^{ \pm} \gamma_{\epsilon}^{ \pm} \chi\left(\frac{|v|^{2}}{K_{\epsilon}}\right)-\bar{g}\right)^{2}\left|\epsilon g_{\epsilon}^{ \pm} \gamma_{\epsilon}^{ \pm}\right| \mathbb{1}_{\left\{\left|\epsilon g_{\epsilon}^{ \pm} \gamma_{\epsilon}^{ \pm}\right| \leq \lambda\right\}} \\
& \quad+\frac{1}{3} g_{\epsilon}^{ \pm} \gamma_{\epsilon}^{ \pm} \chi\left(\frac{|v|^{2}}{K_{\epsilon}}\right) \bar{g}\left|\epsilon g_{\epsilon}^{ \pm} \gamma_{\epsilon}^{ \pm}\right| \mathbb{1}_{\left\{\left|\epsilon g_{\epsilon}^{ \pm} \gamma_{\epsilon}^{ \pm}\right| \leq \lambda\right\}} \\
& \quad \leq\left(\frac{1}{\epsilon^{2}} h\left(\epsilon g_{\epsilon}^{ \pm}\right)-g_{\epsilon}^{ \pm} \gamma_{\epsilon}^{ \pm} \chi\left(\frac{|v|^{2}}{K_{\epsilon}}\right) \bar{g}+\frac{1}{2} \bar{g}^{2}\right) \mathbb{1}_{\left\{\left|\epsilon g_{\epsilon}^{ \pm} \gamma_{\epsilon}^{ \pm}\right| \leq \lambda\right\}} \\
& \quad+\frac{\lambda}{6}\left(g_{\epsilon}^{ \pm} \gamma_{\epsilon}^{ \pm} \chi\left(\frac{|v|^{2}}{K_{\epsilon}}\right)-\bar{g}\right)^{2} \mathbb{1}_{\left\{\left|\epsilon g_{\epsilon}^{ \pm} \gamma_{\epsilon}^{ \pm}\right| \leq \lambda\right\}}+C K \epsilon|\log \epsilon| \hat{g}_{\epsilon}^{ \pm 2}
\end{aligned}
$$

whence, fixing $0<\lambda<3$,

(12.16)

$$
\begin{aligned}
\left(\frac{1}{2}-\frac{\lambda}{6}\right) & \left(g_{\epsilon}^{ \pm} \gamma_{\epsilon}^{ \pm} \chi\left(\frac{|v|^{2}}{K_{\epsilon}}\right)-\bar{g}\right)^{2} \mathbb{1}_{\left\{\left|\epsilon g_{\epsilon}^{ \pm} \gamma_{\epsilon}^{ \pm}\right| \leq \lambda\right\}} \\
\leq & \left(\frac{1}{\epsilon^{2}} h\left(\epsilon g_{\epsilon}^{ \pm}\right)-g_{\epsilon}^{ \pm} \gamma_{\epsilon}^{ \pm} \chi\left(\frac{|v|^{2}}{K_{\epsilon}}\right) \bar{g}+\frac{1}{2} \bar{g}^{2}\right) \mathbb{1}_{\left\{\left|\epsilon g_{\epsilon}^{ \pm} \gamma_{\epsilon}^{ \pm}\right| \leq \lambda\right\}}+C K \epsilon|\log \epsilon| \hat{g}_{\epsilon}^{ \pm 2}
\end{aligned}
$$


Therefore, combining estimates (12.15) and 12.16 and using the uniform bound on $\hat{g}_{\epsilon}^{ \pm}$from Lemma 5.2 , we obtain that

$$
\begin{aligned}
\min \left\{\frac{1}{C_{0}},\left(1-\frac{\lambda}{3}\right)\right\} \frac{1}{2} & \left(g_{\epsilon}^{ \pm} \gamma_{\epsilon}^{ \pm} \chi\left(\frac{|v|^{2}}{K_{\epsilon}}\right)-\bar{g}\right)^{2} \\
& \leq\left(\frac{1}{\epsilon^{2}} h\left(\epsilon g_{\epsilon}^{ \pm}\right)-g_{\epsilon}^{ \pm} \gamma_{\epsilon}^{ \pm} \chi\left(\frac{|v|^{2}}{K_{\epsilon}}\right) \bar{g}+\frac{1}{2} \bar{g}^{2}\right) \\
& +O(\epsilon|\log \epsilon|)_{L^{\infty}\left(d t ; L^{1}(M d x d v)\right)},
\end{aligned}
$$

which, upon integrating against $M d x d v$ and adding the contributions of the electromagnetic field and the defect measures to the energy, concludes the proof of the lemma.

The following result establishes the renormalized modulated entropy inequality at the order $\epsilon$, which will eventually allow us to deduce the crucial weak-strong stability of the limiting thermodynamic fields, thus defining dissipative solutions.

Proposition 12.2. One has the stability inequality

$$
\begin{aligned}
& \delta \mathcal{H}_{\epsilon}(t)+\frac{1}{2} \int_{0}^{t} \delta \mathcal{D}_{\epsilon}(s) e^{\int_{s}^{t} \lambda(\sigma) d \sigma} d s \\
& \leq \delta \mathcal{H}_{\epsilon}(0) e^{\int_{0}^{t} \lambda(s) d s} \\
& +\int_{0}^{t} \int_{\mathbb{R}^{3}} \mathbf{A} \cdot\left(\begin{array}{c}
\tilde{u}_{\epsilon}-\bar{u} \\
\frac{3}{2} \tilde{\theta}_{\epsilon}-\tilde{\rho}_{\epsilon}-\frac{5}{2} \bar{\theta} \\
-\frac{\sigma}{2} \int_{\mathbb{R}^{3} \times \mathbb{R}^{3} \times \mathbb{S}^{2}}\left(\hat{q}_{\epsilon}^{+,-}-\hat{q}_{\epsilon}^{-,+}\right) v M M_{*} d v d v_{*} d \sigma-\bar{j} \\
E_{\epsilon}-\bar{E}+\bar{u} \wedge\left(B_{\epsilon}-\bar{B}\right) \\
B_{\epsilon}-\bar{B}+\left(E_{\epsilon}-\bar{E}\right) \wedge \bar{u}
\end{array}\right)(s) d x \\
& \times e^{\int_{s}^{t} \lambda(\sigma) d \sigma} d s+o(1)_{L_{\mathrm{loc}}^{\infty}(d t)},
\end{aligned}
$$

where the acceleration operator is defined by

$$
\mathbf{A}(\bar{u}, \bar{\theta}, \bar{j}, \bar{E}, \bar{B})=\left(\begin{array}{c}
\mathbf{A}_{1} \\
\mathbf{A}_{2} \\
\mathbf{A}_{3} \\
\mathbf{A}_{4} \\
\mathbf{A}_{5}
\end{array}\right)=\left(\begin{array}{c}
-2\left(\partial_{t} \bar{u}+P\left(\bar{u} \cdot \nabla_{x} \bar{u}\right)-\mu \Delta_{x} \bar{u}\right)+P(\bar{j} \wedge \bar{B}) \\
-2\left(\partial_{t} \bar{\theta}+\bar{u} \cdot \nabla_{x} \bar{\theta}-\kappa \Delta_{x} \bar{\theta}\right) \\
-\frac{1}{\sigma} \bar{j}+P(\bar{E}+\bar{u} \wedge \bar{B}) \\
-\left(\partial_{t} \bar{E}-\operatorname{rot} \bar{B}+\bar{j}\right) \\
-\left(\partial_{t} \bar{B}+\operatorname{rot} \bar{E}\right)
\end{array}\right)
$$

and the growth rate is given by

$$
\begin{aligned}
\lambda(t)=C\left(\frac{\|\bar{u}(t)\|_{W^{1, \infty}(d x)}+\left\|\partial_{t} \bar{u}(t)\right\|_{L^{\infty}(d x)}+\|\bar{j}(t)\|_{L^{\infty}(d x)}}{1-\|\bar{u}(t)\|_{L^{\infty}(d x)}}\right. & \\
& \left.+\|\bar{\theta}(t)\|_{W^{1, \infty}(d x)}+\|\bar{\theta}(t)\|_{W^{1, \infty}(d x)}^{2}\right),
\end{aligned}
$$

with a constant $C>0$ independent of test functions and $\epsilon$.

Proof. The main ingredients of the proof of this stability inequality are : 
- The scaled entropy inequality 4.35

$$
\begin{aligned}
& \frac{1}{\epsilon^{2}} H\left(f_{\epsilon}^{+}\right)+\frac{1}{\epsilon^{2}} H\left(f_{\epsilon}^{-}\right)+\frac{1}{2 \epsilon^{2}} \int_{\mathbb{R}^{3}} \operatorname{Tr} m_{\epsilon} d x+\frac{1}{2} \int_{\mathbb{R}^{3}}\left(\left|E_{\epsilon}\right|^{2}+\left|B_{\epsilon}\right|^{2}+\operatorname{Tr} a_{\epsilon}\right) d x \\
& \quad+\frac{1}{4} \int_{0}^{t} \int_{\mathbb{R}^{3} \times \mathbb{R}^{3} \times \mathbb{R}^{3} \times \mathbb{S}^{2}}\left(\left(\hat{q}_{\epsilon}^{+}\right)^{2}+\left(\hat{q}_{\epsilon}^{-}\right)^{2}+\left(\hat{q}_{\epsilon}^{+,-}\right)^{2}+\left(\hat{q}_{\epsilon}^{-,},+\right)^{2}\right) M M_{*} d x d v d v_{*} d \sigma d s \\
& \quad \leq \frac{1}{\epsilon^{2}} H\left(f_{\epsilon}^{+ \text {in }}\right)+\frac{1}{\epsilon^{2}} H\left(f_{\epsilon}^{- \text {in }}\right)+\frac{1}{2} \int_{\mathbb{R}^{3}}\left(\left|E_{\epsilon}^{\text {in }}\right|^{2}+\left|B_{\epsilon}^{\text {in }}\right|^{2}\right) d x
\end{aligned}
$$

which is naturally satisfied by renormalized solutions of the scaled two species Vlasov-Maxwell-Boltzmann system 4.34 (provided they exist) and where we have used the inequality (5.7) from Lemma 5.3 in order to conveniently simplify the dissipation terms.

- The approximate conservation of energy obtained in Proposition 9.5

$$
\partial_{t}\left(\frac{3}{2} \tilde{\theta}_{\epsilon}-\tilde{\rho}_{\epsilon}\right)+\nabla_{x} \cdot\left(\frac{5}{2} \tilde{u}_{\epsilon} \tilde{\theta}_{\epsilon}-\int_{\mathbb{R}^{3} \times \mathbb{R}^{3} \times \mathbb{S}^{2}} \frac{\hat{q}_{\epsilon}^{+}+\hat{q}_{\epsilon}^{-}}{2} \tilde{\psi} M M_{*} d v d v_{*} d \sigma\right)=R_{\epsilon, 1}
$$

where the remainder $R_{\epsilon, 1}$ satisfies

$$
\left\|R_{\epsilon, 1}\right\|_{W_{\mathrm{loc}}^{-1,1}(d x)} \leq C \delta \mathcal{E}_{\epsilon}(t)+C\left(\delta \mathcal{E}_{\epsilon}(t) \delta \mathcal{D}_{\epsilon}(t)\right)^{\frac{1}{2}}+o(1)_{L_{\mathrm{loc}}^{1}(d t)},
$$

for some $C>0$. Note that we do not employ the approximate conservation of momentum from Proposition 9.5.

- The approximate conservation of momentum law from Proposition 9.6

$$
\begin{aligned}
& \partial_{t}\left(\tilde{u}_{\epsilon}+\frac{1}{2} E_{\epsilon} \wedge B_{\epsilon}+\frac{1}{2}\left(\begin{array}{c}
a_{\epsilon 26}-a_{\epsilon 35} \\
a_{\epsilon 34}-a_{\epsilon 16} \\
a_{\epsilon 15}-a_{\epsilon 24}
\end{array}\right)\right) \\
& \quad+\nabla_{x} \cdot\left(\tilde{u}_{\epsilon} \otimes \tilde{u}_{\epsilon}-\frac{\left|\tilde{u}_{\epsilon}\right|^{2}}{3} \mathrm{Id}+\frac{1}{2 \epsilon^{2}} m_{\epsilon}-\int_{\mathbb{R}^{3} \times \mathbb{R}^{3} \times \mathbb{S}^{2}} \frac{\hat{q}_{\epsilon}^{+}+\hat{q}_{\epsilon}^{-}}{2} \tilde{\phi} M M_{*} d v d v_{*} d \sigma\right) \\
& \quad-\frac{1}{2} \nabla_{x} \cdot\left(E_{\epsilon} \otimes E_{\epsilon}+e_{\epsilon}+B_{\epsilon} \otimes B_{\epsilon}+b_{\epsilon}\right)+\nabla_{x}\left(\frac{\left|E_{\epsilon}\right|^{2}+\left|B_{\epsilon}\right|^{2}+\operatorname{Tr} a_{\epsilon}}{4}\right) \\
& \quad=-\frac{1}{\epsilon} \nabla_{x}\left(\tilde{\rho}_{\epsilon}+\tilde{\theta}_{\epsilon}\right)+\partial_{t}\left(R_{\epsilon, 2}\right)+R_{\epsilon, 3},
\end{aligned}
$$

where the remainders $R_{\epsilon, 2}$ and $R_{\epsilon, 3}$ satisfy

$$
\begin{aligned}
R_{\epsilon, 2} & =o(1)_{L^{\infty}\left(d t ; L_{\mathrm{loc}}^{1}(d x)\right)} \\
\left\|R_{\epsilon, 3}\right\|_{W_{\mathrm{loc}}^{-1,1}(d x)} & \leq C_{1} \delta \mathcal{H}_{\epsilon}(t)+C_{2} \delta \mathcal{E}_{\epsilon}(t)+o(1)_{L_{\mathrm{loc}}^{1}(d t)},
\end{aligned}
$$

for some $C_{1}, C_{2}>0$.

- The approximate Ohm's law

$\pm \int_{\mathbb{R}^{3} \times \mathbb{R}^{3} \times \mathbb{S}^{2}} \hat{q}_{\epsilon}^{ \pm, \mp} v M M_{*} d v d v_{*} d \sigma=\nabla_{x} \bar{p}_{\epsilon}-\left(E_{\epsilon}+\tilde{u}_{\epsilon} \wedge B_{\epsilon}\right)+R_{\epsilon, 4}+R_{\epsilon, 5}$

where the remainder $R_{\epsilon, 4}$ vanishes weakly

$$
R_{\epsilon, 4}=o(1)_{w-L_{\mathrm{loc}}^{1}(d t d x)},
$$

whereas $R_{\epsilon, 5}$ satisfies

$$
\left\|R_{\epsilon, 5}\right\|_{L^{1}(d x)} \leq \frac{C \delta \mathcal{E}_{\epsilon}(t)}{1-\|\bar{u}\|_{L^{\infty}(d x)}}+o(1)_{w-L_{\mathrm{loc}}^{1}(d t)}
$$


This approximate law is obtained directly from the limiting laws derived in Proposition 8.1. Indeed, it is easily deduced from 8.2 that 12.23 holds with the remainders

$$
\begin{aligned}
R_{\epsilon, 4} & =\left(E_{\epsilon}-E\right) \pm \int_{\mathbb{R}^{3} \times \mathbb{R}^{3} \times \mathbb{S}^{2}}\left(\hat{q}_{\epsilon}^{ \pm, \mp}-q^{ \pm, \mp}\right) v M M_{*} d v d v_{*} d \sigma \\
& +\left(\tilde{u}_{\epsilon}-u\right) \wedge B+u \wedge\left(B_{\epsilon}-B\right) \\
R_{\epsilon, 5} & =\left(\tilde{u}_{\epsilon}-u\right) \wedge\left(B_{\epsilon}-B\right)=\frac{O\left(\delta \mathcal{E}_{\epsilon}(t)+\delta \mathcal{E}(t)\right)_{L^{1}(d x)}}{1-\|\bar{u}\|_{L^{\infty}(d x)}},
\end{aligned}
$$

where we have used that

(12.26)

$$
\begin{aligned}
& \frac{1-\|\bar{u}\|_{L^{\infty}(d x)}}{2}\left(\left\|E_{\epsilon}-\bar{E}\right\|_{L^{2}(d x)}^{2}+\left\|B_{\epsilon}-\bar{B}\right\|_{L^{2}(d x)}^{2}\right) \\
& \leq \frac{1}{2}\left(\left\|E_{\epsilon}-\bar{E}\right\|_{L^{2}(d x)}^{2}+\left\|B_{\epsilon}-\bar{B}\right\|_{L^{2}(d x)}^{2}\right)-\int_{\mathbb{R}^{3}}\left(\left(E_{\epsilon}-\bar{E}\right) \wedge\left(B_{\epsilon}-\bar{B}\right)\right) \cdot \bar{u} d x .
\end{aligned}
$$

The above estimate on $R_{\epsilon, 5}$ is then readily improved to 12.25 upon noticing from 12.13 that

$$
\delta \mathcal{E}(t) \leq \delta \mathcal{E}_{0}(t)
$$

where $\delta \mathcal{E}_{0}(t)$ is the limit, up to extraction of subsequences, of $\delta \mathcal{E}_{\epsilon}(t)$ in $w^{*}-L^{\infty}(d t)$, and then writing

$$
\begin{aligned}
\delta \mathcal{E}_{\epsilon}(t)+\delta \mathcal{E}(t) & \leq 2 \delta \mathcal{E}_{\epsilon}(t)+\delta \mathcal{E}_{0}(t)-\delta \mathcal{E}_{\epsilon}(t) \\
& =2 \delta \mathcal{E}_{\epsilon}(t)+o(1)_{w^{*}-L^{\infty}(d t)}
\end{aligned}
$$

It is to be emphasized that it would be possible to derive the above approximate Ohm's law directly from Proposition 9.5. However, the method used here is more robust and we find it much more satisfiying to derive the consistency of the approximate law from the knowledge of the limiting equation. Indeed, morally, it is much more difficult to the derive limiting equations, which require some kind of weak stability property, than to merely establish the consistency of approximate equations.

- Maxwell's equations

$$
\left\{\begin{aligned}
\partial_{t} E_{\epsilon}-\operatorname{rot} B_{\epsilon} & =-j_{\epsilon}=-\tilde{j}_{\epsilon}+R_{\epsilon, 6} \\
\partial_{t} B_{\epsilon}+\operatorname{rot} E_{\epsilon} & =0 \\
\operatorname{div} E_{\epsilon} & =\delta n_{\epsilon} \\
\operatorname{div} B_{\epsilon} & =0
\end{aligned}\right.
$$

where the remainder $R_{\epsilon, 6}=\tilde{j}_{\epsilon}-j_{\epsilon}$ satisfies, thanks to the convergence (12.6),

$$
R_{\epsilon, 6}=o(1)_{L_{\mathrm{loc}}^{1}(d t d x)}
$$

Notice that we cannot rigorously write the identities 1.10 and 1.12 for the above system, because the source terms $j_{\epsilon}$ and $n_{\epsilon}$ do not belong to $L_{\text {loc }}^{2}(d t d x)$ a priori. Nevertheless, one has the following modulated identities :

$$
\begin{aligned}
\partial_{t}\left(E_{\epsilon} \cdot \bar{E}+B_{\epsilon} \cdot \bar{B}\right) & +\nabla_{x} \cdot\left(E_{\epsilon} \wedge \bar{B}+\bar{E} \wedge B_{\epsilon}\right) \\
& =-\left(\tilde{j}_{\epsilon}-R_{\epsilon, 6}\right) \cdot \bar{E}-\left(\bar{j}+\mathbf{A}_{4}\right) \cdot E_{\epsilon}-\mathbf{A}_{5} \cdot B_{\epsilon},
\end{aligned}
$$


and

$$
\begin{aligned}
\partial_{t} & \left(\left(E_{\epsilon}-\bar{E}\right) \wedge\left(B_{\epsilon}-\bar{B}\right)\right)+\frac{1}{2} \nabla_{x}\left(\left|E_{\epsilon}-\bar{E}\right|^{2}+\left|B_{\epsilon}-\bar{B}\right|^{2}\right) \\
& -\nabla_{x} \cdot\left(\left(E_{\epsilon}-\bar{E}\right) \otimes\left(E_{\epsilon}-\bar{E}\right)+\left(B_{\epsilon}-\bar{B}\right) \otimes\left(B_{\epsilon}-\bar{B}\right)\right) \\
& =\partial_{t}\left(E_{\epsilon} \wedge B_{\epsilon}\right)+\frac{1}{2} \nabla_{x}\left(\left|E_{\epsilon}\right|^{2}+\left|B_{\epsilon}\right|^{2}\right)-\nabla_{x} \cdot\left(E_{\epsilon} \otimes E_{\epsilon}+B_{\epsilon} \otimes B_{\epsilon}\right) \\
& +\left(\bar{j}+\mathbf{A}_{4}\right) \wedge\left(B_{\epsilon}-\bar{B}\right)+\left(E_{\epsilon}-\bar{E}\right) \wedge \mathbf{A}_{5} \\
& +\left(\tilde{j}_{\epsilon}-R_{\epsilon, 6}\right) \wedge \bar{B}+\delta n_{\epsilon} \bar{E} .
\end{aligned}
$$

- The asymptotic characterization 6.7 of the limiting collision integrands $q^{ \pm}$from Proposition 6.2, which implies that

$$
\begin{array}{r}
\left(\int_{\mathbb{R}^{3} \times \mathbb{R}^{3} \times \mathbb{S}^{2}} \frac{\hat{q}_{\epsilon}^{+}+\hat{q}_{\epsilon}^{-}}{2} \tilde{\phi} M M_{*} d v d v_{*} d \sigma\right)-\mu\left(\nabla_{x} \tilde{u}_{\epsilon}+\nabla_{x}^{t} \tilde{u}_{\epsilon}\right) \rightarrow 0, \\
\left(\int_{\mathbb{R}^{3} \times \mathbb{R}^{3} \times \mathbb{S}^{2}} \frac{\hat{q}_{\epsilon}^{+}+\hat{q}_{\epsilon}^{-}}{2} \tilde{\psi} M M_{*} d v d v_{*} d \sigma\right)-\frac{5}{2} \kappa \nabla_{x} \tilde{\theta}_{\epsilon} \rightarrow 0,
\end{array}
$$

in the sense of distributions, where $\mu, \kappa>0$ are defined by 2.56 .

- The asymptotic characterization 6.11 of the limiting collision integrands $q^{ \pm, \mp}$ from Proposition 6.3 which implies that

$$
\sigma\left(\int_{\mathbb{R}^{3} \times \mathbb{R}^{3} \times \mathbb{S}^{2}} \frac{\hat{q}_{\epsilon}^{+,-}-\hat{q}_{\epsilon}^{-,+}}{2} v M M_{*} d v d v_{*} d \sigma\right)+\tilde{j}_{\epsilon} \rightarrow 0,
$$

in the sense of distributions, where $\sigma>0$ is defined by 2.70). Moreover, since $j$ is solenoidal, it holds that

$$
P^{\perp}\left(\int_{\mathbb{R}^{3} \times \mathbb{R}^{3} \times \mathbb{S}^{2}} \frac{\hat{q}_{\epsilon}^{+,-}-\hat{q}_{\epsilon}^{-,+}}{2} v M M_{*} d v d v_{*} d \sigma\right) \rightarrow 0 \quad \text { in } L^{2}(d t d x) .
$$

Now, by definition of the acceleration operator A, straightforward energy computations, similar to those performed in the proof of Proposition 3.3. applied to the test functions $(\bar{u}, \bar{\theta}, \bar{j}, \bar{E}, \bar{B})$, show that the following energy identity holds :

$$
\frac{d}{d t} \overline{\mathcal{E}}(t)+\overline{\mathcal{D}}(t)=-\int_{\mathbb{R}^{3}} \mathbf{A} \cdot\left(\begin{array}{c}
\bar{u} \\
\frac{5}{2} \bar{\theta} \\
\bar{j} \\
\bar{E} \\
\bar{B}
\end{array}\right) d x
$$

where the energy $\overline{\mathcal{E}}$ and energy dissipation $\overline{\mathcal{D}}$ are defined by

$$
\begin{aligned}
\overline{\mathcal{E}}(t) & =\|\bar{g}\|_{L^{2}(M d x d v)}^{2}+\frac{1}{2}\|\bar{E}\|_{L^{2}(d x)}^{2}+\frac{1}{2}\|\bar{B}\|_{L^{2}(d x)}^{2} \\
& =\|\bar{u}\|_{L^{2}(d x)}^{2}+\frac{5}{2}\|\bar{\theta}\|_{L^{2}(d x)}^{2}+\frac{1}{2}\|\bar{E}\|_{L^{2}(d x)}^{2}+\frac{1}{2}\|\bar{B}\|_{L^{2}(d x)}^{2},
\end{aligned}
$$

and

$$
\begin{aligned}
\overline{\mathcal{D}}(t) & =2 \mu\left\|\nabla_{x} \bar{u}\right\|_{L_{x}^{2}}^{2}+5 \kappa\left\|\nabla_{x} \bar{\theta}\right\|_{L_{x}^{2}}^{2}+\frac{1}{\sigma}\|\bar{j}\|_{L_{x}^{2}}^{2} \\
& =\frac{1}{4}\left\|\bar{q}^{+}\right\|_{L^{2}\left(M M_{*} d x d v d v_{*} d \sigma\right)}^{2}+\frac{1}{4}\left\|\bar{q}^{-}\right\|_{L^{2}\left(M M_{*} d x d v d v_{*} d \sigma\right)}^{2} \\
& +\frac{1}{4}\left\|\bar{q}^{+,-}\right\|_{L^{2}\left(M M_{*} d x d v d v_{*} d \sigma\right)}^{2}+\frac{1}{4}\left\|\bar{q}^{-,+}\right\|_{L^{2}\left(M M_{*} d x d v d v_{*} d \sigma\right)}^{2}
\end{aligned}
$$


Next, similar duality computations applied to the approximate conservation of energy 12.19 yield that

$$
\begin{aligned}
\frac{d}{d t} & \int_{\mathbb{R}^{3}}\left(\frac{3}{2} \tilde{\theta}_{\epsilon}-\tilde{\rho}_{\epsilon}\right) \cdot \bar{\theta} d x+\int_{\mathbb{R}^{3}}\left(\bar{u} \cdot \nabla_{x} \bar{\theta}\left(\frac{3}{2} \tilde{\theta}_{\epsilon}-\tilde{\rho}_{\epsilon}\right)-\kappa \Delta_{x} \bar{\theta}\left(\frac{3}{2} \tilde{\theta}_{\epsilon}-\tilde{\rho}_{\epsilon}\right)\right) d x \\
& -\int_{\mathbb{R}^{3}}\left(\frac{5}{2} \tilde{u}_{\epsilon} \tilde{\theta}_{\epsilon}-\int_{\mathbb{R}^{3} \times \mathbb{R}^{3} \times \mathbb{S}^{2}} \frac{\hat{q}_{\epsilon}^{+}+\hat{q}_{\epsilon}^{-}}{2} \tilde{\psi} M M_{*} d v d v_{*} d \sigma\right) \cdot \nabla_{x} \bar{\theta} d x \\
& =\int_{\mathbb{R}^{3}} R_{\epsilon, 1} \cdot \bar{\theta}-\frac{1}{2} \mathbf{A}_{2}\left(\frac{3}{2} \tilde{\theta}_{\epsilon}-\tilde{\rho}_{\epsilon}\right) d x .
\end{aligned}
$$

Further reorganizing the preceding equation so that all remainder terms appear on its right-hand side, we find that

$$
\begin{aligned}
\frac{d}{d t} & \int_{\mathbb{R}^{3}}\left(\frac{3}{2} \tilde{\theta}_{\epsilon}-\tilde{\rho}_{\epsilon}\right) \cdot \bar{\theta} d x \\
& +\int_{\mathbb{R}^{3}}\left(\int_{\mathbb{R}^{3} \times \mathbb{R}^{3} \times \mathbb{S}^{2}}\left(\hat{q}_{\epsilon}^{+}+\hat{q}_{\epsilon}^{-}\right) \tilde{\psi} M M_{*} d v d v_{*} d \sigma\right) \cdot \nabla_{x} \bar{\theta} d x \\
& =\int_{\mathbb{R}^{3}} R_{\epsilon, 1} \cdot \bar{\theta}-\frac{1}{2} \mathbf{A}_{2}\left(\frac{3}{2} \tilde{\theta}_{\epsilon}-\tilde{\rho}_{\epsilon}\right)+\frac{5}{2}\left(\tilde{u}_{\epsilon}-\bar{u}\right) \cdot \nabla_{x} \bar{\theta}\left(\tilde{\theta}_{\epsilon}-\bar{\theta}\right) d x \\
& +\int_{\mathbb{R}^{3}} \frac{5}{4} P^{\perp} \tilde{u}_{\epsilon} \cdot \nabla_{x}\left(\bar{\theta}^{2}\right)+\left(\bar{u} \cdot \nabla_{x} \bar{\theta}-\kappa \Delta_{x} \bar{\theta}\right)\left(\tilde{\theta}_{\epsilon}+\tilde{\rho}_{\epsilon}\right) d x \\
& +\int_{\mathbb{R}^{3}} \frac{5}{2} \kappa \tilde{\theta}_{\epsilon} \Delta_{x} \bar{\theta}+\left(\int_{\mathbb{R}^{3} \times \mathbb{R}^{3} \times \mathbb{S}^{2}} \frac{\hat{q}_{\epsilon}^{+}+\hat{q}_{\epsilon}^{-}}{2} \tilde{\psi} M M_{*} d v d v_{*} d \sigma\right) \cdot \nabla_{x} \bar{\theta} d x .
\end{aligned}
$$

It then follows, using the convergences 12.4, 12.5, 12.31, the estimate 12.20 and Lemma 12.1 (allowing to control the energy by the entropy), that (12.35)

$$
\begin{aligned}
\frac{d}{d t} \int_{\mathbb{R}^{3}}\left(\frac{3}{2} \tilde{\theta}_{\epsilon}-\tilde{\rho}_{\epsilon}\right) \cdot \bar{\theta} d x & +\int_{\mathbb{R}^{3}}\left(\int_{\mathbb{R}^{3} \times \mathbb{R}^{3} \times \mathbb{S}^{2}}\left(\hat{q}_{\epsilon}^{+}+\hat{q}_{\epsilon}^{-}\right) \tilde{\psi} M M_{*} d v d v_{*} d \sigma\right) \cdot \nabla_{x} \bar{\theta} d x \\
& \geq-C\|\bar{\theta}\|_{W^{1, \infty}(d x)}\left(\delta \mathcal{E}_{\epsilon}(t)+\left(\delta \mathcal{E}_{\epsilon}(t) \delta \mathcal{D}_{\epsilon}(t)\right)^{\frac{1}{2}}\right) \\
& -\frac{1}{2} \int_{\mathbb{R}^{3}} \mathbf{A}_{2}\left(\frac{3}{2} \tilde{\theta}_{\epsilon}-\tilde{\rho}_{\epsilon}\right) d x+o(1)_{w-L_{\mathrm{loc}}^{1}(d t)} \\
& \geq-C\left(\|\bar{\theta}\|_{W^{1, \infty}(d x)}+\|\bar{\theta}\|_{W^{1, \infty}(d x)}^{2}\right) \delta \mathcal{H}_{\epsilon}(t)-\frac{1}{4} \delta \mathcal{D}_{\epsilon}(t) \\
& -\frac{1}{2} \int_{\mathbb{R}^{3}} \mathbf{A}_{2}\left(\frac{3}{2} \tilde{\theta}_{\epsilon}-\tilde{\rho}_{\epsilon}\right) d x+o(1)_{w-L_{\mathrm{loc}}^{1}(d t)} .
\end{aligned}
$$


Likewise, using the solenoidal property $\operatorname{div} \bar{u}=0$, analogous duality computations applied to the approximate conservation of momentum 12.21 yield that

$$
\begin{aligned}
& \frac{d}{d t} \int_{\mathbb{R}^{3}}\left(\tilde{u}_{\epsilon} \cdot \bar{u}+\frac{1}{2}\left(E_{\epsilon} \wedge B_{\epsilon}\right) \cdot \bar{u}+\frac{1}{2}\left(\begin{array}{c}
a_{\epsilon 26}-a_{\epsilon 35} \\
a_{\epsilon 34}-a_{\epsilon 16} \\
a_{\epsilon 15}-a_{\epsilon 24}
\end{array}\right) \cdot \bar{u}-R_{\epsilon, 2} \cdot \bar{u}\right) d x \\
& \quad-\int_{\mathbb{R}^{3}}\left(\frac{1}{2}\left(E_{\epsilon} \wedge B_{\epsilon}\right) \cdot \partial_{t} \bar{u}+\frac{1}{2}\left(\begin{array}{c}
a_{\epsilon 26}-a_{\epsilon 35} \\
a_{\epsilon 34}-a_{\epsilon 16} \\
a_{\epsilon 15}-a_{\epsilon 24}
\end{array}\right) \cdot \partial_{t} \bar{u}-R_{\epsilon, 2} \cdot \partial_{t} \bar{u}\right) d x \\
& +\int_{\mathbb{R}^{3}}\left(\left(P \tilde{u}_{\epsilon}\right) \otimes \bar{u}-\tilde{u}_{\epsilon} \otimes \tilde{u}_{\epsilon}-\frac{1}{2 \epsilon^{2}} m_{\epsilon}\right): \nabla_{x} \bar{u} d x \\
& +\int_{\mathbb{R}^{3}}\left(\int_{\mathbb{R}^{3} \times \mathbb{R}^{3} \times \mathbb{S}^{2}} \frac{\hat{q}_{\epsilon}^{+}+\hat{q}_{\epsilon}^{-}}{2} \tilde{\phi} M M_{*} d v d v_{*} d \sigma\right): \nabla_{x} \bar{u}-\mu \Delta_{x} \bar{u} \cdot \tilde{u}_{\epsilon} d x \\
& +\frac{1}{2} \int_{\mathbb{R}^{3}}\left(E_{\epsilon} \otimes E_{\epsilon}+e_{\epsilon}+B_{\epsilon} \otimes B_{\epsilon}+b_{\epsilon}\right): \nabla_{x} \bar{u} d x \\
& =\int_{\mathbb{R}^{3}} R_{\epsilon, 3} \cdot \bar{u}+\frac{1}{2} P(\bar{j} \wedge \bar{B}) \cdot \tilde{u}_{\epsilon}-\frac{1}{2} \mathbf{A}_{1} \cdot \tilde{u}_{\epsilon} d x,
\end{aligned}
$$

whence, reorganizing some terms so that remainders are moved to the right-hand side,

$$
\begin{aligned}
\frac{d}{d t} & \int_{\mathbb{R}^{3}}\left(\tilde{u}_{\epsilon} \cdot \bar{u}+\frac{1}{2}\left(E_{\epsilon} \wedge B_{\epsilon}\right) \cdot \bar{u}+\frac{1}{2}\left(\begin{array}{c}
a_{\epsilon 26}-a_{\epsilon 35} \\
a_{\epsilon 34}-a_{\epsilon 16} \\
a_{\epsilon 15}-a_{\epsilon 24}
\end{array}\right) \cdot \bar{u}\right) d x \\
& +\int_{\mathbb{R}^{3}}\left(\int_{\mathbb{R}^{3} \times \mathbb{R}^{3} \times \mathbb{S}^{2}}\left(\hat{q}_{\epsilon}^{+}+\hat{q}_{\epsilon}^{-}\right) \tilde{\phi} M M_{*} d v d v_{*} d \sigma\right): \nabla_{x} \bar{u} d x \\
& =\int_{\mathbb{R}^{3}} R_{\epsilon, 3} \cdot \bar{u}+\frac{1}{2} P(\bar{j} \wedge \bar{B}) \cdot \tilde{u}_{\epsilon}-\frac{1}{2} \mathbf{A}_{1} \cdot \tilde{u}_{\epsilon} d x \\
& +\int_{\mathbb{R}^{3}}\left(\bar{u} \otimes\left(P^{\perp} \tilde{u}_{\epsilon}\right)+\left(P^{\perp} \tilde{u}_{\epsilon}\right) \otimes \bar{u}+\left(\tilde{u}_{\epsilon}-\bar{u}\right) \otimes\left(\tilde{u}_{\epsilon}-\bar{u}\right)+\frac{1}{2 \epsilon^{2}} m_{\epsilon}\right): \nabla_{x} \bar{u} d x \\
& +\int_{\mathbb{R}^{3}} \mu \tilde{u}_{\epsilon} \cdot \Delta_{x} \bar{u}+\left(\int_{\mathbb{R}^{3} \times \mathbb{R}^{3} \times \mathbb{S}^{2}} \frac{\hat{q}_{\epsilon}^{+}+\hat{q}_{\epsilon}^{-}}{2} \tilde{\phi} M M_{*} d v d v_{*} d \sigma\right): \nabla_{x} \bar{u} d x \\
& +\int_{\mathbb{R}^{3}}\left(\frac{1}{2}\left(E_{\epsilon} \wedge B_{\epsilon}\right) \cdot \partial_{t} \bar{u}+\frac{1}{2}\left(\begin{array}{l}
a_{\epsilon 26}-a_{\epsilon 35} \\
a_{\epsilon 34}-a_{\epsilon 16} \\
a_{\epsilon 15}-a_{\epsilon 24}
\end{array}\right) \cdot \partial_{t} \bar{u}-R_{\epsilon, 2} \cdot \partial_{t} \bar{u}\right) d x \\
& -\frac{1}{2} \int_{\mathbb{R}^{3}}\left(E_{\epsilon} \otimes E_{\epsilon}+e_{\epsilon}+B_{\epsilon} \otimes B_{\epsilon}+b_{\epsilon}\right): \nabla_{x} \bar{u} d x+\frac{d}{d t} \int_{\mathbb{R}^{3}} R_{\epsilon, 2} \cdot \bar{u} d x .
\end{aligned}
$$

Then, using the convergences 12.4, 12.31), the estimates 4.19, (4.24), 12.22 and Lemmas 4.3 and 12.1 (allowing to control the energy by the entropy), we arrive 
at

$$
\begin{aligned}
& \frac{d}{d t} \int_{\mathbb{R}^{3}}\left(\tilde{u}_{\epsilon} \cdot \bar{u}+\frac{1}{2}\left(E_{\epsilon} \wedge B_{\epsilon}\right) \cdot \bar{u}+\frac{1}{2}\left(\begin{array}{c}
a_{\epsilon 26}-a_{\epsilon 35} \\
a_{\epsilon 34}-a_{\epsilon 16} \\
a_{\epsilon 15}-a_{\epsilon 24}
\end{array}\right) \cdot \bar{u}\right) d x \\
& \quad+\int_{\mathbb{R}^{3}}\left(\int_{\mathbb{R}^{3} \times \mathbb{R}^{3} \times \mathbb{S}^{2}}\left(\hat{q}_{\epsilon}^{+}+\hat{q}_{\epsilon}^{-}\right) \tilde{\phi} M M_{*} d v d v_{*} d \sigma\right): \nabla_{x} \bar{u} d x \\
& \quad \geq-C\left(\|\bar{u}\|_{W^{1, \infty}(d x)}+\frac{\left\|\partial_{t} \bar{u}\right\|_{L^{\infty}(d x)}}{1-\|\bar{u}\|_{L^{\infty}(d x)}}\right) \delta \mathcal{H}_{\epsilon}(t)-\frac{1}{2} \int_{\mathbb{R}^{3}} \mathbf{A}_{1} \cdot \tilde{u}_{\epsilon} d x \\
& \quad+o(1)_{w-L_{\text {loc }}^{1}(d t)}+\frac{d}{d t}\left(o(1)_{L^{\infty}(d t)}\right)+\frac{1}{2} \int_{\mathbb{R}^{3}} P(\bar{j} \wedge \bar{B}) \cdot \tilde{u}_{\epsilon} d x \\
& \quad+\frac{1}{2} \int_{\mathbb{R}^{3}}\left(E_{\epsilon} \wedge B_{\epsilon}\right) \cdot \partial_{t} \bar{u}-\left(E_{\epsilon} \otimes E_{\epsilon}+e_{\epsilon}+B_{\epsilon} \otimes B_{\epsilon}+b_{\epsilon}\right): \nabla_{x} \bar{u} d x .
\end{aligned}
$$

The next step consists in combining the preceding inequality with the identity 12.30 in order to modulate the Poynting vector $E_{\epsilon} \wedge B_{\epsilon}$. This yields

$$
\begin{aligned}
\frac{d}{d t} & \int_{\mathbb{R}^{3}}\left(\tilde{u}_{\epsilon} \cdot \bar{u}+\frac{1}{2}\left(\left(E_{\epsilon}-\bar{E}\right) \wedge\left(B_{\epsilon}-\bar{B}\right)\right) \cdot \bar{u}+\frac{1}{2}\left(\begin{array}{c}
a_{\epsilon 26}-a_{\epsilon 35} \\
a_{\epsilon 34}-a_{\epsilon 16} \\
a_{\epsilon 15}-a_{\epsilon 24}
\end{array}\right) \cdot \bar{u}\right) d x \\
& +\int_{\mathbb{R}^{3}}\left(\int_{\mathbb{R}^{3} \times \mathbb{R}^{3} \times \mathbb{S}^{2}}\left(\hat{q}_{\epsilon}^{+}+\hat{q}_{\epsilon}^{-}\right) \tilde{\phi} M M_{*} d v d v_{*} d \sigma\right): \nabla_{x} \bar{u} d x \\
& \geq-C\left(\|\bar{u}\|_{W^{1, \infty}(d x)}+\frac{\left\|\partial_{t} \bar{u}\right\|_{L^{\infty}(d x)}}{1-\|\bar{u}\|_{L^{\infty}(d x)}}\right) \delta \mathcal{H}_{\epsilon}(t) \\
& -\frac{1}{2} \int_{\mathbb{R}^{3}} \mathbf{A}_{1} \cdot \tilde{u}_{\epsilon} d x+\frac{1}{2} \int_{\mathbb{R}^{3}}\left(\mathbf{A}_{4} \wedge\left(B_{\epsilon}-\bar{B}\right)+\left(E_{\epsilon}-\bar{E}\right) \wedge \mathbf{A}_{5}\right) \cdot \bar{u} d x \\
& +o(1)_{w-L_{\text {loc }}^{1}(d t)}+\frac{d}{d t}\left(o(1)_{L^{\infty}(d t)}\right) \\
& +\frac{1}{2} \int_{\mathbb{R}^{3}}\left(\delta n_{\epsilon} \bar{E}-R_{\epsilon, 6} \wedge \bar{B}\right) \cdot \bar{u}-\left(\tilde{u}_{\epsilon} \wedge B_{\epsilon}\right) \cdot \bar{j}-(\bar{u} \wedge \bar{B}) \cdot \tilde{j}_{\epsilon}-(\bar{j} \wedge \bar{B}) \cdot P^{\perp} \tilde{u}_{\epsilon} d x \\
& +\frac{1}{2} \int_{\mathbb{R}^{3}}\left(\bar{j} \wedge\left(B_{\epsilon}-\bar{B}\right)\right) \cdot\left(\bar{u}-\tilde{u}_{\epsilon}\right)+\left(\left(E_{\epsilon}-\bar{E}\right) \wedge\left(B_{\epsilon}-\bar{B}\right)\right) \cdot \partial_{t} \bar{u} d x \\
& -\frac{1}{2} \int_{\mathbb{R}^{3}}\left(\left(E_{\epsilon}-\bar{E}\right) \otimes\left(E_{\epsilon}-\bar{E}\right)+e_{\epsilon}+\left(B_{\epsilon}-\bar{B}\right) \otimes\left(B_{\epsilon}-\bar{B}\right)+b_{\epsilon}\right): \nabla_{x} \bar{u} d x
\end{aligned}
$$

It then follows, using the convergence (12.4), the estimates 4.24, 12.28) and Lemmas 4.3 and 12.1 (allowing to control the energy by the entropy), that

$$
\begin{aligned}
\frac{d}{d t} & \int_{\mathbb{R}^{3}}\left(\tilde{u}_{\epsilon} \cdot \bar{u}+\frac{1}{2}\left(\left(E_{\epsilon}-\bar{E}\right) \wedge\left(B_{\epsilon}-\bar{B}\right)\right) \cdot \bar{u}+\frac{1}{2}\left(\begin{array}{c}
a_{\epsilon 26}-a_{\epsilon 35} \\
a_{\epsilon 34}-a_{\epsilon 16} \\
a_{\epsilon 15}-a_{\epsilon 24}
\end{array}\right) \cdot \bar{u}\right) d x \\
& +\int_{\mathbb{R}^{3}}\left(\int_{\mathbb{R}^{3} \times \mathbb{R}^{3} \times \mathbb{S}^{2}}\left(\hat{q}_{\epsilon}^{+}+\hat{q}_{\epsilon}^{-}\right) \tilde{\phi} M M_{*} d v d v_{*} d \sigma\right): \nabla_{x} \bar{u} d x \\
& \geq-C \frac{\|\bar{u}\|_{W^{1, \infty}(d x)}+\left\|\partial_{t} \bar{u}\right\|_{L^{\infty}(d x)}+\|\bar{j}\|_{L^{\infty}(d x)}}{1-\|\bar{u}\|_{L^{\infty}(d x)}} \delta \mathcal{H}_{\epsilon}(t) \\
& -\frac{1}{2} \int_{\mathbb{R}^{3}} \mathbf{A}_{1} \cdot \tilde{u}_{\epsilon} d x+\frac{1}{2} \int_{\mathbb{R}^{3}}\left(\mathbf{A}_{4} \wedge\left(B_{\epsilon}-\bar{B}\right)+\left(E_{\epsilon}-\bar{E}\right) \wedge \mathbf{A}_{5}\right) \cdot \bar{u} d x \\
& +o(1)_{w-L_{\mathrm{loc}}^{1}(d t)}+\frac{d}{d t}\left(o(1)_{L^{\infty}(d t)}\right)-\frac{1}{2} \int_{\mathbb{R}^{3}}\left(\tilde{u}_{\epsilon} \wedge B_{\epsilon}\right) \cdot \bar{j}+(\bar{u} \wedge \bar{B}) \cdot \tilde{j}_{\epsilon} d x .
\end{aligned}
$$


Now, for mere convenience of notation, we introduce the following integrand :

$$
\begin{aligned}
\mathcal{I} & =\tilde{u}_{\epsilon} \cdot \bar{u}+\frac{1}{2}\left(E_{\epsilon} \cdot \bar{E}+B_{\epsilon} \cdot \bar{B}\right) \\
& +\frac{1}{2}\left(\left(E_{\epsilon}-\bar{E}\right) \wedge\left(B_{\epsilon}-\bar{B}\right)\right) \cdot \bar{u}+\frac{1}{2}\left(\begin{array}{c}
a_{\epsilon 26}-a_{\epsilon 35} \\
a_{\epsilon 34}-a_{\epsilon 16} \\
a_{\epsilon 15}-a_{\epsilon 24}
\end{array}\right) \cdot \bar{u}
\end{aligned}
$$

Thus, further employing the identity 12.29 , we find that

$$
\begin{aligned}
& \frac{d}{d t} \int_{\mathbb{R}^{3}} \mathcal{I} d x+\int_{\mathbb{R}^{3}}\left(\int_{\mathbb{R}^{3} \times \mathbb{R}^{3} \times \mathbb{S}^{2}}\left(\hat{q}_{\epsilon}^{+}+\hat{q}_{\epsilon}^{-}\right) \tilde{\phi} M M_{*} d v d v_{*} d \sigma\right): \nabla_{x} \bar{u} d x \\
& \quad \geq-C \frac{\|\bar{u}\|_{W^{1, \infty}(d x)}+\left\|\partial_{t} \bar{u}\right\|_{L^{\infty}(d x)}+\|\bar{j}\|_{L^{\infty}(d x)} \delta \mathcal{H}_{\epsilon}(t)}{1-\|\bar{u}\|_{L^{\infty}(d x)}} \\
& \quad-\frac{1}{2} \int_{\mathbb{R}^{3}} \mathbf{A}_{1} \cdot \tilde{u}_{\epsilon}+\mathbf{A}_{4} \cdot\left(E_{\epsilon}+\bar{u} \wedge\left(B_{\epsilon}-\bar{B}\right)\right)+\mathbf{A}_{5} \cdot\left(B_{\epsilon}+\left(E_{\epsilon}-\bar{E}\right) \wedge \bar{u}\right) d x \\
& \quad+o(1)_{w-L_{\text {loc }}^{1}(d t)}+\frac{d}{d t}\left(o(1)_{L^{\infty}(d t)}\right) \\
& \quad+\frac{1}{2} \int_{\mathbb{R}^{3}} R_{\epsilon, 6} \cdot \bar{E}-\left(E_{\epsilon}+\tilde{u}_{\epsilon} \wedge B_{\epsilon}\right) \cdot \bar{j}-(\bar{E}+\bar{u} \wedge \bar{B}) \cdot \tilde{j}_{\epsilon} d x,
\end{aligned}
$$

whence, in view of the estimate 12.28,

$$
\begin{aligned}
& \frac{d}{d t} \int_{\mathbb{R}^{3}} \mathcal{I} d x+\int_{\mathbb{R}^{3}}\left(\int_{\mathbb{R}^{3} \times \mathbb{R}^{3} \times \mathbb{S}^{2}}\left(\hat{q}_{\epsilon}^{+}+\hat{q}_{\epsilon}^{-}\right) \tilde{\phi} M M_{*} d v d v_{*} d \sigma\right): \nabla_{x} \bar{u} d x \\
& \quad \geq-C \frac{\|\bar{u}\|_{W^{1, \infty}(d x)}+\left\|\partial_{t} \bar{u}\right\|_{L^{\infty}(d x)}+\|\bar{j}\|_{L^{\infty}(d x)} \delta \mathcal{H}_{\epsilon}(t)}{1-\|\bar{u}\|_{L^{\infty}(d x)}} \\
& \quad-\frac{1}{2} \int_{\mathbb{R}^{3}} \mathbf{A}_{1} \cdot \tilde{u}_{\epsilon}+\mathbf{A}_{4} \cdot\left(E_{\epsilon}+\bar{u} \wedge\left(B_{\epsilon}-\bar{B}\right)\right)+\mathbf{A}_{5} \cdot\left(B_{\epsilon}+\left(E_{\epsilon}-\bar{E}\right) \wedge \bar{u}\right) d x \\
& \quad+o(1)_{w-L_{\text {loc }}^{1}(d t)}+\frac{d}{d t}\left(o(1)_{L^{\infty}(d t)}\right) \\
& \quad-\frac{1}{2} \int_{\mathbb{R}^{3}}\left(E_{\epsilon}+\tilde{u}_{\epsilon} \wedge B_{\epsilon}\right) \cdot \bar{j}+(\bar{E}+\bar{u} \wedge \bar{B}) \cdot \tilde{j}_{\epsilon} d x .
\end{aligned}
$$


Using then the approximate Ohm's law (12.23) and reorganizing the resulting inequality so that all remainder terms appear on its right-hand side, we obtain

$$
\begin{aligned}
& \frac{d}{d t} \int_{\mathbb{R}^{3}} \mathcal{I} d x+\int_{\mathbb{R}^{3}}\left(\int_{\mathbb{R}^{3} \times \mathbb{R}^{3} \times \mathbb{S}^{2}}\left(\hat{q}_{\epsilon}^{+}+\hat{q}_{\epsilon}^{-}\right) \tilde{\phi} M M_{*} d v d v_{*} d \sigma\right): \nabla_{x} \bar{u} d x \\
& \quad-\int_{\mathbb{R}^{3}}\left(\int_{\mathbb{R}^{3} \times \mathbb{R}^{3} \times \mathbb{S}^{2}} \frac{\left.\frac{\hat{q}_{\epsilon}^{+,--}-\hat{q}_{\epsilon}^{-,+}}{2} v M M_{*} d v d v_{*} d \sigma\right) \cdot \bar{j} d x}{1-\|\bar{u}\|_{L^{\infty}(d x)}}\right. \\
& \quad \geq-C \frac{\|\bar{u}\|_{W^{1, \infty}(d x)}+\left\|\partial_{t} \bar{u}\right\|_{L^{\infty}(d x)}+\|\bar{j}\|_{L^{\infty}(d x)} \delta \mathcal{H}_{\epsilon}(t)}{2} \\
& \quad-\frac{1}{2} \int_{\mathbb{R}^{3}} \mathbf{A}_{1} \cdot \tilde{u}_{\epsilon}+\mathbf{A}_{4} \cdot\left(E_{\epsilon}+\bar{u} \wedge\left(B_{\epsilon}-\bar{B}\right)\right)+\mathbf{A}_{5} \cdot\left(B_{\epsilon}+\left(E_{\epsilon}-\bar{E}\right) \wedge \bar{u}\right) d x \\
& +\frac{\sigma}{2} \int_{\mathbb{R}^{3}} \mathbf{A}_{3} \cdot\left(\int_{\mathbb{R}^{3} \times \mathbb{R}^{3} \times \mathbb{S}^{2}} \frac{\hat{q}_{\epsilon}^{+,-}-\hat{q}_{\epsilon}^{-,},+}{2} v M_{*} d v d v_{*} d \sigma\right) d x \\
& +o(1)_{w-L_{\mathrm{loc}}^{1}(d t)}+\frac{d}{d t}\left(o(1)_{L^{\infty}(d t)}\right)-\frac{1}{2} \int_{\mathbb{R}^{3}}\left(R_{\epsilon, 4}+R_{\epsilon, 5}\right) \cdot \bar{j} d x \\
& +\frac{\sigma}{2} \int_{\mathbb{R}^{3}}(\bar{E}+\bar{u} \wedge \bar{B}) \cdot P^{\perp}\left(\int_{\mathbb{R}^{3} \times \mathbb{R}^{3} \times \mathbb{S}^{2}} \frac{\hat{q}_{\epsilon}^{+,-}-\hat{q}_{\epsilon}^{-},+}{2} v M M_{*} d v d v_{*} d \sigma\right) d x \\
& \quad-\frac{1}{2} \int_{\mathbb{R}^{3}}\left(\sigma\left(\int_{\mathbb{R}^{3} \times \mathbb{R}^{3} \times \mathbb{S}^{2}} \frac{\hat{q}_{\epsilon}^{+,-}-\hat{q}_{\epsilon}^{-,+}}{2} v M M_{*} d v d v_{*} d \sigma\right)+\tilde{j}_{\epsilon}\right) \cdot(\bar{E}+\bar{u} \wedge \bar{B}) d x .
\end{aligned}
$$

Thus, in view of the convergences $12.32,12.33$, the estimates 12.24, 12.25 and Lemma 12.1 (allowing to control the energy by the entropy), we infer that (12.36)

$$
\begin{aligned}
\frac{d}{d t} & \int_{\mathbb{R}^{3}} \mathcal{I} d x+\int_{\mathbb{R}^{3}}\left(\int_{\mathbb{R}^{3} \times \mathbb{R}^{3} \times \mathbb{S}^{2}}\left(\hat{q}_{\epsilon}^{+}+\hat{q}_{\epsilon}^{-}\right) \tilde{\phi} M M_{*} d v d v_{*} d \sigma\right): \nabla_{x} \bar{u} d x \\
& -\int_{\mathbb{R}^{3}}\left(\int_{\mathbb{R}^{3} \times \mathbb{R}^{3} \times \mathbb{S}^{2}} \frac{\hat{q}_{\epsilon}^{+,-}-\hat{q}_{\epsilon}^{-,+}}{2} v M M_{*} d v d v_{*} d \sigma\right) \cdot \bar{j} d x \\
& \geq-C \frac{\|\bar{u}\|_{W^{1, \infty}(d x)}+\left\|\partial_{t} \bar{u}\right\|_{L^{\infty}(d x)}+\|\bar{j}\|_{L^{\infty}(d x)}}{1-\|\bar{u}\|_{L^{\infty}(d x)}} \delta \mathcal{H}_{\epsilon}(t) \\
& -\frac{1}{2} \int_{\mathbb{R}^{3}} \mathbf{A}_{1} \cdot \tilde{u}_{\epsilon}+\mathbf{A}_{4} \cdot\left(E_{\epsilon}+\bar{u} \wedge\left(B_{\epsilon}-\bar{B}\right)\right)+\mathbf{A}_{5} \cdot\left(B_{\epsilon}+\left(E_{\epsilon}-\bar{E}\right) \wedge \bar{u}\right) d x \\
& +\frac{\sigma}{2} \int_{\mathbb{R}^{3}} \mathbf{A}_{3} \cdot\left(\int_{\mathbb{R}^{3} \times \mathbb{R}^{3} \times \mathbb{S}^{2}} \frac{\hat{q}_{\epsilon}^{+,-}-\hat{q}_{\epsilon}^{-,+}}{2} v M M_{*} d v d v_{*} d \sigma\right) d x \\
& +o(1)_{w-L_{\mathrm{loc}}^{1}(d t)}+\frac{d}{d t}\left(o(1)_{L^{\infty}(d t)} .\right.
\end{aligned}
$$

At last, we may now combine the inequalities 12.35 and 12.36 to deduce, employing the symmetries of collision integrands and (12.12) to rewrite dissipation 
terms, that

$$
\begin{aligned}
& \frac{d}{d t} \int_{\mathbb{R}^{3}}\left(\left(g_{\epsilon}^{+} \gamma_{\epsilon}^{+}+g_{\epsilon}^{-} \gamma_{\epsilon}^{-}\right) \chi\left(\frac{|v|^{2}}{K_{\epsilon}}\right) \bar{g}+E_{\epsilon} \cdot \bar{E}+B_{\epsilon} \cdot \bar{B}\right. \\
& \left.+\left(\left(E_{\epsilon}-\bar{E}\right) \wedge\left(B_{\epsilon}-\bar{B}\right)+\left(\begin{array}{c}
a_{\epsilon 26}-a_{\epsilon 35} \\
a_{\epsilon 34}-a_{\epsilon 16} \\
a_{\epsilon 15}-a_{\epsilon 24}
\end{array}\right)\right) \cdot \bar{u}\right) d x \\
& +\frac{1}{2} \int_{\mathbb{R}^{3} \times \mathbb{R}^{3} \times \mathbb{R}^{3} \times \mathbb{S}^{2}}\left(\hat{q}_{\epsilon}^{+} \bar{q}^{+}+\hat{q}_{\epsilon}^{-} \bar{q}^{-}+\hat{q}_{\epsilon}^{+,-} \bar{q}^{+,-}+\hat{q}_{\epsilon}^{-,+} \bar{q}^{-,+}\right) M M_{*} d x d v d v_{*} d \sigma \\
& \geq-\lambda(t) \delta \mathcal{H}_{\epsilon}(t)-\int_{\mathbb{R}^{3}} \mathbf{A} \cdot\left(\begin{array}{c}
-\frac{\sigma}{2} \int_{\mathbb{R}^{3} \times \mathbb{R}^{3} \times \mathbb{S}^{2}}\left(\hat{q}_{\epsilon}^{+,-}-\hat{q}_{\epsilon}^{-},+\right) v M \tilde{\theta}_{\epsilon}-\tilde{\rho}_{\epsilon} d v d v_{*} d \sigma \\
E_{\epsilon}+\bar{u} \wedge\left(B_{\epsilon}-\bar{B}\right) \\
B_{\epsilon}+\left(E_{\epsilon}-\bar{E}\right) \wedge \bar{u} \\
1
\end{array}\right) d x \\
& +o(1)_{w-L_{\mathrm{loc}}^{1}(d t)}+\frac{d}{d t}\left(o(1)_{L^{\infty}(d t)}\right)-\frac{1}{2} \delta \mathcal{D}_{\epsilon}(t) .
\end{aligned}
$$

Next, assembling the preceding inequality with the scaled entropy inequality (12.18) and the energy estimate 12.34, we finally obtain

$$
\begin{aligned}
& \frac{d}{d t} \delta \mathcal{H}_{\epsilon}(t)+\delta \mathcal{D}_{\epsilon}(t)
\end{aligned}
$$

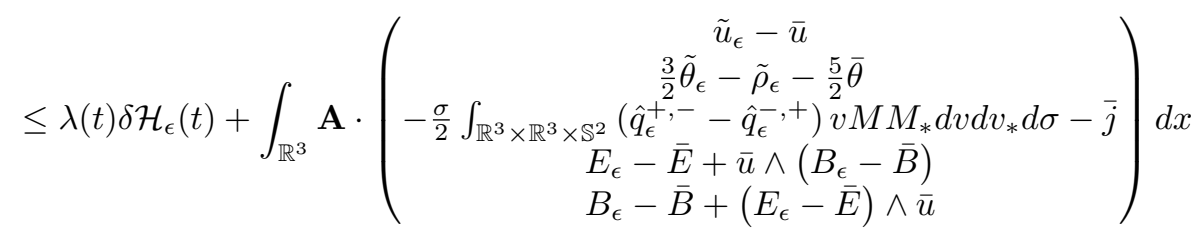

$$
\begin{aligned}
& +o(1)_{w-L_{\mathrm{loc}}^{1}(d t)}+\frac{d}{d t}\left(o(1)_{L^{\infty}(d t)}\right)+\frac{1}{2} \delta \mathcal{D}_{\epsilon}(t),
\end{aligned}
$$

which, with a straightforward application of Grönwall's lemma (carefully note that this is valid even though $\delta \mathcal{H}_{\epsilon}(t)$ may be negative), concludes the proof of the proposition.

REMARK. The proof of Proposition 12.2 is based on the construction of the stability inequality 3.31 from Proposition 3.13 for the two-fluid incompressible Navier-Stokes-Maxwell system with solenoidal Ohm's law (3.6). This approach has the great advantage of using the approximate macroscopic conservation of momentum established in Proposition 9.6 rather than the one from Proposition 9.5.

Indeed, if we were to use the latter approximate conservation law from Proposition 9.5 we would have to modulate the nonlinear term $\tilde{j}_{\epsilon} \wedge B_{\epsilon}$ into $\left(\tilde{j}_{\epsilon}-\bar{j}\right) \wedge$ $\left(B_{\epsilon}-B\right)$ (much like in the proof of Proposition 3.11 ; see $(3.28)$ ). The term $\left(B_{\epsilon}-\bar{B}\right)$ would then have to be absorbed (through Grönwall's lemma) by a renormalized modulated energy (or entropy), whereas $\left(\tilde{j}_{\epsilon}-\bar{j}\right)$ would need to be controlled by a renormalized modulated entropy dissipation provided $\tilde{j}_{\epsilon}$ is replaced by the collision integrands $-\sigma\left(\int_{\mathbb{R}^{3} \times \mathbb{R}^{3} \times \mathbb{S}^{2}} \frac{\hat{q}_{\epsilon}^{+,-}-\hat{q}_{\epsilon}^{-,+}}{2} v M M_{*} d v d v_{*} d \sigma\right)$. However, this last step produces remainders which may not belong to $L^{2}(d t d x)$ and, therefore, cannot multiply $B_{\epsilon}$. Thus, this procedure would fail.

It is therefore not possible (at least, we do not know how to make it work) to establish a similar renormalized relative entropy inequality for renormalized solutions of the scaled two species Vlasov-Maxwell-Boltzmann system (4.34) based on the construction of the stability inequality (3.26) from Proposition 3.11. 
Using the strategy of Proposition 3.13 removes this difficulty altogether by expressing the Lorentz force $\tilde{j}_{\epsilon} \wedge B_{\epsilon}$ with the Poynting vector $E_{\epsilon} \wedge B_{\epsilon}$ (and some other terms). However, the drawback of this approach resides in the necessity of the restriction $\|\bar{u}\|_{L_{t, x}^{\infty}}<1$. Recall, nevertheless, that this restriction is physically relevant, since it merely entails that the modulus of the velocity $\bar{u}$ be less than the speed of light (see comments after the proofs of Propositions 3.10 and 3.13 .).

12.2.4. Convergence and conclusion of proof. We may now pass to the limit in the approximate stability inequality 12.17 and, thus, derive the crucial modulated energy inequality for the limiting system 4.38). To this end, we simply integrate 12.17) in time against non-negative test functions and then let $\epsilon \rightarrow 0$, which yields, in view of the well-preparedness of the initial data (4.41), the weak convergences 12.1), 12.3) and the lower semi-continuities 12.13), (12.14), that

$$
\begin{aligned}
\delta \mathcal{E}(t) & +\frac{1}{2} \int_{0}^{t} \delta \mathcal{D}(s) e^{\int_{s}^{t} \lambda(\sigma) d \sigma} d s \\
& \leq \delta \mathcal{E}(0) e^{\int_{0}^{t} \lambda(s) d s} \\
& +\int_{0}^{t} \int_{\mathbb{R}^{3}} \mathbf{A} \cdot\left(\begin{array}{c}
u-\bar{u} \\
-\frac{\sigma}{2} \int_{\mathbb{R}^{3} \times \mathbb{R}^{3} \times \mathbb{S}^{2}}\left(q^{+,-}-q^{-,+}\right) v M M_{*} d v d v_{*} d \sigma-\bar{j} \\
E-\bar{E}+\bar{u} \wedge(B-\bar{B}) \\
B-\bar{B}+(E-\bar{E}) \wedge \bar{u}
\end{array}\right)(s) d x \\
& \times e^{\int_{s}^{t} \lambda(\sigma) d \sigma} d s .
\end{aligned}
$$

Finally, using 12.2 and the characterization 6.11 of the limiting collision integrands $q^{ \pm, \mp}$ from Proposition 6.3 , we deduce that

$$
\begin{aligned}
& \delta \mathcal{E}(t)+\frac{1}{2} \int_{0}^{t} \delta \mathcal{D}(s) e^{\int_{s}^{t} \lambda(\sigma) d \sigma} d s \\
& \leq \delta \mathcal{E}(0) e^{\int_{0}^{t} \lambda(s) d s}+\int_{0}^{t} \int_{\mathbb{R}^{3}} \mathbf{A} \cdot\left(\begin{array}{c}
u-\bar{u} \\
\frac{5}{2}(\theta-\bar{\theta}) \\
j-\bar{j} \\
E-\bar{E}+\bar{u} \wedge(B-\bar{B}) \\
B-\bar{B}+(E-\bar{E}) \wedge \bar{u}
\end{array}\right)(s) d x e^{\int_{s}^{t} \lambda(\sigma) d \sigma} d s,
\end{aligned}
$$

which is precisely the stability inequality we were after.

As for the temporal continuity of $\left(u, \frac{5}{2} \theta, E, B\right)$, it is readily seen from the approximate macroscopic conservation laws from Proposition 9.5 and Maxwell's equations 12.27 that $\partial_{t} P \tilde{u}_{\epsilon}, \partial_{t}\left(\frac{3}{2} \tilde{\theta}_{\epsilon}-\tilde{\rho}_{\epsilon}\right), \partial_{t} E_{\epsilon}$ and $\partial_{t} B_{\epsilon}$ are uniformly bounded, in $L_{\text {loc }}^{1}$ in time and in some negative index Sobolev space in $x$. It is therefore possible to show (see [57, Appendix C]) that $\left(P \tilde{u}_{\epsilon}, \frac{3}{2} \tilde{\theta}_{\epsilon}-\tilde{\rho}_{\epsilon}, E_{\epsilon}, B_{\epsilon}\right)$ converges to $\left(u, \frac{5}{2} \theta, E, B\right) \in C\left([0, \infty) ; w-L^{2}\left(\mathbb{R}^{3}\right)\right)$ weakly in $L^{2}(d x)$ uniformly locally in time.

At last, the proof of Theorem 4.5 is complete.

\subsection{Proof of Theorem 4.6 on strong interactions}

This demonstration closely follows the method of proof of Theorem 4.5 presented in the preceding section. However, the asymptotic limit treated in Theorem 4.6 is more singular than the one from Theorem 4.5. Some steps in the coming proof will therefore require some greater care than their counterparts from the previous section.

As before, we begin our proof by appropriately gathering previous results together. 
12.3.1. Weak convergence of fluctuations, collision integrands and electromagnetic fields. Thus, we are considering here a family of renormalized solutions $\left(f_{\epsilon}^{ \pm}, E_{\epsilon}, B_{\epsilon}\right)$ to the scaled two species Vlasov-Maxwell-Boltzmann system (4.34), in the regime of strong interspecies interactions, i.e. $\delta=1$, satisfying the scaled entropy inequality 4.35 .

According to Lemmas 5.1 and 5.2 , the corresponding families of fluctuations $g_{\epsilon}^{ \pm}$ and renormalized fluctuations $\hat{g}_{\epsilon}^{ \pm}$are weakly compact in $L_{\text {loc }}^{1}\left(d t d x ; L^{1}\left(\left(1+|v|^{2}\right) M d v\right)\right)$ and $L_{\text {loc }}^{2}\left(d t ; L^{2}(M d x d v)\right)$, respectively, while, in view of Lemma 5.3 the corresponding collision integrands $\hat{q}_{\epsilon}^{ \pm}$and $\hat{q}_{\epsilon}^{ \pm, \mp}$ are weakly compact in $L^{2}\left(\overline{M M}_{*} d t d x d v d v_{*} d \sigma\right)$. Thus, using Lemma 5.1 again and the decomposition (5.5), we know that there exist $g^{ \pm} \in L^{\infty}\left(d t ; L^{2}(M d x d v)\right),(E, B) \in L^{\infty}\left(d t ; L^{2}(d x)\right)$ and $q^{ \pm}, q^{ \pm, \mp} \in L^{2}\left(M M_{*} d t d x d v d v_{*} d \sigma\right)$, such that, up to extraction of subsequences,

$$
\begin{array}{cl}
g_{\epsilon}^{ \pm} \rightarrow g^{ \pm} & \text {in } L_{\mathrm{loc}}^{1}\left(d t d x ; L^{1}\left(\left(1+|v|^{2}\right) M d v\right)\right), \\
\hat{g}_{\epsilon}^{ \pm} \stackrel{*}{\rightarrow} g^{ \pm} & \text {in } L^{\infty}\left(d t ; L^{2}(M d x d v)\right), \\
\left(E_{\epsilon}, B_{\epsilon}\right) \stackrel{*}{\rightarrow}(E, B) & \text { in } L^{\infty}\left(d t ; L^{2}(d x)\right), \\
\hat{q}_{\epsilon}^{ \pm} \rightarrow q^{ \pm} & \text {in } L^{2}\left(M M_{*} d t d x d v d v_{*} d \sigma\right), \\
\hat{q}_{\epsilon}^{ \pm, \mp} \rightarrow q^{ \pm, \mp} & \text { in } L^{2}\left(M M_{*} d t d x d v d v_{*} d \sigma\right),
\end{array}
$$

as $\epsilon \rightarrow 0$. Therefore, one also has the weak convergence of the densities $\rho_{\epsilon}^{ \pm}$, bulk velocities $u_{\epsilon}^{ \pm}$and temperatures $\theta_{\epsilon}^{ \pm}$corresponding to $g_{\epsilon}^{ \pm}$:

$$
\rho_{\epsilon}^{ \pm} \rightarrow \rho^{ \pm}, \quad u_{\epsilon}^{ \pm} \rightarrow u^{ \pm} \quad \text { and } \quad \theta_{\epsilon}^{ \pm} \rightarrow \theta^{ \pm} \quad \text { in } L_{\mathrm{loc}}^{1}(d t d x) \text { as } \epsilon \rightarrow 0,
$$

where $\rho^{ \pm}, u^{ \pm}, \theta^{ \pm} \in L^{\infty}\left(d t ; L^{2}(d x)\right)$ are, respectively, the densities, bulk velocities and temperatures corresponding to $g^{ \pm}$. In fact, Lemma 5.10 implies that

$$
g^{ \pm}=\Pi g^{ \pm}=\rho^{ \pm}+u^{ \pm} \cdot v+\theta^{ \pm}\left(\frac{|v|^{2}}{2}-\frac{3}{2}\right) .
$$

Next, we further introduce the scaled fluctuations

$$
h_{\epsilon}=\frac{1}{\epsilon}\left[\left(g_{\epsilon}^{+}-g_{\epsilon}^{-}\right)-n_{\epsilon}\right],
$$

where $n_{\epsilon}=\rho_{\epsilon}^{+}-\rho_{\epsilon}^{-}$is the charge density, and the electrodynamic variables

$$
j_{\epsilon}=\frac{1}{\epsilon}\left(u_{\epsilon}^{+}-u_{\epsilon}^{-}\right), \quad w_{\epsilon}=\frac{1}{\epsilon}\left(\theta_{\epsilon}^{+}-\theta_{\epsilon}^{-}\right),
$$

which are precisely the bulk velocity and temperature associated with the scaled fluctuations $h_{\epsilon}$. In view of Lemma 5.13, the electric current $j_{\epsilon}$ and the internal electric energy $w_{\epsilon}$ are uniformly bounded in $L_{\text {loc }}^{1}(d t d x)$, which necessarily implies, letting $\epsilon \rightarrow 0$, that $u^{+}=u^{-}$and $\theta^{+}=\theta^{-}$. Carefully note, though, that the limiting densities $\rho^{+}$and $\rho^{-}$may be distinct here. Therefore, we appropriately rename the limiting macroscopic variables

$$
\rho=\frac{\rho^{+}+\rho^{-}}{2}, \quad n=\rho^{+}-\rho^{-}, \quad u=u^{+}=u^{-}, \quad \theta=\theta^{+}=\theta^{-},
$$

whence

$$
\begin{aligned}
g^{ \pm} & =\rho^{ \pm}+u \cdot v+\theta\left(\frac{|v|^{2}}{2}-\frac{3}{2}\right), \\
\frac{g^{+}+g^{-}}{2} & =\rho+u \cdot v+\theta\left(\frac{|v|^{2}}{2}-\frac{3}{2}\right), \\
g^{+}-g^{-} & =n .
\end{aligned}
$$


Now, according to Lemma 5.14 , it holds that $\frac{h_{\epsilon}}{1+\left\|\hat{g}_{\epsilon}^{+}-\hat{g}_{\epsilon}^{-}\right\|_{L^{2}(M d v)}}$ is weakly compact in $L_{\mathrm{loc}}^{1}\left(d t d x ; L^{1}((1+|v|) M d v)\right)$ and that $\frac{j_{\epsilon}}{1+\left\|\hat{g}_{\epsilon}^{+}-\hat{g}_{\epsilon}^{-}\right\|_{L^{2}(M d v)}}$ is weakly compact in $L_{\text {loc }}^{2}(d t d x)$. Moreover, Lemma 7.11 indicates that, up to extraction of subsequences, there is $h \in L_{\mathrm{loc}}^{1}\left(d t d x ; L^{1}\left(\left(1+|v|^{2}\right) M d v\right)\right)$ such that

$$
\frac{h_{\epsilon}}{1+\left\|\hat{g}_{\epsilon}^{+}-\hat{g}_{\epsilon}^{-}\right\|_{L^{2}(M d v)}} \rightarrow \frac{h}{1+|n|} \text { in } L_{\mathrm{loc}}^{1}\left(d t d x ; L^{1}((1+|v|) M d v)\right),
$$

as $\epsilon \rightarrow 0$. Note, however, that $h$ is not characterized by an infinitesimal Maxwellian form. Here, we only have that

$$
\Pi h=j \cdot v+w\left(\frac{|v|^{2}}{2}-\frac{3}{2}\right)
$$

where the electric current $j$ and the internal electric energy $w$ are defined by

$$
j=\int_{\mathbb{R}^{3}} h v M d v, \quad w=\int_{\mathbb{R}^{3}} h\left(\frac{|v|^{2}}{3}-1\right) M d v .
$$

In particular, it holds that

$$
\frac{j_{\epsilon}}{1+\left\|\hat{g}_{\epsilon}^{+}-\hat{g}_{\epsilon}^{-}\right\|_{L^{2}(M d v)}} \rightarrow \frac{j}{1+|n|} \text { in } L_{\mathrm{loc}}^{2}(d t d x) .
$$

Finally, since $n \in L^{\infty}\left(d t ; L^{2}(d x)\right)$, setting

$$
r_{\epsilon}=\frac{1+|n|}{1+\left\|\hat{g}_{\epsilon}^{+}-\hat{g}_{\epsilon}^{-}\right\|_{L^{2}(M d v)}} \in L^{\infty}\left(d t ; L_{\mathrm{loc}}^{2}(d x)\right),
$$

which, according to Lemma 7.11 and up to extraction of subsequences, converges almost everywhere towards the constant function 1, we see that

$$
r_{\epsilon} j_{\epsilon} \rightarrow j \quad \text { in } L_{\text {loc }}^{1}(d t d x) \text {. }
$$

Similarly, since $\frac{h_{\epsilon}}{1+\left\|\hat{g}_{\epsilon}^{+}-\hat{g}_{\epsilon}^{-}\right\|_{L^{2}(M d v)}}$ is bounded in $L_{\text {loc }}^{2}\left(d t d x ; L^{1}((1+|v|) M d v)\right)$ uniformly, by virtue of Lemma 5.14, it is possible to show that

$$
r_{\epsilon} h_{\epsilon} \rightarrow h \quad \text { in } L_{\text {loc }}^{1}\left(d t d x ; L^{1}((1+|v|) M d v)\right) .
$$

12.3.2. Constraint equations, Maxwell's system and energy inequality. In view of Proposition 8.3, we already know that the limiting thermodynamic fields $\rho, u$ and $\theta$ satisfy the incompressibility and Boussinesq relations

$$
\operatorname{div} u=0, \quad \rho+\theta=0 .
$$

Moreover, the discussion in Section 6.4 shows that the limiting electromagnetic field satisfies the Faraday equation and Gauss' laws :

$$
\left\{\begin{aligned}
\partial_{t} B+\operatorname{rot} E & =0 \\
\operatorname{div} E & =n \\
\operatorname{div} B & =0
\end{aligned}\right.
$$

Recall, however, that we do not know from Section 6.4 whether Ampère's equation is necessarily satisfied in the sense of distributions in the limit.

Finally, Proposition 8.5 further establishes that the electrodynamic variables $j$ and $w$ satisfy Ohm's law and the internal electric energy equilibrium relation

$$
j-n u=\sigma\left(-\frac{1}{2} \nabla_{x} n+E+u \wedge B\right), \quad w=n \theta,
$$

where the electric conductivity $\sigma>0$ is defined by 2.65 . 
As for the energy bound, Proposition 8.6 states that, for almost every $t \geq 0$,

$$
\begin{aligned}
& \frac{1}{2}\left(\frac{1}{2}\|n\|_{L_{x}^{2}}^{2}+2\|u\|_{L_{x}^{2}}^{2}+5\|\theta\|_{L_{x}^{2}}^{2}+\|E\|_{L_{x}^{2}}^{2}+\|B\|_{L_{x}^{2}}^{2}\right)(t) \\
& +\int_{0}^{t}\left(2 \mu\left\|\nabla_{x} u\right\|_{L_{x}^{2}}^{2}+5 \kappa\left\|\nabla_{x} \theta\right\|_{L_{x}^{2}}^{2}+\frac{1}{\sigma}\|j-n u\|_{L_{x}^{2}}^{2}\right)(s) d s \leq C^{\mathrm{in}},
\end{aligned}
$$

where the viscosity $\mu>0$, thermal conductivity $\kappa>0$ and electric conductivity $\sigma>0$ are respectively defined by 2.56 and 2.65 . In particular, it holds that

$$
\begin{aligned}
(n, u, \theta, E, B) & \in L^{\infty}\left([0, \infty), d t ; L^{2}\left(\mathbb{R}^{3}, d x\right)\right), \\
(u, \theta) & \in L^{2}\left([0, \infty), d t ; \dot{H}^{1}\left(\mathbb{R}^{3}, d x\right)\right), \\
j-n u & \in L^{2}\left([0, \infty) \times \mathbb{R}^{3}, d t d x\right) .
\end{aligned}
$$

This energy bound can be improved to the actual energy inequality

$$
\begin{aligned}
\frac{1}{2}\left(\frac{1}{2} \|\right. & \left.n\left\|_{L_{x}^{2}}^{2}+2\right\| u\left\|_{L_{x}^{2}}^{2}+5\right\| \theta\left\|_{L_{x}^{2}}^{2}+\right\| E\left\|_{L_{x}^{2}}^{2}+\right\| B \|_{L_{x}^{2}}^{2}\right)(t) \\
& +\int_{0}^{t}\left(2 \mu\left\|\nabla_{x} u\right\|_{L_{x}^{2}}^{2}+5 \kappa\left\|\nabla_{x} \theta\right\|_{L_{x}^{2}}^{2}+\frac{1}{\sigma}\|j-n u\|_{L_{x}^{2}}^{2}\right)(s) d s \\
& \leq \frac{1}{2}\left(\frac{1}{2}\left\|n^{\mathrm{in}}\right\|_{L_{x}^{2}}^{2}+2\left\|u^{\mathrm{in}}\right\|_{L_{x}^{2}}^{2}+5\left\|\theta^{\mathrm{in}}\right\|_{L_{x}^{2}}^{2}+\left\|E^{\mathrm{in}}\right\|_{L_{x}^{2}}^{2}+\left\|B^{\mathrm{in}}\right\|_{L_{x}^{2}}^{2}\right)
\end{aligned}
$$

using the well-preparedness of the initial data 4.44.

12.3.3. The renormalized modulated entropy inequality. We move on now to the rigorous derivation of a stability inequality encoding the asymptotic macroscopic evolution equations for $u$ and $\theta$ and the Ampère equation in the spirit of the weak-strong stability inequalities used in Section 3.2 .3 to define dissipative solutions for some Navier-Stokes-Maxwell systems. Recall that, as explained therein, such systems are in general not known to display weak stability so that their weak solutions in the energy space are not known to exist.

The strategy used here closely follows the method employed in the case of weak interactions detailed in Section 12.2.3.

Thus, as in Section 9.2 , we define the renormalized fluctuations $g_{\epsilon}^{ \pm} \gamma_{\epsilon}^{ \pm} \chi\left(\frac{|v|^{2}}{K_{\epsilon}}\right)$, with $K_{\epsilon}=K|\log \epsilon|$, for some large $K>0$, and $\chi \in C_{c}^{\infty}([0, \infty))$ a smooth compactly supported function such that $\mathbb{1}_{[0,1]} \leq \chi \leq \mathbb{1}_{[0,2]}$, and where $\gamma_{\epsilon}^{ \pm}=\gamma\left(G_{\epsilon}^{ \pm}\right)$for some renormalization $\gamma \in C^{1}([0, \infty) ; \mathbb{R})$ satisfying $(9.36)$.

Since, up to further extraction of subsequences, $\gamma_{\epsilon}^{ \pm} \chi\left(\frac{|v|^{2}}{K_{\epsilon}}\right)$ converges almost everywhere towards $1, g_{\epsilon}^{ \pm}$is weakly compact in $L_{\text {loc }}^{1}\left(d t d x ; L^{1}\left(\left(1+|v|^{2}\right) M d v\right)\right)$ and $g_{\epsilon}^{ \pm} \gamma_{\epsilon}^{ \pm}$is uniformly bounded in $L^{\infty}\left(d t ; L^{2}(M d x d v)\right)$, we deduce, by the Product Limit Theorem, that

$$
g_{\epsilon}^{ \pm} \gamma_{\epsilon}^{ \pm} \chi\left(\frac{|v|^{2}}{K_{\epsilon}}\right) \stackrel{*}{\rightarrow} g^{ \pm} \quad \text { in } L^{\infty}\left(d t ; L^{2}(M d x d v)\right) .
$$

Therefore, one has the weak convergence of the densities $\tilde{\rho}_{\epsilon}^{ \pm}$, bulk velocities $\tilde{u}_{\epsilon}^{ \pm}$and temperatures $\tilde{\theta}_{\epsilon}^{ \pm}$corresponding to $g_{\epsilon}^{ \pm} \gamma_{\epsilon}^{ \pm} \chi\left(\frac{|v|^{2}}{K_{\epsilon}}\right)$ :

$$
\tilde{\rho}_{\epsilon}^{ \pm} \stackrel{*}{\rightarrow} \rho^{ \pm}, \quad \tilde{u}_{\epsilon}^{ \pm} \stackrel{*}{\rightarrow} u \quad \text { and } \quad \tilde{\theta}_{\epsilon}^{ \pm} \stackrel{*}{\rightarrow} \theta \quad \text { in } L^{\infty}\left(d t ; L^{2}(d x)\right) \text { as } \epsilon \rightarrow 0 .
$$

In particular, the hydrodynamic variables $\tilde{\rho}_{\epsilon}=\frac{\tilde{\rho}_{\epsilon}^{+}+\tilde{\rho}_{\epsilon}^{-}}{2}, \tilde{u}_{\epsilon}=\frac{\tilde{u}_{\epsilon}^{+}+\tilde{u}_{\epsilon}^{-}}{2}$ and $\tilde{\theta}_{\epsilon}=\frac{\tilde{\theta}_{\epsilon}^{+}+\tilde{\theta}_{\epsilon}^{-}}{2}$ also obviously verify

$$
\tilde{\rho}_{\epsilon} \stackrel{*}{\rightarrow} \rho, \quad \tilde{u}_{\epsilon} \stackrel{*}{\rightarrow} u \quad \text { and } \quad \tilde{\theta}_{\epsilon} \stackrel{*}{\rightarrow} \theta \quad \text { in } L^{\infty}\left(d t ; L^{2}(d x)\right) \text { as } \epsilon \rightarrow 0,
$$


while the charge density $\tilde{n}_{\epsilon}=\tilde{\rho}_{\epsilon}^{+}-\tilde{\rho}_{\epsilon}^{-}$satisfies

$$
\tilde{n}_{\epsilon} \stackrel{*}{\rightarrow} n \quad \text { in } L^{\infty}\left(d t ; L^{2}(d x)\right) \text { as } \epsilon \rightarrow 0 .
$$

It follows that, since $u$ is solenoidal,

$$
P^{\perp} \tilde{u}_{\epsilon} \stackrel{*}{\rightarrow} 0 \quad \text { in } L^{\infty}\left(d t ; L^{2}(d x)\right) \text { as } \epsilon \rightarrow 0,
$$

and, in view of the limiting Boussinesq relation,

$$
\tilde{\rho}_{\epsilon}+\tilde{\theta}_{\epsilon} \stackrel{*}{\rightarrow} 0 \quad \text { in } L^{\infty}\left(d t ; L^{2}(d x)\right) \text { as } \epsilon \rightarrow 0 \text {. }
$$

Here, in constrast with the convergence properties of the electric current established in Section 12.2.3 for weak interactions, we cannot show the convergence of the electric current $j_{\epsilon}=\frac{1}{\epsilon}\left(\tilde{u}_{\epsilon}^{+}-\tilde{u}_{\epsilon}^{-}\right)$towards $j$ unless we renormalize it as in 12.38. Instead, we establish below in 12.64 a useful consistency relation for $\tilde{j}_{\epsilon}$ by suitably controlling remainders in the spirit of Section 9.2

Now, just as in the case of weak interspecies interactions, the $L^{2}(M d x d v)$ norm of $g_{\epsilon}^{ \pm} \gamma_{\epsilon}^{ \pm} \chi\left(\frac{|v|^{2}}{K_{\epsilon}}\right)$ is not a Lyapunov functional but it is nevertheless controlled by the relative entropy

$$
\frac{1}{2}\left\|g_{\epsilon}^{ \pm} \gamma_{\epsilon}^{ \pm} \chi\left(\frac{|v|^{2}}{K_{\epsilon}}\right)\right\|_{L^{2}(M d x d v)}^{2} \leq \frac{C}{\epsilon^{2}} H\left(f_{\epsilon}^{ \pm}\right)
$$

for some $C>1$, and therefore by the initial data 4.42. One may therefore try, in a preliminary attempt to show an asymptotic stability inequality, to modulate the approximate energy associated with $g_{\epsilon}^{ \pm} \gamma_{\epsilon}^{ \pm} \chi\left(\frac{|v|^{2}}{K_{\epsilon}}\right)$, i.e. its $L^{2}(M d x d v)$ norm, by introducing a test functions $\bar{g}^{ \pm}$in infinitesimal Maxwellian form :

$$
\bar{g}^{ \pm}=\bar{\rho}^{ \pm}+\bar{u} \cdot v+\bar{\theta}\left(\frac{|v|^{2}}{2}-\frac{3}{2}\right)
$$

where

$$
\bar{\rho}^{ \pm}(t, x), \bar{u}(t, x), \bar{\theta}(t, x) \in C_{c}^{\infty}\left([0, \infty) \times \mathbb{R}^{3}\right) \quad \text { with } \operatorname{div} \bar{u}=0, \frac{\bar{\rho}^{+}+\bar{\rho}^{-}}{2}+\bar{\theta}=0
$$

and then establishing a stability inequality for the modulated energies

$$
\frac{1}{2}\left\|g_{\epsilon}^{ \pm} \gamma_{\epsilon}^{ \pm} \chi\left(\frac{|v|^{2}}{K_{\epsilon}}\right)-\bar{g}^{ \pm}\right\|_{L^{2}(M d x d v)}^{2}
$$


Notice that it holds, utilizing the elementary identity $a^{2}+\frac{3}{2} b^{2}=\frac{3}{5}(a+b)^{2}+$ $\frac{5}{2}\left(\frac{3 b-2 a}{5}\right)^{2}$, for any $a, b \in \mathbb{R}$,

$$
\begin{aligned}
\sum_{ \pm} & \left\|g_{\epsilon}^{ \pm} \gamma_{\epsilon}^{ \pm} \chi\left(\frac{|v|^{2}}{K_{\epsilon}}\right)-\bar{g}^{ \pm}\right\|_{L^{2}(M d x d v)}^{2} \\
& \geq \sum_{ \pm}\left\|\Pi\left(g_{\epsilon}^{ \pm} \gamma_{\epsilon}^{ \pm} \chi\left(\frac{|v|^{2}}{K_{\epsilon}}\right)\right)-\bar{g}^{ \pm}\right\|_{L^{2}(M d x d v)}^{2} \\
& =\sum_{ \pm}\left(\left\|\tilde{\rho}_{\epsilon}^{ \pm}-\bar{\rho}^{ \pm}\right\|_{L^{2}(d x)}^{2}+\left\|\tilde{u}_{\epsilon}^{ \pm}-\bar{u}\right\|_{L^{2}(d x)}^{2}+\frac{3}{2}\left\|\tilde{\theta}_{\epsilon}^{ \pm}-\bar{\theta}\right\|_{L^{2}(d x)}^{2}\right) \\
& \left.=2\left\|\tilde{\rho}_{\epsilon}-\bar{\rho}\right\|_{L^{2}(d x)}^{2}+\frac{1}{2}\left\|\tilde{n}_{\epsilon}-\bar{n}\right\|_{L^{2}(d x)}^{2}\right) \\
& +\sum_{ \pm}\left(\left\|\tilde{u}_{\epsilon}^{ \pm}-\bar{u}\right\|_{L^{2}(d x)}^{2}+\frac{3}{2}\left\|\tilde{\theta}_{\epsilon}^{ \pm}-\bar{\theta}\right\|_{L^{2}(d x)}^{2}\right) \\
& \left.=\frac{1}{2}\left\|\tilde{n}_{\epsilon}-\bar{n}\right\|_{L^{2}(d x)}^{2}\right) \\
& +\sum_{ \pm}\left(\frac{3}{5}\left\|\tilde{\rho}_{\epsilon}+\tilde{\theta}_{\epsilon}^{ \pm}\right\|_{L^{2}(d x)}^{2}+\left\|\tilde{u}_{\epsilon}^{ \pm}-\bar{u}\right\|_{L^{2}(d x)}^{2}+\frac{5}{2}\left\|\frac{3 \tilde{\theta}_{\epsilon}^{ \pm}-2 \tilde{\rho}_{\epsilon}}{5}-\bar{\theta}\right\|_{L^{2}(d x)}^{2}\right)
\end{aligned}
$$

where we have denoted $\bar{\rho}=\frac{\bar{\rho}^{+}+\bar{\rho}^{-}}{2}$ and $\bar{n}=\bar{\rho}^{+}-\bar{\rho}^{-}$.

As before, it turns out that this approach is not quite suitable for our purpose because, even though, for any $0 \leq t_{1}<t_{2}$ (see the proof of Lemma 5.1),

$$
\int_{t_{1}}^{t_{2}} \frac{1}{2}\left\|g^{ \pm}\right\|_{L^{2}(M d x d v)}^{2} d t \leq \liminf _{\epsilon \rightarrow 0} \int_{t_{1}}^{t_{2}} \frac{1}{\epsilon^{2}} H\left(f_{\epsilon}^{ \pm}\right) d t
$$

it is not possible to set $C=1$ in 12.44. Indeed, the first term in the polynomial expansion of the function $h(z)=(1+z) \log (1+z)-z$ defining the entropy is $\frac{1}{2} z^{2}$, but the second term is $-\frac{1}{6} z^{3}$ and may be negative.

Some entropy (or energy) is therefore lost by considering the modulated energies 12.45). These considerations lead us to introduce a more precise modulated functional in replacement of $(12.45)$ capturing more information on the fluctuations. To be precise, instead of (12.45), we consider now the renormalized modulated entropies

$$
\frac{1}{\epsilon^{2}} H\left(f_{\epsilon}^{ \pm}\right)-\int_{\mathbb{R}^{3} \times \mathbb{R}^{3}} g_{\epsilon}^{ \pm} \gamma_{\epsilon}^{ \pm} \chi\left(\frac{|v|^{2}}{K_{\epsilon}}\right) \bar{g}^{ \pm} M d x d v+\frac{1}{2}\left\|\bar{g}^{ \pm}\right\|_{L^{2}(M d x d v)}^{2} .
$$

Note that the above functional may be negative for fixed $\epsilon>0$. However, in view of 12.46, it recovers asymptotically a non-negative quantity, which is precisely the asymptotic modulated energy :

$$
\begin{aligned}
\int_{t_{1}}^{t_{2}} \frac{1}{2} & \left(\left\|\rho^{ \pm}-\bar{\rho}^{ \pm}\right\|_{L^{2}(d x)}^{2}+\|u-\bar{u}\|_{L^{2}(d x)}^{2}+\frac{3}{2}\|\theta-\bar{\theta}\|_{L^{2}(d x)}^{2}\right) d t \\
& =\int_{t_{1}}^{t_{2}} \frac{1}{2}\left\|g^{ \pm}-\bar{g}^{ \pm}\right\|_{L^{2}(M d x d v)}^{2} d t \\
& \leq \liminf _{\epsilon \rightarrow 0} \int_{t_{1}}^{t_{2}}\left(\frac{1}{\epsilon^{2}} H\left(f_{\epsilon}^{ \pm}\right)\right. \\
& \left.-\int_{\mathbb{R}^{3} \times \mathbb{R}^{3}} g_{\epsilon}^{ \pm} \gamma_{\epsilon}^{ \pm} \chi\left(\frac{|v|^{2}}{K_{\epsilon}}\right) \bar{g}^{ \pm} M d x d v+\frac{1}{2}\left\|\bar{g}^{ \pm}\right\|_{L^{2}(M d x d v)}^{2}\right) d t
\end{aligned}
$$

for all $0 \leq t_{1}<t_{2}$. 
The first term in 12.47 is precisely the entropy of $f_{\epsilon}^{ \pm}$and will be controlled by the scaled entropy inequality (4.35), whereas the last term in 12.47 only involves smooth quantities and will therefore be controlled directly. As for the middle term in the modulated entropy (12.47), its time derivative will involve the approximate macroscopic conservation laws for $g_{\epsilon}^{ \pm} \gamma_{\epsilon}^{ \pm} \chi\left(\frac{|v|^{2}}{K_{\epsilon}}\right)$.

Now, in order to establish the renormalized modulated entropy inequality leading to the convergence stated in Theorem 4.6. we introduce further test functions

$$
\bar{E}(t, x), \bar{B}(t, x), \bar{j}(t, x) \in C_{c}^{\infty}\left([0, \infty) \times \mathbb{R}^{3}\right) \quad \text { with } \operatorname{div} \bar{E}=\bar{n}, \operatorname{div} \bar{B}=0,
$$

and we define the renormalized modulated entropy

$$
\begin{aligned}
\delta \mathcal{H}_{\epsilon}(t) & =\frac{1}{\epsilon^{2}} H\left(f_{\epsilon}^{+}\right)+\frac{1}{\epsilon^{2}} H\left(f_{\epsilon}^{-}\right) \\
& -\int_{\mathbb{R}^{3} \times \mathbb{R}^{3}}\left(g_{\epsilon}^{+} \gamma_{\epsilon}^{+} \chi\left(\frac{|v|^{2}}{K_{\epsilon}}\right) \bar{g}^{+}+g_{\epsilon}^{-} \gamma_{\epsilon}^{-} \chi\left(\frac{|v|^{2}}{K_{\epsilon}}\right) \bar{g}^{-}\right) M d x d v \\
& +\frac{1}{2}\left\|\bar{g}^{+}\right\|_{L^{2}(M d x d v)}^{2}+\frac{1}{2}\left\|\bar{g}^{-}\right\|_{L^{2}(M d x d v)}^{2} \\
& +\frac{1}{2}\left\|E_{\epsilon}-\bar{E}\right\|_{L^{2}(d x)}^{2}+\frac{1}{2}\left\|B_{\epsilon}-\bar{B}\right\|_{L^{2}(d x)}^{2}+\frac{1}{2} \int_{\mathbb{R}^{3}}\left(\frac{1}{\epsilon^{2}} \operatorname{Tr} m_{\epsilon}+\operatorname{Tr} a_{\epsilon}\right) d x \\
& -\int_{\mathbb{R}^{3}}\left(\left(E_{\epsilon}-\bar{E}\right) \wedge\left(B_{\epsilon}-\bar{B}\right)+\left(\begin{array}{c}
a_{\epsilon 26}-a_{\epsilon 35} \\
a_{\epsilon 34}-a_{\epsilon 16} \\
a_{\epsilon 15}-a_{\epsilon 24}
\end{array}\right)\right) \cdot \bar{u} d x,
\end{aligned}
$$

where the matrix measures $m_{\epsilon}$ and $a_{\epsilon}$ are the defects introduced in Section 4.1.4 and controlled by the scaled entropy inequality 4.35 .

We also define the renormalized modulated energy

$$
\begin{aligned}
\delta \mathcal{E}_{\epsilon}(t) & =\frac{1}{2}\left\|g_{\epsilon}^{+} \gamma_{\epsilon}^{+} \chi\left(\frac{|v|^{2}}{K_{\epsilon}}\right)-\bar{g}^{+}\right\|_{L^{2}(M d x d v)}^{2}+\frac{1}{2}\left\|g_{\epsilon}^{-} \gamma_{\epsilon}^{-} \chi\left(\frac{|v|^{2}}{K_{\epsilon}}\right)-\bar{g}^{-}\right\|_{L^{2}(M d x d v)}^{2} \\
& +\frac{1}{2}\left\|E_{\epsilon}-\bar{E}\right\|_{L^{2}(d x)}^{2}+\frac{1}{2}\left\|B_{\epsilon}-\bar{B}\right\|_{L^{2}(d x)}^{2}+\frac{1}{2} \int_{\mathbb{R}^{3}}\left(\frac{1}{\epsilon^{2}} \operatorname{Tr} m_{\epsilon}+\operatorname{Tr} a_{\epsilon}\right) d x \\
& -\int_{\mathbb{R}^{3}}\left(\left(E_{\epsilon}-\bar{E}\right) \wedge\left(B_{\epsilon}-\bar{B}\right)+\left(\begin{array}{c}
a_{\epsilon 26}-a_{\epsilon 35} \\
a_{\epsilon 34}-a_{\epsilon 16} \\
a_{\epsilon 15}-a_{\epsilon 24}
\end{array}\right)\right) \cdot \bar{u} d x
\end{aligned}
$$

which is asymptotically equivalent to $\delta \mathcal{H}_{\epsilon}(t)$, at least formally. Note that $\delta \mathcal{H}_{\epsilon}(t)$ controls more accurately the large values of the fluctuations $g_{\epsilon}^{ \pm}$than $\delta \mathcal{E}_{\epsilon}(t)$. Lemma 12.3 below shows how the modulated entropy $\delta \mathcal{H}_{\epsilon}(t)$ controls the modulated energy $\delta \mathcal{E}_{\epsilon}(t)$.

Finally, we introduce the renormalized modulated entropy dissipation

$$
\begin{aligned}
\delta \mathcal{D}_{\epsilon}(t) & =\frac{1}{4}\left\|\hat{q}_{\epsilon}^{+}-\bar{q}^{+}\right\|_{L^{2}\left(M M_{*} d x d v d v_{*} d \sigma\right)}^{2}+\frac{1}{4}\left\|\hat{q}_{\epsilon}^{-}-\bar{q}^{-}\right\|_{L^{2}\left(M M_{*} d x d v d v_{*} d \sigma\right)}^{2} \\
& +\frac{1}{4}\left\|\hat{q}_{\epsilon}^{+,-}-\bar{q}^{+,-}\right\|_{L^{2}\left(M M_{*} d x d v d v_{*} d \sigma\right)}^{2}+\frac{1}{4}\left\|\hat{q}_{\epsilon}^{-,+}-\bar{q}^{-,+}\right\|_{L^{2}\left(M M_{*} d x d v d v_{*} d \sigma\right)}^{2}
\end{aligned}
$$


where

$$
\begin{aligned}
\bar{q}^{ \pm} & =\frac{1}{2} \nabla_{x} \bar{u}:\left(\tilde{\phi}+\tilde{\phi}_{*}-\tilde{\phi}^{\prime}-\tilde{\phi}_{*}^{\prime}\right)+\frac{1}{2} \nabla_{x} \bar{\theta} \cdot\left(\tilde{\psi}+\tilde{\psi}_{*}-\tilde{\psi}^{\prime}-\tilde{\psi}_{*}^{\prime}\right) \\
& \mp \frac{1}{\sigma}(\bar{j}-\bar{n} \bar{u}) \cdot\left(\tilde{\Phi}+\tilde{\Phi}_{*}-\tilde{\Phi}^{\prime}-\tilde{\Phi}_{*}^{\prime}\right), \\
\bar{q}^{ \pm, \mp} & =\frac{1}{2} \nabla_{x} \bar{u}:\left(\tilde{\phi}+\tilde{\phi}_{*}-\tilde{\phi}^{\prime}-\tilde{\phi}_{*}^{\prime}\right)+\frac{1}{2} \nabla_{x} \bar{\theta} \cdot\left(\tilde{\psi}+\tilde{\psi}_{*}-\tilde{\psi}^{\prime}-\tilde{\psi}_{*}^{\prime}\right) \\
& \mp \frac{1}{\sigma}(\bar{j}-\bar{n} \bar{u}) \cdot\left(\tilde{\Phi}-\tilde{\Phi}_{*}-\tilde{\Phi}^{\prime}+\tilde{\Phi}_{*}^{\prime}\right),
\end{aligned}
$$

so that

$$
\begin{aligned}
\frac{1}{2} \int_{\mathbb{R}^{3} \times \mathbb{S}^{2}}\left(\bar{q}^{+}+\bar{q}^{-}+\bar{q}^{+,-}+\bar{q}^{-,+}\right) M_{*} d v_{*} d \sigma & =\nabla_{x} \bar{u}: \mathcal{L} \tilde{\phi}+\nabla_{x} \bar{\theta} \cdot \mathcal{L} \tilde{\psi} \\
& =\nabla_{x} \bar{u}: \phi+\nabla_{x} \bar{\theta} \cdot \psi \\
\int_{\mathbb{R}^{3} \times \mathbb{S}^{2}}\left(\bar{q}^{+}-\bar{q}^{-}\right) M_{*} d v_{*} d \sigma & =-\frac{2}{\sigma}(\bar{j}-\bar{n} \bar{u}) \cdot \mathcal{L}(\tilde{\Phi}), \\
\int_{\mathbb{R}^{3} \times \mathbb{S}^{2}}\left(\bar{q}^{+,-}-\bar{q}^{-,+}\right) M_{*} d v_{*} d \sigma & =-\frac{2}{\sigma}(\bar{j}-\bar{n} \bar{u}) \cdot \mathfrak{L}(\tilde{\Phi}), \\
\bar{q}^{+}+\bar{q}^{-}-\bar{q}^{+,-}-\bar{q}^{-,+} & =0,
\end{aligned}
$$

with $\phi, \psi, \tilde{\phi}$ and $\tilde{\psi}$ defined by 2.12 and 2.13 and $\tilde{\Phi}$ defined by 2.63 .

Then, assuming from now on that $\|\bar{u}\|_{L^{\infty}(d t d x)}<1$ and using the lower weak sequential semi-continuity of the entropies 12.48 and of the electromagnetic energy (3.24) together with Lemma 4.3 , we find that, for all $0 \leq t_{1}<t_{2}$,

$$
\int_{t_{1}}^{t_{2}} \delta \mathcal{E}(t) d t \leq \liminf _{\epsilon \rightarrow 0} \min \left\{\int_{t_{1}}^{t_{2}} \delta \mathcal{H}_{\epsilon}(t) d t, \int_{t_{1}}^{t_{2}} \delta \mathcal{E}_{\epsilon}(t) d t\right\},
$$

where

$$
\begin{aligned}
\delta \mathcal{E}(t) & =\frac{1}{2}\left\|g^{+}-\bar{g}^{+}\right\|_{L^{2}(M d x d v)}^{2}+\frac{1}{2}\left\|g^{-}-\bar{g}^{-}\right\|_{L^{2}(M d x d v)}^{2} \\
& +\frac{1}{2}\|E-\bar{E}\|_{L^{2}(d x)}^{2}+\frac{1}{2}\|B-\bar{B}\|_{L^{2}(d x)}^{2}-\int_{\mathbb{R}^{3}}\left(\left(E_{\epsilon}-\bar{E}\right) \wedge\left(B_{\epsilon}-\bar{B}\right)\right) \cdot \bar{u} d x \\
& =\|\rho-\bar{\rho}\|_{L^{2}(d x)}^{2}+\frac{1}{4}\|n-\bar{n}\|_{L^{2}(d x)}^{2}+\|u-\bar{u}\|_{L^{2}(d x)}^{2}+\overline{\frac{3}{2}}\|\theta-\bar{\theta}\|_{L^{2}(d x)}^{2} \\
& +\frac{1}{2}\|E-\bar{E}\|_{L^{2}(d x)}^{2}+\frac{1}{2}\|B-\bar{B}\|_{L^{2}(d x)}^{2}-\int_{\mathbb{R}^{3}}\left(\left(E_{\epsilon}-\bar{E}\right) \wedge\left(B_{\epsilon}-\bar{B}\right)\right) \cdot \bar{u} d x \\
& =\frac{1}{4}\|n-\bar{n}\|_{L^{2}(d x)}^{2}+\|u-\bar{u}\|_{L^{2}(d x)}^{2}+\frac{5}{2}\|\theta-\bar{\theta}\|_{L^{2}(d x)}^{2} \\
& +\frac{1}{2}\|E-\bar{E}\|_{L^{2}(d x)}^{2}+\frac{1}{2}\|B-\bar{B}\|_{L^{2}(d x)}^{2}-\int_{\mathbb{R}^{3}}\left(\left(E_{\epsilon}-\bar{E}\right) \wedge\left(B_{\epsilon}-\bar{B}\right)\right) \cdot \bar{u} d x
\end{aligned}
$$

while, repeating mutatis mutandis the computations leading to 8.47 and 8.49 in the proof of Proposition 8.6. we obtain, for all $0 \leq t_{1}<t_{2}$,

$$
\int_{t_{1}}^{t_{2}} \delta \mathcal{D}(t) d t \leq \liminf _{\epsilon \rightarrow 0} \int_{t_{1}}^{t_{2}} \delta \mathcal{D}_{\epsilon}(t) d t
$$

where

$$
\begin{aligned}
\delta \mathcal{D}(t) & =2 \mu\left\|\nabla_{x}(u-\bar{u})\right\|_{L_{x}^{2}}^{2}+5 \kappa\left\|\nabla_{x}(\theta-\bar{\theta})\right\|_{L_{x}^{2}}^{2}+\frac{1}{\sigma}\|(j-n u)-(\bar{j}-\bar{n} \bar{u})\|_{L_{x}^{2}}^{2} \\
& \leq \frac{1}{4}\left\|q^{+}-\bar{q}^{+}\right\|_{L^{2}\left(M M_{*} d x d v d v_{*} d \sigma\right)}^{2}+\frac{1}{4}\left\|q^{-}-\bar{q}^{-}\right\|_{L^{2}\left(M M_{*} d x d v d v_{*} d \sigma\right)}^{2} \\
& +\frac{1}{4}\left\|q^{+,-}-\bar{q}^{+,-}\right\|_{L^{2}\left(M M_{*} d x d v d v_{*} d \sigma\right)}^{2}+\frac{1}{4}\left\|q^{-,+}-\bar{q}^{-,+}\right\|_{L^{2}\left(M M_{*} d x d v d v_{*} d \sigma\right)}^{2} .
\end{aligned}
$$


The following lemma shows how the modulated entropy $\delta \mathcal{H}_{\epsilon}$ controls the modulated energy $\delta \mathcal{E}_{\epsilon}$ up to a small remainder. It is obtained by repeating the proof of Lemma 12.1 and, thus, we skip the details of its demonstration.

LEMMA 12.3. It holds that

$$
\delta \mathcal{E}_{\epsilon}(t) \leq C \delta \mathcal{H}_{\epsilon}(t)+o(1)_{L^{\infty}(d t)}
$$

for some fixed constant $C>1$.

The following result establishes the renormalized modulated entropy inequality at the order $\epsilon$, which will eventually allow us to deduce the crucial weak-strong stability of the limiting thermodynamic fields, thus defining dissipative solutions.

Proposition 12.4. One has the stability inequality

$$
\begin{aligned}
& \delta \mathcal{H}_{\epsilon}(t)+\frac{1}{2} \int_{0}^{t} \delta \mathcal{D}_{\epsilon}(s) e^{\int_{s}^{t} \lambda(\sigma) d \sigma} d s \\
& \leq \delta \mathcal{H}_{\epsilon}(0) e^{\int_{0}^{t} \lambda(s) d s} \\
& +\int_{0}^{t} \int_{\mathbb{R}^{3}} \mathbf{A} \cdot\left(\begin{array}{c}
\tilde{u}_{\epsilon}-\bar{u} \\
-\int_{\mathbb{R}^{3} \times \mathbb{R}^{3} \times \mathbb{S}^{2}} \sum_{ \pm \pm}\left( \pm \hat{q}_{\epsilon}^{ \pm} \pm \hat{q}_{\epsilon}^{ \pm}, \mp\right) \tilde{\Phi} M M_{*} d v d v_{*} d \sigma-(\bar{j}-\bar{n} \bar{u}) \\
E_{\epsilon}-\overline{\bar{E}}+\bar{u} \wedge\left(B_{\epsilon}-\bar{B}\right)-\frac{1}{2} \nabla_{x}\left(\tilde{n}_{\epsilon}-\bar{n}\right) \\
B_{\epsilon}-\bar{B}+\left(E_{\epsilon}-\bar{E}\right) \wedge \bar{u}
\end{array}\right)(s) d x \\
& \times e^{\int_{s}^{t} \lambda(\sigma) d \sigma} d s+o(1)_{L_{\mathrm{loc}}^{\infty}(d t),}
\end{aligned}
$$

where the acceleration operator is defined by

$$
\begin{aligned}
\mathbf{A}(\bar{u}, \bar{\theta}, \bar{n}, \bar{j}, \bar{E}, \bar{B}) & =\left(\begin{array}{l}
\mathbf{A}_{1} \\
\mathbf{A}_{2} \\
\mathbf{A}_{3} \\
\mathbf{A}_{4} \\
\mathbf{A}_{5}
\end{array}\right) \\
& =\left(\begin{array}{c}
-2\left(\partial_{t} \bar{u}+P\left(\bar{u} \cdot \nabla_{x} \bar{u}\right)-\mu \Delta_{x} \bar{u}\right)+P(\bar{n} \bar{E}+\bar{j} \wedge \bar{B}) \\
-2\left(\partial_{t} \bar{\theta}+\bar{u} \cdot \nabla_{x} \bar{\theta}-\kappa \Delta_{x} \bar{\theta}\right) \\
-\frac{1}{\sigma}(\bar{j}-\bar{n} \bar{u})-\frac{1}{2} \nabla_{x} \bar{n}+\bar{E}+\bar{u} \wedge \bar{B} \\
-\left(\partial_{t} \bar{E}-\operatorname{rot} \bar{B}+\bar{j}\right) \\
-\left(\partial_{t} \bar{B}+\operatorname{rot} \bar{E}\right)
\end{array}\right),
\end{aligned}
$$

and the growth rate is given by

$$
\begin{aligned}
& \lambda(t)= \\
& C\left(\frac{\|\bar{u}(t)\|_{W^{1, \infty}(d x)}+\left\|\partial_{t} \bar{u}(t)\right\|_{L^{\infty}(d x)}+\|\bar{\theta}(t)\|_{W^{1, \infty}(d x)}+\|(\bar{j}-\bar{n} \bar{u})(t)\|_{L^{\infty}(d x)}}{1-\|\bar{u}(t)\|_{L^{\infty}(d x)}}\right. \\
& \left.\quad+\|\bar{\theta}(t)\|_{W^{1, \infty}(d x)}^{2}+\left\|\left(\frac{1}{2} \nabla_{x} \bar{n}-\bar{E}-\bar{u} \wedge \bar{B}\right)(t)\right\|_{L^{\infty}(d x)}\right),
\end{aligned}
$$

with a constant $C>0$ independent of test functions and $\epsilon$.

Proof. The main ingredients of the proof of this stability inequality are : 
- The scaled entropy inequality 4.35

$$
\begin{aligned}
& \frac{1}{\epsilon^{2}} H\left(f_{\epsilon}^{+}\right)+\frac{1}{\epsilon^{2}} H\left(f_{\epsilon}^{-}\right)+\frac{1}{2 \epsilon^{2}} \int_{\mathbb{R}^{3}} \operatorname{Tr} m_{\epsilon} d x+\frac{1}{2} \int_{\mathbb{R}^{3}}\left(\left|E_{\epsilon}\right|^{2}+\left|B_{\epsilon}\right|^{2}+\operatorname{Tr} a_{\epsilon}\right) d x \\
& \quad+\frac{1}{4} \int_{0}^{t} \int_{\mathbb{R}^{3} \times \mathbb{R}^{3} \times \mathbb{R}^{3} \times \mathbb{S}^{2}}\left(\left(\hat{q}_{\epsilon}^{+}\right)^{2}+\left(\hat{q}_{\epsilon}^{-}\right)^{2}+\left(\hat{q}_{\epsilon}^{+,-}\right)^{2}+\left(\hat{q}_{\epsilon}^{-,},+\right)^{2}\right) M M_{*} d x d v d v_{*} d \sigma d s \\
& \quad \leq \frac{1}{\epsilon^{2}} H\left(f_{\epsilon}^{+\mathrm{in}}\right)+\frac{1}{\epsilon^{2}} H\left(f_{\epsilon}^{-\mathrm{in}}\right)+\frac{1}{2} \int_{\mathbb{R}^{3}}\left(\left|E_{\epsilon}^{\mathrm{in}}\right|^{2}+\left|B_{\epsilon}^{\mathrm{in}}\right|^{2}\right) d x
\end{aligned}
$$

which is naturally satisfied by renormalized solutions of the scaled two species Vlasov-Maxwell-Boltzmann system (4.34) (provided they exist) and where we have used the inequality (5.7) from Lemma 5.3 in order to conveniently simplify the dissipation terms.

- The approximate conservation of energy obtained in Proposition 9.5

$$
\partial_{t}\left(\frac{3}{2} \tilde{\theta}_{\epsilon}-\tilde{\rho}_{\epsilon}\right)+\nabla_{x} \cdot\left(\frac{5}{2} \tilde{u}_{\epsilon} \tilde{\theta}_{\epsilon}-\int_{\mathbb{R}^{3} \times \mathbb{R}^{3} \times \mathbb{S}^{2}} \frac{\hat{q}_{\epsilon}^{+}+\hat{q}_{\epsilon}^{-}}{2} \tilde{\psi} M M_{*} d v d v_{*} d \sigma\right)
$$

where the remainder $R_{\epsilon, 1}$ satisfies

$$
\left\|R_{\epsilon, 1}\right\|_{W_{\mathrm{loc}}^{-1,1}(d x)} \leq \frac{C \delta \mathcal{E}_{\epsilon}(t)}{1-\|\bar{u}\|_{L^{\infty}(d x)}}+C\left(\delta \mathcal{E}_{\epsilon}(t) \delta \mathcal{D}_{\epsilon}(t)\right)^{\frac{1}{2}}+o(1)_{L_{\mathrm{loc}}^{1}(d t)},
$$

for some $C>0$, where we have used 12.26. Note that we do not employ the approximate conservation of momentum from Proposition 9.5 .

- The approximate conservation of momentum law from Proposition 9.6

$$
\begin{aligned}
& \partial_{t}\left(\tilde{u}_{\epsilon}+\frac{1}{2} E_{\epsilon} \wedge B_{\epsilon}+\frac{1}{2}\left(\begin{array}{c}
a_{\epsilon 26}-a_{\epsilon 35} \\
a_{\epsilon 34}-a_{\epsilon 16} \\
a_{\epsilon 15}-a_{\epsilon 24}
\end{array}\right)\right) \\
& \quad+\nabla_{x} \cdot\left(\tilde{u}_{\epsilon} \otimes \tilde{u}_{\epsilon}-\frac{\left|\tilde{u}_{\epsilon}\right|^{2}}{3} \mathrm{Id}+\frac{1}{2 \epsilon^{2}} m_{\epsilon}-\int_{\mathbb{R}^{3} \times \mathbb{R}^{3} \times \mathbb{S}^{2}} \frac{\hat{q}_{\epsilon}^{+}+\hat{q}_{\epsilon}^{-}}{2} \tilde{\phi} M M_{*} d v d v_{*} d \sigma\right) \\
& \quad-\frac{1}{2} \nabla_{x} \cdot\left(E_{\epsilon} \otimes E_{\epsilon}+e_{\epsilon}+B_{\epsilon} \otimes B_{\epsilon}+b_{\epsilon}\right)+\nabla_{x}\left(\frac{\left|E_{\epsilon}\right|^{2}+\left|B_{\epsilon}\right|^{2}+\operatorname{Tr} a_{\epsilon}}{4}\right) \\
& \quad=-\frac{1}{\epsilon} \nabla_{x}\left(\tilde{\rho}_{\epsilon}+\tilde{\theta}_{\epsilon}\right)+\partial_{t}\left(R_{\epsilon, 2}\right)+R_{\epsilon, 3},
\end{aligned}
$$

where the remainders $R_{\epsilon, 2}$ and $R_{\epsilon, 3}$ satisfy

$$
\begin{aligned}
R_{\epsilon, 2} & =o(1)_{L^{\infty}\left(d t ; L_{\mathrm{loc}}^{1}(d x)\right)} \\
\left\|R_{\epsilon, 3}\right\|_{W_{\mathrm{loc}}^{-1,1}(d x)} & \leq C_{1} \delta \mathcal{H}_{\epsilon}(t)+C_{2} \delta \mathcal{E}_{\epsilon}(t)+o(1)_{L_{\mathrm{loc}}^{1}(d t)},
\end{aligned}
$$

for some $C_{1}, C_{2}>0$.

- The approximate Ohm's law

$$
\begin{aligned}
\frac{1}{\sigma} \int_{\mathbb{R}^{3} \times \mathbb{R}^{3} \times \mathbb{S}^{2}}\left(\hat{q}_{\epsilon}^{+}-\hat{q}_{\epsilon}^{-}+\hat{q}_{\epsilon}^{+,-}\right. & \left.-\hat{q}_{\epsilon}^{-,+}\right) \tilde{\Phi} M M_{*} d v d v_{*} d \sigma \\
& =\frac{1}{2} \nabla_{x} \tilde{n}_{\epsilon}-\left(E_{\epsilon}+\tilde{u}_{\epsilon} \wedge B_{\epsilon}\right)+R_{\epsilon, 4}+\nabla_{x} R_{\epsilon, 5}+R_{\epsilon, 6},
\end{aligned}
$$

where $\sigma>0$ is defined by 2.65 and the remainders $R_{\epsilon, 4}$ and $R_{\epsilon, 5}$ vanish weakly

$$
R_{\epsilon, 4}=o(1)_{w-L_{\mathrm{loc}}^{1}(d t d x)} \quad \text { and } \quad R_{\epsilon, 5}=o(1)_{w-L_{\mathrm{loc}}^{1}(d t d x)},
$$


whereas $R_{\epsilon, 6}$ satisfies

$$
\left\|R_{\epsilon, 6}\right\|_{L^{1}(d x)} \leq \frac{C \delta \mathcal{E}_{\epsilon}(t)}{1-\|\bar{u}\|_{L^{\infty}(d x)}}+o(1)_{w-L_{\mathrm{loc}}^{1}(d t)} .
$$

This approximate law is obtained directly from the limiting laws derived in Proposition 8.3. Indeed, it is easily deduced from 8.32 that 12.58 holds with the remainders

$$
\begin{aligned}
R_{\epsilon, 4} & =\frac{1}{\sigma} \int_{\mathbb{R}^{3} \times \mathbb{R}^{3} \times \mathbb{S}^{2}}\left(\hat{q}_{\epsilon}^{+}-\hat{q}_{\epsilon}^{-}+\hat{q}_{\epsilon}^{+,-}-\hat{q}_{\epsilon}^{-,+}\right) \tilde{\Phi} M M_{*} d v d v_{*} d \sigma \\
& -\frac{1}{\sigma} \int_{\mathbb{R}^{3} \times \mathbb{R}^{3} \times \mathbb{S}^{2}}\left(q^{+}-q^{-}+q^{+,-}-q^{-,+}\right) \tilde{\Phi} M M_{*} d v d v_{*} d \sigma \\
& +\left(E_{\epsilon}-E\right)+\left(\tilde{u}_{\epsilon}-u\right) \wedge B+u \wedge\left(B_{\epsilon}-B\right), \\
R_{\epsilon, 5} & =-\frac{1}{2}\left(\tilde{n}_{\epsilon}-n\right), \\
R_{\epsilon, 6} & =\left(\tilde{u}_{\epsilon}-u\right) \wedge\left(B_{\epsilon}-B\right)=\frac{O\left(\delta \mathcal{E}_{\epsilon}(t)+\delta \mathcal{E}(t)\right)_{L^{1}(d x)}}{1-\|\bar{u}\|_{L^{\infty}(d x)}},
\end{aligned}
$$

where we have used 12.26 . The above estimate on $R_{\epsilon, 6}$ is then readily improved to 12.60 upon noticing from 12.50 that

$$
\delta \mathcal{E}(t) \leq \delta \mathcal{E}_{0}(t)
$$

where $\delta \mathcal{E}_{0}(t)$ is the limit, up to extraction of subsequences, of $\delta \mathcal{E}_{\epsilon}(t)$ in $w^{*}-L^{\infty}(d t)$, and then writing

$$
\begin{aligned}
\delta \mathcal{E}_{\epsilon}(t)+\delta \mathcal{E}(t) & \leq 2 \delta \mathcal{E}_{\epsilon}(t)+\delta \mathcal{E}_{0}(t)-\delta \mathcal{E}_{\epsilon}(t) \\
& =2 \delta \mathcal{E}_{\epsilon}(t)+o(1)_{w^{*}-L^{\infty}(d t)}
\end{aligned}
$$

As in the case of the approximate solenoidal Ohm's law 12.23 for weak interspecies interactions, it would be possible to derive the above approximate Ohm's law employing the methods of proof of Proposition 9.5. Nevertheless, the method presented here is more robust.

- Maxwell's equations

$$
\left\{\begin{aligned}
\partial_{t} E_{\epsilon}-\operatorname{rot} B_{\epsilon} & =-j_{\epsilon}=-\tilde{j}_{\epsilon}+R_{\epsilon, 7} \\
\partial_{t} B_{\epsilon}+\operatorname{rot} E_{\epsilon} & =0 \\
\operatorname{div} E_{\epsilon} & =n_{\epsilon}=\tilde{n}_{\epsilon}-R_{\epsilon, 8} \\
\operatorname{div} B_{\epsilon} & =0
\end{aligned}\right.
$$

where the remainders $R_{\epsilon, 7}=\tilde{j}_{\epsilon}-j_{\epsilon}$ and $R_{\epsilon, 8}=\tilde{n}_{\epsilon}-n_{\epsilon}$ satisfy

$$
\left\|R_{\epsilon, 7}\right\|_{L_{\mathrm{loc}}^{1}(d x)} \leq C \delta \mathcal{H}_{\epsilon}(t)+o(1)_{L_{\mathrm{loc}}^{1}(d t)},
$$

and

$$
R_{\epsilon, 8}=o(1)_{L^{\infty}\left(d t ; L_{\mathrm{loc}}^{1}(d x)\right)}
$$

The convergence $(12.63)$ straightforwardly follows from 9.62 . As for the control 12.62 , it is obtained through the following estimate. First, since $G_{\epsilon}^{ \pm} \geq 2$ and $\epsilon \hat{g}_{\epsilon}^{ \pm} \geq 2(\sqrt{2}-1)$ on the support of $1-\gamma_{\epsilon}^{ \pm}$, we easily 
deduce, using Lemma 9.9 , that

$$
\begin{aligned}
\left\|\frac{1}{\epsilon} g_{\epsilon}^{ \pm}\left(1-\gamma_{\epsilon}^{ \pm}\right)\right\|_{L_{\mathrm{loc}}^{1}\left(d x ; L^{1}\left((1+|v|)^{2} M d v\right)\right)} & \left\|\frac{1}{\epsilon}\left(\hat{g}_{\epsilon}^{ \pm}+\frac{\epsilon}{4} \hat{g}_{\epsilon}^{ \pm 2}\right)\left(1-\gamma_{\epsilon}^{ \pm}\right)\right\|_{L_{\mathrm{loc}}^{1}\left(d x ; L^{1}\left((1+|v|)^{2} M d v\right)\right)} \\
& \leq C\left\|\mathbb{1}_{\left\{G_{\epsilon}^{ \pm} \geq 2\right\}} \hat{g}_{\epsilon}^{ \pm}\right\|_{L_{\mathrm{loc}}^{2}\left(d x ; L^{2}\left((1+|v|)^{2} M d v\right)\right)}^{2} \\
& \leq C_{1} \int_{\mathbb{R}^{3} \times \mathbb{R}^{3}}\left(\frac{1}{\epsilon^{2}} h\left(\epsilon g_{\epsilon}^{ \pm}\right)-\frac{1}{2}\left(g_{\epsilon}^{ \pm} \gamma_{\epsilon}^{ \pm} \chi\left(\frac{|v|^{2}}{K_{\epsilon}}\right)\right)^{2}\right) M d x d v \\
& +C_{2}\left\|g_{\epsilon}^{ \pm} \gamma_{\epsilon}^{ \pm} \chi\left(\frac{|v|^{2}}{K_{\epsilon}}\right)-\bar{g}^{ \pm}\right\|_{L^{2}(M d x d v)}^{2}+o(1)_{L_{\mathrm{loc}}^{1}(d t)} \\
& \leq C_{1} \delta \mathcal{H}_{\epsilon}(t)+C_{2} \delta \mathcal{E}_{\epsilon}(t)+o(1)_{L_{\mathrm{loc}}^{1}(d t)} .
\end{aligned}
$$

Furthermore, using the Gaussian decay 8.8 and that $g_{\epsilon}^{ \pm} \gamma_{\epsilon}^{ \pm}$is comparable to $\hat{g}_{\epsilon}$, we also obtain

$$
\begin{aligned}
\| \frac{1}{\epsilon} g_{\epsilon}^{ \pm} \gamma_{\epsilon}^{ \pm}\left(1-\chi\left(\frac{|v|^{2}}{K_{\epsilon}}\right)\right) & \|_{L^{1}\left((1+|v|)^{2} M d v\right)} \\
& \leq C\left\|\frac{1}{\epsilon} \hat{g}_{\epsilon}^{ \pm}\left(1-\chi\left(\frac{|v|^{2}}{K_{\epsilon}}\right)\right)\right\|_{L^{1}\left((1+|v|)^{2} M d v\right)} \\
& \leq C\left\|\hat{g}_{\epsilon}^{ \pm}\right\|_{L^{2}(M d v)}\left\|\frac{1}{\epsilon}\left(1-\chi\left(\frac{|v|^{2}}{K_{\epsilon}}\right)\right)\right\|_{L^{2}\left((1+|v|)^{4} M d v\right)} \\
& =O\left(K^{\frac{5}{4}}|\log \epsilon|^{\frac{5}{4}} \epsilon^{\frac{K}{4}-1}\right)_{L^{\infty}\left(d t ; L^{2}(d x)\right)} \cdot
\end{aligned}
$$

Thus, further using Lemma 12.3 , we infer, provided $K>4$, that

$$
\frac{1}{\epsilon}\left\|g_{\epsilon}^{ \pm}-g_{\epsilon}^{ \pm} \gamma_{\epsilon}^{ \pm} \chi\left(\frac{|v|^{2}}{K_{\epsilon}}\right)\right\|_{L_{\mathrm{loc}}^{1}\left(d x ; L^{1}\left((1+|v|)^{2} M d v\right)\right)} \leq C \delta \mathcal{H}_{\epsilon}(t)+o(1)_{L_{\mathrm{loc}}^{1}(d t)},
$$

whence

$$
\left\|\tilde{j}_{\epsilon}-j_{\epsilon}\right\|_{L_{\mathrm{loc}}^{1}(d x)} \leq C \delta \mathcal{H}_{\epsilon}(t)+o(1)_{L_{\mathrm{loc}}^{1}(d t)},
$$

which establishes 12.62 .

Notice that we cannot rigorously write the identities 1.10 and 1.12 for the above system, because the source terms $j_{\epsilon}$ and $n_{\epsilon}$ do not belong to $L_{\text {loc }}^{2}(d t d x)$ a priori. Nevertheless, one has the following modulated identities :

$$
\begin{aligned}
\partial_{t}\left(E_{\epsilon} \cdot \bar{E}+B_{\epsilon} \cdot \bar{B}\right) & +\nabla_{x} \cdot\left(E_{\epsilon} \wedge \bar{B}+\bar{E} \wedge B_{\epsilon}\right) \\
& =-\left(\tilde{j}_{\epsilon}-R_{\epsilon, 7}\right) \cdot \bar{E}-\left(\bar{j}+\mathbf{A}_{4}\right) \cdot E_{\epsilon}-\mathbf{A}_{5} \cdot B_{\epsilon},
\end{aligned}
$$

and

$$
\begin{aligned}
\partial_{t} & \left(\left(E_{\epsilon}-\bar{E}\right) \wedge\left(B_{\epsilon}-\bar{B}\right)\right)+\frac{1}{2} \nabla_{x}\left(\left|E_{\epsilon}-\bar{E}\right|^{2}+\left|B_{\epsilon}-\bar{B}\right|^{2}\right) \\
& -\nabla_{x} \cdot\left(\left(E_{\epsilon}-\bar{E}\right) \otimes\left(E_{\epsilon}-\bar{E}\right)+\left(B_{\epsilon}-\bar{B}\right) \otimes\left(B_{\epsilon}-\bar{B}\right)\right) \\
& =\partial_{t}\left(E_{\epsilon} \wedge B_{\epsilon}\right)+\frac{1}{2} \nabla_{x}\left(\left|E_{\epsilon}\right|^{2}+\left|B_{\epsilon}\right|^{2}\right)-\nabla_{x} \cdot\left(E_{\epsilon} \otimes E_{\epsilon}+B_{\epsilon} \otimes B_{\epsilon}\right) \\
& +\left(\bar{j}+\mathbf{A}_{4}\right) \wedge\left(B_{\epsilon}-\bar{B}\right)+\left(E_{\epsilon}-\bar{E}\right) \wedge \mathbf{A}_{5} \\
& +\left(\tilde{j}_{\epsilon}-R_{\epsilon, 7}\right) \wedge \bar{B}+\left(\tilde{n}_{\epsilon}-R_{\epsilon, 8}\right) \bar{E}+\bar{n}\left(E_{\epsilon}-\bar{E}\right) .
\end{aligned}
$$


Finally, taking the divergence of the approximate Ampère equation from 12.61, we obtain the approximate conservation of charge (or approximate continuity equation)

$$
\partial_{t} \tilde{n}_{\epsilon}+\nabla_{x} \cdot \tilde{j}_{\epsilon}=\partial_{t} R_{\epsilon, 8}+\nabla_{x} \cdot R_{\epsilon, 7} .
$$

Note that we could just as well use the approximate conservation of charge from Proposition 9.5 .

- The asymptotic characterization (8.31) of the limiting collision integrands from Proposition 8.3 combined with 8.40 from Proposition 8.4, which implies that

$$
\begin{array}{r}
\left(\int_{\mathbb{R}^{3} \times \mathbb{R}^{3} \times \mathbb{S}^{2}} \frac{\hat{q}_{\epsilon}^{+}+\hat{q}_{\epsilon}^{-}}{2} \tilde{\phi} M M_{*} d v d v_{*} d \sigma\right)-\mu\left(\nabla_{x} \tilde{u}_{\epsilon}+\nabla_{x}^{t} \tilde{u}_{\epsilon}\right) \\
\left(\int_{\mathbb{R}^{3} \times \mathbb{R}^{3} \times \mathbb{S}^{2}} \frac{\hat{q}_{\epsilon}^{+}+\hat{q}_{\epsilon}^{-}}{2} \tilde{\psi} M M_{*} d v d v_{*} d \sigma\right)-\frac{5}{2} \kappa \nabla_{x} \tilde{\theta}_{\epsilon} \rightarrow 0, \\
\hat{q}_{\epsilon}^{+}+\hat{q}_{\epsilon}^{-}-\hat{q}_{\epsilon}^{+,-}-\hat{q}_{\epsilon}^{-,+} \rightarrow 0,
\end{array}
$$

in the sense of distributions, where $\mu, \kappa>0$ are defined by $(2.56)$.

- The asymptotic characterizations 8.38 and 8.39 of the limiting collision integrands from Proposition 8.4. whose proofs imply that

$$
\int_{\mathbb{R}^{3} \times \mathbb{R}^{3} \times \mathbb{S}^{2}}\left(\hat{q}_{\epsilon}^{+}-\hat{q}_{\epsilon}^{-}+\hat{q}_{\epsilon}^{+,-}-\hat{q}_{\epsilon}^{-,+}\right) \tilde{\Phi} M M_{*} d v d v_{*} d \sigma+\tilde{j}_{\epsilon}-\tilde{n}_{\epsilon} \tilde{u}_{\epsilon}=R_{\epsilon, 9},
$$

where the remainder $R_{\epsilon, 9}$ satisfies

$$
\left\|R_{\epsilon, 9}\right\|_{L_{\mathrm{loc}}^{1}(d x)} \leq C \delta \mathcal{H}_{\epsilon}(t)+o(1)_{L_{\mathrm{loc}}^{1}(d t)} .
$$

Indeed, we first obtain from (8.41), using Lemmas 9.10 and 12.3 , that

$$
\begin{aligned}
& \left\|h_{\epsilon}-\hat{h}_{\epsilon}-\frac{1}{4} \hat{n}_{\epsilon}\left(\hat{g}_{\epsilon}^{+}-\hat{\rho}_{\epsilon}^{+}+\hat{g}_{\epsilon}^{-}-\hat{\rho}_{\epsilon}^{-}\right)\right\|_{L_{\mathrm{loc}}^{1}\left(d x ; L^{1}\left(\left(1+|v|^{2}\right) M d v\right)\right)} \\
& \leq C \delta \mathcal{H}_{\epsilon}(t)+o(1)_{L_{\text {loc }}^{1}(d t)},
\end{aligned}
$$

where $\hat{h}_{\epsilon}=\frac{1}{\epsilon}\left[\left(\hat{g}_{\epsilon}^{+}-\hat{g}_{\epsilon}^{-}\right)-\hat{n}_{\epsilon}\right], \hat{n}_{\epsilon}=\hat{\rho}_{\epsilon}^{+}-\hat{\rho}_{\epsilon}^{-}$and $\hat{\rho}_{\epsilon}^{ \pm}$are the densities associated with the fluctuations $\hat{g}_{\epsilon}^{ \pm}$. Next, combining 8.44 with 8.45), straightforward computations yield that

$$
\begin{aligned}
& (\mathcal{L}+\mathfrak{L})\left(h_{\epsilon}-\frac{1}{2} \hat{n}_{\epsilon}\left(\hat{g}_{\epsilon}^{+}+\hat{g}_{\epsilon}^{-}\right)\right)+\int_{\mathbb{R}^{3} \times \mathbb{S}^{2}}\left(\hat{q}_{\epsilon}^{+}-\hat{q}_{\epsilon}^{-}+\hat{q}_{\epsilon}^{+,-}-\hat{q}_{\epsilon}^{-,+}\right) M_{*} d v_{*} d \sigma \\
& =\frac{1}{2} \mathcal{Q}\left(\hat{g}_{\epsilon}^{+}-\hat{g}_{\epsilon}^{-}-\hat{n}_{\epsilon}, \hat{g}_{\epsilon}^{+}+\hat{g}_{\epsilon}^{-}\right)+(\mathcal{L}+\mathfrak{L})\left(h_{\epsilon}-\hat{h}_{\epsilon}-\frac{1}{4} \hat{n}_{\epsilon}\left(\hat{g}_{\epsilon}^{+}-\hat{\rho}_{\epsilon}^{+}+\hat{g}_{\epsilon}^{-}-\hat{\rho}_{\epsilon}^{-}\right)\right) \\
& -\frac{1}{2} \hat{n}_{\epsilon} \mathcal{L}\left(\hat{g}_{\epsilon}^{+}+\hat{g}_{\epsilon}^{-}-\Pi\left(\hat{g}_{\epsilon}^{+}+\hat{g}_{\epsilon}^{-}\right)\right),
\end{aligned}
$$

which implies, using $(2.63)$ and the self-adjointness of $\mathcal{L}+\mathfrak{L}$ and then employing Lemmas 9.7, 9.8 (on consistency estimates) and 12.3 (allowing to control the energy by the entropy) with the estimates (5.14) and (12.71), that

$$
\begin{aligned}
\left\|j_{\epsilon}-\hat{n}_{\epsilon} \hat{u}_{\epsilon}+\int_{\mathbb{R}^{3} \times \mathbb{R}^{3} \times \mathbb{S}^{2}}\left(\hat{q}_{\epsilon}^{+}-\hat{q}_{\epsilon}^{-}+\hat{q}_{\epsilon}^{+,-}-\hat{q}_{\epsilon}^{-,+}\right) \tilde{\Phi} M M_{*} d v d v_{*} d \sigma\right\|_{L_{\mathrm{loc}}^{1}(d x)} \\
\leq C \delta \mathcal{H}_{\epsilon}(t)+o(1)_{L_{\mathrm{loc}}^{1}(d t)},
\end{aligned}
$$

where $\hat{u}_{\epsilon}=\frac{1}{2} \int_{\mathbb{R}^{3}}\left(\hat{g}_{\epsilon}^{+}+\hat{g}_{\epsilon}^{-}\right) v M d v$. Finally, utilizing the control 12.64 with yet another application of Lemma 9.7 allows us to deduce the validity of 12.69 from the preceding estimate. 
Now, by definition of the acceleration operator A, straightforward energy computations, similar to those performed in the proof of Proposition 3.3, applied to the test functions $(\bar{u}, \bar{\theta}, \bar{n}, \bar{j}, \bar{E}, \bar{B})$, show that the following energy identity holds :

$$
\frac{d}{d t} \overline{\mathcal{E}}(t)+\overline{\mathcal{D}}(t)=-\int_{\mathbb{R}^{3}} \mathbf{A} \cdot\left(\begin{array}{c}
\bar{u} \\
\overline{\frac{5}{2}} \bar{\theta} \\
\bar{j}-\bar{n} \bar{u} \\
\bar{E}-\frac{1}{2} \nabla_{x} \bar{n} \\
\bar{B}
\end{array}\right) d x,
$$

where the energy $\overline{\mathcal{E}}$ and energy dissipation $\overline{\mathcal{D}}$ are defined by

$$
\begin{aligned}
\overline{\mathcal{E}}(t) & =\frac{1}{2}\left\|\bar{g}^{+}\right\|_{L^{2}(M d x d v)}^{2}+\frac{1}{2}\left\|\bar{g}^{-}\right\|_{L^{2}(M d x d v)}^{2}+\frac{1}{2}\|\bar{E}\|_{L^{2}(d x)}^{2}+\frac{1}{2}\|\bar{B}\|_{L^{2}(d x)}^{2} \\
& =\|\bar{\rho}\|_{L^{2}(d x)}^{2}+\frac{1}{4}\|\bar{n}\|_{L^{2}(d x)}^{2}+\|\bar{u}\|_{L^{2}(d x)}^{2}+\frac{3}{2}\|\bar{\theta}\|_{L^{2}(d x)}^{2} \\
& +\frac{1}{2}\|\bar{E}\|_{L^{2}(d x)}^{2}+\frac{1}{2}\|\bar{B}\|_{L^{2}(d x)}^{2}, \\
& =\frac{1}{4}\|\bar{n}\|_{L^{2}(d x)}^{2}+\|\bar{u}\|_{L^{2}(d x)}^{2}+\frac{5}{2}\|\bar{\theta}\|_{L^{2}(d x)}^{2}+\frac{1}{2}\|\bar{E}\|_{L^{2}(d x)}^{2}+\frac{1}{2}\|\bar{B}\|_{L^{2}(d x)}^{2},
\end{aligned}
$$

and

$$
\begin{aligned}
\overline{\mathcal{D}}(t) & =2 \mu\left\|\nabla_{x} \bar{u}\right\|_{L_{x}^{2}}^{2}+5 \kappa\left\|\nabla_{x} \bar{\theta}\right\|_{L_{x}^{2}}^{2}+\frac{1}{\sigma}\|\bar{j}-\bar{n} \bar{u}\|_{L_{x}^{2}}^{2} \\
& =\frac{1}{16}\left\|\bar{q}^{+}+\bar{q}^{-}+\bar{q}^{+,-}+\bar{q}^{-,+}\right\|_{L^{2}\left(M M_{*} d x d v d v_{*} d \sigma\right)}^{2} \\
& +\frac{1}{8}\left\|\bar{q}^{+}-\bar{q}^{-}\right\|_{L^{2}\left(M M_{*} d x d v d v_{*} d \sigma\right)}^{2}+\frac{1}{8}\left\|\bar{q}^{+,-}-\bar{q}^{-,+}\right\|_{L^{2}\left(M M_{*} d x d v d v_{*} d \sigma\right)}^{2} \\
& =\frac{1}{4}\left\|\bar{q}^{+}\right\|_{L^{2}\left(M M_{*} d x d v d v_{*} d \sigma\right)}^{2}+\frac{1}{4}\left\|\bar{q}^{-}\right\|_{L^{2}\left(M M_{*} d x d v d v_{*} d \sigma\right)}^{2} \\
& +\frac{1}{4}\left\|\bar{q}^{+,-}\right\|_{L^{2}\left(M M_{*} d x d v d v_{*} d \sigma\right)}^{2}+\frac{1}{4}\left\|\bar{q}^{-,+}\right\|_{L^{2}\left(M M_{*} d x d v d v_{*} d \sigma\right)}^{2} .
\end{aligned}
$$

Next, notice that a slight variant of the estimate 12.35 derived in the proof of Proposition 12.2 on weak interactions is also valid here in the case of strong interactions. Indeed, reproducing the very same duality computations preceding 12.35 onto the approximate conservation of energy 12.54 and, then, using the convergences 12.42, 12.43, 12.68), the estimate 12.55) and Lemma 12.3 (allowing to control the energy by the entropy), yields that (12.73)

$$
\begin{aligned}
\frac{d}{d t} \int_{\mathbb{R}^{3}} & \left(\frac{3}{2} \tilde{\theta}_{\epsilon}-\tilde{\rho}_{\epsilon}\right) \cdot \bar{\theta} d x \\
& +\frac{1}{2} \int_{\mathbb{R}^{3}}\left(\int_{\mathbb{R}^{3} \times \mathbb{R}^{3} \times \mathbb{S}^{2}}\left(\hat{q}_{\epsilon}^{+}+\hat{q}_{\epsilon}^{-}+\hat{q}_{\epsilon}^{+,-}+\hat{q}_{\epsilon}^{-,+}\right) \tilde{\psi} M M_{*} d v d v_{*} d \sigma\right) \cdot \nabla_{x} \bar{\theta} d x \\
& \geq-C\|\bar{\theta}\|_{W^{1, \infty}(d x)}\left(\frac{\delta \mathcal{E}_{\epsilon}(t)}{1-\|\bar{u}\|_{L^{\infty}(d x)}}+\left(\delta \mathcal{E}_{\epsilon}(t) \delta \mathcal{D}_{\epsilon}(t)\right)^{\frac{1}{2}}\right) \\
& -\frac{1}{2} \int_{\mathbb{R}^{3}} \mathbf{A}_{2}\left(\frac{3}{2} \tilde{\theta}_{\epsilon}-\tilde{\rho}_{\epsilon}\right) d x+o(1)_{w-L_{\mathrm{loc}}^{1}(d t)} \\
& \geq-C\left(\frac{\|\bar{\theta}\|_{W^{1, \infty}(d x)}}{1-\|\bar{u}\|_{L^{\infty}(d x)}}+\|\bar{\theta}\|_{W^{1, \infty}(d x)}^{2}\right) \delta \mathcal{H}_{\epsilon}(t)-\frac{1}{4} \delta \mathcal{D}_{\epsilon}(t) \\
& -\frac{1}{2} \int_{\mathbb{R}^{3}} \mathbf{A}_{2}\left(\frac{3}{2} \tilde{\theta}_{\epsilon}-\tilde{\rho}_{\epsilon}\right) d x+o(1)_{w-L_{\mathrm{loc}}^{1}(d t)} .
\end{aligned}
$$


Likewise, following the proof of Proposition 12.2, using the solenoidal property $\operatorname{div} \bar{u}=0$, analogous duality computations applied to the approximate conservations of momentum (12.56) and charge 12.67) yield that

$$
\begin{aligned}
& \frac{d}{d t} \int_{\mathbb{R}^{3}}\left(\frac{1}{4} \tilde{n}_{\epsilon} \bar{n}+\tilde{u}_{\epsilon} \cdot \bar{u}+\frac{1}{2}\left(E_{\epsilon} \wedge B_{\epsilon}\right) \cdot \bar{u}\right. \\
& \left.+\frac{1}{2}\left(\begin{array}{c}
a_{\epsilon 26}-a_{\epsilon 35} \\
a_{\epsilon 34}-a_{\epsilon 16} \\
a_{\epsilon 15}-a_{\epsilon 24}
\end{array}\right) \cdot \bar{u}-R_{\epsilon, 2} \cdot \bar{u}-\frac{1}{4} R_{\epsilon, 8} \bar{n}\right) d x \\
& -\int_{\mathbb{R}^{3}}\left(\frac{1}{2}\left(E_{\epsilon} \wedge B_{\epsilon}\right) \cdot \partial_{t} \bar{u}+\frac{1}{2}\left(\begin{array}{l}
a_{\epsilon 26}-a_{\epsilon 35} \\
a_{\epsilon 34}-a_{\epsilon 16} \\
a_{\epsilon 15}-a_{\epsilon 24}
\end{array}\right) \cdot \partial_{t} \bar{u}-R_{\epsilon, 2} \cdot \partial_{t} \bar{u}-\frac{1}{4} R_{\epsilon, 8} \partial_{t} \bar{n}\right) d x \\
& +\int_{\mathbb{R}^{3}} \frac{1}{4}\left(\tilde{n}_{\epsilon} \nabla_{x} \cdot \bar{j}-\tilde{j}_{\epsilon} \nabla_{x} \bar{n}\right)+\left(\left(P \tilde{u}_{\epsilon}\right) \otimes \bar{u}-\tilde{u}_{\epsilon} \otimes \tilde{u}_{\epsilon}-\frac{1}{2 \epsilon^{2}} m_{\epsilon}\right): \nabla_{x} \bar{u} d x \\
& +\int_{\mathbb{R}^{3}}\left(\int_{\mathbb{R}^{3} \times \mathbb{R}^{3} \times \mathbb{S}^{2}} \frac{\hat{q}_{\epsilon}^{+}+\hat{q}_{\epsilon}^{-}}{2} \tilde{\phi} M M_{*} d v d v_{*} d \sigma\right): \nabla_{x} \bar{u}-\mu \Delta_{x} \bar{u} \cdot \tilde{u}_{\epsilon} d x \\
& +\frac{1}{2} \int_{\mathbb{R}^{3}}\left(E_{\epsilon} \otimes E_{\epsilon}+e_{\epsilon}+B_{\epsilon} \otimes B_{\epsilon}+b_{\epsilon}\right): \nabla_{x} \bar{u} d x \\
& =\int_{\mathbb{R}^{3}} R_{\epsilon, 3} \cdot \bar{u}-\frac{1}{4} R_{\epsilon, 7} \cdot \nabla_{x} \bar{n} d x \\
& +\int_{\mathbb{R}^{3}} \frac{1}{2} P(\bar{n} \bar{E}+\bar{j} \wedge \bar{B}) \cdot \tilde{u}_{\epsilon}-\frac{1}{2} \mathbf{A}_{1} \cdot \tilde{u}_{\epsilon}-\frac{1}{4} \tilde{n}_{\epsilon} \nabla_{x} \cdot \mathbf{A}_{4} d x,
\end{aligned}
$$

whence, reorganizing some terms so that remainders are moved to the right-hand side,

$$
\begin{aligned}
\frac{d}{d t} & \int_{\mathbb{R}^{3}}\left(\frac{1}{4} \tilde{n}_{\epsilon} \bar{n}+\tilde{u}_{\epsilon} \cdot \bar{u}+\frac{1}{2}\left(E_{\epsilon} \wedge B_{\epsilon}\right) \cdot \bar{u}+\frac{1}{2}\left(\begin{array}{c}
a_{\epsilon 26}-a_{\epsilon 35} \\
a_{\epsilon 34}-a_{\epsilon 16} \\
a_{\epsilon 15}-a_{\epsilon 24}
\end{array}\right) \cdot \bar{u}\right) d x \\
& +\int_{\mathbb{R}^{3}}\left(\int_{\mathbb{R}^{3} \times \mathbb{R}^{3} \times \mathbb{S}^{2}}\left(\hat{q}_{\epsilon}^{+}+\hat{q}_{\epsilon}^{-}\right) \tilde{\phi} M M_{*} d v d v_{*} d \sigma\right): \nabla_{x} \bar{u} d x \\
& =\int_{\mathbb{R}^{3}} R_{\epsilon, 3} \cdot \bar{u}-\frac{1}{4} R_{\epsilon, 7} \cdot \nabla_{x} \bar{n}-\frac{1}{2} \mathbf{A}_{1} \cdot \tilde{u}_{\epsilon}-\frac{1}{4} \tilde{n}_{\epsilon} \nabla_{x} \cdot \mathbf{A}_{4} d x \\
& +\int_{\mathbb{R}^{3}} \frac{1}{2} P(\bar{n} \bar{E}+\bar{j} \wedge \bar{B}) \cdot \tilde{u}_{\epsilon}+\frac{1}{4}\left(\tilde{j}_{\epsilon} \nabla_{x} \bar{n}-\tilde{n}_{\epsilon} \nabla_{x} \cdot \bar{j}\right) d x \\
& +\int_{\mathbb{R}^{3}}\left(\bar{u} \otimes\left(P^{\perp} \tilde{u}_{\epsilon}\right)+\left(P^{\perp} \tilde{u}_{\epsilon}\right) \otimes \bar{u}+\left(\tilde{u}_{\epsilon}-\bar{u}\right) \otimes\left(\tilde{u}_{\epsilon}-\bar{u}\right)+\frac{1}{2 \epsilon^{2}} m_{\epsilon}\right): \nabla_{x} \bar{u} d x \\
& +\int_{\mathbb{R}^{3}} \mu \tilde{u}_{\epsilon} \cdot \Delta_{x} \bar{u}+\left(\int_{\mathbb{R}^{3} \times \mathbb{R}^{3} \times \mathbb{S}^{2}} \frac{\hat{q}_{\epsilon}^{+}+\hat{q}_{\epsilon}^{-}}{2} \tilde{\phi}_{M} M_{*} d v d v_{*} d \sigma\right): \nabla_{x} \bar{u} d x \\
& +\int_{\mathbb{R}^{3}}\left(\frac{1}{2}\left(E_{\epsilon} \wedge B_{\epsilon}\right) \cdot \partial_{t} \bar{u}+\frac{1}{2}\left(\begin{array}{l}
a_{\epsilon 26}-a_{\epsilon 35} \\
a_{\epsilon 34}-a_{\epsilon 16} \\
a_{\epsilon 15}-a_{\epsilon 24}
\end{array}\right) \cdot \partial_{t} \bar{u}-R_{\epsilon, 2} \cdot \partial_{t} \bar{u}-\frac{1}{4} R_{\epsilon, 8} \partial_{t} \bar{n}\right) d x \\
& -\frac{1}{2} \int_{\mathbb{R}^{3}}\left(E_{\epsilon} \otimes E_{\epsilon}+e_{\epsilon}+B_{\epsilon} \otimes B_{\epsilon}+b_{\epsilon}\right): \nabla_{x} \bar{u} d x+\frac{d}{d t} \int_{\mathbb{R}^{3}} R_{\epsilon, 2} \cdot \bar{u}+\frac{1}{4} R_{\epsilon, 8} \bar{n} d x
\end{aligned}
$$

Then, using the convergences (12.42), 12.68), the estimates 4.19, 4.24), 12.57), 12.63 and Lemmas 4.3 and 12.3 (allowing to control the energy by the entropy), 
we arrive at

$$
\begin{aligned}
& \frac{d}{d t} \int_{\mathbb{R}^{3}}\left(\frac{1}{4} \tilde{n}_{\epsilon} \bar{n}+\tilde{u}_{\epsilon} \cdot \bar{u}+\frac{1}{2}\left(E_{\epsilon} \wedge B_{\epsilon}\right) \cdot \bar{u}+\frac{1}{2}\left(\begin{array}{c}
a_{\epsilon 26}-a_{\epsilon 35} \\
a_{\epsilon 34}-a_{\epsilon 16} \\
a_{\epsilon 15}-a_{\epsilon 24}
\end{array}\right) \cdot \bar{u}\right) d x \\
& \quad+\int_{\mathbb{R}^{3}}\left(\int_{\mathbb{R}^{3} \times \mathbb{R}^{3} \times \mathbb{S}^{2}}\left(\hat{q}_{\epsilon}^{+}+\hat{q}_{\epsilon}^{-}\right) \tilde{\phi} M M_{*} d v d v_{*} d \sigma\right): \nabla_{x} \bar{u} d x \\
& \quad \geq-C\left(\|\bar{u}\|_{W^{1, \infty}(d x)}+\frac{\left\|\partial_{t} \bar{u}\right\|_{L^{\infty}(d x)}}{1-\|\bar{u}\|_{L^{\infty}(d x)}}\right) \delta \mathcal{H}_{\epsilon}(t)-\frac{1}{4} \int_{\mathbb{R}^{3}} R_{\epsilon, 7} \cdot \nabla_{x} \bar{n} d x \\
& \quad-\int_{\mathbb{R}^{3}} \frac{1}{2} \mathbf{A}_{1} \cdot \tilde{u}_{\epsilon}+\frac{1}{4} \tilde{n}_{\epsilon} \nabla_{x} \cdot \mathbf{A}_{4} d x+o(1)_{w-L_{\text {loc }}^{1}(d t)}+\frac{d}{d t}\left(o(1)_{L^{\infty}(d t)}\right) \\
& \quad+\int_{\mathbb{R}^{3}} \frac{1}{2} P(\bar{n} \bar{E}+\bar{j} \wedge \bar{B}) \cdot \tilde{u}_{\epsilon}+\frac{1}{4}\left(\tilde{j}_{\epsilon} \nabla_{x} \bar{n}-\tilde{n}_{\epsilon} \nabla_{x} \cdot \bar{j}\right) d x \\
& \quad+\frac{1}{2} \int_{\mathbb{R}^{3}}\left(E_{\epsilon} \wedge B_{\epsilon}\right) \cdot \partial_{t} \bar{u}-\left(E_{\epsilon} \otimes E_{\epsilon}+e_{\epsilon}+B_{\epsilon} \otimes B_{\epsilon}+b_{\epsilon}\right): \nabla_{x} \bar{u} d x .
\end{aligned}
$$

The next step consists in combining the preceding inequality with the identity 12.66 in order to modulate the Poynting vector $E_{\epsilon} \wedge B_{\epsilon}$. This yields

$$
\begin{aligned}
\frac{d}{d t} & \int_{\mathbb{R}^{3}}\left(\frac{1}{4} \tilde{n}_{\epsilon} \bar{n}+\tilde{u}_{\epsilon} \cdot \bar{u}+\frac{1}{2}\left(\left(E_{\epsilon}-\bar{E}\right) \wedge\left(B_{\epsilon}-\bar{B}\right)\right) \cdot \bar{u}+\frac{1}{2}\left(\begin{array}{l}
a_{\epsilon 26}-a_{\epsilon 35} \\
a_{\epsilon 34}-a_{\epsilon 16} \\
a_{\epsilon 15}-a_{\epsilon 24}
\end{array}\right) \cdot \bar{u}\right) d x \\
& +\int_{\mathbb{R}^{3}}\left(\int_{\mathbb{R}^{3} \times \mathbb{R}^{3} \times \mathbb{S}^{2}}\left(\hat{q}_{\epsilon}^{+}+\hat{q}_{\epsilon}^{-}\right) \tilde{\phi} M M_{*} d v d v_{*} d \sigma\right): \nabla_{x} \bar{u} d x \\
& \geq-C\left(\|\bar{u}\|_{W^{1, \infty}(d x)}+\frac{\left\|\partial_{t} \bar{u}\right\|_{L^{\infty}(d x)}}{1-\|\bar{u}\|_{L^{\infty}(d x)}}\right) \delta \mathcal{H}_{\epsilon}(t) \\
& +\int_{\mathbb{R}^{3}} \frac{1}{2}\left(\mathbf{A}_{4} \wedge\left(B_{\epsilon}-\bar{B}\right)+\left(E_{\epsilon}-\bar{E}\right) \wedge \mathbf{A}_{5}\right) \cdot \bar{u}-\frac{1}{2} \mathbf{A}_{1} \cdot \tilde{u}_{\epsilon}-\frac{1}{4} \tilde{n}_{\epsilon} \nabla_{x} \cdot \mathbf{A}_{4} d x \\
& +o(1)_{w-L_{\text {loc }}}(d t)+\frac{d}{d t}\left(o(1)_{L^{\infty}(d t)}\right) \\
& +\frac{1}{2} \int_{\mathbb{R}^{3}}\left(\left(\bar{u} \wedge \bar{B}-\frac{1}{2} \nabla_{x} \bar{n}\right) \cdot R_{\epsilon, 7}-R_{\epsilon, 8} \bar{E} \cdot \bar{u}\right)+(\bar{n} \bar{E}+\bar{j} \wedge \bar{B}) \cdot P^{\perp} \tilde{u}_{\epsilon} d x \\
& +\frac{1}{2} \int_{\mathbb{R}^{3}} \bar{n} \bar{E} \cdot \tilde{u}_{\epsilon}+\tilde{n}_{\epsilon} \bar{E} \cdot \bar{u}+\bar{n}\left(E_{\epsilon}-\bar{E}\right) \cdot \bar{u} d x \\
& +\frac{1}{2} \int_{\mathbb{R}^{3}}\left(\frac{1}{2} \nabla_{x} \tilde{n}_{\epsilon}-\tilde{u}_{\epsilon} \wedge B_{\epsilon}\right) \cdot \bar{j}+\left(\frac{1}{2} \nabla_{x} \bar{n}-\bar{u} \wedge \bar{B}\right) \cdot \tilde{j}_{\epsilon} d x \\
& +\frac{1}{2} \int_{\mathbb{R}^{3}}\left(\bar{j} \wedge\left(B_{\epsilon}-\bar{B}\right)\right) \cdot\left(\bar{u}-\tilde{u}_{\epsilon}\right)+\left(\left(E_{\epsilon}-\bar{E}\right) \wedge\left(B_{\epsilon}-\bar{B}\right)\right) \cdot \partial_{t} \bar{u} d x \\
& -\frac{1}{2} \int_{\mathbb{R}^{3}}\left(\left(E_{\epsilon}-\bar{E}\right) \otimes\left(E_{\epsilon}-\bar{E}\right)+e_{\epsilon}+\left(B_{\epsilon}-\bar{B}\right) \otimes\left(B_{\epsilon}-\bar{B}\right)+b_{\epsilon}\right): \nabla_{x} \bar{u} d x
\end{aligned}
$$


It then follows, using the convergence 12.42, the estimates 4.24, 12.63 and Lemma 4.3 that

$$
\begin{aligned}
\frac{d}{d t} \int_{\mathbb{R}^{3}}\left(\frac{1}{4} \tilde{n}_{\epsilon} \bar{n}+\tilde{u}_{\epsilon} \cdot \bar{u}+\frac{1}{2}\left(\left(E_{\epsilon}-\bar{E}\right) \wedge\left(B_{\epsilon}-\bar{B}\right)\right) \cdot \bar{u}+\frac{1}{2}\left(\begin{array}{l}
a_{\epsilon 26}-a_{\epsilon 35} \\
a_{\epsilon 34}-a_{\epsilon 16} \\
a_{\epsilon 15}-a_{\epsilon 24}
\end{array}\right) \cdot \bar{u}\right) d x \\
\quad+\int_{\mathbb{R}^{3}}\left(\int_{\mathbb{R}^{3} \times \mathbb{R}^{3} \times \mathbb{S}^{2}}\left(\hat{q}_{\epsilon}^{+}+\hat{q}_{\epsilon}^{-}\right) \tilde{\phi} M M_{*} d v d v_{*} d \sigma\right): \nabla_{x} \bar{u} d x \\
\quad \geq-C\left(\frac{\|\bar{u}\|_{W^{1, \infty}(d x)}+\left\|\partial_{t} \bar{u}\right\|_{L^{\infty}(d x)}}{1-\|\bar{u}\|_{L^{\infty}(d x)}}\right) \delta \mathcal{H}_{\epsilon}(t) \\
+\int_{\mathbb{R}^{3}} \frac{1}{2}\left(\mathbf{A}_{4} \wedge\left(B_{\epsilon}-\bar{B}\right)+\left(E_{\epsilon}-\bar{E}\right) \wedge \mathbf{A}_{5}\right) \cdot \bar{u}-\frac{1}{2} \mathbf{A}_{1} \cdot \tilde{u}_{\epsilon}-\frac{1}{4} \tilde{n}_{\epsilon} \nabla_{x} \cdot \mathbf{A}_{4} d x \\
+o(1)_{w-L_{\text {loc }}^{1}(d t)}+\frac{d}{d t}\left(o(1)_{L^{\infty}(d t)}\right)+\frac{1}{2} \int_{\mathbb{R}^{3}}\left(\bar{u} \wedge \bar{B}-\frac{1}{2} \nabla_{x} \bar{n}\right) \cdot R_{\epsilon, 7} d x \\
+\frac{1}{2} \int_{\mathbb{R}^{3}} \bar{n} \bar{E} \cdot \tilde{u}_{\epsilon}+\tilde{n}_{\epsilon} \bar{E} \cdot \bar{u}+\bar{n}\left(E_{\epsilon}-\bar{E}\right) \cdot \bar{u} d x \\
+\frac{1}{2} \int_{\mathbb{R}^{3}}\left(\frac{1}{2} \nabla_{x} \tilde{n}_{\epsilon}-\tilde{u}_{\epsilon} \wedge B_{\epsilon}\right) \cdot \bar{j}+\left(\frac{1}{2} \nabla_{x} \bar{n}-\bar{u} \wedge \bar{B}\right) \cdot \tilde{j}_{\epsilon} d x \\
+\frac{1}{2} \int_{\mathbb{R}^{3}}\left(\bar{j} \wedge\left(B_{\epsilon}-\bar{B}\right)\right) \cdot\left(\bar{u}-\tilde{u}_{\epsilon}\right) d x .
\end{aligned}
$$

Now, for mere convenience of notation, we introduce the following integrand :

$$
\begin{aligned}
\mathcal{I} & =\frac{1}{4} \tilde{n}_{\epsilon} \bar{n}+\tilde{u}_{\epsilon} \cdot \bar{u}+\frac{1}{2}\left(E_{\epsilon} \cdot \bar{E}+B_{\epsilon} \cdot \bar{B}\right) \\
& +\frac{1}{2}\left(\left(E_{\epsilon}-\bar{E}\right) \wedge\left(B_{\epsilon}-\bar{B}\right)\right) \cdot \bar{u}+\frac{1}{2}\left(\begin{array}{c}
a_{\epsilon 26}-a_{\epsilon 35} \\
a_{\epsilon 34}-a_{\epsilon 16} \\
a_{\epsilon 15}-a_{\epsilon 24}
\end{array}\right) \cdot \bar{u} .
\end{aligned}
$$

Thus, further employing the identity 12.65, we find that

$$
\begin{aligned}
& \frac{d}{d t} \int_{\mathbb{R}^{3}} \mathcal{I} d x+\int_{\mathbb{R}^{3}}\left(\int_{\mathbb{R}^{3} \times \mathbb{R}^{3} \times \mathbb{S}^{2}} \sum_{ \pm} \hat{q}_{\epsilon}^{ \pm} \tilde{\phi} M M_{*} d v d v_{*} d \sigma\right): \nabla_{x} \bar{u} d x \\
& \geq-C\left(\frac{\|\bar{u}\|_{W^{1, \infty}(d x)}+\left\|\partial_{t} \bar{u}\right\|_{L^{\infty}(d x)}}{1-\|\bar{u}\|_{L^{\infty}(d x)}}\right) \delta \mathcal{H}_{\epsilon}(t)-\frac{1}{2} \int_{\mathbb{R}^{3}} \mathbf{A}_{1} \cdot \tilde{u}_{\epsilon} d x \\
& -\frac{1}{2} \int_{\mathbb{R}^{3}} \mathbf{A}_{4} \cdot\left(E_{\epsilon}+\bar{u} \wedge\left(B_{\epsilon}-\bar{B}\right)-\frac{1}{2} \nabla_{x} \tilde{n}_{\epsilon}\right)+\mathbf{A}_{5} \cdot\left(B_{\epsilon}+\left(E_{\epsilon}-\bar{E}\right) \wedge \bar{u}\right) d x \\
& +o(1)_{w-L_{\text {loc }}^{1}(d t)}+\frac{d}{d t}\left(o(1)_{L^{\infty}(d t)}\right)+\frac{1}{2} \int_{\mathbb{R}^{3}}\left(\frac{1}{2} \nabla_{x} \tilde{n}_{\epsilon}-E_{\epsilon}-\tilde{u}_{\epsilon} \wedge B_{\epsilon}\right) \cdot(\bar{j}-\bar{n} \bar{u}) d x \\
& +\frac{1}{2} \int_{\mathbb{R}^{3}}\left(\frac{1}{2} \nabla_{x} \bar{n}-\bar{E}-\bar{u} \wedge \bar{B}\right) \cdot\left(\tilde{j}_{\epsilon}-\tilde{n}_{\epsilon} \tilde{u}_{\epsilon}\right) d x \\
& +\frac{1}{2} \int_{\mathbb{R}^{3}}\left(\left(\tilde{u}_{\epsilon}-\bar{u}\right) \wedge\left(B_{\epsilon}-\bar{B}\right)\right) \cdot(\bar{j}-\bar{n} \bar{u})+\frac{1}{4} P^{\perp} \tilde{u}_{\epsilon} \cdot \nabla_{x}\left(\bar{n}^{2}\right) d x \\
& +\frac{1}{2} \int_{\mathbb{R}^{3}}\left((n-\bar{n})(u-\bar{u})-R_{\epsilon, 7}\right) \cdot\left(\frac{1}{2} \nabla_{x} \bar{n}-\bar{E}-\bar{u} \wedge \bar{B}\right) d x
\end{aligned}
$$


whence, in view of the convergence 12.42 , the estimate 12.62 and Lemma 12.3 (allowing to control the energy by the entropy),

$$
\begin{aligned}
& \frac{d}{d t} \int_{\mathbb{R}^{3}} \mathcal{I} d x+\int_{\mathbb{R}^{3}}\left(\int_{\mathbb{R}^{3} \times \mathbb{R}^{3} \times \mathbb{S}^{2}} \sum_{ \pm} \hat{q}_{\epsilon}^{ \pm} \tilde{\phi} M M_{*} d v d v_{*} d \sigma\right): \nabla_{x} \bar{u} d x \\
& \geq-C\left(\frac{\|\bar{u}\|_{W^{1, \infty}(d x)}+\left\|\partial_{t} \bar{u}\right\|_{L^{\infty}(d x)}+\|\bar{j}-\bar{n} \bar{u}\|_{L^{\infty}(d x)}}{1-\|\bar{u}\|_{L^{\infty}(d x)}}\right. \\
& \left.+\left\|\frac{1}{2} \nabla_{x} \bar{n}-\bar{E}-\bar{u} \wedge \bar{B}\right\|_{L^{\infty}(d x)}\right) \delta \mathcal{H}_{\epsilon}(t)-\frac{1}{2} \int_{\mathbb{R}^{3}} \mathbf{A}_{1} \cdot \tilde{u}_{\epsilon} d x \\
& -\frac{1}{2} \int_{\mathbb{R}^{3}} \mathbf{A}_{4} \cdot\left(E_{\epsilon}+\bar{u} \wedge\left(B_{\epsilon}-\bar{B}\right)-\frac{1}{2} \nabla_{x} \tilde{n}_{\epsilon}\right)+\mathbf{A}_{5} \cdot\left(B_{\epsilon}+\left(E_{\epsilon}-\bar{E}\right) \wedge \bar{u}\right) d x \\
& +o(1)_{w-L_{\text {loc }}^{1}(d t)}+\frac{d}{d t}\left(o(1)_{L^{\infty}(d t)}\right)+\frac{1}{2} \int_{\mathbb{R}^{3}}\left(\frac{1}{2} \nabla_{x} \tilde{n}_{\epsilon}-E_{\epsilon}-\tilde{u}_{\epsilon} \wedge B_{\epsilon}\right) \cdot(\bar{j}-\bar{n} \bar{u}) d x \\
& +\frac{1}{2} \int_{\mathbb{R}^{3}}\left(\frac{1}{2} \nabla_{x} \bar{n}-\bar{E}-\bar{u} \wedge \bar{B}\right) \cdot\left(\tilde{j}_{\epsilon}-\tilde{n}_{\epsilon} \tilde{u}_{\epsilon}\right) d x .
\end{aligned}
$$

Using then the approximate Ohm's law 12.58 with the control 12.69 and reorganizing the resulting inequality so that all remainder terms appear on its righthand side, we obtain

$$
\begin{aligned}
\frac{d}{d t} & \int_{\mathbb{R}^{3}} \mathcal{I} d x+\int_{\mathbb{R}^{3}}\left(\int_{\mathbb{R}^{3} \times \mathbb{R}^{3} \times \mathbb{S}^{2}} \sum_{ \pm} \hat{q}_{\epsilon}^{ \pm} \tilde{\phi} M M_{*} d v d v_{*} d \sigma\right): \nabla_{x} \bar{u} d x \\
& -\frac{1}{\sigma} \int_{\mathbb{R}^{3}}\left(\int_{\mathbb{R}^{3} \times \mathbb{R}^{3} \times \mathbb{S}^{2}} \sum_{ \pm}\left( \pm \hat{q}_{\epsilon}^{ \pm} \pm \hat{q}_{\epsilon}^{ \pm, \mp}\right) \tilde{\Phi} M M_{*} d v d v_{*} d \sigma\right) \cdot(\bar{j}-\bar{n} \bar{u}) d x \\
& \geq-C\left(\frac{\|\bar{u}\|_{W^{1, \infty}(d x)}+\left\|\partial_{t} \bar{u}\right\|_{L^{\infty}(d x)}+\|\bar{j}-\bar{n} \bar{u}\|_{L^{\infty}(d x)}}{1-\|\bar{u}\|_{L^{\infty}(d x)}}\right. \\
& \left.+\left\|\frac{1}{2} \nabla_{x} \bar{n}-\bar{E}-\bar{u} \wedge \bar{B}\right\|_{L^{\infty}(d x)}\right) \delta \mathcal{H}_{\epsilon}(t)+o(1)_{w-L_{\mathrm{loc}}(d t)}+\frac{d}{d t}\left(o(1)_{L^{\infty}(d t)}\right) \\
& -\frac{1}{2} \int_{\mathbb{R}^{3}} \mathbf{A}_{4} \cdot\left(E_{\epsilon}+\bar{u} \wedge\left(B_{\epsilon}-\bar{B}\right)-\frac{1}{2} \nabla_{x} \tilde{n}_{\epsilon}\right)+\mathbf{A}_{5} \cdot\left(B_{\epsilon}+\left(E_{\epsilon}-\bar{E}\right) \wedge \bar{u}\right) d x \\
& +\frac{1}{2} \int_{\mathbb{R}^{3}} \mathbf{A}_{3} \cdot\left(\int_{\mathbb{R}^{3} \times \mathbb{R}^{3} \times \mathbb{S}^{2}} \sum_{ \pm}\left( \pm \hat{q}_{\epsilon}^{ \pm} \pm \hat{q}_{\epsilon}^{ \pm, \mp}\right) \tilde{\Phi} M M_{*} d v d v_{*} d \sigma\right)-\mathbf{A} \mathbf{A}_{1} \cdot \tilde{u}_{\epsilon} d x \\
& -\frac{1}{2} \int_{\mathbb{R}^{3}}\left(R_{\epsilon, 4}+\nabla_{x} R_{\epsilon, 5}+R_{\epsilon, 6}\right) \cdot(\bar{j}-\bar{n} \bar{u})-\left(\frac{1}{2} \nabla_{x} \bar{n}-\bar{E}-\bar{u} \wedge \bar{B}\right) \cdot R_{\epsilon, 9} d x
\end{aligned}
$$


Thus, in view of the estimates 12.59, 12.60, 12.68, 12.70 and Lemma 12.3 (allowing to control the energy by the entropy), we infer that

$$
\begin{aligned}
\frac{d}{d t} \int_{\mathbb{R}^{3}} \mathcal{I} d x+\frac{1}{2} \int_{\mathbb{R}^{3}}\left(\int_{\mathbb{R}^{3} \times \mathbb{R}^{3} \times \mathbb{S}^{2}} \sum_{ \pm}\left(\hat{q}_{\epsilon}^{ \pm}+\hat{q}_{\epsilon}^{ \pm, \mp}\right) \tilde{\phi} M M_{*} d v d v_{*} d \sigma\right): \nabla_{x} \bar{u} d x \\
\quad-\frac{1}{\sigma} \int_{\mathbb{R}^{3}}\left(\int_{\mathbb{R}^{3} \times \mathbb{R}^{3} \times \mathbb{S}^{2}} \sum_{ \pm}\left( \pm \hat{q}_{\epsilon}^{ \pm} \pm \hat{q}_{\epsilon}^{ \pm, \mp}\right) \tilde{\Phi} M M_{*} d v d v_{*} d \sigma\right) \cdot(\bar{j}-\bar{n} \bar{u}) d x \\
\quad \geq-C\left(\frac{\|\bar{u}\|_{W^{1, \infty}(d x)}+\left\|\partial_{t} \bar{u}\right\|_{L^{\infty}(d x)}+\|\bar{j}-\bar{n} \bar{u}\|_{L^{\infty}(d x)}}{1-\|\bar{u}\|_{L^{\infty}(d x)}}\right. \\
\left.\quad+\left\|\frac{1}{2} \nabla_{x} \bar{n}-\bar{E}-\bar{u} \wedge \bar{B}\right\|_{L^{\infty}(d x)}\right) \delta \mathcal{H}_{\epsilon}(t)+o(1)_{w-L_{\text {loc }}^{1}(d t)}+\frac{d}{d t}\left(o(1)_{L^{\infty}(d t)}\right) \\
\quad-\frac{1}{2} \int_{\mathbb{R}^{3}} \mathbf{A}_{4} \cdot\left(E_{\epsilon}+\bar{u} \wedge\left(B_{\epsilon}-\bar{B}\right)-\frac{1}{2} \nabla_{x} \tilde{n}_{\epsilon}\right)+\mathbf{A}_{5} \cdot\left(B_{\epsilon}+\left(E_{\epsilon}-\bar{E}\right) \wedge \bar{u}\right) d x \\
+\frac{1}{2} \int_{\mathbb{R}^{3}} \mathbf{A}_{3} \cdot\left(\int_{\mathbb{R}^{3} \times \mathbb{R}^{3} \times \mathbb{S}^{2}} \sum_{ \pm}\left( \pm \hat{q}_{\epsilon}^{ \pm} \pm \hat{q}_{\epsilon}^{ \pm, \mp}\right) \tilde{\Phi} M M_{*} d v d v_{*} d \sigma\right)-\mathbf{A}_{1} \cdot \tilde{u}_{\epsilon} d x .
\end{aligned}
$$

At last, we may now combine the inequalities 12.73 and 12.74 to deduce, employing the symmetries of collision integrands and (12.49) to rewrite dissipation terms, that

$$
\begin{aligned}
& \frac{d}{d t} \int_{\mathbb{R}^{3}}\left(\left(g_{\epsilon}^{+} \gamma_{\epsilon}^{+} \chi\left(\frac{|v|^{2}}{K_{\epsilon}}\right) \bar{g}^{+}+g_{\epsilon}^{-} \gamma_{\epsilon}^{-} \chi\left(\frac{|v|^{2}}{K_{\epsilon}}\right) \bar{g}^{-}\right)+E_{\epsilon} \cdot \bar{E}+B_{\epsilon} \cdot \bar{B}\right. \\
& \left.+\left(\left(E_{\epsilon}-\bar{E}\right) \wedge\left(B_{\epsilon}-\bar{B}\right)+\left(\begin{array}{c}
a_{\epsilon 26}-a_{\epsilon 35} \\
a_{\epsilon 34}-a_{\epsilon 16} \\
a_{\epsilon 15}-a_{\epsilon 24}
\end{array}\right)\right) \cdot \bar{u}\right) d x \\
& +\frac{1}{2} \int_{\mathbb{R}^{3} \times \mathbb{R}^{3} \times \mathbb{R}^{3} \times \mathbb{S}^{2}}\left(\hat{q}_{\epsilon}^{+} \bar{q}^{+}+\hat{q}_{\epsilon}^{-} \bar{q}^{-}+\hat{q}_{\epsilon}^{+,-} \bar{q}^{+,-}+\hat{q}_{\epsilon}^{-,+} \bar{q}^{-,+}\right) M M_{*} d x d v d v_{*} d \sigma \\
& \geq-\lambda(t) \delta \mathcal{H}_{\epsilon}(t)+o(1)_{w-L_{\text {loc }}^{1}(d t)}+\frac{d}{d t}\left(o(1)_{L}(d t)\right)-\frac{1}{2} \delta \mathcal{D}_{\epsilon}(t) \\
& \tilde{u}_{\epsilon} \\
& \left.\begin{array}{c}
\frac{3}{2} \tilde{\theta}_{\epsilon}-\tilde{\rho}_{\epsilon} \\
-\int_{\mathbb{R}^{3} \times \mathbb{R}^{3} \times \mathbb{S}^{2}}\left(\hat{q}_{\epsilon}^{+}-\hat{q}_{\epsilon}^{-}+\hat{q}_{\epsilon}^{+,-}-\hat{q}_{\epsilon}^{-,+}\right) \tilde{\Phi} M M_{*} d v d v_{*} d \sigma \\
E_{\epsilon}+\bar{u} \wedge\left(B_{\epsilon}-\bar{B}\right)-\frac{1}{2} \nabla_{x} \tilde{n}_{\epsilon} \\
B_{\epsilon}+\left(E_{\epsilon}-\bar{E}\right) \wedge \bar{u}
\end{array}\right) d x .
\end{aligned}
$$

Next, assembling the preceding inequality with the scaled entropy inequality 12.53 and the energy estimate 12.72 , we finally obtain

$$
\begin{aligned}
& \frac{d}{d t} \delta \mathcal{H}_{\epsilon}(t)+\delta \mathcal{D}_{\epsilon}(t) \\
& \leq \lambda(t) \delta \mathcal{H}_{\epsilon}(t)+o(1)_{w-L_{\mathrm{loc}}^{1}(d t)}+\frac{d}{d t}\left(o(1)_{L^{\infty}(d t)}\right)+\frac{1}{2} \delta \mathcal{D}_{\epsilon}(t) \\
& +\int_{\mathbb{R}^{3}} \mathbf{A} \cdot\left(\begin{array}{c}
\tilde{u}_{\epsilon}-\bar{u} \\
-\int_{\mathbb{R}^{3} \times \mathbb{R}^{3} \times \mathbb{S}^{2}}\left(\hat{q}_{\epsilon}^{+}-\hat{q}_{\epsilon}^{-}+\hat{q}_{\epsilon}^{+,-}-\hat{\theta}_{\epsilon}^{-,+}\right) \tilde{\Phi} M M_{*} d v d v_{*} d \sigma-(\bar{j}-\bar{n} \bar{u}) \\
E_{\epsilon}-\bar{E}+\bar{u} \wedge\left(B_{\epsilon}-\bar{B}\right)-\frac{1}{2} \nabla_{x}\left(\tilde{n}_{\epsilon}-\bar{n}\right) \\
B_{\epsilon}-\bar{B}+\left(E_{\epsilon}-\bar{E}\right) \wedge \bar{u} \\
\tilde{\theta}
\end{array}\right) d x,
\end{aligned}
$$


which, with a straightforward application of Grönwall's lemma (carefully note that this is valid even though $\delta \mathcal{H}_{\epsilon}(t)$ may be negative), concludes the proof of the proposition.

REMARK. The proof of Proposition 12.4 is based on the construction of the stability inequality 3.21 from Proposition 3.10 for the two-fluid incompressible Navier-Stokes-Maxwell system with Ohm's law (3.5). As in the proof of Proposition 12.2, this approach has the great advantage of using the approximate macroscopic conservation of momentum established in Proposition 9.6 rather than the one from Proposition 9.5 and, thus, removes the difficulties associated with the nonlinear Lorentz force $\tilde{n}_{\epsilon} E_{\epsilon}+\tilde{j}_{\epsilon} \wedge B_{\epsilon}$ by expressing it with the Poynting vector $E_{\epsilon} \wedge B_{\epsilon}$ (and some other terms).

However, the drawback of this approach resides in the necessity of the restriction $\|\bar{u}\|_{L_{t, x}^{\infty}}<1$. Recall, nevertheless, that this restriction is physically relevant, since it merely entails that the modulus of the velocity $\bar{u}$ be less than the speed of light (see comments after the proofs of Propositions 3.10 and 3.13 .

Note finally that it is not possible (at least, we do not know how to make it work) to establish a similar renormalized relative entropy inequality for renormalized solutions of the scaled two species Vlasov-Maxwell-Boltzmann system (4.34) based on the construction of the stability inequality (3.17) from Proposition 3.8 (see the remark following the proof of Proposition 12.2).

12.3.4. Convergence and conclusion of proof. We may now pass to the limit in the approximate stability inequality 12.52 and, thus, derive the crucial modulated energy inequality for the limiting system 4.37). To this end, we simply integrate 12.52 in time against non-negative test functions and then let $\epsilon \rightarrow$ 0 , which yields, in view of the well-preparedness of the initial data (4.44), the weak convergences 12.37, 12.40, 12.41) and the lower semi-continuities (12.50), 12.51, that

$$
\begin{aligned}
& \delta \mathcal{E}(t)+\frac{1}{2} \int_{0}^{t} \delta \mathcal{D}(s) e^{\int_{s}^{t} \lambda(\sigma) d \sigma} d s \\
& \leq \delta \mathcal{E}(0) e^{\int_{0}^{t} \lambda(s) d s} \\
& +\int_{0}^{t} \int_{\mathbb{R}^{3}} \mathbf{A} \cdot\left(\begin{array}{c}
u-\bar{u} \\
-\int_{\mathbb{R}^{3} \times \mathbb{R}^{3} \times \mathbb{S}^{2}} \sum_{ \pm}\left( \pm q^{ \pm} \pm q^{ \pm, \mp}\right) \tilde{\Phi} M M_{*} d v d v_{*} d \sigma-(\bar{j}-\bar{n} \bar{u}) \\
E-\bar{E}+\bar{u} \wedge(B-\bar{B})-\frac{1}{2} \nabla_{x}(n-\bar{n}) \\
B-\bar{B}+(E-\bar{E})^{\prime} \wedge \bar{u}
\end{array}\right)(s) d x \\
& \times e^{\int_{s}^{t} \lambda(\sigma) d \sigma} d s .
\end{aligned}
$$

Finally, using (12.39) and the characterizations 8.38 , 8.39 of the limiting collision integrands $q^{ \pm}, q^{ \pm, \mp}$ from Proposition 8.4 . we deduce that (see also the proof of Proposition 8.5 for more detailed computations yielding the term $j-n u$ )

$$
\begin{aligned}
\delta \mathcal{E}(t) & +\frac{1}{2} \int_{0}^{t} \delta \mathcal{D}(s) e^{\int_{s}^{t} \lambda(\sigma) d \sigma} d s \\
& \leq \delta \mathcal{E}(0) e^{\int_{0}^{t} \lambda(s) d s} \\
& +\int_{0}^{t} \int_{\mathbb{R}^{3}} \mathbf{A} \cdot\left(\begin{array}{c}
u-\bar{u} \\
\frac{5}{2}(\theta-\bar{\theta}) \\
j-\bar{E}+\bar{u} \wedge(B-\bar{j}-\bar{n} \bar{u}) \\
B-\bar{B}+(E-\bar{E})_{x} \wedge \bar{u}(n-\bar{n})
\end{array}\right)(s) d x e^{\int_{s}^{t} \lambda(\sigma) d \sigma} d s,
\end{aligned}
$$


which is precisely the stability inequality we were after.

As for the temporal continuity of $\left(u, n, \frac{5}{2} \theta, E, B\right)$, it is readily seen from the approximate macroscopic conservation laws from Proposition 9.5 and Maxwell's equations 12.61 that $\partial_{t} P \tilde{u}_{\epsilon}, \partial_{t} \tilde{n}_{\epsilon}, \partial_{t}\left(\frac{3}{2} \tilde{\theta}_{\epsilon}-\tilde{\rho}_{\epsilon}\right), \partial_{t} E_{\epsilon}$ and $\partial_{t} B_{\epsilon}$ are uniformly bounded, in $L_{\text {loc }}^{1}$ in time and in some negative index Sobolev space in $x$. It is therefore possible to show (see [57, Appendix C]) that $\left(P \tilde{u}_{\epsilon}, \tilde{n}_{\epsilon}, \frac{3}{2} \tilde{\theta}_{\epsilon}-\tilde{\rho}_{\epsilon}, E_{\epsilon}, B_{\epsilon}\right)$ converges to $\left(u, n, \frac{5}{2} \theta, E, B\right) \in C\left([0, \infty) ; w-L^{2}\left(\mathbb{R}^{3}\right)\right)$ weakly in $L^{2}(d x)$ uniformly locally in time.

At last, the proof of Theorem 4.6 is complete. 
Part 3

Unconditional convergence results : long-range interactions 



\section{Part 4}

\section{Unconditional convergence results : short-range interactions}



Appendices 



\section{APPENDIX A}

\section{Cross-section for momentum and energy transfer}

The cross-section for momentum and energy transfer $m(z)=m(|z|) \in$ $L_{\text {loc }}^{1}\left(\mathbb{R}^{3}\right)$, such that $m(z) \geq 0$, is defined by

$$
\begin{gathered}
\int_{\mathbb{S}^{2}}\left(v-v^{\prime}\right) b\left(v-v_{*}, \sigma\right) d \sigma=m\left(v-v_{*}\right)\left(v-v_{*}\right) \\
\int_{\mathbb{S}^{2}}\left(\frac{|v|^{2}}{2}-\frac{\left|v^{\prime}\right|^{2}}{2}\right) b\left(v-v_{*}, \sigma\right) d \sigma=m\left(v-v_{*}\right)\left(\frac{|v|^{2}}{2}-\frac{\left|v_{*}\right|^{2}}{2}\right) .
\end{gathered}
$$

Clearly, it is defined as the average transfer of momentum and energy in any collision between any two particles having pre-collisional velocities $v \in \mathbb{R}^{3}$ and $v_{*} \in \mathbb{R}^{3}$. The following proposition guarantees that $m(z)$ is well-defined by the relations above.

Proposition A.1. Let

$$
m\left(v-v_{*}\right)=m\left(\left|v-v_{*}\right|\right)=\frac{1}{2} \int_{\mathbb{S}^{2}}(1-\cos \theta) b\left(\left|v-v_{*}\right|, \cos \theta\right) d \sigma,
$$

with $\cos \theta=\frac{v-v_{*}}{\left|v-v_{*}\right|} \cdot \sigma$.

It holds that

$$
\begin{gathered}
\int_{\mathbb{S}^{2}}\left(v-v^{\prime}\right) b\left(v-v_{*}, \sigma\right) d \sigma=m\left(v-v_{*}\right)\left(v-v_{*}\right), \\
\int_{\mathbb{S}^{2}}\left|v-v^{\prime}\right|^{2} b\left(v-v_{*}, \sigma\right) d \sigma=m\left(v-v_{*}\right)\left|v-v_{*}\right|^{2}, \\
\int_{\mathbb{S}^{2}}\left(|v|^{2}-\left|v^{\prime}\right|^{2}\right) b\left(v-v_{*}, \sigma\right) d \sigma=m\left(v-v_{*}\right)\left(|v|^{2}-\left|v_{*}\right|^{2}\right),
\end{gathered}
$$

and

$$
\begin{array}{rl}
\int_{\mathbb{R}^{3} \times \mathbb{R}^{3} \times \mathbb{S}^{2}}\left(v-v^{\prime}\right) \otimes\left(v-v^{\prime}\right) b & \left.v-v_{*}, \sigma\right) M M_{*} d v d v_{*} d \sigma \\
= & \frac{1}{3} \int_{\mathbb{R}^{3} \times \mathbb{R}^{3}} m\left(v-v_{*}\right)\left|v-v_{*}\right|^{2} M M_{*} d v d v_{*} \mathrm{Id} \\
\int_{\mathbb{R}^{3} \times \mathbb{R}^{3} \times \mathbb{S}^{2}}\left(v-v^{\prime}\right)\left(|v|^{2}-\left|v^{\prime}\right|^{2}\right) & b\left(v-v_{*}, \sigma\right) M M_{*} d v d v_{*} d \sigma \\
& =0 \\
\int_{\mathbb{R}^{3} \times \mathbb{R}^{3} \times \mathbb{S}^{2}}\left(|v|^{2}-\left|v^{\prime}\right|^{2}\right)^{2} & b\left(v-v_{*}, \sigma\right) M M_{*} d v d v_{*} d \sigma \\
& =\int_{\mathbb{R}^{3} \times \mathbb{R}^{3}} m\left(v-v_{*}\right)\left(|v|^{2}-\left|v_{*}\right|^{2}\right)^{2} M M_{*} d v d v_{*}
\end{array}
$$

Proof. Note first that, using the spherical symmetries of the cross-section $b$,

$$
b\left(v-v_{*}, \sigma\right)=b\left(v-v_{*}, \tilde{\sigma}\right),
$$

where

$$
\tilde{\sigma}=2\left(\sigma \cdot \frac{v-v_{*}}{\left|v-v_{*}\right|}\right) \frac{v-v_{*}}{\left|v-v_{*}\right|}-\sigma \in \mathbb{S}^{2} .
$$


Hence, we compute

$$
\begin{aligned}
\int_{\mathbb{S}^{2}}\left(v-v^{\prime}\right) b\left(v-v_{*}, \sigma\right) d \sigma & =\int_{\mathbb{S}^{2}}\left(\left(v-v^{\prime}\right) \cdot \frac{v-v_{*}}{\left|v-v_{*}\right|}\right) \frac{v-v_{*}}{\left|v-v_{*}\right|} b\left(v-v_{*}, \sigma\right) d \sigma \\
& =\frac{1}{2} \int_{\mathbb{S}^{2}}(1-\cos \theta)\left(v-v_{*}\right) b\left(v-v_{*}, \sigma\right) d \sigma
\end{aligned}
$$

Next, since $\left|v-v^{\prime}\right|^{2}=\frac{1}{2}(1-\cos \theta)\left|v-v_{*}\right|^{2}$, we easily find that

$$
\int_{\mathbb{S}^{2}}\left|v-v^{\prime}\right|^{2} b\left(v-v_{*}, \sigma\right) d \sigma=\frac{1}{2} \int_{\mathbb{S}^{2}}(1-\cos \theta)\left|v-v_{*}\right|^{2} b\left(v-v_{*}, \sigma\right) d \sigma .
$$

Further straightforward computations yield, employing the previous identities, that

$$
\begin{aligned}
\int_{\mathbb{S}^{2}}\left(|v|^{2}-\left|v^{\prime}\right|^{2}\right) b\left(v-v_{*}, \sigma\right) d \sigma & =\int_{\mathbb{S}^{2}}\left(2 v \cdot\left(v-v^{\prime}\right)-\left|v-v^{\prime}\right|^{2}\right) b\left(v-v_{*}, \sigma\right) d \sigma \\
& =m\left(v-v_{*}\right)\left(2 v \cdot\left(v-v_{*}\right)-\left|v-v_{*}\right|^{2}\right) \\
& =m\left(v-v_{*}\right)\left(|v|^{2}-\left|v_{*}\right|^{2}\right) .
\end{aligned}
$$

Finally, we obtain, using the pre-post-collisional change of variables and the previous identities,

$$
\begin{aligned}
\int_{\mathbb{R}^{3} \times \mathbb{R}^{3} \times \mathbb{S}^{2}} & \left(v-v^{\prime}\right) \otimes\left(v-v^{\prime}\right) b\left(v-v_{*}, \sigma\right) M M_{*} d v d v_{*} d \sigma \\
& =2 \int_{\mathbb{R}^{3} \times \mathbb{R}^{3} \times \mathbb{S}^{2}}\left(v-v^{\prime}\right) \otimes v b\left(v-v_{*}, \sigma\right) M M_{*} d v d v_{*} d \sigma \\
& =2 \int_{\mathbb{R}^{3} \times \mathbb{R}^{3}} m\left(v-v_{*}\right)\left(v-v_{*}\right) \otimes v M M_{*} d v d v_{*} \\
& =\int_{\mathbb{R}^{3} \times \mathbb{R}^{3}} m\left(v-v_{*}\right)\left(v-v_{*}\right) \otimes\left(v-v_{*}\right) M M_{*} d v d v_{*} \\
& =\frac{1}{3} \int_{\mathbb{R}^{3} \times \mathbb{R}^{3}} m\left(v-v_{*}\right)\left|v-v_{*}\right|^{2} M M_{*} d v d v_{*} \mathrm{Id} \\
& \left(v-v^{\prime}\right)\left(|v|^{2}-\left|v^{\prime}\right|^{2}\right) b\left(v-v_{*}, \sigma\right) M M_{*} d v d v_{*} d \sigma \\
& =2 \int_{\mathbb{R}^{3} \times \mathbb{R}^{3} \times \mathbb{S}^{2}}\left(v-v^{\prime}\right)|v|^{2} b\left(v-v_{*}, \sigma\right) M M_{*} d v d v_{*} d \sigma \\
& =2 \int_{\mathbb{R}^{3} \times \mathbb{R}^{3}} m\left(v-v_{*}\right)\left(v-v_{*}\right)|v|^{2} M M_{*} d v d v_{*} \\
& =\int_{\mathbb{R}^{3} \times \mathbb{R}^{3}} m\left(v-v_{*}\right)\left(v-v_{*}\right)\left(|v|^{2}-\left|v_{*}\right|^{2}\right) M M_{*} d v d v_{*} \\
& =0,
\end{aligned}
$$

and

$$
\begin{aligned}
\int_{\mathbb{R}^{3} \times \mathbb{R}^{3} \times \mathbb{S}^{2}} & \left(|v|^{2}-\left|v^{\prime}\right|^{2}\right)^{2} b\left(v-v_{*}, \sigma\right) M M_{*} d v d v_{*} d \sigma \\
& =2 \int_{\mathbb{R}^{3} \times \mathbb{R}^{3} \times \mathbb{S}^{2}}\left(|v|^{2}-\left|v^{\prime}\right|^{2}\right)|v|^{2} b\left(v-v_{*}, \sigma\right) M M_{*} d v d v_{*} d \sigma \\
& =2 \int_{\mathbb{R}^{3} \times \mathbb{R}^{3}} m\left(v-v_{*}\right)\left(|v|^{2}-\left|v_{*}\right|^{2}\right)|v|^{2} M M_{*} d v d v_{*} \\
& =\int_{\mathbb{R}^{3} \times \mathbb{R}^{3}} m\left(v-v_{*}\right)\left(|v|^{2}-\left|v_{*}\right|^{2}\right)^{2} M M_{*} d v d v_{*},
\end{aligned}
$$

which concludes the justification of the proposition. 


\section{APPENDIX B}

\section{Young inequalitites}

The use of generalized Young inequalities has been ubiquitous in the theory of hydrodynamic limits of the Boltzmann equation since its early treatment in $\mathbf{9}$. In its most general version, Young's inequality (also known as Fenchel-Young inequality or Fenchel's inequality, in this case) states that

$$
\langle z, y\rangle_{E, E^{*}} \leq f(z)+f^{*}(y)
$$

where $E$ is a real vector space, $E^{*}$ is its algebraic dual space, $f(z)$ is a real-valued functional defined on a domain $D \subset E$ and $f^{*}(y)$ is its Legendre transform (or Legendre-Fenchel transform) defined by

$$
f^{*}(y)=\sup _{z \in D}\left(\langle z, y\rangle_{E, E^{*}}-f(z)\right)
$$

on the dual domain

$$
D^{*}=\left\{y \in E^{*}: \sup _{z \in D}\left(\langle z, y\rangle_{E, E^{*}}-f(z)\right)<\infty\right\} .
$$

Note that $D^{*}$ is convex and that $f^{*}$ is lower semi-continuous and convex, for it is defined as the supremum of affine functions. Thus, the transform $f^{*}$ is also called the convex conjugate of $f$. Clearly, the inequality $(\mathrm{B} .1)$ is an obvious consequence of the definition $\mathrm{B} .2$.

The Young inequalities are fundamental in extracting useful information and controls from the entropy and the entropy dissipation bounds in $(2.6)$ and $(2.33)$. To this end, following $[\mathbf{9}$, we introduce the non-negative convex functions

$$
\begin{aligned}
& h(z)=(1+z) \log (1+z)-z, \\
& r(z)=z \log (1+z),
\end{aligned}
$$

defined over $z>-1$. Notice that $h(z) \leq r(z)$. In this notation, we may then recast the entropy as

$$
\frac{1}{\epsilon^{2}} H\left(f_{\epsilon}\right)=\frac{1}{\epsilon^{2}} H\left(f_{\epsilon} \mid M\right)=\int_{\mathbb{R}^{3} \times \mathbb{R}^{3}} \frac{1}{\epsilon^{2}} h\left(\epsilon g_{\epsilon}\right) M d x d v,
$$

where $f_{\epsilon}=M\left(1+\epsilon g_{\epsilon}\right)$, and the entropy dissipation as

$$
\frac{1}{\epsilon^{4}} D\left(f_{\epsilon}\right)=\frac{1}{4 \epsilon^{4}} \int_{\mathbb{R}^{3} \times \mathbb{R}^{3} \times \mathbb{S}^{2}} r\left(\frac{f_{\epsilon}^{\prime} f_{\epsilon *}^{\prime}}{f_{\epsilon} f_{\epsilon *}}-1\right) f_{\epsilon} f_{\epsilon *} b\left(v-v_{*}, \sigma\right) d v d v_{*} d \sigma .
$$

We recall now some useful properties, which are already found in $\mathbf{9}$, of the convex functions $h(z)$ and $r(z)$. Thus, we consider the Legendre transformations $h^{*}(y)$ and $r^{*}(y)$ of $h(z)$ and $r(z)$, respectively, well-defined for any $y \in \mathbb{R}$ by

$$
h^{*}(y)=e^{y}-1-y,
$$

and

$$
r^{*}(y)=\frac{z_{0}^{2}}{1+z_{0}}
$$


where $z_{0}>-1$ is the unique solution to $y=\log \left(1+z_{0}\right)+\frac{z_{0}}{1+z_{0}}$. Since $h(z) \leq r(z)$, notice that $h^{*}(y) \geq r^{*}(y)$. Then, of course, for any $z>-1$ and $y \in \mathbb{R}$, the Young inequalities hold :

$$
\begin{aligned}
& z y \leq h(z)+h^{*}(y)=[(1+z) \log (1+z)-z]+\left[e^{y}-1-y\right], \\
& z y \leq r(z)+r^{*}(y)=[z \log (1+z)]+r^{*}(y) .
\end{aligned}
$$

It is also possible to show that $r(z)$ and $h(z)$ satisfy the reflection inequalities, for any $z>-1$,

$$
\begin{aligned}
& h(|z|) \leq h(z), \\
& r(|z|) \leq r(z),
\end{aligned}
$$

and that $h^{*}(y)$ and $r^{*}(y)$ have the following exponential growth control, for any $y \geq 0$,

$$
\begin{aligned}
h^{*}(y) & \leq e^{y}, \\
r^{*}(y) & \leq e^{y},
\end{aligned}
$$

and the superquadratic homogeneity, for any $y \geq 0$ and $0 \leq \lambda \leq 1$,

$$
\begin{aligned}
& h^{*}(\lambda y) \leq \lambda^{2} h^{*}(y), \\
& r^{*}(\lambda y) \leq \lambda^{2} r^{*}(y),
\end{aligned}
$$

which is easily obtained by proving that $\lambda \mapsto \frac{h^{*}(\lambda y)}{\lambda^{2}}$ and $\lambda \mapsto \frac{r^{*}(\lambda y)}{\lambda^{2}}$ are increasing functions.

Thus, combining the above properties we arrive at the most useful inequalities

$$
\begin{gathered}
|z y \beta| \leq \frac{\alpha}{\epsilon^{2}}\left(h(\epsilon|z|)+h^{*}\left(\frac{\epsilon|\beta y|}{\alpha}\right)\right) \leq \frac{\alpha}{\epsilon^{2}} h(\epsilon z)+\frac{\beta^{2}}{\alpha} e^{|y|}, \\
\quad \text { for any } \quad z>-\frac{1}{\epsilon}, \quad y \in \mathbb{R}, \quad \alpha \geq \epsilon|\beta|>0,
\end{gathered}
$$

and

$$
\begin{gathered}
|z y \beta| \leq \frac{\alpha}{\epsilon^{4}}\left(r\left(\epsilon^{2}|z|\right)+r^{*}\left(\frac{\epsilon^{2}|\beta y|}{\alpha}\right)\right) \leq \frac{\alpha}{\epsilon^{4}} r\left(\epsilon^{2} z\right)+\frac{\beta^{2}}{\alpha} e^{|y|}, \\
\text { for any } z>-\frac{1}{\epsilon^{2}}, \quad y \in \mathbb{R}, \quad \alpha \geq \epsilon^{2}|\beta|>0 .
\end{gathered}
$$

The above Young inequalities $\mathrm{B} .3$ and $\mathrm{B} .4$ are intensively used throughout this work to extract bounds and compactness properties from the various entropy and entropy dissipation bounds.

Finally, for reference, we list some elementary inequalities in connection with the convex functions $h(z)$ and $r(z)$ and their corresponding Young inequalities :

- For every $z>-1$, it holds that

$$
(\sqrt{1+z}-1)^{2} \leq h(z),
$$

which follows straightforwardly from the identity, for all $z>-1$,

$$
(\sqrt{1+z}-1)^{2}+2 h(\sqrt{1+z}-1) \sqrt{1+z}=h(z),
$$

or by showing that the function defined on $z>-1$ by

$$
f(z)= \begin{cases}\frac{h(z)}{(\sqrt{1+z}-1)^{2}} & \text { if } z \neq 0, \\ 2 & \text { if } z=0\end{cases}
$$

is increasing and reaches the value 1 as $z \rightarrow-1$. 
- For every $z>-1$, it holds that

$$
\frac{1}{2} z^{2} \leq h(z)+\frac{1}{6} z^{3}
$$

which is obtained by integrating twice the elementary inequality

$$
1 \leq \frac{1}{1+z}+z=h^{\prime \prime}(z)+z,
$$

valid for every $z>-1$.

- For every $z>-1$, it holds that

$$
(\sqrt{1+z}-1)^{2} \leq|z|
$$

which is a consequence of the direct computation

$$
|z|-(\sqrt{1+z}-1)^{2}= \begin{cases}2(\sqrt{1+z}-1) & \text { if } z \geq 0 \\ 2 \sqrt{1+z}(1-\sqrt{1+z}) & \text { if } z \leq 0\end{cases}
$$

- For every $z>-1$, it holds that

$$
(\sqrt{1+z}-1)^{2} \leq \frac{1}{4} r(z)
$$

which follows straightforwardly by integrating twice the inequality

$$
\frac{1}{(1+z)^{\frac{3}{2}}} \leq \frac{1}{2}\left(\frac{1}{1+z}+\frac{1}{(1+z)^{2}}\right) .
$$

- For every $z>-1$ and $y \in \mathbb{R}$, it holds that

$$
\begin{aligned}
z y & =z y \mathbb{1}_{\{y \geq 0, y \leq \log (1+z)\} \cup\{y<0, y>\log (1+z)\}} \\
& +z y \mathbb{1}_{\left\{y \geq 0, z<e^{y}-1\right\} \cup\left\{y<0, z \geq e^{y}-1\right\}} \\
& \leq z \log (1+z)+\left(e^{y}-1\right) y=r(z)+\left(e^{y}-1\right) y .
\end{aligned}
$$

This implies, in particular, that $r^{*}(y) \leq\left(e^{y}-1\right) y$, for every $y \in \mathbb{R}$. 



\section{APPENDIX C}

\section{End of proof of Lemma 7.7 on hypoelliptic transfer of compactness}

The justification of Lemma 7.7 has not been fully completed in Chapter 7 lest it become unclear and tedious. Instead, we complete it now with a full justification of Lemma 7.7. when the equi-integrability of $\left\{\phi_{\epsilon}(t, x, v)\right\}_{\epsilon>0}$ is not known a priori and when each $\phi_{\epsilon}$ is non-negative, based on the proof of Theorem 2.4 from [6. Recall that, according to the partial proof following the statement of Lemma 7.7. it is sufficient in this case to establish the equi-integrability of $\left\{\phi_{\epsilon}(t, x, v)\right\}_{\epsilon>0}$ in all variables.

For convenience of the reader, we first recall the precise result which we are about to justify.

Lemma C.1. Let the bounded family of non-negative functions

$$
\left\{\phi_{\epsilon}(t, x, v)\right\}_{\epsilon>0} \subset L^{1}\left(\mathbb{R}_{t} \times \mathbb{R}_{x}^{3} ; L^{r}\left(\mathbb{R}_{v}^{3}\right)\right),
$$

for some $1<r<\infty$, be locally relatively compact in $v$ and such that

$$
\left(\epsilon \partial_{t}+v \cdot \nabla_{x}\right) \phi_{\epsilon}=\left(1-\Delta_{x}\right)^{\frac{\beta}{2}}\left(1-\Delta_{v}\right)^{\frac{\alpha}{2}} S_{\epsilon},
$$

for all $\epsilon>0$ and for some bounded family

$$
\left\{S_{\epsilon}(t, x, v)\right\}_{\epsilon>0} \subset L^{1}\left(\mathbb{R}_{t} \times \mathbb{R}_{x}^{3} ; L^{r}\left(\mathbb{R}_{v}^{3}\right)\right),
$$

where $\alpha \geq 0$ and $0 \leq \beta<1$. We further assume that, for any compact set $K \subset$ $\mathbb{R}^{3} \times \mathbb{R}^{3}$,

$$
\left.\left\{\int_{K} \phi_{\epsilon}(t, x, v) d x d v\right\}_{\epsilon>0} \quad \text { is equi-integrable (in } t\right) .
$$

Then, $\left\{\phi_{\epsilon}(t, x, v)\right\}_{\epsilon>0}$ is equi-integrable (in all variables).

We advise the reader of the difficulty to grasp the full content of the proof below without any prior knowledge of the work from [6]. Therefore, we suggest that this appendix be read in parallel with the article [6].

Proof. We first notice, repeating the proof of Lemma 3.1 from $[\mathbf{6}$, that we have the following interpolation formula (compare with (5.17) in $\mathbf{6}$ ), for any $R>0$ and $\delta>0$, denoting by $(\tau, \eta, \xi)$ the Fourier variables of $(t, x, v)$ and by $\mathcal{F}$ the Fourier 
transforms :

(C.1)

$$
\begin{aligned}
{\left[\mathcal{F}_{x, v}^{-1}(1-\chi)\right.} & \left.\left(\frac{\eta}{R}\right) \chi\left(\frac{\xi}{\lambda}\right) \mathcal{F}_{x, v}\right] \phi_{\epsilon}(t, x, v) \\
& =\frac{1}{(2 \pi)^{3}} \int_{\mathbb{R} \times \mathbb{R}^{3} \times \mathbb{R}^{3}} \lambda^{3} \hat{\chi}(\lambda(u-v)) K_{1}(t-s, x-y, u) \\
& {\left[\mathcal{F}_{x, v}^{-1}(1-\chi)\left(\frac{\eta}{R}\right) \psi\left(\frac{\xi}{\lambda}\right) \mathcal{F}_{x, v}\right] \phi_{\epsilon}(s, y, u) d s d y d u } \\
& -\frac{2 \delta i}{(2 \pi)^{3}} \int_{\mathbb{R} \times \mathbb{R}^{3} \times \mathbb{R}^{3}} \lambda^{3} \hat{\chi}(\lambda(u-v)) K_{2}(t-s, x-y, u) \\
& {\left[\mathcal{F}_{x, v}^{-1}(1-\chi)\left(\frac{\eta}{R}\right) R^{1-\beta} \frac{\langle\eta\rangle^{\beta}}{|\eta|} \chi\left(\frac{\xi}{10 \lambda}\right) \frac{\langle\xi\rangle^{\alpha}}{\lambda^{\alpha}} \mathcal{F}_{x, v}\right] S_{\epsilon}(s, y, u) d s d y d u, }
\end{aligned}
$$

where $\lambda=\delta^{\frac{1}{1+\alpha}} R^{\frac{1-\beta}{1+\alpha}}, \chi, \psi \in C_{c}^{\infty}\left(\mathbb{R}^{3}\right)$ are cutoff functions such that $\mathbb{1}_{\left\{|r| \leq \frac{1}{2}\right\}} \leq$ $\chi(r) \leq \mathbb{1}_{\{|r| \leq 1\}}$ and $\mathbb{1}_{\{1 \leq|r| \leq 5\}} \leq \psi(r) \leq \mathbb{1}_{\left\{\frac{1}{2} \leq|r| \leq \frac{11}{2}\right\}}$, and the singular kernels $K_{i}$, $i=1,2$, are defined by

$$
\begin{aligned}
& K_{1}(t, x, u)=\mathcal{F}_{t, x}^{-1}\left[\rho\left(\frac{2 \lambda}{|\eta|}(\eta \cdot u+\epsilon \tau)\right)\right], \\
& K_{2}(t, x, u)=\mathcal{F}_{t, x}^{-1}\left[\gamma\left(\frac{2 \lambda}{|\eta|}(\eta \cdot u+\epsilon \tau)\right)\right],
\end{aligned}
$$

where $\rho \in \mathcal{S}(\mathbb{R})$ is such that $\rho(0)=\frac{1}{2 \pi} \int_{\mathbb{R}} \hat{\rho}(\sigma) d \sigma=1$ and $\operatorname{supp} \hat{\rho} \subset\{1 \leq|\sigma| \leq 2\}$, and $\gamma(r)=\frac{1-\rho(r)}{r}$ for all $r \in \mathbb{R}$.

The rather deep meaning of the above interpolation formula (C.1) resides in the presence of the frequency cutoff functions $\psi\left(\frac{\xi}{\lambda}\right)$ and $\chi\left(\frac{\xi}{10 \lambda}\right)$ in its right-hand side, which is only possible through a precise analysis of dispersive and hypoelliptic phenomena, i.e. the transport of frequencies, in the kinetic transport equation. This requires that the support of $\hat{\rho}(\sigma)$ be restricted to $\{1 \leq|\sigma| \leq 2\}$, though.

Now, we claim that the mappings

$$
f(t, x, v) \mapsto \frac{1}{(2 \pi)^{3}} \int_{\mathbb{R} \times \mathbb{R}^{3} \times \mathbb{R}^{3}} \lambda^{3} \hat{\chi}(\lambda(u-v)) K_{i}(t-s, x-y, u) f(s, y, u) d s d y d u,
$$

with $i=1,2$, have bounded extensions between

$$
L^{1}\left(\mathbb{R}_{t} \times \mathbb{R}_{x}^{3} ; L^{r}\left(\mathbb{R}_{v}^{3}\right)\right) \longrightarrow L^{r}\left(\mathbb{R}_{v}^{3} ; L^{1, \infty}\left(\mathbb{R}_{t} \times \mathbb{R}_{x}^{3}\right)\right)
$$

As shown in [6], this boundedness follows from an application of Theorem 5.2 therein (or a very slight variant of it allowing different dimensions for different variables, i.e. $(t, x) \in \mathbb{R}^{4}$ and $\left.v \in \mathbb{R}^{3}\right)$ provided we establish that

$$
\begin{aligned}
& \sup _{v \in \mathbb{R}^{3}} \sup _{(s, y) \neq 0} \int_{\{|(t, x)| \geq 2|(s, y)|\}} \\
& \left|A_{v} \sharp K_{i}(t-s, x-y, u)-A_{v} \sharp K_{i}(t, x, u)\right| d t d x \|_{L^{r^{\prime}\left(\left|\lambda^{3} \hat{\chi}(\lambda(u-v))\right| d u\right)}}<\infty,
\end{aligned}
$$

where $A_{v} \sharp K_{i}(t, x, u)=\left|\operatorname{det} A_{v}\right| K_{i}\left(A_{v}(t, x), u\right)$, for some family of automorphisms $A_{v}$ of $\mathbb{R} \times \mathbb{R}^{3}$ fixing the origin (the remaining hypotheses of Theorem 5.2 from 6 being easily verified through standard arguments from the analysis of Fourier multipliers). Here, we will consider, for each $v \in \mathbb{R}^{3}$, the automorphism $A_{v}$ of 
$\mathbb{R} \times \mathbb{R}^{3}$ defined by

$$
A_{v}(t, x)=(\lambda \epsilon t, x+\lambda t v)
$$

so that

$$
\begin{aligned}
& A_{v} \sharp K_{1}(t, x, u)=\mathcal{F}_{t, x}^{-1}\left[\rho\left(2 \lambda \frac{\eta \cdot(u-v)}{|\eta|}+2 \frac{\tau}{|\eta|}\right)\right], \\
& A_{v} \sharp K_{2}(t, x, u)=\mathcal{F}_{t, x}^{-1}\left[\gamma\left(2 \lambda \frac{\eta \cdot(u-v)}{|\eta|}+2 \frac{\tau}{|\eta|}\right)\right] .
\end{aligned}
$$

In turn, the estimate C.4 is established employing classical methods from harmonic analysis (in 6], the corresponding step is performed in Lemmas 5.3 and 5.4). Thus, it can be shown that C.4 will hold as soon as the following HörmanderMikhlin condition for homogeneous Fourier multipliers is verified :

$$
\int_{1<|(\tau, \eta)|<2}\left|\partial_{\tau, \eta}^{\nu} \mathcal{F}_{t, x}\left(A_{v} \sharp K_{i}\right)(\tau, \eta, u)\right|^{2} d \tau d \eta \leq C(1+\lambda|u-v|)^{N},
$$

for some independent constant $C>0$ and some possibly very large $N \in \mathbb{N}$, and for any multi-index $\nu \in \mathbb{N}^{4}$ such that $\nu_{1} \in\{0,1\}$ and $0 \leq \nu_{2}+\nu_{3}+\nu_{4} \leq 2$.

The preceding control is easily verified for $K_{1}$ through a straightforward calculation using that $\rho(r)$ decays rapidly. As for $K_{2}$, this step requires some greater care because $\gamma^{(n)}(r)$ only decays as $\frac{1}{|r|^{n+1}}$ for large values of $r$, for all $n \in \mathbb{N}$. Nevertheless, it is also straightforwardly verified upon noticing, for any multi-index $\nu$ as before, that

$$
\begin{aligned}
|(\tau, \eta)|^{|\nu|} \mid & \partial_{\tau, \eta}^{\nu}\left[\gamma\left(2 \lambda \frac{\eta \cdot(u-v)}{|\eta|}+2 \frac{\tau}{|\eta|}\right)\right] \mid \\
& \leq C \sum_{n=0}^{3}(1+\lambda|u-v|)^{n}\left(1+\frac{|\tau|}{|\eta|}\right)^{n+2}\left|\gamma^{(n)}\left(2 \lambda \frac{\eta \cdot(u-v)}{|\eta|}+2 \frac{\tau}{|\eta|}\right)\right| \\
& \leq C\left(1+\frac{|\tau|}{|\eta|}\right) \sum_{n=0}^{3}(1+\lambda|u-v|)^{2 n+1} \\
& \left|\left(1+\left|\lambda \frac{\eta \cdot(u-v)}{|\eta|}+\frac{\tau}{|\eta|}\right|\right)^{n+1} \gamma^{(n)}\left(2 \lambda \frac{\eta \cdot(u-v)}{|\eta|}+2 \frac{\tau}{|\eta|}\right)\right| \\
& \leq C\left(1+\frac{|\tau|}{|\eta|}\right)(1+\lambda|u-v|)^{7} \leq \frac{C}{|\eta|}(1+\lambda|u-v|)^{7},
\end{aligned}
$$

which implies that C.5 holds with $N=14$, for $\frac{1}{|\eta|^{2}}$ is locally integrable in $\mathbb{R}^{3}$.

So far, we have thus established the weak type boundedness on the spaces (C.3) of the mappings (C.2). Therefore, we conclude from the interpolation formula (C.1) that

$$
\begin{aligned}
\left\|\left[\mathcal{F}_{x}^{-1}(1-\chi)\left(\frac{\eta}{R}\right) \mathcal{F}_{x}\right] \phi_{\epsilon}\right\|_{L_{v}^{r} L_{t, x}^{1, \infty}} & \leq C\left\|\left[\mathcal{F}_{v}^{-1}(1-\chi)\left(\frac{\xi}{\lambda}\right) \mathcal{F}_{v}\right] \phi_{\epsilon}\right\|_{L_{t, x}^{1} L_{v}^{r}} \\
& +C\left\|\left[\mathcal{F}_{v}^{-1} \psi\left(\frac{\xi}{\lambda}\right) \mathcal{F}_{v}\right] \phi_{\epsilon}\right\|_{L_{t, x}^{1} L_{v}^{r}} \\
& +C \delta\left\|S_{\epsilon}\right\|_{L_{t, x}^{1} L_{v}^{r}}
\end{aligned}
$$

whence, since the $\phi_{\epsilon}$ 's are relatively compact in $v$, since $\lim _{R \rightarrow \infty} \lambda=\infty$ and by the arbitrariness of $\delta>0$,

$$
\lim _{R \rightarrow \infty} \sup _{\epsilon>0}\left\|\left[\mathcal{F}_{x}^{-1}(1-\chi)\left(\frac{\eta}{R}\right) \mathcal{F}_{x}\right] \phi_{\epsilon}\right\|_{L_{v}^{r} L_{t, x}^{1, \infty}}=0 .
$$


Next, applying spatial Riesz transforms $f \mapsto \mathcal{F}_{x}^{-1} \frac{\eta_{i}}{|\eta|} \mathcal{F}_{x} f, i=1,2,3$, to the identity C.1 and repeating the preceding arguments, we deduce that it also holds

$$
\lim _{R \rightarrow \infty} \sup _{\epsilon>0}\left\|\left[\mathcal{F}_{x}^{-1}(1-\chi)\left(\frac{\eta}{R}\right) \frac{\eta_{i}}{|\eta|} \mathcal{F}_{x}\right] \phi_{\epsilon}\right\|_{L_{v}^{r} L_{t, x}^{1, \infty}}=0 .
$$

Consequently, we obtain that the $\phi_{\epsilon}$ 's are relatively compact in $x$ in the quasiBanach space defined by the quasi-norm

$$
\|f\|_{L_{v}^{r} L_{t, x}^{1, \infty}}+\sum_{i=1}^{3}\left\|\mathcal{F}_{x}^{-1} \frac{\eta_{i}}{|\eta|} \mathcal{F}_{x} f\right\|_{L_{v}^{r} L_{t, x}^{1, \infty}},
$$

which can be shown, repeating standard (but difficult) arguments from the analysis of weak Hardy spaces (see [6] and the references therein for details), to be equivalent to the quasi-norm

$$
\left\|\sup _{s>0}\left|\varphi_{s} *_{x} f\right|\right\|_{L_{v}^{r} L_{t, x}^{1, \infty}},
$$

where $\varphi(x) \in C_{c}^{\infty}\left(\mathbb{R}^{3}\right)$ is such that $\int_{\mathbb{R}^{3}} \varphi(x) d x \neq 0$ and $\varphi_{s}(x)=\frac{1}{s^{3}} \varphi\left(\frac{x}{s}\right)$.

Finally, following 6, we explain how the ensuing spatial compactness in the topology given by the quasi-norm C.8 is sufficient to entail the equi-integrability of the $\phi_{\epsilon}$ 's, provided they are non-negative. To this end, we note first, for every $a, b>0$ and any compact subset $K \subset \mathbb{R} \times \mathbb{R}^{3} \times \mathbb{R}^{3}$, that

$$
\begin{aligned}
& \int_{K \cap\left\{\left|\phi_{\epsilon}\right|>a\right\}}\left|\phi_{\epsilon}(t, x, v)\right| d t d x d v \\
& \leq \int_{K \cap\left\{\left|\phi_{\epsilon}\right|>a,\left\|f_{\lambda}\right\|_{L_{x}^{1}} \leq b\right\}}\left|\phi_{\epsilon}(t, x, v)\right| d t d x d v+\int_{\left\{\left\|\phi_{\epsilon}\right\|_{L_{x}^{1}}>b\right\}}\left\|\mathbb{1}_{K} \phi_{\epsilon}(t, x, v)\right\|_{L_{x}^{1}} d t d v .
\end{aligned}
$$

Clearly, in view of the equi-integrability hypotheses, the family $\left\|\phi_{\epsilon}(t, x, v)\right\|_{L_{x}^{1}}$ is locally equi-integrable in $t$ and $v$, so that the last term above can be made uniformly small by choosing $b>0$ arbitrarily large.

Thus, the proof will be finished upon showing that, for each fixed $b>0$ and every compact subset $K \subset \mathbb{R} \times \mathbb{R}^{3} \times \mathbb{R}^{3}$,

$$
\lim _{a \rightarrow \infty} \sup _{\epsilon>0} \int_{\left\{(t, x, v) \in K:\left|\phi_{\epsilon}\right|>a,\left\|\phi_{\epsilon}\right\|_{L_{x}^{1}} \leq b\right\}}\left|\phi_{\epsilon}(t, x, v)\right| d t d x d v=0,
$$

which, following the arguments from the end of the proof of Theorem 2.4 in 6 based on Proposition 5.5 therein (which is only valid for non-negative functions), is a direct consequence of the compactness estimates (C.6) and (C.7).

The justification of the lemma is thus complete. 


\section{Bibliography}

[1] R. Alexandre and C. Villani. On the Boltzmann equation for long-range interactions. Comm. Pure Appl. Math., 55(1):30-70, 2002.

[2] Luigi Ambrosio. Transport equation and Cauchy problem for BV vector fields. Invent. Math., 158(2):227-260, 2004.

[3] Diogo Arsénio. On the global existence of mild solutions to the Boltzmann equation for small data in $L^{D}$. Comm. Math. Phys., 302(2):453-476, 2011.

[4] Diogo Arsénio. From Boltzmann's Equation to the Incompressible Navier-Stokes-Fourier System with Long-Range Interactions. Arch. Ration. Mech. Anal., 206(2):367-488, 2012.

[5] Diogo Arsénio, Slim Ibrahim, and Nader Masmoudi. A derivation of the magnetohydrodynamic system from Navier-Stokes-Maxwell systems. Arch. Ration. Mech. Anal., 216(3):767$812,2015$.

[6] Diogo Arsénio and Laure Saint-Raymond. Compactness in kinetic transport equations and hypoellipticity. J. Funct. Anal., 261(10):3044-3098, 2011.

[7] Diogo Arsénio and Laure Saint-Raymond. Solutions of the Vlasov-Maxwell-Boltzmann system with long-range interactions. C. R. Math. Acad. Sci. Paris, 351(9-10):357-360, 2013.

[8] Jean-Pierre Aubin. Un théorème de compacité. C. R. Acad. Sci. Paris, 256:5042-5044, 1963.

[9] Claude Bardos, François Golse, and C. David Levermore. Fluid dynamic limits of kinetic equations. II. Convergence proofs for the Boltzmann equation. Comm. Pure Appl. Math., 46(5):667-753, 1993

[10] Claude Bardos, François Golse, and David Levermore. Fluid dynamic limits of kinetic equations. I. Formal derivations. J. Statist. Phys., 63(1-2):323-344, 1991.

[11] F. Bouchut. Hypoelliptic regularity in kinetic equations. J. Math. Pures Appl. (9), 81(11):1135-1159, 2002.

[12] François Bouchut and Laurent Desvillettes. A proof of the smoothing properties of the positive part of Boltzmann's kernel. Rev. Mat. Iberoamericana, 14(1):47-61, 1998.

[13] François Bouchut, François Golse, and Mario Pulvirenti. Kinetic equations and asymptotic theory, volume 4 of Series in Applied Mathematics (Paris). Gauthier-Villars, Éditions Scientifiques et Médicales Elsevier, Paris, 2000. Edited and with a foreword by Benoit Perthame and Laurent Desvillettes.

[14] Yann Brenier. Convergence of the Vlasov-Poisson system to the incompressible Euler equations. Comm. Partial Differential Equations, 25(3-4):737-754, 2000.

[15] Yann Brenier, Camillo De Lellis, and László Székelyhidi, Jr. Weak-strong uniqueness for measure-valued solutions. Comm. Math. Phys., 305(2):351-361, 2011.

[16] Tristan Buckmaster. Onsager's conjecture almost everywhere in time. Comm. Math. Phys., 333(3):1175-1198, 2015

[17] Tristan Buckmaster, Camillo De Lellis, Philip Isett, and László Székelyhidi, Jr. Anomalous dissipation for 1/5-Hölder Euler flows. Ann. of Math. (2), 182(1):127-172, 2015.

[18] Tristan Buckmaster, Camillo De Lellis, and László Székelyhidi, Jr. Dissipative Euler flows with Onsager-critical spatial regularity. arXiv.org, math.AP, april 2014.

[19] Carlo Cercignani. The Boltzmann equation and its applications, volume 67 of Applied Mathematical Sciences. Springer-Verlag, New York, 1988

[20] Carlo Cercignani, Reinhard Illner, and Mario Pulvirenti. The mathematical theory of dilute gases, volume 106 of Applied Mathematical Sciences. Springer-Verlag, New York, 1994.

[21] A. Cheskidov, P. Constantin, S. Friedlander, and R. Shvydkoy. Energy conservation and Onsager's conjecture for the Euler equations. Nonlinearity, 21(6):1233-1252, 2008.

[22] Antoine Choffrut, Camillo De Lellis, and László Székelyhidi, Jr. Dissipative continuous Euler flows in two and three dimensions. arXiv.org, math.AP, May 2012.

[23] Peter Constantin, Weinan E, and Edriss S. Titi. Onsager's conjecture on the energy conservation for solutions of Euler's equation. Comm. Math. Phys., 165(1):207-209, 1994.

[24] C. M. Dafermos. The second law of thermodynamics and stability. Arch. Rational Mech. Anal., 70(2):167-179, 1979 
[25] Camillo De Lellis and László Székelyhidi, Jr. On admissibility criteria for weak solutions of the Euler equations. Arch. Ration. Mech. Anal., 195(1):225-260, 2010.

[26] Camillo De Lellis and László Székelyhidi, Jr. Dissipative continuous Euler flows. Invent. Math., 193(2):377-407, 2013.

[27] Camillo De Lellis and László Székelyhidi, Jr. Dissipative Euler flows and Onsager's conjecture. J. Eur. Math. Soc. (JEMS), 16(7):1467-1505, 2014.

[28] L. Desvillettes and F. Golse. A remark concerning the Chapman-Enskog asymptotics. In Advances in kinetic theory and computing, volume 22 of Ser. Adv. Math. Appl. Sci., pages 191-203. World Sci. Publ., River Edge, NJ, 1994.

[29] R. J. DiPerna and P.-L. Lions. Global weak solutions of Vlasov-Maxwell systems. Comm. Pure Appl. Math., 42(6):729-757, 1989.

[30] R. J. DiPerna and P.-L. Lions. On the Cauchy problem for Boltzmann equations: global existence and weak stability. Ann. of Math. (2), 130(2):321-366, 1989

[31] R. J. DiPerna and P.-L. Lions. Ordinary differential equations, transport theory and Sobolev spaces. Invent. Math., 98(3):511-547, 1989.

[32] R. J. DiPerna and P.-L. Lions. Global solutions of Boltzmann's equation and the entropy inequality. Arch. Rational Mech. Anal., 114(1):47-55, 1991.

[33] R. J. DiPerna, P.-L. Lions, and Y. Meyer. $L^{p}$ regularity of velocity averages. Ann. Inst. H. Poincaré Anal. Non Linéaire, 8(3-4):271-287, 1991.

[34] Ronald J. DiPerna and Andrew J. Majda. Oscillations and concentrations in weak solutions of the incompressible fluid equations. Comm. Math. Phys., 108(4):667-689, 1987.

[35] Hiroshi Fujita and Tosio Kato. On the Navier-Stokes initial value problem. I. Arch. Rational Mech. Anal., 16:269-315, 1964.

[36] Pierre Germain, Slim Ibrahim, and Nader Masmoudi. Well-posedness of the Navier-StokesMaxwell equations. Proc. Roy. Soc. Edinburgh Sect. A, 144(1):71-86, 2014.

[37] François Golse and Laure Saint-Raymond. Velocity averaging in $L^{1}$ for the transport equation. C. R. Math. Acad. Sci. Paris, 334(7):557-562, 2002.

[38] François Golse and Laure Saint-Raymond. The incompressible Navier-Stokes limit of the Boltzmann equation for hard cutoff potentials. J. Math. Pures Appl. (9), 91(5):508-552, 2009.

[39] Harold Grad. On the kinetic theory of rarefied gases. Comm. Pure Appl. Math., 2:331-407, 1949.

[40] Harold Grad. Principles of the kinetic theory of gases. In Handbuch der Physik (herausgegeben von S. Flügge), Bd. 12, Thermodynamik der Gase, pages 205-294. Springer-Verlag, BerlinGöttingen-Heidelberg, 1958.

[41] Yan Guo. The Vlasov-Maxwell-Boltzmann system near Maxwellians. Invent. Math., 153(3):593-630, 2003.

[42] David Hilbert. Begründung der kinetischen Gastheorie. Math. Ann., 72(4):562-577, 1912.

[43] Slim Ibrahim and Sahbi Keraani. Global small solutions for the Navier-Stokes-Maxwell system. SIAM J. Math. Anal., 43(5):2275-2295, 2011.

[44] Slim Ibrahim and Tsuyoshi Yoneda. Local solvability and loss of smoothness of the NavierStokes-Maxwell equations with large initial data. J. Math. Anal. Appl., 396(2):555-561, 2012.

[45] John David Jackson. Classical electrodynamics. John Wiley \& Sons Inc., New York, second edition, 1975.

[46] Juhi Jang and Nader Masmoudi. Derivation of Ohm's law from the kinetic equations. SIAM J. Math. Anal., 44(5):3649-3669, 2012.

[47] C. Le Bris and P.-L. Lions. Renormalized solutions of some transport equations with partially $W^{1,1}$ velocities and applications. Ann. Mat. Pura Appl. (4), 183(1):97-130, 2004.

[48] Jean Leray. Sur le mouvement d'un liquide visqueux emplissant l'espace. Acta Math., 63(1):193-248, 1934

[49] C. David Levermore and Weiran Sun. Compactness of the gain parts of the linearized Boltzmann operator with weakly cutoff kernels. Kinet. Relat. Models, 3(2):335-351, 2010

[50] J.-L. Lions. Équations différentielles opérationnelles et problèmes aux limites. Die Grundlehren der mathematischen Wissenschaften, Bd. 111. Springer-Verlag, Berlin, 1961.

[51] P.-L. Lions. Compactness in Boltzmann's equation via Fourier integral operators and applications. I. J. Math. Kyoto Univ., 34(2):391-427, 1994.

[52] P.-L. Lions. Compactness in Boltzmann's equation via Fourier integral operators and applications. II. J. Math. Kyoto Univ., 34(2):429-461, 1994.

[53] P.-L. Lions. Compactness in Boltzmann's equation via Fourier integral operators and applications. III. J. Math. Kyoto Univ., 34(3):539-584, 1994.

[54] P.-L. Lions. Conditions at infinity for Boltzmann's equation. Comm. Partial Differential Equations, 19(1-2):335-367, 1994. 
[55] P.-L. Lions and N. Masmoudi. From the Boltzmann equations to the equations of incompressible fluid mechanics. I, II. Arch. Ration. Mech. Anal., 158(3):173-193, 195-211, 2001.

[56] P.-L. Lions and N. Masmoudi. Uniqueness of mild solutions of the Navier-Stokes system in $L^{N}$. Comm. Partial Differential Equations, 26(11-12):2211-2226, 2001.

[57] Pierre-Louis Lions. Mathematical topics in fluid mechanics. Vol. 1, volume 3 of Oxford Lecture Series in Mathematics and its Applications. The Clarendon Press Oxford University Press, New York, 1996. Incompressible models, Oxford Science Publications.

[58] Nader Masmoudi. Global well posedness for the Maxwell-Navier-Stokes system in 2D. J. Math. Pures Appl. (9), 93(6):559-571, 2010.

[59] Nader Masmoudi and Laure Saint-Raymond. From the Boltzmann equation to the StokesFourier system in a bounded domain. Comm. Pure Appl. Math., 56(9):1263-1293, 2003.

[60] Stéphane Mischler. On the initial boundary value problem for the Vlasov-Poisson-Boltzmann system. Comm. Math. Phys., 210(2):447-466, 2000.

[61] Stéphane Mischler. Kinetic equations with Maxwell boundary conditions. Ann. Sci. Éc. Norm. Supér. (4), 43(5):719-760, 2010.

[62] D.C. Montgomery and D.A. Tidman. Plasma Kinetic Theory. McGraw-Hill advanced physics monograph series. McGraw-Hill, 1964

[63] François Murat. Compacité par compensation. Ann. Scuola Norm. Sup. Pisa Cl. Sci. (4), $5(3): 489-507,1978$.

[64] François Murat. Compacité par compensation: condition nécessaire et suffisante de continuité faible sous une hypothèse de rang constant. Ann. Scuola Norm. Sup. Pisa Cl. Sci. (4), 8(1):69-102, 1981.

[65] Marjolaine Puel and Laure Saint-Raymond. Quasineutral limit for the relativistic VlasovMaxwell system. Asymptot. Anal., 40(3-4):303-352, 2004.

[66] H.L. Royden and P.M. Fitzpatrick. Real Analysis. Prentice Hall PTR, 2010.

[67] Laure Saint-Raymond. Convergence of solutions to the Boltzmann equation in the incompressible Euler limit. Arch. Ration. Mech. Anal., 166(1):47-80, 2003.

[68] Laure Saint-Raymond. Hydrodynamic limits of the Boltzmann equation, volume 1971 of Lecture Notes in Mathematics. Springer-Verlag, Berlin, 2009.

[69] Laure Saint-Raymond. Hydrodynamic limits: some improvements of the relative entropy method. Ann. Inst. H. Poincaré Anal. Non Linéaire, 26(3):705-744, 2009.

[70] A. Shnirelman. Weak solutions with decreasing energy of incompressible Euler equations. Comm. Math. Phys., 210(3):541-603, 2000.

[71] Jacques Simon. Compact sets in the space $L^{p}(0, T ; B)$. Ann. Mat. Pura Appl. (4), 146:65-96, 1987.

[72] Michael Struwe. Variational methods, volume 34 of Ergebnisse der Mathematik und ihrer Grenzgebiete. 3. Folge. A Series of Modern Surveys in Mathematics [Results in Mathematics and Related Areas. 3rd Series. A Series of Modern Surveys in Mathematics]. SpringerVerlag, Berlin, fourth edition, 2008. Applications to nonlinear partial differential equations and Hamiltonian systems.

[73] L. Tartar. Compensated compactness and applications to partial differential equations. In Nonlinear analysis and mechanics: Heriot-Watt Symposium, Vol. IV, volume 39 of Res. Notes in Math., pages 136-212. Pitman, Boston, Mass., 1979.

[74] Cédric Villani. A review of mathematical topics in collisional kinetic theory. In Handbook of mathematical fluid dynamics, Vol. I, pages 71-305. North-Holland, Amsterdam, 2002.

[75] Bernt Wennberg. Regularity in the Boltzmann equation and the Radon transform. Comm. Partial Differential Equations, 19(11-12):2057-2074, 1994.

[76] Horng-Tzer Yau. Relative entropy and hydrodynamics of Ginzburg-Landau models. Lett. Math. Phys., 22(1):63-80, 1991. 